Stefan Engelberg, Heidrun Kämper und Petra Storjohann (Hrsg.)

Wortschatz: Theorie, Empirie, Dokumentation 


\section{Germanistische Sprachwissenschaft um 2020}

Herausgegeben von

Albrecht Plewnia und Andreas Witt

\section{Band 2}




\section{Wortschatz:}

Theorie, Empirie,

Dokumentation

Herausgegeben von

Stefan Engelberg, Heidrun Kämper

und Petra Storjohann

\section{DE GRUYTER}


Die Open-Access-Publikation dieses Bandes wurde gefördert vom Institut für Deutsche Sprache, Mannheim.

ISBN 978-3-11-053671-3

e-ISBN (PDF) 978-3-11-053858-8

e-ISBN (EPUB) 978-3-11-053682-9

\section{(c) BY}

Dieses Werk ist lizenziert unter der Creative Commons Attribution 4.0 Lizenz. Weitere Informationen finden Sie unter http://creativecommons.org/licenses/by/4.0/.

\section{Bibliografische Information der Deutschen Nationalbibliothek}

Die Deutsche Nationalbibliothek verzeichnet diese Publikation in der Deutschen Nationalbibliografie; detaillierte bibliografische Daten sind im Internet über http://dnb.dnb.de abrufbar.

(c) 2018 Stefan Engelberg, Heidrun Kämper und Petra Storjohann, publiziert von Walter de Gruyter GmbH, Berlin/Boston

Foto Einbandabbildung: (๑) Oliver Schonefeld, Institut für Deutsche Sprache, Mannheim Portrait Ludwig M. Eichinger, Seite V: () David Ausserhofer, Leibniz-Gemeinschaft Satz: Meta Systems Publishing \& Printservices GmbH, Wustermark Druck und Bindung: CPI books GmbH, Leck

www.degruyter.com 


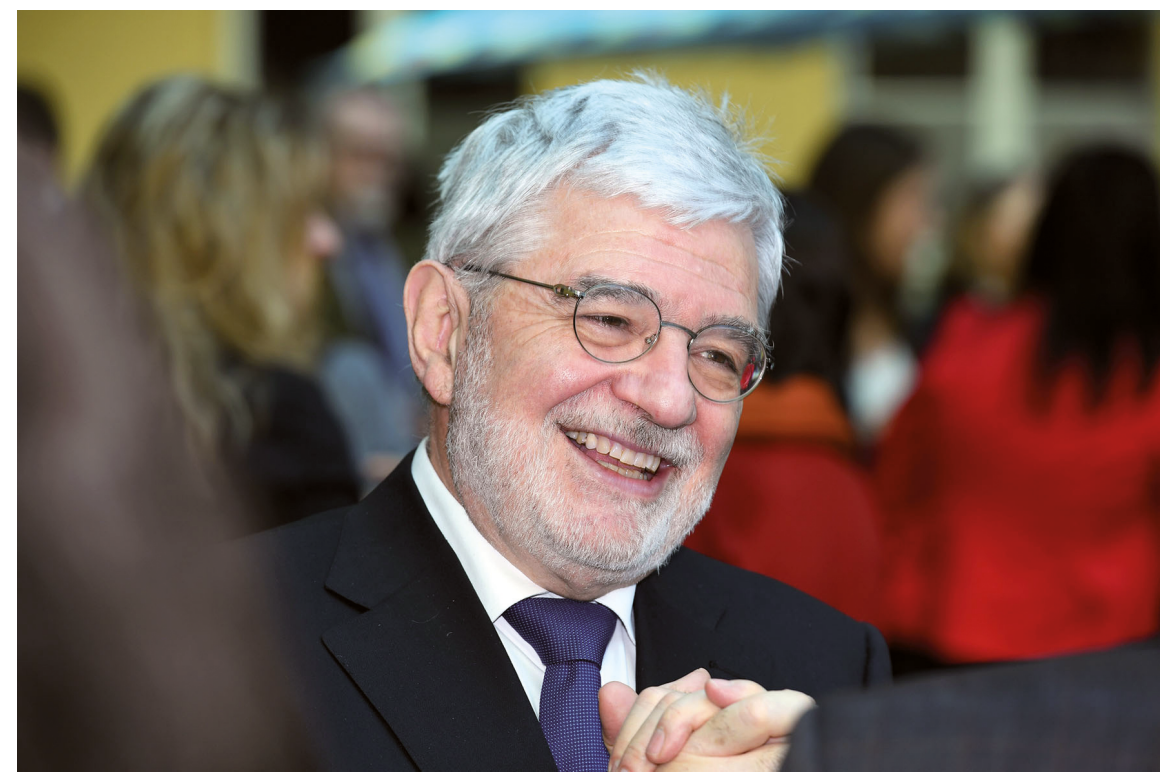

Ludwig M. Eichinger gewidmet 



\section{Vorwort}

Wo steht die germanistische Sprachwissenschaft aktuell? Der vorliegende Band mit dem Titel „Wortschatz: Theorie, Empirie, Dokumentation“ ist der zweite Teil einer auf sechs Bände angelegten Reihe, die eine zwar nicht exhaustive, aber doch umfassende Bestandsaufnahme derjenigen Themenfelder innerhalb der germanistischen Linguistik bieten will, die im Kontext der Arbeiten des Instituts für Deutsche Sprache in den letzten Jahren für das Fach von Bedeutung waren und in den kommenden Jahren von Bedeutung sein werden (und von denen nicht wenige auch vom Institut für Deutsche Sprache bedient wurden und werden). Jeder einzelne Band behandelt ein abgeschlossenes Themengebiet und steht insofern für sich; in der Zusammenschau aller Bände ergibt sich ein Panorama der „Germanistischen Sprachwissenschaft um 2020“.

Anlass des Erscheinens dieser Bände ist der Eintritt des langjährigen Direktors des Instituts für Deutsche Sprache, Ludwig M. Eichinger, in den Ruhestand. Ludwig M. Eichinger leitete das Institut von 2002 bis 2018. Seine akademische Laufbahn begann er als Wissenschaftlicher Assistent an der Universität Bayreuth; anschließend war er Heisenberg-Stipendiat an der LudwigMaximilians-Universität München. Ab 1990 hatte er eine Fiebiger-Professur für Deutsche Sprachwissenschaft an der Universität Passau inne, 1997 wurde er auf den Lehrstuhl für Deutsche Philologie an der Christian-Albrechts-Universität zu Kiel berufen. Mit seiner Ernennung zum Direktor des Instituts für Deutsche Sprache im Jahr 2002 wurde er auch Ordinarius für Germanistische Linguistik an der Universität Mannheim. Ludwig M. Eichinger ist Ehrendoktor der Pannonischen Universität Veszprém und der Universität Bukarest. Er ist Mitglied der Akademie der Wissenschaften und der Literatur zu Mainz und der Österreichischen Akademie der Wissenschaften; außerdem ist er Ständiger Gastprofessor an der Beijing Foreign Studies University.

Ludwig M. Eichinger hat das Institut in den Jahren seines Wirkens entscheidend geprägt; in Anerkennung und Dankbarkeit seien ihm diese Bände gewidmet.

Albrecht Plewnia und Andreas Witt

- Reihenherausgeber - 



\section{Inhalt}

Vorwort — VII

Stefan Engelberg, Heidrun Kämper und Petra Storjohann

Einleitung -1

\section{Lexikalische Theorien}

Manfred Krifka

1 Das Lexikon in der formalen Semantik - 9

Dietrich Busse

2 Historisch-semantische Epistemologie - 31

Rainer Perkuhn

3 Kontexte und ihre Verteilung - 61

Klaus Fischer

4 Grammatische Komplexität und semantische Transparenz in deutschen und englischen Satzstrukturen -91

\section{Lexemtypen}

Damaris Nübling

5 Neue Ansätze in der Namenforschung: Plädoyer für eine Gender-

Onomastik 127

Dmitrij Dobrovol'skij

6 Phraseme aus kognitiver und kontrastiver Sicht -151

Thorsten Roelcke

7 Die Konstitution terminologischer Systeme in Fachsprachen - 171

Birte Kellermeier-Rehbein

8 Plurizentrische Wortschatzvariation des Deutschen innerhalb und außerhalb Europas — 189 
X Inhalt

\section{Lexikografie}

Rufus H. Gouws

9 Internet lexicography in the 21st century - 215

Marie Vachková

10 Methoden zur korpuslinguistischen Erforschung von semantischen Relationen - 237

Sven Staffeldt

11 jetzt verstehen -267

Nicola McLelland

12 Deutsch als Fremdsprache und die deutsch-englische Lexikographie bis $1900-295$

Register - 321

Autorinnen und Autoren -325 


\section{Stefan Engelberg, Heidrun Kämper und Petra Storjohann Einleitung}

\section{Aufgaben der Lexikologie}

Die Beschäftigung mit dem Wortschatz des Deutschen steht gegenwärtig vor zwei sehr interessanten Aufgaben. Zum einen wirft die theoretische Lexikologie die Frage auf, inwieweit die Einheit ihres Forschungsgegenstands als komplex verknüpftes System lexikalischer Einheiten verschiedenster Typen in Abgrenzung zur Grammatik der Deutschen eigentlich gegeben ist. Die Analyse großer Korpora, die sprachliche Konventionalisierungen insbesondere im Mehrwortbereich aufgedeckt hat, und die konstruktionsgrammatisch inspirierten Theorien, die eine Auflösung der Grenzen zwischen Lexikon und Grammatik anstreben, haben die Lexikologie in eine Phase der Neubestimmung geführt.

Zum anderen steht die deskriptive Lexikologie und die damit einhergehende lexikographische Praxis vor der Aufgabe, die Fülle an Daten, die aus großen Textkorpora gewonnen werden, in adäquate lexikalische Sprachbeschreibungen zu überführen. Auch hier sind die starren Trennlinien zwischen Lexikologie und Kulturwissenschaft, aber auch die Grenzen zu anderen Disziplinen, wie z. B. der Kognitionswissenschaft sowie zu bestimmten IT-Bereichen, in Auflösung begriffen.

Die gegenwärtig größten Textkorpora des Deutschen enthalten Einwortlexeme in der Größenordnung von vermutlich über zehn Millionen. Mehrwortlexeme in ihrer Varianz und Muster im Wortbildungs-, Redewendungs- und Argumentstrukturbereich ergänzen diese Vielfalt, und Frequenz-, Kookkurrenz- und Produktivitätsdaten decken die dynamischen Prozeduren in solchen Großwortschätzen auf. Mit welchen Methoden diese Datenmengen analytisch explorativ und effektiv analysiert werden können, wie die aufgedeckten Strukturen und die vorhandene sprachliche Dynamik angemessen beschrieben werden können und zu welchen theoretischen Modellen sie uns verhelfen können, werden die großen Fragen der nächsten Jahre sein.

Stefan Engelberg, Institut für Deutsche Sprache Mannheim, R5, 6-13, D-68161 Mannheim, E-Mail: engelberg@ids-mannheim.de

Heidrun Kämper, Institut für Deutsche Sprache Mannheim, R5, 6-13, D-68161 Mannheim, E-Mail: kaemper@ids-mannheim.de

Petra Storjohann, Institut für Deutsche Sprache Mannheim, R5, 6-13, D-68161 Mannheim, E-Mail: storjohann@ids-mannheim.de

○ Open Access. ๑ 2018 Stefan Engelberg, Heidrun Kämper und Petra Storjohann, publiziert von De Gruyter. (cc) BY Dieses Werk ist lizenziert unter der Creative Commons Attribution 4.0 Lizenz. https://doi.org/10.1515/9783110538588-001 
Der vorliegende Band versucht, Schlaglichter auf die gegenwärtige lexikologische Forschung zu werfen und dabei ein Spektrum an theoretischen und empirischen Fragestellungen aufzuzeigen. Der Band ist in drei Abschnitte gegliedert. Er beginnt mit Beiträgen, in deren Zentrum die Theoriebildung in der Lexikonforschung steht, gefolgt von Aufsätzen, die sich mit bestimmten Typen von Lexemen befassen. Den Abschluss bilden Arbeiten zu Entwicklungen in der Lexikographie.

\section{Lexikalische Theorien}

Theorien, die die Bedeutung von lexikalischen Einheiten zum Gegenstand haben, sind oft stark von prädikatenlogischen Notationen geprägt. Dies hat seinen Ursprung in der wahrheitskonditionalen Semantik, die in den 70er Jahren von ihren satzsemantischen Ursprüngen aus immer stärker auf die lexikalische Semantik auszustrahlen begann. Insbesondere lexikalische Dekompositionen haben die folgenden Jahrzehnte stark geprägt, ausgehend von strukturalistischen merkmalssemantischen Theorien und der Generativen Semantik über Dekompositionsansätze wie in der Conceptual Semantics hin zu Ansätzen mit ereignissemantischer Grundlage und zu Theorien, die auf die lexikalische Semantik-Pragmatik-Schnittstelle fokussieren. Manfred Krifka leitet den vorliegenden Band ein mit einem Blick auf die Leistungen, die die formale Semantik bezüglich unseres Verständnisses des Lexikons erbracht hat. Dabei geht er auf Wortbedeutungen, semantische Relationen und natürlichsprachliche Ontologien ein - bis hin zu Konnotationen und diskursbezogenen Bedeutungen. Dass neben formalsemantischen Ansätzen zur Wortbedeutung aber gerade in jüngerer Zeit auch eher hermeneutisch-interpretative Ansätze ihren Platz haben, macht Dietrich Busse in seinem Beitrag deutlich, der ausgehend von begriffsgeschichtlichen, diskursanalytischen und framesemantischen Ansätzen die Bedingungen einer historisch-semantischen Epistemologie auslotet. Dabei zeigt der Autor insbesondere, wie Bedeutungskonstitution als unter spezifischen historischen Bedingungen sich vollziehender sozialer Akt $\mathrm{zu}$ verstehen ist und insofern einen komplexen sprachwissenschaftlichen Gegenstand darstellt, der als historische Semantik die Grenzen der traditionellen linguistischen Zugänge und Ansätze überschreitet.

Mit der empirischen Wende in der Linguistik hat sich lexikalischen Theorien nicht nur eine neue empirische Grundlage erschlossen, sondern der Blick aufs Lexikon hat sich unter den neuen quantitativen Methoden der Korpusanalyse insgesamt deutlich verändert. Rainer Perkuhn vergleicht in seinem Beitrag kontextbasierte Ansätze der Korpus- und Computerlinguistik hinsichtlich 
ihrer Leistung bei der Ermittlung polysemer Strukturen und semantischer Beziehungen zwischen Wörtern. Er zeigt insbesondere, welche Rolle distributionelle Ansätze aus dem Information Retrieval für die Auffassung und Interpretation von Beziehungsnähe und Kontext spielen. Eine Blüte erlebt die Lexikonforschung zurzeit auch in der typologischen Linguistik, wo sprachübergreifende Gesetzmäßigkeiten der Lexikalisierung von Konzepten erforscht werden, und in der kontrastiven Linguistik, in der Parameter des Sprachvergleichs erforscht werden. Klaus Fischer befasst sich in seinem deutsch-englisch kontrastiven Aufsatz mit dem Zusammenhang zwischen semantischer Transparenz und grammatischer Komplexität und der Frage, welchen Beitrag grammatische versus lexikalische Mittel dabei leisten.

\section{Lexemtypen}

Die gegenwärtige Dekade hat - zum Teil bedingt durch empirisch-methodische Innovationen - ein Interesse an bestimmten Typen von Lexemen geweckt, die in den Zeiten zuvor eher wenig zur Entwicklung lexikalischer Theorien beigetragen haben. Dazu gehören etwa Eigennamen, Mehrwortlexeme und die gesprochensprachliche Lexik (siehe den Beitrag von Sven Staffeldt in Abschnitt 3 dieses Bandes). Die Onomastik hat mit einer Verknüpfung system- und soziolinguistischer Ansätze in jüngerer Zeit einen deutlichen Aufschub erfahren. In dem vorliegenden Band zeigt Damaris Nübling, Trägerin des Konrad-DudenPreises 2014, beispielhaft, wie detaillierte Analysen zum Genusverhalten von Personennamen und zum phonologischen Gendering von Rufnamen gegenwärtige Veränderungen in der Namensgebung erhellen können. Redewendungen, Sprichwörter und andere Mehrwortlexeme konstituieren eine Gruppe von Lexemen, deren Untersuchung auf der Basis großer Textkorpora zu ganz neuen Erkenntnissen geführt hat. Insbesondere die unerwartet hohe Varianz von vermeintlich festen Wendungen und die dabei auftretenden Musterbildungen lassen Mehrwortlexeme heute als paradigmatisch bei der Konstitution lexikalischer Theorien im Spannungsfeld von Lexikon und Grammatik erscheinen. Dmitrij Dobrovol'skij zeigt in seinem Beitrag, wie korpus- und psycholinguistische Methoden in einer kognitiv ausgerichteten kontrastiven Phrasemforschung eingesetzt werden können. Diese Kontrastierung von Idiomen, die der Autor am Beispiel des Deutschen und des Russischen vornimmt, eröffnet auch eine neue Perspektive der lexikografischen Darstellung.

Sprachliche Varianz ist $\mathrm{zu}$ einem zentralen Gegenstand in der Grammatikwie der Lexikonforschung geworden. Dabei spielt sowohl die varietäteninterne Varianz eine Rolle, wie zum Beispiel die gerade angesprochene Varianz im Aus- 
druck von Mehrwortlexemen, als auch die varietätenübergreifende Varianz, die in zwei Beiträgen behandelt wird. Thorsten Roelcke greift die Abgrenzung zwischen Standard- und Fachsprache auf und betont dabei, dass die Fachwortlexikologie nicht nur mit der Definition einzelner Termini befasst ist, sondern auch die Prinzipien der Konstitution terminologischer Systeme im Auge haben muss. Ausgehend von der systemlinguistischen, pragmalinguistischen und der kognitionslinguistischen fachsprachlichen Konzeption macht der Autor die Perspektivenbedingtheit terminologischer Systeme und das Erklärungspotenzial insbesondere des kognitiven Ansatzes deutlich. Birte Kellermeier-Rehbein befasst sich mit lexikalischen Aspekten des Deutschen als plurizentrischer Sprache. Sie erläutert Aspekte der Erzeugung nationaler Identität und staatlicher Autonomie, die sie vor allem für die Sprecher jeweiliger Nationalvarietäten herausstellt. Darüber hinaus fokussiert sie in ihrem Beitrag auf die Besonderheiten der standardnahen Lexik in deutschsprachigen Zentren, in denen das Deutsche keine Amtssprache ist (Rumänien, Namibia, Nordamerika) und begründet die Frage der Entwicklung einer eigenen Standardvarietät unter anderem mit der Isolierung von der Herkunftssprache und ihrer Veränderungen.

\section{Lexikographie}

Kaum ein Bereich der Linguistik ist so grundlegend durch die Digitalisierung des Fachs verändert worden, wie die Lexikographie. Das betrifft zum einen die Verfügbarkeit großer elektronischer Textkorpora, die nicht nur eine erhebliche Verbesserung der empirischen Grundlage lexikographischer Beschreibung bedeuten, sondern über die Fokussierung auf Frequenzen, Kookkurrenzen und Varianz auch eine Veränderung des Blicks auf lexikalische Strukturen insgesamt bewirkt haben. Zum anderen hat die Digitalisierung die Präsentationsformen lexikographischer Daten und den Zugriff aufs Wörterbuch grundlegend erneuert: Das Wörterbuch des 21. Jahrhunderts ist eine elektronische, multimediale, im Internet verfügbare Ressource, die eine Bandbreite an Beschreibungsebenen sowie eine Fülle verschiedener Zugriffsmöglichkeiten bieten kann. Rufus Gouws leitet den dritten Teil des vorliegenden Bandes mit einem Artikel ein, der diese Besonderheiten der Wörterbuchstruktur und der Zugriffsstrukturen in den Mittelpunkt rückt und auf der Grundlage eines ausführlichen Vergleichs konzeptionell sehr unterschiedlicher Online-Wörterbücher die nutzeradäquate Modellierung solcher Strukturen als wichtige Aufgabe der Metalexikographie benennt. Marie Vachková beschreibt die Vorteile explorativer korpusanalytischer Methoden zur Ermittlung von semantischen Ähnlichkeitsprofilen und als Vergleichsbasis emergenter Strukturen des Sprachgebrauchs anhand von selbstor- 
ganisierenden Merkmalskarten. Sie zeigt, wie diese gewinnbringend für die Wörterbucharbeit, die Didaktik, aber auch für die Theoriebildung eingesetzt werden können. Die Verfügbarkeit gesprochensprachlicher Korpora hat der Erforschung lexikalischer Aspekte des gesprochenen Deutsch einen großen Auftrieb verliehen. Sven Staffeldt demonstriert, wie detaillierte Analysen von jetzt im gesprochenen Deutsch neben temporalen insbesondere adversativ-kontrastive Lesarten erkennen lassen. Der Beitrag beleuchtet dabei, wie entsprechende Wörterbucheinträge modelliert werden können. Den Abschluss des Bandes bildet der Aufsatz von Nicola McLelland, die über Forschungslücken in der historischen bilingualen Lexikografie und der Sprachvermittlung berichtet und die Geschichte der deutsch-englischen Wörterbuchtraditionen skizziert. Neben gut dokumentierten Entwicklungen von verschiedenen Glossaren, Lehrbüchern und Grammatiken gestattet sie zudem einen Blick auf weniger bekannte sprachreflexive, sprachpuristische oder sprachvergleichende Unternehmungen und gibt auch der ersten deutsch-englischen Lexikografin einen besonderen Platz in ihrem Beitrag.

\section{Würdigung}

Mit den Aufsätzen in diesem Band soll der Beitrag Ludwig M. Eichingers zur Lexikologie des Deutschen gewürdigt werden. Ludwig M. Eichinger hat nicht nur zahlreiche Arbeiten zur lexikologischen Forschung beigesteuert, insbesondere in den Bereichen Wortbildung, Valenz und Varietätenlinguistik; er hat in seiner Eigenschaft als Direktor des Instituts für Deutsche Sprache auch über lange Jahre hinweg die empirische Wende in der lexikologischen Forschung gefördert und den lexikographischen Wandel vom gedruckten Wörterbuch zum Internetportal unterstützend begleitet. 

I Lexikalische Theorien 



\title{
1 Das Lexikon in der formalen Semantik
}

\begin{abstract}
Der Artikel gibt einen Überblick über den Beitrag, den die formale Semantik zu unserem Verständnis des Lexikons geleistet hat. Die zentrale Rolle der Wahrheitsbedingungen wird dabei besonders hervorgehoben. Näher besprochen werden Wörter mit einer logisch definierbaren Bedeutung, die Darstellung von Bedeutungsbeziehungen wie der Hyponymie, Graduierung und antonyme Ausdrücke, Fragen der natürlichsprachlichen Ontologie, die Dekomposition von lexikalischen Bedeutungen, der Alternativenbezug und negative Polaritätselemente sowie schließlich expressive Konnotationen und diskursbezogene Bedeutungen.
\end{abstract}

Keywords: Bedeutungsbeziehungen, Dekomposition, Kompositionalitätsprinzip, Satzsemantik, Wahrheitsbedingungen, wahrheitsfunktionale Bedeutungsanalyse, Wahrheitswert

\section{Grundlagen}

Unter formaler Semantik versteht man eine Richtung in der Erforschung der Bedeutung natürlichsprachlicher Ausdrücke, die wesentliche Einflüsse der formalen Logik verdankt. Sie kann zurückgeführt werden auf das Werk Gottlob Freges, der sowohl in der Logik als auch in der Sprachphilosophie bahnbrechende Arbeiten geleistet hat. Darauf gründet sich über die Vermittlung von Rudolf Carnap das Werk von Richard Montague, David Lewis und Max Cresswell, das vor allem über die Arbeiten von Barbara Partee großen Einfluss in der modernen sprachwissenschaftlichen Forschung gewonnen hat. Im deutschen Sprachraum wurden diese Ansätze bald aufgegriffen (Montague 1972; Löbner 1976; Link 1979), und sie haben sich an vielen Forschungsinstitutionen durchgesetzt.

Das Epitheton formal ist dabei ein wenig ungenau, da es auf einen eigentlich von allen wissenschaftlichen Ansätzen einzulösenden Präzisionsstandard abzielt. Die Ansätze, die unter diesem Begriff zusammengefasst werden, haben gemeinsam, dass sie den Begriff der Wahrheitsbedingungen in den Mittelpunkt stellen und damit den Träger von Wahrheitsbedingungen, nämlich den Satz.

Manfred Krifka, Leibniz-Zentrum Allgemeine Sprachwissenschaft (ZAS) und HumboldtUniversität zu Berlin, Schützenstraße 18, D-10117 Berlin, E-Mail: krifka@leibniz-zas.de

Ә Open Access. (C) 2018 Manfred Krifka, publiziert von De Gruyter. (c) BY Dieses Werk ist lizenziert unter der Creative Commons Attribution 4.0 Lizenz.

https://doi.org/10.1515/9783110538588-002 
Die Bedeutung eines Satzes zu verstehen heißt ja mindestens, die Bedingungen zu kennen, unter denen der Satz wahr oder falsch ist. Treffendere Ausdrücke für die Semantik, die in diesem Artikel behandelt werden, sind damit wahrheitsfunktionale Semantik, englisch truthconditional semantics, oder eben Satzsemantik (vgl. für einen Überblick Krifka 2014).

Was hat nun aber die Satzsemantik mit der Lexik, also mit der Bedeutung von Wörtern zu tun? Die Bedeutungen der Wörter tragen zu der Bedeutung des Satzes bei, in denen sie vorkommen. Dem liegt ein fundamentales Prinzip zugrunde, dem die Satzsemantik folgt, das Kompositionalitätsprinzip, wonach sich die Bedeutung eines zusammengesetzten Ausdrucks aus den Bedeutungen der unmittelbaren syntaktischen Teile und der Art ihrer syntaktischen Verknüpfung ergibt. Man kann damit von der Satzbedeutung ausgehend den Bedeutungsbeitrag der Wörter, die zu der Satzbedeutung beitragen, „zurückrechnen“. Die Bedeutung der Wörter ein, Mädchen und singt in dem Satz Ein Mädchen singt lässt sich beispielsweise bestimmen, indem man die Wahrheitsbedingungen dieses Satzes mit den Wahrheitsbedingungen von minimalen Alternativen, wie Jedes Mädchen singt, Ein Junge singt und Ein Mädchen lacht vergleicht. Auf diese Weise werden die Wahrheitsbedingungen von Sätzen zu einem Untersuchungsinstrument für die Bedeutungen von Wörtern.

Für die Beschreibung der Wahrheitsbedingungen der natürlichen Sprache verwendet die formale Semantik dasselbe Werkzeug, das auch bei den formalen Sprachen der Logik zum Einsatz kommt, die Modelltheorie (vgl. Zimmermann 2011). Man nimmt auf der einen Seite eine Menge von sprachlichen Ausdrücken an, wie sie durch eine Grammatik erzeugt werden. Diese werden in einem modelltheoretischen Rahmen interpretiert, der typischerweise drei Bestandteile enthält: Erstens eine Menge von Wahrheitswerten (wahr 1 und falsch 0, man kann aber auch Zwischenwerte oder einen Wert für undefiniert annehmen), zweitens eine Menge von Entitäten, das Diskursuniversum, und drittens eine Menge von Möglichkeiten, wie die Welt beschaffen sein kann. Hier unterscheidet man zwischen möglichen Welten und Zeiten, wobei man jeweils eine Welt und eine Zeit als einen Index zusammenfassen kann.

Unter einem Modell versteht man eine Interpretation der sprachlichen Ausdrücke in einem solchen Rahmen. Beispielsweise wird ein Satz wie Ein Hund jault interpretiert als eine Funktion, die jedem Index i einen Wahrheitswert zuweist, je nachdem, ob der Satz an diesem Index als wahr oder falsch verstanden werden soll. Die Interpretation eines Ausdrucks wird oft so geschrieben, dass man Doppelklammern um ihn setzt. Wir haben zum Beispiel «ein Hund jault $\|=\left\{\mathrm{i}_{0} \mapsto 0, \mathrm{i}_{1} \mapsto 0, \mathrm{i}_{2} \mapsto 1, \mathrm{i}_{3} \mapsto 0, \ldots\right\}$ für eine Interpretation, in welcher der Satz ein Hund jault bei den Indizes $\mathrm{i}_{0}$, $\mathrm{i}_{1}$ und $\mathrm{i}_{3}$ falsch und bei $\mathrm{i}_{2}$ wahr ist. Solche Funktionen werden oft in der Lambda-Schreibweise angegeben, zum Beispiel 
$\lambda$ i[ein Hund jault bei i]. Hier wurde der Wert der Funktion der Einfachheit halber umgangssprachlich angegeben. Man kann sich so eine Funktion auch als Menge vorstellen: die Menge aller möglicher Indizes, bei denen ein Hund jault, \{i | ein Hund jault bei i\}. Aber die Funktionenschreibweise ist auf viele andere Fälle verallgemeinerbar und wird daher bevorzugt.

Die Bedeutung der einzelnen Wörter trägt zu dieser Gesamtbedeutung bei. Wortbedeutungen sind gemäß dem Kompositionalitätsprinzip so gestaltet, dass sie eine andere Bedeutung erwarten, mit der zusammen sie dann eine neue Bedeutung ergeben. Das beste Verfahren, dies zu modellieren, sind wiederum Funktionen im mathematischen Sinn. Die Bedeutung von jault kann man modellieren als eine Funktion, die uns für jeden Index i eine Funktion gibt, die für jedes Objekt aus dem Diskursuniversum x sagt, ob $\mathrm{x}$ bei $\mathrm{i}$ jault. Wir schreiben: [jault $\rrbracket=\lambda \mathrm{i} \lambda \mathrm{x}[\mathrm{x}$ jault bei $\mathrm{i}]$. Wenn wir diese Funktion in Index $\mathrm{i}_{7}$ auf die Entität $\mathrm{x}_{13}$ anwenden, dann ergibt das einen Wahrheitswert: $\lambda \mathrm{i} \lambda \mathrm{x}[\mathrm{x}$ jault bei $i]\left(i_{7}\right)\left(i_{13}\right)=\left[x_{13}\right.$ jault bei $\left.i_{7}\right]=1$ (wahr), falls $i_{13}$ bei $i_{7}$ jault, = 0 (falsch), falls $\mathrm{x}_{13}$ bei $\mathrm{i}_{7}$ nicht jault. Ähnlich gibt die Bedeutung von Hund für jeden Index eine Funktion, die für jedes Objekt sagt, ob es ein Hund ist: $\llbracket$ Hund $\rrbracket=\lambda \mathrm{i} \lambda \mathrm{x}[\mathrm{x}$ ist ein Hund bei i]. Der indefinite Artikel ein hat nun eine Bedeutung, welche für jeden Index i eine Funktion liefert, welche die Bedeutung des Nomens und des Verbs bei i nimmt, hier $\lambda x[\mathrm{x}$ ist ein Hund bei i] und $\lambda \mathrm{x}$ [x jault bei $\mathrm{i}$, und uns sagt, ob die Hund-Funktion und die jault-Funktion auf mindestens ein Objekt gleichzeitig zutreffen oder nicht. Die Bedeutung von ein kann man damit angeben als: $\llbracket e i n \rrbracket=\lambda \mathrm{i} \lambda \mathrm{N} \lambda \mathrm{V} \exists \mathrm{x}[\mathrm{N}(\mathrm{x}) \wedge \mathrm{V}(\mathrm{x})]$, wobei $\mathrm{N}$ für Nomenbedeutungen und $\mathrm{V}$ für die Bedeutung eines intransitiven Verbs bei i stehen.

Das Lexikon spielt eine besondere Rolle in der formalen Semantik: Wenn die Bedeutungen der Grundausdrücke festgelegt sind, dann sind sofort auch die Bedeutungen aller Ausdrücke festgelegt, die man damit nach den Regeln der Grammatik aufbauen kann. Dies schließt nicht aus, dass es Idiome gibt, nämlich zusammengesetzte Ausdrücke, die eine irreduzible Bedeutung besitzen; diese Bedeutung muss dann eben durch eine eigene lexikalische Regel angegeben werden. Im Folgenden werden wir zunächst verschiedene Arten von logischen Wörtern und ihre Behandlung in formal-semantischen Ansätzen näher betrachten.

\section{Logische Wörter}

Der geistesgeschichtlichen Herkunft der formalen Semantik entsprechend haben sich die frühen Arbeiten vor allem auf die Bedeutung von Wörtern konzentriert, die für den Ausdruck logische Beziehungen von unmittelbarer Rele- 
vanz waren. Dazu gehören die Negation, die Koordinationen und und oder und die Determinatoren wie ein, der, jeder, kein. Montague (1973) hat dafür nicht einmal eigene Lexikoneinheiten angenommen, sondern diese Wörter durch syntaktische Regeln, also synkategorematisch, eingeführt. In späteren Ansätzen wurden solche Wörter durchaus interpretiert. Im Unterschied zu Wörtern wie Hund und jaul- hängt ihre Bedeutung aber nicht von einem Index ab. Zum Beispiel kann man als Bedeutung von und eine Funktion angeben, die einen Index i als Argument nimmt und eine Funktion von zwei Satzbedeutungen $S$, $\mathrm{S}^{\prime}$ gibt. Es gilt dabei: $\llbracket u n d \rrbracket=\lambda \mathrm{i} \lambda \mathrm{S} \lambda \mathrm{S}^{\prime}\left[\mathrm{S} \wedge \mathrm{S}^{\prime}\right]$. Wenn etwa es blitzt als $\lambda \mathrm{i}[\mathrm{es}$ blitzt bei i] interpretiert wird und es donnert als $\lambda \mathrm{i}$ [es donnert bei i], dann wird der Satz es blitzt und es donnert interpretiert als : $\lambda \mathrm{i}[\llbracket$ und $\rrbracket(\mathrm{i})(\llbracket e s$ blitzt $\rrbracket(\mathrm{i}))(\llbracket e s$ donnert $\rrbracket(i))]$, was sich zu $\lambda \mathrm{i}[[$ es blitzt bei i] $\wedge$ [es donnert bei i]] reduziert. Wichtig ist hier, dass die Bedeutung von und selbst nicht von i abhängt; das heißt, bei verschiedenen Indizes bedeutet und immer das gleiche. Das ist anders als etwa bei dem Wort jault, dessen Bedeutung wir angegeben haben durch die Beschreibung, dass ein $\mathrm{x}$ bei $\mathrm{i}$ jault.

Was hat man in der formalen Semantik über die logischen Ausdrücke herausgefunden? Am beeindruckendsten sind wohl die Erkenntnisse über Determinatoren. Wir haben am Ende des letzten Abschnitts gesehen, wie die Bedeutung des indefiniten Artikels ein dargestellt werden kann. Wir können die NomenFunktion und die Verb-Funktion etwas vereinfacht auch als Mengen auffassen, nämlich als die Menge der Objekte, auf die das Nomen bei i und das Verb bei i zutrifft. Die Bedeutung von ein ist dann $\lambda \mathrm{i} \lambda \mathrm{N} \lambda \mathrm{V}[\mathrm{N} \cap \mathrm{V} \neq \varnothing]$, d. h. der Schnitt der N-Menge mit der V-Menge ist nicht leer. Der Satz ein Hund jault ist wahr, wenn es etwas gibt, was sowohl ein Hund ist als auch jault. Die Bedeutung von jeder ist dann $\lambda \mathrm{i} \lambda \mathrm{N} \lambda \mathrm{V}[\mathrm{N} \subseteq \mathrm{V}], \mathrm{d}$. h. die N-Menge ist in der V-Menge enthalten. Der Satz Jeder Hund jault ist schließlich genau dann wahr bei einem Index i, wenn die Hunde-Menge in der Menge der Jaulenden enthalten ist. Wir können die Bedeutung von kein angeben als: $\lambda \mathrm{i} \lambda \mathrm{N} \lambda \mathrm{V}[\mathrm{N} \cap \mathrm{V}=\varnothing]$, d. h. der Schnitt der N-Menge mit der V-Menge ist leer. Und die Bedeutung von die meisten kann man angeben als: $\lambda \mathrm{i} \lambda \mathrm{N} \lambda \mathrm{V}[\#(\mathrm{~N} \cap \mathrm{V})>\#(\mathrm{~N}-\mathrm{V})]$, also: Die Zahl der Elemente in dem Schnitt von $\mathrm{N}$ und $\mathrm{V}$ ist größer als die Zahl der Elemente in $\mathrm{N}$, die nicht in $\mathrm{V}$ sind. Es gibt mehr Hunde, die jaulen, als Hunde, die nicht jaulen.

Es hat sich in der Forschungsrichtung der Theorie der generalisierten Quantoren (Barwise \& Cooper 1981; Keenan 2011) herausgestellt, dass sich alle Determinatoren auf diese Weise darstellen lassen, sogar zusammengesetzte und höchst komplexe, wie z. B. zwischen drei und sieben, eine ungerade Anzahl von, oder weniger als $10 \%$ der (Hunde jaulen). Determinatoren wie der oder beide können ebenfalls in diesem Format dargestellt werden, wobei deren Bedeutung eine Vorbedingung oder Präsupposition an die N-Funktion stellt - 
nämlich unter der Voraussetzung, dass sie auf genau ein Objekt zutrifft (im Fall von der) bzw. auf genau zwei (im Fall von beide). Auf der anderen Seite hat sich gezeigt, dass die Zahl der theoretisch möglichen Determinatorbedeutungen ungleich viel größer ist als die Zahl derjenigen, die man sprachlich ausdrücken kann. Warum gibt es in keiner Sprache einen Determinator mit der Bedeutung $\lambda \mathrm{w} \lambda \mathrm{N} \lambda \mathrm{V}[\#(\mathrm{~N})<\#(\mathrm{~V})]$, also ein Determinator, der ausdrückt, dass es weniger Entitäten in der Nomen-Bedeutung als in der Verb-Bedeutung gibt? Oder einen Determinator mit der Bedeutung $\lambda w \lambda \mathrm{N} \lambda \mathrm{V}[\#(\mathrm{~N} \cap \mathrm{V})>\#(\mathrm{~V}-\mathrm{N})]$, also dass es mehr Entitäten gibt, die sowohl N und V sind, als Entitäten, die V, aber keine $\mathrm{N}$ sind?

Man konnte eine Reihe von sehr allgemeinen Prinzipien identifizieren, welche die möglichen Determinatorbedeutungen stark einschränken. Insbesondere genügt es für viele natürlichsprachlichen Quantoren, sich auf die Nomenmenge $\mathrm{N}$ alleine zu konzentrieren. Die syntaktische Struktur [[D N] V] ist gewissermaßen ein Signal dafür, dass man sich bei der Bestimmung der Wahrheitsbedingungen auf die N-Entitäten beschränken kann. Man nennt diese Eigenschaft Konservativität. Es gibt Quantoren, für die es ausschließlich relevant ist, wie viele Elemente sich in der Schnittmenge $\mathrm{N} \cap \mathrm{V}$ befinden, die sogenannten intersektiven Quantoren wie ein, drei oder kein. Bei einer anderen Klasse von Quantoren ist es relevant, wie das Verhältnis der Nomenmenge N zu der Schnittmenge $\mathrm{N} \cap \mathrm{V}$ aussieht, wie bei jeder (100\%) oder die meisten (> 50\%), die sogenannten proportionalen Quantoren. Allerdings spielt manchmal auch die Betonung eine Rolle. Erst kürzlich wurde eine Art von Quantorbedeutung entdeckt, welche nicht konservativ ist: Der Satz 60 Prozent Frauen arbeiten im Bundeskanzleramt hat bei Betonung auf Frauen eine Lesart, nach der $60 \%$ der Personen, die im Bundeskanzleramt arbeiten, Frauen sind. Hier kommt es also auf das Verhältnis von $\mathrm{N} \cap \mathrm{V}$ und $\mathrm{V}$ an; sie sind damit konservativ im Hinblick auf die Bedeutung des verbalen Prädikats (vgl. Ahn \& Sauerland 2015). Da bei vielen Quantorsätzen die Hauptbetonung auf dem verbalen Prädikat liegt, scheint es also eher auf die Informationsstruktur als auf die syntaktische Struktur anzukommen. Diese Rolle der Informationsstruktur bei der Quantifikation ist mittlerweile gut erforscht; vgl. Partee (1991) für ein einflussreiches frühes Werk und Krifka (2016) für einen aktuellen Überblick.

\section{Beziehungen zwischen Bedeutungen}

$\mathrm{Zu}$ den Grundbegriffen der lexikalischen Semantik gehören diejenigen, welche die Beziehungen zwischen den Bedeutungen von lexikalischen Ausdrücken beschreiben, und zwar von Wörtern, die in paradigmatischer syntaktischer Bezie- 
hung zueinander stehen, wie zum Beispiel Hund, Tiger, Mond oder bellen, singen, leuchten oder einige, dreizehn, wenige. Da solche Wörter in einem Satz füreinander einstehen können - z. B. \{einige / dreizehn / wenige $\{$ Hunde / Tiger / Monde\} \{bellen / singen / leuchten $\}$ - müssen sie grundsätzlich ähnliche Bedeutungen haben, sonst könnte man sie ja nicht zu einer Satzbedeutung zusammenfügen. Sie müssen vom selben semantischen „Typ“ sein. So sind Hund, Tiger, Mond vom Typ einstelliger nominaler Prädikate, und bellen, singen, leuchten vom Typ einstelliger verbaler Prädikate. Der Typ der intransitiven Verben unterscheidet sich vom Typ der transitiven Verben wie sehen und kennen; erstere haben Bedeutungen der Art $\lambda \mathrm{i} \lambda \mathrm{x}[. .$.$] , also mit einer Argumentstelle$ $\mathrm{x}$; letztere haben Bedeutungen der Art $\lambda \mathrm{i} \lambda \mathrm{y} \lambda \mathrm{x}[. .$.$] , also mit zwei Argument-$ stellen, zum Beispiel $\lambda \mathrm{i} \lambda \mathrm{y} \lambda \mathrm{x}[\mathrm{x}$ kennt y bei $\mathrm{i}]$.

Zwischen lexikalischen Ausdrücken desselben Typs können nun systematische Beziehungen bestehen (vgl. Cann 2011). Grundsätzlich kann eine Bedeutung in einer anderen eingeschlossen sein, in der Hyponymie; zwei Bedeutungen können sich ausschließen, in der Antonymie; und zwei Bedeutungen können identisch sein, in der Synonymie. Diese Verhältnisse können systematisch modelliert werden. Zum Beispiel ist Hund ein Hyponym von Tier; alles, was unter den Begriff Hund fällt, fällt notwendigerweise auch unter den Begriff Tier: $\forall \mathrm{i} \forall \mathrm{x}[\llbracket$ Hund $\rrbracket(\mathrm{i})(\mathrm{x}) \rightarrow \llbracket$ Tier $\rrbracket(\mathrm{i})(\mathrm{x})]$. In Worten: Für alle Indizes i und für alle Objekte $\mathrm{x}$ gilt: Wenn $\mathrm{x}$ bei $\mathrm{i}$ ein Hund ist, dann ist $\mathrm{x}$ bei $\mathrm{i}$ ein Tier. Dies ist eine Festlegung über alle mögliche Welten und drückt daher eine notwendige Beziehung aus. Es könnte zum Beispiel sein, dass bei einem bestimmten Index $\mathrm{i}_{7}$ alle Hunde schwarzweiß gefleckt sind, dass also $\forall \mathrm{x}\left[\llbracket\right.$ Hund $\rrbracket\left(\mathrm{i}_{7}\right)(\mathrm{x}) \rightarrow$ «schwarzweiß gefleckt $\left.\rrbracket\left(\mathrm{i}_{7}\right)(\mathrm{x})\right]$ gilt. Aufgrund dieses zufälligen Sachverhalts wird aber Hund nicht zu einem Hyponym zu schwarzweiß gefleckt. Die Beziehung gilt nämlich nicht für alle Indizes $i$.

Auch Tiger ist ein Hyponym zu Tier. Als sogenannte Ko-Hyponyme schließen sich Tiger und Hund systematisch aus: $\forall \mathrm{i} \neg \exists \mathrm{x}[\llbracket$ Hund $\rrbracket(\mathrm{i})(\mathrm{x}) \wedge \llbracket$ Tiger $\rrbracket(\mathrm{i})(\mathrm{x})]$; das heißt, für alle Indizes i gibt es kein $\mathrm{x}$, das sowohl Hund als auch Tiger ist. Das Lexikon einer Sprache ist zumindest in Teilen in Form von Taxonomien gegliedert; so hat Hund selbst wiederum Hyponyme, wie zum Beispiel Pudel; es gilt nämlich $\forall \mathrm{i} \forall \mathrm{x}[\llbracket$ Pudel $\rrbracket(\mathrm{i})(\mathrm{x}) \rightarrow \llbracket$ Hund $\rrbracket(\mathrm{i})(\mathrm{x})]$. Aus dieser Modellierung können wir dann ableiten, dass jeder Pudel ein Tier ist, und dass kein Pudel ein Tiger ist. Auf diese Weise kann ein sehr großer Bereich lexikalischen Wissens mit einfachen, gut etablierten Methoden erfasst werden.

Regeln wie die eben angegebenen, welche Begriffe wie Pudel und Hund zueinander fixieren, nennt man Bedeutungspostulate. Sie haben ihren Ursprung in dem Werk von Montague, der damit systematische Beziehungen zwischen Wörtern oder auch Gruppen von Wörtern erfassen wollte; er hat das an 
dem Beispiel seek und try to find illustriert. Bedeutungspostulate schränken die möglichen Interpretationen von lexikalischen Ausdrücken systematisch ein. Formal-semantisch geprägte Ansätze sind typischerweise nicht daran interessiert, was genau nicht-logische Wörter wie Hund oder rennen bedeuten; sie wollen aber erfassen, in welchen Beziehungen diese Wörter zueinander stehen. Der Grund an diesem Interesse liegt daran, dass für solche Ansätze das logische Schlussverhalten von Sätzen zentral ist. Mithilfe des Bedeutungspostulates, das die Interpretation von Pudel und Hund relativ zueinander fixiert, kann man dann ableiten, dass aus Ein Pudel bellt der Satz Ein Hund bellt folgt, und aus dem Satz Kein Hund bellt der Satz Kein Pudel bellt.

Hyponymiebeziehungen gibt es nicht nur bei Nomina, sondern auch bei Verben (z. B. rennen - laufen - sich bewegen) und bei Adjektiven (z. B. heiß - warm). Letzteres Beispiel unterscheidet sich systematisch von Hund - Tier: Während wir jederzeit einen Hund als Tier klassifizieren würden, sind wir vielleicht eher zurückhaltend damit, eine heiße Herdplatte als warm zu bezeichnen. Da wir jedoch ohne Widerspruch sagen können: Die Herdplatte ist warm, sogar heiß, muss man wohl gelten lassen, dass warm nicht in Widerspruch zu heiß steht. Ein systematischer Unterschied zu Hund / Tier besteht darin, dass Tier eine Reihe von parallelen Ko-Hyponymen besitzt (Hund, Tiger, Maulwurf, ...), dies aber bei warm / heiß nicht so ist. Dies scheint der Grund dafür zu sein, dass bei der Verwendung von warm im Allgemeinen angedeutet wird, dass das spezifische Prädikate heiß nicht zutreffen würde - worauf in dem Abschnitt zu Alternativen noch einmal zurückzukommen sein wird.

Im nominalen Bereich finden wir Fälle wie Katze und Kater, die an den Fall warm / heiß erinnern. Hier hat Katze nur ein Hyponym, Kater, für die männliche Katze; ein Kohyponym für die weibliche Katze fehlt, sieht man mal von der sehr seltenen Bildung Kätzin ab. Katze wird als Autohyponym bezeichnet, als Hyponym seiner selbst (Becker 2002). Oft bezieht sich die hyponymische Verwendungsweise, also bei Katze die Bedeutung ,weibliche Katze‘, auf diejenigen Vertreter des Oberbegriffs, die eher als prototypisch verstanden werden.

\section{Graduierung und Antonymie}

Wörter wie warm und kalt stehen ebenfalls in einer systematischen Beziehung zueinander: Sie schließen sich stets aus. Was als warm bezeichnet wird, kann nicht kalt sein, und umgekehrt; das kann mit den Bedeutungspostulaten $\forall \mathrm{i} \forall \mathrm{x}[\llbracket$ warm $\rrbracket(\mathrm{i})(\mathrm{x}) \rightarrow \neg \llbracket k a l t \rrbracket(\mathrm{i})(\mathrm{x})]$ und $\forall \mathrm{i} \forall \mathrm{x}[\llbracket k a l t \rrbracket(\mathrm{i})(\mathrm{x}) \rightarrow \neg \llbracket$ warm $\rrbracket(\mathrm{i})(\mathrm{x})]$ erfasst werden, die es natürlich zulassen, dass Objekte $\mathrm{x}$ in einer Welt weder als warm noch als kalt klassifiziert werden. Solche Antonyme sind für die Wort- 
klasse der Adjektive typisch, und in vielen Fällen sind die Ausdrücke dann graduierbar.

Das Konzept der Graduierbarkeit und seine Ausprägung in verschiedenen Sprachen wurde in formal-semantischen Arbeiten detailliert untersucht; hier können nur einige Grundideen vorgestellt werden. Eine einflussreiche Vorstellung, die auf Lewis (1970) zurückgeht und von Klein (1980) weiterentwickelt wurde, ist, dass es neben dem Parameter für Indizes i einen zweiten gibt, der ausdrückt, wie strikt oder lax graduierbare Begriffe interpretiert werden. Nennen wir diesen Parameter s, für Striktheit. Bei der Interpretation eines Ausdrucks wie warm hängt es davon ab, wie dieser Parameter gesetzt ist: Es sei $\mathrm{x}$ in $\mathrm{w}$ eine Suppe von $30^{\circ} \mathrm{C}$; unter der Striktheit $\mathrm{s}_{1}$ gilt $\mathrm{x}$ als warm,

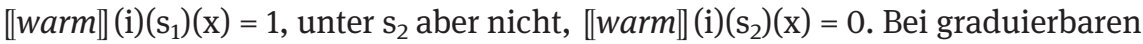
Begriffen kann man nun eine zugrundeliegende Dimension rekonstruieren, bei warm etwa die der Temperatur. Wenn y in i eine Suppe von $40^{\circ} \mathrm{C}$ ist, dann kann y unter der größeren Striktheit $s_{2}$ als warm gelten: $\llbracket w a r m \rrbracket(i)\left(s_{2}\right)(y)=1$. Und dann muss y natürlich auch unter der geringeren Striktheit $s_{1}$ als warm gelten. Man kann nun auch die Bedeutung des Komparativsatz $y$ ist wärmer als $x$ ausdrücken, nämlich durch $\exists \mathrm{s}[\llbracket$ warm $\rrbracket(\mathrm{i})(\mathrm{s})(\mathrm{y})=1 \wedge \llbracket$ warm $\rrbracket(\mathrm{i})(\mathrm{s})(\mathrm{x})=0]$; d. h., es gibt eine Striktheit s, unter der y in i warm ist, $x$ aber nicht.

Für graduierbare Ausdrücke nimmt man aber meist an, dass sie sich auf einen Grad in einer Dimension beziehen (vgl. Bierwisch 1987; vgl. Beck 2011). Beispielsweise können wir eine Dimension der Temperatur $\mathrm{T}$ annehmen, die eine linear geordnete Menge von Temperaturgraden enthält; $t<t^{\prime}$ stehe für „der Temperaturgrad $t$ ist kleiner als der Temperaturgrad $t^{\prime \prime}$. In manchen, eher seltenen Fällen, gibt es die Möglichkeit, solche Grade zu bezeichnen, zum Beispiel Temperaturgrade mit dem ${ }^{\circ} \mathrm{C}$-System. Der graduierbare Begriff warm weist dann bei einem Index i einem Objekt $x$ einen Temperaturgrad zu, zum Beispiel $\llbracket$ warm $\rrbracket(\mathrm{i})(\mathrm{x})=30^{\circ} \mathrm{C}$. Das erklärt Verwendungen wie Die Suppe ist $30^{\circ} \mathrm{C}$ warm. Die Wahrheitsbedingungen von Komparativsätzen wie $y$ ist wärmer als $x$ kann

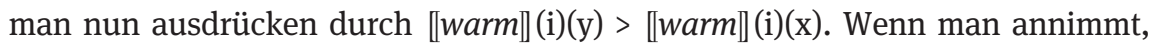
dass man von Wärmegraden Differenzen bilden kann, dann wird $y$ ist $10^{\circ} \mathrm{C}$ wärmer als $x$ ausgedrückt durch $\llbracket$ warm $\rrbracket(\mathrm{i})(\mathrm{y})=\llbracket$ warm $\rrbracket(\mathrm{i})(\mathrm{x})+10^{\circ} \mathrm{C}$. Interessanterweise wird nun aber der einfache Positivsatz, Die Suppe ist warm, semantisch komplexer. Er muss nämlich nun als Vergleich mit einem kontextabhängigen

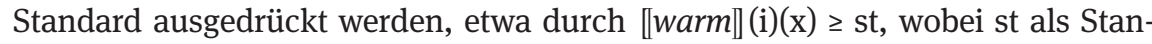
dard gilt, ab dem man eine Suppe als „warm“ bezeichnen kann. Das hat Konsequenzen für die intersubjektive Beurteilung der Wahrheit von Sätzen: Ob der Satz $y$ ist wärmer als $x$ wahr ist, kann man objektiv feststellen; ob der Satz $x$ ist warm wahr ist, hängt hingegen von der subjektiven Setzung des Standards ab.

Die positiven Verwendungen von graduierbaren Ausdrücken sind Musterbeispiele für die Vagheit der natürlichen Sprache. Vagheit wird manchmal als 
eine Eigenschaft genannt, die es verhindern würde, die präzisen Mittel der formalen Semantik anzuwenden. Aber wie wir gesehen haben, ist das nicht der Fall. In der formalen Semantik kann man vielmehr die Rolle der Vagheit genau lokalisieren, nämlich als kontextuell angenommene Schwellenwerte. Diese Schwellenwerte sind pragmatischer Natur und verhandelbar; das macht die menschliche Sprache gerade so flexibel. Wenn beispielsweise eine Situation zwei Asteroide enthält, einer mit $5 \mathrm{~km}$ Durchmesser, der andere mit $10 \mathrm{~km}$ Durchmesser, wird man wohl beide nicht als „groß“ bezeichnen. Wir können uns aber jederzeit auf den zweiten mit der große Asteroid beziehen, weil wir den Standard für Asteroide zweckgebunden so setzen können, dass er zwischen $5 \mathrm{~km}$ und $10 \mathrm{~km}$ Durchmesser liegt (Kyburg \& Morreau 2000).

\section{Sortale und ontologische Beziehungen}

Wie wir gesehen haben, legt die formale Semantik für die Interpretation ein Modell zugrunde, das insbesondere aus einer Menge von Entitäten, dem Diskursuniversum, besteht. Diese stehen für die Dinge, über die wir sprechen können. In der ursprünglichen Form der modelltheoretischen Semantik bei Montague wurden dabei keine weiteren Annahmen gemacht. Es wurde eine Menge von Individuen $U$ angenommen, wobei es dann von der Interpretation «.』 abhängt, ob ein gegebenes $\mathrm{x}$ aus der Menge $\mathrm{U}$ an einem Index i ein Hund, ein Asteroid oder ein Teller Suppe ist, oder ob es unter gar kein lexikalisches Prädikat fällt. Wir können allerdings in unserem Universum bestimmte sortale Unterschiede annehmen und etwa spezielle Sorten für Personen, Dinge oder Flüssigkeiten unterscheiden. Es werden dann nur solche Interpretationen zugelassen, in denen

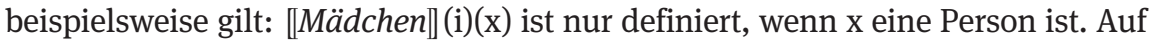
diese Weise können sortale Einschränkungen in der Verwendung von Wörtern erfasst werden - wie z. B., dass das Interrogativpronomen wer nur für Personen verwendbar ist und das Verb verschütten nur für direkte Objekte, welche sich auf Flüssigkeiten oder Substanzen beziehen, deren Teile gegeneinander beweglich sind (vgl. Krifka, im Erscheinen).

Normalerweise denken wir bei Entitäten an konkrete Personen, Tiere, Dinge oder Substanzen. Aber der Bereich der Entitäten ist tatsächlich viel größer. Wir haben in Abschnitt 3 taxonomische Beziehungen wie die zwischen den nominalen Prädikaten Pudel, Hund und Tier diskutiert. In der natürlichen Sprache können wir uns aber auch anders auf solche Gattungen beziehen. Wenn wir sagen Der Hund ist seit Jahrtausenden ein Begleiter des Menschen oder Der Pudel ist gelehrig, dann referieren wir mit einer definiten Beschreibung auf Hunde oder Pudel. Dies wurde so beschrieben, dass manche der Funktionen 
$\lambda \mathrm{i} \lambda \mathrm{x}[. .$.$] selbst wieder als Individuen auftauchen, genauer: als Gattungsindivi-$ duen. Zum Beispiel steht die Eigenschaft $\lambda \mathrm{i} \lambda \mathrm{x}[\mathrm{x}$ ist ein Hund bei $\mathrm{i}]$ mit einem Individuum - nennen wir es Canis - in Beziehung. Bei Gattungsindividuen wie canis gibt es eine Exemplar-Relation R, und es gilt: $\forall \mathrm{i} \forall \mathrm{x}[\llbracket H u n d](\mathrm{i})(\mathrm{x}) \leftrightarrow$ $\mathrm{R}(\mathrm{i})($ Canis)(x)]; d.h., $\mathrm{x}$ fällt unter das Prädikat Hund genau dann, wenn $\mathrm{x}$ eine Realisation der Gattung Canis ist. Solche Verdinglichungen oder Reifizierungen von Prädikaten finden dann statt, wenn den Exemplaren der Art essentielle Eigenschaften zugeschrieben werden. Die Bedeutung von solchen und anderen generischen Sätzen wurde in der formal-semantischen Literatur detailliert behandelt (Carlson 1977, Chierchia 1998). Wichtig im gegenwärtigen Zusammenhang ist, dass Entitäten wie Canis gewissermaßen abstrakte Individuen darstellen, die mit konkreten Entitäten wie einzelnen Hunden in systematischer Beziehung stehen.

Bei graduierbaren Ausdrücken haben wir einen anderen Typ von abstrakten Entitäten angenommen, nämlich Grade. Was sind Grade? Wir können sie, wie Cresswell (1977) vorgeschlagen hat, als Mengen von Entitäten konstruieren. Der Temperaturgrad $30^{\circ} \mathrm{C}$ ist dann die Menge der Objekte, welche die Temperatur $30^{\circ} \mathrm{C}$ besitzen; die Dimension der Temperatur ist folglich eine Menge von solchen Mengen. Wir können Temperaturgrade aber auch als abstrakte Entitäten in unserem Modell selbst postulieren, die durch eine lineare Ordnungsrelation < miteinander verbunden sind. Das haben wir bereits mit Notationen wie $\llbracket$ warm $\rrbracket(i)(x)<\llbracket w a r m \rrbracket(i)(y)$ gemacht, wo die beiden Teilausdrücke jeweils für einen solchen Temperaturgrad stehen.

Wir haben hier in unserem modelltheoretischen Rahmen also eine bestimmte Sorte von Entitäten angenommen: Temperaturgrade, die durch eine bestimmte Relation geordnet sind. Solche Anreicherungen und Ausspezifizierungen der Entitäten eines Modells wurden vielfach vorgenommen. Der Montague-Schüler Daniel Gallin fasste bereits die möglichen Welten und Zeiten als eine Sorte von Entitäten auf, die durch Relationen wie die der zeitlichen Abfolge geordnet sind (Gallin 1975). Für die Darstellung der Semantik von Pluralausdrücken schlug Link (1983) vor, dass die Objekte eines Modells zu Summen zusammengefasst werden können. Wenn immer wir etwa zwei Objekte $\mathrm{x}, \mathrm{y}$ in unserem Diskursuniversum haben, dann gibt es auch ein Objekt $\mathrm{x} \oplus \mathrm{y}$, die Zusammenfassung der beiden Objekte. Wenn etwa $\mathrm{x}$ und y unter das nominale Prädikat Hund fällt, dann fällt $\mathrm{x} \oplus \mathrm{y}$ notwendig unter Hunde, als Bedeutungspostulat: $\forall \mathrm{i} \forall \mathrm{x} \forall \mathrm{y}[\llbracket H u n d \rrbracket(\mathrm{i})(\mathrm{x}) \wedge \llbracket$ Hund $\rrbracket(\mathrm{i})(\mathrm{y}) \rightarrow \llbracket$ Hunde $\rrbracket(\mathrm{i})(\mathrm{x} \oplus \mathrm{y})]$. Die Summenoperation $\oplus$ hat dabei bestimmte, mathematisch beschreibbare Eigenschaften; auf ihrer Grundlage kann man die Beziehung der Teilbeziehung $\sqsubseteq$ definieren. Zum Beispiel gilt, dass $\mathrm{x}$ ein Teil von $\mathrm{x} \oplus \mathrm{y}$ ist, also $\mathrm{x} \sqsubseteq \mathrm{x} \oplus \mathrm{y}$.

Die Einführung der Teilbeziehung ist von großer Relevanz für die Beschreibung lexikalischer Beziehungen. Der Wortschatz wird nämlich unter anderem 
durch sogenannte Meronyme gegliedert: Der menschliche Körper wird strukturiert aufgefasst mit den Teilen Kopf, Rumpf, Arme und Beine; die Arme wiederum bestehen aus Oberarm, Unterarm und Hand, die Hand aus Daumen, Finger und Handfläche (vgl. Champollion \& Krifka 2016). Eine weitere wichtige lexikalische Unterscheidung ist die zwischen Zählnomina und Massennomina. Die Extension von Zählnomina ist so beschaffen, dass sie kleinste Teile haben. Der Begriff Hund ist so beschaffen, dass er etwa auf einen Hund x zutrifft, nicht aber auf Teile wie den Schwanz dieses Hundes. Bei Massennomina ist das anders: Bei dem natürlichen, vor-wissenschaftlichen Verständnis von Wasser kann man nicht davon ausgehen, dass dieser Ausdruck eine Extension mit kleinsten Teilen besitzt. Die Unterscheidung zwischen Zählnomina und Massennomina kann auch wiederum als eine sortale Unterscheidung der Entitäten des Modellrahmens gewertet werden, wie es Link (1983) vorgeschlagen hat. Das erlaubt es beispielsweise, zwischen einem Ring und dem Gold, aus dem der Ring besteht, zu unterscheiden - was notwendig ist, da diese beiden Dinge verschiedene Eigenschaften haben können.

Eine weitere Sorte von Individuen, deren Einführung sich als essentiell für die linguistische Semantik erwiesen hat, sind Ereignisse. Im Gegensatz zu Objekten sind Ereignisse eher ephemerer Natur; aber wir können uns doch manchmal direkt auf sie mit beziehen, manchmal sogar mit referierenden Bezeichnungen wie der Zweite Weltkrieg oder sogar Namen wie Pam, der Zyklon, der 2015 den Inselstaat Vanuatu verwüstete. Wir verwenden unterschiedliche Prädikate für Objekte und Ereignisse - erstere existieren, letztere passieren, geschehen oder dauern und beginnen, enden oder werden unterbrochen. Vor allem weichen die typischen lexikalischen Mittel, mit denen wir uns typischerweise auf Ereignisse beziehen, ab - es sind nämlich Verben. Wenn wir auf Ereignisse referieren, geschieht dies oft mit nominalisierten Verben wie zum Beispiel die Eroberung Roms. Aber es gibt auch gute Argumente dafür, dass bei dem normalen, prädikativen Gebrauch von Verben Ereignisse involviert sind. Der Satz Hasso bellt drückt nach dieser Analyse nicht aus, dass das Prädikat bellt auf den Träger des Namens Hasso angewendet wird, sondern vielmehr, dass es ein Ereignis gibt, das ein Bellens-Ereignis ist, und zu dem Hasso in Agens-Beziehung steht: $\exists \mathrm{e}[\llbracket$ bellen $\rrbracket(\mathrm{i})(\mathrm{e}) \wedge \mathrm{AG}(\mathrm{i})(\llbracket$ Hasso $\rrbracket(\mathrm{i}), \mathrm{e})]$. Diese ereignissemantische Analyse ist sehr plausibel; sie kann z. B. Adverbien wie laut als Prädikate über Ereignisse erklären, und sagt unter anderem voraus, dass aus Hasso bellt laut logisch folgt: Hasso bellt (vgl. Davidson 1967; Engelberg 2000; Maienborn \& Wöllstein 2005).

Der ereignissemantische Ansatz hat sich aber auch für die feinere Analyse lexikalischer Bedeutungen als sehr fruchtbar erwiesen, da man mit seiner Hilfe zwischen den Bedeutungen verschiedener Verbtypen unterscheiden kann. Ein 
Aktivitätsverb wie sehen etwa trifft auf Ereignisse e zu, in denen ein Experiencer einen Stimulus visuell wahrnimmt. Es gilt: Wenn e ein Sehens-Ereignis ist, dann gilt dies auch für Teile von e. Dies ist anders bei dem inchoativen Verb erblicken. Es trifft nur auf den Anfang eines Sehens-Ereignisses zu, und damit gilt, dass Erblickens-Ereignisse gar keine echten Teile haben können. Die Dinge verhalten sich wiederum anders bei gesunden. Ein Gesundens-Ereignis ist zeitlich ausgedehnt, und ein echter zeitlicher Teil eines solchen Ereignisses gilt nicht mehr als ein Gesundens-Ereignis. Bei einem transitiven Verb wie lesen spielt die Art und Weise, wie Ereignis-Teile und Objekt-Teile zueinander stehen, eine wesentliche Rolle: Bei ein Buch lesen wird nahegelegt, dass die Teile des Lesen-Ereignisses und die Teile des Buchs in einer Eins-zu-eins-Beziehung zueinander stehen; wenn der letzte Teil des Buches gelesen ist, dann ist das Ereignis zu Ende. Das ist anders etwa bei eine Wolke sehen. Diese Unterschiede haben grammatische Konsequenzen: Wir können sagen Maria las das Buch in drei Stunden, aber nicht Maria sah die Wolke in drei Stunden. Solche Beobachtungen kann man zwar mit reinen Merkmalsregeln erfassen, aber es liegen ihnen doch systematische semantische Verhältnisse aufgrund der Teil-GanzesBeziehung zugrunde; und in formal-semantischen Ansätzen konnten hierzu präzise Erklärungen entwickelt werden (vgl. Filip 2011).

Es gibt eine Reihe von weiteren Möglichkeiten, das Universum durch neue Sorten von Entitäten anzureichern und so die Ontologie zu konstruieren, die der natürlichen Sprache zugrundeliegt. Ein Beispiel sind Strecken, gerichtete Pfade und Wege zur Darstellung von Bewegung im realen dreidimensionalen Raum, aber auch von der Veränderung von Eigenschaften in anderen Räumen (vgl. Krifka 1998). Ein anderes Beispiel sind Vektoren, wie sie Zwarts (1997) zur Beschreibung von räumlichen Präpositionalphrasen wie in den Park vorgeschlagen hat. Schließlich wurde der Begriff der force, wie er von Talmy (1988) für kausative Konzepte eingeführt wurde, mit formal-semantischen Methoden rekonstruiert, z. B. bei Lambalgen \& Hamm (2005) und Copley (2016).

Mit dieser Strukturierung des Individuenbereichs schlägt die formale Semantik eine Brücke zu Vorstellungen, wie sie in der kognitiven Semantik entwickelt wurden (vgl. Croft \& Cruse 2004). Die Sorten und die Relationen, die zwischen den Individuen bestimmter Sorten bestehen, kann man als Modellierungen von konzeptuellen Strukturen verstehen; die lexikalischen Einheiten selbst werden dann innerhalb einer Sorte interpretiert. Zum Beispiel kann es keine lexikalische Bedeutung geben, die querbeet auf Entitäten verschiedener Sorten zutrifft. Der Vorteil der formal-semantischen Methode ist dabei, dass sie es erlaubt, die Konsistenz von semantischen Theorien zu überprüfen, dass sie einen Begriff dafür hat, dass eine Bedeutung aus einer anderen folgt oder diese ausschließt, und dass sie mit einer ausgearbeiteten Theorie 
darüber versehen ist, wie die Bedeutung komplexer Ausdrücke aus den Bedeutungen der Teilausdrücke abgeleitet werden kann.

\section{Dekomposition von lexikalischer Bedeutung}

Im einleitenden Abschnitt 1 wurde gesagt, dass die lexikalischen Einheiten die Bausteine seien, aus denen sich die Bedeutungen komplexer Ausdrücke ergeben, und dass deren Bedeutungen somit die Interpretation von komplexen Ausdrücken induzieren. Das ist zwar richtig; es zwingt uns aber nicht, als die Atome der Bedeutungen lexikalische Wörter zu sehen. Man kann annehmen, dass auch die Bedeutungen von Ausdrücken wie Junge oder Frau auf der Kombination von Bedeutungsatomen beruhen, etwa $\llbracket$ Mädchen $\rrbracket=\lambda \mathrm{i} \lambda \mathrm{x}[\operatorname{HUMAN}(\mathrm{i})(\mathrm{x}) \wedge$ FEMALE(i)(x) ^ YOUNG(i)(x)]. Dabei ist es nicht nötig, dass eine Sprache diese primitiven Bedeutungsbestandteile selbst als eigene lexikalische Ausdrücke besitzt. Auf diese Weise kann man auf die Einsichten von kombinatorischen Bedeutungstheorien wie der Merkmalssemantik (Katz \& Fodor 1963) und der „Natural Semantic Metalanguage“ (Goddard 2008) zurückgreifen und sie in die formale Semantik integrieren. Es ist aber nicht zwingend, dass der gesamte Wortschatz einer Sprache sich durch solche semantischen Primitive darstellen lässt. Für manche Bereiche spiegelt eine solche Darstellung plausiblerweise unser lexikalisches Wissen wider, weil die semantischen Primitive wiederholt eingesetzt werden - man denke an die Verwandtschaftsterminologie und Merkmale wie MALE/FEMALE, OFFSPRING oder MARRIED. Bei anderen aber ist das nicht der Fall; zwischen Schwalbe und Mauersegler durch ein Merkmal SPLITTAIL unterscheiden zu wollen, scheint verfehlt.

Die lexikalische Dekomposition ist dabei nicht nur eine Sache, die auf die wahrheitsfunktionale Bedeutungsanalyse draufgesetzt wird. Es gibt Beispiele, die man so verstehen kann, dass die Bedeutung eines Ausdrucks nur einen Teil der Bedeutung eines anderen Ausdrucks modifiziert, wie dies insbesondere David Dowty gezeigt hat (vgl. Dowty 1979). Dowty integriert Ansichten, die in der generativen Semantik entwickelt wurden, in die modelltheoretische Semantik. Beispielsweise analysiert er töten als: $\lambda \mathrm{i} \lambda \mathrm{y} \lambda \mathrm{x}[\mathrm{DO}(\mathrm{i})(\mathrm{x})(\mathrm{CAUSE}(\mathrm{BE}-$ $\operatorname{COME}(\neg \operatorname{ALIVE}(\mathrm{y})))]$ - das Subjekt $\mathrm{x}$ tut etwas, was zur Folge hat, dass y tot wird. Ein Argument für diese Analyse ist, dass sich das Adverb fast auf verschiedene Ebenen dieser Repräsentation beziehen kann. Karl hat Anton fast getötet kann heißen: Karl hat etwas getan, was verursacht hat, dass Anton fast tot ist: Er hat etwa so auf ihn eingeprügelt, dass er kaum noch am Leben ist: $\lambda \mathrm{i} \lambda \mathrm{y} \lambda \mathrm{x}[\mathrm{DO}(\mathrm{i})(\mathrm{x})(\operatorname{CAUSE}(\mathrm{BECOME}(\llbracket$ fast $\rrbracket(\neg \operatorname{ALIVE}(\mathrm{i})(\mathrm{y}))))]$. Der Satz kann aber auch heißen, dass Karl etwas getan hat, was fast verursacht hat, dass Anton 
nicht mehr lebt. Er hat etwa auf ihn gezielt, ihn aber verfehlt. Dieser Lesart entspricht die Struktur $\lambda \mathrm{i} \lambda \mathrm{y} \lambda \mathrm{x}[\mathrm{DO}(\mathrm{i})(\mathrm{x}) \llbracket$ ffast $\rrbracket(\mathrm{BECOME}(\mathrm{i})(\neg \operatorname{ALIVE}(\mathrm{i})(\mathrm{y}))))]$. Ferner kann der Satz auch meinen, dass Karl den Vorsatz hatte, Anton zu töten, es dann aber gelassen hat: $\lambda \mathrm{i} \lambda \mathrm{y} \lambda \mathrm{x}[\llbracket$ fast $\rrbracket(\mathrm{DO}(\mathrm{i})(\mathrm{x})(\mathrm{BECOME}(\mathrm{i})(\neg \mathrm{ALIVE}(\mathrm{i})(\mathrm{y}))))]$. Das heißt, das Adverb fast kann an verschiedenen Stellen in der semantischen Interpretation von töten andocken.

Das ist kein Einzelfall. Wir finden beispielsweise auch eine Ambiguität in Karl hat die Türe wieder geschlossen: Sie war vorher bereits einmal zu, oder er hat sie vorher bereits einmal zugemacht. Ein drittes Beispiel, auf das Jacobs (1980) hingewiesen hat, ist der Satz Jeder Zahnarzt fährt keinen Mercedes, gesprochen mit der sogenannten Hutkontur - steigender Akzent auf jeder, fallender Akzent auf keinen. Den Satz kann man dann so paraphrasieren: Es ist nicht der Fall, dass jeder Zahnarzt einen Mercedes fährt. Daran ist seltsam, dass die Negation, die in keinen steckt, den ganzen Satz betrifft, das Objekt aber im semantischen Bereich des Subjekts stehen muss - in prädikatenlogischer Darstellung: $\neg \forall \mathrm{x}[$ Zahnarzt(i)(x) $\rightarrow \exists y[$ Mercedes(i)(y) $\wedge$ fährt(i)(y)(x)]]. Man kann sich vorstellen, dass keinen zusammengesetzt ist aus der Negation $\neg$ und dem Existenzquantor $\exists$, und dass diese beiden Bedeutungsbestandteile offenbar unabhängig voneinander platziert werden können.

Damit Operatoren wie fast oder wieder an die richtige Bedeutungsebene andocken können, muss man annehmen, dass der Aufbau von Bedeutungen syntaktisch transparent ist. Das heißt, ein Verb wie töten hängt nicht nur an einem syntaktischen Knoten, sondern unter mehreren. Nur dann können fast und wieder die entsprechenden Bedeutungsbestandteile modifizieren. Auf der anderen Seite muss es möglich sein, eine solche komplexe syntaktische Repräsentation eines Wortes durch ein Lexem zu äußern. Dies entspricht den Annahmen, die man in der distributionellen Morphologie macht, die somit besonders gut zur Bedeutungskomposition passt. Es soll hier aber betont werden, dass die wahrheitsfunktionale Semantik nicht zwingend eine distribuierte Repräsentation voraussagt, und dass es durchaus auch in anderen Theorien distributionelle Repräsentationen gibt. Für eine weitergehende Behandlung sei auf den Handbuchartikel von Engelberg (2011) verwiesen.

\section{Alternativenbezug und Polaritätselemente}

Es gibt eine Reihe von Fällen, in denen die kommunizierte Bedeutung eines Ausdrucks nicht nur davon abhängt, was er wörtlich sagt, sondern auch davon, welche Ausdrucksalternativen nicht gesagt wurden. Ein bekanntes Beispiel sind skalare Implikaturen. Aus dem Satz Maria fährt einen BMW oder 
einen Porsche schließt man im Allgemeinen, dass es nicht der Fall ist, dass Maria einen BMW und einen Porsche fährt. Dies liegt nicht daran, dass die Disjunktion oder die ausschließende wäre. Es ist vielmehr so, dass oder und und in einer engen lexikalischen Beziehung zueinander stehen. Wenn eine Sprecherin oder gewählt hat, dann hat sie bewusst nicht und gewählt - in der Regel deshalb, weil für die Behauptung des entsprechenden Satzes, hier Maria fährt einen BMW und einen Porsche, die Evidenz fehlt. Wie kann man zeigen, dass oder die einschließende Disjunktion ist, also dem logischen $\vee$ entspricht? Beobachten wir, was passiert, wenn wir den Satz negieren: Es stimmt nicht, dass Maria einen BMW oder einen Porsche fährt. Der Satz sagt, dass weder das eine noch das andere der Fall ist. Wäre die Negation tatsächlich die ausschließende, dann wäre der negierte Satz aber auch dann wahr, wenn Maria einen BMW und einen Porsche fährt. Dies wird aber höchstens dann mitgeteilt, wenn oder speziell betont wird und damit eine vorhergehende Äußerung zurückgewiesen wird: Es stimmt nicht, dass Maria einen BMW oder einen Porsche fährt - sie fährt beides! Diese „metalinguistische“ Negation (Horn 1985) ist aber von einer anderen Art als die normale: Sie negiert nicht eine Proposition, sondern sie weist die Art und Weise zurück, wie eine Vorgängeräußerung ausgedrückt wurde.

Für unsere Vorstellung vom Lexikon heißt das, dass es nicht genügt zu lernen, was und und oder bedeuten. Es genügt auch nicht, zu lernen, was die Numeralia ein, zwei, drei, ... bedeuten, oder quantifizierende Determinatoren wie einige und alle, oder skalare Begriffe wie lauwarm, warm, heiß. Man beherrscht diese Begriffe erst dann, wenn man weiß, dass sie in einer Austauschklasse zueinander stehen, also dass jemand, der oder sagt, bewusst nicht und sagt, und dass jemand, der drei sagt, bewusst nicht vier sagt. Solche Austauschklassen werden Horn-Skalen genannt. Dies ist bei anderen Begriffen nicht der Fall, auch wenn sie systematisch in einer Inklusionsbeziehung zueinander stehen. Wenn man sagt, dass man einen Hund gesehen hat, schließt man nicht aus, einen Pudel gesehen zu haben.

Zur Beschreibung dieses Phänomens sind die Wahrheitsbedingungen, welche die formale Semantik in das Zentrum rückt, ganz unvermeidlich. Man kann skalare Implikaturen nicht beschreiben ohne anzunehmen, dass „A oder B“ schwächere Wahrheitsbedingungen hat als „A und B“. Und das Verschwinden von regulären skalaren Implikaturen unter der Negation bleibt ein völliges Rätsel, wenn man nicht berücksichtigt, dass sich die Stärke der Wahrheitsbedingungen unter der Negation umkehrt, dass also „Nicht: A oder B“ unter weniger Umständen wahr ist als „Nicht: A und B“. Die Negation schafft einen sogenannten abwärts-implizierenden Kontext. Es gibt auch andere semantische Operatoren, die solche abwärts-implizierenden Kontexte erzeugen, zum Beispiel Konditionalsätze; und auch bei ihnen finden wir einen ähnlichen Effekt. Auf den DM- 
Geldscheinen war zu lesen: Wer Banknoten fälscht oder gefälschte Banknoten in den Verkehr bringt, wird mit Zuchthaus nicht unter 2 Jahren bestraft. Wäre das oder exklusiv zu verstehen, könnte sich ein Geldfälscher damit rausreden, Banknoten sowohl gefälscht als auch in den Verkehr gebracht zu haben!

Abwärts-implizierende Kontexte sind für das grammatische Verhalten einer kleinen, aber hochinteressanten Klasse lexikalischer Ausdrücke von höchstem Interesse, den sogenannten negativen Polaritätselementen. Dies sind Wörter wie jemals oder Idiome wie einen Finger rühren, die nicht in einfachen positiven Sätzen vorkommen können; Hans war jemals im Jemen ist ungrammatisch, hingegen sind grammatisch Sätze wie Es stimmt nicht, dass Hans jemals im Jemen war oder Wenn Hans jemals im Jemen war, dann kennt er die Hauptstadt Sanaa. Ladusaw (1983) hat entdeckt, dass negative Polaritätselemente in abwärts-implizierenden Kontexten vorkommen. Aber warum gibt es diese Beschränkung? Nach Krifka (1995) führen sie systematisch alternative Bedeutungen ein - jemals etwa alternative Zeitangaben, einen Finger rühren alternative Arbeitsleistungen, ein Haar krümmen alternative feindselige Akte. Das negative Polaritätselement selbst bezeichnet die jeweils allgemeinste oder schwächste Alternative: jemals trifft auf alle Zeiten zu, einen Finger rühren ist die minimale Arbeitsleistung, ein Haar krümmen der minimale feindselige Akt, ein roter Heller der minimale Geldbetrag. Es handelt sich hier um infinitesimale Größen (vgl. Eckardt 2008). Und über solche eine Aussage zu treffen ist auf systematische Weise völlig uninformativ, was wiederum zu einer Einschätzung solcher Sätze als ungrammatisch führt (vgl. Abrusán 2007 für andere Fälle dieser Art).

\section{Expressive Konnotationen}

Frege hat bestimmte Arten von Bedeutungen aus seiner Betrachtung ausgeschlossen, die er Färbungen genannt hat. Sie haben in den letzten Jahren eine sehr intensive Beachtung in der formalen Semantik gefunden. Es handelt sich um diejenigen Bedeutungselemente, die ,auf das Gefühl, die Stimmung des Hörers wirken oder seine Einbildungskraft anregen“ (Frege 1918: 63). Aus seinen verstreuten Bemerkungen dazu (vgl. Freitag 2014) wird klar, dass darunter ganz verschiedene Dinge fallen: Pejorative wie Köter und Gaul, Satzadverbien, die eine Einstellung des Sprechers ausdrücken wie leider und gottlob, emotionale Modulation der Stimme, aber auch Konjunktionen, die sich nicht mit logischen Mitteln erfassen lassen, wie aber, was - wenn wir nur die wahrheitsfunktionalen Aspekte betrachten - dasselbe heißt wie und.

Für expressive Konnotationen, wie sie durch Köter, leider oder auch Ausrufe wie $a c h$ ! ausgedrückt werden, gibt es verschiedene Möglichkeiten, wie diese 
mit einer wahrheitswertfunktionalen Semantik verknüpft werden können. Eine wurde von Potts (2007) vorgeschlagen. Nach dieser Theorie gibt es neben der wahrheitsfunktionalen Bedeutung eine zweite Ebene der semantischen Repräsentation für expressive Bedeutungen. Wir können sie aber auch als Präsuppositionen werten. Nehmen wir an, Ausdrücke werden nicht nur in Bezug auf einen Index, sondern auch auf einen Kontext, in dem sie geäußert werden, interpretiert. Dies braucht man für deiktische Ausdrücke wie ich, heute, hier oder auch für das Präteritum als vergangenheitsbezogene Tempusform ohnehin. Ein Satz wie Ich kam gestern hier an würde dann, stark vereinfacht, interpretiert werden als: $\lambda c \lambda \mathrm{i}[\mathrm{i}$ in $\operatorname{Vortag}(\mathrm{c}) \wedge \operatorname{Sprecher}(\mathrm{c})$ kommt bei i an Ort(c) an]. Hier steht c für den Kontext; die Bedeutung des Satzes ist damit eine Funktion von Kontexten in eine Funktion von Indizes in Wahrheitswerte, was Kaplan (1978) „Charakter“ genannt hat. Dann können wir als Bedeutung für Köter angeben: $\lambda c \lambda i \lambda x$ : Sprecher(c) hat eine abwertende Einstellung $\mathrm{zu} x$ in $\mathrm{c}[\mathrm{x}$ ist ein Hund bei i]. Der Charakter von Hund ist also gar nicht definiert, wenn der Sprecher keine negative Einstellung zu dem bezeichneten Objekt hat. Und der Satz Leider regnet es würde wie folgt ausgedrückt: $\lambda c \lambda \mathrm{i}$ : Sprecher(c) bedauert, dass es bei Zeit(c) regnet $[i=\operatorname{Zeit}(c) \wedge$ es regnet bei i]. Dem entspricht, dass man gegen ausgedrückte Einstellungen ähnlich protestieren kann wie gegen Präsuppositionen, zum Beispiel mit Du liebst doch den Regen. Eine einfache Antwort Nein, es regnet nicht würde die Einstellung des Sprechers nicht zurückweisen.

\section{Diskursbezogene Bedeutungen}

Der wohl folgenreichste Schub in der Entwicklung der formalen Semantik war die Verlagerung von der Konzentration auf Wahrheitsbedingungen auf die Effekte, die Äußerungen auf den Informationszustand von Adressaten haben. Für diese Art von Fragestellung hat sich der Begriff dynamische Semantik eingebürgert. Der zentrale Begriff ist der Common Ground, die Information, welche von den Teilnehmern einer Konversation als geteilt vorausgesetzt wird; es ist das Ziel einer Konversation, diesen Common Ground zu verändern. Eingeführt wurde der Begriff von Robert Stalnaker (z. B. Stalnaker 1978). Der Ansatz erwies sich als äußerst tragfähig für die Behandlung von Präsuppositionen als Informationen, die bereits im Common Ground vorausgesetzt werden (z. B. Heim 1983), oder von anaphorischer Referenz, vermittelt durch die Einführung und Aufnahme von Diskursreferenten (z. B. Kamp \& Reyle 1993). Jüngere Ansätze setzen elaborierte Modelle des Common Grounds ein. Zum Beispiel nehmen Farkas \& Bruce (2010) an, dass es einen Bereich gibt, den Table, in dem die Partizipanten verhandeln, was in den Common Ground überführt werden soll. 
Krifka (2015) schlägt eine andere Art der Elaboration des Modells vor, nämlich dass nicht nur erfasst werden soll, was die Gesprächsteilnehmer tatsächlich als geteilte Information annehmen, sondern auch, wie sich der Common Ground weiterentwickeln soll. Zum Beispiel stellen Fragen eine solche Einschränkung dar, welche den Fortgang der Konversation beeinflussen (vgl. Onea 2016).

In diesen diskursbezogenen Theorien können nunmehr auch Ausdrücke abgehandelt werden, die Frege (1918) zu den „Färbungen“ gerechnet hat, wie zum Beispiel aber: Ein Satz wie Es regnet, aber es ist warm drückt über die Anreicherung des Common Grounds mit den Informationen, dass es regnet und dass es warm ist, folgendes aus: Die Anreicherung durch es regnet würde aus dem Common Ground bei einer herrschenden Fragestellung wie etwa der, ob man einen Spaziergang machen will, zu einem bestimmten Schluss führen, nämlich dass man keinen machen sollte. Der mit aber markierte zweite Beitrag drückt hingegen aus, dass man diese Schlussfolgerung aber womöglich nicht ziehen sollte. Auf diese Weise drückt aber die Relevanz des Satzes für den weiteren Entwicklungsverlauf des Common Grounds aus. Ähnliches gilt auch für Modalpartikeln wie ja und doch, die auf schon bekannte oder möglicherweise kontroverse Beiträge zum Common Ground hinweisen (vgl. z. B. Döring 2016).

Es ist dabei bemerkenswert, dass die Berücksichtigung von Färbungen und von Diskursphänomenen die ursprüngliche wahrheitsfunktionale Auffassung der formalen Semantik nicht beeinträchtigt hat. Man kann vielmehr argumentieren, dass sie die Grundlagen für die Entwicklung dieser weiterführenden Modelle bereitgestellt hat. Denn um zu beschreiben, wie der Informationszustand eines Adressaten angereichert wird, muss man zwingend auf die eine oder andere Weise auf Wahrheitsbedingungen zurückgreifen.

\section{Rückblick}

In diesem kurzen Artikel habe ich versucht, den Beitrag der formalen Semantik zu unserem Verständnis des Lexikons anhand einiger Beispiele aus verschiedenen Bereichen zu illustrieren. Insgesamt handelt es sich ohne Zweifel um eine Erfolgsgeschichte: Dank formal-semantischer Ansätze auf der Basis von Wahrheitsbedingungen haben wir heute eine sehr viel bessere Vorstellung von der Bedeutung verschiedener Arten lexikalischer Ausdrücke. Insbesondere erlauben es diese Ansätze zu verstehen, wie lexikalische Bedeutungen zur Satzbedeutung beitragen. Und sie erwiesen sich als flexibel genug, um auch solche Bedeutungsaspekte zu erfassen, die nicht unmittelbar die Wahrheitsbedingungen betreffen und etwa den Fortgang des Diskurses regeln. 
Damit soll aber nicht geleugnet werden, dass manche wichtige Aspekte des lexikalischen Wissens mithilfe von formal-semantischen Methoden schwer zu fassen sind. Dazu gehört beispielsweise die Tatsache, dass es bei den Entitäten, die unter einen sprachlichen Begriff fallen, solche gibt, die mehr oder weniger typisch sind. Solche Graduierungen legt die Prototypensemantik zugrunde (Taylor 2011); hier liegt möglicherweise eine Verwendung von abgestuften Wahrheitswerten nahe. Allerdings ist es nicht klar, wie sich dies mit der Kompositionalität von Bedeutung vereinbaren lässt: Ein typischer steinerner Löwe hat wenig mit dem zu tun, was ein typischer Stein ist, oder was ein typischer Löwe ist (Kamp \& Partee 1995). Ein weiterer Bereich, der über die strikt wahrheitsfunktionale Bedeutungsanalyse hinausgeht, sind die Schemata oder Frames, welche von Bedeutungen evoziert werden (Löbner 2014), und die für die natürlichsprachliche Kommunikation unerlässlich sind. Frames erlauben uns beispielsweise, den Ausdruck die Rechnung zu interpretieren, wenn von einem Restaurantbesuch die Rede ist. Eine Möglichkeit, dieses Wissen in die modelltheoretische Semantik zu integrieren besteht darin, die Ontologie des Diskursuniversums entsprechend durch Relationen anzureichern. Angesichts der Vielfalt von Frames stellt dies aber sicher eine Herausforderung für die Zukunft dar.

\section{Literatur}

Abrusán, Márta (2007): Contradiction and grammar: The case of weak islands. Doctoral dissertation. Cambridge, Mass.: Massachusetts Institute of Technology.

Ahn, Dorothy \& Uli Sauerland (2015): The grammar of relative measurement. SALT 25, 125-142.

Barwise, Jon \& Robin Cooper (1981): Generalized quantifiers and natural language. Linguistics and Philosophy 4, 159-219.

Beck, Sigrid (2011): Comparison constructions. In Claudia Maienborn, Klaus von Heusinger \& Paul Portner (Hrsg.), Semantics: An international handbook of natural language and meaning. Vol. 2, 1341-1389. Berlin: Mouton de Gruyter.

Becker, Thomas (2002): Autohyponymy: Implicature in lexical semantics, word formation, and grammar. Journal of Germanic Linguistics 14, 105-136.

Bierwisch, Manfred (1969): Certain problems of semantic representations. Foundations of Language 5, 153-184.

Bierwisch, Manfred (1987): Semantik der Graduierung. In Manfred Bierwisch \& Ewald Lang (Hrsg.), Grammatische und konzeptuelle Aspekte von Dimensionsadjektiven, 91-286. Berlin: Akademie-Verlag.

Cann, Ronnie (2011): Sense relations. In Claudia Maienborn, Paul Portner \& Klaus von Heusinger, (Hrsg.), Semantics: An international handbook of natural language meaning. Vol. 1, 456-478. Berlin: Mouton de Gruyter.

Carlson, Greg N. (1977): A unified analysis of the English bare plural. Linguistics and Philosophy 1, 413-456. 
Champollion, Lucas \& Manfred Krifka (2016): Mereology. In Maria Aloni (Hrsg.), The Cambridge handbook of formal semantics, 513-541. Cambridge: Cambridge University Press.

Chierchia, Gennaro (1998): Reference to kinds across languages. Natural Language Semantics 6, 339-405.

Copley, Bridget (2016): Force dynamics. In Rob Truswell (Hrsg.), Oxford handbook of event structure. Oxford: Oxford University Press.

Cresswell, Max (1976): The semantics of degree. In Barbara Partee (Hrsg.), Montague grammar, 261-292. New York: Academic Press.

Croft, William \& Alan D. Cruse (2004): Cognitive linguistics. Cambridge: Cambridge University Press.

Davidson, Donald (1967): The logical form of action sentences. In Nicholas Rescher (Hrsg.), The logic of decision and action, 81-95. Pittsburgh: Pittsburgh University Press.

Döring, Sophia (2016): Modal particles, discourse structure, and common ground management: theoretical and empirical approaches. Doctoral dissertation. Berlin: Humboldt-Universität zu Berlin.

Dowty, David (1979): Word meaning and Montague grammar. The semantics of verbs and times in generative semantics and in Montague's PTQ. Dordrecht: Reidel.

Eckardt, Regine (2008): The lower part of the event ontology. In Johannes Dölling, Tatjana Heyde-Zybatow \& Martin Schäfer (Hrsg.), Event structures in linguistic form and interpretation, 477-491. Berlin: de Gruyter.

Engelberg, Stefan (2000): Verben, Ereignisse und das Lexikon. Tübingen: Niemeyer.

Engelberg, Stefan (2011): Lexical decomposition: Foundational issues. In Claudia Maienborn, Klaus von Heusinger \& Paul Portner (Hrsg.), Semantics: An international handbook of natural language meaning (1), 71-112. Berlin: de Gruyter.

Farkas, Donka F. \& Kim B. Bruce (2010): On reacting to assertions and polar questions. Journal of Semantics 27, 81-118.

Filip, Hana (2011): Verb phrase semantics. In Claudia Maienborn, Klaus von Heusinger \& Paul Portner (Hrsg.), Semantics: An international handbook of natural language and meaning, 1186-1217. Berlin: Mouton de Gruyter.

Frege, Gottlob (1918): Der Gedanke. Eine logische Untersuchung. Beiträge zur Philosophie des Deutschen Idealismus 2.

Freitag, Wolfgang (2014): Frege über "leider" und "gottlob". In Bastian Reichardt \& Alexander Samans (Hrsg.), Freges Philosophie nach Frege, 161-174. Münster: mentis.

Gallin, Daniel (1975): Intensional and higher-order modal logic. Amsterdam: North-Holland.

Goddard, Cliff (2008): Natural semantic metalanguage: The state of art. In Cliff Goddard (Hrsg.), Cross-linguistic semantics, 1-34. Amsterdam: John Benjamins.

Heim, Irene (1983): On the projection problem for presuppositions. WCCFL. 2. Stanford, 114-125.

Horn, Laurence R. (1985): Metalinguistic negation and pragmatic ambiguity. Language 61, 121-174.

Jacobs, Joachim (1980): Lexical decomposition in Montague-grammar. Theoretical Linguistics 7, 121-136.

Kamp, Hans \& Uwe Reyle (1993): From discourse to logic. Introduction to model theoretic semantics of natural language, formal logic, and discourse representation theory. Dordrecht: Kluwer.

Kamp, Hans \& Barbara Partee (1995): Prototype theory and compositionality. Cognition 57, 129-191. 
Kaplan, David (1978): On the logic of demonstratives. Journal of Philosophical Logic 8, 81-98.

Katz, Jerrold J. \& Jerry A. Fodor (1963): The structure of a semantic theory. Language 39, 170-210.

Keenan, Edward (2011): Quantifiers. In Claudia Maienborn, Klaus von Heusinger \& Paul Portner, (Hrsg.), Semantics: An international handbook of natural language and meaning, 1058-1087. Berlin: Mouton de Gruyter.

Klein, Ewan (1980): A semantics for positive and comparative adjectives. Linguistics and Philosophy 4, 1-45.

Krifka, Manfred (1995): The semantics and pragmatics of polarity items. Linguistic Analysis 25, 209-257.

Krifka, Manfred (1998): The origins of telicity. In Susan Rothstein (Hrsg.), Events and grammar, 197-235. Dordrecht: Kluwer.

Krifka, Manfred (2014): Fünfzig Jahre Satzsemantik - am Beispiel der Negation. In Ludwig Eichinger (Hrsg.), Sprachwissenschaft im Fokus. Positionsbestimmungen und Perspektiven, 247-276. Berlin: Mouton de Gruyter.

Krifka, Manfred (2015): Bias in Commitment Space Semantics: Declarative questions, negated questions, and question tags. SALT. 25. LSA Open Journal Systems, 328-345.

Krifka, Manfred (2016): Quantification and information structure. In Caroline Féry \& Shinichiro Ishihara (Hrsg.), handbook of information structure, 251-269. Oxford: Oxford University Press.

Krifka, Manfred (im Erscheinen): Individuating matter over time. In Hana Filip (Hrsg.), Counting and measuring in natural language. Cambridge: Cambridge University Press.

Kyburg, Alice \& Michael Morreau (2000): Fitting words: Vague language in context. Linguistics and Philosophy 23, 577-597.

Ladusaw, William A. (1983): Logical form and conditions on grammaticality. Linguistics and Philosophy 6, 373-392.

Lambalgen, Michiel van \& Fritz Hamm (2005): The proper treatment of events. Oxford: Blackwell Publishers.

Lewis, David (1970): General semantics. Synthese 22, 18-67.

Link, Godehard (1979): Montague-Grammatik. Die logischen Grundlagen. München: Fink.

Link, Godehard (1983): The logical analysis of plurals and mass terms: A Lattice-theoretical approach. In Rainer Bäuerle, Christoph Schwarze \& Arnim v. Stechow (Hrsg.), Meaning, use, and interpretation of language, 302-335. Berlin: de Gruyter.

Löbner, Sebastian (1976): Einführung in die Montague-Grammatik. Kronberg: Scriptor.

Löbner, Sebastian (2014): Evidence for frames in natural language. In Thomas Gamerschlag, Doris Gerland, Rainer Oswald \& Wiebke Petersen (Hrsg.), Frames and concept types. Applications in language and philosophy. Heidelberg: Springer.

Maienborn, Claudia \& Angelika Wöllstein (Hrsg.) (2005): Event arguments: Foundations and applications. Berlin: de Gruyter.

Montague, Richard (1972): Universale Grammatik. Übersetzt von Helmut Schnelle. Braunschweig: Vieweg.

Montague, Richard (1973): The proper treatment of quantification in ordinary English. In Patrick Suppes, Julius Moravcsik \& Jaakko Hintikka (Hrsg.), Approaches to natural language, 221-242. Dordrecht: Reidel.

Onea, Edgar (2016): Potential questions at the semantics-pragmatics interface. Leiden: Brill

Partee, Barbara H. (1991): Topic, focus and quantification. SALT 1, Cornell Working Papers in Linguistics, 159-188. 
Potts, Christopher (2007): The expressive dimension. Theoretical Linguistics 33, 165-198.

Stalnaker, Robert (1978): Assertion. In Peter Cole (Hrsg.), Pragmatics, 315-323. New York: Academic Press.

Talmy, Leonard (1988): Force dynamics in language and cognition. Cognitive Science 12, 49-100.

Taylor, John R. (2011): Prototype theory. In Claudia Maienborn, Klaus von Heusinger \& Paul Portner (Hrsg.), Semantics: An international handbook of natural language and meaning, 643-664. Berlin: Mouton de Gruyter.

Zimmermann, Thomas Ede (2011): Model-theoretic semantics. In Claudia Maienborn, Klaus von Heusinger \& Paul Portner (Hrsg.), Semantics: An international handbook of natural language and meaning, 762-802. Berlin: Mouton de Gruyter.

Zwarts, Joost (1997): Vectors as relative positions: A compositional semantics of modified PPs. Journal of Semantics $14,57-86$. 


\title{
Dietrich Busse
}

\section{Historisch-semantische Epistemologie}

\begin{abstract}
Der Aufsatz diskutiert die Möglichkeit und Rahmenbedingungen einer historisch-semantischen Epistemologie als Weiterentwicklung von kulturlinguistisch orientierten Ansätzen wie der Begriffsgeschichte (nach dem Vorbild Kosellecks), der Diskursanalyse (nach den Anregungen Foucaults) sowie verwandter Ansätze einer tiefensemantisch orientierten, synchron wie diachron anwendbaren Analyse, die sämtliche Sparten und Erscheinungsformen des Wissens, das ein Verstehen von Sprachzeichen und Texten ermöglicht, in den Fokus der linguistisch-semantischen Analyse einbezieht. Vorgeschlagen wird, dabei schemaoder frame-theoretische Modelle der Semantik zu benutzen, da sie besser als die reduktionistischen Modelle der traditionellen linguistischen oder sprachlogischen Semantik geeignet erscheinen, die Vielfalt und den gesamten Umfang des verstehensrelevanten Wissens in den integrativen Blick einer Analyseform zu bringen, die immer Sprachanalyse und Wissensanalyse zugleich ist.
\end{abstract}

Keywords: Begriffsgeschichte, Diskursanalyse, Epistemologie, Foucault, Frame, Frame-Semantik, Historische Semantik, Koselleck, verstehensrelevantes Wissen, Wissen.

\section{Einleitung}

Unter historisch-semantischer Epistemologie (als Teil einer umfassenderen, auch synchrone Aspekte einbeziehenden linguistischen Epistemologie) kann eine Forschungsorientierung verstanden werden, die das Wissen (individuell und/oder gesellschaftlich) in den Mittelpunkt nicht nur der praktisch-empirischen Analysen, sondern auch der sprachbezogenen Theoriebildung stellt, das Menschen aktivieren, wenn sie die an sich zunächst inhaltslosen, physisch realisierten Wahrnehmungsanlässe interpretieren, die wir sprachliche Zeichen nennen. ${ }^{1}$ Eine

1 Von einer „historisch-semantischen“ oder „linguistischen Epistemologie“ in dem in diesem Aufsatz behandelten Sinne wurde erstmals explizit in Busse (2000, 2003, 2004) gesprochen. In nachfolgenden Aufsätzen (Busse 2006, 2007a, b, 2008a, b, 2013a) und Buchkapiteln (Busse 2012: Kap. 8.2; Busse 2014: Kap. 7) wurde dieser Gedanke immer weiter ausgeführt und begründet; zur Rezeption dieses Ansatzes siehe auch Lotz (2011).

Dietrich Busse, Heinrich Heine Universität Düsseldorf, Institut für Germanistik, Universitätsstraße 1, D-40225 Düsseldorf, E-Mail: d.busse@uni-duesseldorf.de

Ә Open Access. (c) 2018 Dietrich Busse, publiziert von De Gruyter. (c) BY Dieses Werk ist lizenziert unter der Creative Commons Attribution 4.0 Lizenz.

https://doi.org/10.1515/9783110538588-003 
linguistische Epistemologie wird dann verstanden als eine unter anderem mit genuin linguistischen Methoden arbeitende Analyse der Beziehungen, die zwischen sprachlichen Einheiten (Wörtern, Wortteilen, Sätzen, Satzteilen, Texten, Textteilen, Textnetzen und Textbeziehungen) einerseits und Elementen des durch diese sprachlichen Einheiten angezielten gesellschaftlichen Wissens andererseits bestehen. Dieses Wissen wird aus linguistischer Perspektive als bedeutungsrelevantes oder verstehensrelevantes bzw. verstehensermöglichendes Wissen in den Blick genommen. Die Beziehungen, die auf der sprachlichen Ausdrucksebene zwischen Wörtern/Begriffen, Sätzen und Texten (und ihren jeweiligen Teileinheiten) bestehen, erscheinen auf der Seite des Wissens als Beziehungen zwischen Wissenselementen; sprachlichen und textuellen Strukturen entsprechen Strukturen im (gesellschaftlichen) Wissen.

An dieser Stelle sind zum besseren Verständnis zunächst zwei terminologische Anmerkungen notwendig:

(a) Die in diesem Aufsatz (und anderen erwähnten Arbeiten des Verf.) erfolgende Verwendung des Ausdrucks Epistemologie weicht in gewisser Weise von der üblichen ab und ist stärker als die internationalen Vorbilder durch den Begriff des Wissens als épistémè bei Foucault geprägt. Als Entlehnung aus dem altgrie-

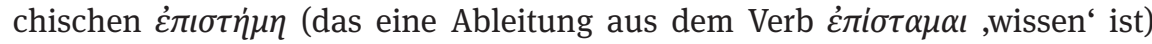
wird épistémè in französischen Wörterbüchern definiert als ensemble des connaissances scientifiques, du savoir d'une époque et ses présupposés (http:// fr.wiktionary.org/wiki/épistémè [letzter Zugriff 13.5. 2017]). Die darin mitschwingende Verkürzung des Begriffs auf,wissenschaftliches Wissen oder ,Wissenschaft ' hat dazu geführt, dass fr. épistémologie und engl. epistemology heute meist verkürzend nur als ,Wissenschaftstheorie‘, ,Wissenschaftsgeschichte oder ,Erkenntnistheorie ‘ verstanden werden. Begründet wird dies damit,

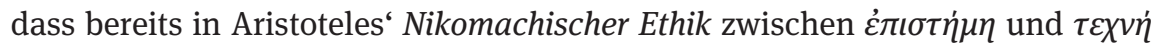
(techne) als ,theoretischem Wissen' und ,praktischem Können' unterschieden werde. $^{2}$ Auch bei Foucault scheint zunächst noch in Les mots et les choses (1966a, dt. Die Ordnung der Dinge) der übliche französische Gebrauch von épis-

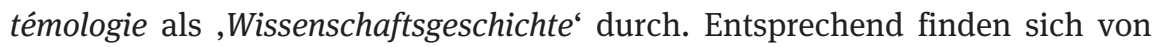
ihm Äußerungen, in denen er épistémè in diesem eingeschränkten Sinne benutzt. Aufgrund meiner eigenen Lektüre von Foucaults L'archéologie du savoir (1969, dt. Archäologie des Wissens) gehe ich jedoch davon aus, dass Foucault spätestens in diesem Werk épistémè im Sinne eines erweiterten, allgemeineren

2 Eine gründliche Analyse des Wissensbegriffs bei Aristoteles müsste aber noch mindestens

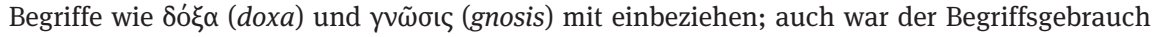
schon bei Aristoteles selbst alles andere als konstant. 
Wissensbegriff verwendet, der es erlaubt, auch das nicht-theoretische, nichtwissenschaftliche Wissen, also das Alltagswissen, mit einzubeziehen. Nur so macht seine überaus bemerkenswerte Aussage Sinn:

Ich habe versucht [...], die Geschichte nun nicht des Denkens allgemein, sondern alles dessen zu schreiben, was in einer Kultur Gedanken enthält. (Foucault 1966b, Spalte 4; dt. 1973: 156)

Es mag jedoch sein, dass der in meiner eigenen Verwendung von Episteme und Epistemologie mitschwingende allgemeine Wissensbegriff (der noch nicht vorab zwischen wissenschaftlichem und nicht-wissenschaftlichem Wissen unterscheidet und es reduktionistisch auf ersteres verkürzt) mindestens ebenso stark von Theorien des Alltagswissens im Kontext der angelsächsischen Ethnomethodologie-Schule (z. B. Garfinkel 1973; Gumperz 1978; Goffman 1974) und/ oder der Wissenssoziologie der Linie Schütz (1932 und 1971) bis Berger \& Luckmann (1969) beeinflusst ist wie von Foucaults Archäologie des Wissens. (Eine weitere Quelle wäre die in Ludwig Wittgensteins Sprachspiel- und LebensformBegriffen aufscheinende Wissenskonzeption; vgl. Wittgenstein 1971). Für Zwecke einer allgemeinen, verstehenstheoretisch reflektierten interpretativen Semantik, und einer auf dieser aufbauenden historisch-semantischen Wissensanalyse scheint es mir jedoch sinnvoller zu sein, die Wirkung des Wissens und von Wissensrahmen in Sprache und Textverstehen zunächst einmal auf einer allgemeinen, grundbegrifflichen und grundlagentheoretischen Ebene zu diskutieren und zu klären, bevor man dann anfängt, verschiedene Sorten von Wissen typologisch $\mathrm{zu}$ unterscheiden (und deren möglicherweise typologisch unterschiedlichen Einflüsse auf Sprache, Semantik und Diskurse zu differenzieren). In diesem Sinne meint Episteme bei mir zunächst einmal: ,menschliches Wissen, gleich welcher Art, Herkunft und Entstehungsform', und Epistemologie meint dementsprechend die Erforschung und Beschreibung des (in unserem Kontext: bedeutungs- bzw. verstehensrelevanten) Wissens noch vor und unabhängig von jeglicher Sorteneinteilung. Das Verhältnis von individuellem und gesellschaftlichem Wissen ist dann ein Spezialthema, das im Rahmen dieses Textes nicht ausführlich diskutiert werden kann.

(b) Der Gedanke, das verstehensrelevante Wissen in den Mittelpunkt jeder semantischen Analyse zu rücken, wurde (zunächst im Rahmen der historischen Semantik und Begriffsgeschichte) erstmals in Busse (1987) explizit artikuliert (dort noch als „bedeutungsrelevante epistemische Momente“; vgl. Busse 1987: 305) und dann in Busse (1991) - im Kontext der Idee einer explikativen Semantik - im Zuge der Einführung von Wissensrahmen / Frames in die Überlegungen zu einer adäquaten Semantik und Sprachverstehenstheorie explizit einge- 
führt (Busse 1991: 78-93, 121-122, 139-166) (12 $^{3}$ und in Hinblick auf eine heuristische Typologie dieses Wissens (Busse 1991: 139-166) explizit ausgeführt. ${ }^{4}$ Vergleichbare Überlegungen hatten bereits zuvor Fillmore (1985) dazu veranlasst, seinen Ansatz der frame-analytischen Semantik als „understanding semantics“ oder „interpretive semantics“ zu charakterisieren. Freilich rückt bei ihm das bedeutungsrelevante Wissen nie als solches (theoretisch oder methodisch) in den Mittelpunkt der Überlegungen, sondern wird nur in den praktischen Analysen erwähnt und in Anschlag gebracht.

Im Begriff des bedeutungs- bzw. verstehensrelevanten Wissens wie im Konzept einer linguistischen Epistemologie werden unter anderem Überlegungen zusammengeführt, die ursprünglich aus verschiedenen Forschungssträngen und aus Überlegungen zu unterschiedlichen Zielsetzungen stammen, sich aber im Nachhinein als Facetten ein und derselben Grundproblematik erwiesen haben, nämlich der Frage, in welcher Form und Struktur Wissen im Gebrauch von Sprache (in gleich welcher Form) zum Zuge kommt. Da ist zum einen die Begriffsgeschichte (etwa im Sinne der Überlegungen des Historikers Koselleck 1972 und 1978), deren kritische Betrachtung aus linguistischer Perspektive unter Aufnahme ihrer wissensanalytischen Intentionen in Busse (1987) zum Programm einer linguistisch reflektierten und verfahrenden Diskursanalyse weiterentwickelt wurde (siehe dazu zuletzt auch Busse \& Teubert 2013), und zum anderen das Programm einer umfassenden Theorie des Sprachverstehens, der Textinterpretation und des (interpretierenden und applizierenden) Umgangs mit Texten (siehe dazu Busse 1991, 2014) - damals konkret in den Kontext einer Juristischen Semantik (siehe dazu Busse 1993/2010) gestellt. Den beiden genannten Forschungs- und Gedankensträngen ist gemeinsam, dass sie beide von Anfang an auch mit dem Ziel einer Neubegründung der in allen ihren Sparten als unzulänglich, weil zeichentheoretisch und wissensanalytisch defizitär erkannten, linguistischen Semantik verbunden waren, und letztlich allein aus diesem Grunde überhaupt angegangen wurden. Die im vorliegenden Aufsatz skizzierte Idee einer linguistischen Epistemologie ist daher immer auch zu verstehen als Beitrag zu einer Weiterentwicklung (und, wie der Verf. meint, adäquateren Fassung) der in der Vergangenheit $\mathrm{zu}$ sehr durch Reduktionismen unterschiedlicher Provenienz eingeschränkten linguistischen Semantik.

3 Wieder abgedruckt in der erweiterten Neuausgabe Busse (2014: 145-167, 282-283, 321-344). 4 Vgl. zu letzterem auch Busse (1997: 19 und passim); unter anderem Ziem (2008: 150-187 und öfter) hat später systematisch auf diesem Gedanken aufgebaut. 


\section{Von der Begriffsgeschichte zur Diskursanalyse: (Historische) Semantik als Epistemologie}

Ende der 1970er Jahre entstand beim Verfasser (zuerst noch unter dem etwas missverständlichen Titel Bedeutungssysteme) die Idee eines systematischen bedeutungstheoretischen und analytisch-semantischen Ansatzes zur Erklärung und Beschreibung des funktionalen Zusammenhanges zwischen sprachlichen Zeichen (verstanden zunächst als reine Zeichenausdrucksseiten) und dem „hinter“ den Zeichen stehenden (diese, im ursprünglichen, Saussure'schen Sinne „motivierenden“) Wissen. Inspiriert war diese Idee einerseits durch langjährige Beschäftigung mit sprach- und bedeutungstheoretischen Grundlagenfragen, andererseits durch die Überzeugung, dass Sprache erheblich dazu beiträgt, wie Menschen die Welt und ihre Dinge und Phänomene sehen und wahrnehmen, wie sie das, was sie als „Wirklichkeit“ begreifen, letztlich über Begriffe (und das heißt implizit immer auch: über den Gebrauch von - meist sprachlichen - Zeichen und Zeichenketten) konstituieren bzw. „konstruieren“. Immer mitschwingend stand dabei im Hintergrund die Überzeugung, dass dieser Zusammenhang ohne eine Einbeziehung der Dimension des Sozialen, Gesellschaftlichen schlechterdings nicht angemessen erklärt werden kann. Auch wenn unbezweifelbar ist, dass „Wissen“ in den Prozess des Zeichengebrauchs und -verstehens immer zunächst und „phänomenhaft“ als persönliches, also individuelles Wissen eingreift, so kann doch ebensowenig bezweifelt werden, dass dieses Wissen (als gesellschaftlich konstituiertes und auch inhaltlich geprägtes) immer ein Reflex des Sozialen (des sozialen Wissens) ist und nur so theoretisch gedacht werden kann. In diese Überlegungen flossen also sprachtheoretische, erkenntnistheoretische und wissensanalytische Überlegungen gleichermaßen ein. Somit waren die ersten Überlegungen ein Synergieeffekt unterschiedlichster Einflüsse:

a) einer kritischen Reflexion des üblichen linguistischen Zeichenbegriffs und der völlig ungenügenden Bestimmung dessen, was in klassischen Zeichenkonzeptionen oft als „Inhaltsseite des Zeichens“ apostrophiert wird;

b) einer kritischen Reflexion der stark reduktionistischen Tendenzen der traditionellen, damals strukturalistisch dominierten linguistischen Semantik (mit ihren Merkmallisten und dem vergeblichen Versuch der Trennung von „wesentlichen“ und „akzidentiellen“ semantischen Merkmalen); ${ }^{5}$

5 Kleiber (1998) nennt diese Richtung in seiner Einführung in die Prototypensemantik recht treffend nur „notwendige-und-hinreichende-Bedingungen-Semantik“, kurz „NHB-Semantik“. Fillmore (1975) spricht sie ebenso treffend als „checklist-semantics“ an. 
c) des Versuchs, solchen aporetischen Modellen eine adäquatere sprach- und bedeutungstheoretische Sichtweise auf der Basis der in Wittgensteins Spätwerk (vor allem Philosophische Untersuchungen und Über Gewissheit) entwickelten sprach-, erkenntnis- und regeltheoretischen Überlegungen entgegenzusetzen;

d) der durch Wittgenstein II ebenso wie durch Herder und Humboldt (und ihre Nachfolger Sapir 1929 und Whorf 1963) inspirierten Idee des konstitutiven, konstruktiven Charakters des menschlichen Wissens und der menschlichen Wirklichkeitsdeutung und des engen Zusammenhangs, den diese mit dem Zeichengebrauch eingehen;

e) der in der Zeichen- und Erkenntnistheorie von Charles Sanders Peirce (1931-1935) artikulierten Idee: „Jeder Gedanke ist ein Zeichen“;

f) der in einigen Grundgedanken bei Husserl (1913) zeichentheoretisch angelegten, bei Mead (1934), Bühler (1934) und vor allem Schütz (1932, 1953) dann sozialpsychologisch begründeten Annahmen über Funktionsweise und Prozesse symbolischer Interaktion, gesellschaftlicher Symbolkonstitution und auf Symbole reagierender Deutungs- und Wissenskonstitutionsprozesse, kumulierend in Berger \& Luckmanns (1969) - letztlich nur Schütz (1932) ausbuchstabierenden - Hypothese von der „gesellschaftlichen Konstruktion der Wirklichkeit“ und der zentralen Rolle, die Sprache und sprachlich verankerte Deutungsmuster und -systeme dabei spielen;

g) den Überlegungen zu einer historisch-genealogisch interessierten, durchaus aber auch synchron-systematisch ausbeutbaren „Archäologie des Wissens“, wie sie im Werk von Michel Foucault (1966a, 1969, 1971) entwickelt und mit dem Begriff der Episteme ebenso wie mit dem Begriff des Diskurses in einen Zusammenhang gebracht worden war.

Später hinzukommende Einflüsse waren dann insbesondere die Auseinandersetzung mit der „bewusstseinsgeschichtlich“ “6 angelegten Begriffsgeschichte als sozio-historiographischer Methode nach Koselleck (1972, 1978), Stierle (1973, 1978), Reichardt (1985) und anderen, das Modell einer die klassischen semantischen Reduktionismen von Linguistik und logikfixierter Sprachphilosophie überwindenden Satzsemantik von Peter von Polenz (1985), sowie die schon bei von Polenz einflussreiche, später vor allem nach Fillmore (1977, 1982, 1985, 2006), Minsky (1974) und Barsalou (1992) modellierte Idee der „Wissensrahmen“ bzw. „Frames“ (als Modellen zur Rekonstruktion des „verstehensrelevanten Wissens“).

6 So der noch in Busse (1987: 11) dafür verwendete Terminus. 
Die Weiterentwicklung der linguistischen Semantik zu einer „linguistischen Epistemologie“ (als deren diachrone Variante die im Titel des vorliegenden Aufsatzes angesprochene „historisch-semantische Epistemologie“ begriffen werden kann) wurde also von der Auseinandersetzung mit der historiographischen Begriffsgeschichte nach dem durch Reinhart Koselleck begründeten (und empirisch bzw. praktisch-analytisch mit dem Lexikonwerk Geschichtliche Grundbegriffe teilweise umgesetzten) Modell ${ }^{7}$ angeregt, sowie dem zunächst eigenständig entwickelten, dann im Umkreis von Kosellecks Bemühungen auch bei Autoren wie Stierle (1973, 1978) oder Günther (1978) artikulierten Gedanken, dass der ganze Umfang von Kosellecks Intentionen am besten durch eine Erweiterung des zunächst begriffsgeschichtlichen Vorhabens auf die Ebene der Diskurse zu realisieren sein würde. Erstes Ergebnis dieser Überlegungen war dann in Busse (1987) das Modell einer historisch-semantischen, wissensanalytisch (d. h. „epistemologisch“) motivierten „linguistischen Diskurssemantik“ bzw. „linguistischen Diskursanalyse“.

Eine historische Semantik im Sinne der zunächst von Koselleck $(1972,1978)$ als Begriffsgeschichte formulierten, dann in Busse (1987) zu einer historisch-semantischen Diskursanalyse weiterentwickelten Zielsetzungen war von Beginn an sozialgeschichtlich, kulturhistorisch und wissensanalytisch motiviert - kurz: Sie zielte und zielt auf eine historische Epistemologie, eine Wissens- und Bewusstseinsgeschichte, die die Repräsentation des gesellschaftlichen Wissens einer Zeit (vermittelt über sprachlich vorliegende Quellen und Ausdruckeinheiten) in ihrer Genese, ihren Konstitutionsbedingungen, ihren kulturhistorischen Traditionslinien und ihren epistemischen Tiefenströmungen offenlegt.

In einer linguistisch reflektierten Aufnahme und Weiterentwicklung einer Begriffsgeschichte im Sinne Kosellecks konvergieren die auf beiden beteiligten Seiten unabhängig voneinander entwickelten und unterschiedlich begründeten Überzeugungen, dass für die Ziele einer so verstandenen kulturhistorischsozialgeschichtlich-wissensanalytischen Semantik die herkömmliche linguistische Semantik zu weit davon entfernt ist, die Werkzeuge und Grundbegriffe bereitstellen $\mathrm{zu}$ können, die für eine solche Art von Forschung nötig wären. Beide Herangehensweisen sind daher nicht $\mathrm{zu}$ trennen von einer fundamentalen Kritik an den Reduktionismen klassischer linguistischer, philosophischer und logizistischer Bedeutungstheorien und -modelle. ${ }^{8}$ Auch Foucault fand in

7 Siehe dazu die Darstellung und Diskussion in Busse (1987: 50-60, Kap. 2). - Ich bin meinem akademischen Lehrer Rainer Wimmer (damals Direktor des Instituts für deutsche Sprache in Mannheim) noch heute außerordentlich dankbar dafür, dass er mir diese dann so fruchtbar werdende Perspektive aufgezeigt hat.

8 So beklagte der Historiker Reinhart Koselleck als Begründer der neuen historiographischen Begriffsgeschichte zu Anfang der 1970er Jahre, dass die dringend gesuchten Gesprächspartner 
der strukturalistisch dominierten Linguistik der 1960er und 1970er Jahre keinerlei Anregungen für die von ihm angezielte Epistemologie, obwohl doch klar war, dass deren Quellen weit überwiegend sprachlicher Natur waren und sie daher linguistische, d.h. vor allem semantische Aspekte notwendigerweise würde berühren müssen. ${ }^{9}$

Aus fachinterner Sicht kann diese fachexterne Kritik mit folgenden Gesichtspunkten unterstützt werden:

a) Fast alle Bedeutungstheorien und bedeutungsanalytischen Methodenvorschläge der „Mainstream“-Positionen nicht nur der Linguistik, sondern auch weiter Teile der Sprachphilosophie sowie der formalen Logik unterschätzen um Dimensionen den Umfang, die Komplexität und die Subtilität des verstehensrelevanten Wissens in Bezug auf Sprachzeichen und Zeichenketten (gleich, ob man es nun Inhaltselemente, semantische Merkmale, epistemische Elemente, bedeutungsrelevantes Wissen, oder wie auch immer nennen mag). ${ }^{10}$

b) Fast alle Mainstream-Modelle der Semantik unterschätzen sträflich die zentrale Rolle dessen, was meist Kontext (manchmal auch: Situation) des Zeichengebrauchs genannt wird, und fixieren sich auf einzelne Wortformen und ein auf einem wortformenfixierten Bedeutungsverständnis ruhendes additives Verständnis von Phrasen- oder Satzbedeutungen. Sie tragen (wenn überhaupt) nicht genügend dem Umstand Rechnung, dass Bedeutungen auch über mehrere Wortformen hinweg identisch sein (oder mindestens epistemisch eng beieinanderliegen) können.

c) Die von Koselleck kritisierten linguistischen Bedeutungsmodelle hatten damals (der Mainstream tut dies heute noch) die diachrone Perspektive auf

für die linguistische Fundierung des von ihm entwickelten Methodenkonzepts sich in der damaligen Sprachwissenschaft partout nicht einstellen wollten. Entweder blieben diese im Methodenspektrum enggefaßter traditioneller Wortgeschichte befangen und konnten das Potential kulturhistorischer und epistemologischer Ausweitungen ihres Forschungsgebiets nicht erkennen; oder sie hatten infolge des Siegeszugs strukturalistischer, formalgrammatischer und generativistischer Modellierungen alle diachrone Sprachforschung vollständig hinter sich gelassen. Die Begriffsgeschichte und kulturhistorische Semantik musste daher außerhalb der Linguistik ausformuliert und praktiziert werden, bis sie dann - einmal als erfolgreich erwiesen - von jüngeren Forscherinnen und Forschern in die Sprachwissenschaft sozusagen reimportiert wurde.

9 Zur Analyse des anti-linguistischen Tenors bei Foucault und deren Einschätzung aus nicht reduktionistischer linguistischer (semantischer) Sicht siehe Busse (1987: 242-245).

10 Man könnte hier mit Bezug auf die Mainstream-Semantik metaphorisch auch von einer „Eisbergspitzen-Semantik“ sprechen, die nur erfasst, was oberhalb der Wasseroberfläche zu sehen ist; bekanntlich aber befindet sich die Mehrheit der Masse eines Eisbergs (80-90\%) unterhalb der Wasseroberfläche und ist dem allerersten Blick entzogen. 
Zeichen und Zeichenbedeutungen, die noch für Ferdinand de Saussure so wichtig gewesen war, völlig hinter sich gelassen und als ernsthaften Forschungsgegenstand der Sprachwissenschaft quasi völlig abgeschafft.

Zwar gab es außerhalb des linguistischen, logizistischen und sprachphilosophischen Mainstreams durchaus Positionen, denen die geschilderten Fehler des Reduktionismus nicht attestiert werden können, so insbesondere und fast ausschließlich die in Wittgensteins Spätwerk entwickelte Konzeption, welche Sprache, die Funktion von Zeichen, sowie auch Bedeutungen auf der Basis einer Theorie des Gebrauchs erklärt (und ein weitgefasstes Verständnis der verstehensrelevanten Wissensaspekte über Konzepte wie Sprachspiel oder Lebensform einzuholen versucht); doch sind solche Positionen bedeutungstheoretisch und vor allem methodisch gesehen viel zu unspezifisch gewesen, als dass sie direkten Einfluss auf eine auch praktisch-analytisch ausgerichtete historische Semantik (z. B. im Sinne Kosellecks) hätten haben können.

Kosellecks Ansatz der historischen Semantik (bei ihm als Begriffsgeschichte gefasst - eine Schublade, in der er später sein Werk gar nicht mehr gerne verortet $\operatorname{sah}^{11}$ ) unterscheidet sich deutlich von anderen, älteren Versionen der Begriffsgeschichte. Begriffsgeschichte in seinem Sinne soll weit über die traditionelle Wortgeschichte (wie die historische Wortforschung und Etymologie) hinaus gehen, soll sich aber auch von der sich häufig als Wortartikel tarnenden Sach- und Ideengeschichte erkennbar unterscheiden. Ausgehend von dem Gedanken, dass es in Wörtern ausgedrückte Begriffe sind, und damit sprachliche Einheiten, die das gesellschaftliche Denken in allen Wissensbereichen prägen, soll dem gesellschaftlichen und historischen Wirken von als „Begriffe“ konzipierten Wissenskomplexen und damit ihrer Wirklichkeit konstituierenden und Wirklichkeit verändernden Kraft nachgespürt werden. ${ }^{12}$

Berühmt geworden ist Kosellecks Diktum, wonach die historisch-sozialen Grundbegriffe nicht nur „Indikatoren“ für gesellschaftliche und historische Prozesse sind, sondern historische Veränderungen direkt als „Faktoren“ dieser Prozesse beeinflussen können. ${ }^{13}$ In dieser Denkweise gesehen werden Begriffe selbst $\mathrm{zu}$ einem Movens historischer Prozesse. ${ }^{14}$ Historische Begriffe (hier ver-

11 Siehe Koselleck (2006: 529).

12 Dabei unterscheidet Koselleck (1972: XXII) nach wie vor zwischen „Begriff“ und „Wort“, freilich auf eine linguistisch gesehen nicht nachvollziehbare und nicht akzeptable Weise (siehe dazu ausführlicher Busse 1987: 52-57 und 80-85).

13 Koselleck (1972: XXIII-IV); vgl. auch Koselleck (1978).

14 Koselleck ging es dann, wie durch spätere Aussagen deutlich wurde, wohl um nicht mehr und nicht weniger als um ein Panoptikum, eine Typologie von Begriffen als Movens, als Agenten des historischen Prozesses. Seine Version der Begriffsgeschichte ist darum in vollem, bes- 
standen als historisch wirksame, geschichtsmächtig werdende Begriffe) sind Indikator historischer Entwicklungen, indem sie historische Erfahrungen, ganze komplexe Zusammenhänge historischer Zustände und Bewegungen in einem einzigen Begriffswort bündeln können. Sie sind daneben aber häufig auch wirkungsmächtige Faktoren des historischen Prozesses selbst, siehe etwa Begriffe wie Sozialismus, Freiheit usw. ${ }^{15}$ „Begriffe“ in diesem Sinne sind Ordnungsleistungen bzw. Strukturierungsgrößen semantisch verankerten (und damit sprachbezogenen) gesellschaftlichen Wissens.

Das Verhältnis von Begriff und sprachlichem Zeichen (Wort) ist dabei, wie schon Koselleck hervorgehoben hat, ein vielschichtiges: Im Sinne der Historiker sind historische Schlüsselbegriffe zwar immer auch durch zugehörige Begriffswörter ausgedrückt. Jedoch kann der historische Begriff sich auch in den Bedeutungen anderer, benachbarter Wörter auswirken oder niederschlagen. Es kommt der Begriffsgeschichte daher immer auch auf die Analyse ganzer Begriffsnetze an. Drückt man diese Zielsetzung der Begriffsgeschichte in den Termini der Epistemologie (der Wissensanalyse) aus, dann sind solche Begriffsnetze gleichbedeutend mit semantisch verankerten Netzwerken, gestützt auf konzeptuelle Strukturen und die mit ihnen verknüpften Wissensrahmen bzw. Frames.

Auch wenn Koselleck diesen Begriff meines Wissens nie verwendet hat, geht es ihm letztlich immer um so etwas wie eine Wissensgeschichte, eine Geschichte des in (von ihm Begriffe genannten) Wissenskomplexen gefassten, jeweils zeit- bzw. epochen-gebundenen gesellschaftlichen Wissens. Anders als etwa historisch-semantische Untersuchungen in der Sprachwissenschaft zielten seine Untersuchungen dabei weniger auf eine Diachronie im Sinne einer

tem Sinne historisch und historiographisch, weil sie Begriffe quasi als Individuen, als Antriebskräfte im historischen Prozess analysieren will. In diesem Sinne sind Begriffe für Koselleck epistemische Größen, die nicht an einzelne Wörter und Wortbedeutungen gebunden sind, auch wenn sie mit Leitvokabeln benannt und identifiziert werden können und oft genug gerade durch diese plakativ-propagandistische Identifizierbarkeit ihre historische Antriebsfunktion erhalten. (Gegenüber einer solchen - wenn man es so nennen darf - „Historie einer belebten, animierten, dynamischen Begriffswelt“ stellt sich die nüchterne Perspektive einer linguistischanalytischen Betrachtungsweise als pure Deskription historisch-epistemischer Möglichkeitsbedingungen dar.)

15 Für solche Begriffe wurden manchmal auch Ausdrücke wie Erwartungsbegriffe, Zielbegriffe, oder - unspezifischer - Fahnenwörter benutzt. Der Linguist Fritz Hermanns (1986, 1989, hier zitiert nach dem Wiederabdruck in Hermanns 2012: 182, 190-191, 202-205) hat dafür den mittlerweile gebräuchlich gewordenen Terminus deontische Begriffe geprägt, im Sinne von: Begriffe, die benennen, „was sein soll“ (Deontik), d.h., was man durch politisch-historische Bewegungen allererst zu erreichen hofft, was aber im Moment des Sprechens bzw. Schreibens noch nicht Wirklichkeit ist. 
Entwicklung, als vielmehr auf eine genaue Erfassung der Begriffe genannten Wissenskomplexe und Wirklichkeitsdeutungen in ihrem jeweiligen Zustand und Eigenwert in der jeweiligen Epoche bzw. dem jeweiligen Zeithorizont.

Es war dann kein Zufall, dass viele Rezipienten des Koselleck'schen Modells in den 1970er Jahren sehr schnell sahen, dass einige der Ziele, die Koselleck auf seine Weise formulierte (und als Begriffsgeschichte konzipierte) mit Zielen identisch waren, wie sie auch Michel Foucault in der von ihm in den 1960er und 1970er Jahren entworfenen historischen Genealogie oder Archäologie des Wissens (so der Titel seines bezüglich dieser Ziele systematischsten Textes; vgl. Foucault 1969) artikuliert hatte, die in der Rezeption meistens mit dem ebenfalls von ihm lancierten Begriff des Diskurses und seiner Ordnung(en ${ }^{16}$ in Zusammenhang gebracht wurden.

Mit Genealogie ist gemeint, dass Foucault seine Diskursanalyse als Analyse der Genese und Genesebedingungen gesellschaftlichen Wissens in diskursiven Formationen verstanden hat. Diskurse stellen sich demnach als Formationssysteme von Wissenssegmenten dar, die, wie er hervorgehoben hat, die Bedingungen der Möglichkeit der Produktion bestimmter Äußerungen steuern. Diskurse stellen ein epistemisch wirksames „historisches Apriori“ dar, welches die Produktion, das Erscheinen, die Serienbildung, die Formation und die Wirkungskraft von Aussagen steuert. Berühmt geworden ist die Bestimmung der Diskurse als Zwischenebene zwischen Denken und Sprechen, die Foucault (1971) in der Ordnung des Diskurses hervorgehoben hat. Auf dieser Zwischenebene sind vor allem die diskursiven Mechanismen wirksam, etwa als Ausschließungsmechanismen, als Mechanismen von Produktionszwängen diskursiver Ereignisse, als Strukturierungsmechanismen der Episteme und als Formationssysteme des Wissens. ${ }^{17}$

Das Diskurskonzept von Foucault gründet auf folgenden vier Grundbegriffen: Ereignis, Serie, Regelhaftigkeit und Möglichkeitsbedingung. Der Begriff des Ereignisses betrifft das spontane und häufig unvorhersehbare Auftreten eines epistemischen Elements in einer Äußerung, in einem Text usw. Dieses epistemische Element (bei Foucault - aus linguistischer Sicht etwas missverständlich - enoncé genannt) muss nicht rundweg neu sein (ist es tatsächlich eher selten); ${ }^{18}$ es reicht für die Ereignishaftigkeit das unvorhergesehene Auftreten

16 Siehe der Titel von Foucault (1971): Die Ordnung des Diskurses.

17 Am plausibelsten ist die Annahme, dass Foucault mit dieser „dritten Ebene“ die Ebene des Sozialen im Feld von Wissen und Sprache markieren wollte; siehe zu diesem Zusammenhang ausführlicher (Busse 2013b: 149-151).

18 Zur Problematik des Begriffs enoncé aus linguistischer Sicht und der Entscheidung, ihn als „epistemisches Element“ zu interpretieren, siehe ausführlich die Begründung bei Busse (1987: 227-232). 
in einer neuen diskursiven Umgebung. Treten solche Ereignisse häufiger auf, bilden sie Serien und werden damit zu Keimzellen diskursiver Formationen. Das Stadium der Etablierung neuer diskursiver Strukturen ist erreicht, wenn Serien diskursiver Ereignisse sich zu einer Regelhaftigkeit verdichtet haben. Als Systeme von Regelhaftigkeiten wirken die einmal etablierten diskursiven Formationen bzw. Strukturen schließlich als Möglichkeitsbedingungen der Produktion zukünftiger, thematisch benachbarter diskursiver Ereignisse. Sie steuern nicht nur das aktuale Auftreten, sondern die Auftretensmöglichkeit einzelner epistemischer Elemente in bestimmten Kontexten überhaupt.

Diskurse werden dann von Foucault auch als „Dispersionssysteme von Aussagen“ aufgefasst. Die Diskursanalyse untersucht also diskursive Ereignisse in einem Feld des Wissens und achtet dabei vor allem auf die Bedingungen des Erscheinens einzelner epistemischer Elemente in gegebenen epistemischdiskursiven Kontexten. Diskurse erweisen sich also als geregelte und diskrete Serien von diskursiven Ereignissen, in deren Analyse es vor allem auf die Identifizierung von Regelmäßigkeiten ankommt. In deren Analyse soll - mit den Worten Foucaults - herausgefunden werden ,wie es kommt, dass eine bestimmte Aussage (an einem gegebenen Punkt) erschienen ist und keine andere an ihrer Stelle“ (Foucault 1969: 143; dt. 1973: 159). Im Sinne der im vorliegenden Aufsatz verwendeten Terminologie kann man auch von Serien des Auftretens von Wissenselementen sprechen, und müsste Foucaults Aussage dann reformulieren als Frage, wie es kommt, dass an einem bestimmten Punkt eines Diskurses (Textes) ein bestimmtes Wissenselement aufgetreten ist, und kein anderes an seiner Stelle.

Auf der Grundlage der auf die Rolle der Diskurse und ihrer Bedingungen, Formationen und Bewegungen sowie insbesondere auf die Bedingungen des Erscheinens und der regelhaften Verdichtung des Auftretens von Wissenselementen in den Diskursen bezogenen Überlegungen von Foucault sowie in Aufnahme einiger Intentionen der Begriffsgeschichte nach Koselleck wurde dann in Busse (1987) und Busse \& Teubert (1994) erstmals im deutschen Sprachraum das Modell einer linguistisch-semantischen, diachron orientierten Diskursanalyse formuliert, ${ }^{19}$ das etwa seit der Jahrtausendwende mit Begriffen wie historisch-semantische Epistemologie oder linguistische Epistemologie verbunden ist.

Historisch-semantische Diskursanalyse in der von Busse (1987) vorgeschlagenen Form, also im Sinne einer linguistisch fundierten historischen Epistemologie, ist gemeint als Methode einer deskriptiv und analytisch zugleich verfah-

19 In der einschlägigen Forschungsliteratur wird sie oft auch als historisch-semantische Diskursanalyse, als Diskurssemantik, als Düsseldorfer Schule der linguistischen Diskursanalyse oder ähnlich bezeichnet. 
renden Wissensanalyse. Dieser Wissensanalyse mit linguistischen Mitteln kommt es nicht so sehr (oder nicht hauptsächlich) darauf an, Wissensquanten zu beschreiben (also quasi den Fundus des Wissens, der Episteme, einer Epoche in der Deskription zu duplizieren), als vielmehr darauf, Relationen, Prädispositionen, historische Aprioris zu explizieren und damit sichtbar zu machen, d.h. sie zielt auf die Offenlegung der epistemischen Fundierung einer Aussagen- und Textsemantik (und wohl auch Wortsemantik), und zwar in jeglicher Hinsicht. Diese Fundierung betrifft historisch-epistemische Wirkungsfaktoren, die sowohl Bewusstes als auch Nicht-Bewusstes, nicht explizit Reflektiertes umfassen können - im Sinne der vielzitierten Verortung der Diskurse als „zwischen Denken und Sprechen liegend“ bei Foucault.

Eine historische Epistemologie in diesem Sinne (ob als Begriffsgeschichte im Sinne Kosellecks ausgeführt oder als Diskursanalyse im Anschluss an Foucault) ist dabei immer - ob gewollt oder ungewollt - Teil einer Semantik im weitesten Sinne. Ob sie nun analytisch-deskriptiv und explizit semantisch aufgefasst wird, wie bei Busse (1987) und Busse \& Teubert (1994), oder stärker als machtkritisch verstanden wird (wie bei anderen Forschern) -: Stets verbleibt die Diskursanalyse im Rahmen einer Semantik, d. h. der Entfaltung von gesellschaftlich konstituiertem, historisch bedingtem und relativem Sinn. Schon aufgrund dieses Verständnisses von Semantik muss sich die Diskursanalyse gegen reduktionistische Semantik-Konzeptionen wenden, wie sie etwa in der systembezogenen, in der formalen Linguistik oder in der logischen Sprachphilosophie favorisiert werden. Eher lässt sie sich schon an eine moderne kognitive Semantik anschließen (wie nachfolgend noch weiter ausgeführt werden wird).

Als Teil einer Epistemologie ist die so aufgefasste semantische Diskursanalyse im Rahmen der Kulturwissenschaften verankert. Als Genealogie, wie sie Foucault verortete, hat sie spezifisch historische Anteile. Sie ist eine der Methoden einer „Ethnographie unserer eigenen Kultur“. Ihr konkretes Ziel besteht in der Sichtbarmachung der (zugleich als Wissenselemente in den Blick genommenen) diskursiven Elemente, Strömungen und Relationen, welche das in einer gegebenen Epoche (einem gegebenen diskursiven Rahmen) zu denken und zu sagen Mögliche prädeterminieren und begrenzen. Ihr Ansatz ist analytisch-deskriptiv (wobei Analyse und Deskription nicht voneinander getrennt werden können), weil nur eine sorgfältige, analytisch gelenkte Beschreibung diskursiv-epistemischer Verhältnisse eine Erklärung gegebener Zustände, nämlich der zu beschreibenden Episteme in ihren Konstitutionsbedingungen zu leisten vermag. 


\section{Sprache und Wissen: Systematische Grundlagen einer linguistisch-semantischen Epistemologie}

Die Verknüpfung einer so verstandenen historischen Wissensanalyse mit Zielsetzungen, Aufgaben und Arbeitsweisen einer linguistischen Semantik ergibt sich zunächst über die unabweisbare Tatsache, dass historische Wissensbestände immer nur über Quellen, die als Texte und somit Komplexe sprachlicher Zeichen vorliegen, zugänglich sind. Anders ausgedrückt: Wissen (insbesondere - aber nicht nur - historisches, in vergangenen Epochen ausgebildetes und artikuliertes Wissen) ist immer nur über seine sprachlichen Ausdrucksmittel überhaupt zugänglich und nur über deren Analyse und Interpretation erfassbar und beschreibbar. Im Kontext einer adäquaten Fassung des Begriffs der (sprachzeichengestützten) Bedeutung kommt das menschliche Wissen dabei wie gesehen - als „bedeutungsrelevantes“ oder „verstehensrelevantes“ (oder „verstehensermöglichendes“ oder „bedeutungskonstituierendes“) Wissen ins Spiel.

Eine konsequent wissensanalytisch orientierte Perspektive in der (linguistischen) Semantik muss die Rolle des Wissens, seine Strukturen, Funktionen und sein Eingreifen in den semasiologischen Prozess für alle drei Hauptbereiche jeder Bedeutungstheorie, die erklärungsadäquat sein will, erklären können: für die Konstitution von Bedeutungen, für die Frage, wie etablierte Bedeutungen relativ konstant sein oder werden können (der Aspekt der Bedeutungskonstanz bzw. Konventionalität bzw. der Etablierung und Tradierung von Zeichenverwendungsregeln), und schließlich, mit letzterem eng zusammenhängend: für den Prozess des Bedeutungswandels. Dabei scheint mir vor dem Hintergrund der Forschungsziele der historischen Semantik unstrittig zu sein, dass das Spektrum des bedeutungsrelevanten Wissens, das sowohl bei einer so ausgerichteten Bedeutungstheorie, aber auch bei der praktischen semantischen Analyse (sei sie historisch oder synchron) berücksichtigt werden muss, sehr viel weiter gezogen werden muss, eine größere Menge und eine größere Reichweite von epistemischen Voraussetzungen der textuellen Bedeutungskonstitution in die Analyse einbeziehen muss, als es der eng gefasste Bedeutungsbegriff der herkömmlichen linguistischen Semantik (strukturalistische Merkmalsemantik, klassische lexikalische Semantik, logische Semantik in der Prägung Freges und Carnaps) nahelegt. Eine semantische Analyse, die das, was man üblicherweise „Bedeutung“ sprachlicher Zeichen oder Zeichenketten nennt, auch nur annähernd zureichend erfassen will, muss das bedeutungsrelevante bzw. verstehensrelevante Wissen, 
das in einer vollständigen ${ }^{20}$ semantischen Analyse expliziert werden muss, daher möglichst weitreichend (wenn nicht vollständig) $\mathrm{zu}$ erfassen versuchen. ${ }^{21}$

Der Punkt der Grenzüberschreitung, an dem die Grenzen der traditionellen linguistischen Semantik in Richtung auf eine wissensorientierte Semantik (auch jenseits des - für die Linguistik ja zunächst fachfremden - Kontextes von Koselleck'scher Begriffsgeschichte oder Foucault'scher Diskursanalyse und Wissensarchäologie) überschritten wurden, kann genau markiert werden. Ich demonstriere ihn nicht (wie es auch möglich wäre) bei Foucault; ${ }^{22}$ ich zeige ihn auch nicht bei Wittgenstein; ${ }^{23}$ ich zeige ihn, weil es sich hier um ein linguistisches Forum handelt, bei dem unzweifelhaften Linguisten (und Begründer der Frame-Semantik) Charles J. Fillmore, in dessen Werk man ihn glücklicherweise präzise benennen kann. Man kann diese Grenzüberschreitung, die ich

20 Die Frage, was eine „,vollständige“ Darstellung bedeutungsrelevanten Wissen für ein Zeichen oder eine Zeichenfolge ist, was „vollständig“ in diesem Zusammenhang heißt oder überhaupt heißen kann, und ob „Vollständigkeit“ in irgendeinem Sinne dieses Wortes in semantischen oder begriffsanalytischen Dingen im Sinne einer Darstellung des von Wörtern evozierten (und für sie verstehensnotwendigen) Wissens überhaupt möglich ist, ist eine äußerst schwierige und komplexe, sowohl grundlagentheoretische als auch methodische Aspekte berührende Frage, die hier aus Platzgründen nicht hinreichend diskutiert werden kann. Es sei jedoch angemerkt, dass berechtigte Zweifel bestehen, ob „Vollständigkeit“ in epistemischem Sinne überhaupt jemals erreicht werden kann. Das Prinzip der „unendlichen Verfeinerbarkeit“, wie es etwa der Frame-Theoretiker Barsalou für die von ihm postulierten Frames oder Wissensrahmen immer wieder deutlich herausgestellt hat, spricht stark dagegen; siehe dazu Barsalou (1992: 34, 41-42, 1993: 40-45) und Busse (2012: 379-381, 401-405) mit weiteren Nachweisen, sowie zusammenfassend (Busse 2012: 624-627).

21 Eine „reiche“ Semantik oder „Tiefensemantik“ in diesem Sinne kann sich nicht auf die Explizierung der sozusagen „offen zu Tage liegenden“ epistemischen Elemente von Wort- und Textbedeutungen beschränken, sondern muss gerade auch das zugrundeliegende, versteckte, normalerweise übersehene, weil als selbstverständlich unterstellte Wissen explizieren. Zu dieser Analyse gehört daher auch die Explizierung von in sprachlichen Äußerungen transportierten oder insinuierten epistemischen Elementen, von deren Vorhandensein die Sprecher und Rezipienten der Texte möglicherweise gar kein reflektiertes Bewusstsein haben - das in der analytischen Sprachphilosophie und Pragmatik so bezeichnete „tacit knowledge“ (,stillschweigendes Wissen“). Jede Tiefensemantik, ob als Wortsemantik, Begriffsgeschichte, Satzsemantik, Textanalyse oder Diskursanalyse angelegt, erfordert die Explizitmachung solchen bedeutungskonstitutiven Wissens.

22 Foucault würde von den meisten Linguisten hinsichtlich linguistischer Phänomene ohnehin nicht als satisfaktionsfähig akzeptiert; auch hat er diese Grenzen in allem seinen Schreiben eh schon von allem Anfang an hinter sich gelassen. Ich erinnere in diesem Kontext nur an seinen exzellenten Essay Vorrede zur Überschreitung (Foucault 1974).

23 „Und der Begriff des Wissens ist mit dem des Sprachspiels verkoppelt.“ (Wittgenstein 1970: $\S 560)$ - „Die unsägliche Verschiedenheit aller der tagtäglichen Sprachspiele kommt uns nicht zum Bewußtsein, weil die Kleider unserer Sprache alles gleichmachen.“ (Wittgenstein 1971: 360). 
lieber eine „epistemologische Wende“ in der linguistischen Semantik nennen würde, datieren mit jenem Moment im Jahre 1971, in dem Fillmore für die linguistische Semantik vorschlägt, die übliche (und seiner Ansicht nach falsche) Frage: „Was ist die Bedeutung dieser Form?“ (d. h. dieses Wortes, Satzes) durch die Frage zu ersetzen: „Was muss ich wissen, um eine sprachliche Form angemessen verwenden $\mathrm{zu}$ können und andere Leute $\mathrm{zu}$ verstehen, wenn sie sie verwenden?“ (Fillmore 1971a: 274). ${ }^{24}$ Die ganze (von Fillmore selbst wohl nicht als solche gesehene) epistemologische Radikalität der von ihm vorgenommenen Neubestimmung der Aufgabe der linguistischen Semantik kommt dort zum Ausdruck, wo er die semantische Aufgabe beschreibt als die Erschließung des „vollen Set[s] von Präsuppositionen [...], der erfüllt sein muss für jede aufrichtige Äußerung [eines] Satzes“ (Fillmore 1971a: 277). In neutralerer Terminologie ausgedrückt kann man auch sagen: Bedingung jeder angemessenen semantischen Analyse und Beschreibung ist immer die Explizierung des vollen Sets an epistemischen Voraussetzungen für die Verstehbarkeit eines Wortes, Satzteils, Satzes oder Texts.

Für die Strukturbeschreibung von Wissen jeglicher Art (nicht nur des bei uns hier im Vordergrund stehenden semantisch relevanten bzw. verstehensermöglichenden Wissens) haben sich in der Kognitionswissenschaft und in Teilen der jüngeren Linguistik schematheoretische Modelle durchgesetzt. Eine schematheoretische Modellfamilie, deren analytisches (Wissen und Wissensaspekte aufschließendes) und deskriptives Potential bereits mehrfach unter Beweis gestellt und erprobt wurde, ist die auf dem Begriff Frame ${ }^{25}$ beruhende

24 Den umfassenden, die Grenzen der traditionellen lexikalischen Semantik transzendierenden Anspruch einer epistemisch gewendeten linguistischen Semantik formuliert Fillmore bereits in diesem frühen Stadium seines Werkes mit einer Radikalität, deren Auswirkungen ihm womöglich zu diesem Zeitpunkt selbst noch nicht vollständig klar waren. Jedenfalls erwecken seine Formulierungen immer den Anschein, als sei ihm ihre Radikalität nicht bewusst gewesen; zumindest will er sie wohl nicht - vielleicht aus strategischen Gründen? - explizit propagandistisch formulieren und ausschlachten.

25 Für die systematische Beschreibung der Strukturen und Formen, in denen diese Wissensaktualisierung (und das dazugehörige Schlussfolgern, technisch gesprochen: das Vollziehen von „Inferenzen“) verläuft, ist schon früh der Begriff Rahmen (frame) angeboten worden. Etwa zeitgleich verwenden sowohl der Linguist Fillmore (siehe einführend und als Überblick dazu Busse 2012: 23-132 oder sehr knapp Busse 2009: 80-90) als auch der Kognitionswissenschaftler Minsky (1974) diesen Terminus, den sie beide (wohl unabhängig voneinander) auf den Schema-Begriff des Psychologen und Gedächtnis-Forschers Bartlett (1932) zurückführen. (Parallele Begriffe aus der Kognitionswissenschaft sind etwa scenes, scripts, mit denen jeweils verschiedene spezielle Rahmentypen bezeichnet werden.) Ich habe an anderem Ort vorgeschlagen, als Oberbegriff für die verschiedenen Typen des verstehensrelevanten Wissens den Ausdruck Wissensrahmen zu verwenden. Dieser Terminus lässt es noch offen, wie stark der sprachbezogene Anteil des jeweils betrachteten verstehensrelevanten Wissens bemessen ist oder wird, bzw., ob man die Rahmen 
Analyse, die in der Linguistik als Frame-Semantik in Aktion tritt. In Verbindung mit den spezifischen Zielsetzungen einer linguistischen Epistemologie (sei es im Sinne der historiographischen Begriffsgeschichte oder der in Aufnahme von deren Zielen entwickelten historisch-semantischen Diskursanalyse) können aus der Frame-Semantik spezifische Analyseziele und -schritte in Hinblick auf das historisch-semantische bzw. diskursive Wissen und seine Beziehungen, Strukturen und Tendenzen abgeleitet werden. Die Verbindung zur linguistischen (lexikalischen) Semantik schafft dabei der durch Fillmore prominent gemachte Begriff der Evokation. Wörter (in Sätzen, Texten) evozieren Wissen. ${ }^{26}$ (Die Aktualisierung verstehensrelevanten Wissens „unterläuft“ dabei den Rezipienten häufig genug quasi ,automatisch“ im Zuge selbstverständlichen, „unbewussten“, meist nicht explizit reflektierten Verstehens; sie kann aber auch Ergebnis von das Verstehen vorbereitenden schlussfolgernden geistigen Akten sein.)

Nach Fillmore wie Minsky stellt jeder Wissensrahmen eine standardisierte (prototypikalisch organisierte) Formation von Wissenselementen dar (verbunden durch Strukturen, die als quasi-prädikative Relationen aufgefasst werden können ${ }^{27}$ ), die bestimmte feste Elemente enthält und diese mit Anschlussstellen für variable Elemente kombiniert. ${ }^{28}$ Wissensrahmen sind daher von ihrem Grundaufbau her immer durch Stabilität und Variabilität zugleich gekennzeichnet. ${ }^{29}$ Die zentrale Rolle der Wissensrahmen für jede Art von Semantik (und damit auch für die historische Semantik und eine linguistische Diskursanalyse nach Foucault und die daraus abgeleitete historisch-semantische Epistemologie) liegt nun darin, dass buchstäblich jedes einzelne Wissenselement, das die Bedeutung eines Wortes, Satzes, Textbestandteils ausmacht und für deren Verstehen relevant und unabdingbare Voraussetzung ist, nur durch seine Position in einem Wissensrahmen seine bedeutungskonstitutive Funktion erhält. Zudem bildet auch das kleinste in einem Rahmen positionierte Wissens-

zum „sprachlichen Wissen im engeren Sinne“ oder doch lieber zum „außersprachlichen enzyklopädischen Wissen“ rechnen will, wie es den Mainstream-Linguisten in ihrer aporetischen Dichotomisierung der Semantik vorschwebt; siehe als umfassende Einführung und Überblick zur Frame-Semantik Busse (2012) oder als Kurzdarstellung Busse (2009: 80-90).

26 Sie spielen auf Wissen an, verweisen darauf, operieren damit, ja, man könnte sogar sagen, sie „spielen mit Wissen“. Der dafür meines Erachtens treffendste Ausdruck ist Allusion.

27 Siehe zu diesem Aspekt ausführlich Busse (2012: 687-704).

28 In der üblich gewordenen Terminologie der Kognitionsforschung nennt man dies heute eine slots-and-fillers-Struktur.

29 Das wechselvolle Verhältnis von Stase und Dynamik, welches für die gesellschaftliche wie individuelle Episteme gleichermaßen charakteristisch ist, ist daher bereits in der Grundstruktur der elementaren Bausteine des Wissens angelegt. 
element selbst letztlich wieder eine Art Rahmen niedrigerer Organisationsstufe. ${ }^{30}$ Eine Semantik (eine Bedeutung, einen Begriff, eine Vorstellung) ohne Rahmenstruktur und Einbindung in übergeordnete Wissensrahmen kann es nach dieser Auffassung daher gar nicht geben. ${ }^{31}$ Jedes sprachliche Zeichen erhält seine kommunikative Funktion (nur) durch die Position, die es in einem vorausgesetzten (im sprachlich geäußerten Satz meistens nur teilweise verbalisierten) Wissensrahmen ausfüllt.

Kern jeder Frame-Theorie ist die Bestimmung der inneren Struktur solcher Wissensrahmen als Relationsgefüge aus sog. Leerstellen und ihren Füllungen. ${ }^{32}$ In kognitivistischer Sichtweise sind Frames Strukturen des Wissens, die eine „Kategorie“ (als den Frame-Kern oder Bezugspunkt) inhaltlich bzw. epistemisch näher spezifizieren. Um diese Kategorie, als einem strukturellen FrameKern, der auch als „Gegenstand“ oder „Thema“ des Frames aufgefasst werden kann, ist eine bestimmte Konstellation von Wissenselementen gruppiert, die in dieser Perspektive als frame-konstituierende Frame-Elemente fungieren. Diese Wissenselemente (oder Frame-Elemente) sind keine epistemisch mit konkreten

30 Jedenfalls, wenn man der Gedächtnistheorie von Bartlett (1932) folgt.

31 Ganz abgesehen davon, dass letztlich jede Begriffstheorie eine Art rudimentärer Vorstufe einer Rahmentheorie darstellt (dies kann jedenfalls implizit für die hierarchischen Begriffsgebäude des 17./18. Jahrhunderts gelten, wie sie etwa im 19. und 20. Jahrhundert noch in der sog. Begriffsjurisprudenz nachwirken), ist diese Tatsache den Semantikern, Lexikologen und Begriffshistorikern vor allem deshalb nie aufgefallen, weil ein Großteil des rahmenspezifischen Wissens - wie bereits oben erwähnt - zum Bereich des als selbstverständlich Vorausgesetzten, Nicht-Thematisierten, häufig genug nicht explizit Bewussten gehört. - Einer angemessenen theoretischen Erfassung der rahmenspezifischen Grundstruktur jedes Verstehens und jeder Semantik stand und steht daher eine als natürlich empfundene Alltags-Auffassung von (sprachlicher) Kommunikation im Wege, wonach Sprache in ihrer Grundfunktion gleichbedeutend sei mit dem expliziten Verbalisieren der gemeinten (und $\mathrm{zu}$ kommunizierenden) Inhalte. Man könnte dies die „Explizitheits-Prämisse“ der sog. „relativ-natürlichen Weltanschauung“ (Terminus nach Schütz in Schütz \& Luckmann 1975: 248; vgl. auch Schütz 1971) nennen - eine Annahme, die versteckt auch den meisten wissenschaftlichen Bedeutungskonzeptionen und Sprachtheorien zugrunde liegt. Diese Prämisse kann einer sprachwissenschaftlichen und verstehenstheoretischen Überprüfung jedoch in keiner Weise Stand halten und erweist sich eindeutig als aporetischer Irrtum. Vor allem Fillmore liefert in seinen Texten eine Fülle von anschaulichen Beispielen der Rahmen-Abhängigkeit des semantischen (sprachlichen) Wissens bis weit in die Kernbereiche der Grammatik hinein; vgl. etwa Fillmore (1977).

32 Vgl. Minsky (1974). Es gibt unterschiedliche Termini: Leerstellen (im engl. slots) wurden zum Teil auch als Anschlussstellen (engl. terminals) bezeichnet; bei Barsalou (1992) heißen sie Attribute; Füllungen (engl. fillers) werden auch als Angeschlossenes oder Zuschreibungen (engl. ascriptions) bezeichnet; bei Barsalou (1992) heißen sie Werte (values). Die Grundidee dazu stammt aus der linguistischen (satzgrammatischen) Valenztheorie, die ihrerseits diesen Gedanken als Metapher aus der Valenz der Atome in der Chemie entlehnt hat. 
Daten vollständig „gefüllte“ Größen, sondern fungieren als Anschlussstellen (Slots), denen in einer epistemischen Kontextualisierung (Einbettung, „Ausfüllung“) des Frames konkrete (,ausfüllende“, konkretisierende) Wissenselemente (sogenannte „Füllungen“ oder Zuschreibungen) jeweils zugewiesen werden. Wichtig ist dabei unter anderem, dass Frames (und damit Begriffsstrukturen bzw. die als Bedeutungsstrukturen analysierten Wissensstrukturen) als rekursive Strukturen aufgefasst werden. Jeder Frame ist danach selbst wieder eine Struktur aus Frames, oder, in der Terminologie Barsalous: Jedes Konzept (jeder Begriff) muss selbst wieder als eine Struktur aus Konzepten (Begriffen) aufgefasst werden. ${ }^{33}$

Die Zahl und Art der Attribute eines Frames ist nicht zwingend für immer festgelegt, sondern kann variieren. So können z. B. neue Attribute hinzukommen. Frames werden dann, wie gesehen, meist verstanden als Strukturen aus (hier als rein epistemische Größen aufgefassten) Konzepten, die sich, da alle Konzepte selbst wiederum in Form von Frames strukturiert sind, als Strukturen aus Frames herausstellen. Insofern Frames im Wesentlichen (epistemische) Anschlussmöglichkeiten und -zwänge (für weitere Detail-Frame-Elemente) spezifizieren, ist ihre Struktur beschreibbar als ein Gefüge aus epistemischen Relationen (zu den angeschlossenen Elementen und unter diesen). Der Nutzen des FrameModells für die Semantik besteht nun insbesondere darin, dass bedeutungskonstituierende Relationen zwischen (durch die Zeichen eines Textes eingeführten / evozierten) Wissenselementen sehr viel präziser identifiziert, herausgearbeitet und bestimmten Typen und Funktionen von Relationen zugeordnet werden können als mit herkömmlichen, eher intuitiv-hermeneutischen Verfahren.

\section{Einige methodische Aspekte einer wissensanalytisch basierten historischen Semantik und Diskursanalyse}

Der Gewinn eines Frame-semantischen Vorgehens bei der (historisch-) semantischen Analyse von Textbedeutungen liegt vor allem in Folgendem:

33 Den dieser Überlegung zugrundeliegenden Gedanken der Rekursivität aller Framestrukturen bzw. Konzeptstrukturen bzw. Wissensstrukturen entlehnt der Kognitionswissenschaftler Barsalou (1992) übrigens aus der linguistischen Syntax-Theorie. Rekursivität im syntaktischen Sinn meint die Einbettung einer Sub-Struktur mit einem bestimmten Aufbau in eine (Ober-)Struktur desselben Typs. So enthält etwa eine Nominalgruppe wie das Haus des Bruders des Vaters des Freundes selbst eine Attribut-Nominalgruppe des Bruders des Vaters des Freundes, die wiederum eine Attribut-Nominalgruppe des Vaters des Freundes enthält. - Nach Barsalou sind solche 
1. Das Frame-Modell erlaubt es, wissens- und verstehensrelevante Elemente aufzuspüren, die dem analytischen Blick sonst leicht entgehen könnten; es fungiert dabei vor allem als Such- und Frage-Strategie. Dies gilt z. B. für die Analyse jeglicher Arten von semantischen, intratextuellen, intertextuellen oder diskursiven Beziehungen in der „Bedeutung“ von einzelnen Zeichen wie in Texten oder Diskursen.

2. Das Frame-Modell ermöglicht es, die Art, den Verlauf und die Struktur von Beziehungen/Relationen zwischen einzelnen Wissens-Elementen einer „Wortbedeutung“, eines „Begriffs“, oder eines Textes (einer Textbedeutung) sehr viel präziser $\mathrm{zu}$ erfassen und $\mathrm{zu}$ kategorisieren; das Frame-Modell gibt dabei das Strukturmuster für die Analyse von Relationen vor, indem die einzelnen Elemente einer solchen Bedeutung (ob explizit verbalisiert oder implizit „mitzudenken“) den Ebenen einer rekursiven Frame-Struktur eindeutig zugeordnet werden können. ${ }^{34}$

3. Das Frame-Modell kann das integrierende Moment für eine Vielzahl unterschiedlicher Analysestrategien (wortsemantisch, anaphernanalytisch, argumentationsanalytisch, metaphernanalytisch) sein. Eine semantische Nutzung der Frame-Analyse besitzt mithin wohl ein erhebliches Entwicklungspotential.

Welchen Nutzen kann nun die Frame-Semantik im Kontext einer historischen Semantik (z. B. einer linguistischen Diskursanalyse in dem vom Verf. vorgeschlagenen Sinne) haben ${ }^{35}$ Eine solche historische Semantik, sei es als Begriffsgeschichte, sei es als Diskursanalyse, hat es vielleicht nicht ausschließlich, aber doch weit überwiegend mit sprachlich verfassten Untersuchungsobjekten (Historiker und Philologen nennen sie Quellen) zu tun. Das - in der Regel sprachlich vorliegende - Material ist dabei der Prüfstein und damit Ausgangspunkt jeglicher Analyse. Diskursanalyse ist also zunächst und vor allem Sprachanalyse (oder, wem diese Abschwächung mehr behagt: sprachgestützte Analyse). Zentraler Gegenstandsbereich der Diskursanalyse ist das, was Foucault épistémè nennt. Die von ihm als Grundeinheit der Diskursanalyse eingeführte enoncé kann als eine bestimmte Konstellation von epistemischen Elementen (Wissenselementen) begriffen werden, die als diese Konstellation an

Rekursionen, wenn man das Prinzip innerhalb von Frames bzw. Begriffsstrukturen beschreibt, prinzipiell unendlich möglich, d.h. jeder Frame, jedes Wissenselement (verstanden als Wissensstruktur; bei Barsalou: Attribut-Werte-Struktur) ist ihm zufolge im Prinzip unendlich aufspaltbar bzw. verfeinerbar.

34 Dass dies eine erhebliche „Interpretations-Arbeit“ voraussetzt, soll hier nicht verschwiegen werden.

35 Immer verstanden als „Diskursanalyse nach Foucault“. 
unterschiedlichen Orten, $\mathrm{zu}$ unterschiedlichen Zeiten, in unterschiedlichen Kontexten, Zweckeinbindungen, Interessen vorkommen und durchaus auch in unterschiedlicher sprachlicher oder zeichenhafter Gestalt auftreten kann. Da die enoncés nicht sozusagen frei flottierend in einem platonischen oder fregeschen Reich der puren Gedanken vor sich hin existieren, sondern notwendigerweise des Ausgesprochen-Werdens, Geschrieben-Werdens, Verstanden- und Gedacht-Werdens bedürfen, müssen sie sich mit den Mitteln der Sprach- bzw. Textanalyse, der Semantik, der kulturellen Interpretation dingfest machen lassen können. Ein Format, mit dem man dies besonders gut leisten kann, ist nun das Format der Wissensrahmen oder Frames.

In Bezug auf die Analyse der enoncés hat Foucault (1971: 55; dt. 1973: 37) wie gesehen die vier Kategorien Ereignis, Serie, Regelhaftigkeit und Möglichkeitsbedingung eingeführt. Diese vier Begriffe stützen die Deutung, dass es hier um jeweils spezifische Konstellationen von Wissenselementen geht. Da mit dem Modell der Wissensrahmen Strukturen aus Wissenselementen beschrieben werden, die sich in bestimmter Weise zueinander verhalten, scheint das Frame-Modell geeignet zu sein, enoncés und damit Strukturen und Bewegungen von und in Diskursen zu erfassen. Beispielsweise kann man dasjenige, was Foucault mit dem „Ereignishaften“ des Auftretens einer enoncé meint, recht plausibel mit den im Zusammenhang der Frame-Theorie beschriebenen Synergieeffekten bei Frame-Erweiterungen, Frame-Kombinationen usw. erklären. Die von Foucault erwähnten Regelmäßigkeiten im Auftreten diskursiver Ereignisse können sich auf den verschiedensten Ebenen der Organisation diskursiver Einheiten einstellen. Auch eine Zeichenverwendungskonvention (eine „Bedeutung“) ist eine Regelmäßigkeit im epistemologischen Sinne. Da das Modell der Wissensrahmen aber gerade dafür entwickelt wurde, „Bedeutungen“ sprachlicher Einheiten besser zu beschreiben als ältere Bedeutungsmodelle, findet das Modell der Wissensrahmen auch in dieser Hinsicht ein Anwendungsfeld im Rahmen der Analyse von Diskursen und diskursiven Beziehungen. Auch die vierte von Foucault genannte Analysekategorie für Diskurse, die der Möglichkeitsbedingung, lässt sich mit Bezug auf Wissensrahmen erklären und beschreiben. Gemeint ist damit: Bestimmte epistemische Ereignisse (im diskurstheoretischen Sinne) können überhaupt nur eintreten, wenn in einer vorherigen epistemischen Konstellation die Bedingungen dafür geschaffen wurden, dass diese möglich wurden. Auch diesen Effekt kann man frame-theoretisch gut erklären: Ein Synergieeffekt durch eine Interrelation von zwei zunächst distanten Frames (oder Frame-Komplexen) kann nur dann entstehen, wenn diese Frames (oder Frame-Komplexe) im System des geltenden Wissens überhaupt aufeinander beziehbar waren. Diese Bedingung der Aufeinander-Beziehbarkeit muss aber erst geschaffen werden; sie ist logisch und epistemologisch unabhängig 
davon, ob diese Beziehung tatsächlich hergestellt wird (ob also das diskursive Ereignis, der Synergieeffekt tatsächlich eintritt).

Geht man davon aus, dass es in Diskursen immer um epistemische Phänomene (um Wissen und seine Bewegungen) geht, dann sind diskursive Elemente als epistemische Elemente (Wissenselemente) und diskursive Ereignisse als das konkrete, kontextualisierte und situierte Auftreten solcher Wissenselemente aufzufassen. Dies bildet den Konnex zu den Wissensrahmen als Strukturen des Wissens, in denen die Position und Funktion von Wissenselementen (FrameElementen und Teil-Frames) dingfest gemacht werden können.

Diskursive bzw. diskursanalytisch interessante Aspekte in oder in Bezug auf Frames (Wissensrahmen) können etwa folgende sein:

1. Frame-Elemente (Attribute/Anschlussstellen/Slots) können aufgrund diskursiver Bewegungen/Mechanismen/Zwänge Teil der Frame-Struktur geworden sein.

2. Analog gilt, dass auch bestimmte Füllungen/Werte/Wertebereiche, z. B. insbesondere auch prototypische oder sog. Standardwerte (default values), aufgrund diskursiver Bewegungen/Mechanismen/Zwänge Teil der FrameStruktur geworden sein können.

3. Sowohl Attribute/Anschlussstellen/Slots als auch bestimmte Füllungen/ Werte/Wertebereiche können aufgrund diskursiver Bewegungen/Mechanismen/Zwänge aus einer Frame-Struktur getilgt/ausgeschlossen werden.

4. Diskurswandel kann dazu führen, dass Attribute/Anschlussstellen/Slots wie auch bestimmte Füllungen/Werte/Wertebereiche auch ohne explizit als solche feststellbare Bewegungen/Mechanismen/Zwänge aus einer Frame-Struktur entfallen (Ideenwandel, Wissenswandel, Begriffswandel, Bedeutungswandel ohne explizit strategische Implemente).

5. Diskursive Bewegungen/Zwänge können dazu führen, dass bestimmte Frame-Elemente (sowohl Attribute/Anschlussstellen/Slots als auch Füllungen/Werte/Wertebereiche) in der Aktualisierung von Frames (Instantiierung, token-Ebene) nicht fokussiert oder nicht aktiviert (unterdrückt) werden (ohne vollständig getilgt worden $\mathrm{zu}$ sein). Es gibt sprachliche Strategien, die solche diskursiven Wirkungen unterstützen oder ermöglichen (z. B. die in der linguistischen Sprachkritik ausführlich beschriebene Tendenz zur Ent-Agentivierung, die insbesondere im politischen Sprachgebrauch häufig genutzt wird).

6. Diskursive Bewegungen/Mechanismen können $\mathrm{zu}$ einer internen Umstrukturierung einer Frame-Struktur führen; Frame-Elemente (Attribute/ Anschlussstellen/Slots) können in der Struktur verschoben, anders ,angebunden“ werden, um sie nicht vollständig aufgeben zu müssen.

7. Frame-Elemente (Attribute/Anschlussstellen/Slots) können innerhalb eines bestimmten Frames unterschiedliche Grade an Salienz aufweisen; 
diskursive Bewegungen/Mechanismen können dann eine interne Umstrukturierung in der Salienz-Hierarchie einer Frame-Struktur bewirken, was heißt, dass einzelne Elemente salienter werden als vorher, oder andere ihre Salienz verlieren bzw. ihren Grad an Salienz reduzieren. (Hier besteht wohl ein Zusammenhang mit dem unter 5. angesprochenen Aspekt der diskursgesteuerten Fokussierung.)

8. Diskursive Bewegungen/Mechanismen können Quer-Relationen zwischen Frames bzw. zwischen Frame-Elementen verschiedener Frames etablieren, tilgen oder verschieben.

9. Eine intensive Form der Etablierung solcher Quer-Relationen kann zur Verschmelzung (Amalgamierung oder Frame-Blending) zweier Frames führen. (Frame-Blending findet z.B. regelmäßig beim Entstehen von Metaphern statt.)

10. Möglicherweise können diskursive Bewegungen/Mechanismen/Zwänge zu Umordnungen in Begriffs-Hierarchien (sogenannte Ontologien, frametechnisch gesprochen: Relationen der Frame-Vererbung) führen, etwa dergestalt, dass ein Frame (ein Begriff) seinen übergeordneten Frame (OberBegriff) wechselt, ohne in seiner internen Struktur vollständig umgestaltet $\mathrm{zu}$ werden.

Weitere Auswirkungen diskursiver Bewegungen/Mechanismen/Zwänge auf Wissensrahmen/Frames wären denkbar, die hier noch nicht aufgeführt sind. $\mathrm{Ob}$ alle vorgenannten Beispiele wirklich Relevanz haben, könnte erst eine intensive integrierte frame- und diskursanalytische Forschung ergeben, die bisher noch nicht existiert.

\section{Sprache - Kognition - Kultur: Ziele und Methoden einer historisch- semantischen Wissensanalyse}

Eine epistemologisch ausgerichtete Semantik versucht, das verstehensrelevante Wissen möglichst umfassend zu explizieren und aufzuklären. Da sich in diesem Wissen vorgängige, gesellschaftlich konstituierte und damit kulturell vorgeprägte Schematisierungen (und Frame-Strukturen) niederschlagen, ist eine auf Verstehensbedingungen zielende semantische Forschung schon von allem Anfang her genuin kulturwissenschaftlich orientiert. Indem die epistemologische Semantik über die Suche nach dem verstehensermöglichenden Wissen die Rahmenstruktur des gesellschaftlich geprägten Denkens selbst zu erschließen 
sucht, erschließt sie nicht nur Aspekte des kulturellen Wissens als solchem, sondern erfasst mit ihren spezifischen Mitteln das, was „Kultur“ als solche im Kern ausmacht. Dabei stehen die kulturellen Artefakte, die wir Sprache, Zeichen, Wörter, Begriffe, Texte, Diskurse nennen, prinzipiell auf derselben Ebene wie andere kulturelle Artefakte, die andere Medien benutzen (wie z. B. die bildende Kunst). Die verschiedenen Formen der Kultur benutzen größerenteils dasselbe gesellschaftliche Wissen, setzen dieselben Rahmen- und SchemaStrukturen der Episteme voraus, wie die medial jeweils von ihnen differierenden Formen. Auch die sogenannten „Praktiken“, „Performanzen“, die in neueren kulturwissenschaftlichen Forschungsansätzen so gerne untersucht werden, setzen Schemabildungen, Wissensrahmungen voraus, die sich strukturell von anderen Formen des Wissens im Kern nicht unterscheiden.

Sprache ist (wenn man so will) das „Medium“, in dem sich nicht nur die Artikulation und Kommunikation des gesellschaftlichen Wissens vollzieht, sondern in dem dieses zugleich als solches (d.h. als gesellschaftliches) konstituiert und strukturiert wird. Sprache und die sprachlichen Erzeugnisse (z. B. Wörter, Begriffe, Texte) sind dabei aber keineswegs das „Archiv“ dieses Wissens. Wollte man eine archivalische Metapher in Bezug auf die Sprache überhaupt verwenden, so könnte man sie noch am ehesten als das „Findebuch“, als das „Register“ des Archivs des gesellschaftlichen Wissens charakterisieren. Dieses „Suchregister“ enthält nur Verweise; und zwar Verweise auf etwas, was jeder Sprachverstehende für sich im Prozess des Verstehens (genauer: in den Schlussfolgerungs-/Inferenz-Prozessen, die zum Verstehen führen) allererst epistemisch realisieren und damit konkretisieren muss. Man kann dies im Sinne Husserls (1913: 38) als den Prozess der „Sinnerfüllung“ bezeichnen. Die Sprache als Register des Wissens erfüllt ihre Aufgabe, indem die einzelnen Zeichen und ihre spezifischen Kombinationen jeweils Wissen (Rahmen, Schemata und Rahmen-/Schemakomplexe) „evozieren“ (Fillmore 1982: 117). (Damit wird zugleich deutlich, dass die Zeichen das verstehensrelevante Wissen, ihre „Bedeutung“ nicht „enthalten“ oder „transportieren“.) In dieser Funktion ist die Sprache, wollte man diese Metapher fortspinnen, „zweistufig“: Sprache als Inventar an Zeichen (nach Saussure sagte man: System) ist sozusagen die erste Stufe des wissensverweisenden „Registers“; dessen zweite Stufe stellen die aus (mit) den Zeichen erzeugten Texte dar. Auch Texte sind daher keineswegs „bedeutungserfüllt“ im Sinne Husserls. Sie sind nur Verweisungsmittel sozusagen höherer Aggregationsstufe, die zu ihrem Verstehen ebenfalls erst massiv mit Frame- bzw. Schemawissen ,aufgefüllt“ werden müssen.

Das Rahmenmodell des Wissens (hier als Frame-Semantik genutzt) ist hervorragend geeignet, kognitive, linguistische und kulturwissenschaftliche Perspektiven $\mathrm{zu}$ integrieren. Indem die Rahmenstruktur des gesellschaftlichen 
Wissens in der Funktion des verstehensrelevanten (verstehensermöglichenden) Wissens direkt an die Funktionen von Sprachzeichen (und Sprachzeichenkomplexen wie Sätzen, Texten, Diskursen) gekoppelt ist, kann einer sprachbezogenen Analyse eine Schlüsselrolle für die Untersuchung des kulturellen Wissens zukommen. Innerhalb des Theorie- und Methodenspektrums der modernen Kulturwissenschaften ist dies zum ersten Mal in der Begriffsgeschichte nach dem Konzept des Historikers Koselleck so gesehen und projektiert worden. Aus einer Kritik an der einzelwort-bezogenen Beschränkung von dessen Modell sind - wie gezeigt - alsbald Konzepte einer „Diskursanalyse nach Foucault“ entwickelt worden, die auch in die Linguistik Eingang gefunden haben. All solchen Konzepten gemeinsam ist das Ziel, das in der Benutzung sprachlicher Elemente (Wörter, Sätze, Texte) zum Vorschein kommende, ihr Erscheinen (an diesem Punkt, zu dieser Zeit) ermöglichende Wissen möglichst umfassend zu erschließen. Die methodisch teilweise sehr unterschiedlichen Ansätze einer kulturwissenschaftlichen Semantik konvergieren also in dem Ziel der Erschließung des verstehensrelevanten (verstehensermöglichenden) Wissens. Dieses Wissen geht, wie gesehen, weit über das hinaus, was in traditionellen Modellen der linguistischen Semantik noch als zur Bedeutung gehörig gerechnet wurde. Die Grenzen der klassischen linguistischen Analyse werden in diesen Ansätzen quasi zwangsläufig transzendiert, da eine Beschränkung auf die reduktionistischen Bedeutungsmodelle der formalen Linguistik ungeeignet wäre, eine kulturwissenschaftlich motivierte semantische Analyse auch nur annähernd zu stützen. Da bei einer solchen Form der sprach- und textbezogenen Analyse das Wissen im Vordergrund des Interesses steht, verwende ich für diese Neuorientierung einer kulturwissenschaftlich ausgerichteten linguistischen Semantik die Bezeichnung Linguistische Epistemologie; ist diese historisch ausgerichtet, tritt sie als historisch-semantische Epistemologie auf.

Eine Linguistische Epistemologie in diesem Sinne steht im Kreuzungspunkt von kognitiver, sprachwissenschaftlicher und kulturwissenschaftlicher Perspektive, weil sie einerseits die kognitiven Bedingungen der Möglichkeit der Erzeugung von sprachgestütztem Sinn ernst zu nehmen hat. Zum anderen muss sie aber auch die sozialen, kulturell konstituierten Bedingungen von (kollektivem) Sinn berücksichtigen, da (aufgrund der aus der Arbitrarität erwachsenden Konventionalität sprachlicher Zeichen und anderer kultureller Symbolsysteme) nur über die Sozialität des verstehensermöglichenden Wissens kommunikativer Austausch, Diskursivität, Kulturalität möglich wird. Über die Funktion des „Alter Ego“36 ist Sozialität (und damit Kultur) tief in die Mög-

36 Den der Sozialpsychologe George H. Mead (1934: 152 und passim) einen „generalisierten Anderen" genannt hat. 
lichkeit kommunikativer Verständigung eingeschrieben. Da jeder, der sich sprachlich verständigen will, seine kommunikativen Intentionen und die im Abgleich zu diesen aus dem Repertoire herangezogenen Sprachzeichen und Sprachzeichen-Anordnungen nach dem Kriterium eines hypothetisch unterstellten „generalisierten Anderen“ bildet (bzw. auswählt), ist jeglicher Sprachverwendung (und geschehe sie auch im ,innersten Monolog“) unhintergehbar ein Moment der Sozialität und Kulturalität eingeschrieben.

Jede historische Semantik ist notwendig eine Form von historischer Epistemologie, indem sie das Wissen beschreibt, das für das Verstehen von Wörtern, Texten, Aussagen (enoncés) und kulturellen Artefakten jeglicher Art notwendig ist. Sie unterscheidet sich von herkömmlichen Formen der Semantik (der Geschichtsschreibung, der Textinterpretation) dadurch, dass sie nicht nur das Offenkundige beschreibt, sondern die impliziten Voraussetzungen, das mitschwingende Wissen, das als selbstverständlich Vorausgesetzte und damit nicht bewusst Gemachte, nicht explizit Thematisierte deskriptiv zu erfassen sucht. Wie der Verlauf der neueren linguistischen Semantik gezeigt hat, reicht dieses „implizite“ Wissen (diese nicht explizit thematisierten Wissensvoraussetzungen) weit über den Bereich desjenigen hinaus, das in herkömmlichen Modellen von „Semantik“ und „Textbedeutung“ noch erfasst und als Gegenstandsbereich einer Semantik überhaupt zugestanden wird. Begriffsgeschichte, Diskursanalyse, Mentalitätsgeschichte haben den Blick für dieses quasi „subkutan“ wirkende Wissen erheblich geschärft. Dazu haben unterschiedlichste Disziplinen einen Beitrag geleistet (in der Reihenfolge des historischen Auftretens in diesem Forschungsfeld: Geschichtswissenschaft, Linguistik, Literaturwissenschaft, Wissenssoziologie, neuerdings auch Philosophie und Sozialpsychologie sowie die entstehenden Kognitionswissenschaften, ganz zu schweigen von dem sich erst in Umrissen andeutenden Spektrum der modernen „Kulturwissenschaft“). Vielleicht ist es an der Zeit, eine solche Forschungsperspektive theoretisch wie methodisch „auf den Begriff zu bringen“. Dafür ist aus Sicht des Verfassers am besten eine strikt epistemologische Perspektive geeignet, die Strukturen und Formationsregeln des verstehensrelevanten Wissens direkt in den Blick nimmt, gleich in welcher Form und welchen Zusammenhängen es auftritt, und gleich zu welchen Zwecken und in welchen disziplinären und paradigmatischen Einbindungen es erforscht wird.

\section{Literatur}

Barsalou, Lawrence W. (1992): Frames, concepts, and conceptual fields. In Adrienne Lehrer \& Eva F. Kittay (Hrsg.), Frames, fields, and contrasts, 21-71. Hillsdale, N.J.: Lawrence Erlbaum. 
Barsalou, Lawrence W. (1993): Flexibility, structure, and linguistic vagary in concepts: Manifestations of a compositional system of perceptual symbols. In Alan F. Collins, Susan E. Gathercole, Martin A. Conway \& Peter E. Morris (Hrsg.), Theories of memory, 29-101. Hove, UK/Hillsdale, N.J.: Lawrence Erlbaum.

Bartlett, Frederick C. (1932): Remembering: A study in experimental and social psychology. Cambridge: University Press.

Berger, Peter L. \& Thomas Luckmann (1969): Die gesellschaftliche Konstruktion der Wirklichkeit. Eine Theorie der Wissenssoziologie. Frankfurt a. M.: Fischer.

Bühler, Karl (1934): Sprachtheorie. Die Darstellungsfunktion der Sprache. Jena: G. Fischer. [Neuauflage Stuttgart, New York: Gustav Fischer 1982].

Busse, Dietrich (1987): Historische Semantik. Stuttgart: Verlag Klett-Cotta. (= Sprache und Geschichte Bd. 13, hrsg. von Reinhart Koselleck \& Karlheinz Stierle).

Busse, Dietrich (1991): Textinterpretation. Sprachtheoretische Grundlagen einer explikativen Semantik. Opladen: Westdeutscher Verlag. [stark erweiterte und überarbeitete Neuausgabe als Busse 2014].

Busse, Dietrich (1993/2010): Juristische Semantik. Grundfragen der juristischen Interpretationstheorie in sprachwissenschaftlicher Sicht. Berlin: Duncker \& Humblot 1993. [2. Auflage 2010].

Busse, Dietrich (1997): Semantisches Wissen und sprachliche Information. Zur Abgrenzung und Typologie von Faktoren des Sprachverstehens. In Inge Pohl (Hrsg.), Methodologische Aspekte der Semantikforschung, 13-34. (= Sprache - System und Tätigkeit 22). Frankfurt am Main u. a.: Peter Lang.

Busse, Dietrich (2000): Historische Diskurssemantik. Ein linguistischer Beitrag zur Analyse gesellschaftlichen Wissens. Sprache und Literatur in Wissenschaft und Unterricht, Heft 86 (31), 39-53.

Busse, Dietrich (2003): Begriffsgeschichte oder Diskursgeschichte? Zu theoretischen Grundlagen und Methodenfragen einer historisch-semantischen Epistemologie. In Carsten Dutt (Hrsg.), Herausforderungen der Begriffsgeschichte, 17-38. Heidelberg: Winter.

Busse, Dietrich (2004): Architekturen des Wissens. Zum Zusammenhang von Semantik und Epistemologie. In Ernst Müller (Hrsg.), Begriffsgeschichte im Umbruch, 85-99. (Archiv für Begriffsgeschichte, Sonderheft). Hamburg: Meiner.

Busse, Dietrich (2006): Text - Sprache - Wissen. Perspektiven einer linguistischen Epistemologie als Beitrag zur Historischen Semantik. In Lutz Danneberg, Wilhelm Schmidt-Biggemann, Horst Thomé \& Friedrich Vollhardt (Hrsg.), Scientia Poetica. 10. Jahrbuch für Geschichte der Literatur und der Wissenschaften, 101-137. Berlin, New York: de Gruyter.

Busse, Dietrich (2007a): Sprache - Kognition - Kultur. Der Beitrag einer linguistischen Epistemologie zur Kognitions- und Kulturwissenschaft. In: Jahrbuch der Heinrich-HeineUniversität Düsseldorf 2007, 267-279. Düsseldorf: Universitätsverlag.

Busse, Dietrich (2007b): Diskurslinguistik als Kontextualisierung: Methodische Kriterien. Sprachwissenschaftliche Überlegungen zur Analyse gesellschaftlichen Wissens. In Ingo Warnke (Hrsg.), Diskurslinguistik nach Foucault. Theorie und Gegenstände, 81-105. (= Linguistik - Impulse und Tendenzen 25). Berlin, New York: de Gruyter.

Busse, Dietrich (2008a): Linguistische Epistemologie. Zur Konvergenz von kognitiver und kulturwissenschaftlicher Semantik am Beispiel von Begriffsgeschichte, Diskursanalyse und Frame-Semantik. In Heidrun Kämper \& Ludwig Eichinger (Hrsg.), Sprache Kognition - Kultur. Sprache zwischen mentaler Struktur und kultureller Prägung, 
73-114. (= Jahrbuch 2007 des Instituts für deutsche Sprache). Berlin New York: de Gruyter.

Busse, Dietrich (2008b): Begriffsgeschichte - Diskursgeschichte - Linguistische

Epistemologie. Bemerkungen zu den theoretischen und methodischen Grundlagen einer Historischen Semantik in philosophischem Interesse anlässlich einer Philosophie der Person. In Alexander Haardt \& Nikolaj Plotnikov (Hrsg.), Diskurse der Personalität: Die Begriffsgeschichte der ,Person “ aus deutscher und russischer Perspektive, 115-142. München: Wilhelm Fink.

Busse, Dietrich (2009): Semantik. Eine Einführung. (= UTB 3280 LIBAC Linguistik Bachelor). München: Fink.

Busse, Dietrich (2012): Frame-Semantik - Ein Kompendium. Berlin, Boston: de Gruyter. Busse, Dietrich (2013a): Diskurs - Sprache - Gesellschaftliches Wissen. Perspektiven einer Diskursanalyse nach Foucault im Rahmen einer Linguistischen Epistemologie. In Dietrich Busse \& Wolfgang Teubert (Hrsg.), Linguistische Diskursanalyse: neue Perspektiven, 147-185. (= Reihe Interdisziplinäre Diskursforschung). Wiesbaden: Springer VS.

Busse, Dietrich (2013b): Linguistische Diskursanalyse. Die Macht der Sprache und die soziale Konstruktion der Wirklichkeit aus der Perspektive einer linguistischen Epistemologie. In Willy Viehoever, Reiner Keller \& Werner Schneider (Hrsg.), Diskurs Sprache - Wissen. Interdisziplinäre Beiträge zum Verhältnis von Sprache und Wissen in der Diskursforschung, 51-77. (= Reihe Interdisziplinäre Diskursforschung). Wiesbaden: VS-Verlag.

Busse, Dietrich (2014): Sprachverstehen und Textinterpretation. Grundzüge einer verstehenstheoretisch reflektierten interpretativen Semantik. Wiesbaden: Springer VS.

Busse, Dietrich \& Wolfgang Teubert (1994): Ist Diskurs ein sprachwissenschaftliches Objekt? Zur Methodenfrage der historischen Semantik. In Dietrich Busse, Fritz Hermanns \& Wolfgang Teubert (Hrsg.), Begriffsgeschichte und Diskursgeschichte. Methodenfragen und Forschungsergebnisse der historischen Semantik, 10-28. Opladen: Westdeutscher Verlag, [Nachdruck in Busse \& Teubert (Hrsg.) 2013, 13-30].

Busse, Dietrich \& Wolfgang Teubert (Hrsg.) (2013): Linguistische Diskursanalyse: neue Perspektiven. (= Reihe Interdisziplinäre Diskursforschung).Wiesbaden: Springer VS.

Fillmore, Charles J. (1971a): Verbs of judging: An exercise in semantic description. In Charles J. Fillmore \& D. Terence Langendoen (Hrsg.), Studies in linguistic semantics, 27-289. New York: Holt, Rinehart and Winston.

Fillmore, Charles J. (1975): An alternative to checklist theories of meaning. In Cathy Cogen et al. (Hrsg.), Proceedings of the first annual meeting of the Berkeley Linguistics Society, 123-129. Berkeley: Berkeley Linguistics Society.

Fillmore, Charles J. (1977): Scenes-and-frames semantics. In Antonio Zampolli (Hrsg.), Linguistic Structures Processing. Vol. 5, 55-81. Amsterdam, New York, Oxford: North Holland.

Fillmore, Charles J. (1982): Frame semantics. Linguistics in the morning calm, 111-137. Seoul: Hanshin Publishing Corp.

Fillmore, Charles J. (1985): Frames and the semantics of understanding.: Quaderni di Semantica 6, 222- 254.

Fillmore, Charles (2006): Frame semantics. In Keith Brown (Hrsg.), Encyclopedia of language and linguistics. 2nd Edition, 613-620. Amsterdam: Elsevier.

Foucault, Michel (1966a): Les mots et les choses. Une archéologie des sciences humaines. Paris: Gallimard. (Dt.: Die Ordnung der Dinge. Frankfurt a. M.: Suhrkamp 1971). 
Foucault, Michel (1966b): Entretien: Michel Foucault, ,les mots et les choses‘. In: Les lettres françaises 1125. (Dt. in: Adelbert Reif (Hrsg.) (1973): Antworten der Strukturalisten: Roland Barthes, Michel Foucault, Francois Jacob, Roman Jakobson, Claude Levi-Strauss, 147 - 156. Hamburg: Hoffmann und Campe).

Foucault, Michel (1969): L'archéologie du savoir. Paris: Gallimard. (Dt.: Archäologie des Wissens. Frankfurt am Main: Suhrkamp 1973).

Foucault, Michel (1971): L'ordre du discours. (Leçon inaugurale au Collège de France prononcée le 2 decembre 1970). Paris: Gallimard. (Dt.: Die Ordnung des Diskurses. München: Hanser 1974).

Foucault, Michel (1974): Vorrede zur Überschreitung. In Michel Foucault, Von der Subversion des Wissens. Hrsg. und übersetzt von Walter Seitter. München: Hanser.

Garfinkel, Harold (1973): Das Alltagswissen über soziale und innerhalb sozialer Strukturen. In Arbeitsgruppe Bielefelder Soziologen (Hrsg.), Alltagswissen, Interaktion und gesellschaftliche Wirklichkeit, Bd. 1, 189-261. Reinbek: Rowohlt.

Goffman, Erving (1974): Frame Analysis. An Essay on the Organization of Experience. Boston: North-eastern University Press. [Dt.: Rahmenanalyse. Ein Versuch über die Organisation von Alltagserfahrungen. Frankfurt am Main: Suhrkamp].

Günther, Horst (1978): Auf der Suche nach der Theorie der Begriffsgeschichte. In Reinhart Koselleck (Hrsg.), Historische Semantik und Begriffsgeschichte, 10 -120. Stuttgart: Klett-Cotta.

Gumperz, John J. (1978): Sprache, soziales Wissen und interpersonale Beziehungen. In Uta Quasthoff (Hrsg.), Sprachstruktur - Sozialstruktur. Zur linguistischen Theorienbildung, 114-127. Königstein/Ts.: Athenäum.

Herder, Johann Gottfried (1960): Sprachphilosophische Schriften. Herausgegeben von Erich Heintel. Hamburg: Meiner.

Hermanns, Fritz (1986): Appellfunktion und Wörterbuch. Ein lexikographischer Versuch. In Herbert Ernst Wiegand (Hrsg.), Studien zur neuhochdeutschen Lexikographie, Bd. 6/1 (= Germanistische Linguistik 84-86), 151-182. Hildesheim, Zürich, New York: Olms.

Hermanns, Fritz (1989): Deontische Tautologien. Ein linguistischer Beitrag zur Interpretation des Godesberger Programms (1959) der Sozialdemokratischen Partei Deutschlands. In Josef Klein (Hrsg.), Politische Semantik, 69-149. Opladen: Westdeutscher Verlag.

Hermanns, Fritz (2012): Der Sitz der Sprache im Leben. Beiträge zu einer kulturanalytischen Linguistik. Herausgegeben von Heidrun Kämper, Angelika Linke \& Martin Wengeler. Berlin, New York: de Gruyter

Humboldt, Wilhelm von (1835): Über die Verschiedenheit des menschlichen Sprachbaus und ihren Einfluß auf die Entwicklung des Menschengeschlechts. In: Humboldt 1963, 368-756.

Humboldt, Wilhelm von (1963): Schriften zur Sprachphilosophie. Werke Bd. 3. Darmstadt: Wiss. Buchgesellschaft.

Humboldt, Wilhelm von (1985): Über die Sprache. Ausgewählte Schriften. Herausgegeben von Jürgen Trabant. München: Fink.

Husserl, Edmund (1913): Logische Untersuchungen. 6. Aufl. 1980. Tübingen: Niemeyer. Kleiber, Georges (1998): Prototypensemantik. Eine Einführung. 2., überarb. Aufl. Tübingen: Narr. Koselleck, Reinhart (1972): Einleitung. In Otto Brunner, Werner Conze \& Reinhart Koselleck (Hrsg.), Geschichtliche Grundbegriffe. Historisches Lexikon zur politisch-sozialen Sprache in Deutschland, Bd. 1. Stuttgart: Klett-Cotta, XIII-XXVII.

Koselleck, Reinhart (1978): Begriffsgeschichte und Sozialgeschichte. In Reinhart Koselleck (Hrsg.), Historische Semantik und Begriffsgeschichte, 19-36. Stuttgart: Klett-Cotta. 
Koselleck, Reinhart (2006): Begriffsgeschichten. Studien zur Semantik und Pragmatik der politischen und sozialen Sprache. Mit zwei Beiträgen von Ulrike Spee und Willibald Steinmetz sowie einem Nachwort zu Einleitungsfragmenten Reinhart Kosellecks von Carsten Dutt. Frankfurt am Main: Suhrkamp.

Lotz, Brigitte (2011): Dietrich Busses linguistische Epistemologie. In DER BROCKHAUS Bildung ${ }^{21}$. Wissen für das 21. Jahrhundert, 354-355. Gütersloh, München:

F. A. Brockhaus.

Mead, George Herbert (1934): Mind, self and society. Chicago: Univ. Press. (Dt.: Geist, Identität und Gesellschaft. Frankfurt am Main: Suhrkamp 1968).

Minsky, Marvin (1974): A framework for representing knowledge. In Artificial Intelligence Memo No. 306, M. I. T. Artificial Intelligence Laboratory. [Abgedruckt in: Patrick H. Winston (Hrsg.), The psychology of computer vision, 211-277. New York: McGraw-Hill, 1975. - Auszug abgedruckt in Dieter Metzing (Hrsg.), Frame conceptions and text understanding, 1-25. Berlin, New York: de Gruyter 1980. - Dt. in Dieter Münch (Hrsg.), Kognitionswissenschaft. Grundlagen, Probleme, Perspektiven, 92-133. Frankfurt am Main: Suhrkamp, 1992].

Peirce, Charles Sanders (1931-1935): Collected papers. Vol. 1-8. Cambridge, MA: Harvard University Press 1931-1935 und 1958. [Zitierweise: Bandnummer und Nummer des Textstücks, z. B. 2.275].

Polenz, Peter von (1985): Deutsche Satzsemantik. Grundbegriffe des Zwischen-den-ZeilenLesens. Berlin, New York: de Gruyter.

Reichardt, Rolf (1985): Einleitung. In: Reichardt/Schmitt (Hrsg.) (1985 ff.), 39-148.

Reichardt, Rolf \& Eberhard Schmitt (Hrsg.) (1985 ff.): Handbuch politisch-sozialer Grundbegriffe in Frankreich 1680-1820. München: Oldenbourg.

Sapir, Edward (1929): The status of linguistics as a science. In Edward Sapir, Culture, language and personality. Berkeley, Los Angeles 1952, 65-77. (Zuerst in: Language 5, 1929, 207-214).

Schütz, Alfred (1932): Der sinnhafte Aufbau der sozialen Welt. Eine Einleitung in die verstehende Soziologie. Wien: Springer.

Schütz, Alfred (1953): Wissenschaftliche Interpretation und Alltagsverständnis menschlichen Handelns. In Schütz (1971), 3-54.

Schütz, Alfred (1971): Das Problem der sozialen Wirklichkeit. Gesammelte Aufsätze, Bd. I. Den Haag: Nijhoff.

Schütz, Alfred \& Thomas Luckmann (1975): Strukturen der Lebenswelt. Neuwied, Darmstadt: Luch-terhand.

Stierle, Karlheinz (1973): Geschehen, Geschichte, Text der Geschichte. In Reinhart Koselleck \& Wolf-Dieter Stempel (Hrsg.), Geschichte - Ereignis und Erzählung, 530-535 (= Poetik und Hermeneutik 5). München: Fink.

Stierle, Karlheinz (1978): Historische Semantik und die Geschichtlichkeit der Bedeutung. In Reinhart Koselleck (Hrsg.), Historische Semantik und Begriffsgeschichte, 154-189. Stuttgart: Klett-Cotta.

Whorf, Benjamin Lee (1963): Sprache, Denken, Wirklichkeit. Beiträge zur Metalinguistik und Sprachphilosophie. Reinbek: Rowohlt.

Wittgenstein, Ludwig (1970): Über Gewißheit. Frankfurt am Main: Suhrkamp. [Sigle: ÜG].

Wittgenstein, Ludwig (1971): Philosophische Untersuchungen. Frankfurt am Main: Suhrkamp. [Sigle: PU],

Ziem, Alexander (2008): Frames und sprachliches Wissen. Kognitive Aspekte der semantischen Kompetenz. Berlin, Boston: de Gruyter. 


\title{
Rainer Perkuhn \\ 3 Kontexte und ihre Verteilung
}

\author{
Kookkurrenz und Distribution
}

\begin{abstract}
Die typischen sprachlichen Kontexte, in denen ein Wort verwendet wird, spannen den Rahmen auf, über den sowohl Sprecher als auch Forscher einer Sprache wesentliche Aspekte der Bedeutung des Wortes erschließen und vermitteln. Über große Korpora und entsprechende korpus-, aber auch computerlinguistische Methoden stehen nunmehr systematische Zugänge zu den typischen Verwendungsweisen zur Verfügung, am Institut für Deutsche Sprache etwa über die Kookkurrenzanalyse seit 1995. Auf den Ergebnissen des letztgenannten Verfahrens operieren weitere Methoden, die Bedeutungsbeziehungen zwischen Wörtern auf Ähnlichkeitsbeziehungen des Kontextverhaltens zurückführen. In jüngerer Zeit werden Ansätze vor allem aus der Computerlinguistik und dem information retrieval diskutiert, die mit einem ähnlichen Ziel antreten. Dieser Beitrag soll einen prinzipiellen Überblick bieten, wie die verschiedenen Forschungsstränge den Begriff Kontext interpretieren, wie sie ihn systematisch erfassen und zum Vergleich einsetzen. Neben Bedeutungsnähe wird vor allem Mehrdeutigkeit besondere Beachtung finden.
\end{abstract}

Keywords: Bedeutungsnähe, Distribution, Kontext, Kookkurrenz, Polysemie/ Homonymie, vector space models, word embeddings

\section{Einleitung}

Am Institut für Deutsche Sprache werden seit seiner Gründung 1964 Daten für insbesondere schriftsprachliche Korpora gesammelt und aufbereitet (Teubert \& Belica 2014). Auf welche Art und Weise Nutzer Zugang zu den Daten hatten, war zunächst den technischen Randbedingungen geschuldet - auf die Idee,

Anmerkung: Die überzeugenden Argumente in diesem Bericht gehen zurück auf die gemeinsame Arbeit mit Cyril Belica, Marc Kupietz, Holger Keibel, Harald Lüngen und Peter Fankhauser. Die übrigen Gedankengänge verantwortet der Autor alleine.

Rainer Perkuhn, IDS Mannheim, R5, 6-13, D-68161 Mannheim, E-Mail: perkuhn@ids-mannheim.de

Ә Open Access. (c) 2018 Rainer Perkuhn, publiziert von De Gruyter. (c) BY Dieses Werk ist lizenziert unter der Creative Commons Attribution 4.0 Lizenz.

https://doi.org/10.1515/9783110538588-004 
eine Kopie eines Stapels Lochkarten mit nach Hause nehmen zu wollen, wäre in der Anfangszeit sicher kein Nutzer gekommen. Lange Zeit, bis weit in die Achtzigerjahre des letzten Jahrhunderts, wurden interessierte Linguisten im Umgang mit einfachen Rechercheinstrumentarien geschult, so wie sie von den Rechnerumgebungen bereits mit bereitgestellt wurden. Um diesen Aufwand zu reduzieren, aber auch, um auf die Anfrage- und Analysebedürfnisse der linguistischen Nutzer besser eingehen zu können, wurden und werden eigene Recherchesysteme entwickelt und eingesetzt, beginnend mit REFER (Brückner 1983, 1988/1989) über Cosmas I (al-Wadi 1994) und Cosmas II (Bodmer 2005, 2014) - bis hin zu dem aktuell im Beta-Betrieb anlaufenden KorAP (Bański et al. 2013). Das Angebot der dedizierten Schnittstellen ermöglichte, die Daten vor dem unmittelbaren Zugriff abzuschirmen; im Zuge der technischen Fortentwicklung ergab sich dann quasi automatisch die Recherchemöglichkeit über das zunächst lokale Netzwerk des IDS, später dann über das Internet. Die in den Neunzigerjahren eingeführte Registrierungspflicht für Endnutzer erlaubte die Überwachung individueller Rechercheaktivitäten. Dies war die Voraussetzung dafür, dass bei den Lizenzverhandlungen mit den Textspendern eingeräumt werden konnte, dass die Nutzung der Daten auf rein wissenschaftliche und nicht-kommerzielle Zwecke für einen begrenzten Nutzerkreis (und im Sinne des Zitationsrechts) beschränkt wird. Dieser Auflage müssen Nutzer durch Akzeptieren der Endnutzervereinbarung zustimmen und anerkennen, dass die Zuwiderhandlung sanktioniert wird.

Die Zugangsbündelung unter ein System unter eigener Kontrolle bot und bietet weiterhin die Möglichkeit, die Funktionalität des Systems um fremdoder eigenentwickelte Methoden nach und nach für alle Nutzer zu erweitern. Das zukünftige System KorAP setzt dies par excellence um, indem es Andockmöglichkeiten für externe Module anbietet. Bei den bisherigen Systemen wurde diese Integration noch IDS-intern bewerkstelligt. Außer eigenen Verfahren der Tokenisierung und einem auch heute noch durchaus wettbewerbfähigen Lemmatisierer (Belica 1994) hat das IDS im traditionellen Bereich vor allem extern entwickelte Werkzeuge eingesetzt - zum einen, weil es vermessen gewesen wäre, zu glauben, mit den sehr begrenzten Kapazitäten am IDS etwas noch Besseres entwickeln zu können, zum anderen aber auch, weil jedes Werkzeug nur eine angenäherte Umsetzung einer linguistischen Sichtweise darstellen kann, insbesondere abgesteckt durch den Rahmen des Formalisierbaren und Operationalisierbaren. Insofern ist es durchaus sinnvoll, für einen vorsichtigen Umgang mit derartigen Angaben zu plädieren. Mit dem neuen System KorAP sind nun erstmals die Voraussetzungen geschaffen, mit mehreren konkurrierenden Angaben mehrerer Werkzeuge zu arbeiten, um die Übereinstimmung oder Widersprüchlichkeit dahinterliegender Annahmen oder Meinungen mit untersuchen zu können. 
Die meisten Anfrageszenarien zielten darauf, eine übersichtliche Treffermenge zu erzielen, mit der eine Hypothese überprüft werden konnte oder aus der Nutzer Belege zur Dokumentation und zur Illustration einer bestimmten Verwendungsweise auswählen konnten. Für die Formulierung entsprechender Suchanfragen haben die Anfragesprachen immer ausgefeiltere Ausdrucksmöglichkeiten bereitgestellt. Die Ergebnisse einer Recherche konnten - je nach verfügbaren Metadaten - in verschiedenen Übersichtsformen präsentiert werden oder in der Gesamtdarstellung - etwa gemäß der Dimension „Zeit“ - nach diesen sortiert werden. Kontextuelle Beziehungen ließen sich bei der Sortierung der Ergebnisgesamtansicht über benachbarte Wörter ansatzweise erahnen oder durch die Formulierung von Mehrwortsuchausdrücken vorgeben, in denen wort- und/oder satzbezogene Abstände festgelegt werden.

Mit den immer größer werdenden Datenmengen wuchs das Bedürfnis nach Filter- und Sortierangeboten; gleichzeitig ermöglichten diese den Einsatz statistischer Verfahren, z.B. zur thematischen Klassifikation. Während hierbei wieder im Wesentlichen auf externe Vorarbeiten zurückgegriffen werden konnte, musste in einem anderen Bereich viel Eigenentwicklung betrieben werden, da es noch kein ausreichend elaboriertes Verfahren gab, das den Besonderheiten der deutschen Sprache gerecht geworden wäre. Die Methode der Kookkurrenzanalyse (Belica 1995) ermöglicht es, zu einer gegebenen sprachlichen Einheit herauszufinden, in welchen typischen Kontexten sie verwendet wird. Die wichtigsten Begleiter eines Wortes treten dabei aus der Analyse hervor; sie müssen nicht vorher erahnt und als Teil einer Mehrwortsuchanfrage benannt werden. Bevor die Methode in Kapitel 3 kurz beschrieben wird, soll im folgenden Kapitel zunächst die Tragweite der Dimension „Kontext“ motiviert werden.

\section{Kontext}

Wird das Korpus in einer herkömmlichen Art und Weise genutzt, um Belege zu einer sprachlichen Einheit, einem Wort oder einer Mehrworteinheit, oder $\mathrm{zu}$ einem grammatischen Konstrukt $\mathrm{zu}$ finden, hoffen Nutzer auf eine kleine Treffermenge oder eine schnelle Reduktion, um dann hermeneutisch die einzelnen (Gesamt-)Trefferkontexte auf die Eignung der Belege zu sichten. Für lexikographische Zwecke können dann daraus Belegbeispiele gewählt oder andere illustrative Angaben abgeleitet werden. Bei größeren Treffermengen kommt diese Vorgehensweise schnell an ihre Grenzen; dafür bräuchte es ein Ordnungssystem der Menge anhand kontextueller Eigenschaften. Wenn wir den Blick ein wenig weiter fassen und nicht nur auf Lexikographie begrenzen, so kann man durchaus die These aufstellen, dass jeder Muttersprachler in der Lage ist, dieses Ordnungssystem aus dem eigenen Spracherleben abzuleiten. 
What people know when they know a word is not how to recite its dictionary definition they know how to use it (when to produce it and how to understand it) in everyday discourse [...]. [...] people learn how to use words by observing how words are used. And because words are used together in phrases and sentences, this starting assumption directs attention immediately to the importance of context. (Miller \& Charles 1991: 4)

Auch wenn wir bisher mit keinem Korpus die Datenmenge simulieren können, der ein einzelner Sprecher in seinem Spracherwerb ausgesetzt ist, schon gar nicht in einer chronologisch-inkrementellen Abfolge, so ist es doch einen Versuch wert, das Deutsche Referenzkorpus (Institut für Deutsche Sprache 2017b) als eine halbwegs vertretbare Annäherung an den rezeptiven Sprachstand eines Erwachsenen zu betrachten. Ganz im Sinne von Firth' berühmtem Zitat wäre ein systematischer Zugang zum Kontextverhalten eines Wortes der Schlüssel zu seinem Verständnis:

You shall know a word by the company it keeps! (Firth 1957: 11)

Wie der Begriff Wort aus empirischer Sicht, insbesondere auf die deutsche Sprache angewandt, zu deuten ist, stellen wir noch kurz zurück. Für das folgende Beispiel schauen wir auf Wortformen. Auch wie konkret der Begriff Kontext auszulegen ist, wie viele Wörter oder Phrasen um das betrachtete Wort herum er umfassen soll und ob weitere hierarchisch-syntaktische oder kategorielle Bedingungen betrachtet werden, vereinfachen wir - wie durchaus üblich - zu einem einfachen Textfenster von fünf Wörtern vor und fünf Wörtern nach dem Bezugswort. Etwas ausführlicher klären müssen wir aber, was sich hinter „company“ verbirgt. Es geht Firth offensichtlich nicht um zufälliges, gelegentliches Miteinandervorkommen, sondern um die übliche Gesellschaft, in der sich die Wörter miteinander befinden. Er nennt dies „habitual collocation“ und führt dazu aus:

The habitual collocations in which words [...] appear are quite simple the mere accompaniment, the other word-material in which they are most commonly or most characteristically embedded. (Firth 1957: 11f.)

Firth spielt mit den beiden Adverbien im Superlativ natürlich auf häufiges oder systematisches Miteinandervorkommen an, ohne dass er an irgendeiner Stelle andeuten würde, wie sich die beiden Eigenschaften quantitativ genau fassen ließen. Um einen Eindruck zu bekommen, betrachten wir ein Beispiel. Wir haben mit dem Suchausdruck

Weichsel /w1:5,s0 <Umgebungswort>

mit Hilfe von Cosmas II (Institut für Deutsche Sprache 2017a) die absoluten Häufigkeiten für die entsprechenden Wortkombinationen im Deutschen Refe- 
renzkorpus ermittelt. Für den Ausdruck <Umgebungswort> ist eines der Wörter der ersten Zeile aus Tabelle 3.1 einzusetzen. Der Suchausdruck setzt die beiden Teilbegriffe zueinander in eine positionelle Beziehung, die mindestens ein, höchstens fünf Wörter betragen soll und auf denselben Satz begrenzt wird.

Tab. 3.1: Anzahl Vorkommen verschiedener Umgebungswörter im Kontext von Weichsel.

\begin{tabular}{llllllllll}
\hline & Rhein & Elbe & Wechsel & fließt & Mündung & Ufer & gelegen & Kirsche & total \\
\hline Weichsel & 58 & 91 & 28 & 16 & 41 & 102 & 11 & 10 & 6109 \\
\hline
\end{tabular}

Die Auswahl der betrachteten Umgebungswörter ist handverlesen und durch die später weiter darauf aufbauenden Ausführungen primär didaktisch motiviert. Abgesehen von dem letzten Wort Kirsche, auf das wir später eingehen, sind alle anderen Umgebungswörter auch naheliegend, sofern man weiß, dass mit dem Wort Weichsel ein Fluss bezeichnet wird. Wie können jetzt aber die Zahlen gedeutet werden, gerade auch mit Blick darauf, ob sie charakteristisch oder besonders geläufig sind - zumal andere, hier nicht gezeigte Wörter mit ähnlichen Häufigkeiten in der Umgebung auftreten (z. B. Wochen 13 Mal). Firth und auch später Harris sind sich dieser Vagheit auch bewusst; letzterer deutet aber auch nur die Richtung an, in der bis heute die beste Lösung gesucht wird:

It is rather a question of the relative frequency of such environments ... (Harris 1970: 786)

Was heißt aber hier „relativ“? Der relative Anteil am Vorkommen des Bezugswortes oder der relative Anteil am Vorkommen des Umgebungswortes? Oder eine Mischung aus beidem? Genau genommen geht es darum, zu bewerten, ob die beobachtete Häufigkeit einer Wortkombination größer ist, als sie sein dürfte, wenn die beteiligten Wörter nur zufällig in den Umgebungen voneinander so verteilt wären. Folgendes Zitat bringt es auf den Punkt:

[...] the aim is to compile a list of those syntagmatic items ('collocates') significantly cooccurring with a given lexical item ('node') within a specified linear distance ('span'). 'Significant collocation' can be defined in statistical terms as the probability of the item $\mathrm{x}$ co-occurring with the items $\mathrm{a}, \mathrm{b}, \mathrm{c}, \ldots$ being greater than might be expected from pure chance. (Berry-Rogghe 1973: 103)

Wie groß aber der erwartbare, zufällig noch vertretbare Wert bemessen wird und mit welcher Metrik die Abweichung davon erfasst werden soll, wird immer noch durchaus kontrovers diskutiert. Übereinstimmung herrscht nur bei dem Eingeständnis, dass die Verteilung sprachlicher Phänomene eigenwilliger Cha- 


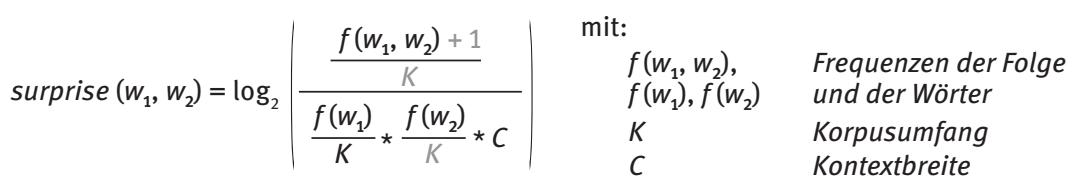

Formel 3.1: Häufigkeit der Kombination: Erwartungswertüberschreitung (ohne grau), Pendant zu pointwise mutual information (mit grau).

Tab. 3.2: Werte gem. Formel 3.1, ohne grau.

\begin{tabular}{lllllllll}
\hline & Rhein & Elbe & Wechsel & fließt & Mündung & Ufer & gelegen & Kirsche \\
\hline Weichsel & 25,80 & 160,84 & 667,09 & 18,44 & 239,49 & 103,50 & 7,73 & 233,73 \\
\hline
\end{tabular}

rakteristika unterliegt, sodass die Anwendung üblicher statistischer Modelle teilweise fragwürdig ist. Trotzdem wird dies in Kauf genommen, um einfache Ansätze entwickeln zu können, so auch an dieser Stelle unserer Argumentation für die Herleitung eines einfachen Maßes.

Um abzuschätzen, wie oft ein Wort (hier: w1) zufällig vorkommen dürfte, berechnen wir seine relative Häufigkeit, indem wir die absolute Häufigkeit durch den Korpusumfang teilen.

Wir ziehen aber nicht nur einmal ein Wort aus dem Korpus, das mit eben dieser Wahrscheinlichkeit das Wort $w 1$ sein könnte (wenn wir ein ganz simples Modell zugrunde legen), sondern wir betrachten alle Wörter in der Umgebung aller Vorkommen des Bezugswortes $w_{2}$ auf so viel Positionen, wie es die Kontextdefinition vorgegeben hat. Diese Berechnung entspricht dem Nenner in Formel 3.1 ohne die grauen Bestandteile. Setzt man diesen nun ins Verhältnis zu der tatsächlich beobachteten Häufigkeit der Kombination (der Zähler ohne grau), erhält man den Faktor, um wie viel häufiger diese auftritt, als es erwartbar wäre; die entsprechenden Werte sind in Tabelle 3.2 wiedergegeben.

Offen gesagt, sind auch diese Angaben nur schwierig genauer zu deuten. Alle Umgebungswörter kommen häufiger vor, als bei einem zufälligen Vorkommen zu erwarten wäre. Der Faktor „um wie viel häufiger“ liegt allerdings um Größenordnungen auseinander. Man ahnt aber schon, dass das Umgebungswort Kirsche trotz eigentlich geringer absoluter Häufigkeit durch diese Bewertung an Bedeutung gewonnen hat.

Anstelle der beiden Terme für absolute Häufigkeiten können wir auch äquivalent relative Angaben einsetzen, indem wir beide durch den Korpusumfang teilen. Nun sind wir noch zwei kleine Schritte von einer Formel entfernt, die tatsächlich in verschiedenen Varianten häufig Verwendung findet. Um die 
Tab. 3.3: Werte gem. Formel 3.1, mit grau.

\begin{tabular}{lllllllll}
\hline & Rhein & Elbe & Wechsel & fließt & Mündung & Ufer & gelegen & Kirsche \\
\hline Weichsel & 4,71 & 7,35 & 9,43 & 4,29 & 7,94 & 6,71 & 3,08 & 8,01 \\
\hline
\end{tabular}

zunehmenden Größensprünge zu dämpfen, wenden wir den Zweierlogarithmus auf den gesamten Term an. Auch wenn es dazu selten eine Begründung gibt, könnte dessen Plausibilität auf eine Parallele zum Weber-Fechner-Gesetz zurückzuführen sein, nach dem unser Einschätzungsvermögen Unterschiede auf höheren Skalenordnungen nicht mehr linear, sondern nur noch nahezu exponentiell abzugrenzen vermag (Fechner 1860/1907). Da das Logarithmisieren des Wertes 0 nicht definiert ist, bedient man sich an der Stelle eines weiteren Tricks und erhöht die Häufigkeiten der Kombinationen um 1, sodass die einzige Gefahr, dass der Zähler Null werden könnte, vermieden wird.

Die Werte dieser Berechnung finden sich in Tabelle 3.3 für die Formel 3.1 mit allen Bestandteilen, mit der wir uns quasi an das Maß pointwise mutual information (pointwise MI) angenähert haben. Dass das Umgebungswort Kirsche nunmehr schon auf den zweiten Rang aufgerückt ist, ist ein Artefakt der Erhöhung der Häufigkeit der Kombination um 1: 11 statt 10 macht sich bei Kirsche stärker bemerkbar als 42 statt 41 bei Mündung. Ansonsten hat sich das Spektrum der Werte nur enger zusammengeschoben; die Rangfolge der Ausschläge ist gleich geblieben. In welcher Weise diese oder andere derartige Werte für eine menschliche Interpretation oder eine weitere automatische Auswertung einfließen können, wird in unterschiedlichen Szenarien unterschiedlich gehandhabt.

\section{Kookkurrenzanalyse des IDS}

Bei der Kookkurrenzanalyse des IDS (Belica 1995) geht es primär um eine erkenntnisleitende Bewertung von Wortkombinationen. Diese werden intern in einer Struktur analog zu Tabelle 3.3 erfasst. Im Standardfall startet ein Nutzer die Analyse selber im Rahmen einer Cosmas II-Sitzung im Anschluss an eine Recherche. Das Verfahren gruppiert dann die Textstellen der Treffermenge anhand der darin entdeckten auffälligen Wortkombinationen und sortiert diese Gruppen nach der gemessenen Auffälligkeit.

Der Nutzer kann hierbei durch die Wahl der Suchanfrage vorgeben, welche Art sprachlicher Einheit untersucht werden soll. In den gängigsten Konstellati- 
onen einer Einwortsuchanfrage ist dies entweder eine konkrete Wortform oder eine Grundform, ein sogenanntes Lemma, oder als Mischung davon eine Aufzählung mehrerer Formen, etwa eine Teilmenge eines Flexionsparadigmas. Zu den Trefferobjekten kann dann der Kontext frei definiert werden, wie viele Wörter links und rechts betrachtet werden sollen und ob Satzgrenzen den Kontext auf jeden Fall beschneiden. Die Häufigkeiten aller in diesem Kontext beobachteten Umgebungswörter werden dann gezählt und vor dem Hintergrund ihrer Frequenz im Gesamtkorpus statistisch bewertet - allerdings nicht mit dem oben hergeleiteten Maß, sondern mit dem Loglikelihood Ratio (Dunning 1993), dessen Formel wesentlich komplizierter aufgebaut ist und hier nicht weiter erklärt werden kann, aber sich für viele Fragestellungen in unserem Umfeld bewährt hat. Im Grunde versucht es aber auch, auf eine etwas andere Art und Weise $\mathrm{zu}$ bewerten, wie überraschend es ist, dass Wörter gemeinsam so oft vorkommen im Vergleich zu einem zufälligen Nebeneinanderstehen. Für die Partnerwörter kann der Nutzer wählen, ob Funktionswörter mit betrachtet oder ausgeblendet werden sollen. Dies hat aber keinen sonstigen Einfluss auf die übrigen im Kontext betrachteten Wörter. Des Weiteren kann - unabhängig von der sprachlichen Einheit, nach der gesucht wurde - festgelegt werden, ob die Umgebungswörter getrennt nach Wortformen bewertet werden, oder ob mehrere Formen, die einem gemeinsamen Lemma zugeordnet werden können, zusammen betrachtet und kumuliert bewertet werden sollen. Für diese Vorgaben wird dann die statistische Bewertung vorgenommen, sodass eine Tabellenzeile entsteht, vergleichbar zu dem Beispiel aus dem vorherigen Kapitel. Die Zellen dieser Zeile werden dann nach dem Assoziationsmaß sortiert. In Abhängigkeit von der Zuverlässigkeit der statistischen Bewertung (sozusagen „der Zuversicht in sich selbst“) kann der Nutzer entscheiden, wie lang der vordere Abschnitt der sortierten Liste ist, die zunächst festgehalten werden soll. Das Verfahren wiederholt jetzt quasi für jedes Ergebnis eines vorhergehenden Durchlaufs eine etwas modifizierte Bewertung, ob zu den bereits erkannten auffälligen Wortkombinationen noch weitere Wörter hinzutreten, die in der Umgebung der Wortkombination besonders auffällig oft beobachtet wurden. Auch hierbei kann der Nutzer vorgeben, wie sicher die Auffälligkeit eingeschätzt werden soll, sodass unterschiedlich granulare Strukturen entstehen.

Das Gesamtergebnis einer Analyse, alle ermittelten Angaben zur Liste der auffälligen Umgebungswörter, wird in Cosmas II in einer eventuell sehr umfangreichen Tabelle, einem sogenannten Kookkurrenzprofil, dargestellt, aus der wir hier nur einen kleinen Ausschnitt zeigen (vgl. Abb. 3.1) zu der Zweierkombination Elbe o gelegen, sowie der feiner granularen Dreierkombination Elbe 。 gelegen o idyllisch.

Neben den bereits erläuterten Angaben werden zu den Wortkombinationen stets die Textstellen einblendbar angeboten (verborgen hinter dem $⿴$ ), die den 


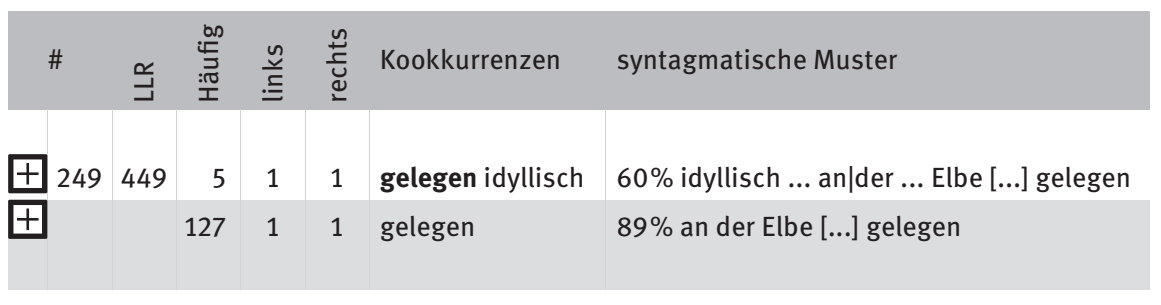

Abb. 3.1: Eine ausgewählte Kookkurrenzstruktur zu dem Bezugswort Elbe.

analytischen Befund legitimieren. Somit kann das Kookkurrenzanalyseverfahren als ein Gruppier- und Sortierverfahren gedeutet werden, das Textstellen einer Treffermenge mit ähnlichen Kontextmustern zusammenfasst, d. h. nach dem Ordnungssystem, das wir uns im ersten Kapitel gewünscht hatten. Aus den jeweiligen Textstellen einer Gruppe wird als Interpretationshilfe ein syntagmatisches Muster abgeleitet, mit dem versucht wird, die hervorstechende lineare Anordnung relevanter Bestandteile des Musters zu rekonstruieren. Mit all diesen Optionen kann der Nutzer im Grunde vielerlei unterschiedliche Kookkurrenzanfragen an den jeweils zugrunde gelegten Datenbestand formulieren; die Kookkurrenzanalyse ist somit nicht ein einziges Verfahren, sondern steht für eine ganze Sammlung verschiedener Instantiierungen. Ausführlicher beschrieben ist dies in Perkuhn, Keibel \& Kupietz (2012: Kap. 8).

Eine grundsätzliche Eigenschaft der Herangehensweise ist jedoch, dass im Zweifelsfall lieber viele Wortverbindungen angeboten werden, ohne dass diese durch gewisse Vorgaben des Nutzers vorweg gefiltert werden - etwa durch syntaktische oder dependentielle Vorgaben und auch nicht durch Angaben wie Wortklassen. Auch wenn aus Sicht vieler Anwendungen gerade dies oft wünschenswert erscheint, nimmt der Ansatz den Nutzer in die Pflicht, selber seine Vorstellungen in qualitative Entscheidungen umzusetzen und die Analyseergebnisse zu interpretieren. Viele der dabei einfließenden menschlichen Bewertungen lassen sich nicht in einem zufriedenstellenden Maß operationalisieren; gerade bei Wortverbindungen lassen sich Beispiele mit einer Genauigkeit von weniger als 0,1\% angeben (Recall eines kommerziellen Tools bei werden plus sein als Pronomen). Unabhängig davon sollte es für Forscher auch durchaus lohnend sein, zu schauen, wie die Typen von Wortverbindungen, die sie gerne sehen und untersuchen möchten, sich im Verhältnis zu anderen Wortverbindungen verhalten. Sind alle Verbindungen, die als höher auffällig bewertet wurden, für sie nicht relevant, können diese ignoriert werden. Wenn dies aber nicht durchgängig gilt, wäre ein vorheriges automatisches Ausblenden fatal gewesen. 
In speziell einer Hinsicht ist die Untersuchung von Wortverbindungen der deutschen Sprache besonders schwierig: Es lässt sich kaum vorgeben, in welchen positionellen Beziehungen die Elemente zueinander stehen. Man könnte zwar gewisse Erwartungen in Abhängigkeit von Wortklassen grob schematisch in bestimmte Typen einteilen, zumindest was die Beziehungen innerhalb von Präpositional- oder Nominalphrasen angeht, sofern die Wortklassen verlässlich bestimmt werden konnten. Aber selbst dabei kann nicht ausgeschlossen werden, dass z. B. flektierte Adjektivformen nicht immer nur eine Position vor dem Substantiv besonders auffällig sind, sondern durch eine typische Adjektivreihung auch systematisch weiter davor stehen können (z. B. blonde [glattel gelockte/gewellte] Haare). Für andere Konstruktionen, wie die prädikative Verwendung von Adjektiven oder Wortpartnerschaften zu Verben, ist dies ohnehin komplizierter. Der gewählte Ansatz ist, den Kontext als Textfenster definieren zu können. Bei großer Unsicherheit kann dieses Fenster auch vorsichtshalber sehr weit ausgedehnt werden, auf Kosten der Antwortzeit auch durchaus größer als der Standardwert von fünf Wörtern links und fünf Wörtern rechts. Da das statistische Verfahren aber die Vorkommen innerhalb dieses (nunmehr deutlich größeren) Ausschnitts mit dem Vorkommen im Korpus insgesamt vergleicht, benachteiligt der große Kontext Phänomene, für die ein kleiner Kontext ausreichend gewesen wäre. Anstatt verschiedene Kontextdefinitionen selber ausprobieren und vergleichen zu müssen, übernimmt diese Funktionalität ein zusätzlicher Mechanismus, der die anderen Optionen ergänzt: der Autofokus. Wählt der Nutzer diesen mit hinzu, so wird der definierte Kontext als maximaler Kontext interpretiert, zu dem alle denkbaren, zusammenhängenden Unterabschnitte getrennt ausgewertet und die darin beobachteten Vorkommen statistisch bewertet werden. Die Grenzen des am höchsten bewerteten Abschnitts werden mit angezeigt (in Abb. 3.1 die Spalten „links“ und „rechts“, mit der Bedeutung: mit Vorzeichen Minus = vor dem Bezugswort, ohne Vorzeichen = nach dem Bezugswort). Das Gesamtbild der nach dem so ermittelten Auffälligkeitsmaß sortierten Kookkurrenzen wird dadurch gewissermaßen leicht verzerrt. Dies kann man vertreten, da die Phänomene, für die ein kleinerer Kontext besser gewesen wäre, davon profitieren und in dem Sinne an Relevanz aufsteigen. Beim Vergleich der verschiedenen Elemente innerhalb eines Kookkurrenzprofils sollte man aber trotzdem im Hinterkopf behalten, dass diese zum Teil Antworten auf verschiedene Fragen darstellen.

Beim Betrachten eines Gesamtkookkurrenzprofils (wie z. B. in Abb. 3.2) zeigt sich der Effekt des sehr weiten, im Wesentlichen statistischen Kookkurrenzbegriffs: Es finden sich verschiedene Arten von Wortverbindungen, die alle statistisch legitimiert, aber sprachlich teilweise sehr unterschiedlich motiviert sind. Neben syntaktischen Konstruktionen (östlich [der] Elbe), Paar- 


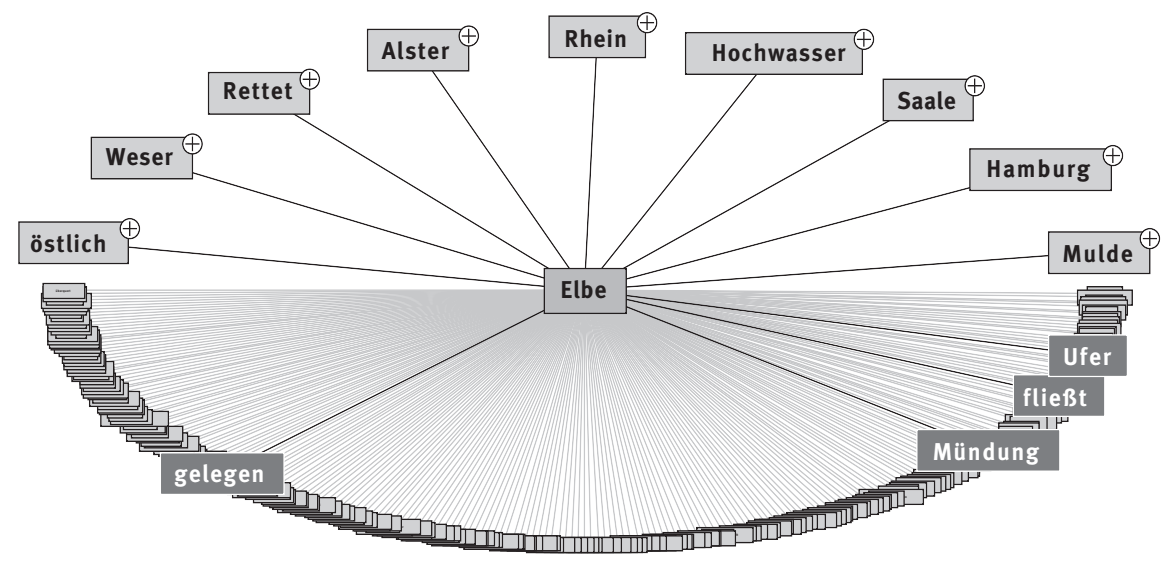

Abb. 3.2: Kookkurrenzprofil des Wortes Elbe (CCDB, visualisiert nach Perkuhn 2007a).

formeln oder Ausschnitten aus Aufzählungen, meist von Kohyponymen (Weser [und] Elbe) finden sich auch Floskeln oder Namen von Bündnissen oder Ähnliches (Rettet [die] Elbe). Einzelne Partnerwörter dazwischen lassen sich über die geografische Verortung der Elbe erklären. Für weitere Mehrwortfügungen wie Kollokationen (im engeren Sinne) und Redewendungen ist unser Beispiel schlecht gewählt; diese ließen sich bei entsprechenden Verben, Adjektiven oder Nomina, die keine Eigennamen sind, analog entdecken. Um das Kookkurrenzverhalten eines Wortes zu erkunden und zur Gänze zu erschließen, bedürfte es also weiterer methodischer Unterstützung (vgl. Perkuhn 2007a, b). Trotzdem ahnt man beim Betrachten schon, dass einige Partnerwörter Hinweise auf die Bedeutung liefern, unabhängig davon, ob es nicht-transparente Fügungen sind, die lange Zeit den Schwerpunkt der Kollokationsforschung ausgemacht haben, oder transparente Kombinationen, die gezielt aus diesem Schwerpunkt herausdefiniert wurden, deren Stellenwert aber gerade für die Fremdsprachendidaktik schon früh gewürdigt wurde - gerade weil sie eben auch zu der Vermittlung von Bedeutung im Kontext beitragen (Bahns 1997: 48). Mittlerweile lässt sich sicher auch erahnen, warum ausgerechnet die fünf Wörter als Umgebungswörter für die Beispiele im ersten Abschnitt gewählt wurden. Vier davon sind auch in dem Kookkurrenzprofil des Wortes Elbe vorhanden und in Abbildung 3.2 hervorgehoben, da wir für unseren roten Argumentationsfaden postulieren, dass diese Wörter zu der Bedeutung ,Fluss' beitragen.

Auch wenn es nach dem vorher Gesagten etwas widersinnig klingt, da wir die Kookkurrenzanalyse als eine Vielzahl von Verfahren beschrieben haben, so haben wir doch eine Standardkonfiguration als prototypisch ausgewählt. Mit dieser wurde bis 2007 eine Datenbank von Kookkurrenzprofilen aufgebaut. In 
dieser Kookkurrenzdatenbank CCDB (Belica 2007) werden Methoden entwickelt und experimentell angeboten, die versuchen, die Beziehungen zwischen Wörtern auf Ähnlichkeiten ihrer Profile zurückzuführen.

\section{Kookkurrenz(profil)-auswertende Methoden}

Nicht nur in der Kookkurrenzdatenbank CCDB, auch in anderen Ansätzen ist ein zentrales Konzept eine zusammenfassende Beschreibung des Kontextverhaltens eines Wortes. Bei diesen ist der Erkenntnismehrwert der Struktur aber untergeordnet unter nachfolgende Auswertungsschritte, die auf dieser Struktur operieren sollen. Die Gesamtheit dieser Struktur wird in Anlehnung an Harris mit Distribution bezeichnet:

The DISTRIBUTION of an element is the total of all environments in which it occurs, i.e. the sum of all the (different) positions (or occurrences) of an element relative to the occurrence of other elements. (Harris 1960: 15 f., Hervorhebung im Original)

Eine Unterscheidung der Verteilung von Instanzen, d. h. von konkreten Formulierungen, die sich zu Kookkurrenzen zusammenfassen lassen, oder von Kookkurrenzen (im Sinne von Kontexttypen), die ein Kookkurrenzprofil manifestieren, wird in diesem Zusammenhang nicht vordergründig thematisiert. Das Hauptanliegen dieser Ansätze ist, die Beziehung zwischen zwei Wörtern auf einen Vergleich ihrer Distributionen zurückzuführen:

If $\mathrm{A}$ and $\mathrm{B}$ have almost identical environments [...], we say they are synonyms [...]. If A and B have some environments in common and some not [...] we say that they have different meanings, the amount of meaning difference corresponding roughly to the amount of difference in their environments. (Harris 1970: 786)

Auch wenn wir mit Kookkurrenzprofilen als eine Abstraktion über dem Gesamtkontextverhalten gedanklich schon einen Schritt weiter waren, wollen wir die Idee des Vergleichs analog zu der Struktur in Kapitel 2 noch einmal elementar entwickeln.

Sollte mit Distribution tatsächlich die absolute Frequenz von Vorkommen von Umgebungswörtern gemeint sein, so ergäben sich für die Wörter Rhein und Elbe die beiden Säulenabschnitte links in Abbildung 3.3 für die Umgebungswörter, die wir auch in Kapitel 3 schon nicht ganz zufällig ausgewählt hatten. In Kapitel 2 war noch dasselbe Wort sowohl als Bezugswort als auch als Umgebungswort aufgeführt. Welchen Beitrag allerdings eine zweite Instanz desselben Wortes in seinem typischen Kontextverhalten zu dessen „Beschrei- 


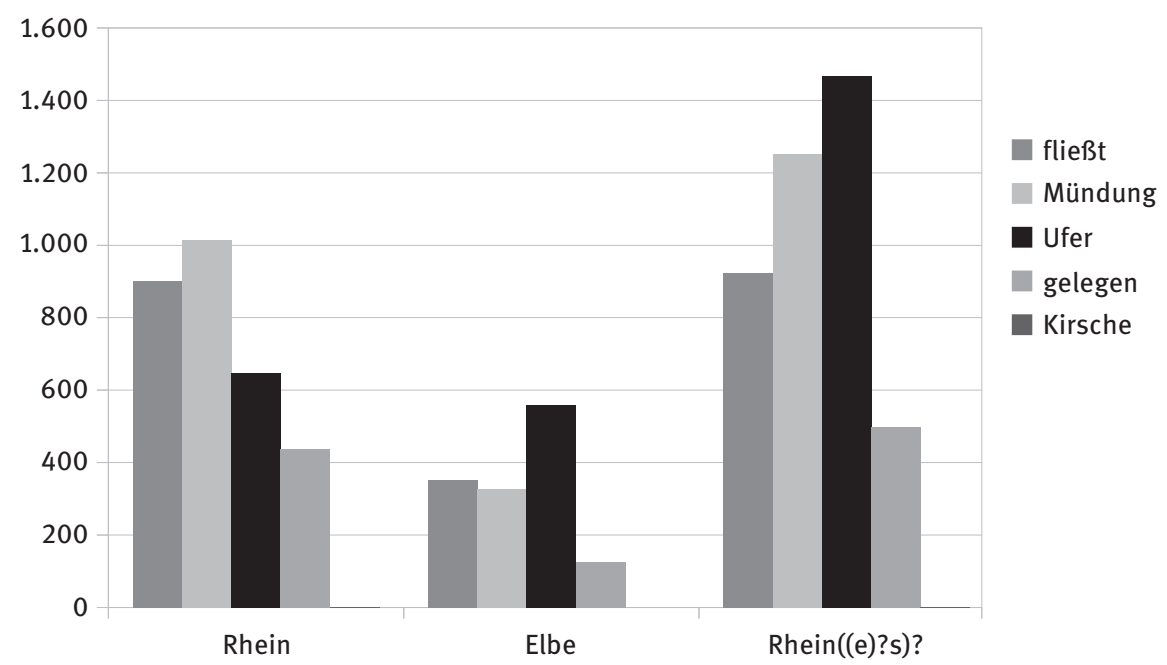

Abb. 3.3: Absolute Häufigkeiten von fünf Umgebungswörtern dreier Bezugswörter.

bung“ beitragen kann, ist vollkommen unklar. In manchen anderen Fällen lässt es sich vielleicht plausibel sinnbringend erklären ([von einem] Ufer [zum anderen] Ufer); einen neuen Beitrag zum besseren Verständnis des Wortes liefert es aber vermutlich nicht. In dieser und manch anderer Hinsicht sind unsere gewählten Beispiele, insbesondere auch als Eigennamen, etwas speziell für den eigentlichen Kerngedanken der Argumentation aber sehr gut geeignet.

Wenn es im Folgenden dann aber darum geht, zwei Beschreibungen miteinander $\mathrm{zu}$ vergleichen, wird es etwas kritischer, wenn das Vorkommen des Wortes Rhein in der Nähe von Rhein eine andere Qualität hat als dasselbe Wort in der Nähe von Elbe: Während der erste Fall quasi redundant ist, liefert der zweite durchaus einen Hinweis darauf, dass die Wörter paarig oder als Teil einer Aufzählung verwendet werden könnten, was für Kohyponyme durchaus nicht ungewöhnlich ist. Dieser besondere Status der am Vergleich beteiligten Wörter sollte entweder im Vergleichsverfahren, spätestens aber bei der Interpretation berücksichtigt werden. Der Einfachheit halber konzentrieren wir uns im Weiteren nur auf die übrigen Wörter.

Da das Wort Rhein viel frequenter ist als das Wort Elbe, ließe sich analog zu dem in Kapitel 2 Gesagten schon nicht annehmen, dass die beiden Wörter kongruente Silhouetten ergeben. Denn das wäre das grafische Pendant für den (hier künstlich) kleinen Ausschnitt an Umgebungen, die wir betrachten, zur distributionellen Hypothese. Der nahezu gleichgroße Ausschlag bei dem Partnerwort Ufer ist sogar eher störend, da er den Gesamteindruck der Kontur 
durchbricht, weil er unverhältnismäßig niedrig liegt. Hintergrund dazu ist ganz einfach, dass die Kombination von Ufer typischerweise mit dem Flussnamen im Genitiv vorkommt. Dieser ist bei der Elbe aber nicht markiert ([am] Ufer [der] Elbe), somit bereits mit berücksichtigt. Beim Rhein ist dies allerdings nicht der Fall ([am] Ufer [des] Rheins). So, wie die Suchanfrage formuliert war, haben wir den Rhein in der Hinsicht benachteiligt. Nehmen wir Genitivendungen explizit mit hinzu (in Abb. 3.3 mit einem regulären Ausdruck für „Rhein oder Rheins oder Rheines“), stellen wir faire Verhältnisse her. Da nun die Treffermenge noch einmal vergrößert wurde, sind alle Kombinationshäufigkeiten und dementsprechend die Säulen höher. Auch wenn sich jetzt alles auf einem höheren Niveau abspielt, haben wir doch einen sichtbaren Erfolg: Das Umgebungswort Ufer ordnet sich nunmehr anders in das Gesamtbild ein und bildet wie bei Elbe die höchste Säule. Dass die Säule zum Umgebungswort Mündung von der Maßnahme nicht so stark betroffen ist und im Kontrast zu Elbe immer noch häufiger als die übrigen Umgebungswörter gebucht ist, liegt einfach daran, dass im Vergleich zu seiner eigenen Mündung (Mündung [des] Rheins) viel öfter über andere Flüsse berichtet wird, die in den Rhein münden - und davon gibt es einige (Mündung [des/der Neckars/Main/Mosel/Ruhr in den] Rhein). Dieser Effekt ist offensichtlich den Zusammenhängen in der realen Welt geschuldet (und unserer Entscheidung, Eigennamen als Beispiele zu wählen); er verzerrt insbesondere die absoluten Häufigkeiten unabhängig von der (Kern-)Bedeutung des Bezeichneten. Eine vergleichbare Verzerrung können wir uns aber für jedes Wort einhandeln, insbesondere in Abhängigkeit von der Zusammensetzung des zugrunde gelegten Korpus. Je nach Ausrichtung (Textsorte, Textgenre) oder auch durch jeweils aktuellen Einfluss des Tagesgeschehens auf insbesondere Zeitungskorpora, können in bestimmten Diskursen Wörter, Lesarten oder Wortkombinationen (zumindest zeitweise) besonders intensiv genutzt werden.

Zumindest vor den punktuellen Einflüssen kann man sich durch eine breitere und somit meist auch größere Datengrundlage schützen; grundsätzlich gilt aber immer, dass es - wie schon in Kapitel 2 dargelegt - besser ist, mit relativen Häufigkeiten zu argumentieren. Aus diesen lässt sich dann auch der Wert ableiten, der für die Kombination zu erwarten wäre, sowie der Faktor, um wie viel öfter die Kombination beobachtet wurde. In Abbildung 3.4 ist dieser Wert für die Bezugswörter Elbe, Rhein (im Folgenden nun stets inklusive Genitiv) und Weichsel und die bekannten fünf Umgebungswörter dargestellt.

Die Konturen von Elbe und Rhein haben sich nun wunderbar angenähert. Für Weichsel ahnt man ansatzweise auch eine Ähnlichkeit für die ersten vier Säulen. Da das Wort Weichsel aber im Vergleich zu den anderen beiden sehr selten im Korpus vorkommt, schlagen die Säulen etwas überbewertet aus. Im Sinne der gleichen Argumentation wie in Kapitel 2 schaffen wir durch Logarithmisieren eine bessere Vergleichbarkeit, wie sie sich in Abbildung 3.5 zeigt. 


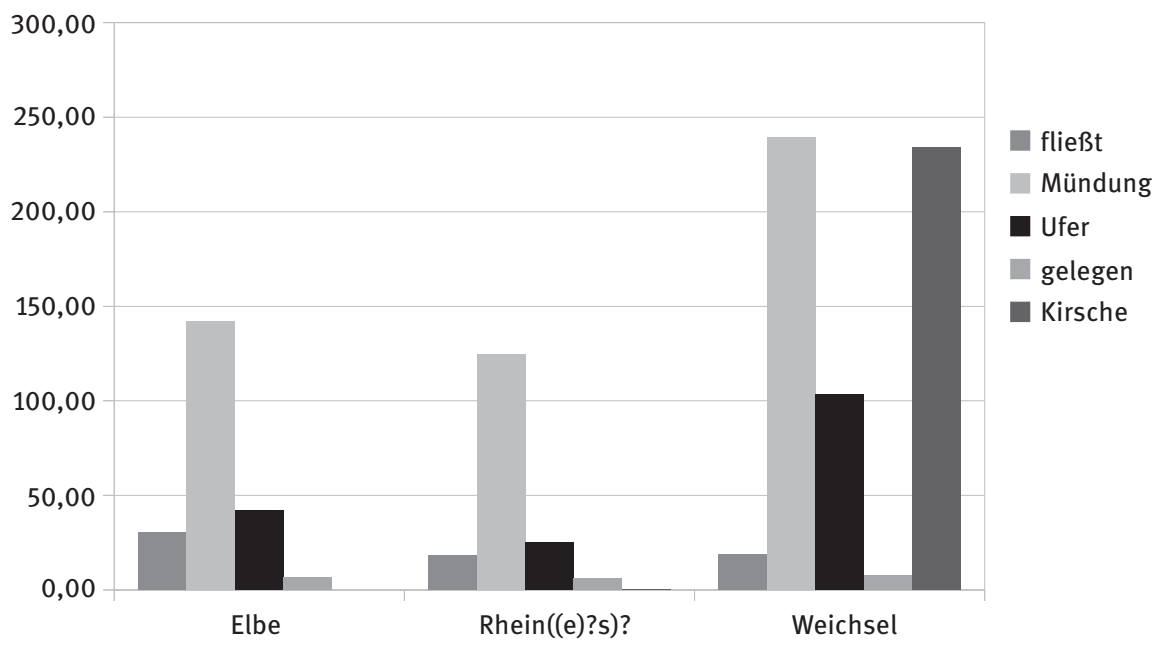

Abb. 3.4: Verhältnis des beobachteten zu dem erwarteten Wert.

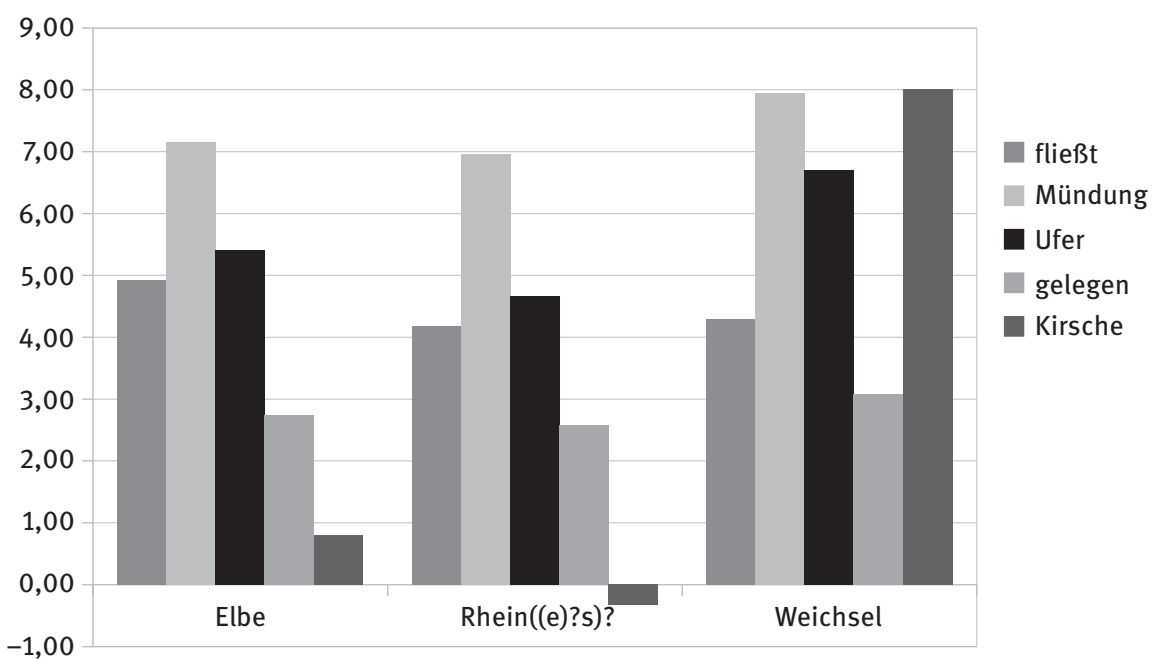

Abb. 3.5: Angaben aus Abbildung 3.4, nur als Zweierlogarithmus, quasi pointwise mutual information.

Mit einer gewissen Toleranz zeigen die ersten vier Säulen ein sehr ähnliches Schema. Die fünfte Säule für das Umgebungswort Kirsche fällt aus dem Rahmen und zeigt zudem, wie der Trick des letzten Schritts bei kleinen Zahlen plötzlich eine (eigentlich nicht erwünschte) Wirkung zeigt. Der Erwartungswert 


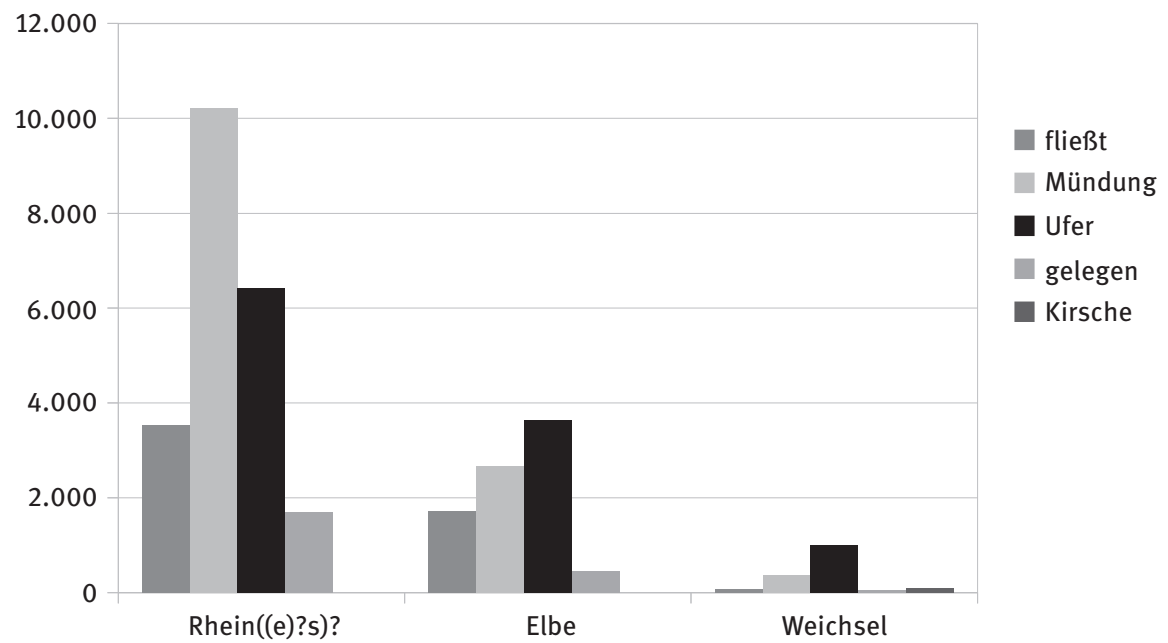

Abb. 3.6: LLR der betrachteten Wortkombinationen.

für Kirsche in der Umgebung von Elbe lag bei 0,58 und wurde mit tatsächlich beobachteten 0 Vorkommen unterschritten. Durch die Addition von 1 sieht es nun fälschlicherweise so aus, als ob der Erwartungswert leicht überschritten wäre. Der Erwartungswert für Kirsche in der Umgebung von Rhein lag bei 2,51 und wurde mit tatsächlich beobachtetem 1 Vorkommen unterschritten. In diesem Fall hat der von uns beschönigte Wert von 2 sich nicht so sichtbar niedergeschlagen, da er immer noch unter dem Erwartungswert geblieben ist. Der einzige wirklich beobachtete Treffer ist dabei zwar berechtigt, aber eher kein Stellvertreter für eine systematische, bedeutungsstiftende Verwendung:

Am Rhein galt die Kirsche einst als Markenzeichen. Allein fünf alte Sorten gab es dort in Hülle und Fülle. [RHZ08/JUL.04389 Rhein-Zeitung, 2. 7. 2008; Filsen will die Kirsche] ${ }^{1}$

Wenn wir einen kurzen Blick auf analoge Darstellungen werfen, die sich aus den quantitativen Angaben der Kookkurrenzanalyse des IDS ableiten lassen, so sehen diese zunächst deutlich weniger beeindruckend aus. Weder das Diagramm, das das Assoziationsmaß LLR aufträgt (Abb. 3.6), noch die Darstellung des inversen Rangs (damit kleine, wichtige Ränge höher abgebildet werden und nicht in der Nähe von 0; Abb. 3.7) zeigen ansatzweise eine schematische Vergleichbarkeit.

1 Die Referenz auf Korpusbelege wird laut bibliographischer Angabe gemäß DEREKo-/ Cosmas II-Konvention übernommen. 


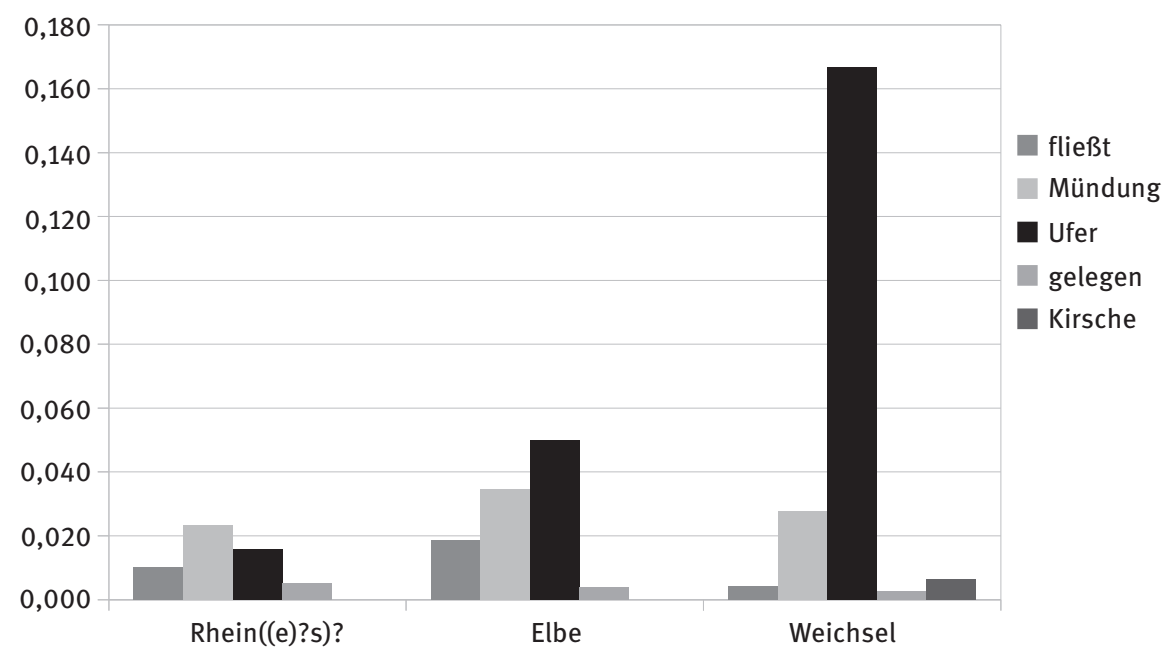

Abb. 3.7: Inverser Rang der betrachteten Wortkombinationen.

Nichtsdestotrotz konnte Belica $(2007,2011)$ ein Ähnlichkeitsmaß für die Kookkurrenzprofile innerhalb der CCDB entwickeln, das beeindruckende Ergebnisse zeigt - oder vielleicht gerade sogar deswegen, weil ein anderes Modell auf einer abstrakteren Ebene zugrunde gelegt wurde, das die Distribution auf eine weniger starr schematische Weise auswertet.

Für das Ähnlichkeitsmaß werden Werte pro übereinstimmendem Kookkurrenzpartnerwort ermittelt, bei dem sowohl deren pro Analyse berechnete LLRWerte, ihre Ränge innerhalb und der Umfang des jeweiligen Kookkurrenzprofils, als auch $t f$-idf-analoge ${ }^{2}$ Maße einfließen, die bemessen, wie stark der Einfluss des betrachten Kookkurrenzpartners in den übrigen Profilen der Kookkurrenzdatenbank ist - im einfachsten Fall, wie oft er in ihnen vorkommt. Dadurch sollen die Partnerwörter belohnt werden, die nur in ganz wenigen anderen Profilen und auch dort nur mit geringer Auffälligkeit vorkommen. Im Extremfall ist das (gemeinsame) Partnerwort nur in den beiden betrachteten Profilen gebucht und sonst in keinem anderen, sodass es natürlich extrem zu einer Gemeinsamkeit gerade dieser beiden beiträgt. In dem anderen Extremfall, dass das Partnerwort in vielen, womöglich (fast) allen anderen Profilen ebenfalls als besonders auffällig verzeichnet ist, hat es quasi keine diskriminierende

2 Termfrequenz/inverse Dokumentfrequenz: Maß aus dem Information Retrieval, das angibt, wie charakteristisch ein Wort für eine Menge von Dokumenten ist (z. B. Schlüsselwörter/Terme für Texte desselben Themas). 
Wirkung. Es trägt zu dem dedizierten Vergleich wenig bei und kann durch entsprechende Gewichtung marginalisiert werden. Da die Gewichtungen der einzelnen Parameter sowie auch deren Zusammenführung zu einem Gesamtwert sich aus explorativ-experimentellen Studien abgeleitet haben, lässt sich für die Berechnung keine mathematische Formel angeben, höchstens eine pseudoformale Umschreibung des zuvor Gesagten:

$$
\operatorname{Sim}\left(w_{1}, w_{2}\right)=\underset{k_{w_{1} x}, k_{w_{2} y} \mid k_{w_{1} x}=k_{w_{2} y}}{G} \quad \begin{array}{r}
\left(\operatorname{lllr}\left(k_{w_{1} x}\right), \operatorname{llr}\left(k_{w_{2} y}\right), r k\left(k_{w_{1} x}\right), r k\left(k_{w_{2} y}\right),\right. \\
\left(\operatorname{maxrk}\left(w_{1}\right), \operatorname{maxrk}\left(w_{2}\right), \operatorname{tfidf}\left(k_{w_{1}}\right)\right)
\end{array}
$$

Formel 3.2: Berechnung des Ähnlichkeitsmaßes in der CCDB.

Abbildung 3.8 zeigt nach diesem Maß sortiert den ersten Abschnitt des Abgleichs aller Einträge der Kookkurrenzdatenbank mit dem vorgegebenen Wort Weichsel. Die Liste lässt sich noch ein ganzes Stück verlängern (in der CCDB interaktiv), zeigt aber schon im ersten Teil, dass wir das Umgebungswort Kirsche nicht ohne Grund gewählt haben. Bei dem Wort Weichsel handelt es sich um Homonym, das zum einen als Bezeichnung eines Flusses verwendet wird, das zum anderen aber auch als Kurzform für Weichselkirsche regional eine Obstsorte bezeichnet. Im Vergleich zur Flussbezeichnung gibt es verhältnismäßig wenig Textstellen für die zweite Lesart. In diesen schlummern aber genügend Hinweise auf sie, sodass sich dies auch im Kookkurrenzprofil zeigt - und sei es in diesem Fall auch besonders über Aufzählungen verschiedener Obstsorten. Die Häufung des Wortes Kirsche in seiner Umgebung kann aber auch durchaus auf paraphrasische Verwendung zurückzuführen sein.

Da wird die Sauerkirsche a.k.a. Weichsel als Kirsche verkauft, mit einem schlappen Fruchtanteil von zwei Prozent. [FLT13/MAR.00585 Falter, 27.3. 2013, S. 48; Gewagte und weniger gewagte Limos, die erfrischen oder nicht]

Dass das Wort Kirsche in der Umgebung von Weichsel oft beobachtet wird, ist also kein Zufall, genauso wenig wie dies bei Birne, Himbeere oder Marille der Fall ist. Die Kookkurrenzanalyse deckt dies jeweils auf; und als gemeinsames Partnerwort trägt es zu dem Ähnlichkeitsmaß bei, so wie weitere gemeinsame Partnerwörter. Ein Teil der Partnerwörter trägt also zu der Ähnlichkeit der Obstbezeichner bei; ein anderer Teil, unter anderem die übrigen vier Umgebungswörter aus unserem Beispiel, sorgen für eine hohe Ähnlichkeit zu den Flussbezeichnern. Die Ähnlichkeiten werden sozusagen lokal gemessen anhand einer opportunistisch-selektiven Strategie. 


\begin{tabular}{l} 
C Cyril Belica: Modelling Semantic Proximity - Similar Collocation Profiles \\
Folgende verwandte Kookkurrenzprofile zu Weichsel wurden gefunden \\
(anklickbar, absteigend nach Verwandtschaftsgrad sortiert): \\
Elbe \\
Neiße \\
Donau \\
Birne \\
Himbeere \\
Marille \\
Oberlauf \\
\hline Pflaume \\
\hline Pfirsich \\
\hline Aprikose \\
\hline Fluss \\
\hline Fluß \\
\hline Rhein \\
Wolga \\
Kirsche \\
\hline ... \\
\hline
\end{tabular}

Abb. 3.8: Ausgabe der Methode Similar Profiles der CCDB.

Andere Ansätze, die letztendlich auch auf den Vergleich von Wörtern hinarbeiten, gehen zunächst - und dann auch speziell in diesem Punkt - anders vor. Die Kerngedanken dieser Verfahren (vgl. Deerwester et al. 1990) sind stark durch Leitfragen des Information Retrieval geprägt, bei dem es zu Beginn weniger um Wörter in der Umgebung von Wörtern, sondern innerhalb von Dokumenten ging. Ziel war eine Klassifikation nach Textinhalten - insbesondere, um Treffer für Suchanfragen anbieten zu können, in denen der Suchausdruck selber gar nicht vorkommen musste, sondern nur bedeutungsähnliche oder -stiftende Ausdrücke. Dazu wurde für jedes Wort ${ }^{3}$ des Vokabulars eine Zeile

3 Üblicherweise „Wortform X Wortform“ oder „Lemma X Lemma“; Ansätze mit Mischung „Lemma X Wortform“, wie in unserem Beispiel, sind uns nicht bekannt, wäre als Option aber wichtig: „It is important, however, to regard each word separately at first, and not as a member of a paradigm“ (Firth 1957: 12). 
(wie in unseren Tabellen in Kapitel 2), eine komplette Matrix unmittelbar quantitativer oder daraus abgeleiteter Werte ermittelt (vgl. Tabellen im Anhang). Eine einzelne Zeile dieser Matrix wird in dieser Welt als Vektor bezeichnet; jede Spalte der Matrix wird als eine Dimension eines Vektorraums aufgefasst. Aus der distributionellen Hypothese lässt sich dann darauf aufbauend eine schöne Analogie herstellen, bei der die Anschaulichkeit aber jenseits von drei Dimensionen wieder verlorengeht: Jeder Eintrag in einer Zeile entspricht der Koordinate einer Dimension, sodass der durch eine Zeile beschriebene Vektor (nun auch räumlich) einen Punkt in dem durch die Dimensionen aufgespannten Vektorraum darstellt. Haben wir bisher Ähnlichkeit über die Kongruenz der Säulendiagramme diskutiert, so ist das Pendant hier, dass die Vektoren zweier ähnlicher Wörter Punkte beschreiben, die in dem Vektorraum nahe beieinander liegen. Die etwas schwächere Fassung davon dämpft schon die Erwartung, dass man globale Skalierungen für alle Wörter und Dimensionen finden werden kann, und vergleicht nur noch die Richtung der Vektoren, indem die Winkel zwischen ihnen gemessen werden.

Eine große Herausforderung dieser Verfahren, die längst auf Kookkurrenzen übertragen wurden, ist die Erstellung und das Auswerten der Matrix für das gesamte Vokabular. Bei einem geschätzten Umfang unseres Korpusvokabulars von ca. 20.000.000 Token ist selbst bei frequenten Wörtern (z. B. 100.000 Treffer mal 10 Wörter im Kontext) und maximaler Diversität nur ein Bruchteil der Spalten mit Werten gefüllt. Verständlicherweise hat man sich viel damit beschäftigt, diese Matrix auf das zu reduzieren, was wirklich wichtig ist: Hochfrequente, teilweise auch niedrigfrequente Wörter werden ausgeblendet, Wörter, die stets gemeinsam in die gleiche Richtung weisen, zusammengefasst. Die resultierenden Verfahren zeigen gute Ergebnisse für Synonyme, haben aber große Schwierigkeiten bei Polysemie oder Homonymie.

Despite their usefulness, most VSMs [vector space models, Anm. d. Verf.] share a common problem that each word is only represented with one vector, which clearly fails to capture homonymy and polysemy. (Huang et al. 2012: 873)

Auf unser Beispiel übertragen zeigt sich an Elbe und Rhein, dass genügend gemeinsame Umgebungswörter eine hohe Ähnlichkeit nahelegen (nur im Ansatz illustriert durch unsere ausgewählten vier Fluss-Umgebungswörter; vgl. Abb. 3.5). Der Einfluss des Umgebungsworts Kirsche ist hier vernachlässigbar klein. Beim Vergleich von Elbe oder Rhein mit Weichsel ist dessen ausgeprägtes Vorhandensein dort aber nicht mehr von der Hand $\mathrm{zu}$ weisen. Solange Kirsche mittelbar oder unmittelbar zu einer Dimension des Vektorraums beiträgt, zeigt der Vektor für Weichsel in eine deutlich andere Richtung als für die beiden anderen Flüsse. Die Teilähnlichkeit zu einer Lesart lässt sich kaum aus der 
Distanz oder dem Winkel der Vektoren herauslesen. Wird Kirsche (und Konsorten) hingegen als Störgeräusch weggeblendet, rutscht Weichsel dichter an die Flüsse; für die Gesamtbetrachtung wird allerdings eine Lesart, womöglich auch ein Teil der sie konstituierenden Umgebungswörter, generell ignoriert.

\section{Distributionen kondensiert - Word Embeddings}

Die gesamte Wort-Kontext-Information in einer vollständigen Matrix aller denkbaren Kombinationen vorzuhalten, ist nicht nur technisch unhandlich; es spricht auch vieles dafür, dass wir Menschen bei der kognitiven Verarbeitung den gleichen Gehalt auf eine wesentlich kompaktere Repräsentation herunterbrechen:

If all knowledge [...] were stored [...], a person's knowledge of word A would be given by all the contexts in which A had occured. However, most theorists assume [...] that the cognitive representation of a word is some abstraction or generalisation derived from the contexts that have been encountered. (Miller \& Charles 1991: 5)

Seit einiger Zeit hat sich ein Forschungsstrang diese These auf die Fahne geschrieben (Lapesa \& Evert 2014; Levy \& Goldberg 2014). Er knüpft zwar gedanklich an viele Punkte der Vektorraummodelle an, versucht aber vor allem, diese kompakte Repräsentation über neuronale Netze zu lernen. Insbesondere der Methodenapparat word2vec, der in der Forschergruppe um Mikolov (Mikolov et al. 2013) entwickelt wurde, und Ableger davon haben der Richtung einen gewaltigen Schub gegeben und viel Hoffnung geweckt.

Stark vereinfacht kann man sich das neuronale Netz vorstellen wie in Abbildung 3.9: Es gibt eine Eingabe- und eine Ausgabeschicht (input/output layer), die jeweils das gesamte Vokabular abbilden. Interessant ist aber vor allem die mittlere, versteckte Schicht (hidden layer), die wesentlich weniger Knoten enthält als die beiden anderen; in einigen Studien werden kleine Hunderterzahlen (z. B. 300) genannt. Jeder Knoten dieser Schicht enthält so viele Felder, wie es Wörter gibt. Beim Training über die Korpuskontextinformationen lernen diese Felder Gewichtungen, die dahingehend ausgerichtet sind, möglichst gut die Kombinationshäufigkeit mit den Wörtern der Ausgabeschicht vorherzusagen. In einer Modellvariante soll das Bezugswort anhand der Kontexte vorhergesagt werden (CBOW, continous bag of words); in der oben dargestellten Variante soll der Kontext anhand des Bezugswortes vorhergesagt werden (SkipGram, eigentlich für die Beschreibung der vorgegebenen Textfenster-/Abstandsmetrik ${ }^{4}$ ).

4 Vergleichbar mit einer Textfensterdefinition ohne Autofokussierung, unter Umständen aber mit einer Ausdehnung der Kontextbreite bei Filterung von Stoppwörtern. 


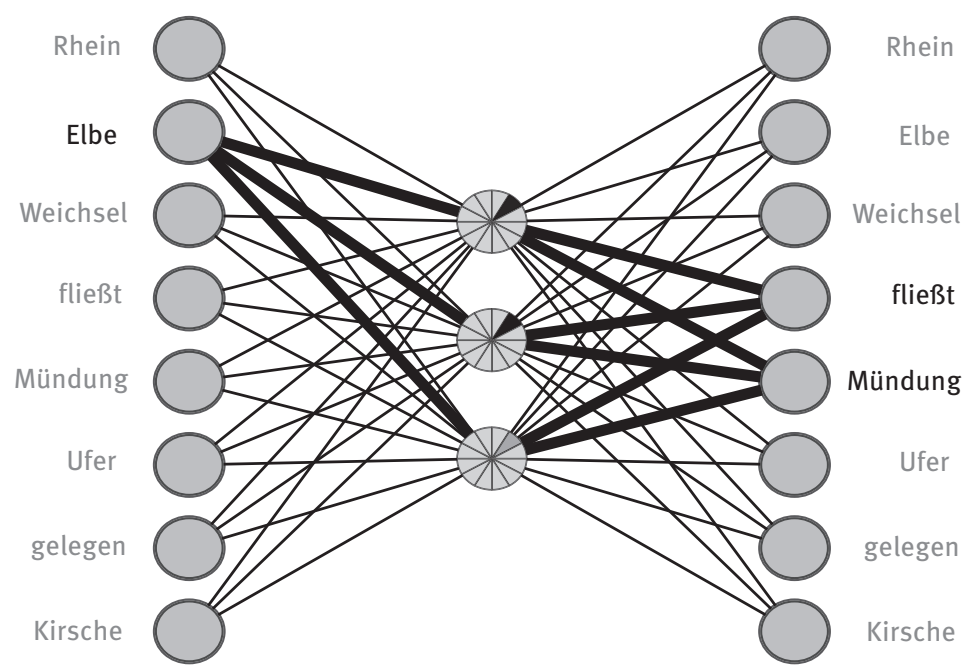

Abb. 3.9: SkipGram-Modell zum Erlernen von Word Embeddings.

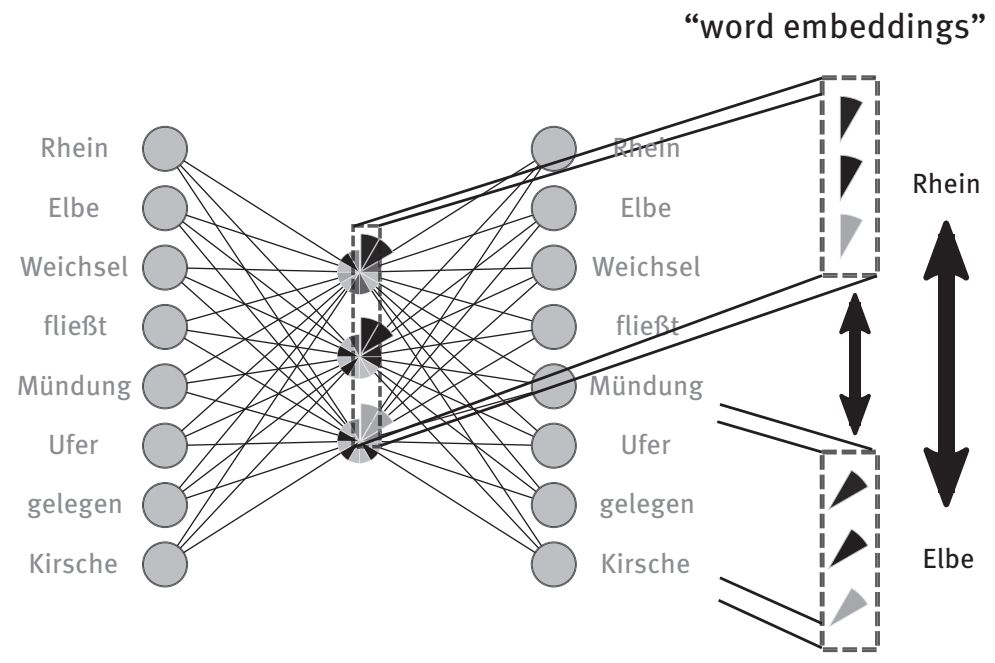

Abb. 3.10: Gelernte Hidden Layer-Gewichte als Word Embeddings zum Vergleich.

Liest man nach dem Training jeweils die Felder aus, die einem Wort zugeordnet sind, erhält man wieder einen Vektor, der zwar wesentlich kleiner ist als der Ausgangsvektor für alle seine Kombinationen, der aber immer noch eine große Vorhersagekraft für das Kontextverhalten hat. Diese gelernten Vektoren, word embeddings genannt, dienen nun zum Vergleich: Eng verwandte Wörter 
sollten auch ähnliche Vektoren haben, die sich wiederum räumlich deuten und eventuell auf zwei Dimensionen verflachen lassen, um diese Beziehung auch $\mathrm{zu}$ visualisieren.

Wofür die Knoten der Zwischenschicht allerdings stehen, ist bisher noch weitestgehend unklar. Obwohl quasi subsymbolisch entstanden, kursiert die Hoffnung, dass sie quasi als Merkmalsbündel operieren, was stichprobenartig durch algebraische Betrachtungen der Vektoren genährt wird: So könnte etwa für die word embeddings für gehen, rennen und schnell die Gleichung aufgestellt werden: $\mathrm{v}($ rennen $)=\mathrm{v}($ gehen $)+\mathrm{v}($ schnell $)$. Studien zum Deutschen stehen allerdings noch aus, für Auswertungen englischer Korpora werden entsprechende Beispiele in der Literatur genannt. Eine systematische Untersuchung liegt jedoch noch nicht vor. Interne experimentelle Studien deuten allerdings an, dass auch diese Ansätze die Herausforderung der Polysemie nicht besser in den Griff bekommen. Sie erben gewissermaßen die Last, ein mehrdeutiges Wort in einem einzigen Vektor darzustellen, der dann in einem einheitlich dimensionierten und skalierten Raum angeordnet werden soll. Vielleicht ist auch gerade die Vorhersagerichtung und das Berücksichtigen negativer Beispiele, was sich für Bedeutungsnähe als besonders geeignet erwiesen hat, für Polysemie gerade nachteilig: Wenn die meisten (auch absolut gezählten) Kontexte auf Weichsel als Fluss hinweisen, ist die Vorhersage von Kirsche aus Weichsel womöglich exotischer, als andersherum. Wenn dann noch bei den meisten Flüssen als negativ dazugelernt wird, dass das Wort Kirsche in der Umgebung quasi nicht vorkommt, spricht die hohe Zahl an Kombinationen dagegen, dass Weichsel ein Fluss sein könnte.

\section{Fazit - Kookkurrenz, Distribution und Polysemie/Homonymie}

In den beiden Welten, in der der Kookkurrenz und in der der Distribution, steht die Auswertung der nahen sprachlichen Kontexte - vollkommen zurecht - im Mittelpunkt. Kookkurrenzbasierte Ansätze wie in der CCDB können den Methodenapparat für das Ermitteln des typischen Kontextverhaltens und den des (nachfolgenden) Vergleichs jeweils getrennt gestalten und so auch auf etwas speziellere Konstellationen eingehen. Durch gewissermaßen lokal angepasste Skalen können Ähnlichkeiten zwischen Weichsel und Rhein, aber auch zwischen Weichsel und Kirsche zunächst getrennt festgestellt werden. Erst auf der Grundlage dieser Mosaiksteinchen entsteht ein Gesamtbild in einem weiteren Schritt. Distributionelle Ansätze legen den Grundriss des Gesamtbildes durch 
eine global-einheitliche Dimensionierung und Skalierung von vornherein fest. Jede sprachliche Einheit muss sich unter dieser Vorgabe an irgendeiner Stelle eindeutig verorten. Bei einem mehrdeutigen Wort lässt sich dieser innere Konflikt nicht so auflösen, dass räumliche Nähe und semantische Nähe miteinander einhergehen. Wird eine Lesart bevorzugt, rückt zwar die Position zu deren Pol; die anderen Lesarten werden aber ignoriert. Werden mehrere Lesarten berücksichtigt, überlagern sich deren Vektoren (oder Embeddings), sodass sich die Position in die Zwischenräume verschiebt. Die Überlegungen, wie man diese Herausforderung in den Griff bekommt, zielen darauf, mehrere Darstellungen der Wörter (sozusagen pro Lesart eine) abzuleiten. Dazu werden dem Verfahren die (Anzahl der) Lesarten vorgegeben, die es über ein Training entsprechend klassifizierter Kontexte auseinanderhalten soll. Dies kann im einfachen Fall die Zuordnung Kontext-Lesart sein. Andere Vorschläge gehen noch einen Schritt weiter und bieten als Informationsressource stattdessen eine Klassifikation der Texte an - nach Themen oder Diskursen ausgerichtet -, davon ausgehend, dass diese sogenannten globalen Kontexte die Manifestation der Lesarten bedingen. Kookkurrenzen können dann nach auffälliger Verteilung über ihre globalen Kontexte zumindest schwerpunktmäßig damit verbundenen Lesarten zugeordnet und die Vektoren entsprechend differenziert werden. In dem der CCDB (und Kookkurrenzanalyse des IDS allgemein) zugrunde liegenden korpuslinguistischen Paradigma wird aber die Vorgabe von Lesarten sehr kritisch gesehen, ähnlich wie es Kilgarriff formuliert hat:

Where 'word senses' have a role to play in a scientific vocabulary, they are to be construed as abstractions over clusters of word usages. (Kilgarriff 1997: 112)

Ähnlich verhält es sich mit der Kraft im Hintergrund, die für ihre Manifestation verantwortlich zeichnet, und die - nicht ganz zufällig - auch im CCDB-Jargon globale Kontexte genannt wird. Unser Ordnungssystem, und hier denken wir speziell auch an das kognitive, mag an vielen Stellen in unserem Weltwissen verwurzelt sein. Abgesehen vom unmittelbar selbst Erlebten leiten wir aber auch dies großenteils aus sprachlich Vermitteltem ab und bauen es ständig aus. Insofern ist auch dieses Ordnungssystem teilweise ein Abbild unseres Spracherlebens. Anstatt zu beanspruchen, dies vorgeben zu können, plädieren wir dafür, dass die jeweils wirkenden globalen Kontexte aus der Sicht des betrachteten Wortes auf die Sprache heraus zu interpretieren sind. Dazu bietet die CCDB ein Verfahren an, das die Liste ähnlicher Profile eines Wortes als eine self-organizing map kart(ograf)iert. Die Elemente der Liste werden paarweise mit derselben Ähnlichkeitsmetrik wie oben verglichen und dann nach diesem Maß gruppiert und räumlich arrangiert. 
(c) Cyril Belica: Modelling Semantic Proximity - Self-Organizing Map (version: 0.32, init tau: 0.04, dist: u, iter: 10000)

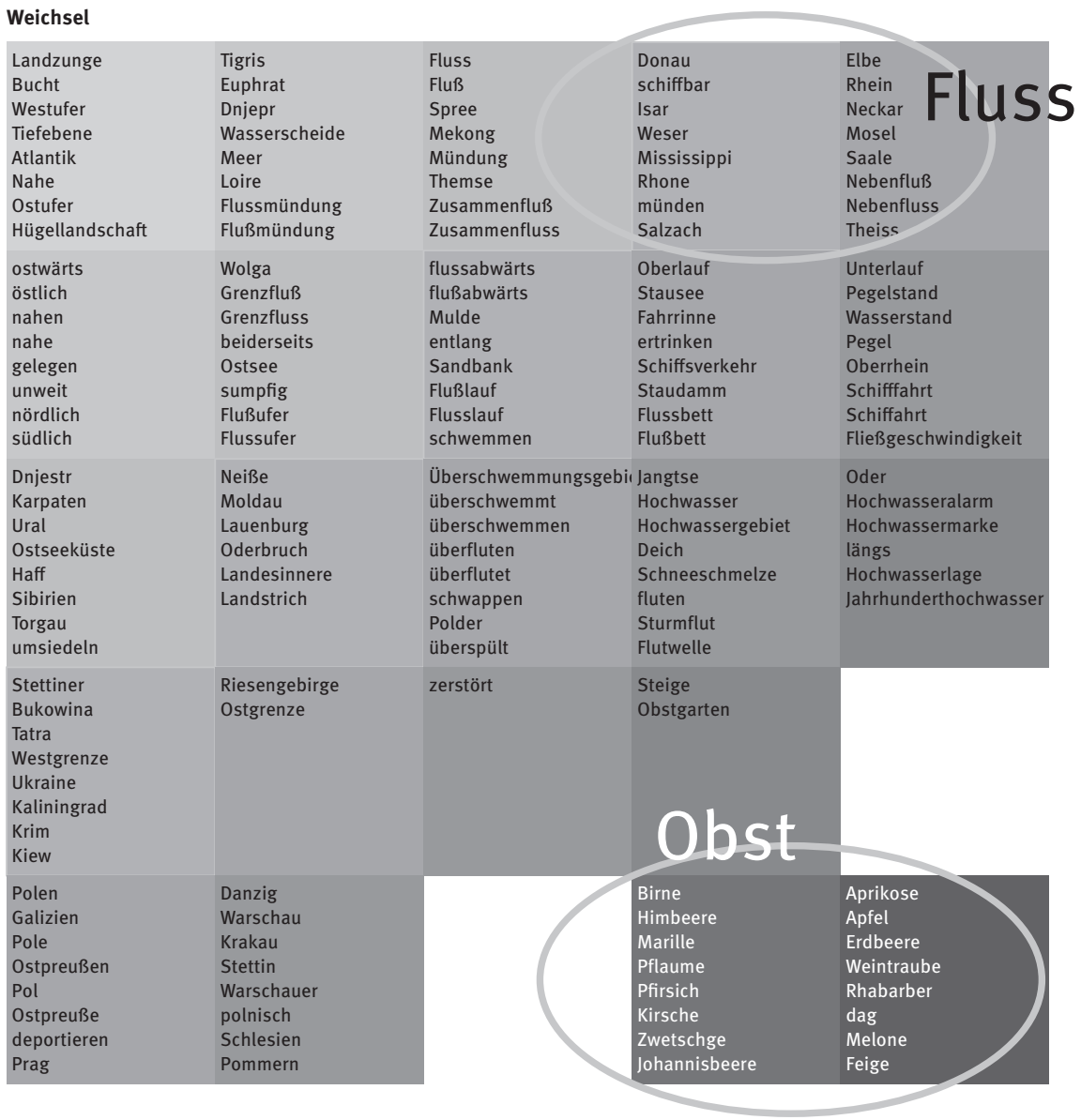

Abb. 3.11: Mit globalen Kontexten annotierte SOM des Wortes Weichsel aus der CCDB.

In der Karte (vgl. Abb. 3.11) zeigt sich die Stärke dieses Ansatzes, dass das Erstellen und das Vergleichen der Kookkurrenzprofile zunächst nach lokalen Prinzipien erfolgt, bevor danach eine räumliche Anordnung angestrebt wird. Und das, obwohl die ermittelten Kennzahlen (vgl. Abb. 3.6 und Abb. 3.7) keine leichte, unmittelbare Vergleichbarkeit suggeriert hätten. Erste interne Studien mit auf embeddings basierenden Darstellungen zeigen keinen Bereich für die Lesart ,Obst', auch wenn es vom Ansatz an dieser Stelle theoretisch auch wieder möglich wäre, wenn die Ähnlichkeiten zu beiden Lesarten, wenngleich stark reduziert, erfasst worden wären. Vielleicht erliegt man in den Vektorraumvorstellungen aber auch gerade der Versuchung, dass die Näheanalogie, 
die sich bei enger Bedeutungsverwandtschaft erzielen lässt (als Kontur des Säulendiagramms oder als Punkte im Vektorraum) auf andere FormBedeutungsbeziehungen übertragen lässt. Inwieweit dies tatsächlich möglich ist, mit wie vielen und welchen externen oder intrinsischen Zusatzinformationen, das wird noch Gegenstand vieler zukünftiger Untersuchungen in beiden Bereichen sein.

\section{Literatur}

al-Wadi, Doris (1994): COSMAS - Ein Computersystem für den Zugriff auf Textkorpora. Version R.1.3-1. Benutzerhandbuch. Mit einem Geleitwort von Prof. Dr. Gerhard Stickel. Mannheim: Institut für deutsche Sprache.

Bahns, Jens (1997): Kollokationen und Wortschatzarbeit im Englischunterricht. Tübingen: Narr.

Bański, Piotr, Joachim Bingel, Nils Diewald, Elena Frick, Michael Hanl, Marc Kupietz, Piotr Pęzik, Carsten Schnober \& Andreas Witt (2013): KorAP: The new corpus analysis platform at IDS Mannheim. In Zygmunt Vetulani \& Hans Uszkoreit (Hrsg.), Human language technologies as a challenge for computer science and linguistics, 586-587. Proceedings of the 6th Language and Technology Conference. Poznań: Fundacja Uniwersytetu im. A.

Belica, Cyril (1994): A German lemmatizer. Final Report MLAP93-21/WP2. Luxemburg. Belica, Cyril (1995): Statistische Kollokationsanalyse und -clustering. Korpuslinguistische Analysemethode. http://corpora.ids-mannheim.de (letzter Zugriff 1. 8. 2017).

Belica, Cyril (2007): Kookkurrenzdatenbank CCDB - V3. Eine korpuslinguistische Denk- und Experimentierplattform für die Erforschung und theoretische Begründung von systemisch-strukturellen Eigenschaften von Kohäsionsrelationen zwischen den Konstituenten des Sprachgebrauchs. http://corpora.ids-mannheim.de/ccdb/ (letzter Zugriff 1. 8. 2017).

Belica, Cyril (2011): Semantische Nähe als Ähnlichkeit von Kookkurrenzprofilen. In Andrea Abel \& Renata Zanin (Hrsg.), Korpora in Lehre und Forschung, 155-178. Bozen-Bolzano: Freie Universität.

Belica, Cyril \& Rainer Perkuhn (2015): Feste Wortgruppen/Phraseologie I: Kollokationen und syntagmatische Muster. In Ulrike Haß \& Petra Storjohann (Hrsg.), Handbuch „Wort und Wortschatz", 201-225 (= Handbücher Sprachwissen 3). Berlin, Boston: de Gruyter.

Berry-Rogghe, Godelieve/Geneviève L.M. (1973): The computation of collocations and their relevance in lexical studies. In Adam J. Aitken, Richard W. Bailey \& Neil Hamilton-Smith (Hrsg.), The computer and literary studies, 103-112. Edinburgh: University Press.

Bodmer, Franck (2005): COSMAS II. Recherchieren in den Korpora des IDS. Sprachreport (3). Mannheim: 2-5.

Bodmer Mory, Franck (2014): Mit COSMAS II »in den Weiten der IDS-Korpora unterwegs«. In Institut für Deutsche Sprache (Hrsg.), Ansichten und Einsichten, 376-385. 50 Jahre Institut für Deutsche Sprache. Redaktion: Melanie Steinle \& Franz Josef Berens. Mannheim: Institut für Deutsche Sprache. 
Brückner, Tobias (1983): Programmdokumentation REFER. LDV-Info (2). Informationsschrift der Arbeitsstelle Linguistische Datenverarbeitung. Mannheim: Institut für deutsche Sprache, 1-26.

Brückner, Tobias (1988/1989): Recherchesystem für Verben. LDV-Info (7). Informationsschrift der Arbeitsstelle Linguistische Datenverarbeitung. Mannheim: Institut für deutsche Sprache, 41-67.

Deerwester, Scott, Susan Dumais, George Furnas, Thomas Landauer \& Richard Harshman (1990): Indexing by Latent Semantic Analysis. Journal of the American Society for information science 41, 391-407.

Dunning, Ted (1993): Accurate methods for the statistics of surprise and coincidence. Computational Linguistics 19, 61-74.

Fechner, Gustav T. (1860/1907): Elemente der Psychophysik. Leipzig: Breitkopf.

Firth, John R. (1957): A synopsis of linguistic theory 1930-1955. In Studies in linguistic analysis, 1-32. Oxford: Blackwell.

Harris, Zellig S. (1960): Structural linguistics. Chicago, London: Phoenix.

Harris, Zellig S. (1970): Distributional structure. In Zellig S. Harris, Papers in structural and transformational linguistics, 775-794. Dordrecht: Reidel. (Erstveröffentlichung: Word 1954, 10, No. 2-3, 146-162).

Huang, Eric H., Richard Socher, Christopher D. Manning, \& Andrew Y. Ng (2012): Improving word representations via global context and multiple word prototypes. In Proceedings of the 50th annual meeting of the Association for Computational Linguistics, 873-882.

Long Papers-Volume 1: Association for Computational Linguistics.

Institut für Deutsche Sprache (2017a): Cosmas II web 2.2.1 (Release Januar 2017). Mannheim: Institut für Deutsche Sprache. https://cosmas2.ids-mannheim.de/cosmas2-web/faces/ home.xhtml (letzter Zugriff 5.7. 2017).

Institut für Deutsche Sprache (2017b): Deutsches Referenzkorpus . Archiv der Korpora geschriebener Gegenwartssprache 2017-I (Release vom 8.3. 2017). Mannheim: Institut für Deutsche Sprache. PID: 10932/00-0373-23CD-C58F-FF01-3. www.ids-mannheim.de/DeReKo.

Keibel, Holger \& Cyril Belica (2007): CCDB: A corpus-linguistic research and development workbench. In Proceedings of corpus linguistics 2007. Birmingham: University of Birmingham. http://corpus.bham.ac.uk/corplingproceedings07/paper/134_Paper.pdf (letzter Zugriff 1. 8. 2017).

Kilgarriff, Adam (1997): I don't believe in word senses. Computers and the Humanities XXXI, 91-113.

Lapesa, Gabriella \& Stefan Evert (2014): A large scale evaluation of distributional semantic models: Parameters, interactions and model selection In Transactions of the Association for Computational Linguistics, vol. 2, 531-545. https://transacl.org/ojs/index.php/tacl/ article/download/457/91 (letzter Zugriff 1. 8. 2017).

Levy, Omer \& Yoav Goldberg (2014): Neural word embedding as implicit matrix factorization. In Advances in neural information processing systems https://papers.nips.cc/paper/ 5477-neural-word-embedding-as-implicit-matrix-factorization.pdf (letzter Zugriff 1. 8. 2017).

Mikolov, Tomas, Ilya Sutskever, Kai Chen, Greg S. Corrado \& Jeff Dean (2013): Distributed representations of words and phrases and their compositionality. In Advances in neural information processing systems, 3111-3119.

Miller, George A. \& Walter G. Charles (1991): Contextual correlates of semantic similarity. Language and Cognitive Processes VI, 1-28. 
Perkuhn, Rainer (2007a): Systematic exploration of collocation profiles. In Proceedings of the 4th Corpus Linguistics Conference (CL 2007), Birmingham. Birmingham: University of Birmingham.

Perkuhn, Rainer (2007b): „Corpus-driven“: Systematische Auswertung automatisch ermittelter sprachlicher Muster. In Heidrun Kämper \& Ludwig M. Eichinger (Hrsg.), Sprach-Perspektiven, 465-491. Germanistische Linguistik und das Institut für Deutsche Sprache (= Studien zur Deutschen Sprache 40). Tübingen: Narr.

Perkuhn, Rainer (2016): Collocation(s) in German minds. In Begoña Sanromán Vilas (Hrsg.), Collocations cross-linguistically. Corpora, dictionaries and language teaching, 167-192. Helsinki: Société Néophilologique.

Perkuhn, Rainer, Holger Keibel \& Marc Kupietz (2012): Korpuslinguistik. (= UTB 3433). Paderborn: Fink.

Perkuhn, Rainer, Cyril Belica, Holger Keibel, Marc Kupietz \& Harald Lüngen (2015): Valenz und Kookkurrenz. In María José Domínguez Vázquez \& Ludwig M. Eichinger (Hrsg.), Valenz im Fokus. Grammatische und lexikographische Studien. Festschrift für Jacqueline Kubczak, 175-196. Mannheim: Institut für Deutsche Sprache.

Teubert, Wolfgang \& Cyril Belica (2014): Von der linguistischen Datenverarbeitung am IDS zur “Mannheimer Schule der Korpuslinguistik". In Institut für Deutsche Sprache (Hrsg.), Ansichten und Einsichten. 50 Jahre Institut für Deutsche Sprache. Redaktion: Melanie Steinle \& Franz Josef Berens, 298-319. Mannheim: Institut für Deutsche Sprache.

\section{Anhang}

Tab. A.3.1: Absolute Häufigkeiten der Umgebungswörter (obere Zeile) zu den Bezugswörtern (erste Spalte).

\begin{tabular}{lrrrrrrrrr}
\hline & Rhein & Elbe & Wechsel & fließt & Mündung & Ufer & gelegen & Kirsche & total \\
\hline Rhein & 2.166 & 1.501 & 58 & 901 & 1.013 & 646 & 437 & 1 & 32.7216 \\
Elbe & 1.501 & 325 & 91 & 352 & 328 & 560 & 127 & 0 & 82.344 \\
Rhein((e)?s)? & 2.303 & 1.541 & 61 & 923 & 1.253 & 1.468 & 498 & 1 & 35.8927 \\
Weichsel & 58 & 91 & 28 & 16 & 41 & 102 & 11 & 10 & 6.109 \\
fließt & 901 & 352 & 16 & 188 & 130 & 59 & 20 & 0 & 126.253 \\
Mündung & 1.013 & 328 & 41 & 130 & 43 & 99 & 92 & 0 & 24.917 \\
Ufer & 646 & 560 & 102 & 59 & 99 & 662 & 380 & 0 & 143.440 \\
gelegen & 437 & 127 & 11 & 20 & 92 & 380 & 25 & 1 & 207.198 \\
Kirsche & 1 & 0 & 10 & 0 & 0 & 0 & 1 & 28 & 6.227 \\
\hline
\end{tabular}


Tab. A.3.2: „Einfache Erwartungswerte“ der Umgebungswörter (obere Zeile) zu den Bezugswörtern (erste Spalte).

\begin{tabular}{lrrrrlllr}
\hline & Rhein & Elbe & Wechsel & fließt & Mündung & Ufer & gelegen & Kirsche \\
\hline Rhein & 17,99 & 49,53 & 25,80 & 19,39 & 110,47 & 12,24 & 5,73 & 0,44 \\
Elbe & 49,53 & 42,62 & 160,84 & 30,10 & 142,14 & 42,16 & 6,62 & 0,00 \\
Rhein((e)?s)? & 17,43 & 46,36 & 24,74 & 18,11 & 124,57 & 25,35 & 5,95 & 0,40 \\
Weichsel & 25,80 & 160,84 & 667,09 & 18,44 & 239,49 & 103,50 & 7,73 & 233,73 \\
fließt & 19,39 & 30,10 & 18,44 & 10,49 & 36,74 & 2,90 & 0,68 & 0,00 \\
Mündung & 110,47 & 142,14 & 239,49 & 36,74 & 61,58 & 24,63 & 15,84 & 0,00 \\
Ufer & 12,24 & 42,16 & 103,50 & 2,90 & 24,63 & 28,61 & 11,37 & 0,00 \\
gelegen & 5,73 & 6,62 & 7,73 & 0,68 & 15,84 & 11,37 & 0,52 & 0,69 \\
Kirsche & 0,44 & 0,00 & 233,73 & 0,00 & 0,00 & 0,00 & 0,69 & 642,05 \\
\hline
\end{tabular}

Tab. A.3.3: Werte aus obiger Tabelle (mit Häufigkeit um 1 erhöht) logarithmisiert.

\begin{tabular}{lrllrlrrr}
\hline & Rhein & Elbe & Wechsel & fließt & Mündung & Ufer & gelegen & Kirsche \\
\hline Rhein & 4,17 & 5,63 & 4,71 & 4,28 & 6,79 & 3,62 & 2,52 & $-0,20$ \\
Elbe & 5,63 & 5,42 & 7,35 & 4,92 & 7,16 & 5,40 & 2,74 & 0,79 \\
Rhein((e)?s)? & 4,12 & 5,54 & 4,65 & 4,18 & 6,96 & 4,67 & 2,58 & $-0,33$ \\
Weichsel & 4,71 & 7,35 & 9,43 & 4,29 & 7,94 & 6,71 & 3,08 & 8,01 \\
fließt & 4,28 & 4,92 & 4,29 & 3,40 & 5,21 & 1,56 & $-0,49$ & 0,18 \\
Mündung & 6,79 & 7,16 & 7,94 & 5,21 & 5,98 & 4,64 & 4,00 & 2,52 \\
Ufer & 3,62 & 5,40 & 6,71 & 1,56 & 4,64 & 4,84 & 3,51 & $-0,01$ \\
gelegen & 2,52 & 2,74 & 3,08 & $-0,49$ & 4,00 & 3,51 & $-0,89$ & 0,46 \\
Kirsche & $-0,20$ & 0,79 & 8,01 & 0,18 & 2,52 & $-0,01$ & 0,46 & 9,38 \\
\hline
\end{tabular}

Tab. A.3.4: Ergebnisse der Kookkurrenzbewertungen der drei Bezugswörter mit fünf Umgebungswörtern (Kookkurrenzanalyse Cosmas II in DeReKo-2017-I).

\begin{tabular}{llccccc}
\hline & & fließt & Mündung & Ufer & gelegen & Kirsche \\
\hline Rhein((e)?s)? & Rang & 98 & 43 & 64 & 195 & - \\
& $1 /$ Rang & 0,01 & 0,02 & 0,02 & 0,01 & \\
& LLR & 3.520 & 10.222 & 6.415 & 1.686 & - \\
\hline Elbe & Rang & 54 & 29 & 20 & 248 & - \\
& 1/Rang & 0,02 & 0,03 & 0,05 & 0 & \\
& LLR & 1.709 & 2.673 & 3.637 & 453 & - \\
\hline \multirow{2}{*}{ Weichsel } & Rang & 242 & 36 & 6 & 376 & 158 \\
& 1/Rang & 0 & 0,03 & 0,17 & 0 & 0,01 \\
& LLR & 69 & 360 & 992 & 48 & 98 \\
\hline
\end{tabular}





\title{
4 Grammatische Komplexität und semantische Transparenz in deutschen und englischen Satzstrukturen
}

\begin{abstract}
Auf dem Hintergrund aktueller Diskussionen zur grammatischen Komplexität und ausgehend von bekannten Charakterisierungen der beiden Sprachen untersucht der Beitrag die semantische Transparenz deutscher und englischer Strukturen und illustriert sie mit Beispielen aus einem parallelen Text. Analysen zu Kasus, syntaktischer und pragmatischer Wortstellung, Verbpräfigierung, Reflexivpronomen, Genus, infiniten Teilsätzen und Spaltsätzen zeigen, dass sich Systemkomplexität nicht eins zu eins auf Textkomplexität abbildet, dass grammatische Komplexität nicht unbedingt semantische Transparenz bedeutet und dass eine Reihe von englischen Strukturen semantisch transparenter ist als ihre deutschen Gegenstücke. In einer kleinen quantitativen Studie wird gezeigt, dass die größere Anzahl deutscher Flexionsmorpheme nicht durch eine größere Anzahl englischer Funktionswörter kompensiert wird. Trotzdem stützt eine Gesamtschau der Ergebnisse ein geringeres Komplexitätsgefälle zwischen den beiden Sprachen als gemeinhin angenommen.
\end{abstract}

Keywords: Äquikomplexitätsthese, Analytizität, deutsch-englische Kontraste, Formsynkretismus, grammatische Komplexität, Kasus, Satzstruktur, semantische Transparenz, Sprachvergleich, Synthetizität, Typologie

\section{Einleitung}

Semantische Transparenz ist ein aktuelles Thema in der gegenwärtigen sprachvergleichenden Forschung. In der Komplexitätsforschung der vergangenen Jahrzehnte gibt es einen weitreichenden Konsens, der die populäre Äquikomplexitätsthese sowohl als widerlegt als auch als ideologisch entschärft sieht: Sprachen hätten ein unterschiedliches Maß an grammatischer und strukturel-

Anmerkung: Ich danke den Gutachtern für wertvolle Anregungen.

Klaus Fischer, London Metropolitan University, 166-220 Holloway Road, London N7 8DB, Great Britain, E-Mail: k.fischer@londonmet.ac.uk

○ Open Access. (C) 2018 Klaus Fischer, publiziert von De Gruyter. (c) BY Dieses Werk ist lizenziert unter der Creative Commons Attribution 4.0 Lizenz. 
ler Komplexität mit nur begrenzten Komplexitätsausgleichen. Das Ergebnis sei nicht so brisant wie einst angenommen, da insbesondere grammatische Komplexität entmystifiziert worden sei: Ein Mangel an sprachlicher Komplexität behindere nicht die Kommunikation komplexer Inhalte; das Minimum an grammatischer und struktureller Komplexität scheint ständig nach unten verschoben zu werden (z. B. McWhorter 2001; Everett 2008; Gil 2009 und 2014).

Der Konsens ist allerdings nicht perfekt: Einmal erlauben sehr abstrakte Komplexitätsmaße den Nachweis weitreichender Komplexitätsausgleiche, z. B. zwischen Wort- und Satzstrukturen, die auch die meist als einfach gesehenen Kreolsprachen einbeziehen (Koplenig et al. 2017). ${ }^{1}$ Sodann gibt es eine Forschungsperspektive, unter der die Äquikomplexitätsthese aus anderen Gründen freundlicher betrachtet wird. Diese Perspektive gesteht erhebliche Unterschiede an grammatischer Komplexität zu, aber sieht komplementäre Unterschiede in der Abbildung sprachlicher Strukturen auf Bedeutungen und Sachverhalte: Eine komplexe Grammatik erlaube eine einfache Abbildung auf Bedeutungen (semantische Transparenz), eine einfache Grammatik führe zu einer komplexen Abbildung auf Bedeutungen (kontextabhängige Interpretation; vgl. Hawkins (2009: 253-254)). Beziehe man diese „versteckte Komplexität“ (Bisang 2009) mit ein, so ergebe sich ein systematischer Komplexitätsausgleich, der die Äquikomplexitätsthese als nicht ganz so abwegig erscheinen lässt, wie es von Vertretern des eingangs beschriebenen Konsenses nahegelegt wird. ${ }^{2}$

In diese Tradition lassen sich eine Reihe von kontrastiven Arbeiten einordnen, die die durch grammatische Komplexität bewirkte größere semantische Transparenz des Deutschen im Vergleich zum grammatisch einfacheren und deshalb kontextabhängigeren Englischen betonen. Insbesondere John A. Hawkins hat in seiner komparativen Typologie des Englischen und Deutschen semantische Transparenz als übergeordnetes Prinzip identifiziert, das die deutsch-englischen Kontraste vereint:

Where the surface structures (morphology and syntax) of English and German contrast, English regularly exhibits greater ,distance' between form and meaning in specifiable ways. (Hawkins 1986: 6)

$1 \mathrm{Zu}$ Kompressionsstudien siehe auch Juola (2008), zu Komplexitätsausgleichen z. B. FenkOczlon \& Fenk (2008), Sinnemäki (2008, 2014), Riddle (2008), Oh (2015).

2 In der Verrechnung von grammatischer Komplexität mit der Komplexität semantischer und pragmatischer Schlüsse wird aber nicht Gleiches mit Gleichem verglichen, wie den Befürwortern der Einbeziehung versteckter Komplexität in den Sprachvergleich auch bewusst ist; siehe dazu Fischer (2017: 30-34). 
Und John McWhorter sieht Englisch als am stärksten kontextabhängige, da am wenigsten „überspezifizierte“ germanische Sprache: ${ }^{3}$

Overall, a comparison with its sisters reveals English to be considerably less overspecified semantically and complexified syntactically. (McWhorter 2002: 2018; Hervorhebungen im Original)

Hawkins' komparative Typologie beruht auf einer einfachen Idee: Während die Typologie seit Joseph Greenbergs Neuausrichtung (siehe z. B. Greenberg 1963) wenige Parameter in vielen Sprachen untersucht, wollte Hawkins viele Parameter in wenigen untersuchen und in einen Zusammenhang stellen. Im Resultat ist Hawkins hier der Typologie des 19. Jahrhunderts verpflichtet, die einen einheitlichen Charakter von Sprachen annahm. Sein Ansatz, eine große Anzahl an Kontrasten auf einen einzigen Nenner zu bringen und einheitlich zu erklären, hat in seiner spezifischen Ausprägung wenig Nachfolger gefunden (vgl. aber De Vogelaer 2007). Seine typologische Perspektive dagegen gab der kontrastiven Linguistik einen neuen Impuls und eine Reibungsfläche (Rohdenburg 1990 und 1991); seine verschiedene existierende Analysen ${ }^{4}$ zusammenfassende Darstellung war in der Einschätzung der deutsch-englischen Kontraste sehr einflussreich (vgl. z. B. König \& Gast 2009: 2-3, 256-257).

McWhorter sucht nach einer historischen Erklärung für das Ausmaß, in dem Englisch grammatisch und syntaktisch vom germanischen Ursprung abweicht. Er findet sie in Sprachkontaktsituationen, in denen eine kritische Anzahl Erwachsener Angelsächsisch unvollständig erworben und eine simplifizierte Version an die nächste Generation weitergegeben habe. Dieses Erklärungsmuster wird von historischen Soziolinguisten als einzige Ursache für einen schnellen und umfassenden Verlust an Komplexität angenommen (vgl. Trudgill 2011: 15-61). McWhorter (2002) sieht die Sprachkontakte mit dem Altnordischen als Hauptursache, McWhorter (2008) verfolgt zusätzlich die keltische Hypothese, die den Substrat-Einfluss des Brythonischen sowohl für Komplexitätsverluste als auch Komplexitätszuwachs (obligatorische do-Umschreibung, obligatorische Verlaufsform) verantwortlich macht. ${ }^{5}$

3 Überspezifizierung bezieht sich auf die grammatische Repräsentation semantischer Kategorien, die nicht in der Vergleichssprache oder in allen Sprachen erfolgt und deshalb zur Kommunikation nicht erforderlich ist; vgl. z. B. McWhorter (2002: 219). Da sich das angenommene grammatische Minimum, wie gesagt, durch die Erforschung von Kreol- und südostasiatischen Sprachen verringert hat, ist in universeller Perspektive fast die ganze Grammatik überspezifiziert.

4 Es handelt sich hier unter anderen um Analysen in Rohdenburg (1974) und Plank (1984).

$5 \mathrm{Zu}$ einer ausgewogenen Darstellung der keltischen Hypothese siehe Hickey (2012). 
Ich werde in meinem Beitrag einige der wichtigeren, von Hawkins und McWhorter angeführten Belege für die größere grammatische Komplexität und Transparenz des Deutschen bzw. größere Kontextabhängigkeit des Englischen darstellen. Sodann werde ich eine Reihe von Argumenten entwickeln, die in Teilbereichen jeweils eine größere semantische Transparenz des Englischen nachweisen. In einem dritten Teil werde ich der Frage nachgehen, wie die größere Synthetizität des Deutschen und Analytizität des Englischen quantifiziert werden kann.

Mein Beitrag hat nicht das Ziel, die insgesamt größere grammatische Komplexität des Deutschen auf Systemebene in Frage zu stellen. Vielmehr möchte ich Evidenz für die folgenden Thesen aufweisen:

- Komplexität auf der Ebene des grammatischen Systems vererbt sich nicht eins zu eins auf Komplexität in der Textrealisierung. Die grammatische Komplexität von Texten muss gesondert festgestellt werden.

- Grammatische Komplexität bewirkt nicht unbedingt semantische Transparenz.

- Synthetische grammatische Komplexität und analytische grammatische Komplexität korrelieren nicht unbedingt negativ miteinander.

- Englisch besitzt sowohl auf Systemebene als auch in der Textrealisierung grammatische Komplexität, die häufig zu größerer semantischer Transparenz führt, als sie die entsprechenden deutschen Strukturen aufweisen.

Als semantische Transparenz zählt nicht nur explizit gegebene Information zum vorliegenden Sachverhalt und seiner lokalen, temporalen, aspektuellen und sonstigen Situierung, sondern auch Information, die die Konstruktion des Gesagten erleichtert (Redundanz, grammatische Bezüge durch Kongruenz und Anaphern), sowie pragmatische Information (Informationsstruktur). Neben Satzstrukturen werden auch grammatische Wort- und in einem Fall Wortbildungsstrukturen einbezogen; semantisch steht aber der Beitrag zur Satzaussage im Vordergrund.

Es ist naheliegend, grammatische Komplexität mit Sprecheraufwand und mangelnde semantische Transparenz mit größerem Höreraufwand gleichzusetzen. Davon zu unterscheiden sind Konsequenzen für den muttersprachlichen sowie zweit- und fremdsprachlichen Lernaufwand. Diesen letztlich empirischen Fragen der Kosten grammatischer Komplexität wird hier nicht systematisch nachgegangen. Allerdings steht die Sprachverarbeitungsperspektive im Hintergrund, und auf Konsequenzen für die sukzessive Bedeutungskonstruktion wird an mehreren Stellen hingewiesen.

Als Quelle für Beispiele und die quantitative Untersuchung im dritten Teil habe ich einen parallelen Text gewählt, und zwar die deutsche und englische Übersetzung von Fred Vargas' Sous les vents de Neptune, einem Kriminalro- 
man. ${ }^{6}$ Der Vorteil meiner Wahl ist, dass Inhalt, Register und Stil konstant sind, der Nachteil, dass beide Texte Übersetzungen sind. Allerdings sind es kommunikative Übersetzungen, die in der Zielsprache jeweils einen natürlichen Text bilden. Abgesehen von letzterem Punkt ist die Textwahl für meine Zwecke nicht so wichtig. Allerdings wäre eine Überprüfung der Befunde an authentischen mündlichen Texten wünschenswert.

\section{Grammatische Komplexität und semantische Transparenz im Deutschen}

Deutsch hat mehr grammatische Morphologie als Englisch. Soweit diese Information trägt, sind die deutschen Wörter isoliert betrachtet semantisch transparenter als ihre englischen Gegenstücke ohne grammatische Morphologie. So geben die deutschen Präsensverbformen gehe, gehst, geht, gehen stärkere Hinweise auf Person und Anzahl als es die zwei englischen Präsensverbformen go und goes tun. Deutsch ist in Bezug auf die genannten Verbformen im Vergleich zum Englischen komplexer, aber im Vergleich z. B. zum Lateinischen weniger komplex, wo eine eineindeutige Zuordnung zu Person und Numerus vorliegt (eo, is, it, imus, itis, eunt). Im Vergleich zu Sprachen ohne Verbflexion, wie z. B. Chinesisch, ist auch Englisch komplexer.

Da Informationen zu Person und Anzahl im Deutschen und Englischen (aber nicht unbedingt im Lateinischen) auch durch die obligatorischen Subjekte gegeben werden, ist die Kennzeichnung am Verb weitgehend redundant. Die Informationen werden bei SV-Stellung wohl nicht noch einmal abgerufen, aber es handelt sich trotzdem um reale mit den Verbformen verbundene semantische Transparenz.

Ich werde nun ausgehend von Hawkins (1986) und McWhorter (2002) eine Reihe von Kontrasten anführen, die eine größere grammatische Komplexität und zum Teil semantische Transparenz deutscher Strukturen zeigen. Dabei werden Grenzen einer funktionalen Interpretation von grammatischer Komplexität aufgewiesen und einige englische Kompensationsstrategien angeführt. Der Vergleich geht über grammatische Morphologie hinaus und bezieht unter anderem Wortbildung ein, da es eine zentrale These beider Forscher ist, dass die Kontraste verschiedener sprachlicher Teilbereiche in einem Zusammenhang zu sehen sind.

6 Quantitative Angaben beziehen sich auf die ersten 1.002 Wörter des deutschen und 1.063 Wörter des englischen Texts, Beispiele mit wenigen Ausnahmen auch. Die Rechtschreibung der deutschen Beispiele wurde an die neuen Regeln angepasst. 


\subsection{Kasus}

Im Mittelpunkt der kontrastiven Typologie von Hawkins steht der englische Kasusabbau, der für eine ganze Reihe von Folgephänomenen verantwortlich gemacht wird. Vorausgesetzt werden im Folgenden die Valenzperspektive, also die Sicht, dass Lexeme Form- und Inhaltsforderungen (semantische Restriktionen und Rollen) an ihre Umgebung stellen, sowie die komplementäre Perspektive, dass Phrasen in einem abstrakte Bedeutung tragenden Konstruktionszusammenhang stehen. Dies sei vorangestellt, um Missverständnisse zu vermeiden, wenn im Folgenden die semantische Leistung von Kasus in isolierten Phrasen angesprochen wird. Unter einer Verarbeitungsperspektive ist die Fragestellung legitim, da Kasusphrasen initial auftreten können, wenn keine Valenz- oder Konstruktionsinformationen zur Verfügung stehen. Ausgeblendet werden nicht valenziell relevante Funktionen von Kasus, etwa in formularhaften autosemantischen Adverbialbestimmungen (Sie schlief den ganzen Tag).

Isoliert betrachtet sind deutsche Nominalphrasen semantisch transparenter, da Kasus einen (natürlich vagen und weiter interpretationsbedürftigen) Hinweis auf ihre semantische Rolle gibt:

(1) Laienhaft begutachtete der Kommissar den Brenner

(1e) The commissaire knew nothing about heating systems, and was examining the silent tank

Die Kasusmorphologie der Artikel der, den in Verbindung mit Genus- und Numerusinformation der folgenden Nomen weist die Nominalphrasen als Subjekt bzw. direktes Objekt aus, was die möglichen semantischen Rollen einschränkt. Die englischen Nominalphrasen besitzen, ebenfalls isoliert betrachtet, keinen morphologischen Hinweis auf ihre syntaktische Funktion und damit mögliche semantische Rolle.

(2) noch war ihm die Situation unangenehm

(2e) nor was he distressed by the situation

Die Dativform ihm schränkt die zu erwartende semantische Rolle auf DativRollen ein. Da Englisch keinen Dativ besitzt, kann dieser Hinweis nicht gegeben werden. Die Realisierung als Subjekt bedeutet nach Hawkins, dass öfters untypische Rollen auf das englische Subjekt abgebildet werden und es deshalb semantisch vager sei als das deutsche Subjekt (Hawkins 1986: 53-73; siehe auch Plank 1983). Gleiches gelte für das englische direkte Objekt im Verhältnis zur Akkusativergänzung: 
(3) clairvoyance which defied the most sophisticated logic

(3d) Vorauswissen, das der raffiniertesten Logik trotzte

Die Objektphrase in (3) realisiert kein prototypisches Patiens; es gibt aber keinen formalen Grund, sie nicht als direktes Objekt anzusprechen. In der deutschen Entsprechung - so kann man es zumindest sehen - ist der mangelnden PatiensPrototypik durch die Realisierung als Dativergänzung Rechnung getragen.

(4) sein freundlicher Blick könnte der Anlage neue Kraft einflößen

(4e) his benign gaze would either restore the boiler's energy

Der Gebrauch der indirekten Objekt-Konstruktion ist im Englischen stark eingeschränkt (dazu ausführlich König \& Gast 2009: 112-121). Insbesondere sind externe Possessoren kaum möglich, sondern werden wie in (4e) phrasenintern als Possessive ausgedrückt. Damit steht in vielen Kontexten die spezifische Semantik der indirekten Objekt-Konstruktion nicht zur Verfügung.

Englisch unterscheidet seltener zwischen Ort und Ziel, eine der Folgen von Kasusverlust:

(5) Er setzte sich auf den Fußboden

(5e) He sat on the floor

Als Kompensation für den Kasusverlust kann die Verwendung komplexer Präpositionen angesehen werden, die eine explizite Identifizierung als Ziel erlauben:

(6) he collapsed on to his bed, fully dressed

(6d) warf er sich angezogen aufs Bett

Bei der Evaluierung der semantischen Leistung von Kasus ist allerdings zu beachten, dass der Synkretismus deutscher Kasusformen erheblich ist. Die Phrasen können mehrheitlich isoliert nicht interpretiert werden, vielmehr müssen Ko- und Kontextschlüsse vorgenommen werden. ${ }^{7}$ Besonders stark ist der For-

7 In einer Korpusuntersuchung deutscher Zeitungstexte fand Evert (2004), dass nur 22,0\% der Nominalphrasen eindeutig kasusgekennzeichnet waren. 40,8\% waren ambig zwischen zwei Kasus (31,0 \% zwischen Nominativ und Akkusativ), 15,8 \% zwischen drei (13,7 \% zwischen Nominativ, Akkusativ und Dativ!) und 21,4\% zwischen allen vier. 
menzusammenfall von Nominativ und Akkusativ, wo nur für das Maskulinum Singular ein Formunterschied besteht. In Kombination mit der häufigen Akkusativergänzung-Subjekt-Stellung führt dies zu temporären Ambiguitäten, die nicht immer formal aufgelöst werden, etwa durch Numeruskongruenz von Subjekt und Verb (Fischer 2013: 217-228). Man betrachte:

(7) der Kälteeinbruch würde die absurden Phantasien entkräften, die die eisigen Weiten Kanadas in den Köpfen seiner Kollegen erzeugten.

Es ist formal nicht angezeigt, dass die Nominalphrase die eisigen Weiten Kanadas Subjekt und deshalb Agens ist. In folgender Fortführung ist die eisigen Weiten Kanadas Akkusativergänzung:

(8) der Kälteeinbruch würde die absurden Phantasien entkräften, die die eisigen Weiten Kanadas attraktiv erschienen ließen.

Typologisch gesehen ist fehlende formale Differenzierung zwischen Subjekt und direktem Objekt häufig. Als Erklärung kann angenommen werden, dass die Differenzierung meist semantisch gut rekonstruierbar ist.

Im englischen Text ist hier eine andere Lösung gewählt, aber wir können die Relativsatzkonstruktionen für die zwei Beispiele nachvollziehen:

(7e) the sudden cold snap would put paid to the absurd phantasies which Canada's icy wastes created in the heads of his colleagues.

(8e) the sudden cold snap would put paid to the absurd phantasies which made Canada's icy wastes look attractive.

In beiden Sätzen ist das englische Subjekt durch seine Stellung unmittelbar vor dem finiten Verb formal gekennzeichnet. Es ist kein Rückgriff auf den weiteren Ko- oder Kontext nötig. Dies ist grundsätzlich so: Die englische Satzgliedstellung erlaubt die Identifizierung von Subjekt, direktem und indirektem Objekt ohne jegliche Ambiguität.

Dies gilt mit minimalen Einschränkungen auch für Sätze mit Subjekt-VerbInversion, deren präverbale Elemente schon formal nicht als prototypische Subjekte aufgefasst werden können. In den meisten Fällen handelt es sich um Zitate (9), Adverbien (10) oder Adverbialbestimmungen. Adverbiale Nominalphrasen sollten möglich sein, kommen aber de facto nicht vor:

(9) 'It only came to me in the night,' said Adamsberg 
(10) And then came the storm. (Buchtitel)

(11) ?? And that evening came the storm.

Wir halten fest, dass Kasus für sich gesehen eher einen bescheidenen Beitrag zur semantischen Transparenz deutscher Sätze leistet. Abgesehen vom Maskulinum Singular werden die Kernergänzungen lediglich entlang der Opposition Nominativ/Akkusativ vs. Dativ unterschieden. Aber auch zur Durchführung dieser Unterscheidung ist zum Teil Genus- und Numerusinformation nötig. Die Kontextabhängigkeit der Identifikation der zentralen Ergänzungen wird in Grammatiken meines Erachtens nicht genügend gewürdigt; das Augenmerk ist eher darauf gerichtet zu zeigen, dass die Unterscheidungen im Zusammenspiel unterschiedlicher formaler Mittel gesichert seien.

Auch nicht gewürdigt wird die nahezu $100 \%$ formale Unterscheidung und damit verbundene semantische Transparenz der zentralen Ergänzungen im Englischen; z. B. diskutiert Hawkins (1986: 37-51) die pragmatische Transparenz der flexiblen deutschen Satzgliedstellung, geht aber auf den semantischen Effekt der Fixierung der englischen Satzgliedfolge nicht ein. Es muss hier hervorgehoben werden, dass Kasusverlust eine Fixierung der Satzgliedfolge begünstigt, aber dass diese kein zwangsläufiges Ergebnis ist und insofern als Grammatikalisierung angesprochen werden sollte (vom Indiz zum Zeichen).

Die weitgehende Einschränkung der englischen externen Possessorkonstruktion wird mit dem Zusammenfall von Dativ und Akkusativ in Zusammenhang gebracht (z. B. König \& Gast 2009: 120, 253). Der Zusammenfall kann aber keine hinreichende Bedingung darstellen, da sonst das Fortbestehen der DoppelObjekt-Konstruktion (give somebody something) nicht erklärbar wäre. Die Fixierung der Satzgliedfolge hätte den semantisch transparenten Fortbestand der externen Possessor-Konstruktion ermöglicht, wie sie von Sprechern des internationalen Englischs immer wieder versucht wird. ${ }^{8}$ McWhorter, der im Rahmen der keltischen Hypothese einen bryonischen Substrateinfluss annimmt (siehe auch Vennemann 2002), weist auf den, wenn auch eingeschränkten, weiteren Fortbestand der externen Possessor-Konstruktion im Niederländischen und in den skandinavischen Sprachen hin (McWhorter 2002: 226).

Hawkins (1986: 53-73) sieht, wie oben angesprochen, eine vagere Semantik des englischen Subjekts und direkten Objekts im Vergleich zu ihren deutschen Entsprechungen. Es lassen sich aber leicht untypische Rollenzuweisungen an

8 As a Vampire he has immortality and regenerative abilities so there's no Problem when someone cut him his arm off. http://aminoapps.com/page/anime/8816261/character-analysisferid-bathory (letzter Zugriff 9. 7. 2017). 
deutsche Subjekte und Akkusativergänzungen finden. Anzuführen sind z. B. Verben mit Stimulus-Subjekten und Experiencer-Akkusativergänzungen, die gleich zwei untypische Rollenzuweisungen zeigen:

(12) Hochwichtige Akten, blutige Verbrechen. Die den gewaltigen Heizkessel indes nicht interessierten.

(12e) Important files, dealing with violent crimes. But the boiler wasn't concerned with all that.

Die Rollenzuweisungen im englischen Pendant sind dagegen regulär.

Man kann das Argument aber auch durch eine abstrakte Überlegung zurückweisen: Deutsch und Englisch haben dieselbe Anzahl an Ergänzungen, wenn man von der ganz niedrigfrequenten Genitivergänzung absieht. ${ }^{9}$ Wären das englische Subjekt und direkte Objekt semantisch vager, wäre zu erwarten, dass bei anderen Ergänzungen schärfere Rollenprofile aufträten. Dies ist bei einer englischen Ergänzung tatsächlich der Fall, nämlich bei dem indirektem Objekt, das viel stärker auf prototypische Dativrollen festgelegt ist als die deutsche Dativergänzung: Das englische indirekte Objekt - dies kann hier nicht im Einzelnen gezeigt werden - akzeptiert nur begrenzt die Rolle des Benefizienten, kaum die Rolle des Malefizienten und überhaupt nicht die Rolle der Quelle bei Verben des Nehmens sowie keine externen Possessoren. Den Dativergänzungen entsprechen häufig englische Präpositionalobjekte, direkte Objekte, Subjekte sowie Possessivphrasen. Während sowohl das Subjekt und direkte Objekt in beiden Sprachen jeweils semantisch vage Großklassen bilden, gibt es im Sprachvergleich tatsächlich eine semantisch transparente Termergänzung: das englische indirekte Objekt (Fischer 2013: 259-268).

Kasus bietet Evidenz für drei der vier oben angeführten Thesen: Die Systemkomplexität (vier Kasus) vererbt sich nicht auf die Textrealisierung, da zwei Kasus überwiegend mit derselben Form realisiert werden und es weitere Formsynkretismen gibt. Deshalb leistet Kasus eine Differenzierung der Kernergänzungen in einem viel geringerem Maße, als Systemdarstellungen es suggerieren. Die Grammatikalisierung von Topologie im Englischen erweist sich dagegen als perfektes Mittel zur Unterscheidung der Kernergänzungen. Die Abbildung der Kernergänzungen auf semantische Rollen ist im Englischen - auch dies konnte hier nicht ausführlich gezeigt werden - nicht vager als im Deut-

9 Fünf Termergänzungen einschließlich Genitivergänzung (Deutsch) bzw. vier (Englisch), einander entsprechende Adverbial- und Prädikativergänzungen sowie Verbativergänzungen. Letztere sind im Englischen stärker formal differenziert; vgl. Fischer (2013: 189-191). 
schen (Fischer 2013: 212-217). Was die Abbildung der zentralen Satzstruktur auf Sachverhalte betrifft, ist Englisch semantisch transparenter.

\subsection{Pragmatische Wortstellung}

Freilich führt die Grammatikalisierung der Topologie der zentralen Ergänzungen zu einer „monofaktorialen Abfolge“ (König \& Gast 2009: 253), die pragmatische Transparenz erschwert. Deutsch besitzt dagegen eine multifaktoriale Abfolge und kann damit stärker pragmatische Information anzeigen:

(13) Eine Not, ein namenloser Schmerz, der ihm die Tränen in die Augen trieb.

Einen Namen aber hätte er gebraucht.

(13e) A distress which brought tears to his eyes, a sorrow without a name.

He had to put a name to it.

Im zweiten Satz von (13) wird auf eine im vorausgehenden Abschnitt eher beiläufige Phrase (namenlos) zurückgegriffen, um ein neues Thema zu etablieren. Das neue Thema erscheint stark fokussiert in Erstposition. Diese Möglichkeit existiert auch im Englischen:

(14) A name he had to put to it.

Sie ist aber stilistisch stark markiert und wird vermieden (vgl. König \& Gast 2009: 161). Ich glaube nicht, dass Kasus- oder Verbzweitverlust schlüssige Erklärungen für die Vermeidung von direkten Objekten in der englischen VorSubjekt-Position darstellen. Die initiale Phrase wird bereits beim Auftreten der nächsten Phrase als Nicht-Subjekt erkannt, im Gegensatz zu satzinitialen deutschen direkten Objekten ohne Akkusativ-Nominativ-Differenzierung (siehe oben). Kasusverlust könnte allenfalls die Prohibition direkter Objekte in Erstposition in den verbliebenen Verbzweitstrukturen erklären, Formensynkretismus verhindert aber nicht präverbale direkte Objekte im Deutschen. Funktional gesehen sollte eine feste Satzgliedfolge die Topikalisierung einzelner Satzglieder durch Herauslösung aus dieser begünstigen. Warum stören zwei satzinitiale Nominalphrasen, wenn sie an anderer Stelle im Satz akzeptabel sind? Eher als V2-Verlust bietet sich ein Fortwirken von V2 bei fester SubjektVerb-Achse als Erklärung an: Es fällt auf, dass satzinitiale Zirkumstanzien vom Restsatz getrennt erscheinen, was schriftlich oft durch ein Komma angezeigt wird. Diese Herauslösung ist für zentrale Ergänzungen aber keine Option. Man 
kann annehmen, dass die Entwicklung einer englischen Topikposition vor dem Subjekt unabgeschlosssen ist.

Neben der Topikalisierung des direkten Objekts erlaubt die häufige Alternanz indirekter Objekte mit Präpositionalobjekten (give sbdy sthg vs. give sthg to sbdy) die Positionierung der Rezipiensphrase hinter dem direktem Objekt. Und für das Subjekt steht in gewissen Kontexten Subjekt-Verb-Inversion zur Verfügung. Für andere englische Satzglieder, z. B. Adverbiale, gibt es mehrere Positionen, so dass auch die englische Satzgliedfolge in den meisten Fällen pragmatisch genutzt werden kann, einschließlich Positionen, die kein Gegenstück im Deutschen haben (Rohdenburg 1991):

\section{(15) Carefully open the sachet}

\section{(15d) ??Vorsichtig öffne/öffen Sie die Tüte}

Die englische Satzgliedstellung ist also nur weniger multifaktorial als die deutsche. Zwar ist die deutsche Wortstellung pragmatisch komplexer und besitzt damit größere informationsstrukturelle Transparenz, aber auch in Bezug auf pragmatische Transparenz zeigt sich ein differenziertes Bild, wobei die theoretisch mögliche satzinitiale Position von englischen Objekten in der Textrealisierung wenig genutzt wird.

\subsection{Verbpräfigierung}

Die Präfigierung von Verben gehört zur Wortbildungskomplexität. Sie ist hier einbezogen, da sie sowohl von Hawkins (1986: 28-35) als auch von McWhorter (2002: 231-234) als Bereich angeführt wird, in dem Englisch erheblich an Komplexität eingebüßt hat (vgl. auch Plank 1984):

(16) sich ein Loch für die Robbenjagd aufhacken

(16e) dig a hole to hunt seals

Das Halbpräfix auf gibt einen Hinweis auf Art und Ziel des Hackens, dig ist dagegen neutral. In dem verglichenen Textabschnitt gibt es 28 Halbpräfixverben (z. B. einstellen, umherstapfen, abwarten; alle Tokens (Exemplare) von unterschiedlichen Types (Lexemen)), denen 21 phrasal verbs gegenüberstehen (z. B. conjure up, put on, break down; 20 Types). Etwas deutlicher ist das Verhältnis bei festen Verbpräfixen: Hier gibt es 33 deutsche Präfixverben (z. B. begutachten, gelangen, unterbrechen; alle Tokens von unterschiedlichen Types), 
aber nur 24 englische (21 Types), wovon 21 Tokens Präfixe lateinischen Ursprungs zeigen (conjure, arrive, restore vs. become, foresee). Auch sind die meisten englischen Präfixverben nicht oder nicht mehr kompositional (z. B. contemplate, differ, offer), in dem vorliegenden Text am ehesten noch remind und foresee, und - wenn man an die wörtlichen, hier aber nicht vorliegenden Lesarten denkt - express und subside. Allerdings stützen die Präfixe durch Reihenbildung die Verbbedeutungen (z. B. arrive, accomplish, arrest; escape, emerge, erupt). Dies alles gilt natürlich auch für die deutschen Präfixverben; aber hier lassen sich klarere und zahlreichere Fälle von Kompositionalität anführen (durchqueren, unterschreiben, verzögern, erreichen, verschwimmen).

Wir halten fest, dass Englisch weniger Präfigierung hat, was sich auch quantitativ in der Textrealisierung niederschlägt, dass der Textkontrast aber nicht so dramatisch ist, wie es der weitgehende Verlust germanischer Verbpräfixe nahelegt.

\subsection{Reflexivpronomen}

Das Reflexivpronomen sich in (17) zeigt den Benefizienten an:

(17) und sich die Zeitung griff

(17e) and picking up the newspaper

Der Gebrauch englischer Reflexivkonstruktionen ist viel eingeschränkter; ein Hinweis auf den Benefizienten durch ein bloßes Reflexivpronomen wäre im gegebenen Kontext ungewöhnlich, ebenso in (16) oben. ${ }^{10}$

Inhärente Reflexivität wird viel seltener angezeigt als im Deutschen (McWhorter 2002: 220-224):

(18) sich um den Kaffeeautomaten drängten

(18e) crowding round the coffee machine

Im folgenden Beispiel findet sich eines der wenigen englischen Verben, bei denen die Anzeige inhärenter Reflexivität obligatorisch ist (vgl. Huddleston \& Pullum 2002: 1488):

10 Zwar ist dig oneself a hole möglich, aber es scheint auf Situationen beschränkt, wo der Protagonist sich in das Loch begibt (dig herself a hole to lie in). 
(19) we have an old man who stumbles and stutters and can't express himself

(19e) wir haben einen zögerlichen alten Mann, der sich in seinen Worten verstrickt.

Im untersuchten Textabschnitt gibt es 21 deutsche, aber nur zwei englische Reflexivpronomen. Reflexivierung ist ein Bereich, in dem Deutsch erheblich komplexer ist. Die mit Reflexivierung verbundene Information ist allerdings nicht besonders hoch zu veranschlagen: Wenn nicht anders angezeigt, macht man etwas für sich (vgl. 17e). Das Anzeigen von inhärenter Reflexivität stellt per definitionem keinen semantischen Mehrwert dar. Allenfalls hilft es, zwischen Verblesarten zu unterscheiden (express oneself vs. express a thought; sich aufmachen vs. etwas aufmachen), wofür sonst ein Schluss vom fehlenden Objekt her nötig ist (crowd vs. crowd somebody in 18e), und bei Verbendstellung eine vage Erwartung auf das zu erwartende Szenario aufzubauen (Ich hatte mich ...: kein Szenario, wo auf ein vom Subjektreferenten unterschiedenes Patiens eingewirkt wird). Der Aufwand für diese marginalen Effekte ist allerdings erheblich.

\subsection{Genus}

Englisch hat kein grammatisches Genus. Dies bedeutet zweifelsohne geringere grammatische Komplexität im Vergleich zum Deutschen; aber es ist nicht so klar, ob damit eine Differenz in der semantischen Transparenz verbunden ist. Zwar kann man sagen, dass die Artikel der, die, das die Information geben, welches Genus das folgende Nomen hat; aber diese Information ist nur hilfreich, wenn Genus semantisch relevant ist. Schließlich müssen englische Sprecher das fehlende Genus nicht kontextuell erschließen. Leiss (2005) geht von einem variablen ide. Genus aus, das Wortstämme quantitativ perspektiviert hätte und deshalb als Flexion anzusehen wäre. Trotz des weitgehenden Verlusts des Mehrfachgenus sei dessen Quantelung der Welt noch wirksam und werde nun über Wortbildung realisiert: Das Maskulinum fasse eine Bedeutung als begrenzt und führe zu Singulativa (der Kristall, der Schrei, der Schreier, der Blauton), das Femininum fasse eine Bedeutung als distribuiert und führe zu Kollektiva (die Schreierei) oder Abstrakta (die Bläue), das Neutrum fasse eine Bedeutung als unbegrenzt und führe $\mathrm{zu}$ Massennomina (das Kristall, das Schreien/Geschreie, das Blaue). Der von Leiss aufgewiesene Zusammenhang soll nicht bestritten werden; aber es stellt sich die Frage, wie stark er ist. Zwar führt Van Mol (2017) ,abstraktes Neutrum‘ als eines der Kriterien an, die bei der Genuszuweisung an französische Lehnwörter wirksam ist; aber es wird von 
neun anderen Kriterien dominiert, wobei allerdings die anderen Kriterien (z. B. ,semantische Äquivalenz‘, ,semantische Gruppe`) den abstrakten quantitativen Prinzipien nicht widersprechen müssen. In Steinmetz' (1986) elaboriertem Regelsystem der Genuszuweisung im Deutschen ist Neutrum mit dem Konzept Oberbegriff verbunden.

Verfügt ein deutscher Hörer über eine mit den drei bestimmten Artikeln verbundene semantische Erwartungshaltung, die einem englischen Hörer vorenthalten ist, der den englischen Einheitsartikel the rezipiert? Die Frage kann hier nicht beantwortet werden; aber meine Vermutung ist, dass die abstrakten Korrelationen und etwaige konkretere mit dem Genus verbundenen semantischen Erwartungshaltungen zu schwach sein dürften, um bei einem Vergleich der semantischen Transparenz verbucht zu werden. Da die semantische Information im folgenden Nomen ebenfalls enthalten ist, dauerte der Vorteil meist nur kurz an - kürzer als z. B. der Nutzen, der aus der durch Kasus gegebenen Valenzinformation bei Verbendstellung entsteht. Davon unbenommen führt die deutsche Wortbildungsmorphologie zu einer größeren semantischen Transparenz im Vergleich zum wortbildungsmorphologisch ärmeren Englischen.

Als Funktion des Genus wird auch angeführt, dass es innertextliche Bezüge durchsichtiger mache und auf diese Weise zur semantischen Transparenz beitrage. Bei nur drei Nomenklassen ist dieser Nebeneffekt nicht sehr häufig, aber real:

(20) ein unsagbares Gefühl von Trauer, wie ein trüber Bodensatz, den die wegströmende Welle hinterlässt

Das Relativpronomen den macht den Bezug auf das maskuline Nomen Bodensatz explizit; man vergleiche eine alternative Fortführung:

(21) ein unsagbares Gefühl von Trauer, wie ein trüber Bodensatz, das über ihn kam

Im von der Konjunktion that eingeleiteten Nebensatz gibt es keine morphologische Disambiguierung der Bezugsphrase; allerdings macht die Interpunktion diesen eindeutig:

(20e) an inexpressible feeling of sorrow, like a dark sediment that the wave leaves behind when it ebbs

In Satz (22) disambiguiert das Relativpronomen zwischen drei Fortführungsmöglichkeiten: 
(22) Da haben wir ein Abendessen im Kreise der Familie, das in einem Gemetzel endet [der diesmal kleiner als üblich war/die vollständig präsent war]

Die folgenden Beispiele zeigen die Chancen und Grenzen der Disambiguierung durch Genus bei der anaphorischen Wiederaufnahme von Nominalphrasen durch Personalpronomen:

(23) Adamsberg sah zu, wie das Bild rucksend aus dem Gerät kam. Er griff es an der Ecke, ließ es rasch in der Luft trocknen und schaltete dann die Lampe an, um es genauer zu betrachten.

Das Pronomen es kann sich formal gesehen auf Bild oder Gerät beziehen, aber nicht auf Adamsberg, Ecke, Luft oder Lampe.

(23e) Adamsberg was watching as the page emerged in stops and starts from the machine. He picked it up by a corner, waved it quickly in the air to dry, then switched on the desk lamp to examine it closely.

Im englischen Text kann it sich dagegen auf page, machine, corner, air und desk lamp beziehen. Es bedurfte einer Suche, um ein Beispiel zu finden, das einen auf Genus zurückzuführenden Vorteil der anaphorischen Wiederaufnahme zeigt; und es ist kein Zufall, dass das Beispiel die Wiederaufnahme eines Neutrums enthält. Erzähltexte handeln von Personen. Das eröffnet im Deutschen die Möglichkeit von Ambiguität zwischen personenbezogenen und nicht personenbezogenen Bezügen bei Wiederaufnahme durch maskuline oder feminine Pronomen, während diese Möglichkeit im Englischen nicht besteht. Man betrachte folgendes Beispiel:

(24) Er hielt ein wenig Abstand und wartete, dass der kräftigste seiner Lieutenants sein Glas von den Zitzen der Maschine zog - denn in der Vorstellungswelt des Kommissars erinnerte der Getränkeapparat an eine Nährkuh, die sich in den Büros der Mordbrigade niedergelassen hatte, eine stille Mutter, die über sie wachte, und dafür liebte er ihn.

Das maskuline Pronomen ihn schließt Bezug auf mehrere Nominalphrasen aus (eine stille Mutter, den Büros ..., der Mordbrigade, eine Nährkuh ..., der Vorstellungswelt..., der Maschine, den Zitzen ..., sein Glas, seiner Lieutenants), aber nicht auf des Kommissars, der kräftigste ..., Abstand. Der korrekte Bezug auf der Getränkeapparat ist also teils formal, teils aber nur kontextuell gesichert. 
(24e) He stood back, waiting for his heftiest officer to take her cup from the machine's udder - since in his mind the drinks dispenser was a kind of dairy cow, tethered inside the Crime Squad's offices, like a silent mother watching over them all, the reason he was so fond of it.

Das unpersönliche Pronomen it schließt Bezug auf a silent mother, the Crime Squad's offices und his heftiest officer aus, aber nicht auf the Crime Squad, a kind of dairy cow und the machine's udder.

Wir halten fest, dass Genus ein Musterbeispiel für grammatische Komplexität ist, die nicht oder nur ganz geringfügig zu semantischer Transparenz beiträgt (vgl. Trudgill 2011: 154-167). Auch was die Disambiguierung der anaphorischen Wiederaufnahme betrifft, scheint der Effekt wenig größer als im Englischen, wo ebenfalls drei, allerdings nach natürlichem Genus sortierte Personalpronomen zur Verfügung stehen. Ein Vorteil ergibt sich aber gegenüber dem häufig neutralen englischen Relativanschluss. ${ }^{11}$ Der Aufwand für diese Effekte scheint allerdings unverhältnismäßig.

\section{Grammatische Komplexität und semantische Transparenz im Englischen}

Ich werde nun auf eine Reihe von Konstruktionen hinweisen, die entweder kein oder ein viel weniger genutztes deutsches Gegenstück haben. In diesen Fällen ergibt sich größere semantische Transparenz im Englischen (vgl. Rohdenburg 1990, 1991).

\subsection{Die -ing Form}

Ins Auge fallen die vielen Verwendungen der aus dem Gerundium entstandenen -ing-Form (Fanego 2004), die auf einer Skala von verbal bis nominal anzusiedeln sind und die eine gemeinsame Semantik um die Bedeutungen ,andauernd', ,zeitgleich“, ,wiederholt' verbindet. Am verbalen Ende des Spektrums besitzt das Englische eine mit -ing gebildete obligatorische Verlaufsform für dynamische Verben:

11 Genus trägt auch zur oben angesprochenen Disambiguierung von Kasusformen bei - aber nur in dem Maße, wie Ambiguität durch die Existenz identischer, aber kasusdifferenter Formen in den drei Genera geschaffen wurde. 
(25) 'What are you thinking about?' she asked after a while.

(26) *'What do you think about?' she asked after a while.

Das simple present in (26) ist in diesem Kontext nicht möglich; man erwartet eine Fortführung, die in eine generelle Frage oder andere Konstruktion mündet:

(27) 'What do you think about first thing in the morning/my suggestion?' she asked after a while.

Im deutschen Text gibt es keinen formalen Hinweis auf den kontemporären Bezug der Frage:

(25e) „Woran denken Sie?“, begann sie nach einer Weile wieder.

Der Bezug muss vielmehr kontextuell erschlossen werden.

Die Obligatorität der englischen Verlaufsform gehört zusammen mit der obligarischen do-Umschreibung und in gewissen Kontexten unvermeidbarem preposition stranding zu den exotischen Merkmalen des Englischen. Unabhängig davon, ob sie eine genuine eigene Entwicklung darstellt (z. B. Visser 1966: 1065-1223) oder durch im schriftlichen Medium erst spät dokumentierten keltischen Substrateinfluss entstanden ist (McWhorter 2008) oder eine kombinierte Entwicklung vorliegt (Hickey 2012), repräsentiert sie (analytische) Formkomplexität, die entweder semantische Transparenz erlaubt oder erzwingt. ${ }^{12}$

Auch eher verbal sind -ing-Verwendungen als Nebensätze, die zum Teil Alternativen $\mathrm{zu}$ Infinitiven bilden:

(28) the enormous central heating boiler which had suddenly stopped working

Working ist Ergänzung zu stopped. Die Form der Ergänzung ist von der vorliegenden Lesart von stop selegiert: stop und work sind nicht zeitlich getrennt. Man vergleiche die zeitlich getrennte Finalangabe in stopped to work (,hielt an, um zu arbeiten'). Die spezifische Semantik der -ing-Form bewirkt eine stärkere Assoziation der Hauptkonkurrenzform, des to-Infinitivs, mit Zukunftsbezogen-

12 Obwohl man die Verlaufsform trotz größerer formaler Komplexität als das simple present als semantisch unmarkiert ansprechen kann, da sie den Bezug auf kontemporäre Tätigkeiten oder Vorgänge bewirkt, stellt sie kein Gegenbeispiel zur Markiertheitshierarchie dar: Sie ist eindeutig weniger frequent als das simple present. 
heit. Wie bei derartigen Grammatikalisierungen zu erwarten sind die semantischen Bezüge nicht perfekt, aber eindeutig gegeben.

Vorkommnisse von -ing haben ganz unterschiedliche deutsche Gegenstücke: Partizipien, Infinitive, Nomen, Präpositionen und gleich- oder untergeordnete finite Teilsätze. Der Kontrast zum deutschen Partizip Präsens ist nicht nur quantitativer Natur, da dieses nicht als Ergänzung fungieren kann (Fischer 2013: 68). Die -ing-Form ist nicht nur eine häufige Ergänzung, sondern ist auch in dieser Funktion mit dem vollen Satz ihrer Ergänzungen einschließlich Subjekt kombinierbar:

(29) Danglard disliked the commissaire reading the newspaper he had just bought.

(29d) Danglard mochte es nicht, wenn der Kommissar seine Zeitung las.

Man beachte, das -ing auch Ergänzung zu Präpositionen sein kann:

(30) And on that terrible day, I despaired of ever being able to clear my brother's name.

(30d) Und an diesem schwarzen Tag habe ich die Hoffnung aufgegeben, jemals die Unschuld meines Bruders beweisen zu können.

Ein direkter infiniter Anschluss an Präpositionen ist im Deutschen nicht möglich. Entweder erscheint die Präposition nicht wie in (30d), oder sie wird in ein Korrelat integriert (Hoffnung darauf, ....). ${ }^{13}$ Im ersteren Fall ist der englische Anschluss grammatisch transparenter, da das Verhältnis von infinitem Teilsatz und Regens (hier: despaired) durch eine konstante, also grammatikalisierte Präposition ( $o f$ ) explizit geregelt ist, während das Verhältnis des Infinitivsatzes in (30d) zum Regens Hoffnung nicht explizit angezeigt ist. Freilich ist mit diesem Mangel an grammatischer Transparenz zumindest in diesem Fall kein Mangel an semantischer Transparenz verbunden, da die Valenz des Nomens Hoffnung einen propositionalen Inhalt verlangt, der durch den Infinitivsatz gegeben ist, und es keinen Zuordnungskonflikt gibt.

Wir können festhalten, dass die englische -ing-Form sowohl in einem Satz von Tempusformen als auch in vielfältigen Teilsatzkonstruktionen verwendet wird, während das deutsche Gegenstück, das Partizip Präsens, eine viel einge-

13 Präpositionen, die einen Teilsatz regieren (ohne Protest/zu protestieren/dass sie protestiert), werden, wenn sie es tun, als Konjunktionen angesprochen (Duden 2006: 861, 1058; Eisenberg 2006: 371-372), im Falle von ohne dass als komplexe (Eisenberg 2006: 203). 
schränktere Verwendung hat. Als Resultat ergibt sich größere Systemkomplexität der englischen analytischen Verbformen und der englischen infiniten Ergänzungssätze (siehe auch Abschnitt 2.2 unten), häufigere Textrealisierung infiniter Angabesätze und in vielen Fällen größere semantische Transparenz.

\subsection{Die for-to-Infinitivkonstruktion}

Englisch besitzt auch eine zusätzliche von der depräpositionalen Konjunktion for ${ }^{14}$ eingeleitete to-Infinitivkonstruktion:

(31) It could take a trifle - an unusual word, an imperfectly formulated idea - for him to launch into an erudite and not always well-timed lecture, which could be stopped by a warning gesture.

(31d) Ein schwacher Anreiz genügte - ein selten verwendetes Wort, ein verschwommener Begriff -, und schon setzte sich bei ihm ein gelehrter Mechanismus in Gang, der nicht unbedingt immer sehr angebracht war, sich jedoch mit einer Handbewegung unterbrechen ließ.

Huddleston \& Pullum (2002: 1183) gestehen der Konjunktion for keine eigene Bedeutung $\mathrm{zu}$, aber Subordination deutet einen engeren Zusammenhang der zwei Sachverhalte an als Koordination, weshalb von for eingeleiteten to-Infinitiv-Sätzen im Deutschen häufig finite Nebensätze entsprechen.

Das System der englischen infiniten Teilsätze besitzt dank der zwei Konstruktionen -ing und for + to-Infinitiv (letztere ohne deutsches Gegenstück) eigene Komplexität, die sich in semantischer oder grammatischer Transparenz niederschlägt. Schränkt man den Vergleich auf infinite Ergänzungssätze ein, so ist das englische System um die zwei genannten Konstruktionen reichhaltiger und dank der -ing-Form semantisch transparenter.

Wir haben bei der Betrachtung von Kasus und des Partizips Präsens gesehen, dass es nicht nur auf das grammatische Inventar ankommt, sondern auch darauf, wie dieses realisiert wird (Formsynkretismus von Kasus), welche Konstruktionen es geriert (present participle als Verlaufsform) und in welchen Kontexten es vorkommt (present participle als satzförmige Ergänzung von Verben, Adjektiven, Nomen und Präpositionen).

14 Alternativ wird auch von einer komplexen distribuierten Konjunktion for ... to gesprochen. Der objective case him in (31) zeigt, dass die Grammatikalisierung als Konjunktion noch nicht ganz abgeschlossen ist. 


\subsection{Spaltsätze}

Wir werden nun eine Klasse englischer Konstruktionen betrachten, die im Deutschen bis auf eine zu vernachlässigende Ausnahme (siehe Fischer 2009) formale Gegenstücke besitzen, die aber im Englischen eine deutlich höhere Frequenz aufweisen:

(32) I'm not in charge of this squad, it's not me that's taking us all over there to fool about in the snow like idiots

(32d) Ich leite doch diese Brigade nicht, und ich schick uns auch nicht los, um die Blödmänner zu spielen im Schnee.

Der zweite Teilsatz in (32) ist ein echter Spaltsatz (it cleft sentence). Der thatSatz gibt den vorerwähnten Hintergrund an (die Dienstreise nach Kanada), allerdings mit offener Subjektreferenz. Der von einem kataphorischen it eingeleitete Obersatz gibt den Wert für die offene Stelle des abhängigen Satzes an bzw. schließt im vorliegenden Beispiel einen Wert aus (not me). Der ausgeschlossene Wert ist die neue Information und deshalb stark fokussiert (in der gesprochenen Sprache mit Kontrastakzent). Die Verwendung des Spaltsatzes ist hier rhetorisch, da beide Protagonisten wissen, wer die Dienstreise geplant hat.

Der deutsche Text enthält keinerlei formalen Hinweis darauf, dass er nicht Sätze mit weitem Neuinformationsfokus enthält. Der Leser muss aus dem Kontext schließen, dass ich kontrastfokussiert ist. Dasselbe gilt für den ersten englischen Satz in (32). In der gesprochenen Sprache würde die Akzentuierung eine Disambiguierung leisten. Die zusätzliche Kennzeichnung durch die Spaltsatzkonstruktion wäre dann als Überspezifizierung bzw. redundante Kennzeichnung zu verbuchen.

Spaltsätze sind in besonderem Maße semantisch transparent: Ein eigener Teilsatz (der Obersatz) dient dazu, die neue Information besonders stark zu fokussieren; die Hintergrundinformation ist ikonisch in einem Nebensatz realisiert. Im echten Spaltsatz erlaubt sie auch Positionierung der neuen Information vor der Hintergrundinformation. Entsprechend groß ist der formale Aufwand (in (32) die fett gedruckten Elemente). Im vorliegenden Beispiel führt die neue Information zugleich das Thema (der Sprecher) fort, aber Spaltsätze als Topikalisierungsmittel anzusprechen verkennt ihre Logik: Der Referent der fokussierten Phrase mag zum Hintergrundwissen gehören oder nicht; neu ist seine Funktion als Wert des offenen Satzes (siehe Fischer 2012).

(33) Yes, Danglard would have been quite capable of that. His fluid intelligence could slip into the narrowest mechanisms of the human mind. As 
long as the mechanisms were those of reason and logic. And it was precisely along that watershed, between reason and instinct, that Adamsberg and his deputy so diametrically differed, and had done for years.

(33d) Ja, zu so etwas war Danglard imstande. Seine bewegliche Intelligenz glitt in jedes noch so feine Räderwerk des menschlichen Geistes: vorausgesetzt natürlich, dass es auf Vernunft und Logik beruhte. Und genau hier, auf dem schmalen Grat zwischen Vernunft und Instinkt, waren Adamsberg und sein Stellvertreter mit den Jahren zu absoluten Gegensätzen geworden.

Der letzte Satz in (33) ist der Form nach ein it-Spaltsatz. Der Wert ist eine „Strecke“, entlang der die beiden Protagonisten diametral entgegengesetzt sind. Aber es ist nicht so klar, was hier die neue Information ist. Zwar hat der Leser die beiden Protagonisten als sehr verschiedene Persönlichkeiten kennen gelernt, aber diametrically differed und had done for years sind zwar vorbereitete, aber vielleicht doch neue Informationen. Dass der Gegensatz Vernunft und Instinkt betrifft, ist noch besser vorbereitet als die im abhängigen Satz enthaltene Information. Würde man den Satz vorlesen, wäre es nur eine von möglichen Lösungen, den Hauptakzent auf precisely oder in die von precisely fokussierte Phrase zu setzen. Alternativ könnte auch diametrically den stärksten Akzent erhalten; auf jeden Fall würde man mehrere Akzente setzen. Man muss nicht, aber kann (33) als „Spaltsatz mit informativer Präsupposition“ (Prince 1978) ansprechen. Diese Spaltsätze haben eine eigene diskursive Dynamik: Dem Leser wird neue, aber vorbereitete Information gegeben, wo Hintergrundinformation erwartet wird. Dies lässt den Leser fragen „Ist das schon gesagt worden?“ und zurückblicken, stärkt also die Textkohäsion und die Erzählerautorität: Die Erzählerin bekommt sozusagen im Spaltsatz eine Stimme. Der Aufwand ist wieder erheblich, wie die größere Anzahl der fett gedruckten Elemente in (33) im Gegensatz zu (33d) zeigt.

Der deutsche Text erreicht eine Kontrastfokussierung durch die Fokuspartikel genau, die eine Auswahlsituation zwischen verschiedenen möglichen Entitäten, hier Orten, schafft, auf die sich die Aussage bezieht. Der zutreffende Ort wird durch die fokussierte Phrase hier gegeben. Liest man den Satz mit Kontrastakzent auf genau hier und ohne zusätzliche Neuinformationsakzente im Rest des Satzes, kann man die oben beschriebene klassische Spaltsatzlogik erzwingen. Die aufwendige englische Spaltsatzkonstruktion wäre ein Beispiel von Überspezifikation ohne Informationsgewinn. Setzt man den stärksten Akzent auf absoluten Gegensätzen, so wechselt der Satz an diesem Punkt in einen 
normalen Satz mit fokussierter Neuinformation. Es gibt für den Hörer keinen Hinweis, die neue Information zugleich als Hintergrundinformation $\mathrm{zu}$ betrachten. Ein aufmerksamer Leser wird freilich die neue Information mit zuvor gegebener verbinden; und natürlich wirkt die implizit immer vorhandene Erzählerautorität. Aber es gibt keinen formalen Hinweis darauf, dass dies hier geleistet werden soll. Der deutsche Leser muss schon aufpassen.

Neben Spaltsätzen, denen im Deutschen ein flacher Satz ohne Fokuspartikel entspricht (siehe 32 vs. 32d), sind auch die Spaltsätze mit informativer Präsupposition semantisch bzw. diskurspragmatisch transparenter, selbst wenn das deutsche Gegenstück zu letzteren eine Fokuspartikel enthält.

\section{Quantifizierung der Kontraste}

Wir haben aufgezeigt, dass Englisch durchaus im Vergleich zum Deutschen zusätzliche grammatische und konstruktionelle Komplexität besitzt, die semantische Transparenz englischer Satzstrukturen befördert. In mehreren Beispielen beruhte unsere Argumentation darauf, dass Deutsch und Englisch zwar dieselbe Form (Partizip Präsens) oder Konstruktion (Spaltsätze) aufweisen, dass ihr Gebrauch aber im Englischen vielfältiger und zahlreicher ist. Aber welchen Effekt haben die angeführten Phänomene auf das kontrastive Gesamtbild? Wir werden nun einige Statistiken aufweisen, die ein Licht auf das Gesamtbild werfen. Dabei konzentrieren wir uns - entsprechend dem Fokus auf Textrealisierung - auf Tokens.

Es ist ein Gemeinplatz, dass Deutsch synthetischer ist als Englisch, und Englisch entsprechend analytischer als Deutsch. Genau zu diesen beiden Begriffen haben Kortmann und Szmrecsanyi Maße entwickelt, die den Grad der Synthetizität und Analytizität von Texten messen (Kortmann \& Szmrecsanyi 2009; Szmrecsanyi \& Kortmann 2009, 2012). Aus einer Zufallsauswahl werden für Synthetizität alle Wörter gezählt, die ein grammatisches Morphem aufweisen (wobei Umlaut als grammatisches Morphem gilt); für Analytizität werden alle grammatischen Wörter (Funktionswörter) gezählt. Es werden dann Synthetizitäts- und Analytizitätsquotienten berechnet. Eine Anzahl von 1000 Wörtern hat sich als ausreichende Basis zur Bestimmung der Quotienten erwiesen (Kortmann \& Szmrecsanyi 2009: 277).

Das Verfahren hat beeindruckende Ergebnisse bei der Untersuchung englischer Varietäten erbracht. In der Anwendung auf unterschiedliche Sprachen ist die arbeitstechnische Entscheidung, Wörter statt Morpheme zu zählen, problematisch. Man vergleiche drückten mit pressed. Beide Wörter weisen ein 
grammatisches Tempusmorphem auf (-t und -ed), aber die deutsche Verbform besitzt zusätzlich ein Numerus/Person-Morphem (-en). Es müssen also grammatische Morpheme, nicht Wörter mit grammatischen Morphemen, gezählt werden. Da es um segmentierbare oder anderweitig real vorhandene Morpheme geht, wird das Numerus/Person-Morphem aber nur einmal gezählt. Zirkumfixmorpheme (gewartet) werden doppelt gezählt, um dem größeren Kennzeichnungsaufwand gegenüber den englischen Partizipien (waited) gerecht zu werden. Auch werden Umlaut und segmentale Kennzeichnungen getrennt gezählt; gesunken weist also drei grammatische Morpheme auf.

Wörter sind nicht nur problematisch als Zähleinheit, sondern auch als Bezugsbasis, da Deutsch und Englisch unterschiedliche Wortbildungsmöglichkeiten und Schreibkonventionen besitzen. Die Gesamtzahl der Morpheme der Zufallsauswahl wäre eine bessere Basis, um einen vergleichbaren Quotienten zu bilden. Eine weniger arbeitsintensive und deshalb realistischere Möglichkeit ist die hier gewählte: Wie oben erklärt, werden Passagen eines parallelen Textes analysiert. Die absoluten Zahlen sind dann aussagekräftig, da sie anzeigen, wie viele grammatische Morpheme und grammatische Wörter benutzt wurden, um dieselbe Information zu kommunizieren.

Jede derartige Zählung setzt eine scharfe Grenzziehung voraus, während es in der Realität fließende Übergänge zwischen den Kategorien gibt. Grammatische Wörter besitzen in unterschiedlichem Ausmaß lexikalischen Inhalt, lexikalische Wörter können weitgehend auf eine grammatische Funktion reduziert sein. ${ }^{15}$ Weniger wichtig ist die Grenzziehung an sich als die konsistente Handhabung in zumindest einer Untersuchung. Die diesbezüglichen Erklärungen sind meist knapp, und auch mein Beitrag kann hier keine Ausnahme bilden. Wortbildungsmorpheme werden hier wie auch in anderen Studien nicht als grammatische Morpheme gerechnet. Wie Leiss (2005: 11-13) anmerkt, ist diese traditionelle Grenzziehung problematisch, da Wortbildungsmorpheme abstrakte Bedeutungen nach Maßgabe grammatischer Morpheme tragen, z. B. Genusinformation. Bei Betrachtung der Ergebnisse ist zu berücksichtigen, dass eine große Quelle morphologischer Komplexität im Deutschen unberücksichtigt bleibt.

In verschiedenen Arbeiten wird Ablaut aus der Zählung der grammatischen Morpheme herausgelöst, um einen eigenen Irregularitätsindex zu bilden (siehe Szmrecsanyi \& Kortmann 2012: 17; Maitz \& Németh 2014: 6-7). Ablaut gilt hier als eine Form von Suppletion, da der Wortstamm verändert wird (siehe

15 Z.B. Verben in Funktionsverbgefügen (eine Entscheidung treffen) oder als Präpositionsäquivalente (pay using/with a credit card). 
Tab. 4.1: Beispiele für grammatische Morpheme und Wörter.

\begin{tabular}{|c|c|c|}
\hline & Deutsch & Englisch \\
\hline $\begin{array}{l}\text { grammatische Morpheme } \\
\text { (segmental) }\end{array}$ & $\begin{array}{l}\text { schwarze, Tage, jede, gewaltigen } \\
\text { (Sg.), wäre, der (Nom. Sg.), den } \\
\text { (Sg.), Füßen (2), gelehnt (2), } \\
\text { gesunken (2), betrachtete (2), } \\
\text { eingestellt (2), hatte (2) }\end{array}$ & $\begin{array}{l}\text { leaning, his, contemplating, } \\
\text { stopped, told, taken, had, } \\
\text { did, been, days, Adamberg's, } \\
\text { his }\end{array}$ \\
\hline $\begin{array}{l}\text { grammatische Morpheme } \\
\text { (suppletiv, } \\
\text { Ablaut/Umlaut) }\end{array}$ & $\begin{array}{l}\text { Füßen, Räumen, gesunken, gab, } \\
\text { könnte, käme, möge, wäre, die } \\
\text { (Sg.) }\end{array}$ & $\begin{array}{l}\text { stood, frozen, told, said, } \\
\text { knew, were, did, done }\end{array}$ \\
\hline $\begin{array}{l}\text { grammatische Morpheme } \\
\text { (suppletiv, Wort) }\end{array}$ & war, waren, wäre, ihm, besser & was, were, went \\
\hline grammatische Wörter & $\begin{array}{l}\text { jede, wäre, der, hatte, möge, } \\
\text { ihm, zuvor, auf, während, } \\
\text { ungefähr, nun, aber, } \\
\text { besonders, schließlich }\end{array}$ & $\begin{array}{l}\text { his, had, did, been, were, at, } \\
\text { against, which, before, } \\
\text { nothing, only, sometimes, } \\
\text { already }\end{array}$ \\
\hline
\end{tabular}

Plank 2016). Abgesehen davon, dass bei Ablaut auch synchron durchschaubare Musterbildungen vorliegen, Irregularität also nicht ein ganz glücklicher Begriff ist, kann man natürlich Suppletion in diesem Sinne gesondert zählen. Hier werden suppletive Formen zu den grammatischen Morphemen dazugerechnet; diese werden dann in segmentale grammatische Morpheme, suppletive grammatische Morpheme (Ablaut, Umlaut) und suppletive grammatische Morpheme (Wort) unterteilt (s. Tab. 4.1). Die Entscheidungen, welche Form zu welcher suppletiv ist, werden ahistorisch aufgrund von Systematik und der Markiertheitsskala getroffen. Die gezählten Fälle sind sicher nicht exhaustiv; es wird z. B. darauf verzichtet, Suppletion in den Numerus- (ich vs. wir etc.) und Genusparadigmen (he vs. she, it) von Personalpronomen zu zählen. Wichtig ist, wie gesagt, dass die gezählten Elemente in beiden Sprachen nach denselben Prinzipien ermittelt werden.

Als grammatische Wörter gelten nur die klassischen Fälle geschlossener und überschaubarer Klassen. Zahlwörter und die meisten Adverbien sind ausgenommen. Schließlich wurde als grammatisches Wort eingeordnet, da es als Textadverb einer geschlossenen Klasse angehört, gleichfalls während als Konjunktion. Die Ergebnisse der Zählung sind wie folgt (s. Tab. 4.2): 
Tab. 4.2: Grammatische Morpheme und Wörter in der deutschen und englischen Übersetzung von F. Vargas Sous Les Vents De Neptune (Ausschnitt aus Kap. 1).

\begin{tabular}{lll}
\hline & Deutsch & Englisch \\
\hline $\begin{array}{l}\text { 1. grammatische Morpheme } \\
\text { (segmental) }\end{array}$ & $508 / 1002$ & $242 / 1063$ \\
\hline 2. grammatische Morpheme & 50,7 & 22,8 \\
(suppletiv: Ablaut/Umlaut) & $102 / 1002$ & $49 / 1063$ \\
\hline 3. grammatische Morpheme & 10,2 & 4,6 \\
(suppletiv: Wort) & $31 / 1002$ & $31 / 1063$ \\
\hline 4. grammatische Wörter & 3,1 & 2,9 \\
(Analytizität) & $545 / 1002$ & $539 / 1063$ \\
\hline & 54,4 & 50,7 \\
\hline 1.+2. grammatische Morpheme & & $291 / 1063$ \\
(Synthetizität) & $610 / 1002$ & 27,4 \\
\hline 2.+3. Suppletion & 60,9 & $80 / 1063$ \\
(Irregularität) & $133 / 1002$ & 7,5 \\
\hline 1.+2.+4. morphologische Grammatizität & 13,3 & $830 / 1063$ \\
& $1155 / 1002$ & 78,1 \\
\hline
\end{tabular}

Wie erwartet, besitzt Deutsch deutlich mehr grammatische Morpheme: Bezogen auf den parallelen Textausschnitt sind es mehr als doppelt so viele (610 vs. 291), bezogen auf die Wortanzahl ist die Differenz noch prägnanter $(60,9$ vs. 27,4 pro 100 Wörter). Hierfür gibt es zwei erwartungsgemäße Gründe: Erstens gibt es in dem deutschen Text mehr als doppelt so viele segmentale grammatische Morpheme wie in dem englischen (508 vs. 242); bezogen auf die Wortanzahl ist die Differenz wiederum noch deutlicher (50,7 vs. 22,8 pro 100 Wörter). Zweitens gibt es im deutschen Text doppelt so viel partielle Suppletion durch Ablaut oder Umlaut (102 vs. 49; 10,2 vs. 4,6 - bezogen auf 100 Wörter). Die Anzahl von Wortsuppletion ist - zumindest nach den hier berücksichtigten Suppletionen - nahezu identisch (31; 3,1 vs. 2,9 pro 100 Wörter).

Überraschend ist die Anzahl grammatischer Wörter. Der etablierte Konsens ist, dass Englisch in einem stärkeren Maße als Deutsch synthetische Kennzeichnung mittels segmentaler grammatischer Morphologie und Ablaut durch analytische Kennzeichnung, also grammatische Wörter, ersetzt hat. Man würde deshalb eine deutlich größere Anzahl grammatischer Wörter im englischen Text erwarten. Dies ist nicht der empirische Befund. Der deutsche Text hat sogar etwas mehr grammatische Wörter (545 vs. 539); bezogen auf die Wortanzahl steht ein Index von 54,4 pro 100 deutsche Wörter einem von 50,7 pro 100 englische Wörter gegenüber. 
Im untersuchten Text hat der stärkere Abbau von grammatischer Morphologie im Englischen also nicht zu größerer morphologischer Analytizität als im Deutschen geführt - anders ausgedrückt: Die Zunahme morphologischer Analytizität im Englischen war nicht größer als die Zunahme morphologischer Analytizität im konservativeren Deutschen.

Der Befund stellt eine weitere Bestätigung der von Kortmann und Szmrecsanyi an englischen Varietäten festgestellten positiven Korrelation zwischen Synthetizität und Analytizität dar: Bei diesen gibt es zwar einen Abfall der Synthetizität von relativ kontaktarmen regionalen Dialekten (z. B. Southeast, East Anglia, Midlands, North) über kontaktreichere L1-Varietäten (z. B. englische Umgangssprache in Großbritannien, den USA und Australien) zu L2-Varietäten (z. B. Hong Kong-Englisch), aber keine entsprechende Zunahme von Analytizität, sondern ebenfalls einen Abfall. Auch Maitz \& Németh (2014: 16) stellen in einer Untersuchung zu deutschen Varietäten (Zimbrisch, gesprochenes Standarddeutsch, Black Namibian German, Unserdeutsch) eine positive Korrelation zwischen Synthetizität und Analytizität fest (siehe auch Maitz 2014: 105).

Diese Ergebnisse haben typologische Brisanz: Der durch viele Einzelbeispiele belegte Ausgleich zwischen synthetischer und analytischer morphologischer Komplexität schlägt nicht unbedingt auf Texte durch. Es wären freilich Untersuchungen an typologisch stärker unterschiedlichen Sprachen nötig, um die Reichweite dieses Nicht-Ausgleichs zu bestimmen.

Mein Befund hat eine weitere Konsequenz: Nimmt man an, dass Grammatizität, also die Frequenz morphologischer grammatischer Kennzeichnung (Morpheme und Wörter) - trotz der in Abschnitt 1 aufgewiesenen Einschränkungen - mit semantischer Transparenz korreliert, so ist der deutsche Text erheblich semantisch transparenter.

In Abschnitt 2 hatten wir eine ganze Reihe englischer Strukturen aufgewiesen, die eine größere semantische Transparenz zeigten als ihre deutschen Gegenstücke. Als eine erhebliche Quelle von semantischer Transparenz im Englischen wurde die Fixierung der Abfolge der zentralen Satzglieder identifiziert. Der untersuchte englische Text hat 149 zentrale Ergänzungen (98 Subjekte ${ }^{16}$, 48 direkte und 3 indirekte Objekte). Die Abfolge in Verbindung mit der Form anderer Phrasen (adverbiale Bestimmungen, etc.) zeigt in allen 149 Fällen eindeutig an, um welche Ergänzung es sich handelt. Betrachtet man jede derartige topologische Kennzeichnung als Äquivalent eines grammatischen Morphems, so erhöhte sich die nun auf zwei grammatischen Mitteln (Morphologie und Topologie) beruhende englische Grammatizität auf 1010, überbrückt also fast die Hälfte der Differenz von 325 grammatischen Kennzeichnungen zwischen

16 Passivsubjekte wurden als solche und nicht als direkte oder indirekte Objekte gezählt. 
den Texten. Zwar stützt auch die deutsche Ergänzungsfolge die Zuordnung zu den zentralen Ergänzungen, aber die zu vergebenden Werte wären erheblich niedriger, da ein Hörer aus der Topologie keine sichere syntaktische Information zur Bestimmung der zentralen Ergänzungen ziehen kann. ${ }^{17}$

Der deutsche Text enthält 143 Determinative und 60 Adjektivattribute, die jeweils eine oder mehrere morphologische Kennzeichnungen tragen $^{18}$, wobei die morphologische Kennzeichnung der Adjektivattribute zum Teil ambig gekennzeichnete Determinative disambiguiert (aufwendige Kennzeichnung durch zwei grammatische Morpheme), zum Teil die gegebene Information wiederholt (Redundanz). Soweit die grammatischen Morpheme von Determinativen und Adjektivattributen das Genus anzeigen, tragen sie, wie oben aufgeführt, wenig zur semantischen Transparenz bei. Ihre Effizienz in der Kennzeichnung von Numerus und insbesondere Kasus ist aufgrund von Formsynkretismus stark beeinträchtigt.

Schauen wir weiter: Der deutsche Text enthält 20 Zirkumfixmorpheme an Partizipien Perfekt, die hier als jeweils zwei Morpheme gezählt wurden. Der semantische Mehrwert der Doppelkennzeichnung im Vergleich zu englischen past participles dürfte sehr gering sein, da letztere entweder eindeutig als solche oder zumindest als Vergangenheitsform gekennzeichnet sind.

Es ist möglich, dass sich der mit dem höheren deutschen Kennzeichnungsaufwand erwartete semantische Mehrwert verflüchtigt, wenn man die real vermittelte Information quantifiziert. Die englischen grammatischen Kennzeichnungen sind jedenfalls überwiegend funktional, indem sie eindeutig eine semantische Information transportieren.

Die nur ansatzweise durchgeführte semantische Interpretation des quantitativen Ergebnisses zeigt, dass letzteres nicht zu dem Schluss zwingt, deutsche Texte seien, was die grammatische Kennzeichnung betrifft, semantisch transparenter und damit weniger kontextabhängig.

\section{Fazit}

Eine Reihe von deutsch-englischen Kontrasten wurde mit dem Ziel diskutiert, Evidenz für drei miteinander verbundene Thesen zur grammatischen Komple-

17 Fischer (2013: 228, 250-251) zählt in Deklarativsätzen nur 56,6\% Subjekte in Vorfeldposition, wobei - dies ist die entscheidende Information - in 4,7 \% der Sätze nominale Akkusativergänzungen die Vorfeldposition einnehmen.

1820 Determinative (ein, sein, kein) besitzen kein grammatisches Morphem und wurden nicht mitgezählt. 
xität vorzustellen: dass sich Systemkomplexität nicht eins zu eins auf Textkomplexität abbildet, dass grammatische Komplexität nicht unbedingt semantische Transparenz bedeutet, und dass synthetische grammatische Komplexität und analytische grammatische Komplexität nicht unbedingt negativ miteinander korrelieren. Außerdem sollten die stereotypen Bilder der zwei Sprachen relativiert werden. Als Quelle für Beispiele und quantitative Untersuchungen diente ein paralleler schriftlicher Text, nämlich die deutsche und englische Übersetzung von Fred Vargas‘ Sous les vents de Neptune, einem Kriminalroman.

Zunächst wurden - ausgehend von John A. Hawkins' und John McWhorters Arbeiten - Kasus, pragmatische Wortstellung, Verbpräfigierung, Reflexivpronomen und Genus als Beispiele für die größere grammatische Komplexität und eventuell semantische Transparenz des Deutschen angeführt und kritisch diskutiert. Dabei wurde auf eine Abmilderung der Kontraste in der Textrealisierung sowie auf Grenzen der grammatisch bewirkten semantischen Transparenz hingewiesen. Beispielsweise vermindert Formsynkretismus die Funktionalität der deutschen Kasus erheblich, während die topologische englische Kennzeichnung der Kernergänzungen optimale Funktionalität bewirkt. Die bescheidene Funktionalität deutscher Kasusformen wurde als Beleg für die These herangezogen, dass sich Systemkomplexität (vier Kasus) nicht eins zu eins auf die Textrealisierung überträgt. Die Diskussion von Reflexivierung und Genus wiederum zeigte, dass grammatische Komplexität nicht immer funktional ist.

Als englische Komplexifizierungen wurden neben der grammatikalisierten Abfolge der drei zentralen Ergänzungen der ausgeweitete Gebrauch der -ingForm (obligatorische Verlaufsform, infiniter Ergänzungssatz) und der for-toInfinitiv angeführt. Bei jeder der Komplexifizierungen konnte ein semantischer oder grammatischer Mehrwert verbucht werden. Außerdem wurden Diskursfunktionen der im Englischen erheblich häufigeren Spaltsätze an kontrastiven Beispielen demonstriert. Es konnte gezeigt werden, dass die englischen Spaltsätze zum Teil informationsstrukturell transparenter sind als ihre flacheren deutschen Entsprechungen.

Eine quantitative Untersuchung eines Abschnitts des parallelen Textes zeigte erwartungsgemäß Deutsch als erheblich synthetischere, aber nicht Englisch als (morphologisch) analytischere Sprache. Dieser Befund bestätigt die von Bernd Kortmann und Benedikt Szmrecsanyi an englischen Varietäten festgestellte positive Korrelation von synthetischer und analytischer Komplexität, die auf der Grundlage von vier deutschen Varietäten auch von Péter Maitz and Attila Németh aufgezeigt wurde. Auch hier zeigt sich, dass typologische Urteile aufgrund von Systemerwägungen und Einzelbeobachtungen nicht unbedingt einer Korpusanalyse standhalten.

Die Erörterung der deutsch-englischen Kontraste erfolgte auf dem Hintergrund aktueller Komplexitätsdiskussionen. Ohne der Äquikomplexitätsthese 
das Wort reden zu wollen, wurde das Komplexitätsgefälle zwischen den beiden Sprachen als geringer veranschlagt als gemeinhin angenommen.

\section{Literatur}

\section{Forschungsliteratur}

Bisang, Walter (2009): On the evolution of complexity: Sometimes less is more in East and mainland Southeast Asia. In Geoffrey Sampson, David Gil \& Peter Trudgill (Hrsg.), Language as an evolving variable, 34-49. Oxford: Oxford University Press.

De Vogelaer, Gunther (2007): Extending Hawkins' comparative typology: Case, word order, and verb agreement in the Germanic languages. Nordlyd 34, 167-182.

Duden (2006): Die Grammatik. Unentbehrlich für richtiges Deutsch. Mannheim u. a.: Dudenverlag.

Eisenberg, Peter (2006): Der Satz. Grundriss der deutschen Grammatik Band 2. 3., durchges. Aufl., Stuttgart, Weimar: J. B. Metzler.

Everett, Daniel (2008): Don't sleep, there are snakes. Life and language in the Amazonian jungle. London: Profile Books.

Evert, Stefan (2004): The statistical analysis of morphosyntactic distributions. In Maria Teresa Lino u. a. (Hrsg.), Fourth international conference on language resources and evaluation, Bd. 3, 1539-1542. Paris: ELRA.

Fanego, Teresa (2004): The rise and development of English verbal gerunds. Diachronica 21 (1), 5-55.

Fenk-Oczlon, Gertrud \& August Fenk (2008): Complexity trade-offs between the subsystems of language. In Matti Miestamo, Kaius Sinnemäki \& Fred Karlsson (Hrsg.), Language complexity. Typology, contact, change, 43-65. Amsterdam: John Benjamins.

Fischer, Klaus (2009). Cleft sentences: form, function and translation. Journal of Germanic Linguistics 21 (2), 167-192.

Fischer, Klaus (2012): Spaltsätze: Summen der valenztheoretischen Teile oder konstruktionelle Unikate? In Klaus Fischer \& Fabio Mollica (Hrsg.): Valenz, Konstruktion und Deutsch als Fremdsprache, 133-166. Frankfurt a. M.: Peter Lang.

Fischer, Klaus (2013): Satzstrukturen im Deutschen und Englischen. Typologie und Textrealisierung. Berlin: Akademie (Konvergenz und Divergenz 1).

Fischer, Klaus (2017): Komplexität - dennoch ein nützlicher Begriff. In Mathilde Hennig (Hrsg.), Linguistische Komplexität - ein Phantom?, 19-52. Tübingen: Stauffenburg.

Gil, David (2009): How much grammar does it take to sail a boat? In Geoffrey Sampson, David Gil \& Peter Trudgill (Hrsg.), Language as an evolving variable, 19-33. Oxford: Oxford University Press.

Gil, David (2014): Sign languages, creoles, and the development of predication. In Frederick J. Newmeyer \& Laurel B. Preston (Hrsg.), Measuring grammatical complexity, 37-64. Oxford: Oxford University Press.

Greenberg, Joseph H. (1963): Some universals of grammar with particular reference to the order of meaningful elements. In Joseph H. Greenberg (Hrsg.), Universals of language, 58-90. Cambridge/MA.: MIT Press. 
Hawkins, John A.(1986): A comparative typology of English and German. Unifying the contrasts. London \& Sydney: Croom Helm.

Hawkins, John A. (2004): Efficiency and complexity in Grammars. Oxford: Oxford University Press.

Hawkins, John A. (2009): An efficiency theory of complexity and related phenomena. In Geoffrey Sampson, David Gil \& Peter Trudgill (Hrsg.), Language as an evolving variable, 252-268. Oxford: Oxford University Press.

Hennig, Mathilde (Hrsg.) (2016): Komplexe Attribution. Ein Nominalstilphänomen aus sprachhistorischer, grammatischer, typologischer und funktionalstilistischer Perspektive. Berlin, Boston: De Gruyter.

Hickey, Raymond (2012): Early English and the Celtic hypothesis. In Terttu Nevalainen \& Elizabeth Closs Traugott, The Oxford handbook of the history of English, 497-507. Oxford, New York: Oxford University Press.

Huddleston, Rodney \& Geoffrey K. Pullum (2002): The Cambridge grammar of the English language. Cambridge: Cambridge Univerity Press.

Juola, Patrick (2008): Assessing linguistic complexity. In Matti Miestamo, Kaius Sinnemäki \& Fred Karlsson (Hrsg.), Language complexity. Typology, contact, change, 89-108. Amsterdam: John Benjamins.

König, Ekkehard \& Volker Gast (2009): Understanding English-German contrasts. 2., neu bearb. Aufl. Berlin: Erich Schmidt. (Grundlagen der Anglistik und Amerikanistik 29).

Koplenig, Alexander, Peter Meyer, Sascha Wolfer \& Carolin Müller-Spitzer (2017): The statistical trade-off between word order and word structure - Large-scale evidence for the principle of least effort. PLoS ONE 12 (3), e0173614. doi:10.1371/journal.

Kortmann, Bernd \& Benedikt Szmrecsanyi (2009): World Englishes between simplification and complexification. In Lucia Siebers \& Thomas Hoffmann (Hrsg.), World Englishes Problems, properties and prospects. Selected papers from the 13th IAWE conference, 265-285. Amsterdam: Benjamins.

Leiss. Elisabeth (2005): Derivation als Grammatikalisierungsbrücke für den Aufbau von Genusdifferenzierungen im Deutschen. In Torsten Leuschner \& Tanja Mortelmans (Hrsg.): Grammatikalisierung im Deutschen, 11-30, Berlin. New York (Linguistik Impulse und Tendenzen 9).

Maitz, Péter (2014): Sprachwandel und sprachliche Komplexität. In Vilmos Ágel \& Andreas Gardt (Hrsg.): Paradigmen der aktuellen Sprachgeschichtsforschung, 94-108. Berlin, Boston/ MA: De Gruyter (Jahrbuch für Germanistische Sprachgeschichte 5).

Maitz, Péter \& Attila Németh (2014): Language contact and morphosyntactic complexity: Evidence from German. Journal of Germanic Linguistics 26 (1), 1-29.

McWhorter, John H. (2001): The world's simplest grammars are creole grammars. Linguistic Typology 5, 125-166.

McWhorter, John H. (2002): What happened to English? Diachronica 19 (2), 217-272.

McWhorter, John H. (2008): Why does a language undress? Strange cases in Indonesia. In Matti Miestamo, Kaius Sinnemäki \& Fred Karlsson (Hrsg.), Language complexity. Typology, contact, change, 167-190. Amsterdam: John Benjamins.

Oh, Yoon Mi (2015): Linguistic complexity and information: Quantitative approaches. Diss. Lyon: CNRS - Université Lumière 2.

Plank, Frans (1983): Transparent versus functional encoding of grammatical relations: A parameter for syntactic change and typology. Linguistische Berichte 86, 1-13.

Plank, Frans (1984): Verbs and objects in semantic agreement: minor differences between English and German that might suggest a major one. Journal of Semantics 3, 305-360. 
Plank, Frans (2016): Vom Suppletiv(un)wesen, in Beziehung zur Paradigmenstruktur. In besonderer Rücksicht der historischen Natur beschränkter Möglichkeiten. In Andreas Bittner \& Klaus-Michael Köpcke (Hrsg.), Prozesse der Regularität und Irregularität in Phonologie und Morphologie, 1-28. Berlin, Boston/MA.: De Gruyter.

Prince, Ellen F. (1978): A comparison of WH-clefts and it-clefts in discourse. Language 54, 883-906.

Riddle, Elizabeth M. (2008): Complexity in isolating languages: Lexical elaboration versus grammatical economy In Matti Miestamo, Kaius Sinnemäki \& Fred Karlsson (Hrsg.), Language complexity. Typology, contact, change, 133-151.Amsterdam: John Benjamins.

Rohdenburg, Günter (1974): Sekundäre Subjektivierungen im Englischen und Deutschen. Vergleichende Untersuchungen zur Verb- und Adjektivsyntax. PAKS-Arbeitsbericht Nr. 8. Bielefeld: Cornelsen Velhagen \& Klasing.

Rohdenburg, Günter (1990): Aspekte einer vergleichenden Typologie des Englischen und Deutschen. Kritische Anmerkungen zu einem Buch von John A. Hawkins. In Claus Gnutzmann (Hrsg.), Kontrastive Linguistik, 133-152. Frankfurt a. M.: Peter Lang (Forum angewandte Linguistik 19).

Rohdenburg, Günter (1991): Weitere Betrachtungen zu einer vergleichenden Typologie des Englischen und Deutschen. Duisburg: Linguistic Agency University of Duisburg (= Paper Series A, 302).

Sinnemäki, Kaius (2008): Complexity trade-offs in core argument marking. In Matti Miestamo, Kaius Sinnemäki \& Fred Karlsson (Hrsg.), Language complexity. Typology, contact, change, 67-88. Amsterdam: John Benjamins.

Sinnemäki, Kaius (2014): Complexity trade-offs: a case study. In Frederick J. Newmeyer \& Laurel B. Preston (Hrsg.), Measuring grammatical complexity, 179-201. Oxford: Oxford University Press.

Steinmetz, Donald (1986): Two principles and some rules for gender in German: Inanimate nouns. WORD 37 (3), 189-217.

Szmrecsanyi, Benedikt \& Bernd Kortmann (2009): Between simplification and complexification: non-standard varieties of English around the world. In Geoffrey Sampson, David Gil \& Peter Trudgill (Hrsg.), Language as an evolving variable, 64-79. Oxford: Oxford University Press.

Szmrecsanyi, Benedikt \& Bernd Kortmann (2012): Introduction: Linguistic complexity. Second language acquisition, indigenization, contact. In Bernd Kortmann \& Szmrecsanyi, Benedikt (Hrsg.), Linguistic complexity. Second language acquisition, indigenization, contact, 6-34. Berlin, Boston/MA: De Gruyter (linguae \& litterae 13).

Trudgill, Peter (2011): Sociolinguistic typology. Social determinants of linguistic complexity. Oxford: Oxford University Press.

Van Mol, Heleen (2017): Das Genus von Gallizismen im Deutschen. Reichweite, Validität und Dominanz von Prinzipien der Genuszuweisung. Germanistische Mitteilungen 43 (1), 5-30.

Vennemann, Theo (2002): On the rise of 'Celtic' syntax in Middle English, In Peter J. Lucas \& Angela M. Lucas (Hrsg.), Middle English from tongue to text. Selected papers from the third international conference on Middle English: Language and Text, held at Dublin, Ireland, 1-4 July 1999, 203-234 Frankfurt a. M.: Peter Lang (Studies in English Medieval Language and Literature 4).

Visser, Frederikus Th. (1966): An historical syntax of the English language. Part II: Syntactical units with one verb. Leiden: $\mathrm{E}$. J. Brill. 


\section{Quellen}

Vargas, Fred (2004): Sous les vents de Neptune. Paris: J'ai lu.

Vargas, Fred (2005): Der vierzehnte Stein. Aus dem Französischen von Julia Schoch. Berlin: Aufbau.

Vargas, Fred (2007): Wash this blood clean from my hand. Translated by Siân Reynolds. London: Random House. 

II Lexemtypen 



\title{
Damaris Nübling \\ 5 Neue Ansätze in der Namenforschung: Plädoyer für eine Gender-Onomastik
}

\begin{abstract}
Personennamen besetzen die Spitze der linguistischen Belebtheitshierarchie und beteiligen sich wie kaum eine andere sprachliche Einheit an der Sortierung von Menschen nach Geschlecht. Dabei befördern sie nicht nur die Geschlechtsbinarisierung, sie vermelden auch durch spezifisches Genusverhalten sozial relevante Subkategorien wie den Übergang vom Mädchen zur (Ehe-)Frau: Während in Dialekten neutrale, nicht-diminuierte Frauennamen wie s Ingrid, et Tanja ursprünglich jungen, sozial abhängigen, dörflichen Frauen galten (soziale Verortung), sind sie heute vertrauten, sympathischen, womöglich mit dem oder der Sprecher/in verwandten Mädchen und Frauen vorbehalten (Beziehungsebene). Das Femininum wirkt eher distanzierend und gilt heute eher unvertrauten, oft zugezogenen Frauen. Umgekehrt geraten Männernamen niemals ins Neutrum ( ${ }^{\star}$ s Otto), selbst dann nicht, wenn diminuiert, was üblicherweise Neutrumzuweisung erzwingt (der Ursli). Dies befestigt umso mehr die Geschlechtergrenze. Außerdem befasst sich der Beitrag mit der phonologischen Gendering von Rufnamen und beobachtet das seit kurzem erfolgende Degendering von Jungennamen auf $-a$, wie Luca oder Mika, die noch vor wenigen Jahren weiblich klassifiziert worden wären, da - $a$ der bislang exklusivste Weiblichkeitsmarker war (Andrea, Nicola). Dies ist als onymischer Reflex sozialer Geschlechtsrollenangleichungen zu werten. Abschließend wird ein Blick auf die Benennung von Haustieren (Hunden) geworfen. Diese kann mit der Entwicklung von anonym > kynonym (ohne Geschlechtskennzeichnung, vgl. ambiges Lumpi) > anthroponym mit zuverlässiger Geschlechtsunterscheidung (Emma vs. Max) charakterisiert werden.
\end{abstract}

Keywords: Genderlinguistik, Geschlechterforschung, Personennamen, Tiernamen, Unisexnamen, Transgender

Damaris Nübling, Johannes Gutenberg-Universität Mainz, FB 05 - Philosophie und Philologie, Deutsches Institut, Historische Sprachwissenschaft des Deutschen, Jakob-Welder-Weg 18, D-55128 Mainz, E-Mail: nuebling@uni-mainz.de

Ә Open Access. () 2018 Damaris Nübling, publiziert von De Gruyter. (c) BY Dieses Werk ist lizenziert unter der Creative Commons Attribution 4.0 Lizenz. 


\section{Der Elefant im Raum}

"One of the most common pieces of information contained in a given name is the sex of the named person“, schreibt der Soziologe Richard Alford (1988: 65) in seiner kulturvergleichenden Untersuchung zur Namengebung weltweit. Von 52 diesbezüglich untersuchten Gesellschaften markieren 37 (72\%) das Geschlecht der benannten Person immer oder üblicherweise am Namen, sieben manchmal und acht nie. Dabei ermittelt Alford eine positive Korrelation zwischen der Größe und Komplexität einer Gesellschaft und namentlicher Geschlechtskennzeichnung. Rufnamen (Vornamen) in Deutschland befassen sich vergleichsweise obsessiv mit der Geschlechtsanzeige; sie wird sogar standesamtlich (wenngleich nicht namenrechtlich) eingefordert. Ambiguitäten irritieren so sehr, dass Namen ohne erkennbares Geschlecht immer noch den Weg in die Medien, ja sogar vor Gerichte finden. Alle kennen das Ungemach, das geschlechtsuneindeutige Namen verursachen, denn unser nominales Anredesystem sieht strikte, overte Geschlechtsbinarität vor: Will man eine unbekannte Person mit fremdem Rufnamen anschreiben, steht nur Frau oder Herr zur Verfügung. Eine dritte Option existiert nicht, ohne in der Stilebene einzubrechen („Hallo!“, „Guten Tag!“). Auch weite Teile der deutschen Grammatik sind von der Geschlechterdichotomie ohne geschlechtsabstrahierende Überdachung durchdrungen. Paradebeispiel sind die singularischen Personalpronomen sie und er, auch die Possessivpronomen ihr- und sein-. An dieser Stelle hat das Schwedische vor einiger Zeit offiziell das dritte, geschlechtsneutrale Pronomen hen eingeführt, von dem reger Gebrauch gemacht wird. In Deutschland würde ein solches (bereits öfter vorgebrachtes) Ansinnen (z. B. sier) zum aktuellen Zeitpunkt keine Chance auf Realisierung haben; der Widerstand ist zu groß, der Glaube an die Geschlechter noch zu tief verwurzelt.

Was die Benennung von Menschen betrifft, so ist es in Deutschland verboten, gegengeschlechtliche Rufnamen zu vergeben. (Anders verhält es sich wieder in Schweden, wo alle Namen für alle Menschen, gleich welchen Geschlechts, offenstehen). Seit dem Kiran-Urteil vom Dezember 2008 sind jedoch geschlechtsneutrale Rufnamen, sog. Unisexnamen, erlaubt, ohne dass ihnen (wie bisher) ein zweiter, geschlechtsdefiniter Name folgen muss. Eine indischstämmige Familie wollte ihrer Tochter den in Indien geschlechtsneutralen Namen Kiran geben und musste damit bis vor das Bundesverfassungsgericht ziehen, nachdem sie vom Standesamt daran gehindert bzw. dazu aufgefordert worden war, dem Namen Kiran einen geschlechtseindeutigen Namen beizufügen. ${ }^{1}$ Anfänglich hatte das Standesamt Kiran sogar als männlich eingeordnet,

1 https://www.bundesverfassungsgericht.de/SharedDocs/Entscheidungen/DE/2008/12/ rk20081205_1bvr057607.html (letzter Zugriff 6.11. 2017). 
und zwar mit Verweis auf seine phonologische Struktur: Der Ausgang -an entspreche dem von Julian, Fabian, Christian und weise den Namen daher als männlich aus. Das Bundesverfassungsgericht erkannte jedoch in dem Unisexnamen Kiran keine Beeinträchtigung des Kindeswohls sowie der Entfaltung der kindlichen Identität und Individualität, was einen Durchbruch in der bisherigen Argumentation markiert (Schmidt-Jüngst 2013).

Die Frage, was einen Namen ,weiblich‘ bzw. ,männlich‘ macht - Phonologie? Morphologie? Konvention? -, wurde bislang kaum gestellt. Weder die Onomastik noch die (Gender-)Linguistik haben bislang erkannt, dass Rufnamen den wohl größten sprachlichen Beitrag zur Herstellung von Geschlecht leisten, das neben der Altersunterscheidung die kulturgeschichtlich älteste Form der Humandifferenzierung leistet (Hirschauer 2014). Erst mit Naming Gender von Susanne Oelkers (2003), die der Kieler Gender Research Group angehörte, ist die erste maßgebliche Arbeit zum Thema „Name und Geschlecht“ vorgelegt worden; der Schwerpunkt liegt in der phonologischen Kodierung von Geschlecht auf Rufnamen. Im gleichen Jahr hat der Kultursoziologe Jürgen Gerhards in Die Moderne und ihre Vornamen die Frage gestellt, ob die Markierung von onymischem Geschlecht im Zeitverlauf als Reflex der gesellschaftlichen Angleichung der Geschlechterrollen abnehme (so wie er onymische Reflexe auf andere soziale Veränderungen wie die Transnationalisierung, Entfamiliarisierung, Säkularisierung etc. nachweisen konnte). Interessanterweise bestätigte sich seine Hypothese nicht. Dies lag jedoch eher daran, dass er nicht mit linguistischen Methoden vertraut war, d. h. nur bestimmte Auslaute zur namentlichen Geschlechtsbestimmung herangezogen hat, was $\mathrm{zu}$ wenig war. (Hinzu kommt, dass nur die Graphie berücksichtigt wurde, auch wenn von Phonetik die Rede ist). Seit 2003 kamen einige Forschungen hinzu, so dass das Thema „Name und Geschlecht“ seit ca. 15 Jahren ein gewisses, doch immer noch zu geringes wissenschaftliches Interesse erfährt.

Dabei kann die Relevanz des Namens als tertiäres Geschlechtsorgan gar nicht hoch genug veranschlagt werden: Für Transgender-Personen genügt es heute, ihre Geschlechtsidentität nur noch durch den Wechsel ihres Vornamens zu deklarieren. Das war in der Vergangenheit anders. Im Transsexuellengesetz (TSG), das auch den Namenwechsel regelt, gab es seit 1981 mehrere Änderungen, die die Relevanz des Rufnamens insofern gestärkt haben, als weitere Bedingungen für die Anerkennung des Geschlechtswechsels gestrichen wurden. 1981 war (neben dem Namenwechsel) eine Hormonbehandlung und eine Operation, die sogar zur Fortpflanzungsunfähigkeit führen musste, verpflichtend. Eine bestehende Ehe wurde annulliert. Nach und nach sind diese und weitere Bedingungen gefallen, was die Relevanz des Namens für den Geschlechtswechsel indirekt erhöht hat. Heute ist somit nur noch der Rufnamenwechsel 
für den offiziellen Geschlechtswechsel erforderlich (denn Trans-Personen dürfen ebenfalls keine gegengeschlechtlichen Rufnamen tragen). Mehr denn je markiert der Name die Transition. Keine andere Transition innerhalb einer sozialen Differenz ist (in Deutschland) zwingend an einen Namenwechsel gekoppelt, weder die religiöse Konversion (allenfalls der Eintritt in einen Orden) noch der Eintritt in eine neue Altersstufe, der Wechsel der Nationalität oder die „Einheirat“ in eine andere Familie (wie dies früher einseitig für die Frau galt). Nur die Elternschaft zwingt ein verheiratetes Paar dazu, einen einheitlichen Familiennamen anzunehmen. Der Name ist für die Geschlechtsidentität von solcher Bedeutung, dass sein Wechsel oft zum Synonym des Geschlechtswechsels selbst wird, wie Bücher und Beiträge über Trans-Personen immer wieder zeigen: Anne wird Tom - Klaus wird Lara (Rauchfleisch 2013), Aus Yvonne wird Balian, Aus Bradley wird Chelsea Manning (siehe hierzu Schmidt-Jüngst 2018). Für viele Trans-Personen performiert in erster Linie der Namenwechsel den Geschlechtswechsel, so wie umgekehrt das Nicht-mehr-in-den-alten-Namen-Passen als Indiz für das falsche Geschlecht gewertet wird (zum Namenwechsel von Transgender-Personen siehe Schmidt-Jüngst 2015; Schmidt-Jüngst 2018; Nübling 2017c). So verhält es sich mit dem Geschlecht von Namen wie mit dem berühmten Elefanten im Raum: Es ist unübersehbar, wird aber nicht thematisiert. Das liegt nicht an seiner Tabuisierung, sondern an der Tatsache, dass die Eigennamenlinguistik im toten Winkel zwischen Onomastik (die sich wenig für Namengrammatik und die Geschlechterdifferenz interessiert) und Linguistik (die sich wenig für Namen interessiert) liegt. Im Folgenden sollen drei unterschiedlich gelagerte gender-onomastische Themen beleuchtet werden: a) Devianzen bei der Genusklassifikation von Personennamen, b) das phonologische Degendering von Jungennamen auf - $a$ seit der Jahrtausendwende, und c) das menschliche Gendering von Tiernamen.

\section{Das Heidi und der Schellen-Ursli: „Verstöße“ gegen Prinzipien der Genuszuweisung}

Wenn es ein semantisches Genuszuweisungsprinzip gibt, auf das Verlass ist und das nicht nur die oft überschaubaren Wörter eines (meist kleinen) semantischen Feldes abdeckt, ${ }^{2}$ dann ist es das Genus-Sexus-Prinzip oder das sog. „natürliche Genus“ (Köpcke \& Zubin 2009: 133), also die Tatsache, dass Be-

2 Dies gilt für die Bezeichnungen von Farben ( $\rightarrow$ N.), Himmelsrichtungen $(\rightarrow$ M.), Winden $(\rightarrow$ M.) oder Früchten ( $\rightarrow$ F. außer Apfel, Pfirsich). 
zeichnungen (Appellativa) für weibliche Menschen feminin und solche für männliche maskulin sind. ${ }^{3}$ Selbst Konversionen ohne formale Kennzeichen (sog. Differentialgenus: die/der Angestellte, Alte, Auszubildende) und Wortbildungssuffixe, die aus männlichen Bezeichnungen weibliche derivieren (der Soldat - die Soldatin) oder umgekehrt (die Hexe - der Hexer), wenden dieses Genus-Sexus-Prinzip produktiv an, ebenso personenbezeichnende Fremd- und Lehnwörter (die Queen - der King). ${ }^{4}$ Einzig das morphologische Prinzip kann das semantische ausstechen, am besten erkennbar an der Diminution, die im Fall von -chen und -lein gemäß dem Kopf-rechts-Prinzip ausnahmslos Neutra generiert (das Fräulein, das Männchen). Allerdings ist das Deutsche bekannt für einige sog. hybrid nouns wie das Weib. Manche zählen (trotz des Diminutivsuffixes, doch wegen seiner Lexikalisierung und fehlenden Grundform) auch das Mädchen dazu (Corbett 1991: 183; Corbett 2006: 213). Diese Genus-SexusDiskordanz wird semantisch „,begradigt“, je weiter entfernt vom genushaltigen Nomen (controller) sich der kongruierende Marker (target) befinden: Während der adjazente Artikel immer neutral ist, d.h. das (grammatische) Genus markiert, kann ein anaphorisches Pronomen im Folgesatz das Wort semantisch als weiblich und damit als Femininum klassifizieren (das Mädchen - sie). Dies bildet die gender agreement hierarchy ab (Corbett 1991: 225-260; Corbett 2006: 214-218; Panther 2009). Abgesehen von diesem hybriden Kongruenzverhalten haben Köpcke \& Zubin früh darauf aufmerksam gemacht, dass mit neutralen Frauenbezeichnungen Abwertungen verbunden sind: Die neutralen Pejorativa für Frauen sind Nomina mit herabsetzender oder bemitleidenswerter Bedeutung, etwa das Weib, das Mensch, das Frauenzimmer (Köpcke \& Zubin 1996: 483; siehe auch Köpcke \& Zubin 2003).

Bislang übersehen wurden Personennamen. Hier ging man von einem besonders strikten Genus-Sexus-Prinzip aus, das wegen seiner Ausnahmslosigkeit keiner Erwähnung bedurfte. Während den meisten Rufnamen sogar ein

3 Um die Verbindung zur Genusforschung zu erhalten, wird hier der biologistische Ausdruck Sexus gebraucht. Gemeint ist das Geschlecht, dem sich ein Mensch zugehörig fühlt. Meistens (wenngleich nicht zwingend) korreliert dieses mit dem biologischen Geschlecht.

4 Merkwürdigerweise besteht Widerstand gegen die Feststellung einer solchen Genus-SexusKorrelation; vermeintliche Gegenbeispiele werden dann in der Tier- oder gar Objektwelt gesucht, obwohl diese Korrelation hierfür nie postuliert wurde. So schreibt Donalies (2008) in der Rubrik „grammis“ des IDS: „Dennoch weiß natürlich jedes Kind, dass das Genus, das grammatische Geschlecht, und der Sexus, das biologische Geschlecht, keineswegs immer übereinstimmen: ,Oder glaubt einer, alle Igel seien männlich und alle Fliegen weiblich? Wir wissen schon Bescheid, aber es interessiert uns eben nicht““ (Heringer 1995: 208). Hinderling (1999) bemüht gar der Apfel und die Birne. Die wirklich interessanten Abweichungen und deren Hintergründe werden dagegen übersehen. 
auf Sexus verweisendes Genus inhärent ist (Ingrid $\rightarrow$ F., Herbert $\rightarrow$ M.), gehören blanke Familiennamen zu den wenigen genuslosen Nomina. Ihnen wird Genus referenziell zugewiesen, d.h. in Abhängigkeit vom Geschlecht der damit bezeichneten Person; ist diese(s) nicht bekannt, dann kann dem Familiennamen auch kein Genus zugewiesen werden (denn das Neutrum als überdachendes Genus steht nicht zur Verfügung). Potentiell noch vorhandene, aber erstarrte appellativisch-lexikalische Strukturen wie -mann, -sohn oder Wiese haben ihr Genus längst gelöscht: die Bergmann, die Mendelsohn, der Wiese sind möglich Bezüge auf konkrete Personen. Nur echte Diminutive überschreiben diese festen Relationen (das Müllerchen). Umso bemerkenswerter ist die in der Überschrift angedeutete Tatsache - es handelt sich um die Protagonisten zweier in der Schweiz spielender Kinderbücher -, dass in manchen Dialekten einerseits (nicht-diminuierte) Frauennamen neutral, andererseits diminuierte Männernamen maskulin sein können: das Ingrid, das Heidi ${ }^{5}$ - der Ursli. Im Alemannischen kommt meist beides zusammen, in anderen Dialekten oft nur eins von beiden. Hier liegen bemerkenswerte Asymmetrien vor, die nur durch soziale Geschlechterrollen und hierarchien erklärt werden können. Im ersten Fall wird Neutrum zugewiesen, obwohl Femininum erwart- und verfügbar wäre; in gewisser Weise zeigen volle weibliche Rufnamen ein Genusverhalten, das man von diminuierten Namen erwarten würde. Spiegelbildlich verhält es sich im zweiten Fall. Hier müsste wegen des Diminutivsuffixes -li das morphologische als stärkstes aller Zuweisungsprinzipien greifen. Ursli müsste also neutral sein, ist aber maskulin. Hier setzt sich das semantisch-referentielle Prinzip gegen das morphologische durch.

Das trinationale DFG-Projekt „Das Anna und ihr Hund - Weibliche Rufnamen im Neutrum. Soziopragmatische vs. semantische Genuszuweisung in Dialekten des Deutschen und Luxemburgischen“ geht solchen Fragen nach (für die Schweiz siehe Christen 1998; für erste Projektergebnisse Busley \& Fritzinger 2018). ${ }^{6}$ Zum einen erhebt es die letzten Relikte solcher onymischer Neutra in

5 Unterschiedlich verhalten sich Ableitungen auf $-i$, die im Standarddeutschen weitgehend der Genus/Sexus-Kongruenz folgen (der Uli, die Uli), in der Schweiz aber bei Frauen das Neutrum auslösen (ds Anni, ds Mueti, ds Tanti). Sogar identische $i$-Bildungen wie Pfusi ,dicker Mensch“ praktizieren eine Art Differentialgenus zwischen Neutrum mit Bezug auf Frauen und Maskulinum mit Bezug auf Männer: ds Pfusi ,dicke Frau', $d r$ Pfusi ,dicker Mann'. Wegen dieses noch nicht abschließend geklärten Status von $-i$ in Heidi wurde das Ingrid hinzugefügt: Frauennamen bedürfen keiner Diminution, um zu Neutra zu werden.

6 Dazu mehr unter https://lettres.unifr.ch/fr/langues-litteratures/germanistik/linguistik/ laufende-forschungsprojekte/das-anna-und-ihr-hund.html und http://www.namenforschung. net/weibliche-rufnamen-im-neutrum/projektvorstellung/. (letzter Zugriff 6.11. 2017) 
möglichst vielen Dialekten (das Areal befindet sich im Westen am Rhein und zieht sich südlich weit in die Schweiz, nördlich ins Niederdeutsche und östlich bis ins Thüringische hinein); siehe Nübling, Busley \& Drenda (2013). Zum anderen versucht es anhand qualitativer Interviews sowie von Online-Befragungen die Funktion dieser Neutra zu ergründen, vor allem dann, wenn daneben auch das Femininum zur Verfügung steht. In vielen Dialekten und im Luxemburgischen ist das Neutrum jedoch fest grammatikalisiert; hier sind Frauenrufnamen per se Neutra. Wo aber das Neutrum neben dem Femininum existiert, erschließt sich die Funktion des Neutrums (sowie des Femininums) am besten. Wenngleich sich je nach Dialekt kleinere Unterschiede ergeben, so lässt sich insgesamt sagen: Vertraute, womöglich mit dem Sprecher/der Sprecherin verwandte, junge, sozial rangniedrigere, im Dorf verbliebene und dialektsprechende Mädchen und Frauen treten ins Neutrum, während „gestandene“, ältere, meist verheiratete und/oder berufstätige Frauen, die womöglich das Dorf verlassen haben oder zugezogen sind, üblicherweise ins Femininum treten immer bezogen auf ihre Rufnamen bzw. Pronomen. Das Alter kann eine Rolle spielen: Während in der Schweiz jegliche weibliche Verwandte, auch Großmütter, Neutra sein können, gilt dies in anderen Dialekten nur für gleichaltrige oder jüngere Verwandte.

Hier bestätigt sich die in Köpcke \& Zubin (2003) für das Deutsche und seine jüngere Geschichte beschriebene Binnendifferenzierung von Frauen in Mädchen und (Ehe-)Frauen, die sogar Genusdubletten wie der Ekel (Abstraktum) vs. das Ekel ,widerwärtige Frau', der Mensch vs. das Mensch ,liederliche Frau', das Fräulein ,ledige Frau'vs. die Fräulein ,Lehrerin' generiert. Dabei vermuten Köpcke \& Zubin (2003) das 17./18. Jh. als Zeitraum für die Entstehung dieser neutrumbasierten Clusterbildung, deren Kern die frequenten neutralen Appellativa Weib, Mädchen und Frauenzimmer bildeten. Im 19. und 20. Jh. habe dieses Cluster einen „dramatic increase“ (Köpcke \& Zubin 2003: 154) erfahren, seine Produktivität hält bis heute an: das Girl, Pin-up, Model, Bunny, Schaf, Ding, Aas, Klappergestell, Loch, Flittchen, Aschenputtel (die Autoren erwähnen ca. 100 solche Neutra in ihrem Sample). Auch wenn man hierfür Metonymien, Metaphern oder einfach nur Diminutive mit ihrer zufälligen Neutrumzugehörigkeit verantwortlich machen möchte, so fällt auf bzw. will erklärt sein, a) warum dieses Ausmaß metaphorischer Neutra nicht für negative Männerbezeichnungen gilt, b) warum Männerbezeichnungen fast nie diminuiert werden, und c) warum es gar eine Art „Differentialneutrum“ bei substantivierten Adjektiven zu geben scheint vom Typ Schau mir in die Augen, Kleines (womit kein Mann oder Junge gemeint sein kann).

Während mit den dialektalen Rufnamenneutra keine negativen Konnotationen verbunden sein müssen (betreffende Frauen stellen sich unter Umstän- 
den selbst im Neutrum vor, z. B. im Saarland, in der Pfalz, im Hunsrück), liefern neutrale Familiennamen, die (überregional) vor allem im Internet verwendet werden, Evidenz für degradierende Effekte. Dies lässt sich beim Gebrauch von das Merkel beobachten. Manchmal wird auch auf Männer im Neutrum referiert, wenn sie Schwäche gezeigt oder versagt haben (z.B. das Diepgen für den gescheiterten Berliner Bürgermeister). Viel mehr als die weitgehend inflationierten (teilweise sogar grammatikalisierten) Rufnamenneutra vermitteln die Familiennameneutra dieses, durch den Genuswechsel bewirkte, alte Pejorationspotential. Bereits im Indogermanischen, aber auch heute bezeichnen neutrale Substantive fast ausschließlich unbelebte Denotate, mit Ausnahme von (noch nicht bzw. gering sexuierten) Kindern und Jungtieren (das Kind, Neugeborene, Kalb, Lamm, Fohlen). Werner (2012) spricht beim Neutrum mit Referenz auf Belebtes von „Asexus [...], und zwar mit intendierter, starker Pejoration“ (Werner 2012: 192). Köpcke (1993: 139) sieht das Neutrum bei Frauenbezeichnungen als „Mittel zur Entkräftung“, Nübling (2014) als „derogatives Genus“ und Mittel zur „Deagentivierung“. Di Meola (2007) bezeichnet das Neutrum als „defizitäres Genus“, da es immer das Fehlen einer Eigenschaft anzeige, unter anderem von Agentivität; er vergleicht Ableitungspaare vom Typ das Gedicht/der Dichter, auch solche mit dem gleichen Suffix (das Diktat/ der Autokrat) und Homonyme wie das Bund/der Bund (Di Meola 2007: 96). In Nübling (2014) wurden die Kontexte von das Merkel-Nennungen in einem WebKorpus dokumentiert, denen allesamt gemein ist, dass sie die Handlungsmacht der Kanzlerin in Frage stellen, indem sie ihr Entschluss- und Handlungsfähigheit absprechen. Das Neutrum dient meines Erachtens primär der Agentivitätsreduktion. Das würde ein Maskulinum nicht leisten.

Interessanterweise werden vermeintlich „unmännliche“ Männer ins Femininum abgeschoben (die Memme, Schwuchtel, Tunte), während Frauen, die nicht die Geschlechterstereotypen erfüllen, ins Neutrum verschoben werden (siehe Abb. 5.1, die auch die sozialen Fallhöhenunterschiede visualisiert). Familiennamen als öffentliche „Distanznamen“, die üblicherweise auch nicht diminuiert werden und die gerade nicht auf Frauen aus dem Nahbereich referieren, transportieren mit dem Neutrum noch diese vermutlich alte, dysphemistische Funktion (mehr in Nübling 2017a).

Umgekehrt ist es von Belang, dass Männerrufnamen nicht nur per se niemals ins Neutrum geraten ( ${ }^{\star}$ s Peter), ${ }^{7}$ sondern sogar auch dann gegen das Neutrum immun sind, wenn sie diminuiert sind. Damit heben sie das stärkste (morphologische) Prinzip aus den Angeln. Dialektbefragungen bestätigen, dass

7 Außer im Wallis sowie in den Walser Mundarten von Gressoney \& Issime; Zürrer (1999: 245156). 


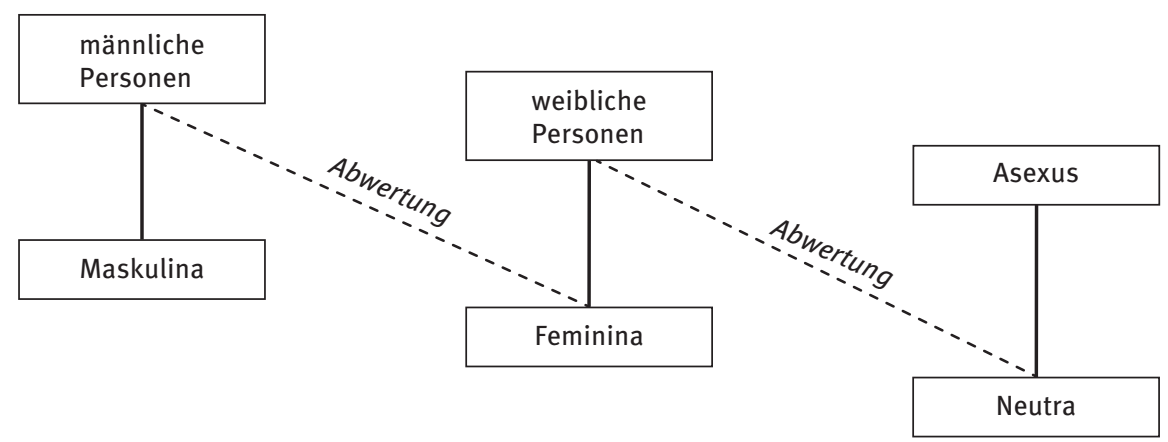

Abb. 5.1: Unterschiedliche Gendereffekte bei Genus-Sexus-Diskordanzen. ${ }^{8}$

Diminutive wie Seppli, Ursli, Peterle, Hänschen zwar verwendet, aber mit maskulinen targets versehen werden, $\mathrm{d}$. h. bei den Pronomen und sogar beim Artikel (der Peterle - er), s. auch Baumgartner \& Christen (2017). Allenfalls bei kleinen Jungen im Vorschulalter wird eingeräumt, dass das Neutrum akzeptabel sein kann, oder es wird, wie eine Online-Umfrage im Rahmen des Projekts erwiesen hat, damit verächtlich auf zu klein oder schmächtig geratene Männer referiert.

Damit erweist sich, dass die Genusklassifikation bei den Animata viel mehr leistet als die Verzahnung von biologischem mit grammatischem Geschlecht: Sie reflektiert Gendervorstellungen einer Gesellschaft, Rollenerwartungen und Geschlechterstereotype. Deren Befolgung korreliert mit Genus-Sexus-Konkordanz, deren Nicht-Befolgung mit Genus-Sexus-Diskordanz. Verstöße gegen das sprachliche Genus-Sexus-Prinzip spiegeln somit Verstöße gegen die soziale Geschlechterordnung. Dabei scheint das Neutrum als „drittes“ Genus nur für Mädchen und Frauen, aber nicht für Jungen und Männer zur Verfügung zu stehen, selbst dann nicht, wenn deren Namen und Appellative diminuiert sind (zur Nutzung anderer Genus-Sexus-Diskordanzen siehe Nübling 2017a). Deshalb ist dafür zu plädieren, die Genusklassifikation nicht nur bei Appellativen, sondern auch bei Eigennamen zu untersuchen. Gerade weil Namen frei von lexikalischer Bedeutung sind, wird die genuine Leistung des Genus umso sichtbarer.

8 Leicht modifiziert nach Nübling (2017a: 204). 


\section{Luca, Noah und Mika: Phonologisches Degendering von Jungennamen auf $-a$}

Informationen über das Geschlecht von Namenträger/inne/n leistet nicht nur das Genus, auch der Namenkörper kann der Geschlechtsauskunft dienen. Am klarsten erweist sich dies im Fall spezifischer Suffixe bzw. phonologischer Segmente wie oben erwähntes, männlich assoziiertes -an in Christian, Fabian, Stefan, das anfänglich die Durchsetzung von Kiran erschwert hatte. Umgekehrt haben Namen auf - $a$, -ette, ine/ina und - $e$ im Deutschen eine sehr hohe Wahrscheinlichkeit, Frauennamen zu sein - erst recht dann, wenn ihre Basis ein Männername ist (unterstrichen): Sabrina, Martina, Annette, Jeanette, Katharina, Janina, Sabine, Wilhelmine, Elke, Christiane. An Männernamen suffigiert kommt diesen Endungen morphologischer Status zu (Martin $\{a\})$; in den anderen Fällen ist der Status nicht richtig geklärt, er oszilliert zwischen phonologisch (Sabrin[a]) und submorphologisch. Tatsache ist, dass Namen auf - $a$ eine fast $100 \%$-ige Wahrscheinlichkeit haben, weiblich zu sein, zumindest bis zur Jahrtausendwende. Umgekehrt haben Einsilber und Namen auf Konsonant oder - $o$ eine sehr hohe Wahrscheinlichkeit, Männernamen zu sein.

Die häufige einseitige Movierung von Männer- zu Frauennamen führt nicht nur zu längeren und vokalreicheren, sondern auch zu anders betonten Namen, denn wie Míchael, Mártin, Chrístian gegenüber Michaéla, Martína, Christiáne zeigen, hat die Movierung häufig den Akzentwechsel auf eine hintere Silbe zur Folge. Dies hat langfristig zu mit Geschlecht aufgeladenen Namenschemata geführt. Die meisten Menschen können auch unbekannten Namen ein Geschlecht zuweisen, und dies mit großer Übereinstimmung, wie die Replizierung eines amerikanischen Tests mit Leipziger Studierenden durch Gerhards (2003) gezeigt hat: Mit klarer Mehrheit wurden Namen wie Lamecca, Maleka, Shatrye weiblich und Oukayod, Cagdas, Rashueen männlich klassifiziert. Auch Oelkers (2003: 199-214) nahm solche Tests vor - mit ähnlichen Resultaten. Das Wissen über geschlechtsindizierende Namenmuster wird somit produktiv angewendet. ${ }^{9}$

9 Dieses Wissen ist relativ neu, denn die dithematischen germanischen Rufnamenkomposita enthalten kaum oder keine phonologischen Unterschiede; vgl. Almut (w.) mit Helmut (m.) oder Gertrud (w.) mit Meinrad (m.). Hier gilt heute pure Konvention, das Wissen um das Namengeschlecht. Im Germanischen galt dagegen folgende Genusregel: Weibliche Namenkomposita mussten ein feminines, männliche ein maskulines Zweitglied enthalten. Auch dieses Faktum spricht für einen engen Konnex zwischen Genus und Geschlecht. Außerdem konnten auch Adjektive verwendet werden. 
Tab. 5.1: Strukturunterschiede der 100 häufigsten Frauen- und Männerrufnamen (transkribiert) in Deutschland.

\begin{tabular}{lll}
\hline & Frauen & Männer \\
\hline 1. Silbenzahl & $\emptyset 2,54$ & $\emptyset 1,92$ \\
\hline 2. Hauptakzent & erste Silbe: $67 \%$ & erste Silbe: $90 \%$ \\
\hline 3. Kons./Vokalanteil & $\mathrm{K}<\mathrm{V}: 22 \%$ & $\mathrm{~K}<\mathrm{V}: 10 \%$ \\
& $\mathrm{~K}=\mathrm{V}: 40,5 \%$ & $\mathrm{~K}=\mathrm{V}: 33 \%$ \\
& $\mathrm{~K}>\mathrm{V}: 37,5 \%$ & $\mathrm{~K}>\mathrm{V}: 57 \%$ \\
\hline 4. Auslaut & auf Vokal: $78,5 \%$ & auf Vokal: $19 \%$ \\
& auf Kons.: $21,5 \%$ & auf Kons.: $81 \%$ \\
\hline
\end{tabular}

Zieht man die 100 häufigsten Rufnamen der zwischen 1930 und 2012 geborenen Personen heran, gelangt man zu den Strukturunterschieden in Tabelle 5.1: Frauennamen sind im Schnitt mehr als eine halbe Silbe länger und zu einem Drittel auf einer nicht-ersten Silbe betont (Männer: 10\%). Sie enthalten insgesamt mehr Vokale, und vor allem lauten sie zu fast $80 \%$ vokalisch aus; Männernamen enden dagegen fast invers zu ca. $80 \%$ konsonantisch. Namenlänge und Auslaut sind damit die am stärksten geschlechtsindizierenden Merkmale. Sie sind kontingent und historisch variabel.

In Nübling (2009) und (2012) konnte anhand der jeweils 20 meistvergebenen Namen zwischen 1945 und 2015 nachgewiesen werden, dass - entgegen Gerhards' (2003) negativen Befunden, die einzig auf dem Namenauslaut basierten - seit ca. 1970 auf dem Namenkörper Genderrückstufungen stattfinden: Die Namenlänge beider Geschlechter hat sich einander angeglichen. Ab 1960 (Mädchen: 2,9 Silben, Jungen: 1,75) wurden die Mädchennamen kürzer und vor allem die Jungennamen länger, was $1980 \mathrm{zu}$ dem minimalen Unterschied von nur 0,1 Silben führte (Mädchen: 2,6; Jungen: 2,5). Seitdem verringern beide Geschlechter ihren Namenumfang; siehe heutige Kindernamen wie Mia, Leah, Lara (weiblich) und Leo, Ben, Finn (männlich). Allerdings existieren so gut wie keine einsilbigen Mädchennamen, abgesehen von Ruth und Kim. Ebenso wenig existierten bis ca. 2000 Jungennamen auf - $a$. Noch 2004 veröffentlichte Oelkers den Aufsatz „Der Fall Luca. Zur Männlichkeit und Weiblichkeit von Vornamen“ (Oelkers 2004), wo sie ein kleines Mädchen in ihrem Bekanntenkreis mit diesem ursprünglich italienischen Männernamen erwähnt. Oelkers prognostiziert, dass Luca - ähnlich wie zuvor Andrea und Gabriele - wegen seines im Deutschen strikt weiblich assoziierten $a$-Auslauts zum Mädchennamen würde. Heute wissen wir, dass das Gegenteil eingetreten ist und dass nicht nur Luca, sondern wenig später auch Noah die obersten Ränge der Jungennamen erobert 


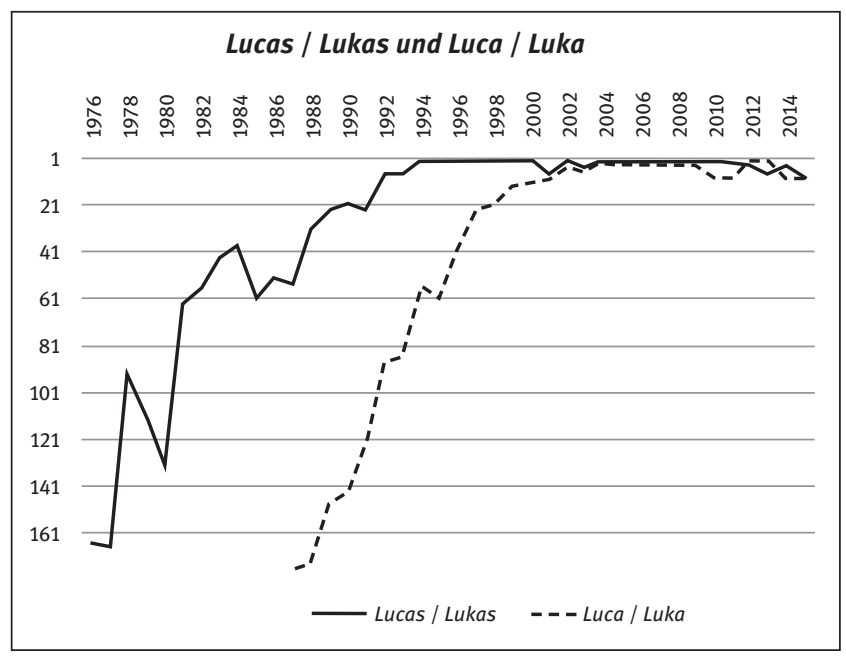

Abb. 5.2: Die Spitzenreiter Luc/kas und Luc/ka (1976 bis 2015). ${ }^{10}$

haben. Dabei handelt es sich genau um die von Gerhards (2003) seit 1960 erwartete sog. Androgynisierung. Genaugenommen liegt eine Rückstufung von Geschlecht vor, ein Degendering oder undoing gender im Sinne von Hirschauer (1994) und (2014). Da Gerhards' Studie nur bis in die 1990er Jahre reichte, konnte er diese Hypothese (noch) nicht bestätigen.

Abbildung 5.2 bezieht sich auf die männlichen Geburtsjahrgänge von 1976 bis 2014 in Zweijahresabständen. Seit 2000 betritt Luca (und damit der erste auf $a$ auslautende Name überhaupt) die Top 10 der Jungennamen. (Genaugenommen handelt es sich um einen Unisexnamen, an dem jedoch nur wenige Mädchen partizipieren). Diese Entwicklung hat sich jedoch erst langsam angebahnt. Zunächst vermehren die Jungennamen ab den 1970er Jahren ihre Silbenzahl und damit auch die Vollvokale in den Nebentonsilben. (Typische Namen um 1945 waren Peter, Dieter, Jürgen, Hans, um 1975 dagegen Michael, Stefan, Matthias, Christian). Doch bleiben diese gefüllten Endsilben noch lange konsonantisch versiegelt. Seit den 1980er Jahren arbeitet sich Lukas stark nach oben und landet schon Anfang der 1990er Jahre in den Top 10, um mehrere Jahre lang den ersten Platz zu besetzen.

Abbildung 5.2 zeigt, dass im Kielwasser von Lukas dicht sein konsonantisch „entsiegeltes“ Pendant, Luca bzw. Luka, folgt. Der parallele, steil aufsteigende Verlauf macht einen Zusammenhang zwischen diesen beiden Namen

10 Knud Bielefeld danke ich sehr für die Erstellung dieser Grafik. 
plausibel. Mittlerweile befindet sich auch Noah (ohne konsonantisch auslautendes Pendant) unter den Top 10, und 2016 sind Jonas auf Platz 1 und Elias auf Platz 2 vorgerückt. Beide besitzen Namenpendants auf offenes - $a$, Jona(h) und Elia(h). Wenn man auf den Seiten von beliebte-vornamen.de deren Verlaufskurven betrachtet, so zeigen beide schon steil nach oben: Jona( $h$ ) hat 2016 mit Platz 42 bereits die Top 50 erreicht, Elia(h) befindet sich auf Platz 116 (mehr siehe in Nübling 2018). Andere Jungennamen wie Joshua und Mika nehmen ebenfalls zu. Wichtig ist, dass sich diese Entwicklungen seit einigen Jahrzehnten im Namenkörper der Jungennamen angekündigt haben; in manchen Fällen besitzen sie sogar ganz konkrete Vorreiter, wie dies für Lukas gegenüber Luca gilt und (vermutlich) für Jonas und Elias gegenüber Jona(h) und Elia(h).

Die Frage ist, ob umgekehrt auch Mädchen von ihren Eltern zunehmend „männlich“ klingende Namen bekommen. Dem entsprächen Einsilber auf Konsonant, z. B. aus dem Amerikanischen entlehnte Namen wie Lynn, Kate, Liv, deren Zunahme jedoch nicht $\mathrm{zu}$ beobachten ist (2015 rangierten diese Namen zwischen Platz 138 und 187). Vielmehr befinden sich unter den 11 häufigsten Mädchennamen ausnahmslos solche auf - a (Mia, Emma, Sophia, Hanna etc.); Platz 12 und 13 besetzen Leonie und Amelie, danach folgen wieder viele Namen auf - $a$. Möglicherweise geht die Gendernivellierung einseitig von den Jungennamen aus, indem diese verstärkt „feminisieren“ und damit langfristig die phonologische Geschlechterdifferenz unterminieren. Diese sich auf den Rufnamen abspielenden Geschlechterdifferenzen und ihre Umbrüche sind noch nicht ausreichend dokumentiert und interpretiert. Hierzu bedarf es transdisziplinärer Expertisen, allen voran von Seiten der Soziologie, die an vielen Stellen eine abnehmende Relevanz von Geschlecht beobachtet. ${ }^{11}$ Gerhards (2003) zufolge sind Rufnamen als Seismographen sozialer Entwicklungen zu betrachten.

\section{Von Lumpi zu Sammy vs. Gina: Zur Sexuierung bzw. Genderisierung von Tiernamen}

In noch stärkerem Maße auf Nachbardisziplinen angewiesen ist man, wenn man die Individualbenennung von Tieren verstehen möchte. Aus onomastischer Sicht ist noch wenig darüber bekannt, noch weniger aus diachroner Perspektive, obwohl das menschliche Interesse an den Namen und der Benennung von Tieren enorm ist. Ob bzw. inwieweit die Benennung auch eine Sexuierung bzw. Gende-

11 Dass Geschlecht innerhalb von Paarbeziehungen nicht nur irrelevant, sondern hinderlich ist, zeigt aus soziologischer Perspektive Hirschauer (2013), aus onomastischer Nübling (2017b). 
risierung des Tieres leistet, ist bislang nicht erforscht. ${ }^{12}$ Es liegen nur wenige und oft indirekte Hinweise vor, denen im Folgenden nachgegangen werden soll (basierend auf Dammel, Nübling \& Schmuck 2015a).

Eine alltägliche Bestätigung für die Relevanz von Geschlecht bei der Tierbenennung liefern Aufrufe von Tiergärten und Zoos, sich an der Namenvergabe für neugeborene Tierjungen zu beteiligen. Dabei wird öfter betont, dass die Geschlechtsbestimmung Voraussetzung für eine Namenvergabe sei und dies bei bestimmten Tierarten (z. B. Kakadus) zu einer späteren Benennung führen könne: ohne Geschlecht kein Name.

Studien zu Haustierbenennungen zeigen, dass dann, wenn ein Tier falsch geschlechtsbestimmt wurde, es zur nachträglichen Umbenennung kommen kann. Für Katzen belegt sind Namenwechsel von Karl zu Karl-Doris, von Peter zu Frau Peter und von Cerberus zu Zerberussa (Kraß 2014). Ein Kaninchen wurde von Hoppel zu Hoppelinchen moviert und diminuiert (Holzschuh 2015). Manchmal kommt es zu Unisexnamen, z. B. Knöpfchen für ein Kaninchen unbekannten Geschlechts. Katzen und Kaninchen werden im Vergleich zu Hunden weniger streng sexuskonform benannt. Dies kann neben ihrer schwerer erkennbaren Geschlechtszugehörigkeit auch daran liegen, dass Kaninchen und Katzen typischerweise Tiere für kleine Kinder sind. Möglicherweise sexuieren Kinder ihre Streicheltiere weniger als dies Erwachsene tun. Vor diesem Hintergrund wären Untersuchungen von Meerschweinchen- oder Hamsternamen interessant.

Wenn Nutztiere benannt werden, beachtet man deren Geschlecht sehr genau, denn ihre Ausbeute ist engstens an ihren Sexus gekoppelt: Kühe geben Milch, Bullen Fleisch. Früher setzte man letztere auch als Arbeits- oder Zugtiere ein. ${ }^{13}$ Auch ist die Verweildauer am Hof und die Tier/MenschKontaktfrequenz - wichtige Faktoren für die Tiernamenvergabe - bei Kühen und Bullen höchst unterschiedlich. Weitere benennungsförderliche Faktoren sind die Menschenähnlichkeit des Tieres, ob es an der Kommunikation teilhat (unter anderem auf seinen Namen reagiert), ob eine emotionale Beziehung zwischen Mensch und Tier besteht, ob das Tier den menschlichen Bewegungs-

12 Bei der Vergeschlechtlichung von Tiernamen dürfte der auf das biologische Geschlecht rekurrierende Terminus Sexuierung der adäquatere sein (besonders bei Nutztieren). Bei den Namen anthropomorphisierter Katzen oder Schoßhunde wäre eher von Genderisierung zu sprechen, da ihre Halter und Halterinnen in ihnen Kinder oder Partner/innen sehen und ihnen oft genderspezifisch-stereotypes Verhalten unterstellen.

13 Interessanterweise können Kühe, wenn sie für - offensichtlich männlich genderisierte - Zugarbeiten eingesetzt werden, ihr Namengeschlecht wechseln. So beobachtet Schönfeld (1987) bei Zugkühen in der Magdeburger Börde, dass ihnen männliche Namen gegeben wurden: „Die Zugkuh wurde auch mit Männernamen bezeichnet, wie das Pferd, also Hans, Max, Moritz, auch Schimmel. Von ihr wurde häufig im männlichen Geschlecht geredet“ (Schönfeld 1987: 217). 
radius teilt und es, wenn in Gruppen gehalten, äußerlich distinkt ist. Im Fall von Nutztieren ist auch ihr Nutzwert relevant, wohingegen ihr intendierter Tod eine Benennung hemmt - umso mehr, je früher geplant.

Seit einigen Jahren liegen empirisch fundierte Einzelbeiträge zur Benennung von Pferden (Schwerdt 2007), Haus- und Zuchthunden (Schaab 2012 bzw. Leppla 2015), Katzen (Kraß 2014) und Kaninchen (Holzschuh 2015) vor; zu weiteren siehe Dammel, Nübling \& Schmuck (2015b, c). Geschlecht wurde dabei meist mitberücksichtigt. Die Frage ist, ob bzw. inwieweit und mit welchen Mitteln eine onymische Geschlechtskennzeichnung vorgenommen wird.

Was das Type/Token-Verhältnis betrifft, so gelangen mehrere dieser Untersuchungen $\mathrm{zu}$ ähnlichen Ergebnissen wie beim Menschen insofern, als bei Tierweibchen aus einem größeren Nameninventar geschöpft wird als bei Männchen: Schaab (2012) hat 1.000 (Haus-) Hundenamen (488 Hündinnen, 512 Rüden) untersucht und festgestellt, dass es bei den Hündinnen nur sieben Namen sind, die fünfmal oder öfter vergeben wurden, während es bei den Rüden mit 14 Namen immerhin doppelt so viele sind. Damit scheinen Hündinnen individueller benannt zu werden, Rüden monotoner - anders bei Zuchthunden, bei denen Singularität des Namens und Markierung des Geschlechts durch die Hundezuchtordnung vorgeschrieben sind. Hier wird Geschlecht überdeutlich am Namen markiert. Zuchttiernamen gleichen Warennamen, da sie auch zum Kauf des Produkts anregen sollen. Dies erklärt die adlige Anmutung vieler Hundezuchtnamen (Tina von den Hochseebächen, Aristo vom Dalbornhof). Bei Katzennamen ist das Type/Token-Verhältnis ausgeglichener, wenngleich weiblichen Katzen ebenfalls eine größere Namenvielfalt zukommt (Kraß 2014). Dagegen kehrt sich dieses Verhältnis bei Kaninchen leicht um (Holzschuh 2015). Dies legt die Vermutung nahe, dass bei der Benennung phänotypischer Geschlechtsdimorphismus eine Rolle spielt: Während das biologische Geschlecht von Nutztieren maximal sichtbar und relevant ist, verringert sich diese Differenz bei Hunden, noch mehr bei den Katzen, und am meisten bei Kaninchen und anderem Kleingetier (siehe Abb. 5.3).

Auch was den Anteil menschlicher Namen an Tiernamen betrifft, ergeben sich Geschlechterunterschiede: Namen für Weibchen schöpfen stärker aus dem Anthroponomastikon als solche für Männchen. Deutlich zeigt dies die Benennung von Zuchtkühen und -bullen. Bausinger (1971) stößt sogar auf spiegelbildliche Verhältnisse:

Die [...] Prozentsätze sind genau seitenverkehrt: bei den weiblichen Tieren tragen $82 \%$ menschliche Vornamen, 18\% andere Namen; bei den männlichen Tieren tauchen $18 \%$ menschliche Vornamen auf, während das Gros von $82 \%$ andere Namen trägt. (Bausinger 1971: 175) 


\begin{tabular}{|c|c|c|c|c|c|}
\hline \multicolumn{2}{|c|}{$\begin{array}{l}\text { gleiche Namen } \\
\text { für beide Geschlechter }\end{array}$} & \multicolumn{2}{|c|}{$\begin{array}{l}\text { wenig distinkte Namen } \\
\text { gemeinsame Namen } \\
\text { für beide Geschlechter }\end{array}$} & \multicolumn{2}{|c|}{$\begin{array}{l}\text { maximal distinkte Namen } \\
\text { keine gemeinsamen Namen } \\
\text { für beide Geschlechter }\end{array}$} \\
\hline $\begin{array}{l}\text { Fische, Reptilien } \\
\text { Insekten }\end{array}$ & $\begin{array}{l}\text { Vögel } \\
\text { Mäuse }\end{array}$ & $\begin{array}{l}\text { Kaninchen } \\
\text { Meerschweinchen }\end{array}$ & Katzen & Hunde & $\begin{array}{l}\text { Pferde Rinder } \\
\text { Nutz-/Zuchttiere } \\
\text { Zoo-/Zirkustiere }\end{array}$ \\
\hline \multicolumn{6}{|l|}{ Faktoren: } \\
\hline \multicolumn{3}{|c|}{$\begin{array}{l}\text { - } \text { kein sichtbarer Geschlechts- } \\
\text { dimorphismus } \\
\text { - } \text { Geschlecht für Nutzeffekt } \\
\text { irrelevant } \\
\text { - } \\
\text { - } \text { merden-/Schwarmtiere } \\
\text { - } \text { max. räumunähnlich Distanz zum Menschen } \\
\text { - }\end{array}$} & & \multicolumn{2}{|c|}{$\begin{array}{l}\text { - } \text { gut sichtbarer Geschlechts- } \\
\text { dimorphismus } \\
\text { - Geschlecht für Nutzeffekt } \\
\text { hochrelevant } \\
\text { - Einzeltiere } \\
\text { - menschenähnlich (Affen) } \\
\text { - räuml. Nähe zu Menschen } \\
\text { - öffentl. Zurschaustellung }\end{array}$} \\
\hline
\end{tabular}

Abb. 5.3: Faktoren des naming gender bei Tieren. ${ }^{14}$

Außerdem entsprechen anthroponymische Kuhnamen eher geläufigen Frauenrufnamen (Bärbel, Gerlinde, Doris, Lotte) ${ }^{15}$, während anthroponymische Bullennamen verstärkt auf seltene, antiquierte Namen zugreifen (Peppo, Meinulf, Rufus). Bausinger (1971) begründet dies damit, dass die Kühe schon auf dem Hof als Kälber benannt und unter diesem Namen auch versteigert werden, während die Benennung von Bullen oft erst kurz vor der Versteigerung erfolge, hier also ein gewisser Zeitdruck bestehe und auch bestimmte Anfangsbuchstaben beachtet werden müssen, was insgesamt zu ausgefalleneren Namen führe.

Für Schweden zeigt Leibring (2015), dass die onymische Mensch/TierGrenze immer durchlässiger wird und sich dabei die Namen von Hündinnen stärker mit aktuellen Mädchennamen überschneiden als die von Rüden mit Jungennamen. Sie begründet dies damit, dass der Hund prototypischerweise männlich konzipiert werde. (Tatsächlich scheinen fast alle fiktiven Hunde in Comics, Literatur und Filmen männlich zu sein; ein genauer Nachweis steht noch aus). Da früher für Hunde noch ein separates Nameninventar (ein sog. Kynonomastikon) bestand und Geschlecht kaum differenziert wurde (siehe Einheitsnamen wie Lumpi, Waldi, Fifi, Rex), heute aber im Zuge der Anthropomorphisierung die soziale Hauptdifferenz Geschlecht immer wichtiger geworden ist, ist das Kynonomastikon, da männlich assoziiert, auf die Rüden

14 Nach Dammel, Nübling \& Schmuck (2015a: 25).

15 Manche Frauennamen wie (der Typus) Liese wurden so häufig als Kuhnamen verwendet, dass sie appellativischen Status erlangten. Ähnliches gilt für Katzennamen (siehe Kraß 2014). 
übergegangen. ${ }^{16}$ Daraus erwuchs ein besonderer Benennungsbedarf für Hündinnen, der mithilfe von Mädchennamen gedeckt wurde und wird. ${ }^{17}$ Ähnliches stellt Schaab (2012: 147) für Deutschland fest, indem Hündinnen zu 63\%, Rüden zu 54\% mit menschlichen Rufnamen bedacht werden. Auch hier scheinen Hundeweibchen eher aktuelle Mädchennamen (Lilly, Mia) zu bekommen als Rüden, bei denen wiederum öfter zu Familiennamen gegriffen wird (Hitchcock, Chagall, Lagerfeld, Bismarck). Insgesamt stößt Schaab (2012) bei den deutschen Hunden auf extrem ausgeprägte namentliche Geschlechterdifferenzen. Die häufigsten Rüdennamen sind Ben(ny), Sam(my), Charly, Merlin, die häufigsten Hündinnennamen Gina, Aimy, Luna, Ronja - fast nur Namen auf - $a$.

Was die formale Seite von Tiernamen betrifft, so scheint das Auslautprinzip noch stärker zu wirken als bei Menschennamen; es scheint sogar alle anderen Prinzipien auszustechen. So kommt es immer wieder dazu, dass Tiere mit Ergonymen benannt werden, z. B. mit Namen beliebter Alkoholika. Schwerdt (2007) stellt bei Pferdebenennungen fest, dass die Namen von (alkoholischen) Getränken, die überwiegend von Frauen konsumiert werden, an Stuten (Baileys, Chablis) und die Namen von Alkoholika, die eher von Männern konsumiert werden, an Hengste vergeben werden (Whisky, Bourbon). Dieses stereotypenbasierte Prinzip kann jedoch vom formalen Auslautprinzip überschrieben werden, indem auf - $a$ oder - $e$ auslautende Ergonyme und Toponyme eher für Stuten und auf -o bzw. Konsonant endende für Hengste verwendet werden (Stuten: Sangria, Wolga, Gascogne, Panama; Hengste: Lambrusco, Cinzano, Woodstock, Lugano). Auch Schaab (2012) bestätigt die Wirksamkeit des Auslautprinzips für Hundenamen: Hündinnen heißen Asia, Bluna, Tequila (trotz maskulinen Genus), Rüden Yukon, Namib (trotz femininen Genus), Jim Beam und Calvados. Kraß (2014: 18) ermittelt für seine 325 Katernamen 70 \% konso-

16 Vom Kynonomastikon einer badischen Kleinstadt um 1900 erfahren wir von Bertsche (1906), der 16 Hunde erwähnt, die zum einen noch Hunde sind, indem sie als Wach- und Jagdhunde hundespezifische Funktionen verrichten; die Hund/Mensch-Distanz ist also noch vorhanden. Zum anderen werden sie auch als Hunde benannt, also nicht anthroponym. Ihre Namen sind, da oft mehrfach vergeben, nur wenig individualisierend. Vor allem ist ihnen kaum das Hundegeschlecht zu entnehmen: viermal Mohr(le), zweimal Schnauzer, zweimal Spitzer(le), dreimal Waldmann (eine Jagdhunderasse), dreimal Scholi. Nur je einfach belegtes Sultan und Ladi (< Lady) können sexusdefinit interpretiert werden.

17 Leibring (2015) berichtet außerdem, dass es derzeit in Schweden auch zur umgekehrten onymischen Überschreitung der Mensch/Tier-Grenze komme, und dies ebenfalls mit geschlechtlicher Schlagseite: Weibliche Tiernamen betreten das Anthroponomastikon, indem literarische Namen wie Ronja und Smilla, aber auch Katzennamen wie Dixie und Nala zuerst an Tiere und später an Mädchen vergeben wurden. Im 19. Jh. war Tindra ein typischer Kuh- und Pferdename; seit den 1990ern wird er zunehmend für Mädchen verwendet, 2004 kam er gar auf Platz 13. Eine solche Entwicklung wurde für Deutschland noch nicht beschrieben. 
nantische und $30 \%$ vokalische Auslaute, während es sich bei den ebenfalls 325 Namen weiblicher Katzen mit 35\% : 65\% fast spiegelbildlich verhält. Ähnlich bei Kaninchen: Namen für Männchen enden zu 68,5\% konsonantisch, die für Weibchen zu $65 \%$ vokalisch (Holzschuh 2015: 110). Prinzipiell haben Namen auf - $a$ und -i(e) eine sehr hohe Wahrscheinlichkeit, auf Weibchen zu referieren, solche auf - o oder Konsonant auf Männchen, obwohl man bei der Tierbenennung an nichts gebunden ist und Tiere in ihrer Geschlechtsidentität nicht allzu verletzbar sein dürften.

Viele Tiernamen gehen auf Appellativa ${ }^{18}$ oder Adjektive zurück und rekurrieren damit auf semantisch reiche Ausdrücke. Im Fall von Appellativen ist ihnen auch ein Genus inhärent. Ob dieses bei Tiernamen zur Sexusanzeige genutzt wird, ist noch nicht untersucht. Wenn Bausinger (1971: 179) für Kühe ausschließlich feminine Deappellativa aufzählt wie Blume, Wicke, Biene, Lerche, Forelle, Koralle, Laune, liegt diese Vermutung nahe. Zum anderen wird aber auch die stereotype Bedeutung dieser Lexeme abgeschöpft (Bausinger erwähnt auch, dass solche Wörter an weibliche Vornamen anklingen). Männliche Rinder tragen Namen wie Rentner, Richter, Kaiser, Pfahl, Pflug, Bussard. Dies bestätigt die doppelte Nutzung von Appellativa. Einen engen Konnex zwischen Genus und Sexus bestätigt auch Leppla (2015: 134) für Hundezuchtnamen: Hier besteht jeweils zu ca. zwei Dritteln Genus/Sexus-Kongruenz. Liegen Genus/ Sexus-Inkongruenzen vor, dann lagert sich das dominantere Auslautprinzip darüber: Mango (f.) für einen Rüden, Panda (m.) für eine Hündin. Bei Neutra entscheidet ebenfalls das Auslautprinzip: Chaos und Atoll sind männliche, Koma und Prisma weibliche (Zucht-)Hundenamen. ${ }^{19}$

Für die Nutzung des appellativischen Genus zur Sexusanzeige spricht auch die Beobachtung, dass bei der (eher seltenen) Benennung von Wildtieren ihr Namengeschlecht dem Genus des (oft beigefügten) Appellativs gleichgeschaltet wird (sofern das biologische Geschlecht nicht offenkundig ist): Problembär Bruno, Kaiman Sammy, Killerwels Kuno, Schildkröte Lotti. Trauerschwänin Petra hieß anfänglich Peter und war damit am Genus von Schwan ausgerichtet. Die Entdeckung ihres weiblichen Geschlechts hat neben dem Geschlechtswechsel des Namens auch den Genuswechsel des Appellativs (durch Movierung) bewirkt.

Abgesehen davon sind deappellativische und deadjektivische Tiernamen für Geschlechterstereotypisierungen prädestiniert. So schreibt Leppla (2015:

18 Gemeint sind Appellativa im weiten Sinn, d.h. nicht nur konkrete Gattungs- oder Klassenbezeichnungen, sondern auch Abstrakta.

19 Wie Fußnote 9 zu entnehmen ist, galt für die germanischen Rufnamen das Prinzip, dass die weiblichen Namenkomposita ein feminines und die männlichen ein maskulines Zweitglied enthalten mussten. Neutra waren inexistent, kamen also für Menschennamen offensichtlich nicht in Frage. 
126) zu Hundezuchtnamen: „Bei Rüden dominieren gewaltige Naturphänomene (Taifun, Donner, Tornado), bei Hündinnen Emotionen (Joy, Happiness)“. Außerdem stellt sie fest, dass Rüden

mit den Stereotypen der Stärke, Größe und Schnelligkeit symbolisiert [werden] (Quick, Stark, Vital), Hündinnen mit Bezeichnungen, die sie als hübsch, süß und zart beschreiben (Belissima, Charming, Nice, Sweet, Belle). (Leppla 2015: 135)

Bei den Adjektiven werden sogar Farbbezeichnungen geschlechtsstereotyp verteilt: dunkle Farben für Rüden (Azur, Blue, Ocker), helle für Hündinnen (Pink, Celeste, Blanca).

Zuletzt sei noch ein Blick auf Unisex-Namen geworfen. Soweit überhaupt erhoben, scheinen sie bei Pferden, Hunden und Katzen selten zu sein. Von den insgesamt 650 Katzen tragen Kraß (2014) zufolge 77 (12\%) einen (von insgesamt 22) Unisex-Namen, wobei auf -[i] endende Namen dominieren (als Unisexnamen wurden mindestens zweimal und dabei an beide Geschlechter vergebene Namen gewertet). Mehr Unisex-Namen sind bei Kaninchen zu beobachten: Holzschuh (2015) schreibt, dass von allen Namen, die mehr als einmal vertreten waren, 23,5\% an beide Geschlechter gehen (Stupsi, Happy, Flocke). Kaninchen als (kaum geschlechtsdimorphe) Streicheltiere für Kinder sind anscheinend geringerer onymischer Sexuierung ausgesetzt als größere, partnerartige Tiere wie Hunde und Pferde. Hierzu sind weitere Studien erforderlich, auch dazu, wie relevant Geschlecht (von Tier und Mensch) bei der Anschaffung eines Tieres ist.

Abbildung 5.3 enthält die wichtigsten Faktoren, die eine Geschlechtsmarkierung bei Tiernamen bedingen. Die größte Rolle dürften sichtbarer Geschlechtsdimorphismus und die Geschlechtsspezifik der Ausbeute von Nutztieren spielen. Außerdem werden Einzeltiere, vor allem wenn in räumlicher Nähe zum Menschen lebend, eher in ihrem Geschlecht wahrgenommen als Herdenoder Wildtiere. Schließlich dürfte die Ähnlichkeit zum Menschen per se eine Rolle spielen. Selten sichtbare Wildtiere werden, wenn überhaupt benannt, oft nach dem Genus ihres Appellativs sexuiert. Wird das (gleiche) Tier dagegen zur Schau gestellt (im Zoo oder Zirkus), bekommt es üblicherweise einen geschlechtsoverten Namen, der sein biologisches Geschlecht deutlich zum Ausdruck bringt (siehe Ewald \& Klager 2007; Fahlbusch \& Schmidt-Jüngst 2015).

\section{Plädoyer für eine Gender-Onomastik}

Weder das grammatische Verhalten von Namen noch ihr Beitrag zur Etablierung und Fixierung der Geschlechterordnung wurde bislang von Linguistik und Onomastik hinreichend berücksichtigt. Dabei geben Personennamen, die 
immerhin die Spitze der Belebtheitshierarchie besetzen, wichtige Hinweise auf noch nicht oder unzureichend geklärte Zusammenhänge, allen voran dem zwischen Genus und sog. Sexus. Noch heute trifft man in der Linguistik auf die Position, dass dieser Zusammenhang ambig sei, zumindest in semantischer Hinsicht - oder dass umgekehrt eine ausnahmslose Eins-zu-eins-Beziehung zwischen Genus und Geschlecht herrsche. Wie deutlich wurde, ist Geschlecht ein schillernder Begriff, der von biologischen Fakten bis zu kontingenten, historisch wie kulturell hochvariablen sozialen Konstruktionen reicht. Die Linguistik reflektiert diese Dimensionen nur unzureichend und spricht verkürzend von Sexus. Im Fall der Eigennamen wird deutlich, dass bei der Benennung von Tieren tatsächlich nur deren Sexus eine Rolle spielen kann (was keineswegs ausschließt, dass wir Tiere und deren Verhalten genderisieren), während dies beim Menschen mit seiner Geschlechtsidentität (oder mehreren) anders ist. Wie es sich mit der Genuszuweisung von animaten Appellativen und Namen verhält, ist hochkomplex und weit entfernt von einer Gleichschaltung von Genus mit Geschlechtsorganen. Anhand der Genusklassifikation von Menschennamen konnte gezeigt werden, dass es erstens zu Genus-Sexus-Diskordanzen kommen kann (die Schwuchtel, der Vamp). Solche Verstöße gegen das sprachliche GenusSexus-Prinzip reflektieren Verstöße gegen die soziale Geschlechterordnung. Zweitens kann auch das dritte Genus, das Neutrum, zur Markierung bestimmter Alters- oder Geschlechtszustände genutzt werden, auffälligerweise nur für Mädchen und bestimmte Frauen. So gilt das Neutrum jungen, unverheirateten, im Dorf ansässigen und vertrauten Mädchen bzw. Frauen, während das Femininum ältere, sozial arrivierte, verheiratete und/oder dem Sprecher bzw. der Sprecherin fernerstehende Frauen klassifiziert (Busley \& Fritzinger 2018). ${ }^{20}$ Männernamen dagegen, selbst diminuierte, sperren sich gegen das Neutrum und heben somit das morphologische als dominantestes Genuszuweisungsprinzip aus den Angeln. Dieser gesamte soziopragmatische Komplex erfordert noch viel Forschung sowie die Expertise vonseiten der Geschlechtersoziologie und kommt nicht umhin, die individuellsten Bezeichnungen der Menschen, ihre Namen, in den Vordergrund rücken.

Wie eng Genus und Sexus bei Tieren zusammenhängen, hat der kursorische Überblick über deren Benennung gezeigt. Von den Rändern her lassen sich zentrale Zusammenhänge oft klarer erkennen - so auch hier, zumal die Benennung von Tieren durch nichts reglementiert ist (außer bei Zuchttieren). Das Genus der appellativischen Tierbezeichnung hat dabei großen Einfluss auf die onymische Geschlechtszuweisung, vor allem dann, wenn das Tiergeschlecht

20 Wie diese beiden Genera in den Grimm'schen Märchen eingesetzt werden, zeigt eindrucksvoll Robinson (2010). Knapp gesagt verhilft die Heirat einer Frau vom Neutrum ins Femininum. 
unbekannt, unsichtbar oder irrelevant ist. Was die Tiernamen selbst betrifft, so hat sich gezeigt, dass ungehemmt stereotypisiert wird. Sehr häufig wird auch das formale Namenauslautprinzip genutzt, das so dominant ist, dass es Genus oder semantische Stereotypen aushebeln kann. Insgesamt kann die zunehmende Berücksichtigung von Geschlecht bei Tiernamen als eine primitive Individualisierung gewertet werden: Vormals überhaupt nicht benannte Tiere werden in einem ersten Schritt durch den Typ Waldi und Lumpi immerhin - wenngleich noch geschlechtsindifferent - benannt, um heute in einem weiteren Schritt onymisch fast ausnahmslos vergeschlechtlicht zu werden. Besonders bei Nutztieren und den uns nächststehenden Haustieren betreiben wir onymisches Gendering, während bei menschlichen Kindernamen schon länger ein Degendering zu beobachten ist, das seit der Jahrtausendwende eine besondere Qualität hinzugewinnt: Immer mehr Jungen bekommen Namen auf bislang weiblich konnotiertes $-a$. Ob es sich dabei um eine Relevanzabstufung von Geschlecht handelt oder um eine prinzipielle Feminisierung heutiger Kindernamen (worauf die immer zahlreicheren Mädchennamen auf - $a$ hindeuten), wird die weitere Entwicklung zeigen.

\section{Literatur}

Alford, Richard (1988): Naming and identity: A cross-cultural study of personal naming practices. New Haven: HRAF Press.

Baumgartner, Gerda \& Helen Christen (2017): Dr Hansjakobli und ds Babettli. Über die Geschlechtstypik diminuierter Rufnamen in der Deutschschweiz. Osnabrücker Beiträge zur Sprachtheorie (OBST) 91. Sprache und Geschlecht. Bd. 2: Empirische Analysen, 111-145.

Bausinger, Hermann (1971): Tierzucht und Namengebung. Zu den Eigennamen des Zuchtviehs. In Maria Bindschedler et al. (Hrsg.), Festschrift für Paul Zinsli, 170-184. Bern: Francke.

Bertsche, Karl (1906): Die Namen der Haustiere in Möhringen. (Amt Engen). Alemannia, N.F. 7, 130-137.

Busley, Simon \& Julia Fritzinger (2018): Em Stefanie sei Mann - Frauen im Neutrum. In Damaris Nübling \& Stefan Hirschauer (Hrsg.), Namen und Geschlechter - Studien zum onymischen Un/doing Gender, 191-212. Berlin, Boston: de Gruyter.

Christen, Helen (1998): Die Mutti oder das Mutti, die Rita oder das Rita? Über Besonderheiten der Genuszuweisung bei Personen- und Verwandtschaftsnamen in schweizerdeutschen Dialekten. In André Schnyder et al. (Hrsg.), Ist mir getroumet mîn leben? Vom Träumen und vom Anderssein, 267-281. Göppingen: Kümmerle.

Corbett, Greville (1991): Gender. Cambridge: University Press.

Corbett, Greville (2006): Agreement. Cambridge: University Press.

Dammel, Antje, Damaris Nübling \& Mirjam Schmuck (2015a): Tiernamen - Zoonyme. Forschungserträge und Forschungsperspektiven zu einer wissenschaftlich vernachlässigten Namenklasse. In Dammel, Nübling \& Schmuck (Hrsg.) (2015b), 1-36. 
Dammel, Antje, Damaris Nübling \& Mirjam Schmuck (Hrsg.) (2015b): Tiernamen - Zoonyme, Band I: Haustiere. Heidelberg: Winter.

Dammel, Antje, Damaris Nübling \& Mirjam Schmuck (Hrsg.) (2015c): Tiernamen - Zoonyme, Band II: Nutztiere. Heidelberg: Winter.

Di Meola, Claudio (2007): Neutrale Genuszuweisung im Deutschen: Das Neutrum als „defizitäres“ Genus. In Claudio di Meola et al. (Hrsg.), Perspektiven Zwei, 87-99. Rom: Istituto Italiano di studi Germanici.

Donalies, Elke (2008): Wen besucht Rotkäppchen, seine oder ihre Großmutter? Korrespondenz zwischen Genus und Sexus. grammis 2.0. Das grammatische Informationssystem des Instituts für deutsche Sprache (IDS). http://hypermedia.idsmannheim.de/call/public/fragen.ansicht?v_kat=3\&v_id=31 (letzter Zugriff 6.11. 2017).

Ewald, Petra \& Christian Klager (2007): Namen von Zootieren. Zum Wesen und Gebrauch einer vernachlässigten Namenklasse. Beiträge zur Namenforschung 42, 325-345.

Fahlbusch, Fabian \& Miriam Schmidt-Jüngst (2015): Manege frei für Kaja, Ramses und Pünktchen. Zur Benennung von Zirkustieren. In Dammel, Nübling \& Schmuck (Hrsg.) (2015c), 385-406.

Gerhards, Jürgen (2003): Die Moderne und ihre Vornamen. Eine Einladung in die Kultursoziologie. 2. Aufl. 2010. Wiesbaden: Opladen.

Heringer, Hans Jürgen (1995): Prinzipien der Genuszuweisung. In Heidrun Popp (Hrsg.), Deutsch als Fremdsprache. An den Quellen eines Faches, 203-216. München: Iudicium. Hinderling, Robert (1999): Das Kind, das Individuum, das Genie. Versuch einer Ehrenrettung des deutschen Neutrums. In Peter Wagener (Hrsg.), Sprachformen, 203-214. Stuttgart: Franz Steiner.

Hirschauer, Stefan (1994): Die soziale Fortpflanzung der Zweigeschlechtlichkeit. Kölner Zeitschrift für Soziologie und Sozialpsychologie 46 (4), 668-692.

Hirschauer, Stefan (2013): Geschlechts(in)differenz in geschlechts(un)gleichen Paaren. Zur Geschlechterunterscheidung in intimen Beziehungen. Gender. Zeitschrift für Geschlecht, Kultur und Gesellschaft, 37-56.

Hirschauer, Stefan (2014): Un/doing Differences. Die Kontingenz sozialer Zugehörigkeiten. Zeitschrift für Soziologie 43 (3), 170-191.

Holzschuh, Melissa (2015): Lilly, Paul und Krümel - Benennungsmotivik und Struktur von Kaninchennamen. In: Dammel, Nübling \& Schmuck (Hrsg.) (2015b), 97-116.

Köpcke, Klaus-Michael (1993): Schemata bei der Pluralbildung im Deutschen. Versuch einer kognitiven Morphologie. Tübingen: Narr.

Köpcke, Klaus-Michael \& David Zubin (1996): Prinzipien für die Genuszuweisung im Deutschen. In Ewald Lang \& Gisela Zifonun (Hrsg.), Deutsch - typologisch. IdS, Jahrbuch 1995, 473-491. Berlin: de Gruyter.

Köpcke, Klaus-Michael \& David Zubin (2003): Metonymic pathways to neuter-gender human nominals in German. In Klaus-Uwe Panther \& Linda Thornberg (Hrsg.), Metonymy and pragmatic inferencing, 149-166. Amsterdam, Philadelphia: Benjamins.

Köpcke, Klaus-Michael \& David Zubin (2009): Genus. In Elke Hentschel \& Petra Vogel (Hrsg.), Deutsche Morphologie, 132-154. Berlin, New York: de Gruyter.

Kraß, Peter (2014): Von Felix, Lilly und Karl-Doris. Zur Benennungsmotivik und zur Struktur von Katzennamen. Beiträge zur Namenforschung 49 (1), 1-26.

Leppla, Carolin (2015): Aristo vom Sonnenhof und Birona von der Herzogsquelle. Zur Motivik, Struktur und Pragmatik von Hundezuchtnamen. In Dammel, Nübling \& Schmuck (Hrsg.) (2015b), 117-141. 
Leibring, Katharina (2015): Zoonyms in the onomasticon - Names of cattle, dogs and cats from a Scandinavian perspective. In Dammel, Nübling \& Schmuck (Hrsg.) (2015b), 37-75.

Nübling, Damaris (2014): Das Merkel - Das Neutrum bei weiblichen Familiennamen als derogatives Genus? In Friedhelm Debus, Rita Heuser \& Damaris Nübling (Hrsg.), Linguistik der Familiennamen (= Germanistische Linguistik 225-227 und 205-232. Hildesheim: Olms).

Nübling, Damaris (2017a): Funktionen neutraler Genuszuweisung bei Personennamen und Personenbezeichnungen im germanischen Vergleich. In Johannes Helmbrecht, Damaris Nübling \& Barbara Schlücker (Hrsg.), Namengrammatik. Linguistische Berichte, Sonderheft 23, 173-211. Hamburg: Buske.

Nübling, Damaris (2017b): Beziehung überschreibt Geschlecht. Zu einem Genderindex von Ruf- und von Kosenamen. In Angelika Linke \& Juliane Schröter (Hrsg.), Sprache und Beziehung, 99-118. Berlin, Boston: de Gruyter.

Nübling, Damaris (2017c): Personennamen und Geschlechter/un/ordnung - Onymisches doing und undoing gender. In Stefan Hischauer (Hrsg.), Un/doing Differences. Praktiken der Humandifferenzierung, 307-335. Weilerswist: Velbrück.

Nübling, Damaris (2018): Luca und Noah - Das phonologische Degendering von Jungennamen seit der Jahrtausendwende. In Damaris Nübling \& Stefan Hirschauer (Hrsg.), Namen und Geschlechter - Studien zum onymischen Un/doing Gender, 239269. Berlin, Boston: de Gruyter.

Nübling, Damaris, Simone Busley \& Juliane Drenda (2013): Dat Anna und s Eva - Neutrale Frauenrufnamen in deutschen Dialekten und im Luxemburgischen zwischen pragmatischer und semantischer Genuszuweisung. Zeitschrift für Dialektologie und Linguistik 80 (2), 152-196.

Oelkers, Susanne (2003): Naming Gender. Empirische Untersuchungen zur phonologischen Struktur von Vornamen im Deutschen. Frankfurt: Peter Lang.

Oelkers, Susanne (2004): Der Fall Luca. Zur Männlichkeit und Weiblichkeit von Vornamen. Bulletin VALS/ASLA 80, 155-170.

Panther, Klaus-Uwe (2009): Grammatische versus konzeptuelle Kongruenz. Oder: Wann siegt das natürliche Geschlecht? In Rita Brdar-Szabó et al. (Hrsg.), An der Grenze zwischen Grammatik und Pragmatik, 67-86. Frankfurt: Peter Lang.

Rauchfleisch, Udo (2013): Anne wird Tom - Klaus wird Lara. Transidentität / Transsexualität verstehen. Mannheim: Patmos.

Robinson, Orrin (2010): Grimm Language: Grammar, Gender and Genuineness in the Fairy Tales. Amsterdam: Benjamins.

Schaab, Eva (2012): Von Bello zu Paul: Zum Wandel und zur Struktur von Hunderufnamen. Beiträge zur Namenforschung 47, 131-161.

Schmidt-Jüngst, Miriam (2013): Von der Öffnung der Zweigeschlechtlichkeit zur Öffnung des Namensrechts? Studia Anthroponymica Scandinavica 31, 111-113.

Schmidt-Jüngst, Miriam (2015): Constructing gender identity. Naming choices of transgender people in Germany. In Emilia Aldrin, Linnea Gustafsson, Maria Löfdahl \& Lena Wenner (Hrsg.), Innovationer i namn och namnmönster, 234-250. Uppsala: Norna-förlaget.

Schmidt-Jüngst, Miriam (2018): Der Rufnamenwechsel als performativer Akt der Transgression. In Damaris Nübling \& Stefan Hirschauer (Hrsg.), Namen und Geschlechter - Studien zum onymischen Un/doing Gender, 45-72. Berlin, Boston: de Gruyter. 
Schönfeld, Helmut (1987): Die Veränderungen in der Sprache und im sprachlichen Verhalten der Dorfbevölkerung seit Beginn des 20. Jahrhunderts. In Hans-Jürgen Rach et al. (Hrsg.), Das Leben der Werktätigen in der Magdeburger Börde, 185-221. Berlin: Akademieverlag.

Schwerdt, Judith (2007): Hipponymie. Zu Benennungsmotiven bei Pferdenamen in Geschichte und Gegenwart. Beiträge zur Namenforschung 42, 1-43.

Werner, Martina (2012): Genus, Derivation und Quantifikation. Zur Funktion der Suffigierung und verwandter Phänomene im Deutschen. Berlin, Boston: de Gruyter.

Zürrer, Peter (1999): Sprachinseldialekte. Walserdeutsch im Aostatal (Italien). Aarau u. a.: Sauerländer. 


\title{
Dmitrij Dobrovol'skij \\ 6 Phraseme aus kognitiver und kontrastiver Sicht
}

\begin{abstract}
Die Anwendung kognitiv orientierter Methoden im Bereich der kontrastiven Phraseologie ermöglicht plausible Erklärungen für die Existenz feiner semantischer Unterschiede zwischen den Idiomen verschiedener Sprachen, die eine sehr ähnliche bis identische lexikalische Struktur haben, d.h. anscheinend auch wörtlich übersetzt werden können. Diese Idiome, die bei einer isolierten Nennung als volläquivalent empfunden werden, erweisen sich in den authentischen Kontexten als Quasi- oder Pseudoäquivalente. Textkorpora liefern viele Beweise dafür. Die betreffenden semantischen Unterschiede lassen sich meistens auf die Besonderheiten der bildlichen Komponenten der lexikalisierten Bedeutungen dieser Idiome zurückführen.

Eine weitere Aufgabe der Phrasemuntersuchung aus kognitiver Sicht besteht in der Berücksichtigung der Ergebnisse psycholinguistischer Experimente. Die relevanten Ergebnisse zeigen, dass die Idiomatik einer Sprache ein sehr heterogenes Feld darstellt. Folglich kann keine allgemeingültige Antwort auf die Frage nach den Prinzipien der Idiom-Verarbeitung im „real time-modus“ gegeben werden. Sowohl die Abfolge einzelner kognitiver Prozeduren bei der Idiom-Verarbeitung als auch ihre Geschwindigkeit hängen von solchen Faktoren ab wie kontextuelle Umgebung, Bekanntheitsgrad des Idioms, seine semantische Motiviertheit sowie Experiment-Design.
\end{abstract}

Keywords: Deutsch, Idiom, kognitive Methoden, kontrastive Idiomforschung, Phraseologie, Russisch

\section{Zielsetzung und theoretische Prämissen}

Als erstes ist zu fragen, welchen Nutzen das kognitive Herangehen an die Untersuchung der Phraseme verschiedener Klassen im Vergleich zur traditionel-

Anmerkung: Die Arbeit ist im Rahmen des RGNF-Projekts 16-04-00291 entstanden. Für ihre sorgfältige Lektüre und ihre wertvollen Kommentare danke ich Elisabeth Piirainen.

Dmitrij Dobrovol'skij, Russische Akademie der Wissenschaften, Institut für russische Sprache, Volkhonka 18/2, 119019 Moskau, Russland, E-Mail: dobrovolskij@gmail.com

ə) Open Access. (c) 2018 Dmitrij Dobrovol'skij, publiziert von De Gruyter. (c) BY Dieses Werk ist lizenziert unter der Creative Commons Attribution 4.0 Lizenz.

https://doi.org/10.1515/9783110538588-007 
len Phraseologieforschung erbringen kann, und zwar nicht nur für die Beschreibung der semantischen und pragmatischen Besonderheiten der Phraseme, sondern auch für deren kontrastive Analyse. Voraussetzung ist hier die Erarbeitung eines kognitiv basierten Inventars von Analysemethoden und -instrumentarien. Um dies zu erreichen, muss zunächst geklärt werden, wie die Grundzüge der kognitiven Linguistik zu verstehen sind. In diesem Bereich gibt es keine einheitliche Meinung; dennoch herrscht in einigen Punkten weitgehend Konsens. Ewa Dąbrowska (2016) zählt dazu drei theoretische Prinzipien, und zwar:

- erstens, dass die zentrale Aufgabe der Sprache darin bestehe, Bedeutungen zu vermitteln und dass sich die Linguistik folglich vor allem mit dem Inhaltsplan sprachlicher Ausdrücke befassen müsse;

- zweitens, dass die linguistischen Postulate den Ergebnissen anderer Disziplinen, die sich mit der Informationsverarbeitung beschäftigen, nicht widersprechen dürften; und

- drittens, dass die Grammatik (wie alle systemhaften Züge der Sprachstruktur) aus dem Usus hervorgehe und demzufolge die Untersuchung des Usus die zentrale Aufgabe der Linguistik sei. ${ }^{1}$

In Bezug auf die Phraseologieforschung sind diese Postulate folgendermaßen $\mathrm{zu}$ interpretieren. Da die wichtigste Besonderheit der Phraseme in ihrer Polylexikalität und Non-Kompositionalität besteht, muss das kognitive Herangehen vor allem auf die Frage eingehen, wie diese Spezifika das Zustandekommen der Phrasembedeutung (die eine Art der lexikalischen Bedeutung darstellt) beeinflussen. Besonders spannend ist es, am Material der Idiome auf diese Frage einzugehen. Idiome unterscheiden sich von den übrigen Phrasemklassen dadurch, dass sie eine semantische Re-Interpretation (oft, wenn auch nicht immer, ist es eine metaphorische Re-Interpretation) voraussetzen. In diesem Sinne ähneln sie den Ein-Wort-Metaphern, unterscheiden sich von ihnen jedoch dadurch, dass sie durch ihre Polylexikalität das zugrundeliegende mentale Bild stärker profilieren und folglich ein größeres Gewicht im Inhaltsplan des betreffenden Ausdrucks bekommen. Aus diesem Grunde werden Idiome in dem vorliegenden Artikel zentral behandelt.

1 „There are at least three central tenets that all cognitive linguists subscribe to, namely: (1) that language exists to convey meaning [...], and that therefore the goal of linguistics is to explain how speakers use linguistic expressions to convey meaning; (2) that 'accounts of human language [should] accord with what is generally known about the mind and brain from disciplines other than linguistics' (Lakoff's 'cognitive commitment': Lakoff 1991: 54); and (3) that grammar emerges from usage, at both developmental and historical level, and therefore usage should be centre stage in all accounts of language“ (Dąbrowska 2016: 479-480). 
Die konstitutiven Merkmale der Idiome sind also Polylexikalität (sie bestehen aus mehr als einem Wort), Stabilität (sie werden jeweils in der nahezu gleichen Form und Bedeutung reproduziert) und Idiomatizität. Die prototypischen Idiome können auf zwei verschiedenen konzeptuellen Ebenen interpretiert werden: in ihrer primären Lesart, d. h. in der „wörtlichen“ Bedeutung, die ihrer inneren Form zugrunde liegt, und der figurativen lexikalisierten Bedeutung. ${ }^{2}$

Diese beiden konzeptuellen Ebenen sind bis zu einem gewissen Grad voneinander unabhängig. Andererseits besteht oft eine semantische Brücke zwischen ihnen. Denn in der lexikalisierten Bedeutung sind oft bestimmte Spuren des mentalen Bildes zu finden. Eben diese Spuren des Bildes in der lexikalisierten Bedeutung des Idioms werden hier als bildliche Bedeutungskomponente bezeichnet (mehr dazu in Dobrovol'skij \& Piirainen 2009; vgl. auch den russischen Terminus obraznaja sostavljajuščcaja in Dobrovol'skij 1996 und den englischen Terminus image component in Dobrovol'skij \& Piirainen 2005). Das Vorhandensein einer zusätzlichen Ebene des Inhaltsplanes rechtfertigt möglicherweise die formale Komplexität der Idiome. Die bildliche Bedeutungskomponente übernimmt dabei die Vermittlungsrolle und konstituiert die Spezifik der Idiomsemantik.

Wenn wir davon ausgehen, dass eine zentrale Aufgabe der Linguistik darin besteht, zu erklären, wie die Bedeutung der sprachlichen Einheiten zustande kommt, muss die kognitive Phrasemforschung erklären, wie sich die figurative Idiombedeutung aus der Re-Interpretation des zugrundeliegenden mentalen Bildes ergibt.

Da eben die bildliche Bedeutungskomponente die Verbindung zwischen diesen beiden Seiten des Inhaltplanes darstellt, ist ihre Ermittlung und sorgfältige Beschreibung das entscheidende Moment der kognitiven Idiomforschung. Das Zustandekommen der figurativen lexikalisierten Bedeutung, d. h. der „eigentlichen“, aktuellen Bedeutung des Idioms, geschieht nicht aufgrund der unmittelbaren Verbindung des Bezeichnenden und des Bezeichneten, sondern sozusagen auf dem Umweg, nämlich über die Re-Interpretation des Bildes. Folglich sehe ich die wichtigste Aufgabe dieser Richtung in der Untersuchung der bildlichen Komponente der Idiombedeutung. Die Rolle dieser Bedeutungskomponente wird besonders beim Vergleich ähnlicher Idiome verschiedener Sprachen evident. Oft haben Idiome der zu vergleichenden Sprachen trotz der vollen Übereinstimmung ihrer lexikalischen Struktur unterschiedliche bildliche Bedeutungskomponenten, weil jede Sprache ganz bestimmte, oft unikale, Elemente des mentalen Bildes fokussiert. Auf diese Problematik wird in Abschnitt 2 anhand einiger deutsch-russischer Idiom-Korrelate eingegangen.

2 Bei polysemen Idiomen gibt es selbstverständlich mehrere lexikalisierte Bedeutungen. 
Ausgehend vom zweiten theoretischen Postulat der kognitiven Linguistik (cognitive commitment) sehe ich eine weitere Aufgabe der Phrasemuntersuchung aus kognitiver Sicht in der Berücksichtigung der Ergebnisse psycholinguistischer Experimente. Diese Problematik wird in Abschnitt 3 besprochen.

Das dritte theoretische Postulat wird in allen Teilen der vorliegenden Arbeit berücksichtigt. Sowohl in Abschnitt 2 als auch in Abschnitt 3 wird auf die Benutzung der Textkorpora bei der Beschreibung der Phraseologie eingegangen. Da die Korpora bestimmte Aspekte des Usus fixieren, entspricht das korpusbasierte Herangehen eher der Forderung, dass die Beschreibung relevanter systemhafter Züge der Sprache auf der Analyse des Gebrauchs sprachlicher Einheiten basieren müsse.

\section{Zwischensprachliche semantische Asymmetrie und ihre kognitiven Grundlagen}

In diesem Abschnitt werden ausgewählte Probleme der semantischen Quasiund Pseudoäquivalenz zwischen Idiomen verschiedener Sprachen besprochen. Die relevanten Unterschiede in der Bedeutung und in den Besonderheiten der Verwendungsnormen der zu vergleichenden Idiome ergeben sich meistens aus der Spezifik der Re-Interpretation des zugrundeliegenden mentalen Bildes und stellen folglich ein kognitives Phänomen dar.

Die Analyse solcher Fälle erfordert zunächst die Klärung bestimmter Grundbegriffe. Dies kann am besten anhand eines Beispiels geschehen. Die Idiome (1-3) haben eine sehr ähnliche, fast identische lexikalische Struktur:

(1) frz. être entre quatre murs, im Gefängnis sein“

(2) dt. in den/seinen eigenen vier Wänden (sein) ,zu Hause sein, in der vertrauten Umgebung, in der Privatsphäre der eigenen Wohnung sein`

(3) russ. сидеть в четырех стенах (wörtlich ,in den vier Wänden sitzen') ,immer zu Hause sein, nicht ausgehen, sich isoliert fühlen‘

Wie lässt sich erklären, dass die Idiome, die in ihrer wörtlichen Lesart angeblich auf das Gleiche hinweisen, unterschiedliche figurative Bedeutungen haben? Handelt es sich möglicherweise um unterschiedliche mentale Bilder, die dahinter stehen? Der Begriff des mentalen Bildes eignet sich kaum für eine exakte Analyse linguistischer Phänomene, weil es eher psychologischer Natur ist und per definitionem individuell sein muss. Grundsätzlich kann sich jeder 
Sprecher beim Produzieren und Rezipieren eines Idioms etwas sehr Konkretes, auf seiner individuellen Erfahrung Basierendes vorstellen. Das überindividuelle, typisierte kognitiv-linguistische Korrelat des mentalen Bildes ist der Begriff des Quellenkonzepts bzw. Ausgangsframes. Der Ausgangsframe ist eine konzeptuelle Struktur, die re-interpretiert wird und folglich die Grundlage der lexikalisierten figurativen Bedeutung liefert. Bei den metaphorischen Idiomen evoziert der Ausgangsframe einen anderen, korrelierenden Frame, den Zielframe. ${ }^{3}$ Bei den metonymischen Idiomen (wie in (1) bis (3)) wird der Ausgangsframe durch einen konzeptuellen Shift modifiziert.

Es fragt sich, ob den Idiomen (1-3) der gleiche Ausgangsframe zugrunde liegt. Das ist der Fall bei (2) und (3), jedoch nicht bei (1). Dem französischen Idiom (1) liegt der Frame „Gefängnis“ zugrunde, während dem deutschen (2) und dem russischen Idiom (3) der Frame „das eigene Zuhause“ zugrunde liegt. Die lexikalische Struktur aller drei Idiome benennt den gleichen Slot „vier Wände“. Dieser Slot ist aber Bestandteil zweier verschiedener Frames und evoziert folglich unterschiedliche konzeptuelle Strukturen. Dies erklärt die Bedeutungsunterschiede zwischen (1) einerseits und (2) und (3) andererseits, nicht aber die Bedeutungsunterschiede zwischen (2) und (3).

Um diese Unterschiede zu erklären, benötigen wir einen weiteren Begriff, und zwar den der bildlichen Bedeutungskomponente. Dieses Element der Idiombedeutung übernimmt die Rolle einer konzeptuellen Brücke zwischen den beiden Makrokomponenten des Inhaltsplanes des Idioms: seiner lexikalisierten Bedeutung und dem Quellenkonzept. Die Unterschiede in der Bedeutung von (2) und (3) lassen sich aufgrund ihrer bildlichen Bedeutungskomponenten erklären. In (2) wird im Frame „das eigene Zuhause“ der Slot „vertraute Umgebung, Privatsphäre“ profiliert, in (3) hingegen der Slot „nicht ausgehen, sich isoliert fühlen“. Diese profilierten Slots bestimmen die Spezifik der bildlichen Komponente der Bedeutungen dieser Idiome. In (2) bildet die Idee der vertrauten Umgebung die semantische Brücke zwischen dem Quellenkonzept bzw. Ausgangframe und der aktuellen lexikalisierten Bedeutung des Idioms; in (3) übernimmt das Gefühl der Einsamkeit, des Isoliertseins diese Funktion.

Eine kognitiv basierte Analyse dieser Art erklärt nicht nur semantische Unterschiede zwischen den formal ähnlichen Idiomen verschiedener Sprachen, sondern ermöglicht eine Formalisierung der intuitiv nachvollziehbaren Grenze zwischen einzelnen Typen der semantischen Non-Äquivalenz in der Idiomatik. In Dobrovol'skij \& Piirainen (2009) unterscheiden wir zwischen den idiomatischen falschen Freunden und den sog. zwischensprachlichen Quasisynonymen.

3 Mehr zu Konzepten, die sich auf die beiden Ebenen der Idiomsemantik beziehen, vgl. Dobrovol'skij (2016b). 
Bei den erstgenannten handelt es sich um Fälle wie (1) vs. (2) und (3), d.h. um Idiome, deren lexikalisierte Bedeutungen nichts miteinander zu tun haben, obwohl sie ähnliche lexikalische Strukturen aufweisen. Die zwischensprachlichen Quasisynonyme sind in gewisser Hinsicht den „falschen Freunden“ ähnlich, denn auch sie weisen bestimmte Abweichungen in der lexikalisierten figurativen Bedeutung auf. Der Unterschied zwischen den beiden Typen besteht vor allem im Grad der betreffenden semantischen Differenzen: Die zwischensprachlichen Quasisynonyme haben einen sehr ähnlichen bis identischen Bedeutungskern. Die Bedeutungen solcher Idiome zeichnen sich durch leichte Divergenzen aus, die oft periphere Komponenten der semantischen Struktur betreffen. Es handelt sich um „falsche Freunde“, wenn verschiedene Frames als Basis der Re-Interpretation dienen. Trotz der Übereinstimmungen in der lexikalischen Struktur evoziert der Ausdrucksplan des Idioms unterschiedliche Konzepte. Die semantischen Unterschiede zwischen den zwischensprachlichen Quasisynonymen gehen hingegen auf die Spezifika ihrer bildlichen Bedeutungskomponenten zurück, wobei die zugrundeliegenden Ausgangsframes identisch sind.

Die Rolle der bildlichen Komponente der Idiombedeutung bei der semantischen Quasiäquivalenz kann am Material aus dem neuen deutsch-russischen Idiom-Wörterbuch illustriert werden. ${ }^{4}$ Ein nichttriviales Beispiel dieser Art stellt das Idiompaar grünes Licht geben vs. дать зелёный свет dar. In vielen Fällen lässt sich das deutsche Idiom grünes Licht geben mit Hilfe des russischen Idioms дать зелёный свет problemlos übersetzen; vgl. (4-6):

(4) Das Arbeitsgericht Frankfurt hat im Tarifstreit beim Flugzeugbauer Airbus grünes Licht für Warnstreiks gegeben. (Rhein-Zeitung, 1.10. 2011)

Суд по трудовым спорам Франкфурта дал зелёный свет предупредительной забастовке рабочих авиастроительного концерна «Аэробус», связанной с тарифным конфликтом.

(5) Die Suche nach der seit Jahrzehnten verschwundenen Leiche von Lolita Brieger auf einer ehemaligen Mülldeponie in der Eifel kann beginnen. Experten gaben grünes Licht. (Rhein-Zeitung, 5.10. 2011)

Поиски исчезнувшего несколько десятков лет назад трупа Лолиты Бригер на бывшей мусорной свалке в Эйфеле могут быть начаты. Эксперты дали зелёный свет.

4 Teile dieses Wörterbuchs sind auf der Homepage des IDS frei online zugänglich: http:// wvonline.ids-mannheim.de/idiome_russ/index.htm. [letzter Zugriff 7.11. 2017]. 
(6) Ein Gericht in Polen gab grünes Licht für die Auslieferung eines mutmaßlichen Agenten des israelischen Geheimdienstes Mossad an Deutschland. (Hamburger Morgenpost, 8. 7. 2010)

Польский суд дал зелёный свет экстрадиции в Германию предполагаемого агента израильской разведки «Моссад».

Es finden sich aber genug Kontexte, in denen diese Äquivalenz auf der Textebene nicht vorhanden ist. Bei der isolierten Nennung scheinen das deutsche und das russische Idiom volläquivalent zu sein, bei ihrer Einbettung in den Kontext der Übersetzung wird das russische Idiom дать зелёный свет als deplatziert und sowohl stilistisch als auch usuell anfechtbar empfunden; vgl. (7-9):

(7) Ben Bernanke (56) kann aufatmen. Er bekommt eine zweite Amtszeit als US-Notenbankchef. Der mächtige Bankenausschuss des Senats gab dafür grünes Licht. (Hamburger Morgenpost, 18.12. 2009)

56-летний Бен Бернанке может вздохнуть с облегчением. Он во второй раз получил должность директора эмиссионного банка США. Вчера влиятельная банковская комиссия сената дала соответствующее разрешение [?? зелёный свет].

(8) Die Börsenaufsichten in Luxemburg, Belgien und Frankreich hatten der Offerte des indischen Stahlunternehmers Lakshmi Mittal grünes Licht gegeben. (Braunschweiger Zeitung, 18. 5. 2006)

Органы биржевого надзора Люксембурга, Бельгии и Франции одобрили предложение [??дали зелёный свет предложению] индийского сталелитейного магната Лакшми Миттала.

(9) Nach einer Zwangspause wegen der Verdachts-Diagnose Gaumenkrebs haben die Ärzte dem Schauspieler Jan Fedder grünes Licht gegeben: Anfang April soll es wieder losgehen mit den Dreharbeiten für das ARD - „Großstadtrevier“. (Braunschweiger Zeitung, 26. 2. 2013)

После вынужденного перерыва из-за подозрения на рак нёба врачи дали актёру Яну Феддеру разрешение снова участвовать [??дали актёру Яну Феддеру снова зелёный свет на участие] в съёмках телесериала «Полицейский участок большого города» для канала «АРД»: они возобновятся в начале апреля.

Diese Unterschiede lassen sich dadurch erklären, dass die zugrundeliegende Ampel-Metapher im russischen Idiom viel stärker profiliert ist. Mit anderen Worten: Der Gebrauch des russischen Idioms дать зелёный свет wird in den 
Kontexten lizenziert, in denen die schon in Gang gesetzten (oder zumindest fest geplanten) Aktivitäten durch höhere Instanzen, Entscheidungsträger usw. zeitweise gestoppt werden. Wenn dann die entsprechende Erlaubnis gegeben wird und diese Aktivitäten fortgesetzt bzw. die bis jetzt noch nicht genehmigten Pläne realisiert werden können, kann das Idiom дать зелёный свет gebraucht werden. Wenn es sich hingegen um Ernennungen, eine Annahme von Angeboten, eine Akzeptierung von Vorschlägen, ärztliche Entscheidungen usw. handelt, muss die Situation mit Hilfe von Verben wie одобрять ,bewilligen', разрешать ,erlauben, genehmigen' oder der Kollokation дать разрешение ,eine Erlaubnis geben, eine Genehmigung erteilen` versprachlicht werden.

In Termini der kognitiven Idiomanalyse heißt es, dass die bildliche Komponente in der Bedeutung des russischen Idioms stärker fokussiert ist. Das den beiden Idiomen zugrundeliegende überindividuelle Quellenkonzept, d.h. der Ausgangsframe, hinterlässt in der lexikalisierten Bedeutung des russischen Idioms noch klar nachvollziehbare Spuren, während in der figurativen Bedeutung des deutschen Idioms diese Spuren verblasst sind und den Gebrauch kaum beeinflussen.

Ein weiteres Idiompaar, das feine semantische Unterschiede aufweist, die auf die Besonderheiten der bildlichen Bedeutungskomponente zurückzuführen sind, ist schwarz auf weiß ,gedruckt, schriftlich, daher offiziell‘ vs. чёрным по белому ,klar, deutlich (nur in Bezug auf Schriftliches, meist offiziell Gedrucktes)‘. Typische Kontexte, in denen diese Idiome vorkommen, sind (10) und (11).

(10) Damit bekämen sportliche Aktivitäten einen verbindlicheren Charakter. Was einmal schwarz auf weiß festgehalten sei, werde nicht so schnell umgestoßen, sagt Mayrhofer. (SN-Lauftagebuch)

(11) Читай внимательней! Там же чёрньм по белому написано, что на что влияет. „Lies aufmerksamer! Dort steht es doch schwarz auf weiß geschrieben, was worauf einwirkt.“

Beiden Idiomen liegen die gleichen Wissensstrukturen als Ausgangsframes zugrunde, nämlich Fragmente des Weltwissens über Druck- bzw. Schrifterzeugnisse. Die lexikalisierten Bedeutungen weisen eine leichte Divergenz auf. Im russischen Idiom bildet die semantische Komponente ,gedruckt‘ eine Präsupposition. Im deutschen Idiom ist der Charakter des ,Offiziellen“ (eines gedruckten oder geschriebenen Dokuments) ein Teil der Assertion, ein zusätzlicher Fokus in der semantischen Struktur, während er im russischen Idiom eher eine mögliche, nicht obligatorische semantische Konsequenz darstellt. Zur Assertion des russischen Idioms gehören vielmehr die semantischen Komponenten 
,Klarheit‘ und ,Deutlichkeit‘. Im Mannheimer Korpus DeReKo finden sich 223 Kontexte mit dem Idiom schwarz auf weiß. Davon beziehen sich 192 Kontexte auf die Situation des Überreichens bzw. Besitzens eines offiziellen Papiers. Im RNC (Russian National Corpus) kommt das Idiom чёрным по белому $278 \mathrm{Mal}$ vor. Nur 18 davon sind ambivalent in dem Sinne, dass sie die semantische Komponente ,offiziell' potentiell zulassen. Daraus ergibt sich eine unterschiedliche Kombinatorik: dt. „jemandem etwas schriftlich (offiziell) geben/zeigen“, „etwas schriftlich (offiziell) haben (wollen)/festhalten“ vs. russ. „etwas steht klar und deutlich geschrieben/gedruckt“. So ist es im Deutschen normal zu sagen: Ich will das schwarz auf weiß haben, während die Übersetzung dieses Satzes ins Russische agrammatisch wäre: ^Дайте мне это чёрньм по белому.

Die Beispiele (12) und (13) zeigen typische Kontexte aus dem neuen deutsch-russischen Idiom-Wörterbuch, in denen das deutsche Idiom schwarz auf weiß nicht mit dem russischen Pseudoäquivalent чёрным по белому übersetzt werden kann:

(12) Jetzt haben wir endlich schwarz auf weiß, was wir immer schon ahnten, aber nie beweisen konnten: Wir zahlen viel zu viel für Benzin - willkürliche Preisaufschläge der Öl-Konzerne. (Hamburger Morgenpost, 22. 3. 2012) Теперь об этом, наконец, пишут официально: то, о чём мы всегда догадывались, но не могли доказать. Мы слишком много платим за бензин из-за произвольных накруток на цену со стороны нефтяных компаний.

(13) Ein Zuhörer legt Bilder vor, die schwarz auf weiß beweisen: Auf den Grundstücken steht auch eine mächtige Trutzburg. (nach Mannheimer Morgen, 18.1. 2012)

Один из слушателей предъявляет фотографии, которые документально доказывают: На земельных участках располагается огромная боевая крепость.

Kontexte, in denen die beiden Idiome als Übersetzungsäquivalente fungieren können, sind eher selten:

(14) Fast stolz präsentiert er sein Zeugnis mit den Worten: „Ich habe elf Sechsen." Und wirklich, dort steht es schwarz auf weiß. (Hamburger Morgenpost, 24.6. 2005)

Почти с гордостью он показывает свой дневник, говоря: «У меня 11 пятёрок». И действительно, там так чёрным по белому и написано. 
Zusammenfassend sei noch einmal darauf hingewiesen, dass sich viele Idiome verschiedener Sprachen, die bei der isolierten Nennung als volläquivalent empfunden werden, in den authentischen Kontexten als Quasi- oder Pseudoäquivalente erweisen. Dank einer systematischen Benutzung der Textkorpora werden relevante semantische Unterschiede zwischen solchen Idiomen offensichtlich. Eine plausible Erklärung für die Existenz dieser semantischen Unterschiede ist mit Hilfe der kognitiven Methoden der Phrasemforschung möglich geworden.

\section{Experimentell basierte Phrasemforschung: Methoden und Ergebnisse}

Bei der kognitiv orientierten Phrasemforschung handelt es sich um zwei grundsätzlich unterschiedliche Forschungsrichtungen, die bedingt als kognitiv-linguistisch und kognitionspsychologisch bezeichnet werden können. Während die erstgenannte Richtung das Ziel hat, mittels kognitivlinguistischer Heuristiken die sprachlichen Besonderheiten der Idiomatik besser $\mathrm{zu}$ verstehen und $\mathrm{zu}$ beschreiben, bemüht sich die letztere um die Verifizierung bestimmter Hypothesen über den Ablauf der kognitiven Verarbeitung der Idiome im „real timemodus“ oder über die psychologische Beschaffenheit der zugrundeliegenden mentalen Bilder. In diesem Forschungsparadigma geht es primär nicht um eine Beschreibung relevanter Systemeigenschaften der Idiome und relevanter Besonderheiten ihres Funktionierens im Diskurs, sondern um die Prozesse der mentalen Verarbeitung bestimmter idiomatischer Lexikoneinheiten. Die traditionelle Phraseologieforschung, die mit rein linguistischen Methoden arbeitete, hat dieser Forschungsrichtung bisher kaum Beachtung geschenkt, um unter anderem anhand deren Ergebnisse die Theorie der Phraseologie weiterzuentwickeln. Das ist bedauerlich, weil die Ergebnisse psychologischer Experimente ohne Zweifel die Aufmerksamkeit der im Bereich der Phraseologie arbeitenden Linguistinnen und Linguisten verdienen und das Erklärungspotenzial relevanter Konzeptionen erhöhen. Die Beachtung experimenteller Ergebnisse ist besonders im Rahmen der kognitiv orientierten Phrasemforschung notwendig, allein schon um den Anforderungen des „cognitive commitment“ gerecht zu werden.

Schon seit den 1970er Jahren versuchen die Psycholinguisten die Frage zu beantworten, welche mentalen Prozeduren in den Verstehensprozess der Idiome involviert sind. In bestimmten Punkten herrscht weitgehend Konsens; im Ganzen bietet dieses Forschungsfeld jedoch ein mosaikartiges Bild mit relativ autonomen Teilbereichen. Dementsprechend wurden im Rahmen der kogni- 
tionspsychologischen Phraseologieforschung mehrere Konzeptionen entwickelt, die jeweils verschiedene Aspekte der mentalen Verarbeitung der Idiome betreffen. In der Regel tragen diese Konzeptionen hypothetischen Charakter, weil sie sich nicht als globale Theorien, sondern als Erklärungsversuche experimenteller Daten verstanden wissen wollen.

Im Ganzen kann man die Arbeiten aus diesem Bereich in drei Gruppen einteilen, die den folgenden drei Aspekten der mentalen Verarbeitung der Idiome entsprechen:

- zeitliche Interaktion der semantischen Ebenen,

- Speicherung der Idiome im mentalen Lexikon,

- $\quad$ semantische Motiviertheit bei der Idiom-Verarbeitung.

Im Folgenden wird der erste der genannten Aspekte besprochen. ${ }^{5}$ Bei diesem Aspekt handelt es sich um die Reihenfolge der mentalen Prozeduren, die bei der Idiom-Verarbeitung aktiviert werden. Wenn ein semantisch transparentes, d.h. motiviertes Idiom wahrgenommen wird, so ist $\mathrm{zu}$ fragen, was als erstes verarbeitet wird: seine wörtliche Bedeutung, seine figurative Bedeutung, oder beides gleichzeitig. Es handelt sich also um das Problem der zeitlichen Interaktion der beiden semantischen Ebenen des Idioms, die traditionell als „direkte“ bzw. „übertragene“ Bedeutung bezeichnet wurden. Entsprechend der Antwort auf diese Frage wurden drei konkurrierende Hypothesen aufgestellt:

- Hypothese „wörtlich vor figurativ“ (literal-first model);

- Hypothese „figurativ vor wörtlich“ (figurative-first model bzw. direct access

- hypothesis oder idiom superiority effect);

- Hypothese der simultanen Verarbeitung (simultaneous processing model). ${ }^{6}$

Die Logik, die der Hypothese „wörtlich vor figurativ“ zugrunde liegt, basiert auf der Unmöglichkeit, bereits beim Rezipieren der ersten Idiom-Konstituente vorauszusehen, dass es sich um ein Idiom handelt, und folglich von dem „wörtlichen“ Verarbeitungsmodus der vorhergehenden Äußerungselemente auf die „figurative“ Interpretation umzuschalten. Wenn das literal-first-Modell zutreffen würde, müssten die wörtlich gebrauchten Idiome schneller verarbeitet werden. Die Experimente haben aber keine signifikanten Unterschiede in

$5 \mathrm{Zu}$ experimentellen Ergebnissen aus dem Bereich der Speicherung der Idiome im mentalen Lexikon und der semantischen Motiviertheit bei der Idiom-Verarbeitung vgl. Dobrovol'skij (1997: 27-36, 43-47; 2016a: 205-207).

6 Zur Übersicht über diese Problematik vgl. unter anderem Cacciari \& Glucksberg (1994), Glucksberg (2001), McElree \& Nordlie (1999); siehe dazu auch bereits Bobrow \& Bell (1973); ausführlicher dazu Dobrovol'skij (1997: 12-18). 
der Verarbeitungszeit aufgezeigt. ${ }^{7}$ Andere Untersuchungsergebnisse (Gibbs 1980 und 1986; Schweigert \& Moates 1988; Cronk \& Schweigert 1992) zeigen, dass die Idiome sogar schneller verstanden werden, wenn sie in ihrer lexikalisierten figurativen Bedeutung gebraucht werden. Dies kann man besonders leicht nachvollziehen, wenn man nicht von isolierten Idiomen, sondern von ihrem Gebrauch in authentischen Kontexten ausgeht, wie die folgenden Belege aus dem Deutschen Referenzkorpus (DeReKo) zeigen:

(15) Dass Museen dezentralisiert werden müssen, ist kalter Kaffee aus den 60er Jahren. (Berliner Zeitung, 28. 8. 2008)

(16) Was soll die ganze Aufregung? Schulschwänzen ist doch kein Kapitalverbrechen. Mal ehrlich - gibt es jemanden unter uns, der statt zu Mathe nicht ins nächste Eiscafé gegangen wäre? Na also. Wollen wir die Kirche mal im Dorf lassen. (Mannheimer Morgen, 25.10. 2005)

Um zu verstehen, dass es sich bei den Ausdrücken kalter Kaffee und die Kirche im Dorf lassen um Idiome und nicht um wörtlich zu verstehende Wortketten handelt, braucht der Rezipient nicht zunächst auf ihre direkte Bedeutung zuzugreifen, um erst dann festzustellen, dass diese Ausdrücke in der sogenannten speaker meaning (im Sinne von Searle) gebraucht wurden. ${ }^{8}$

Die figurative-first-Hypothese geht von der Möglichkeit eines direkten $\mathrm{Zu}$ griffs auf die aktuelle Idiombedeutung aus und ist deshalb auch unter dem Namen direct access hypothesis bekannt (Gibbs 1980; Gibbs 1986). Laut dieser Hypothese soll die Verarbeitung der figurativ gebrauchten Idiome weniger Zeit in Anspruch nehmen. Dieses Modell scheint besser als die literal-first-Hypothese der Tatsache Rechnung zu tragen, dass die Idiome reproduzierbare und nicht regelmäßig generierbare Einheiten par excellence sind und folglich, wie andere Lexikoneinheiten, einen direkten Zugriff von der Form auf die Bedeutung ermöglichen müssen. Beispiele wie (17)-(19) scheinen zunächst diese Hypothese $\mathrm{zu}$ verifizieren:

(17) Dass der Anmeldeschein gefälscht war, sieht ein Blinder mit Stock. (Niederösterreichische Nachrichten, 2.4. 2008)

(18) Ein Gericht in Polen gab grünes Licht für die Auslieferung eines mutmaßlichen Agenten des israelischen Geheimdienstes Mossad an Deutschland. (Hamburger Morgenpost, 8. 7. 2010)

7 Vgl. unter anderem Estill \& Kemper (1982), Glass (1983), Schweigert (1986).

8 „When sentence meaning is defective, look for a speaker meaning that differs from sentence meaning“ (Searle 1979: 114). 
(19) Aufgeschaut haben wir als Schüler $\mathrm{zu}$ jenen Lehrern, die uns die Leviten lasen und uns dennoch spüren ließen, dass sie uns mochten. (nach Kleine Zeitung, 26.2. 1997)

Wenn in der Äußerung eine Wortverbindung sieht ein Blinder (vgl. Beleg 17) vorkommt, ist es für den Rezipienten schon aufgrund des allgemeinen Weltwissens unmittelbar klar, dass das Gesagte nicht wörtlich gemeint sein konnte. Oder wenn in (18) der Satz mit Ein Gericht in Polen gab grünes beginnt, wird mit großer Wahrscheinlichkeit sofort angenommen, dass es sich nicht um irgendeinen grünen Gegenstand handelt. So antizipiert der Rezipient noch vor dem Moment, in dem das ganze Idiom ausgesprochen wird, einen sprachlichen Ausdruck mit figurativem Sinn. Noch klarer ist dies in Belegen wie (19), wenn die erste Konstituente des Idioms ein unikales Wort darstellt, das normalerweise nur in der Struktur des betreffenden Idioms vorkommt.

Die figurative-first-Hypothese ist auch mit der psycholinguistischen Erkenntnis konsistent, dass es „no general priority of the literal“ (Glucksberg 1991: 147) gibt. $^{9}$

In ihrem Forschungsüberblick „Psycholinguistic aspects of phraseology: American tradition“ liefern Gibbs \& Colston (2007: 822-823) mit Verweis auf (Katz \& Ferratti 2003) zusätzliche Argumente zugunsten der Hypothese „figurativ vor wörtlich“:

In general, there is much evidence to suggest that people do not need to first parse conventional phrases according to their literal meanings, before they access any putative phrasal lexicon to retrieve its conventional meaning. Idioms and proverbs, like many conventional expressions, are therefore not difficult to understand because their meanings vary from their so-called literal interpretations. (Gibbs \& Colston 2007: 822-823)

Die Ansichten zur figurative-first-Hypothese sind in sich widersprüchlich. Gegen die Hypothese in ihrer starken Version sprechen verschiedene Ergebnisse der experimentellen Psycholinguistik; vgl. die in Cacciari \& Tabossi (1988) und Tabossi \& Zardon (1992) diskutierten Daten. Wenn bei der kognitiven Verarbeitung der Idiome direkt auf die aktuelle Bedeutung zugegriffen wird, bedeutet dies, dass die Idiome im mentalen Lexikon als wortähnliche Einheiten repräsentiert sind. Wenn das stimmt, sollte die kognitive Verarbeitung der Idiome nach dem gleichen Prinzip ablaufen wie die der Wörter. Es ist inzwischen bewiesen, dass die Aktivierung der Wort-Semantik beginnt, lange bevor das

9 Dunbars „Theory of Parameterised Lexical Concepts“ unterstützt die figurative-first-Hypothese, indem sie von der Annahme ausgeht, „that there is a preliminary scan for idioms which then hands over to a literal processor only if no idiom is found“ (Dunbar 1991: 131). 
Wort-Formativ endgültig erkannt werden kann. Der Rezipient stellt verschiedene Hypothesen über die Semantik auf, die mit dem Zuwachs der akustischen Information reduziert werden, bis nur die eine (d.h. die richtige) übrig bleibt (vgl. dazu Marslen-Wilson 1987; Zwitserlood 1989).

Das folgende Priming-Experiment von Tabossi \& Zardon (1992) legt die Annahme nahe, dass die Idiome nicht nach diesem Prinzip verarbeitet werden. Den Versuchspersonen (Vpn) wurden in mündlicher Form Äußerungen präsentiert, die geläufige Idiome beinhalteten, z. B. After the last tournament, the tennis player was in seventh heaven for the success. Diese Kontexte ließen nur die figurative Lesart zu. Die Vpn erhielten dabei ein schriftlich präsentiertes Wort (visual target), das die idiomatische Interpretation unterstützen sollte, z. B. HAPPY. Dieses Wort wurde in verschiedenen Phasen des Experiments präsentiert:

a) vor dem Idiom, d. h. gleich nach dem Wort player = Kontrollposition,

b) nach dem Verb was = Verbposition,

c) nach dem Idiom, d.h. gleich nach dem Wort heaven = Endposition.

Wenn die figurative-first-Hypothese zutrifft, soll die Präsentation des die figurative Bedeutung evozierenden Wortes HAPPY in der Verbposition die Verarbeitung wesentlich beschleunigen. Wenn die literal-first-Hypothese richtig ist, soll die Verarbeitungszeit dadurch nicht beeinflusst werden, denn die Aktivierung der aktuellen Bedeutung kann nicht beginnen, bevor die ganze Kette verarbeitet und mit dem Kontext abgeglichen wird. In diesem Fall kann die Verarbeitung nur durch die visuelle Unterstützung in der Endposition beschleunigt werden. Das Experiment bestätigte die letztgenannte Annahme und legte die Vermutung nahe, dass

idiom processing may be substantially different from lexical processing, showing a time course of activation for the meaning of these expressions that differs from what one would expect if they were mentally represented as long, complex words. (Tabossi \& Zardon 1992: 125)

Tabossi \& Zardon (1992: 122) interpretieren diese Daten als Bestätigung der in Cacciari \& Tabossi (1988) erstmalig vorgeschlagenen Konfigurationshypothese. Jedes Idiom enthält eine Schlüsselkonstituente (key), d.h. ein Wort, das im Verarbeitungsprozess eine qualitative Grenze markiert. Bevor der key gehört wird, versucht der Rezipient das Idiom wörtlich zu interpretieren; nach dem Hören des key wird die figurative Bedeutung aktiviert. Folglich hat der key eine idiom-evozierende Funktion. Um imstande zu sein, auf die im mentalen Lexikon gespeicherte figurative Idiombedeutung zuzugreifen, muss der Rezipient über genug formale Informationen verfügen. Anderenfalls kann er nicht wissen, dass er es mit einem Idiom und nicht mit einer freien Wortkombination 
zu tun hat. Diese Gewissheit (oder zumindest Ahnung) bekommt er in der Regel nicht gleich nach der ersten Konstituente, wenn der formale Informationsumfang noch zu klein ist. Er braucht aber auch nicht zu warten, bis die ganze Konstituenten-Kette realisiert ist. Daraus erklärt sich, warum die starren, syntaktisch undurchlässigen Idiome (wie kick the bucket) schneller verarbeitet werden als flexible Einheiten (vgl. die Experimente von Gibbs \& Gonzales 1985). Sie lassen sich als Idiome offensichtlich schneller erkennen. Problematisch sind dabei Fälle, in denen die Struktur des Idioms modifiziert wird und das idiom-evozierende Element (key) an einer anderen Stelle erscheint.

Auch die Untersuchungsergebnisse von Gibbs (1980 und 1986), Schweigert \& Moates (1988) und Cronk \& Schweigert (1992) scheinen die figurative-firstHypothese in Frage zu stellen. Laut Cacciari (2014) sind diese Fragen weiterhin offen. Dies kann man anhand der folgenden Beispiele aufzeigen:

(20) Es werden immer wieder Leute auf den Bauch fallen, wenn es bei der Reisebuchung in erster Linie um den Preis geht. Nicht alles im Internet, was glänzt, ist Gold. Mein Tipp: Geht ins Reisebüro und lasst Euch beraten. (Hamburger Morgenpost, 9. 8. 2008)

In (20) sind vor dem Verb fallen auch wörtliche Interpretationen des Ganzen nicht ausgeschlossen. Folglich wird nicht in allen Fällen auf die figurative Interpretation früh genug zugegriffen. Es ist durchaus möglich, dass dem Rezipienten beide Interpretationen recht lange offenstehen.

Eine alternative Hypothese über die zeitliche Interaktion der wörtlichen und lexikalisierten (figurativen) Idiombedeutung, die diesen experimentellen Daten Rechnung zu tragen versucht, ist das Modell der simultanen Verarbeitung der beiden semantischen Ebenen.

Die Hypothese der simultanen Verarbeitung, die unter dem Namen simultaneous processing model bekannt ist (Schweigert 1992), besagt, dass beide Interpretationen (wörtliche und figurative) gleichzeitig verlaufen (vgl. auch Swinney \& Cutler 1979; Estill \& Kemper 1982). Beim Rezipieren einer Äußerung werden allen Elementen semantische Repräsentationen zugeordnet. Diese Elemente sind im Normalfall Wörter; es können aber auch key-Teile eines Idioms (im Sinne von Cacciari \& Tabossi 1988), beliebige Idiom-Konstituenten oder ganze Idiome sein. Die Information darüber, dass das betreffende Element eine Idiom-Konstituente oder ein ganzes Idiom darstellt, bildet eine Komponente seiner semantischen Repräsentation. Wenn mehrere Bedeutungen des Elements im Prinzip möglich sind, werden sie gleichzeitig als potenzielle semantische Versionen aus dem Gedächtnis abgerufen. Im nächsten Schritt werden die Kontextbedingungen überprüft. Wenn nur eine der Bedeutungen 
(wörtliche oder figurative) mit dem Kontext kompatibel ist, wird sie vom Bewusstsein registriert:

(21) Viele Kinder, die zu uns kommen, müssen erst wieder lernen, den Kopf hoch zu tragen. Wer in ein Förderzentrum geht, braucht viel Selbstbewusstsein, um trotzdem stolz auf seine Schule zu sein und gern dorthin zu gehen. Die Schüler wissen, dass ihre Berufschancen nicht rosig sind. (Nürnberger Nachrichten, 10.11. 2006)

Der erste Satz von (21) gestattet die Annahme, dass der Ausdruck den Kopf hoch $z u$ tragen auch wörtlich gemeint werden könnte. Erst nach dem Rezipieren des weiteren Kontexts wird die wörtliche Interpretation ausgeschlossen.

Wenn beide Interpretationen im gegebenen Kontext sinnvoll sind, erfolgt ein „double-take effect“. Potenziell gibt es eine weitere Möglichkeit: Wenn sich die wörtliche Bedeutung mit dem Kontext nicht verträgt, findet der Rezipient in seinem semantischen Gedächtnis auch keine passende figurative Lesart. In diesem Fall muss er selbst eine kontextkompatible aktuelle Bedeutung konstruieren. Auf diese Weise werden Ad-hoc-Metaphern und unbekannte Idiome verarbeitet. Wenn diese Annahme stimmt, muss die Verarbeitung unbekannter Idiome bedeutend mehr Zeit in Anspruch nehmen. Denn um eine unbekannte Kette sinnvoll $\mathrm{zu}$ interpretieren, muss der Rezipient die Inferenzprozeduren selbst nachvollziehen. Auch eine ungewöhnliche, mit den produktiven Regeln der Grammatik kollidierende Form kann die Verarbeitungszeit wesentlich verlängern. In Beispiel (22) kommt das Idiom mit der regionalen Form bei die vor:

(22) Bei uns ist er ein Star, klar. Aber jetzt mal Butter bei die Fische: Wie kommt Elbkoch Tim Mälzer mit seinem Hamburger Schnack bei den Bayern an? (Hamburger Morgenpost, 23. 7. 2007)

Andererseits können gerade verschiedenartige Abweichungen von der grammatischen Norm als Trigger der figurativen Interpretation fungieren. Voraussetzung ist dabei aber, dass der Rezipient die betreffenden Idiome kennt und schnell wiedererkennen kann. Wenn das Idiom ein seltenes Wort (wie z. B applecart) enthält, so begünstigt das grundsätzlich die figurative Interpretation. So versuchten die Teilnehmer des Experiments von Cronk, Lima \& Schweigert (1993) die Kette upset the applecart auch in den Fällen als ein Idiom zu interpretieren, in denen die wörtliche Lesart gemeint war; vgl. auch deutsche unikale Wörter wie fix oder Geldhahn in fix und fertig und jmdm. den Geldhahn zudrehen.

Die „simultane Hypothese“ erklärt, warum die Zeitmessungsexperimente oft widersprüchliche Ergebnisse liefern. Wenn die beiden semantischen Ebe- 
nen gleichzeitig in den Verarbeitungsprozess involviert werden, bedeutet das, dass in den ersten Phasen verschiedene Alternativen offen stehen. Es beginnt ein Rennen um die Wette, ein „horse-race“, wie Gibbs (1990: 428) es beschreibt. So ist die Form an die Flasche in (23) und (24) sowohl als Teil des Idioms an die Flasche geraten, als auch als Teil des Ausdrucks ein Baby an die Flasche gewöhnen interpretierbar.

(23) Er kannte sie also nur aus dem Fernsehen und von den Titelblättern der Zeitschriften, aus jenen Tagen, bevor er an die Flasche geraten war. (Deon Meyer, Dreizehn Stunden: Kriminalroman)

(24) 8. 8. 2011: Könnt ihr uns raten, wie er am besten an die Flasche - oder Alternativen - gewöhnt werden kann? (www.rund-ums-baby.de)

Zusammenfassend ist festzuhalten, dass die Idiomatik einer Sprache ein sehr heterogenes Feld darstellt. Folglich kann keines der besprochenen Modelle den Anspruch auf eine allgemeine Erklärung der Idiom-Verarbeitung im „real timemodus“ erheben. Darauf weist auch Cacciari (2014: 274-275) hin:

In any case, the speed with which idioms are processed depends on contextual information, idiom familiarity, meaning dominance, and on the online vs. offline nature of the experimental task questioning the idea of a generalized idiom superiority effect.

Diese Erkenntnisse werden auch durch neuropsychologische Forschungen bestätigt (vgl. Kutas \& Federmeier 2000; Vespignani et al. 2010).

\section{Fazit und Ausblick}

Kognitive Phrasemanalyse ist keine einheitliche Forschungsrichtung. Vielmehr finden sich einzelne kognitiv orientierte Forschungsansätze verstreut in verschiedenen Arbeiten, die sich mit der Idiomsemantik und Idiomsyntax befassen. Gemeinsam ist ihnen eine Hinwendung zu Kategorien des Wissens und dessen Verarbeitung, zu konzeptuellen Strukturen, die hinter den betreffenden Sprachstrukturen stehen. Dabei handelt es sich oft um korpusbasierte Untersuchungen relevanter Gebrauchsrestriktionen.

Kontrastive Arbeiten zur Phraseologie befassten sich traditionell mit der Typologisierung zwischensprachlicher Idiom-Äquivalente, wobei Typen wie Volläquivalenz, Teiläquivalenz, phraseologische Parallelität und Nulläquivalenz unterschieden wurden. Vor dem Hintergrund dieser Typologie könnte man annehmen, dass alle zwischensprachlichen Korrelate der ersten Gruppe (sog. 
Volläquivalenz) nicht nur eine (fast) identische lexikalische Struktur aufweisen, sondern auch in ihren Bedeutungen völlig übereinstimmen. In Wirklichkeit finden sich aber vollkommene Äquivalenzbeziehungen viel seltener. Die meisten „Volläquivalente“ weisen signifikante Unterschiede auf, die letzten Endes auf ihre systeminhärente Beschaffenheit zurückzuführen sind.

In der vorliegenden Arbeit wurde versucht, diese beiden Aspekte der Phrasemforschung (d.h. kognitiv orientierte Phraseologie und kontrastive Idiomanalyse) miteinander zu verbinden. Es stellte sich heraus, dass relevante semantische Unterschiede mit Hilfe der Methoden der kognitiven Phrasemforschung nachweisbar sind. Eine systematische Benutzung der Textkorpora erweist sich dabei als eine der entscheidenden Voraussetzungen.

Neben der kontrastiven Problematik wurde hier auf einige Ergebnisse psycholinguistischer Experimente im Bereich der kognitiven Idiomforschung eingegangen. Dieser Aspekt korreliert mit der bekannten Forderung der kognitiven Linguistik, die Lakoff (1991) als cognitive commitment bezeichnet: Die linguistischen Postulate dürfen den Ergebnissen anderer Disziplinen, die sich mit der Informationsverarbeitung beschäftigen, nicht widersprechen. Der hier vorgenommene Vergleich verschiedener Modelle zur zeitlichen Interaktion der semantischen Ebenen des Idiom-Inhaltsplanes hat gezeigt, dass keines dieser Modelle den Anspruch auf eine allgemein gültige Erklärung der Idiom-Verarbeitung im „real time-modus“ erheben kann. Dies hängt mit der Heterogenität der Idiomatik einer Sprache zusammen.

Die Perspektiven der kognitiven Phraseologieforschung sehe ich sowohl in der Weiterentwicklung interdisziplinärer Untersuchungen, als auch in der Umsetzung gewonnener Erkenntnisse für die Lösung eigentlich linguistischer Probleme, darunter für die Erarbeitung neuer Methoden der korpusbasierten lexikographischen Darstellung von Phrasemen verschiedener Klassen.

\section{Literatur}

\section{Forschungsliteratur}

Bobrow, Samuel \& Stephen Bell (1973): On catching on to idiomatic expressions. Memory and Cognition 1, 343-346.

Cacciari, Cristina (2014): Processing multiword idiomatic strings: Many words in one? Mental Lexicon 9, 267-293.

Cacciari, Cristina \& Sam Glucksberg (1994): Understanding figurative language. In Morton Ann Gernsbacher (ed.), Handbook of Psycholinguistics, 447-477. New York: Academic Press.

Cacciari, Cristina \& Patrizia Tabossi (1988): The comprehension of idioms. Journal of Memory and Language 27, 668-683. 
Cronk, Brian C. \& Wendy A. Schweigert (1992): The comprehension of idioms: The effects of familiarity, literalness and usage. Applied Psycholinguistics 13, 131-146.

Cronk, Brian C., Susan D. Lima \& Wendy A. Schweigert (1993): Idioms in sentences: Effects of frequency, literalness, and familiarity. Journal of Psycholinguistic Research 22, 59-81.

Dąbrowska, Ewa (2016): Cognitive linguistics' seven deadly sins. Cognitive Linguistics 27 (4), 479-491.

Dobrovol'skij, Dmitrij (1996): Obraznaja sostavljajuščaja v semantike idiom. Voprosy jazykoznanija 1, 71-93.

Dobrovol'skij, Dmitrij (1997): Idiome im mentalen Lexikon: Ziele und Methoden der kognitivbasierten Phraseologieforschung. Trier: WVT Wissenschaftlicher Verlag Trier.

Dobrovol'skij, Dmitrij (2016a): Kognitive Aspekte der Idiom-Semantik. Studien zum Thesaurus deutscher Idiome. 2. aktualisierte und erweiterte Aufl. Tübingen: Stauffenburg.

Dobrovol'skij, Dmitrij (2016b): The notion of „inner form“ and idiom semantics. Études et travaux d'Eur'ORBEM 1, 21-36.

Dobrovol'skij, Dmitrij \& Elisabeth Piirainen (2005): Figurative language: Cross-cultural and cross-linguistic perspectives. Amsterdam u. a.: Elsevier.

Dobrovol'skij, Dmitrij \& Elisabeth Piirainen (2009): Zur Theorie der Phraseologie: Kognitive und kulturelle Aspekte. Tübingen: Stauffenberg.

Dunbar, George (1991): The cognitive lexicon. Tübingen: Narr.

Estill, Robert \& Susan Kemper (1982): Interpreting idioms. Journal of Psycholinguistic Research 9, 559-568.

Gibbs, Raymond W. (1980): Spilling the beans on understanding and memory for idioms in conversation. Memory and Cognition 8 (2), 149-156.

Gibbs, Raymond W. (1986): Skating on thin ice: Literal meaning and understanding idioms in conversation. Discourse Processes 9, 17-30.

Gibbs, Raymond W. (1990): Psycholinguistic studies on the conceptual basis of idiomaticity. Cognitive Linguistics 1 (4), 417-451.

Gibbs, Raymond W. \& Herbert L. Colston (2007): Psycholinguistic aspects of phraseology: American tradition. In Harald Burger, Dmitrij Dobrovol'skij, Peter Kühn \& Neal R. Norrick (Hrsg.), Phraseologie: Ein internationales Handbuch zeitgenössischer Forschung (= Phraseology: An international handbook of contemporary research), Vol. 2, 819-836. Berlin, New York: De Gruyter.

Gibbs, Raymond W. \& Gayble P. Gonzales (1985): Syntactic frozenness in processing and remembering idioms. Cognition 20 (3), 243-259.

Glass, Arnold L. (1983): The comprehension of idioms. Journal of Psycholinguistic Research 12, 429-442.

Glucksberg, Sam (1991): Beyond literal meanings: The psychology of allusion. Psychological Science 2, 146-152.

Glucksberg, Sam (2001): Understanding figurative language. Oxford: Oxford University Press.

Katz, Albert N. \& Todd R. Ferratti (2003): Reading proverbs in context: The role of explicit markers. Discourse Processes 36, 19-46.

Kutas, Marta \& Kara D. Federmeier (2000): Electrophysiology reveals semantic memory use in language comprehension. Trends in Cognitive Sciences 4, 463-470.

Lakoff, George (1991): Cognitive vs generative linguistics: How commitments influence results. Language and Communication 11 (1/2), 53-62.

Marslen-Wilson, William D. (1987): Functional parallelism in spoken word-recognition. Cognition 25, 71-102. 
McElree, Brian \& Johanna Nordlie (1999): Literal and figurative interpretations are computed in equal time. Psychonomic Bulletin \& Review 6, 486-494.

Schweigert, Wendy A. (1986): The comprehension of familiar and less familiar idioms. Journal of Psycholinguistic Research 15 (1), 33-46.

Schweigert, Wendy A. (1992): The muddy waters of idiom comprehension begin to settle. In Martin Everaert, Erik-Jan van der Linden, André Schenk \& Rob Schreuder (eds.), Proceedings of Idioms, 111-118. Tilburg: Katholieke Universiteit Brabant.

Schweigert, Wendy A. \& Danny R. Moates (1988): Familiar idiom comprehension. Journal of Psycholinguistic Research 17 (4), 281-296.

Searle, John R. (1979): Expression and meaning: Studies in the theory of speech acts. Cambridge: Cambridge University Press.

Swinney, David A. \& Anne Cutler (1979): The access and processing of idiomatic expressions. Journal of Verbal Learning and Verbal Behavior 18, 523-534.

Tabossi, Patrizia \& Francesco Zardon (1992): The activation of idiomatic meaning. In Martin Everaert, Erik-Jan van der Linden, André Schenk \& Rob Schreuder (eds.), Proceedings of Idioms, 119-126. Tilburg: Katholieke Universiteit Brabant.

Vespignani, Francesco, Paolo Canal, Nicola Molinaro, Sergio Fonda \& Cristina Cacciari (2010): Predictive mechanisms in idiom comprehension. Journal of Cognitive Neuroscience 22 (8), 1682-1700.

Zwitserlood, Pienie (1989): The locus of the effects of sentential-semantic context in spokenword processing. Cognition 32, 25-64.

\section{Digitale Ressourcen}

DeReKo: Das Deutsche Referenzkorpus des IDS Mannheim im Portal COSMAS II (Corpus Search, Management and Analysis System): https://cosmas2.ids-mannheim.de/ cosmas2-web (letzter Zugriff 1.11. 2017).

Deutsch-russische Idiome online: http://wvonline.ids-mannheim.de/idiome_russ/index.htm (letzter Zugriff 1.11. 2017). 


\title{
Thorsten Roelcke \\ 7 Die Konstitution terminologischer Systeme in Fachsprachen
}

\begin{abstract}
Während die Festlegung einzelner Fachwörter linguistisch gut erforscht ist und dabei konzeptionell unterschiedlich interpretiert wird, liegen bislang nur verhältnismäßig geringe Erkenntnisse über die Einführung ganzer Fachwortschätze vor. Vor diesem Hintergrund stellt ein integrativer Ansatz bei der Konzeption von Terminologie ein wissenschaftliches Desiderat dar. In dem vorliegenden Beitrag werden daher die Definition von Termini einerseits und die Konstitution terminologischer Systeme andererseits aus Perspektive der System-, Pragma- und kognitiven Linguistik analysiert und interpretiert sowie in einen gemeinsamen konzeptionellen Zusammenhang gebracht.
\end{abstract}

Keywords: Definition, Eigentlichkeit, Eindeutigkeit, Exaktheit, kognitive Linguistik, Pragmalinguistik, Systemlinguistik, terminologisches System, Terminologisierung, Terminus

\section{Einleitende Bemerkungen}

In der modernen Fachsprachenforschung lassen sich drei sprachliche Konzeptionen unterscheiden, welche die Art und Weise der wissenschaftlichen Beschäftigung mit fachlicher Lexik (zur Übersicht vgl. Baßler 2002; Fraas 1998; Kageura \& L'Homme (2008); Roelcke 2010: 55-77; Roelcke 2015) maßgeblich bestimmen und eigene forschungsgeschichtliche Etappen ausmachen (vgl. Roelcke 2010: 13-28):

1. Fachsprachen als Zeichensysteme auf der Grundlage eines systemlinguistischen Inventarmodells (seit den 1930er Jahren bis heute);

2. Fachsprachen als Textäußerungen unter Bezug auf ein pragmalinguistisches Kontextmodell (seit den 1970er Jahren bis heute);

3. fachsprachliche Kognitionsfunktionen auf der Basis eines kognitionslinguistischen Funktionsmodells (seit den 1990er Jahren bis heute).

Thorsten Roelcke, Institut für Sprache und Kommunikation, Technische Universität Berlin, Sekr. HBS 2, Hardenbergstraße 16-18, D-10623 Berlin, E-Mail: roelcke@tu-berlin.de

○ Open Access. (๑) 2018 Thorsten Roelcke, publiziert von De Gruyter. (๔)BY Dieses Werk ist lizenziert unter der Creative Commons Attribution 4.0 Lizenz. 
Diese drei Sprachkonzeptionen bedingen jeweils eigene Konzepte zur Konstitution terminologischer Systeme. Diese Konzepte können im Falle des systemlinguistischen Inventarmodells als homogen und statisch sowie im Falle des pragmalinguistischen Kontextmodells als heterogen und dynamisch charakterisiert werden; im Falle des kognitionslinguistischen Funktionsmodells wäre hier von funktional und epistemisch zu sprechen.

Im vorliegenden Beitrag werden die unterschiedlichen Konzeptionen einer Konstitution terminologischer Systeme im Rahmen des system- und des pragmalinguistischen Modells erörtert und einander gegenübergestellt; abschließend folgen einige Überlegungen zu einer Befassung mit der Konstitution von terminologischen Systemen aus Sicht der kognitiven Linguistik. Dabei steht die Frage im Vordergrund, wie durch die Konstitution terminologischer Systeme das Verständnis von und die Verständigung über spezifische Fachbereiche gewährleistet wird.

\section{Das systemlinguistische Inventarmodell}

Die moderne Beschäftigung mit fachsprachlicher Terminologie geht zurück auf Eugen Wüster, dessen Standardwerk Internationale Sprachnormung in der Technik, besonders in der Elektrotechnik (Wüster 1931/1970) die nationale wie internationale Terminologiearbeit (vgl. Arntz, Picht \& Schmitz 2014; Felber \& Budin 1989) und Terminologienormung (vgl. etwa DIN 2330 und ISO 704) bis in die Gegenwart hinein prägt. Die Perspektive der modernen Terminologielehre ist dabei primär systemlinguistisch: So werden im Hinblick auf die Semantik fachsprachlicher Lexik insbesondere drei Eigenschaften postuliert, die durch eine angemessene Definition von Termini gewährleistet werden: Exaktheit, Eindeutigkeit und Eigentlichkeit (zum Folgenden vgl. Arntz, Picht \& Schmitz 2014; Roelcke 2010: 55-77).

Die Exaktheit von Termini besteht hiernach in deren unmissverständlicher Zuordnung hinsichtlich der bezeichneten Gegenstände und Sachverhalte. Im Sinne der klassisch-rhetorischen Unterscheidung zwischen klar (lat. clare) und deutlich (lat. distincte) wird diese referentielle Unmissverständlichkeit entweder durch einen unmittelbaren Sinnesbezug (zum Beispiel der Wörter süß, rot oder kalt) oder mittels einer expliziten Angabe der Bedeutung mit anderen Wörtern (etwa des Wortes Fisch als ,Tier mit Flossen, das im Wasser lebt') gewährleistet.

Demgegenüber besteht die Eindeutigkeit von Termini im Rahmen eines systemlinguistischen Konzeptes in deren unmissverständlicher Zuordnung im Hinblick auf andere Bedeutungen und Ausdrücke. Diese semantische Unmissverständlichkeit besteht hiernach sowohl in einer Monosemie (Ausbleiben von 


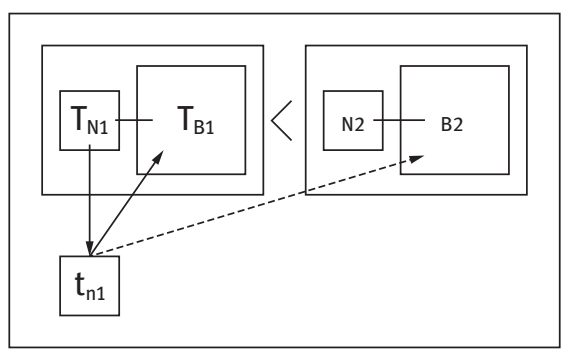

$\mathrm{T}_{\mathrm{N} 1}=$ Terminus-Benennung (System-Ebene)

$\mathrm{T}_{\mathrm{B} 1}=$ Terminus-Begriff (System-Ebene)

$\mathrm{B} 2, \mathrm{~N} 2=$ nicht fachlicher Begriff, Bezeichnung

$\mathrm{t}_{\mathrm{n} 1}=$ Terminus-token Benennung (Individual-Ebene)

$\downarrow=$,ideale“ Repräsentation

= kontaminierte Repräsentation (= Terminologisierung)

$=$ Begriffsverengung

Abb. 7.1: Terminologisierung einzelner Wörter (Gerzymisch-Arbogast 1996: 184).

Polysemie) als auch in einer Heteronymie (Ausbleiben von Synonymie) der betreffenden Wörter. Mit Blick auf diese Bidirektionalität der eindeutigen Beziehung von Ausdruck und Bedeutung ist hier auch in Anlehnung an die Logik von terminologischer Eineindeutigkeit die Rede.

Die semantische Eigentlichkeit von Termini beruht auf deren unmissverständlicher Zuordnung in Bezug auf ihre Ausdrucksseite selbst. Im Rahmen eines systemlinguistischen Konzepts fachsprachlicher Lexik werden übertragene Bedeutungen im Sinne von Metaphern oder Metonymien (vgl. das sog. Metapherntabu von Weinrich 1989; Kretzenbacher 1994) oder uneigentliche Bedeutungen im Falle von Wortbildungen als problematisch angesehen, da die Motivation der entsprechenden Bedeutung durch den Ausdruck als nicht hinreichend geklärt gilt.

Mit dieser Konzeption von Exaktheit als referentieller, Eindeutigkeit als semantischer und Eigentlichkeit als motivierter Unmissverständlichkeit wird von der systemlinguistisch geprägten Terminologielehre und Fachsprachenforschung ein statisches Konzept der Güteeigenschaften einzelner Termini vertreten: Die Unmissverständlichkeit von Fachwörtern innerhalb von Fachtexten wird im kommunikativen Vorfeld, also auf der Systemebene, herbeigeführt, sodass den Produzenten und Rezipienten während der fachsprachlichen Kommunikation selbst letztlich keine Verständigungsprobleme mehr unterlaufen sollten.

Das wesentliche Instrument, diese Unmissverständlichkeit herzustellen bzw. zu gewährleisten, ist dabei die sog. Terminologisierung anhand von Definitionen, wobei mit klassischer bzw. aristotelischer, genetischer bzw. operationaler, synonymischer oder exemplarischer Bestimmung von Termini verschiedene Verfahren $\mathrm{zu}$ unterscheiden sind. Ihnen allen ist jedoch gemeinsam, dass sie eine semantische Transformation einzelner Wörter zu Termini leisten - ein Prozess, der etwa von Gerzymisch-Arbogast (1996: 184) als „Terminologisierung“ im Rahmen einer Begriffsverengung gefasst wird (vgl. Abb. 7.1) 


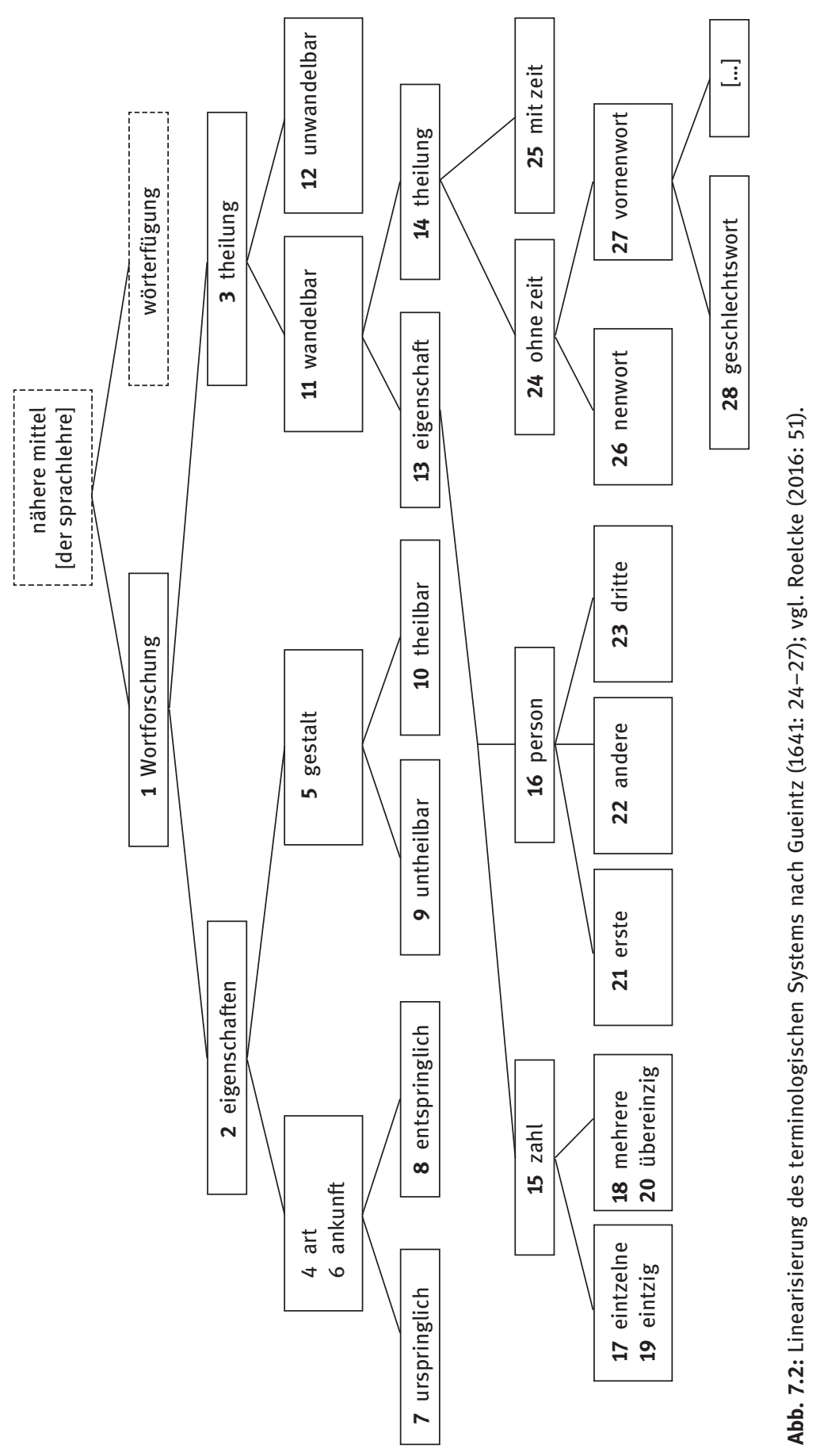


Systemlinguistische Konzeptionen beziehen sich nicht allein auf einzelne Termini, sondern auch auf ganze Terminologien, die sich aus semantisch miteinander vernetzten Termini zusammensetzen. Diese systemlinguistischen Konzeptionen erweisen sich dabei als statisch sowie an dem Inventar und der Struktur von Terminologien ausgerichtet. Beispiele für solche terminologischen Systeme finden sich in vielen Fachbereichen (und lassen sich analog zueinander graphisch darstellen) - so zum Beispiel in der wort- und formbildungsbezogenen Sprachdidaktik des Barock bei Christian Gueintz (vgl. Abb. 7.2), in der erkenntnistheoretischen Philosophie der Spätaufklärung bei Immanuel Kant (vgl. Abb. 7.3), im sachrechtsbezogenen Teil des Bürgerlichen Gesetzbuchs der Bundesrepublik Deutschland (vgl. Abb. 7.4) oder in der terminologischen Grundsatznormung des Deutschen Instituts für Normung (vgl. Abb. 7.5). Diese vier terminologischen Systeme weisen unterschiedliche Strukturen auf (vgl. auch Roelcke 2014a).

Das System, das in Gueintz (1641: 24-27) entwickelt wird, ist hierarchisch angelegt und differenziert dabei in der Regel binär vom Abstrakten zum Konkreten hin aus (Abb. 7.2; vgl. Roelcke 2016: 50): So wird etwa Wortforschung in diejenige der eigenschaften und diejenige der theilung oder die art bzw. ankunft einzelner Wörter in urspringlich und entspringlich unterteilt. Bemerkenswert ist hierbei, dass die Hierarchie nicht allein durch substantivische, sondern auch durch adjektivische Fachwörter zum Ausdruck gebracht wird, wobei die adjektivischen Differenzierungen hyponyme Mehrworttermini mit den hyperonymen Einworttermini bilden können, hier zum Beispiel also urspringliche art oder entspringliche art.

Entsprechendes gilt für das terminologische System aus dem zweiten Abschnitt der DIN-Norm 2330 (Abb. 7.5; vgl. Roelcke 2013a; 20): Hier sind unterhalb des Mehrwortterminus übergeordneter Begriff die Einworttermini Oberbegriff und Verbandsbegriff sowie unterhalb von untergeordneter Begriff dann Unterbegriff und Teilbegriff zu finden; dabei beziehen sich die Termini Oberbegriff und Unterbegriff auf Abstraktionsbeziehungen und die Termini Verbandsbegriff und Teilbegriff auf Teil/Ganzes-Beziehungen, sodass das terminologische System an dieser Stelle nicht zwei-, sondern dreidimensional ausfällt. 


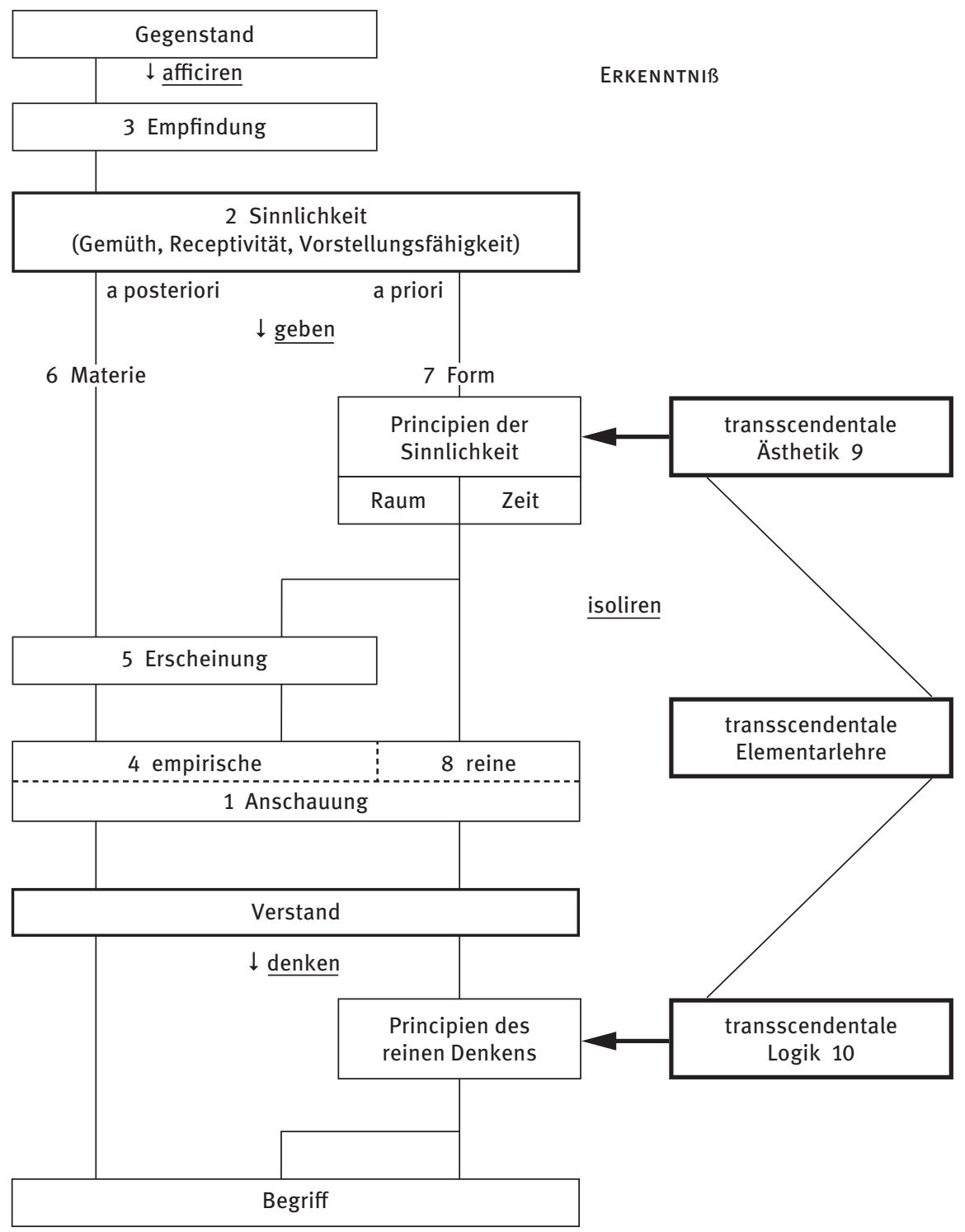

Abb. 7.3: Linearisierung des terminologischen Systems nach Kant (1787: 24-27); vgl. Roelcke (2013a: 31). 


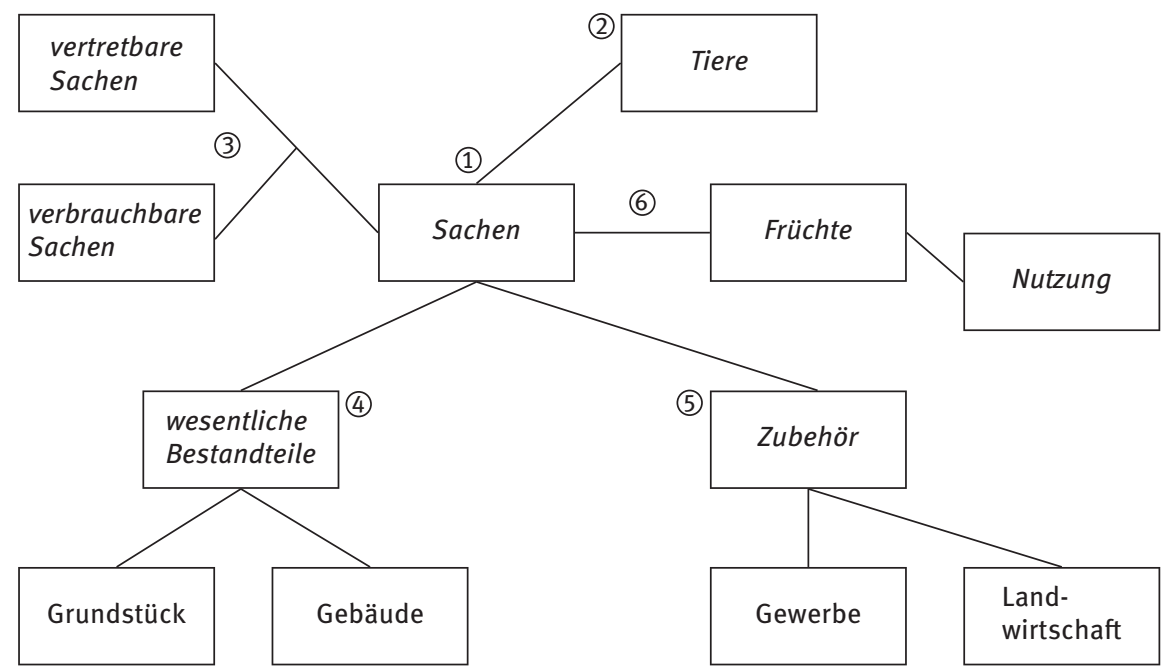

Abb. 7.4: Linearisierung des terminologischen Systems in §§ 90-103 BGB (vgl. Roelcke 2013b: 154).

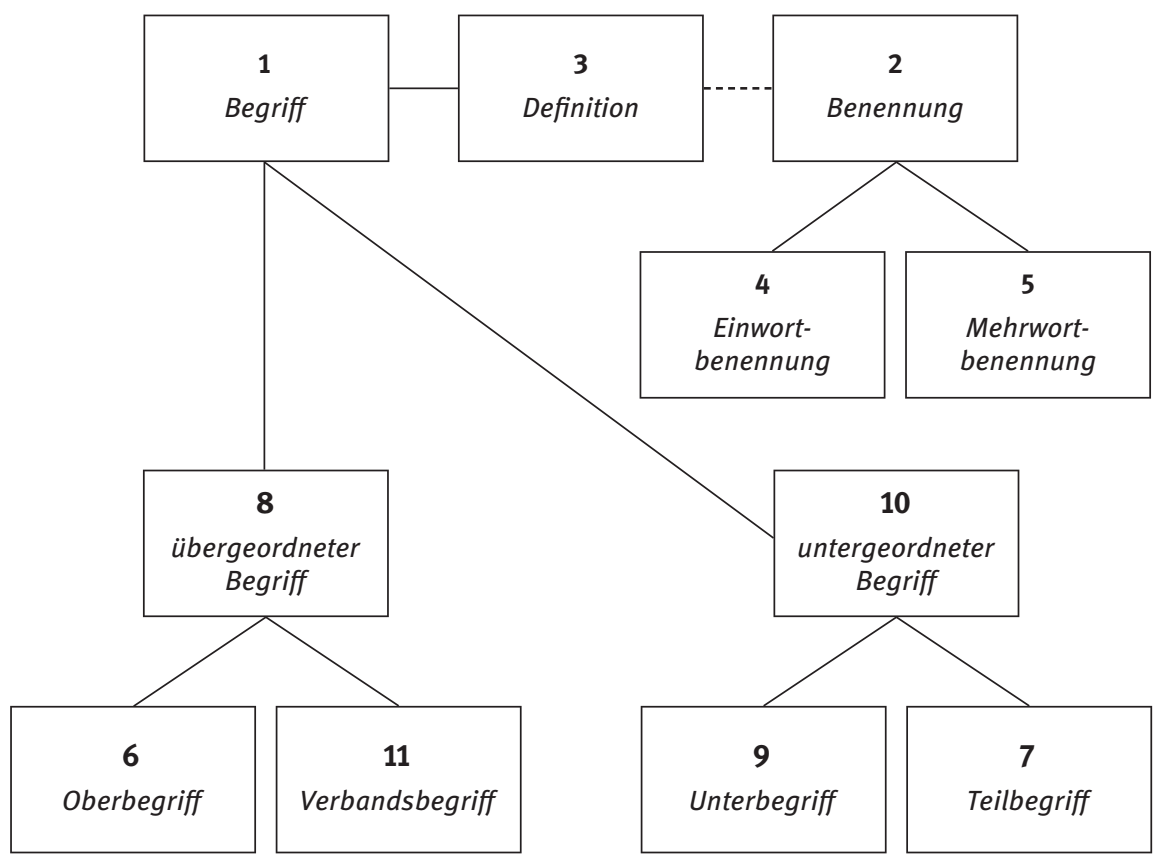

Abb. 7.5: Linearisierung des terminologischen Systems in Abschnitt 2 von DIN 2330 (vgl. Roelcke 2013a: 23). 
Das terminologische System nach BGB weicht demgegenüber deutlich von einer solchen hierarchischen Struktur ab (Abb. 7.4; vgl. Roelcke 2013b: 152), indem es sternförmig um einen zentralen Terminus herum organisiert erscheint: Im Zentrum steht der Terminus Sachen; um diesen herum erscheinen die Mehrworttermini vertretbare Sachen, verbrauchbare Sachen und wesentliche Bestandteile sowie die Einworttermini Tiere und Zubehör; der Terminus Früchte hängt diesem Verbund, der mit Sachen sowie vertretbare Sachen und verbrauchbare Sachen partiell durchaus auch hierarchische Beziehungen umfasst, semantisch nur lose an.

Der Ausschnitt aus dem terminologischen System in Kants Kritik der reinen Vernunft schließlich zeigt eine komplexe Struktur (Abb. 7.3; Roelcke 2013a: 28): Es umfasst Bezeichnungen für verschiedene epistemologische Vermögen wie Sinnlichkeit und Verstand, für deren Erkenntnisprinzipien wie Raum und Zeit oder Principien des reinen Denkens, für Erkenntnisse selbst wie Anschauung oder Begriff sowie auf eine Metaebene Bezeichnungen für erkenntnistheoretische Teildisziplinen, wie etwa transscendentale Ästhetik und transscendentale Logik. Um einige hierarchische Abstraktionsbeziehungen (beispielsweise transscendentale Elementarlehre gegenüber transscendentale Ästhetik und transscendentale Logik) und Teil/Ganzes-Beziehungen (wie Principien der Sinnlichkeit mit Raum und Zeit) herum spiegelt dieses System insbesondere einen Prozess wider, die Genese von Erkenntnis anhand von Anschauungen und Begriffen.

\section{Das pragmalinguistische Kontextmodell}

Eine solch statische Betrachtungsweise der Konstitution terminologischer Systeme greift indessen zu kurz. Empirische Untersuchungen - insbesondere auch zu den Fachsprachen des Deutschen - zeigen, dass Termini in der Regel keine exakte, eindeutige und „eigentliche“ Bedeutung aufweisen, sondern oftmals vage, mehrdeutig und übertragen verwendet werden (vgl. etwa Roelcke 1991, 2010: 68-76). Beispiele hierfür sind insbesondere interfachliche Termini wie Analyse oder System für Vagheit, Freiheit oder Demokratie (aus Politik und Politikwissenschaft) für Mehrdeutigkeit sowie Wurzel (neben Biologie aus Mathematik, Sprachwissenschaft oder Zahnheilkunde) oder Schlag (neben Alltagssprache und Physik in der Elektrotechnik) für fachsprachliche Metaphorik. Darüber hinaus erweist sich eine strukturelle Analyse terminologischer Systeme angesichts deren kommunikativer Verankerung innerhalb von Fachtexten als nicht hinreichend. Vor diesem Hintergrund erscheint eine Konzeption fachsprachlicher Lexik als indiziert, die nicht (alleine) an der unmissverständlichen Definition einzelner Termini und an der Struktur ganzer terminologischer Sys- 


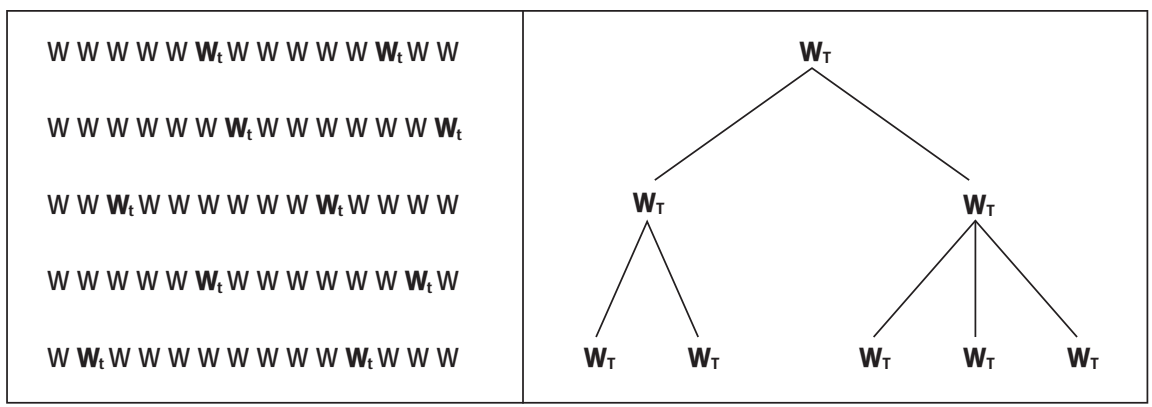

Abb. 7.6: Termini in einem linearen fachlichen Text und in einer hierarchischen terminologischen Struktur; $W=$ Wort, $W_{t}=$ Terminustoken, $W_{T}=$ Terminustype (Roelcke 2013a: 2 ).

teme ansetzt, sondern aus einer pragmalinguistischen Perspektive (auch) die Gestaltung fachsprachlicher Texte in Betracht zieht (eine Übersicht der Ansätze gewähren hier insbesondere Baumann \& Kalverkämper (2004) und Kalverkämper (2016)). Dabei spielen zum einen die lineare Anlage sprachlicher Texte sowie der Kontext, der durch diese geschaffen wird, eine entscheidende Rolle.

Eine solche pragmalinguistische Konzeption lässt nun Vagheit, Mehrdeutigkeit und Übertragung nicht weiter als Eigenschaften von Termini erscheinen, die deren Missverständlichkeit bedingen. Durch den sprachlichen Kontext von Fachtexten werden vielmehr kommunikative Indikatoren gesetzt, die zur Auflösung von Vagheit, Mehrdeutigkeit und Übertragung und somit zu deren Verständlichkeit beitragen. Angesichts dieser Abhängigkeit vom Kontext zeichnen sich Termini aus Sicht eines pragmalinguistischen Kontextmodells durch eine relative Exaktheit und eine relative Eindeutigkeit sowie eine relative Eigentlichkeit aus. Vagheit, Mehrdeutigkeit und Übertragung erweisen sich somit als (beherrschbare) Regel und nicht als (missverständliche) Ausnahme von Terminologie (vgl. auch Roelcke 1995, 2004), sodass eine Abgrenzung Fach- und Allgemeinsprache, wie sie insbesondere in den 1970er Jahren wiederholt versucht wurde (vgl. zum Beispiel Mentrup 1979), kaum mehr sinnvoll erscheint.

Ein weiterer Aspekt der pragmalinguistischen Betrachtungsweise besteht in der Konstitution von Terminologien: Terminologisierung in einem weiteren Sinne erschöpft sich hiernach nicht in der Definition einzelner Termini (vgl. Abb. 7.1), sondern umfasst die Einführung von komplexen terminologischen Systemen in sprachlichen Texten, die in der Regel linear aufgebaut sind (vgl. Abb. 7.6): In fachsprachlichen Texten erscheinen Termini als Token $\left(W_{t}\right)$ in einer eindimensionalen Reihe von Worttoken, während sie in (zwei- oder mehrdimensionalen) fachsprachlichen Systemen als Types $\left(\mathrm{W}_{\mathrm{T}}\right)$ erscheinen (der Beitrag von Abbildungen zur Terminologisierung wird im Folgenden aus- 
geblendet; vgl. hierzu etwa Roelcke (2012 und 2013c). Vor diesem Hintergrund stellt sich also die Frage, wie Terminustypes fachsprachlicher Systeme als Terminustoken in fachsprachlichen Texten konstituiert werden.

Die linguistische Analyse von Terminologisierung kann eine ganze Reihe qualitativ unterschiedlicher Erscheinungen erfassen - insbesondere die Reihenfolge der Einführung einzelner Termini im Text (Linearisierung), die Verwendung verschiedener Definitionsarten oder die implizite und explizite Vernetzung der Termini untereinander. Aus einer quantitativen Betrachtungsweise sind hier darüber hinaus etwa die Anzahl von Termini in Relation zum Gesamtwortschatz des Textes (terminologische Dichte) und die Anzahl der Definition relativ zur Gesamtzahl der Sätze (definitorische Dichte) von Interesse.

Die vier oben beschriebenen terminologischen Systeme (vgl. Abb. 7.2-7.5) zeigen jeweils eine eigene Linearisierung (vgl. jeweils die Ziffern in den Strukturdarstellungen, welche die Reihenfolge anzeigen, in der die Termini im entsprechenden Text eingeführt werden). Bei Gueintz (1641; vgl. Abb. 7.2) werden Termini mit abstrakter vor solchen mit konkreter Bedeutung (von oben nach unten) eingeführt, wobei die Verzweigungen des Systems jeweils einzeln (von links nach rechts) komplettiert werden - also eigenschaften und theilung nach Wortforschung sowie dann art und gestalt wiederum nach eigenschaften. Im Gegensatz zu Gueintz' struktureller Linearisierung erfolgt diejenige bei Kant (1787) inhaltlich (vgl. Abb. 7.3): Zunächst werden Termini eingeführt, die verschiedene Arten (etwa Anschauung, Sinnlichkeit oder Empfindung), und daraufhin solche, die diverse Komponenten menschlichen Erkennens (wie Materie und Form) bezeichnen; zuletzt folgen Termini, die sich auf einzelne Disziplinen der Transzendentalphilosophie beziehen (also transscendentale Ästhetik oder transscendentale Logik). Bei der Linearisierung im BGB (vgl. Abb. 7.4) wird wiederum strukturbezogen vorgegangen: Zunächst wird das Zentrum des sternförmigen Systems eingeführt (Sachen), und im Anschluss hieran folgen nach und nach die einzelnen Randbereiche (Tiere, vertretbare Sachen und verbrauchbare Sachen, wesentliche Bestandteile usw.). In der terminologischen Grundsatznorm der DIN 2330 (vgl. Abb. 7.5) schließlich scheinen die Termini auf den ersten Blick vom Abstrakten zum Konkreten eingeführt zu werden; tatsächlich aber erscheinen diese in alphabetischer Reihenfolge im Text: von Begriff und Benennung bis untergeordneter Begriff und Verbandsbegriff).

Ein weiterer wichtiger Aspekt der Konstitution terminologischer Systeme in Fachsprachen besteht in der Verteilung einzelner Definitionsarten. Im Falle von Gueintz (vgl. Roelcke 2016; 54) lässt sich zunächst feststellen, dass hier Termini auf nahezu sämtlichen Ebenen des terminologischen Systems aristotelisch definiert werden. Allein auf der untersten Ebene sind explikative und dabei recht alltagsnahe Definitionen zu finden; exemplarische Definitionen er- 
scheinen lediglich örtlich. Obwohl das terminologische System bei Kant (Roelcke 2013a: 30-33) nicht hierarchisch angelegt ist, werden auch hier nahe$\mathrm{zu}$ ausschließlich aristotelische Definitionen eingesetzt. Bemerkenswert ist dabei die Verteilung von Real- und Nominaldefinitionen (die sich etwa mit „,x ist y“ auf Wirkliches bzw. mit „x heißt y“ auf Sprachliches beziehen): Realdefinitionen werden hier ausschließlich zur Bestimmung von Termini eingesetzt, über deren Bedeutung zu seiner Zeit Einvernehmen bestanden haben mochte; Nominaldefinitionen finden sich darüber hinaus auch bei solchen Termini, die als spezifisch für Kants Transzendentalphilosophie gelten dürfen. Auch im BGB (Roelcke 2013b: 154) tritt ausschließlich der aristotelische Definitionstyp in Erscheinung. Hier ist indessen die Anzahl von sog. Legaldefinitionen von Interesse: Nur knapp die Hälfte der Definitionen erfolgen ausdrücklich „im Sinne des Gesetzes“, während auch die übrigen durchaus rechtsspezifische Festlegungen beinhalten, selbst wenn diese nicht expressis verbis als solche gekennzeichnet werden. In der DIN-Norm 2330 (Roelcke 2013a: 23) findet sich ein analoger Befund: Hier herrschen wiederum aristotelische Definitionen vor; operationale Bestandteile sind lediglich in den differentiae specificae anzutreffen, deren unterste Ebene unspezifiziert bleibt. - Angesichts der Tatsache, dass in allen vier Texten aristotelische Definitionen vorherrschen, ist die explizite terminologische Vernetzung hier als verhältnismäßig stark anzusehen.

Neben den qualitativen Erscheinungen der Linearisierung des terminologischen Systems und der Verteilung einzelner Definitionsarten sind mit der terminologischen und der definitorischen Dichte im Weiteren auch einige quantitative Gesichtspunkte hinsichtlich der Konstitution terminologischer Systeme von Interesse (vgl. Roelcke 2013a: 205-207, 211-213). Hier bringt ein direkter Vergleich der Textausschnitte aus Kant und DIN bemerkenswerte Befunde: So beläuft sich die relative Häufigkeit von Termini (Terminus-Tokens gemessen an der Gesamtzahl der Wort-Tokens) im DIN-Text auf $12 \%$, diejenige im KantText dagegen lediglich auf 9\%; damit ist die terminologische Dichte im DINText höher als im Kant-Text. Im Weiteren machen $44 \%$ der Sätze in DIN Definitionen aus, während es im Falle von Kant lediglich $38 \%$ sind; angesichts dieses Befundes ist die Definitionsdichte im DIN-Text höher als im Kant-Text. Und schließlich weist die mittlere Zahl an Termini in den Definitionen aus DIN den Wert 2 auf, während diejenige bei Kant beim Wert 2,8 liegt und dabei zudem eine weitaus höhere Varianz zeigt; damit erweisen sich die Definitionen bei Kant gegenüber DIN als terminologisch komplexer. Im Vergleich zeigt also Kant eine relativ niedrige und DIN eine relativ hohe terminologische wie definitorische Dichte - mit Ausnahme der mittleren Zahl an Termini in Definitionen, welche bei Kant diejenige in DIN übersteigt. 


\section{Das kognitionslinguistische Funktionsmodell}

Während die Konstitution terminologischer Systeme aus system- bzw. pragmalinguistischer Perspektive mit dem Postulat diverser Güte bzw. der Analyse tatsächlicher Eigenschaften einzelner Termini und ganzer Terminologien in Verbindung gebracht wird, stellt sich die kognitive Linguistik im Wesentlichen die Aufgabe, diese zu interpretieren bzw. deren Funktion zu erklären. Dabei spielen Fachlichkeit als spezialisierte menschliche Tätigkeit sowie diverse Textfunktionen, die sich aus dieser ableiten lassen, im Vordergrund (vgl. auch Roelcke 2001).

Vagheit bzw. relative Exaktheit von Termini sind hiernach nicht (allein) durch Definitionen gegeben und anhand kontextueller Indikatoren (durchaus) beherrschbar, sondern stellen spezifische sprachliche Mittel dar, mit denen die Kommunikation innerhalb solcher spezialisierten Tätigkeitsbereiche in besonderer Weise bewältigt werden kann. Die Relativität der terminologischen Exaktheit besteht dabei in einer adäquaten Festlegung der Bedeutung, die für den Zweck einerseits notwendig und andererseits hinreichend erscheint, um den kommunikativen Aufwand - gemessen an einem bestimmten kommunikativen Ziel - optimal bzw. effizient zu halten (vgl. Roelcke 2002). Eine zu geringe Exaktheit wäre hiernach ineffektiv, eine zu hohe zwar effektiv, nicht aber effizient.

Entsprechend verhält es sich mit der Ein- bzw. Mehrdeutigkeit von Termini: Polysemie und Synonymie von Termini erfüllen ebenfalls wichtige Funktionen innerhalb der Kommunikation spezialisierter menschlicher Tätigkeitsbereiche: Zum einen führen sie zu einer Erweiterung des fachlexikalischen Inventars und erlauben somit überhaupt erst dessen Spezialisierung (weitere Verfahren sind hier Wortbildung und Entlehnung); zum anderen ermöglichen sie eine Differenzierung von Bedeutungen, anhand derer fachspezifische Aspekte festgemacht werden können. Auch hier gilt, dass der kommunikative Aufwand und das kommunikative Ergebnis in einem optimalen Verhältnis zueinander zu stehen haben.

Diese Bedingung effizienter Kommunikation gilt im Weiteren auch für die fachsprachliche Übertragung lexikalischer Ausdrücke, die neben deren Eigentlichkeit wiederum nicht als missverständlich, sondern als förderlich für fachliche Kommunikation anzusehen ist. Sie dient ebenfalls der terminologischen Inventarerweiterung innerhalb fachlicher Bereiche und sichert dabei durch Äquivalenz deren Anschließbarkeit an bereits bestehende lexikalische Einheiten - seien diese nun allgemein- oder fachsprachlicher Provenienz. Damit erweist sich die Übertragung von lexikalischen Bedeutungen innerhalb von Fachsprachen nicht allein als unmissverständlich, sondern darüber hinaus sogar als förderlich für eine fachliche Kommunikation, die Bestehendes (speziali- 
sierend) weiterentwickelt (Der Unterschied zur Allgemeinsprache ist hiernach also nur graduell, indem die Metaphorik im Rahmen spezialisierter Tätigkeitsbereiche eingesetzt wird).

Die terminologischen Güte bzw. tatsächlichen Eigenschaften Vagheit, Mehrdeutigkeit und Übertragung im Sinne relativer Exaktheit, Eindeutigkeit und Eigentlichkeit erscheinen somit also nicht allein aus Sicht einer pragmatischen Fachsprachenkonzeption beherrschbar, sondern darüber hinaus auch aus der Sicht einer kognitiven Fachsprachenlinguistik unabdingbar, soll die Kommunikation innerhalb spezialisierter menschlicher Tätigkeitsbereiche doch an bestehende Bereiche anknüpfen und diese weiterentwickeln. Eine solche Erklärung vor dem Hintergrund kognitiver Erfordernisse ist nun auch im Hinblick auf die diversen Erscheinungen der Terminologisierung möglich. Dabei ist von verschiedenen Grundfunktionen der Fachtexte auszugehen.

Der didaktische Text von Gueintz (1641) kann im Sinne der Typologie fachsprachlicher Texte nach Roelcke (2014b) als konfirmierend und regulierend charakterisiert werden, da er bestehendes Wissen seiner Zeit mit geringer Verbindlichkeit zusammenfasst. Dies schlägt sich nicht allein in einer streng hierarchischen Anlage des terminologischen Systems nieder, sondern darüber hinaus auch in dessen struktureller Linearisierung von oben nach unten und in der umfassenden Verwendung aristotelischer Definitionen, die auf der untersten Ebene explikativ ergänzt werden. Eine solch systematische und dabei deduktive Vorgehensweise unterstützt eine übersichtliche Exteriorisierung und Interiorisierung von Wissen.

Der philosophische Text von Kant ist im Gegensatz zu demjenigen von Gueintz nicht als konfirmierend, sondern als aktualisierend und regulierend anzusehen, da er nicht gesichertes Wissen didaktisch aufbereitet, sondern neue Erkenntnisse entwickelt. Das komplexe terminologische System wird dabei nicht nach strukturellen, sondern nach inhaltlichen Gesichtspunkten linearisiert; Die aristotelischen Definitionen erscheinen in Bezug auf Bekanntes als Real-, in Bezug auf Unbekanntes als Nominaldefinitionen. Auf diese Weise folgt die Konstitution des terminologischen Systems der Argumentationslinie des Autors, der seine neuen Erkenntnisse unter Anknüpfung an bestehende Wissensbestände nach und nach exteriorisiert und zur Interiorisierung bereitstellt. Dieser eher diskursiven Anlage entspricht eine (im Vergleich mit dem DIN-Text) relativ niedrige Dichte an Termini und Definitionen.

Das BGB (2002/2011) ist als ein konfirmierend sanktionierender Text aufzufassen, der mit hoher Verbindlichkeit bestehende Rechtsnormen zusammenfasst und für die Rechtsprechung zur Verfügung stellt. Das zentriert bzw. sternförmig angelegte terminologische System wird daher (hierin demjenigen von Gueintz vergleichbar) systematisch linearisiert und zugänglich gemacht, wobei 
einige der aristotelischen Definitionen als Legaldefinitionen erscheinen und somit expressis verbis als juristisch ausgewiesen werden.

Der DIN-Text ist im Gegensatz zu demjenigen aus dem BGB weniger als konfirmierend, sondern eher als aktualisierend und sanktionierend aufzufassen, da er neu und eigens entwickelte Grundsatznormen zur Veröffentlichung bringt, dabei jedoch nicht entwickelt, sondern zur Übersicht und zum Nachschlagen zusammenstellt. Um in diesem Zusammenhang eine rasche und punktuelle Interiorisierung von Wissen zu ermöglichen, sind sein terminologisches System hierarchisch und seine Linearisierung unter Verwendung aristotelischer Definitionen alphabetisch angelegt. Der im Grundsatz normativen Anlage entsprechend fällt die Dichte an Termini und Definitionen relativ (im Vergleich zum Text von Kant) hoch aus.

\section{Abschließende Bemerkungen}

Die Konstitution terminologischer Systeme in Fachsprachen zeigt sich zum einen in Merkmalen einzelner Termini und zum anderen in solchen ganzer Terminologien. Deren wissenschaftliche Betrachtung folgt dabei entweder der Konzeption eines systemlinguistischen Inventarmodells, eines pragmalinguistischen Kontextmodells oder eines kognitionslinguistischen Funktionsmodells. Dabei kommen diese drei Betrachtungsweisen zu ganz unterschiedlichen Ergebnissen und Einschätzungen.

Die Verwendung von Termini, die durch Definition oder Konvention festgelegt sind, dient der Unmissverständlichkeit der Kommunikation in spezialisierten menschlichen Tätigkeitsbereichen (vgl. Tab. 7.1). Mit Blick auf die Wirklichkeit ist dabei von einer referentiellen Unmissverständlichkeit $\mathrm{zu}$ sprechen, hinsichtlich der Bedeutung und des Ausdrucks von einer semantischen und einer motivierten Unmissverständlichkeit. Aus systemlinguistischer Perspektive schlägt sich dies in einem Postulat der Exaktheit, der Eindeutigkeit und der Eigentlichkeit von Termini nieder, durch welches deren Unmissverständlichkeit vor dem tatsächlichen Gebrauch innerhalb von Fachtexten sichergestellt werden soll. Die linguistische Analyse im Rahmen eines pragmalinguistischen Modells zeigt indessen, dass Termini oft durch Vagheit, Mehrdeutigkeit und Übertragung gekennzeichnet sind, die jedoch durch kontextuelle Indikatoren kommunikativ beherrschbar sind und somit nicht als missverständlich zu gelten haben. Erst ein kognitionslinguistischer Ansatz erlaubt es schließlich, diese Eigenschaften funktional zu erklären, indem relative Exaktheit als referentielle Adäquatheit, relative Mehrdeutigkeit als semantische Differenzierung und Übertragung als motivierte Äquivalenz interpretiert werden. 
Tab. 7.1: Terminologische Eigenschaften im Überblick.

\begin{tabular}{lllll}
\hline $\begin{array}{l}\text { Typ der Unmiss- } \\
\text { verständlichkeit }\end{array}$ & $\begin{array}{l}\text { Bezug auf } \\
\text { semiotische } \\
\text { Größe }\end{array}$ & $\begin{array}{l}\text { System- } \\
\text { linguistische } \\
\text { Perspektive }\end{array}$ & $\begin{array}{l}\text { Pragma- } \\
\text { linguistische } \\
\text { Perspektive }\end{array}$ & $\begin{array}{l}\text { Perspektive } \\
\text { der kognitiven } \\
\text { Linguistik }\end{array}$ \\
\hline Referenz & Wirklichkeit & Exaktheit & Vagheit & Adäquatheit \\
\hline Semantik & Bedeutung & Eindeutigkeit & Mehrdeutigkeit & Differenzierung \\
\hline Motivation & Ausdruck & Eigentlichkeit & Übertragung & Äquivalenz \\
\hline & $\leftrightarrow$ Definition & $\rightarrow$ Postulat & $\rightarrow$ Analyse & $\rightarrow$ Interpretation \\
& und Konvention & & & \\
\hline
\end{tabular}

Neben den Merkmalen einzelner Termini lassen sich auch solche ganzer Terminologien unter den drei Konzeptionen fassen. So führt eine systemlinguistische Betrachtung hier zunächst zu dem Ergebnis, dass die Terminologie nach Gueintz und in DIN hierarchisch strukturiert ist, während Kants Terminologie komplex und diejenige des BGB zentriert erscheint. Aus pragmalinguistischer Perspektive erweisen sich die Linearisierungen der Systeme von Gueintz und im BGB als strukturell, diejenigen von Kant und in DIN als inhaltlich bzw. alphabetisch. In allen vier Texten herrschen aristotelische Definitionen vor, wobei in Kants Text eine gezielte Verwendung von Nominal- und Realdefinitionen und im BGB der Einsatz von Legaldefinitionen zu beobachten ist.

Diese systematischen und pragmatischen Befunde spiegeln sich aus Sicht der kognitiven Linguistik in den grundsätzlichen Funktionen der Texte wider (vgl. Tab. 7.2): So dienen die hierarchische Anlage und die strukturelle Linearisierung der konfirmierend regulierenden Grundfunktion des didaktischen Textes von Gueintz sowie das komplexe System und die inhaltliche Linearisierung einschließlich der Unterscheidung von Nominal- und Realdefinitionen dem aktualisierend regulierenden Charakter des diskursiven Textes von Kant (der zudem eine entsprechend niedrige Dichte an Termini und Definitionen zeigt). Die beiden sanktionierenden Texte bieten das folgende Bild: Während der eher konfirmierende Text des BGB das zentrierte terminologische System strukturell linearisiert und dabei ausdrückliche Legaldefinitionen verwendet, findet sich in dem eher aktualisierenden Text des DIN eine alphabetische Linearisierung des hierarisch angelegten Systems (wobei eine verhältnismäßig hohe Dichte an Termini und Definitionen festzustellen ist).

Angesichts dieser Postulate, Analysen und Interpretationen wird zum einen deutlich, dass die drei verschiedenen fachsprachenlinguistischen Konzeptionen einen jeweils eigenen Blickwinkel auf die Konstitution terminologischer Systeme in Fachsprachen haben. Zum anderen zeigt sich, dass eine kognitive 
Tab. 7.2: Terminologisierung im Überblick.

\begin{tabular}{|c|c|c|c|c|c|}
\hline & System & Linearisierung & Definitionen & Dichte & Textfunktion \\
\hline $\begin{array}{l}\text { Gueintz } \\
\text { (1641) }\end{array}$ & hierarchisch & strukturell & (explikativ) & $\begin{array}{l}\text { [nicht } \\
\text { ermittelt] }\end{array}$ & $\begin{array}{l}\text { konfirmierend } \\
\text { regulierend }\end{array}$ \\
\hline Kant (1787) & komplex & inhaltlich & $\begin{array}{l}\text { nominal \& } \\
\text { real }\end{array}$ & $\begin{array}{l}\text { relativ } \\
\text { niedrig }\end{array}$ & $\begin{array}{l}\text { aktualisierend } \\
\text { regulierend }\end{array}$ \\
\hline $\begin{array}{l}\text { BGB (2002/ } \\
2011)\end{array}$ & zentriert & strukturell & legal & $\begin{array}{l}\text { [nicht } \\
\text { ermittelt] }\end{array}$ & $\begin{array}{l}\text { konfirmierend } \\
\text { sanktionierend }\end{array}$ \\
\hline DIN (1993) & hierarchisch & alphabetisch & (explikativ) & relativ hoch & $\begin{array}{l}\text { aktualisierend } \\
\text { sanktionierend }\end{array}$ \\
\hline
\end{tabular}

Interpretation hilft, pragmatische Befunde zu erklären, die systematischen Postulaten in der Regel entgegenstehen. Eine solche kognitive und funktionale Perspektive sollte daher die weitere Diskussion um Termini und Terminologie bestimmen - sowohl im Hinblick auf die moderne Terminologielehre, die bis heute weitgehend einem systemlinguistischen Inventarmodell verhaftet geblieben ist, als auch hinsichtlich einer Angewandten Sprachwissenschaft, die sich als Disziplin zur Lösung kommunikativer Probleme (vgl. Knapp \& Antos 2011) versteht.

\section{Literatur}

\section{Quellen}

BGB (2002/2011): Bürgerliches Gesetzbuch in der Fassung der Bekanntmachung vom 2. Januar 2002 (BGBI. I S. 42, 2909; 2003 I S. 738), das zuletzt durch Artikel 1 des Gesetzes vom 27. Juli 2011 (BGBI. I S. 1600) geändert worden ist.

Gueintz, Christian (1641): Deutscher Sprachlehre Entwurf. Köthen [Nachdruck - Hildesheim, New York: Olms 1978 (Documenta Linguistica)].

Kant, Immanuel: Kritik der reinen Vernunft. 2. Aufl. 1787. Berlin: de Gruyter 1968 (Kants Werke. Akademie-Textausgabe. Band III).

DIN 2330: Begriffe und Benennungen; Allgemeine Grundsätze. Berlin, Wien, Zürich: Beuth 1993. Auch in: Gottfried Herzog \& Holger Mühlbauer: Normen für Übersetzer und technische Autoren. 2. überarbeitete und erweiterte Aufl., hrsg. von: DIN Deutsches Institut für Normung e. V. Berlin, Wien, Zürich: Beuth (2007), 105-116. 


\section{Forschungsliteratur}

Arntz, Reiner. Heribert Picht \& Klaus-Dirk Schmitz (2014): Einführung in die Terminologiearbeit. 7., vollständig überarbeitete und aktualisierte Aufl. (= Studien zu Sprache und Technik, Bd. 2). Hildesheim, Zürich, New York: Olms.

Baßler, Harald (2002): Definierte Wörter. Fachsprachliche Terminologie. In Jürgen Dittmann \& Claudia Schmitt (Hrsg.) (2002), 211-231.

Baumann, Klaus-Dieter \& Hartwig Kalverkämper (Hrsg.) (2004): Pluralität in der Fachsprachenforschung. (= Forum für Fachsprachen-Forschung 67). Tübingen: Narr. Candlin, Christopher N. \& Srikant Sarangi (Hrsg.) (2011): Handbook of communication in organisations and professions. (= Handbooks of Applied Linguistics 3). Berlin, Boston: de Gruyter.

Dittmann, Jürgen \& Claudia Schmitt (Hrsg.) (2002): Über Wörter - Grundkurs Linguistik. Freiburg (Rombach Grundkurs 5).

Felber, Helmut \& Gerhard Budin (1989): Terminologie in Theorie und Praxis. (= Forum für Fachsprachen-Forschung 9). Tübingen: Narr.

Fraas, Claudia (1998): Lexikalisch-semantische Eigenschaften von Fachsprachen. In Lothar Hoffmann, Hartwig Kalverkämper \& Herbert Ernst Wiegand (Hrsg.) (1998), 428-438.

Gerzymisch-Arbogast, Heidrun (1996): Termini im Kontext. Verfahren zur Erschließung und Übersetzung der textspezifischen Bedeutung von fachlichen Ausdrücken. (= Forum für Fachsprachen-Forschung 31). Tübingen: Narr.

Haß, Ulrike \& Petra Storjohann (Hrsg.) (2015): Handbuch Wort und Wortschatz.

(= Handbücher Sprachwissen 3). Berlin, Boston: de Gruyter.

Hoffmann, Lothar, Hartwig Kalverkämper \& Herbert Ernst Wiegand (Hrsg.) (1998-1999): Fachsprachen. Languages for Special Purposes. Ein internationales Handbuch zur Fachsprachenforschung und Terminologiewissenschaft. An International Handbook of Special-Language and Terminology Research. 2 Bde. (= Handbücher zur Sprach- und Kommunikationswissenschaft 14.1 und 14.2). Berlin, New York: de Gruyter.

ISO 704: Terminology work - Principles and methods. Geneva: International Organization for Standardization (2000).

Kageura, Kyo \& Marie-Claude L'Homme (2008): Reflecting fifteen years of research and development in terminology. Terminology 14, 153-157.

Kalverkämper, Hartwig (2016): Fachkommunikation im Fokus - Paradigmen, Positionen, Perspektiven. Klaus-Dieter Baumann zum 60. Geburtstag gewidmet. (= Forum für Fachsprachen-Forschung 100). Berlin: Frank \& Timme.

Knapp, Karlfried \& Gerd Antos (2011): Introduction to the handbook series. Linguistics for problem solving. In Christopher Candlin \& Srikant Sarangi (Hrsg.) (2011), v-xv.

Kretzenbacher, Heinz-Leonhard (1994): 'Just Give Us the Facts!' The Connection between the Narrative Taboo, the Ego Taboo and the Metaphor Taboo in Scientific Style. Lingua e Stile 29, 115-130.

Mentrup, Wolfgang (Hrsg.) (1979): Fachsprachen und Gemeinsprache. Jahrbuch 1978 des Instituts für deutsche Sprache. (=Sprache der Gegenwart, 46). Düsseldorf: Schwann.

Roelcke, Thorsten (1991): Das Eineindeutigkeitspostulat der lexikalischen Fachsprachensemantik. Zeitschrift für germanistische Linguistik 19, 194-208.

Roelcke, Thorsten (1995): Fachwortkonzeption und Fachwortgebrauch. Hintergründe einer Diskrepanz zwischen Sprachwissenschaft und Sprachwirklichkeit. Zeitschrift für deutsche Philologie 114, 394-409. 
Roelcke, Thorsten (2001): Was bringt die Kognitive Semantik dem fachlexikalischen Wissenstransfer? In Sigurd Wichter \& Gerd Antos, in Zusammenarbeit mit Daniela Schütte \& Oliver Stenschke (Hrsg.), Wissenstransfer zwischen Experten und Laien. Umrisse einer Transferwissenschaft. (= Transferwissenschaften 1), 51-63. Frankfurt a. M.: Peter Lang.

Roelcke, Thorsten (2002): Kommunikative Effizienz. Eine Modellskizze. (= Sprache - Literatur und Geschichte 23). Heidelberg: Winter.

Roelcke, Thorsten (2004): Stabilität statt Flexibilität? Kritische Anmerkungen zu den semantischen Grundlagen der modernen Terminologielehre. In Inge Pohl \& Klaus-Peter Konerding (Hrsg.), Stabilität und Flexibilität in der Semantik. Strukturelle, kognitive, pragmatische und historische Perspektiven. (= Sprache - System und Tätigkeit 52), 137-150. Frankfurt a. M.: Peter Lang.

Roelcke, Thorsten ( $\left.{ }^{3} 2010\right)$ : Fachsprachen. 3., neu bearb. Aufl. (= Grundlagen der Germanistik 37). Berlin: Schmidt.

Roelcke, Thorsten (2012): Der Beitrag von Text und Bild zur Konstituierung von Fachwortschatz. Eine exemplarische Studie zur terminologischen Grundsatznorm DIN 2330. In Ingelore Oomen-Welke \& Michael Staiger (Hrsg.), Bilder in Medien, Kunst, Literatur, Sprache, Didaktik. Festschrift für Adalbert Wichert, 231-247. Freiburg: Fillibach.

Roelcke, Thorsten (2013a): Definitionen und Termini. Quantitative Studien zur Konstituierung von Fachwortschatz. (= Linguistik - Impulse und Tendenzen 48). Berlin, Boston: de Gruyter.

Roelcke, Thorsten (2013b): Definitionen und Linearisierung in §§ 90-103 BGB. In Marina Wagnerová \& Gerald G. Sander (Hrsg.), Die Rechtssprache in der internationalen Diskussion. (= Schriften zu Mittel- und Osteuropa in der Europäischen Integration 13), 143-162. Hamburg: Kovač.

Roelcke, Thorsten (2013c): Der Beitrag von graphischen Abbildungen zur Konstituierung von Fachwortschatz in der terminologischen Grundsatznorm DIN 2330 des Deutschen Instituts für Normung. Glottotheory 4, 5-35.

Roelcke, Thorsten (2014a): Three ways of terminological linearisation. Glottotheory 5, 85-99.

Roelcke, Thorsten (2014b): Zur Gliederung von Fachsprache und Fachkommunikation. Fachsprache - International Journal of Specialized Communication 37, 154-178.

Roelcke, Thorsten (2015): Besondere Wörter II: Fachwörter, Termini. In Ulrike Haß \& Petra Storjohann (Hrsg.) (2015), 371-393.

Roelcke, Thorsten (2016): Terminologisierung in Gueintz' „Deutscher Sprachlehre Entwurf“ (1641). In Jörg Meier \& Albrecht Greule (Hrsg.), Von der Arznei bis zum Ziegeldach. Historische Fach- und Handwerkersprachen. Deidesheimer Gespräche zur Sprach- und Kulturgeschichte IV, 47-64. Berlin: Weidler.

Weinrich, Harald (1989): Formen der Wissenschaftssprache. Jahrbuch 1988 der Akademie der Wissenschaften zu Berlin, 119-158. Berlin, New York: de Gruyter.

Wüster, Eugen (1931/1970): Internationale Sprachnormung in der Technik, besonders in der Elektrotechnik. (Die nationale Sprachnormung und ihre Verallgemeinerung). [3., abermals ergänzte Aufl. Bonn: Bouvier 1970 (= Sprachforum. Beiheft 2)]. 


\title{
Birte Kellermeier-Rehbein \\ 8 Plurizentrische Wortschatzvariation des Deutschen innerhalb und außerhalb Europas
}

\begin{abstract}
Aufgrund ihrer plurizentrischen Struktur weist die deutsche Sprache Standardvariation auf. Dies gilt insbesondere für die Lexik in den europäischen Staaten und Regionen, in denen Deutsch Amtssprache ist. Der vorliegende Beitrag zeigt, dass auch außerhalb der Amtssprachregion standardsprachliche Wortschatzvarianten existieren. Am Beispiel der deutschsprachigen Minderheiten in Rumänien, Namibia und Mennonitensiedlungen des amerikanischen Kontinents werden Gründe für den Erhalt der deutschen Sprache und für die Entwicklung und Pflege spezifischer standardsprachlicher Lexeme dargestellt.
\end{abstract}

Keywords: Amtssprache, Identität, Mennonitendeutsch, namibisches Deutsch, nationale Varianten, Rumäniendeutsch, Standardvarietäten, Spracherhalt, Sprachzentrum

\section{Einleitung}

Die Standardvarietäten nehmen innerhalb der zahlreichen Varietäten der deutschen Sprache eine zentrale Stellung ein, weil sie überregionale Kommunikation ermöglichen und adäquate Ausdrucksweisen für die öffentliche und formelle Kommunikation sind. Im Volksmund werden sie in der Regel (korrektes) Hochdeutsch oder Schriftsprache genannt. Herkömmliche Bezeichnungen wie Gemein- oder Einheitssprache suggerieren ein einziges homogenes Sprachsystem mit einheitlichem Wortschatz und Grammatik, was aber de facto nicht gegeben ist.

In der Fachwelt herrscht heute die Auffassung vor, dass Deutsch zu den sogenannten plurizentrischen Sprachen gehört. Diese fungieren in mehreren Staaten oder Regionen als Amtssprache und verfügen über mehrere Standard-

Birte Kellermeier-Rehbein, Dr., Bergische Universität Wuppertal, Fakultät für Geistes- und Kulturwissenschaften, Gaußstraße 20, D-42113 Wuppertal, E-Mail: kellermeier-rehbein@uni-wuppertal.de

○ Open Access. (c) 2018 Birte Kellermeier-Rehbein, publiziert von De Gruyter. (c) BY Dieses Werk ist lizenziert unter der Creative Commons Attribution 4.0 Lizenz.

https://doi.org/10.1515/9783110538588-009 
varietäten, die zwar einander sehr ähnlich sind, sich aber dennoch durch gewisse Besonderheiten (Varianten) in Aussprache, Wortschatz, Grammatik und Pragmatik unterscheiden. Gebiete mit eigenen sprachlichen Normen werden als Sprachzentren bezeichnet. Die Standardvarietäten Deutschlands, Österreichs und der Schweiz, wie auch anderer, kleinerer Staatsgebiete und Regionen sind linguistisch gleichwertig, insofern sie sich gleichermaßen für präzise und stilistisch angemessene Formulierungen in sämtlichen Themenbereichen eignen. Ihre Varianten sind keinesfalls als Normabweichungen aufzufassen, sondern entsprechen zentrumseigenen Regeln.

Der vorliegende Beitrag erläutert die Standardvariation des Deutschen bezüglich der Lexik und zeigt, dass sich die Plurizentrik innerhalb und außerhalb Europas jenseits der Gebiete fortsetzt, in denen Deutsch Amtssprache ist (Amtssprachregion).

\section{Sprachlicher Standard und Probleme der Abgrenzung}

Für den Begriff Standardvarietät liegt noch keine allgemein anerkannte Definition vor (vgl. Kellermeier-Rehbein 2013: 4-7). Ein Problem ist, dass er sich nur schwer aus sich selbst heraus definieren lässt, ohne Zuhilfenahme von Begriffen wie Dialekt oder Umgangsvarietät, die ihrerseits wenig scharf umrissen sind. Erschwerend kommt hinzu, dass Dialekte, Umgangsvarietäten und der sprachliche Standard ein Kontinuum mit fließenden Übergängen bilden, wodurch eine exakte Abgrenzung der Sprachschichten nur durch bewusste Entscheidung möglich ist, die sich nicht in jedem Fall zwingend begründen lässt.

Immerhin lassen sich Eigenschaften von Standardvarietäten spezifizieren, die in Richtung einer möglichen allgemein akzeptablen Definition weisen. Nach Ammon (1995: 73 ff.) sind sie die sprachliche Norm für öffentliche und formelle Kommunikationssituationen, wie z. B. das Verlesen der Nachrichten in Rundfunk oder Fernsehen oder öffentliche Ansprachen von hohen Amtsträgern. Ihr Gebrauch in mündlicher und schriftlicher Form ist ferner für die amtliche Kommunikation verpflichtend. Es wird erwartet, dass Behördenmitarbeiter ${ }^{1}$ im dienstlichen Verkehr innerhalb der eigenen, aber auch mit fremden Behörden sowie mit den Bürgern die Standardvarietät verwenden. Schulen und Universitäten sollen sich ebenfalls der sprachlichen Norm bedienen. Dies setzt eine prä-

1 Zur besseren Lesbarkeit verwende ich das generische Maskulinum stellvertretend für beide Geschlechter. 
skriptive Kodifizierung voraus, also die Darstellung der standardsprachlichen Einheiten in Wörterbüchern und Grammatiken in einer Art und Weise, die vorgibt, was als korrekt gilt und was nicht. Solche Kodifizierungen sind in der Regel Grundlage des muttersprachlichen Unterrichts in der Schule, wo die Standardvarietät Unterrichtsmedium und Lehrgegenstand ist. Personen, die ihrer nicht mächtig sind, werden daher oft als ungebildet eingeschätzt.

Die Abgrenzung des sprachlichen Standards sollte in zweierlei Hinsicht vorgenommen werden (vgl. Ammon 1995: 89). Auf einer vertikalen Ebene erfolgt eine sozial-normative Unterscheidung zwischen sprachlichem Standard und Nonstandard (z. B. Umgangsvarietäten, Soziolekte und anderen). Dabei geht es um die Frage, was zur sprachlichen Norm gehört und Erwartungen an die sprachliche Richtigkeit erfüllt bzw. was von der Norm abweicht und folglich nicht als Standard bezeichnet werden kann. Für manche Sprachen gibt es regulierende staatliche Einrichtungen (z. B. Académie Française, Real Academia de la Lengua Española), die solche Abgrenzungsprobleme autoritär regeln. Da es für die deutsche Sprache keine derartige Instanz gibt, übernehmen vier gesellschaftliche Gruppen diese Aufgabe, die von Ammon (1995: 73 ff.) als das „soziale Kräftefeld einer Standardvarietät“ bezeichnet wurden. Dazu gehören Modellschreiber und -sprecher (z. B. Schriftsteller, Journalisten, Nachrichtensprecher und andere), die die standardsprachlichen Einheiten in ihren Modelltexten verwenden. Diese Modelltexte dienen nicht selten als Datenmaterial für empirische Untersuchungen von Sprachexperten (Linguisten), die den Aufbau und die Funktionsweise des Sprachsystems untersuchen. Ihre Forschungsergebnisse fließen in die Arbeit der Kodifizierer (Lexikographen und Autoren von Grammatiken) ein, die allerdings auch selbst Untersuchungen durchführen und ihre Ergebnisse häufig durch Belege aus Modelltexten stützen. Schließlich nutzt die Gruppe der Sprachnormautoritäten (z. B. Lehrer, Hochschuldozenten) den Kodex und die Forschungsergebnisse der Sprachexperten als Grundlage des Unterrichts und zur Rechtfertigung ihrer Korrekturen. Im Idealfall sind sich alle Gruppen bezüglich der Normebene (Standard oder Nonstandard) einer sprachlichen Einheit einig. Wenn aber keine Einigkeit erzielt wird, ist der betreffende sprachliche Ausdruck nicht völlig uneingeschränkt in öffentlichen und formellen Kontexten verwendbar und wird in einem Wörterbuch mit einer entsprechenden Markierung versehen. Natürlich spielt auch die Bevölkerungsmehrheit eine Rolle bei der Festlegung der sprachlichen Norm, denn ohne ihre Akzeptanz werden die Vorschläge des Kräftefeldes nicht zum Sprach-Usus.

Die zweite Ebene der Standard-Abgrenzung ist horizontal. Dabei werden Standardvarietäten verschiedener Nationen oder Regionen voneinander abgegrenzt (z. B. deutscher vs. österreichischer Standard). Mit diesen Problemen befasst sich die variationslinguistische Plurizentrik-Forschung, die im Folgenden dargestellt wird. 


\section{Deutsch als plurizentrische Sprache}

Von einer plurizentrischen Sprache spricht man dann, wenn diese in mehr als einem Land als nationale oder regionale Amtssprache in Gebrauch ist und wenn sich dadurch standardsprachliche Unterschiede herausgebildet haben. (VWD 2016: XXXIX)

So lautet die einschlägige Definition im Variantenwörterbuch des Deutschen (= VWD).

Dass die deutsche Sprache diese Bedingungen erfüllt, lässt sich leicht nachweisen. Sie ist in Deutschland, Österreich, der Schweiz, Liechtenstein, Luxemburg, Ostbelgien und Bozen-Südtirol (Italien) nationale oder regionale Amtssprache. Ferner gibt es in diesen Staaten und Regionen eigene standardsprachliche Besonderheiten, die als nationale Varianten bezeichnet werden. Darunter versteht man

[...] diejenigen Sprachformen, die Bestandteil der Standardvarietät mindestens einer Nation, aber nicht der Standardvarietäten aller Nationen der betreffenden Sprachgemeinschaft sind. Sie müssen zudem [um als Varianten zu gelten (B.K.-R.)] Entsprechungen in den übrigen Standardvarietäten der betreffenden Sprachgemeinschaft haben. (Ammon 1995: 70)

Der Austriazismus Karfiol ist beispielsweise nur in Österreich (A) standardsprachlich, während in Deutschland (D) und der Schweiz (CH) der Ausdruck Blumenkohl gilt. Dem Helvetismus Berufsdiplom entspricht in Österreich und Deutschland die Variante Gesellenbrief. Bundestag ist ein Teutonismus und synonym mit dem österreichischen und schweizerischen Wort Nationalrat. Sprachliche Einheiten, die in allen Zentren zum sprachlichen standardsprachlich gehören (z.B. Mensch, grün, trinken), sind gemeindeutsch und gehören selbstredend nicht zu den nationalen Varianten.

Die geographische Verbreitung von nationalen Varianten kongruiert jedoch nicht immer exakt mit den Staatsgrenzen. Wie oben schon deutlich wurde, gibt es unspezifische Varianten, die in zwei Zentren standardsprachlich sind (z.B. Quark CH D) oder in einem Zentrum und einem Teilgebiet eines weiteren Zentrums (z. B. Kren A D-südost ,Meerrettich'). Wieder andere gelten nur in einem Teilgebiet eines einzigen Zentrums (z. B. Feudel D-nord ,BodenPutzlappen').

Nationale Standardvarianten können auf allen Ebenen der Sprache vorkommen. Aussprache- und Wortschatzvarianten sind besonders auffällig, während morphologische, syntaktische und semantische Varianten eher unscheinbar und häufig nicht auf den ersten Blick erkennbar sind. Der vorliegende Beitrag behandelt ausschließlich die lexikalische Standardvariation des Deut- 
schen. Sie ist besonders gut erforscht und seit der Erstauflage des VWD (2004) gründlich dokumentiert. Es ist das einzige Wörterbuch, das die Wortschatzvariation einer plurizentrischen Sprache umfassend, d.h. unter Berücksichtigung aller (bisher als solche identifizierten) Sprachzentren, darstellt und die nationalen Standardvarianten des Deutschen als hinsichtlich ihrer Geltung im jeweiligen Zentrum gleichwertig präsentiert. Seit 2016 liegt die völlig neu bearbeitete, aktualisierte und erweiterte Zweitauflage des gleichnamigen Wörterbuchs vor.

Die folgenden Beispiele sind nur ein kleiner Ausschnitt aus den ca. 12.000 Einträgen des VWD (2016). Varianten sind kursiv gedruckt, gemeindeutsche Bedeutungserläuterungen stehen zwischen einfachen Anführungszeichen:

Deutschland: $\quad$ Abitur (in A CH: Matura), Bürgersteig (,eine Straße entlang führender [erhöhter] Weg für Fußgänger(innen)'), Möhre (,Karotte'), Tüte (,Plastiktragetasche`)

Österreich: $\quad$ Beistrich (,Komma'), Jänner (in CH D: Januar), Landeshauptmann (Ministerpräsident D), Marille (Aprikose CH D)

Schweiz: $\quad$ Autocar (in A D: Reisebus), Morgenessen (,Frühstück'), Panaché (,helles Bier mit Limonade‘), Velo (,Fahrrad‘)

Ostbelgien: $\quad$ Animation (,Veranstaltung'), Bürgermeisterkollegium (,Gemeinderat'), Heiratsbuch (,Familienbuch')

Luxemburg: Erkennungstafel (,Nummernschild'), es ist gewusst (,es ist bekannt'), Stagiar (,Anwärter, Praktikant')

Liechtenstein: eine Behandlung ziehen (,(eine Gesetzesvorlage) im Parlament behandeln'), Landesphysikus (Amtsarzt A D), Neni (,Großvater')

Südtirol: $\quad$ Basisarzt (,Hausarzt'), Befähigungsdiplom (Gesellenbrief A D), Hydrauliker (,Installateur')

Varianten wie diese sind Erkennungsmerkmale der nationalen Varietäten des Deutschen. Nach Ammon (1995: 71-72) gilt eine Standardvarietät dann als nationale Varietät, wenn sie mindestens eine spezifische nationale Variante enthält oder eine spezifische Kombination von Varianten, die in mehr als einem Zentrum gelten. Dies ist allerdings eine Minimalbedingung, die alle nationalen Varietäten vielfach erfüllen.

Vielfältige Einflüsse trugen zur Entstehung von nationalen Varietäten bei, vor allem historische Gegebenheiten (z. B. Reformation und Gegenreformation, die Zugehörigkeit Österreichs zur Donaumonarchie, die Folgen der Weltkriege und des Dritten Reichs), sprachgeschichtliche Entwicklungen (z. B. der Einfluss des Ostmitteldeutschen, die Herausbildung frühneuzeitlicher regionaler Schreibbzw. Kanzleisprachen), dialektale Grundlagen, Sprachpurismus und andere. 
Es kann zusammengefasst werden, dass die deutsche Sprache beide Bedingungen für plurizentrische Sprachen erfüllt: Sie ist Amtssprache in sieben Staaten und hat standardsprachliche Varianten herausgebildet. Andere europäische Sprachen gehören ebenfalls in die Kategorie der plurizentrischen Sprachen, vor allem solche, die durch Kolonialismus auch auf anderen Kontinenten verbreitet wurden, z. B. Englisch, Französisch, Spanisch, Portugiesisch oder Niederländisch.

Der Terminus Plurizentrik leitet sich von Zentrum, genauer Sprachzentrum, ab. Damit wird ein Staat, eine Nation oder eine Sprechergemeinschaft als Teil einer Nation (z. B. deutschsprachige Schweizer) mit einer eigenen Standardvarietät einer Sprache bezeichnet (vgl. Ammon 1995: 95). Im Falle der deutschen Sprache sind es die oben genannten Staaten und Regionen. Allerdings handelt es sich dabei um unterschiedliche Arten von Sprachzentren, nämlich Voll- und Halbzentren. In beiden Fällen ist Deutsch staatliche Amtssprache. Der Unterschied liegt in der Kodifizierung, denn in den Vollzentren (Deutschland, Österreich, Schweiz) gibt es einen sogenannten Binnenkodex. Dabei handelt es sich um Nachschlagewerke, die im jeweiligen Sprachzentrum verfasst wurden und die eigene Standardvarietät (präskriptiv) darstellen. In Deutschland sind dies vor allem die Dudenbände, in Österreich das Österreichische Wörterbuch und in der Schweiz Werke wie Schweizer Schülerduden oder Schweizer Wahrig. Da es in den Halbzentren keine Binnenkodizes gibt, müssen die Sprecher bei sprachlichen Unsicherheiten auf die Nachschlagewerke der (benachbarten) Vollzentren zurückgreifen. Darüber hinaus beschränkt sich die Standardvariation in den Halbzentren auf den Wortschatz.

Dehnt man das Konzept der Sprachzentren noch weiter aus, kann man auch noch sogenannte Viertelzentren ansetzen. In diesen Gebieten hat die entsprechende Sprache keinen amtlichen Status, sondern ist Sprache einer Minderheit. Dennoch verfügt sie über standardsprachliche Varianten, die in öffentlichen und formellen Kontexten sowie in Modelltexten unbeanstandet verwendet werden können und von Sprachnormautoritäten als korrekt akzeptiert werden (vgl. VWD 2016: XII). Als Viertelzentren des Deutschen gelten bisher Rumänien, Namibia und Mennonitensiedlungen auf dem amerikanischen Kontinent. Ob es weitere Viertelzentren gibt, muss durch zukünftige Forschungen gezeigt werden.

Obwohl die Standardvarietäten des Deutschen (zumindest die der Vollzentren) aus linguistischer Sicht gleichwertig sind, ist in der Sprachwirklichkeit dennoch eine gewisse Dominanz der bundesdeutschen Norm zu beobachten. Dies ist vor allem den demographischen Verhältnissen geschuldet. Während nach Angaben des VWD (2016: XLIII, XLIX, LII) in der BRD ca. 81 Mio. Menschen leben, sind es in Österreich ca. 8,7 Mio. und in der Schweiz nur 4,5 Mio. Deutschspra- 
chige. Österreicher und Schweizer konsumieren häufig Medien des nördlichen Nachbarn, während Deutsche nur verhältnismäßig selten mit denjenigen der anderen Zentren in Berührung kommen. Auch renommierte Wörterbücher und Grammatiken (z. B. Adelung, Grimm, Duden, Siebs) wurden in den Gebieten verfasst, die heute zur BRD gehören, wodurch sich deutsche Normen stärker verbreiten konnten. Diese und weitere Gründe führten zu der vor allem in der Bundesrepublik weit verbreiteten Ansicht, dass es nur ein „korrektes Hochdeutsch“ gebe, und zwar die deutsche Standardvarietät (vgl. Hägi 2014: 71).

In der Fachwelt ist die Standardvariation der deutschen Sprache zwar ziemlich allgemein anerkannt, doch wird die Art der sprachgeographischen Gliederung und ihre Interpretation diskutiert. Es stehen sich dabei im Wesentlichen zwei Positionen gegenüber: einerseits die plurizentrische bzw. plurinationale Deutung, vertreten durch Clyne (1992), Ammon (1995), VWD (2004, 2016) und andere, andererseits das pluriareale Modell, vertreten durch Scheuringer (1996), Pohl (1997) und andere. Erstere fassen Sprachzentren vor allem als Staaten bzw. Nationen auf und verwenden folglich Termini wie nationale Variante und Nationalvarietät. Die Standardvariation sei Folge und Ausdruck der staatlichen Eigenständigkeit und werde durch zentrumseigene Kodifizierung gepflegt und geschützt. Etliche Besonderheiten entstammten zudem der Domäne der staatlichen Verwaltung. Trotzdem müsse das Verbreitungsgebiet der nationalen Varianten nicht immer exakt mit den Staatsgrenzen kongruieren, worauf oben bereits hingewiesen wurde. Die Über- oder Unterschreitung der staatlichen Grenzen durch die Standardvarianten ließ vor allem in Österreich Kritik am plurinationalen Modell entstehen. Der Terminus Plurizentrik sei inadäquat und irreführend, weil er die Bedeutung der staatlichen Zentren für die Variation überbewerte und die Existenz staatlich bzw. national einheitlicher Varietäten suggeriere, die es so nicht gebe (vgl. Schrodt 1997: 16; Scheuringer 1996: 151-152). Der deutsche und österreichische Standard sei weder homogen (Pohl 1997: 68), noch geographisch eindeutig an diese Staaten gekoppelt, weshalb der Terminus pluriareale Variation zu bevorzugen sei.

Nicht jeder folgt dieser angenommenen Dichotomie von Plurinationalität vs. Pluriarealität und fasst die Konzeptionen als Widersprüche auf. Alternativ können beide Auffassungen als sich ergänzende Beschreibungsmodelle der Standardvariation betrachtet werden, die beide gleichermaßen ihre Daseinsberechtigung haben (z.B. in Kellermeier-Rehbein 2014). Im Hinblick auf die schwindende Bedeutung der Nationalstaaten zugunsten von supranationalen Organisationsformen (z. B. Europäische Union) und angesichts der allgemeinen Globalisierung fordert Glauninger (2013: 464-465), die Heterogenität der deutschen Sprache mithilfe einer „»supra-« bzw. "postnational« orientierten Theorie“ zu untersuchen und darzustellen; jedoch bedarf dieser Vorschlag noch der Konkretisierung. 
Zusammenfassend ist festzuhalten, dass aufgrund der Plurizentrik ein national und regional heterogener Standardwortschatz im deutschsprachigen Raum existiert. Die variierende Lexik macht allerdings nur einen geringen prozentualen Anteil der Gesamtlexik aus. Schmidlin (2013: 23) schätzt alle Arten von Standardvarianten (inklusive morphologischer und grammatischer Varianten) auf etwa fünf Prozent, sodass die nationalen Varietäten des Deutschen eine große linguistische Ähnlichkeit aufweisen (vgl. Ammon 1995: 5). Die gegenseitige Verständigung der Sprecher aus unterschiedlichen Zentren ist dadurch gesichert.

\section{Nationale Varietäten und Identität}

Sprache hat neben ihrer Kommunikationsfunktion auch eine Identifikationsfunktion. Durch eine gemeinsame Sprache oder Varietät wird die Zugehörigkeit $\mathrm{zu}$ einer Sprachgemeinschaft und die Abgrenzung von anderen Sprechergruppen zum Ausdruck gebracht. Die nationale Identität spiegelt sich in der Regel in sogenannten ganzen Sprachen (z. B. Deutsch, Polnisch etc.). Dies hat seinen Ursprung in der in Europa traditionell engen Verknüpfung von Sprache und Nation. Besonders zur Zeit der Nationenbildung galt als Idealvorstellung, dass alle Mitglieder einer Nation dieselbe Muttersprache („Nationalsprache“) hätten und alle Muttersprachler einer Sprache eine Nation („Sprachnation“) bildeten. Diese enge Verbindung zeigt sich bis heute in gleichen oder ähnlichen Bezeichnungen für Nation und Sprache: Dänemark - Dänisch, Italien - Italienisch, Polen - Polnisch etc.

Nach Clyne stellen die plurizentrischen Sprachen in diesem Zusammenhang einen Sonderfall dar. Ihre Sprecher zeigen durch den Gebrauch einer Nationalvarietät - willkürlich oder unwillkürlich - die Zugehörig zur betreffenden Sprachgemeinschaft und grenzen sich gleichzeitig von Mitgliedern anderer Nationen mit der gleichen Sprache ab, ohne sich dabei sprachlich-kommunikativ $\mathrm{zu}$ isolieren:

Plurizentrische Sprachen vereinen und grenzen zugleich ab. In dieser Beziehung spiegeln sie eine mehrfache Identität wider [...]. Wer sich einer bestimmten Nationalvarietät des Dt. [Deutschen - B. K.-R.] bedient, verbindet sich dadurch mit allen Mitgliedern der internationalen dt. [deutschen (- B. K.-R.)] Sprachgemeinschaft, drückt aber zugleich seine nationale Identität aus. (Clyne 2000: 2008)

Nationale Varietäten sind aber nicht nur wichtig für die Identität ihrer Sprecher, sondern fungieren auch als Symbole der staatlichen Eigenständigkeit, denn nur eine Nation mit weitgehender Autonomie kann eigene sprachliche Standards als 
verbindlich erklären, schützen und pflegen (vgl. Kellermeier-Rehbein 2014: 178). In Österreich zeigt sich die Bedeutung der nationalen Varietät und der dazugehörigen Varianten für die nationale Identität besonders deutlich. Seit 1951 wird die eigene Varietät im Österreichischen Wörterbuch dokumentiert und geschützt. Inzwischen liegt es in der 43. Auflage (2016, Schulausgabe) vor und ist für den Sprachgebrauch in Schulen und Behörden maßgeblich. Nicht selten verwenden Österreicher ganz bewusst sogenannte Demonstrationsaustriazismen, um sich von Deutschen (seltener auch von Schweizern) abzugrenzen. Der wohl eindrucksvollste Beweis für die Wichtigkeit der Austriazismen für die nationale Identität der Österreicher ist die Festlegung von 23 EU-Austriazismen für die Verwendung in amtlichen Schriftstücken der Europäischen Union. Sie müssen per Schrägstrich den bundesdeutschen Ausdrücken an die Seite gestellt werden (z. B. Quark/Topfen) (vgl. Kellermeier-Rehbein 2014: 180).

Auch in der Schweiz ist die Nationalvarietät nicht unerheblich für die Identität der Deutschschweizer. Allerdings ist das Schweizerhochdeutsche den alemannischen Dialekten als Nationalsymbol nachgeordnet (vgl. Ammon 1995: $301 \mathrm{ff}$.), d. h. die Mundarten sind in noch stärkerem Maße identitätsstiftend. Lediglich in der BRD herrscht kaum ein Bewusstsein von den nationalen Varietäten, weshalb man sich dort nicht über die deutsche Standardvarietät identifiziert.

Dass die im Mutter- und Amtssprachgebiet des Deutschen gelegenen Sprachzentren eigene Standardvarianten und -varietäten entwickelt haben, pflegen und schützen, ist aus den oben genannten Gründen verständlich und gut nachvollziehbar.

In den nächsten Kapiteln geht es um die außerhalb der Amtssprachregion, ja sogar außerhalb Europas gelegenen Viertelzentren, ihre spezifischen Standardvarianten und deren Bedeutung für die Identität der Minderheiten.

\section{Lexikalische Standardvariation des Deutschen außerhalb der Amtssprachregion}

Wenn die Amtssprachregion einer Sprache mehrere Staaten oder Regionen umfasst, wird die Entwicklung von spezifischen nationalen Standardvarianten und -varietäten erleichtert, denn autonome Staaten können dafür Sorge tragen, dass die von Haugen (1997: 350) genannten vier Schritte zur Standardisierung von Varietäten effizient und nachhaltig durchgeführt werden: Auswahl einer Sprachnorm („,selection of norm“), Kodifizierung der Sprachformen („,codification of form“), Ausweitung der Funktion („elaboration of function“) und 
Akzeptanz in der Bevölkerung (,acceptance by the community“). Fehlt der Amtssprachenstatus, wird die Standardvariation zwar erschwert, aber nicht unmöglich gemacht. Im Folgenden wird dies anhand von deutschsprachigen Minderheiten gezeigt, die außerhalb des Amtssprachengebietes leben. Dazu werden die Viertelzentren vorgestellt, deren lexikalische Variation im VWD (2016) dokumentiert ist: Rumänien, Namibia und Mennonitensiedlungen. Die Aufnahme dieser Varianten ins VWD ist ein gewichtiger Indikator für ihre Standardsprachlichkeit, da die Autoren keine dialektale oder umgangssprachliche Lexik lemmatisierten, wie sie schon im Vorwort der Erstauflage deutlich machten (vgl. VWD 2004: XII). Das VWD verzeichnet 79 Rumänismen, 37 Namibismen und 46 Varianten der mexikanischen Mennonitensiedlungen (vgl. VWD 2016: XIII).

\subsection{Rumänien}

Die beiden wichtigsten deutschsprachigen Gruppen in Rumänien sind die Siebenbürger Sachsen und die Banater Schwaben. Die Bezeichnungen Sachsen und Schwaben dürfen nicht wörtlich genommen werden, denn ihre Vorfahren stammten aus verschiedenen deutschen und österreichischen Regionen. Die ersten Deutschsprachigen kamen schon im 12. Jahrhundert auf Geheiß des Königs Géza II. nach Siebenbürgen, um dort das Gebiet ,jenseits der Wälder“ (Transsilvanien) militärisch zu sichern und wirtschaftlich zu entwickeln (vgl. Lăzărescu 2013: 372). Im Zuge weiterer Einwanderungswellen wurden auch andere Gebiete besiedelt, darunter das Banat. Die Siebenbürger Sachsen wandten sich im Zuge der Reformation dem Protestantismus zu, während die Banater Schwaben in der Regel katholisch sind.

Die Anzahl der Deutschstämmigen ist in den letzten Jahrzehnten, besonders aufgrund massenhafter Auswanderungen während der CeauşescuDiktatur und seit 1989, deutlich zurückgegangen (vgl. Lăzărescu 2013: 374). In der Zwischenkriegszeit waren es ca. 850.000, bei der Volkszählung von 1992 noch rund 120.000 und inzwischen bewegen sich die Schätzungen zwischen ca. 30.000 (Lăzărescu 2013: 374) und 50.000 (VWD 2016: LXI). Als Folge davon sind die ursprünglichen Siedlungsgebiete zu Sprachinseln geschrumpft, sofern man aufgrund der Mischsiedlung mit Rumänen überhaupt noch von Sprachinseln im klassischen Sinn sprechen kann, denn sie leben gemeinsam mit der Mehrheitsbevölkerung und 18 weiteren Minderheiten im multilingualen und multiethnischen Rumänien (vgl. Lăzărescu 2013: 373).

Lăzărescu (2013: 375 und 377) beschreibt das Rumäniendeutsche als ein Diasystem mit diversen dialektalen Varietäten, betont aber auch die Existenz einer 
überregionalen Standardvarietät, die durch den Kontakt zu deutschsprachigen Staaten und anderen in Rumänien gebräuchlichen Sprachen geprägt ist.

Zum einen führte das enge historisch-politische Verhältnis zu Österreich dazu, dass sich etliche Übereinstimmungen mit dem österreichischen Deutsch entwickelten: sogenannte Rumäno-Austriazismen wie beispielsweise Topfen (,Quark'), Kren (,Meerrettich'), Staubzucker (,Puderzucker`) und andere (Lǎzǎrescu 2007: 678). Das Rumäniendeutsche war aufgrund der Herkunft der Siedler auch westmitteldeutsch geprägt. Die Siebenbürger Kanzleisprache erfuhr darüber hinaus ostmitteldeutsche Einflüsse durch die Luthersprache und stand unter der Einwirkung der österreichischen kaiserlichen Kanzlei, insbesondere nach der Theresianischen Reform (vgl. Lǎzărescu \& Scheuringer 2013: 421, 427). Später kamen Entlehnungen aus dem Wortschatz der BRD sowie DDR-Einflüsse auf den politischen Wortschatz hinzu, weshalb das Rumäniendeutsche als „Ausgleichsvariante“ bezeichnet wird (vgl. Lǎzǎrescu \& Scheuringer 2013: 424); in der Terminologie des vorliegenden Aufsatzes müsste es Ausgleichsvarietät heißen).

Zum anderen erfolgten zahlreiche Entlehnungen aus Kontaktsprachen (Kontakt-Zentrismen; vgl. Lǎzărescu 2013: 375), die zur Erweiterung des rumäniendeutschen Wortschatzes beitrugen. Dies erklärt sich durch den oben genannten Multilingualismus, durch den das Deutsche über Jahrhunderte mit Rumänisch und anderen Minderheitensprachen in Berührung war. Entlehnungen aus diesen Spendersprachen wurden durch die zwei- oder mehrsprachigen Kompetenzen der Rumäniendeutschen erleichtert, die neben ihrer Muttersprache auch die Mehrheitssprache Rumänisch und zum Teil Ungarisch oder andere Minderheitensprachen beherrschen (vgl. Lǎzărescu 2013: 378-379).

Von den insgesamt 79 im VWD (2016) verzeichneten Rumänismen sind knapp 50 aus dem Rumänischen entlehnt oder in Analogie zum Rumänischen gebildet worden. Im Folgenden werden einige exemplarisch vorgestellt:

\section{Rumänismus}

Ägrisch, der

Amphitheater

Autobahnhof

Bierfabrik

Bizikel, das

Bokantsch, der

Chemiekombinat

Hydrozentrale

\section{Bedeutung oder} deutsche Entsprechung

,Stachelbeere‘

,Hörsaal‘

,Busbahnhof‘

,Bierbrauerei“

,Fahrrad“

,Bergschuh, grober Schnürschuh“

,Großbetrieb zur Herstellung

von Chemikalien`

,Wasserkraftwerk rumänischer

Ursprung

agrişă

amfiteatru

autogară

fabrică de bere

bicicletă

bocanci

combinat chimic

hidrocentrală 


\begin{tabular}{|c|c|c|}
\hline Rumänismus & $\begin{array}{l}\text { Bedeutung oder } \\
\text { deutsche Entsprechung }\end{array}$ & $\begin{array}{l}\text { rumänischer } \\
\text { Ursprung }\end{array}$ \\
\hline Komponenz, die & ,Zusammensetzung“ & componentă \\
\hline konsekriert & ,berühmt, anerkannt‘ & consacrat \\
\hline Milchfabrik & ,Molkerei‘ & fabrică de lapte \\
\hline Muskelfieber & ,Muskelkater & febră musculară \\
\hline Planifizierung & ,Planung“ & planificare \\
\hline Sarmale, die & $=$ Kohlroulade D & sarmale \\
\hline $\begin{array}{l}\text { Turmblock } \\
\text { und andere }\end{array}$ & „Hochhaus“ & bloc turn \\
\hline
\end{tabular}

Die folgenden Rumänismen sind keine Entlehnungen (vgl. VWD 2016):

\begin{tabular}{|c|c|}
\hline Rumänismus & Bedeutung \\
\hline assistieren & ,hospitieren' \\
\hline Aufboden & ,Dachboden` \\
\hline diskriminatorisch & ,diskriminierend' \\
\hline Eingruß & ,Einstand“ \\
\hline Konsolidierung & ,Sanierung, Renovierung“ \\
\hline Mistkorb & ,Mülleimer \\
\hline Theaterchronik & ,Theaterkritik \\
\hline
\end{tabular}

Ursprünglich dialektal sind die Lexeme Hanklich, die (eine Kuchenart) und Palukes, der/die (,Polenta').

Die deutschsprachige Minderheit in Rumänien kann zu Recht als Viertelzentrum der deutschen Sprache aufgefasst werden, denn sie erfüllt die oben genannten Bedingungen. Sie verfügt über eine eigene Standardvarietät, da es spezifische Rumänismen gibt, die in Modelltexten verwendet werden und durch Sprachnormautoritäten als zur sprachlichen Norm gehörend akzeptiert werden (VWD 2016: LX). Zu den Modelltexten gehören in erster Linie deutschsprachige rumänische Zeitungen, wie z. B. die Allgemeine Deutsche Zeitung für Rumänien oder die Hermannstädter Zeitung. Lăzărescu (2013: 385) verweist auch auf den Gebrauch der deutschen Sprache durch rumäniendeutsche Schriftsteller wie Hans Bergel oder die Literaturnobelpreisträgerin Herta Müller. Ob sie in ihren Texten Rumänismen verwenden, wird allerdings nicht erwähnt und müsste eigens überprüft werden. Das Rumäniendeutsche dient ferner als Kirchen- und Schulsprache (Lăzărescu 2013: 381-382). Leider nimmt Lăzărescu keinen Bezug auf das Korrekturverhalten der Lehrer und ihre Bewer- 
tung der Rumänismen. Die Kodifizierung der Rumänismen durch das VWD (2016) ist eine Außenkodifizierung durch Lexikographen aus den Vollzentren, indirekt aber auch eine Binnenkodifizierung, denn die Lemmata wurden den Mitarbeitern des VWD durch Lǎzǎrescu gemeldet.

\subsection{Namibia}

Deutsch kam im Zuge des Kolonialismus nach Namibia, das von 1884 bis 1919 unter der Bezeichnung Deutsch-Südwestafrika einzige Siedlungskolonie des Kaiserreichs war. Im Jahr 1913 befanden sich über 12.000 Deutschsprachige im sogenannten Schutzgebiet (vgl. Gretschel 1993: 44). Deutsch wurde offizielle Amtssprache und Unterrichtssprache in Schulen für weiße Kinder. Nach dem Ersten Weltkrieg verlor es diesen Status. Heute bilden die Nachfahren der damaligen Siedler und andere Deutschstämmige die ca. 20.000 bis 25.000 Personen umfassende deutschsprachige Minderheit. Sie macht zwar nur rund 1\% der Gesamtbevölkerung aus, ist aber wirtschaftlich sehr einflussreich.

Namibia ist ein multilingualer Staat mit ca. 30 Sprachen, die zu drei Sprachfamilien gehören: Bantu- und Khoisan-Sprachen sowie die germanischen Sprachen Afrikaans, Deutsch und Englisch. Letztere ist seit der Unabhängigkeit (1990) die einzige Amtssprache. Deutsch und Afrikaans sind sogenannte Nationalsprachen Namibias, was in etwa der Stellung einer geschützten Minderheitensprache gleichkommt (vgl. Kellermeier-Rehbein 2016; Ammon 2015: 359-369).

Das namibische Deutsch zeichnet sich - ebenso wie das Rumäniendeutsche - durch viele Entlehnungen aus. Seit den 1960er Jahren beschäftigen sich Sprachwissenschaftler (vereinzelt) mit diesem Thema und führen viele lexikalische Varianten vor allem auf den Sprachkontakt mit Afrikaans, Englisch und deutlich weniger ausgeprägt - mit autochthonen afrikanischen Sprachen zurück. Nöckler (1963: 36 ff.) untersuchte das damals so genannte Südwesterdeutsch und fand über 300 lexikalische Besonderheiten, darunter 197 Entlehnungen aus dem Afrikaans und 77 aus dem Englischen. Beide Sprachen waren bis zur Unabhängigkeit Namibias offizielle Amtssprachen und Afrikaans war die numerisch stärkste germanische Sprache im Land. Nach Gretschel (1993: 45) kamen die Deutschen erst nach den Afrikaanssprachigen in das Gebiet, lernten von ihnen und übernahmen Teile ihres Wortschatzes, um lexikalische Lücken zu füllen, die sich in der neuen Umgebung auftaten. Nöckler (1963: 115) zufolge stammen viele Lehnwörter aus den Domänen Tier- und Pflanzenwelt, Geographie, Wirtschaft und anderen. Gretschel (1995: 306) erwähnt darüber hinaus auch solche, die keine Wortschatzlücken füllen, sondern deutsche Wörter ersetzen, was er als Gefahr für die Sprache einstuft. Ursprünglich war Afri- 
kaans die wichtigste Spendersprache, doch seit der Unabhängigkeit und Festlegung des Englischen als solo-offizielle Amtssprache, wird es als Quelle für sprachliche Neuerungen immer wichtiger (vgl. Pütz 1991: 464).

Die zahlreichen Entlehnungen sind zum einen auf die Kompetenzen der in der Regel dreisprachigen Deutschnamibier zurückzuführen (vgl. Pütz 1991: 470), die aufgrund ihrer Sprachkenntnisse die Möglichkeit haben, Lexeme aus diesen Sprachen zu übernehmen. Zum anderen erleichtert die strukturelle Ähnlichkeit der Sprachen die Übernahme und Integration der Lehnwörter ins Deutsche.

Im Folgenden werden nur solche Namibismen exemplarisch präsentiert, die in der aktuellen Forschung als standardsprachlich und spezifisch für das namibische Deutsch anerkannt sowie im VWD (2016) verzeichnet sind. Leider gibt das Wörterbuch keine Informationen über die Spendersprachen der entlehnten Lexeme. Stichproben in einem Afrikaans-Wörterbuch (Trümpelmann \& Erbe 2015) zeigen eine Reihe von Übereinstimmungen, die als Indizien für die Übernahme aus dieser Sprache gedeutet werden können.

\begin{tabular}{|c|c|c|}
\hline Namibismus & Bedeutung & Afrikaans-Ursprung \\
\hline Bokkie, das & ,Ziege“ & bok (,Ziegenbock`) \\
\hline Braai, der & ,Grillparty & braai (,braten') \\
\hline auf Pad gehen & ,unterwegs sein` & pad (,Weg') \\
\hline Ram, der & ,Widder & ram (,Widder') \\
\hline Rivier, das & ,Trockenfluss[bett]‘ & rivier (,Fluss`) \\
\hline Vley, das & $\begin{array}{l}\text {,Senke, die sich in der Regenzeit } \\
\text { mit Wasser füllt" }\end{array}$ & $\begin{array}{l}\text { vallei (,Tal') bzw. } \\
\text { vlei (,Sumpfland, Moor })\end{array}$ \\
\hline
\end{tabular}

Aus dem Englischen, und zwar teilweise aus dem südafrikanischen Englisch (SAE), sind Namibismen wie die folgenden entlehnt.

\begin{tabular}{|c|c|c|}
\hline Namibismus & Bedeutung & englischer Urspung \\
\hline Bakkie, der & ,Pick-up[-truck]' & bakkie (SAE) \\
\hline Biltong, das & ,Trockenfleisch, Dörrfleisch` & biltong (SAE) \\
\hline Depositum, das & ,[Miet]kaution“ & deposit \\
\hline $\begin{array}{l}\text { Permit, das } \\
\text { und andere }\end{array}$ & ,Genehmigung einer Behörde‘ & permit \\
\hline
\end{tabular}

Weitere Namibismen sind nicht auf Entlehnung zurückzuführen, oder ihre Herkunft ist unklar: 


\section{Namibismus}

etwas befestigen

Damm

Einschwörung

Fellchen

Gämsbock

Kamp, der

Klippe

Magistratsgericht

Matrikulant/in

Panga, der/die

Veld, das

und andere

\section{Bedeutung oder deutsche Entsprechung}

,etw. [z. B. einen Termin] bestätigen“

,Speicherbecken eines Stausees“

,Vereidigung“

,Lammfell`

,Oryx-Antilope‘

,eingezäunte Fläche‘

,Stein“

$=$ Amtsgericht $\mathrm{CH} \mathrm{D}$

= Abiturient $\mathrm{D}$

,Buschmesser

,Savanne،

Der wichtigste Modelltext der deutschen Standardvarietät in Namibia ist die Allgemeine Zeitung (AZ, Windhoek, Namibia), die in einer Auflagenstärke von rund 4.000 Exemplaren von montags bis freitags erscheint (vgl. AZ-Profil) und für viele deutschsprachige Namibier eine wichtige Informationsquelle darstellt. In ihren standardsprachlichen Texten erscheinen die oben genannten Namibismen, ohne in irgendeiner Form als umgangs- oder nonstandardsprachlich gekennzeichnet zu sein.

Damm: Mit einem Volumen von 331,7 Millionen $\mathrm{m}^{3}$ beinhalten die südlichen Dämme [...] auf jeden Fall genügend Trinkwasser. (AZ, 23. 3. 2017)

Panga: [...] habe die Einheit am nächsten Tag auf der genannten Farm einen Mann mit einem Panga gesichtet und ihn für einen mutmaßlichen Wilderer gehalten. (AZ, 9.1. 2017)

Rivier: Auch der Fahrer dieses Pickups hat sich offensichtlich überschätzt, als er am vergangenen Sonntag mit seinem Auto das sandige OmaruruRivier durchqueren wollte. $(A Z, 3.4$. 2017)

Veld: Indessen ist die junge, angeschossene Nashornkuh aus Gobabis nun doch im Veld verendet. $(A Z, 8.2 .2017)$

Die Gruppe der Sprachnormautoritäten wird durch namibische Lehrkräfte vertreten, die nach Angaben des VWD (2016: LXII) die Namibismen in ihrem Korrekturverhalten als korrekt anerkennen. Untersuchungen von Sprachexperten zum namibischen Deutsch waren bislang oft eindimensional, da weder der Begriff Namibismus noch die Existenz von mehreren Varietäten des Deutschen in Namibia berücksichtigt wurden (z. B. Namslang; vgl. KellermeierRehbein 2015). Man war sich weitgehend einig, dass das dort verwendete 
Sprachsystem „Südwesterdeutsch“ kein Dialekt sei (vgl. Böhm 2003: 564-565; Pütz 1991: 464). Davon abgesehen gingen die Meinungen jedoch auseinander. Gretschel (1995: 306) bezeichnete es als „Umgangssprache“ und grenzte es explizit vom „Hochdeutschen“ ab. Shah (2007: 21) machte dagegen den zaghaften Versuch, von einer namibischen Standardvarietät des Deutschen zu sprechen, was sie aber sogleich wieder relativierte (vgl. Kellermeier-Rehbein 2016: 226).

Zusammenfassend kann festgehalten werden, dass die Namibismen Bestandteile einer spezifisch namibischen Standardvarietät der deutschen Sprache sind, die man - mit gewissen Einschränkungen - aufgrund ihrer Geschichte mit Clyne (1992: 3) als „(neo)koloniale Nationalvarietät“ bezeichnen könnte. Dabei handelt es sich um eine Varietät, die durch Kolonialismus in ein anderssprachiges Gebiet kam und dort zur offiziellen oder inoffiziellen Landessprache wurde, aber für die Bevölkerungsmehrheit eine Zweit- oder Fremdsprache ist (vgl. Kellermeier-Rehbein 2016: 227). Allerdings wäre im Falle Namibias die Bezeichnung postkoloniale Standardvarietät (statt neokoloniale Nationalvarietät) angemessener, da der Kolonialismus beendet ist und es sich bei den Deutschnamibiern nicht um eine Nation, sondern um eine Minderheit handelt.

\subsection{Mennonitensiedlungen}

Die Mennoniten sind eine christliche Glaubensgemeinschaft, die im Zuge der Reformation in den Niederlanden entstand und aufgrund ihrer religiösen Ansichten (Erwachsenentaufe, Pazifismus und anderes) immer wieder mit der Obrigkeit in Konflikt kam. Daher wanderten sie in der Mitte des 16. Jahrhunderts zunächst nach Westpreußen, wo sie den niederdeutschen Dialekt Plautdietsch übernahmen, und emigrierten dann im 18. Jahrhundert nach Russland. Als ihnen dort gegen Ende des 19. Jahrhunderts die allgemeine Wehrpflicht drohte, ließen sich die Russlandmennoniten auf dem amerikanischen Kontinent nieder (z. B. in Kanada, USA, Mexiko und anderen Staaten) (vgl. Ammon 2015: 382), wo sie sich bis heute von der Mehrheitsbevölkerung fernhalten und in mehr oder weniger isolierten Siedlungsgebieten leben.

Das VWD (2016) verzeichnet Varianten der Mennoniten, die Nachfahren der Russlandmennoniten sind. Die Erhebungen fanden ausschließlich in Mexiko statt, aber die Autoren des VWD (2016: LXII) gehen davon aus, dass diese Ausdrücke auch bei Mennoniten in den oben genannten anderen Staaten des amerikanischen Kontinents gebräuchlich sind. Dies ist darauf zurückzuführen, dass die Mennonitensiedlungen ein Spracharchipel bilden, in dem Deutsch als „muttersprachliche Brückensprache“ (Ammon 2015: 391) dient, um mit Glaubensbrüdern über anderssprachige Gebiete hinweg zu kommunizieren. 
Die Russlandmennoniten sprechen zum Teil bis heute noch Plautdietsch sowie ein archaisch anmutendes Hochdeutsch (vgl. Ammon 2015: 382). Letzteres wird vor allem im Gottesdienst, in der Schule und in anderen formellen Situationen verwendet (vgl. VWD 2016: LXII). Als Modelltexte können die Wochenzeitungen Kurze Nachrichten aus Mexiko, Deutsch-mexikanische Rundschau oder die Zeitschrift Der Leserfreund genannt werden, aus denen das VWD seine Belege bezieht.

Die standardsprachlichen Varianten der Mennoniten beruhen, ebenso wie in den anderen Viertelzentren, zum Teil auf Entlehnungen. Die Spendersprachen sind, wie nicht anders zu erwarten, Englisch und Spanisch; vgl. dazu die folgenden Beispiele aus dem VWD (2016):

\begin{tabular}{|c|c|c|}
\hline $\begin{array}{l}\text { Mennoniten- } \\
\text { Variante }\end{array}$ & Bedeutung & spanischer Ursprung \\
\hline Diputado, der & $\begin{array}{l}\text {,gewähltes Mitglied } \\
\text { eines Parlaments“ }\end{array}$ & diputado (,Abgeordneter') \\
\hline Graduation & $\begin{array}{l}\text {,Abschluss } \\
\text { der Schulausbildung }\end{array}$ & $\begin{array}{l}\text { Graduación } \\
\text { (,Graduierung‘) }\end{array}$ \\
\hline Munizip, das & $\begin{array}{l}\text {,Verwaltungsbezirk, } \\
\text { Gemeinde' }\end{array}$ & $\begin{array}{l}\text { Municipio } \\
\text { (,(Stadt)Gemeinde‘) }\end{array}$ \\
\hline Prepa, die & ,Gymnasium‘ & $\begin{array}{l}\text { Preparatoria } \\
\text { (,Vorbereitungskurs') }\end{array}$ \\
\hline Remolque, der & ,LKW-Anhänger‘ & remolque (,Anhänger`) \\
\hline $\begin{array}{l}\text { Mennoniten- } \\
\text { Variante }\end{array}$ & Bedeutung & $\begin{array}{l}\text { englischer Ursprung/ } \\
\text { Lehnübersetzung aus ... }\end{array}$ \\
\hline Grünhaus & ,Gewächshaus“ & green house \\
\hline Hochweg & ,Autobahn` & highway \\
\hline Luftblase & ,Airbag' & airbag \\
\hline Seida, der & ,Limonade‘ & cidre (,Apfelwein') \\
\hline Trubel, der & ,Unannehmlichkeiten` & trouble \\
\hline
\end{tabular}

Übernahmen aus dem niederdeutschen Mennoniten-Dialekt sind die Wörter drock (,von Arbeitsüberlastung gekennzeichnet'), jankern (,bei jemandem Verlangen auslösen') und Vaspa (,Zwischenmahlzeit am frühen Nachmittag'). Andere Varianten sind nicht auf Entlehnung zurückzuführen: Acker (,4.000 Quadratmeter'), Kornkuchen (,Tortilla'), Ohm (,Prediger einer Gemeinde'), Weltmensch (,nicht zur christlichen bzw. mennonitischen Gemeinde gehörende Person') und andere. 


\section{Spracherhalt und Entstehung von Standardvarietäten außerhalb der Amtssprachregion}

Die oben erfolgte exemplarische Dokumentation der lexikalischen Standardvariation in Rumänien, Namibia und den Mennonitensiedlungen wirft die Frage auf, warum die deutschstämmigen Minderheiten ihre Herkunftssprache nicht nur bewahrten, sondern darüber hinaus sogar jeweils eigene Standardvarietäten entwickelten, anstatt sich einfach der bereits existierenden Schriftsprache Deutschlands oder Österreichs zu bedienen.

Ein wichtiger Grund für den Spracherhalt ist die Sicherung der Kommunikation mit Mitgliedern der internationalen deutschsprachigen Gemeinschaft. Damit können Kontakte zur Bevölkerung der Herkunftsländer aufrecht erhalten oder neu geknüpft werden, was unter Umständen für wirtschaftliche, politische oder kulturelle Zusammenarbeit vorteilhaft ist. Im „neuen“ Lebensraum dient das Festhalten an der Herkunftssprache auch zur Abgrenzung von der Mehrheitsbevölkerung oder anderen Minderheiten.

Die Hauptursache für die Entstehung spezifischer Standardvarietäten ist in der Isolierung vom zusammenhängenden deutschen Sprachgebiet anzunehmen. Historisch gesehen führten die zum Teil beträchtlichen Entfernungen zwischen den alten und neuen Siedlungsgebieten zur Beeinträchtigung oder gar Aufgabe der Kontakte zu den Herkunftsländern. Daher nahmen die Varietäten der Minderheiten nicht an den gleichen Sprachwandelprozessen teil wie diejenigen der Vollzentren, sodass sich Lexik und Grammatik unterschiedlich entwickelten. Insbesondere die geographischen, kulturellen, politischen oder klimatischen Bedingungen der neuen Umgebung erforderten eine Anpassung oder Erweiterung der Lexik, die häufig durch Entlehnungen aus Kontaktsprachen erfolgte.

Ein weiterer wichtiger Grund für die Herausbildung von eigenen Standardvarietäten entspringt aus dem Wunsch, die ethnische, kulturelle oder religiöse Identität der Minderheit auch gegenüber den Bewohnern Deutschlands, Österreichs und der Schweiz sprachlich zu untermauern. Oben wurde bereits deutlich, dass eine wichtige Funktion der nationalen Varietäten die Kenntlichmachung der nationalen Identität und staatlichen Eigenständigkeit ist. Dies spielt in den Viertelzentren keine Rolle, da es sich nicht um eigenständige Nationen handelt, sondern um Minderheiten, die Teil einer Nation sind. Stattdessen kann eine eigene Standardvarietät die Identität einer Minderheit unterstreichen, die sich durch eine bestimmte Herkunft und Geschichte auszeichnet und gleichzeitig Teil einer neuen Gesellschaft ist. 
Nicht zuletzt sind die Gründe für den Erhalt der deutschen Sprache und die Herausbildung spezifischer Standardvarietäten in den Viertelzentren auch in zwei diametral verschiedenen, weltweit zu beobachtenden Prozessen auszumachen. Die Rede ist hier von der Globalisierung und der gleichzeitig sich vollziehenden Regionalisierung, wobei letztere in Form einer Zuwendung zum Regionalen erfolgt. Im Zuge der weltweiten Globalisierung, bei der internationale Zusammenarbeit in Handel, Politik, Kultur, Verkehr, Wissenschaft etc. immer wichtiger und allgegenwärtiger wird, verliert die Nation als Identitätsstifterin für nicht wenige Menschen an Bedeutung. Wer an der Globalisierung teilhat, sieht sich weniger als Bürger einer bestimmten Nation, denn als Mitglied einer transnationalen, über die Staatsgrenzen hinweg agierenden Gemeinschaft (vgl. Ammon 2015: 83). Gleichzeitig ist bei vielen das Bedürfnis nach Einbindung in eine Gruppe auszumachen, deren Mitglieder gleiche oder ähnliche Lebensentwürfe und Wertvorstellungen aufweisen. Der entgrenzenden Globalisierung und dem damit einhergehenden Verlust der nationalen Bindung wird dann nicht selten eine identifikatorische Anbindung an die heimische Region entgegengesetzt. Die Verwendung von Dialekten, Regional- oder Minderheitensprachen kann dabei eine kollektive sprachliche und kulturelle Identität stiften, die dem Individuum die Orientierung an gemeinsamen Werten, Anschauungen und Verhaltensmustern ermöglicht.

Diese allgemeinen Gründe für Spracherhalt und die Herausbildung eigener Standardvarietäten gelten im Grunde für alle Minderheiten. Zusätzlich spielen in jedem Viertelzentrum spezifische Faktoren eine Rolle, die im Folgenden kurz skizziert werden.

Der Spracherhalt der Rumäniendeutschen wurde durch den Umstand erleichtert, dass die Gebiete Siebenbürgen und Banat vom 17. Jahrhundert bis 1918 zu Ungarn gehörten und daher Teil der Habsburgermonarchie waren. Nach Lǎzǎrescu \& Scheuringer (2013: 421-422) konnte die deutschsprachige Bevölkerung unter dem Schutz der österreichischen Staatsmacht ihre Herkunftssprache bewahren und pflegen. Darüber hinaus baute sie eine solide Infrastruktur für deutschsprachige Bildung, Kultur und Presse auf, was auch später von rumänischer Seite nie behindert oder gar sanktioniert wurde. Dies führte nach Ansicht der Autoren (vgl. Lǎzărescu \& Scheuringer 2013: 422) dazu, dass die deutschsprachige Minderheit in Rumänien heutzutage überproportional gebildet und wohlhabend ist. Ihre gute Ausbildung sei ferner mit der großen Auswanderungswelle der Jahre 1990/91 zu begründen, die zu einer Überdimensionierung des muttersprachlichen Bildungssystems geführt habe. Daher ist der Erhalt der Sprache ein Mittel, die Zugehörigkeit zu dieser privilegierten Gruppe zu signalisieren und von dem gut ausgebauten Bildungssystem zu profitieren. Gleichzeitig sind das Schulsystem und die rumäniendeutsche Presse 
ein wirksames Mittel, um die im Laufe der Zeit durch österreichische Einflüsse und Kontakte zu anderen Sprachen entstandenen Rumänismen überregional unter den Mitgliedern der Minderheit zu verbreiten.

Wie oben bereits erwähnt wurde, sind die Deutschstämmigen in Namibia wirtschaftlich sehr einflussreich und stellen viele Geschäftsleute und Arbeitgeber. Daher berichtet Pütz (1991: 469), dass ihre Sprache mit hohem sozialem und wirtschaftlichem Status assoziiert wird, wodurch man sich von anderen ethnischen Gruppen des Landes abgrenzen kann. Aufgrund des wirtschaftlichen Einflusses der Sprecher erleichtert Deutsch auch für andere Bevölkerungsgruppen in Namibia den Zugang zu begehrten Arbeitsplätzen und gesellschaftlichen Aufstieg. Für den Spracherhalt spricht ferner die Tatsache, dass es keine eindeutige Alternative gibt, für die man Deutsch hätte aufgeben können. Es existiert zwar eine Mehrheitssprache (Oshiwambo), doch ist sie Muttersprache von nur knapp der Hälfte der Bevölkerung und war nie staatliche Amtssprache. Im Laufe der Geschichte übernahmen erst Deutsch, dann Afrikaans und Englisch, und seit der Unabhängigkeit nur letztgenannte diese Funktion. Mit ihrer Herkunftssprache demonstrieren die Sprecher nicht zuletzt ihre Auffassung, dass auch sie wahre Repräsentanten der deutschen Kultur sind (vgl. Gretschel 1995: 306).

Die namibische Standardvarietät ist ein wichtiges Symbol für die „südwesterdeutsche“ Identität, wie Gretschel (1995: 306) sie wenige Jahre nach der Unabhängigkeit nannte (heutzutage wäre Identität der Deutschnamibier eine angemessenere Bezeichnung). Sie ergibt sich aus den von Europa stark abweichenden Lebensumständen, der wechselvollen Geschichte der Minderheit, ihrem Prestige sowie der multilingualen Gesellschaft, in der sie lebt. Der namibische Standard unterstreicht die Gemeinschaft der Minderheit und grenzt gleichzeitig von den übrigen Deutschsprachigen in Europa ab. Nicht umsonst nennen sich die Mitglieder der Minderheit selbst „Deutsche“, obwohl sie natürlich die namibische Staatsangehörigkeit haben, während sie die Bürger der Bundesrepublik als „Deutschländer“ bezeichnen (vgl. Gretschel 1995: 306).

Die Mennoniten hätten Deutsch zugunsten der Amtssprachen des amerikanischen Kontinents aufgeben können, denn im Vergleich zu den beiden anderen Minderheiten ist anzunehmen, dass die Religionsgemeinschaft keinen Wert auf die Aufrechterhaltung der Kommunikation mit den Deutschsprachigen in Europa legt, es sei denn mit anderen dort lebenden Mennoniten. Aufgrund der wiederholten Wanderungen innerhalb Europas und zum amerikanischen Kontinent ist die historische und identifikatorische Distanz besonders groß. Darüber hinaus bestehen zwischen Mennoniten und deutschsprachigen Europäern große sozialpsychologische Mentalitätsunterschiede, denn das religiöse Leben ist für Mennoniten besonders wichtig und prägt ihr Alltagsleben nachhaltig. 
Da sie in Europa aufgrund ihrer Ansichten Verfolgungen ausgesetzt waren, erfolgte die Emigration aus dem Wunsch heraus, ihren Glauben gänzlich ausleben zu können, was für sie wichtiger war als nationale, ethnische oder kulturelle Zugehörigkeit. Aus diesen Gründen gibt es zwischen Mennoniten und der internationalen deutschen Sprachgemeinschaft - wenn überhaupt - nur eine geringe Bindung, die Kontakte verzichtbar macht. Zuweilen sind Begegnungen mit Deutschsprachigen nicht einmal gern gesehen, weil man befürchtet, dass unerwünschte Einflüsse von außen eingeschleppt werden könnten, die womöglich den Glauben unterminieren (vgl. Ammon 2015: 369).

Dennoch haben die Mennoniten die deutsche Sprache erhalten, da viele Mitglieder der Religionsgemeinschaft isoliert in sogenannten „Kolonien“ leben (Ammon 2015: 381, 390) und daher nicht unmittelbar auf andere Sprachen angewiesen sind. In Mexiko beherrschen zwar einige, vor allem männliche Angehörige der Minderheit auch die Amtssprache Spanisch (Kaufmann 1997: 144), doch distanzieren sie sich aufgrund ihrer stark religiös geprägten Wertvorstellungen in der Regel von Nicht-Mennoniten. Dadurch ergibt sich weder eine Vermischung mit der Mehrheitsbevölkerung noch eine Assimilation an deren Kultur und Lebensweise.

Der Erhalt des Deutschen ermöglicht der Religionsgemeinschaft darüber hinaus die Aufrechterhaltung der Kommunikation mit anderen auf dem amerikanischen Kontinent siedelnden Mennonitengruppen, mit denen sie im oben genannten Spracharchipel über weite Distanzen hinweg auf Deutsch Kontakte pflegen (Ammon 2015: 390). Nicht zu unterschätzen ist schließlich die Tatsache, dass ihre religiösen Texte auf Deutsch vorliegen (Kaufmann 1997: 67), was den Spracherhalt unterstützt.

Die Entwicklung einer mennonitischen Standardvarietät beruht auf den Lebensumständen auf dem amerikanischen Kontinent und dem, wenn auch nur spärlichen, Kontakt zur Mehrheitsbevölkerung. Sie findet ihre mündliche und schriftliche Anwendung vor allem im Gottesdienst und bei der Rezeption religiöser Texte, aber ebenso in der Schule oder in formellen Situationen (VWD 2016: 388-389) und dient darüber hinaus der Abgrenzung von deutschsprachigen Nicht-Mennoniten.

\section{Fazit}

Der Beitrag präsentierte die plurizentrische Standardvariation des Deutschen innerhalb und außerhalb Europas am Beispiel der Lexik. Staaten und Regionen im Amtssprachgebiet verfügen über individuelle Standardvarianten und -varietäten, so dass diese Gebiete sogenannte Sprachzentren bilden und Deutsch 
zu den plurizentrischen Sprachen gehört. In der Fachwelt ist dies inzwischen weitgehend anerkannt, auch wenn zum Teil Uneinigkeit bezüglich der Frage besteht, ob die Standardvariation als plurizentrisch oder pluriareal zu bezeichnen sei. Unter Laien ist das Bewusstsein von der Standardvariation am wenigsten in Deutschland ausgeprägt, wo man in der Regel irrtümlich davon ausgeht, dass es nur eine einheitliche Sprachnorm für alle Sprecher im gesamten deutschsprachigen Raum gebe. Österreicher und Schweizer sind für die Plurizentrik des Deutschen deutlich stärker sensibilisiert und ihre eigenen Standardvarietäten sind wichtige Symbole ihrer nationalen Identität. Im Ausdruck der Gruppenidentität durch Sprache ist auch die Motivation der deutschsprachigen Minderheiten in Rumänien, Namibia und den Mennonitensiedlungen für die Entwicklung eigener Standardvarietäten des Deutschen zu sehen. Durch die Verwendung einer eigenen Standardvarietät der plurizentrischen Sprache Deutsch drücken sie im Sinne von Clyne (2000: 2008) ihre Identität als deutschstämmige Minderheiten innerhalb anderssprachiger Gesellschaften aus, ohne sich dabei kommunikativ von der internationalen deutschsprachigen Gemeinschaft abzusondern.

\section{Literatur}

Ammon, Ulrich (1995): Die deutsche Sprache in Deutschland, Österreich und der Schweiz. Das Problem der nationalen Varietäten. Berlin, New York: de Gruyter.

Ammon, Ulrich (2005): Standard und Variation: Norm, Autorität, Legitimation. In Ludwig M. Eichinger \& Werner Kallmeyer (Hrsg.), Standardvariation. Wie viel Variation verträgt die deutsche Sprache?, 28-40. Berlin, New York: de Gruyter.

Ammon, Ulrich (2015): Die Stellung der deutschen Sprache in der Welt. Berlin, München, Boston: de Gruyter.

Ammon, Ulrich, Hans Bickel, Jakob Ebner et al. (2004): Variantenwörterbuch des Deutschen. Die Standardsprache in Österreich, der Schweiz und Deutschland sowie in Liechtenstein, Luxemburg, Ostbelgien und Südtirol. Berlin, New York: de Gruyter. [VWD 2004].

Ammon, Ulrich, Hans Bickel \& Alexandra Lenz (Hrsg.) (2016): Variantenwörterbuch des Deutschen. Die Standardsprache in Österreich, der Schweiz, Deutschland, Liechtenstein, Luxemburg, Ostbelgien und Südtirol sowie Rumänien, Namibia und Mennonitensiedlungen. 2. Aufl. Berlin, Boston: de Gruyter. [VWD 2016].

AZ-Profil = Allgemeine Zeitung - ein Kurzprofil. URL: https://www.az.com.na/page/az-profil/ (letzter Zugriff 4. 4. 2017).

Böhm, Michael Anton (2003): Deutsch in Afrika. Die Stellung der deutschen Sprache in Afrika vor dem Hintergrund der bildungs- und sprachpolitischen Gegebenheiten sowie der deutschen Auswärtigen Kulturpolitik. Frankfurt a. M. u. a.: Peter Lang.

Clyne, Michael (1992): Pluricentric languages - Introduction. In Michael Clyne (Hrsg.), Pluricentric languages. Differing norms in different nations, 1-9. Berlin, New York: de Gruyter.

Clyne, Michael (2000): Varianten des Deutschen in den Staaten mit vorwiegend deutschsprachiger Bevölkerung. In Werner Besch, Anne Betten, Oskar Reichmann \& 
Stefan Sonderegger (Hrsg.), Sprachgeschichte. Ein Handbuch zur Geschichte der deutschen Sprache und ihrer Erforschung, 2008-2016 (= HSK-Band 2.2, 2. Aufl.). Berlin, New York: de Gruyter.

Glauninger, Manfred (2013): Deutsch im 21. Jahrhundert: "pluri-«, »supra-« oder "postnational«? In Ingeborg Fiala-Fürst, Jürgen Joachimsthaler \& Walter Schmitz (Hrsg.), Mitteleuropa. Kontakte und Kontroversen. Dokumentation des II. Kongresses des Mitteleuropäischen Germanistenverbandes (MGV) in Olomouc/Olmütz, 459-465. Dresden: Thelem.

Gretschel, Hans-Volker (1993): The lost Umlaut: The German language in Namibia. Logos: A Publication of the Academy Windhoek 13, 44-60.

Gretschel, Hans-Volker (1995): The status and use of the German language in independent Namibia: Can German survive the transition? In Martin Pütz (Hrsg.), Discrimination through language in Africa? Perspectives on the Namibian experience, 299-313. Berlin, New York: de Gruyter.

Hägi, Sara (2014): Eine Tüte voller Leckerbissen. Feinheiten der deutschen Sprache aus plurizentrischer Sicht. ide Informationen zum Deutschunterricht. Zeitschrift für den Deutschunterricht in Wissenschaft und Schule. Themenheft Österreichisches Deutsch und Plurizentrik, hrsg. von Jutta Ransmayr, Andrea Moser-Pacher \& Ilona Elisabeth Fink 38 (3), 69-77.

Haugen, Einar (1997): Language standardization. In Nikolas Coupland \& Adam Jaworski (Hrsg.), Sociolinguistics. A reader and coursebook, 341-352. Basingstoke, New York: Palgrave Macmillan.

Kaufmann, Göz (1997): Varietätendynamik in Sprachkontaktsituationen. Attitüden und Sprachverhalten rußlanddeutscher Mennoniten in Mexiko und den USA. Frankfurt a. M. u. a.: Peter Lang.

Kellermeier-Rehbein, Birte (2013): Standard oder Nonstandard? Ungelöste Probleme der Abgrenzung. In Karina Schneider-Wiejowski, Birte Kellermeier-Rehbein \& Jakob Haselhuber (Hrsg.), Vielfalt, Variation und Stellung der deutschen Sprache, 3-22. Berlin, Boston: de Gruyter.

Kellermeier-Rehbein, Birte (2014): Plurizentrik. Einführung in die nationalen Varietäten des Deutschen. Berlin: Erich Schmidt.

Kellermeier-Rehbein, Birte (2015): Namslang - Deutsche Jugendsprache in Namibia? In Corinna Peschel \& Kerstin Runschke (Hrsg.), Sprachvariation und Sprachreflexion in interkulturellen Kontexten, 41-62. Frankfurt a. M. u. a.: Peter Lang.

Kellermeier-Rehbein, Birte (2016): Sprache in postkolonialen Kontexten II: Varietäten der deutschen Sprache in Namibia. In Thomas Stolz, Ingo H. Warnke \& Daniel SchmidtBrücken (Hrsg.), Sprache und Kolonialismus. Eine interdisziplinäre Einführung zu Sprache und Kommunikation in kolonialen Kontexten, 213-234. Berlin, Boston: de Gruyter.

Lăzărescu, loan (2007): Wie stark österreichisch geprägt ist das heutige Rumäniendeutsch? In Walter Schmitz \& Jürgen Joachimsthaler (Hrsg.), Zwischeneuropa/Mitteleuropa. Sprache und Literatur in interkultureller Konstellation, 675-686. Dresden: w.e.b. Thelem.

Lăzărescu, loan (2013): Rumäniendeutsch - eine eigenständige, besondere Varietät der deutschen Sprache. In Karina Schneider-Wiejowski, Birte Kellermeier-Rehbein \& Jakob Haselhuber (Hrsg.), Vielfalt, Variation und Stellung der deutschen Sprache, 369-389. Berlin, Boston: de Gruyter. 
Lăzărescu, loan \& Hermann Scheuringer (2013): Rumänisches Deutsch im Kontakt mit Binnenraumvarietäten. Zu einem neuen Wörterbuch und zu einem neuen Forschungsprojekt. In Ingeborg Fiala-Fürst, Jürgen Joachimsthaler \& Walter Schmitz (Hrsg.), Mitteleuropa. Kontakte und Kontroversen, 421-428. Dresden: w.e.b. Thelem. Nöckler, Herbert C. (1963): Sprachmischung in Südwestafrika. München: Max Huber.

Österreichisches Wörterbuch [1951] (2016). Herausgegeben im Auftrag des

Bundesministeriums für Bildung; Redaktion: Christiane M. Pabst, Herbert Fussy \& Ulrike Steiner. 43. Aufl. Wien: öbv.

Pohl, Heinz Dieter (1997): Gedanken zum Österreichischen Deutsch (als Teil der „pluriarealen“ deutschen Sprache). In Rudolf Muhr \& Richard Schrodt (Hrsg.), Österreichisches Deutsch und andere nationale Varietäten europäischer Sprachen in Europa, 67-87. Wien: Hölder-Pichler-Tempsky.

Pütz, Martin (1991): „Südwesterdeutsch“ in Namibia: Sprachpolitik, Sprachplanung und Spracherhalt. Linguistische Berichte 136, 455-476.

Scheuringer, Hermann (1996): Das Deutsche als pluriareale Sprache: Ein Beitrag gegen staatlich begrenzte Horizonte in der Diskussion um die deutsche Sprache in Österreich. Die Unterrichtspraxis 29 (2), 147-153.

Schmidlin, Regula (2013): Gebrauch und Einschätzung des Deutschen als plurizentrische Sprache. In Karina Schneider-Wiejowski, Birte Kellermeier-Rehbein \& Jakob Haselhuber (Hrsg.), Vielfalt, Variation und Stellung der deutschen Sprache, 23-41. Berlin, Boston: de Gruyter.

Schrodt, Richard (1997): Nationale Varianten, areale Unterschiede und der ,Substandard': An den Quellen des Österreichischen Deutsch. In Rudolf Muhr \& Richard Schrodt (Hrsg.), Österreichisches Deutsch und andere nationale Varietäten europäischer Sprachen in Europa, 12-39. Wien: Hölder-Pichler-Tempsky.

Shah, Sheena (2007): German in a contact situation: The case of Namibian German. eDUSA 2, 20-45.

Trümpelmann, Georg P. J. \& Elfriede Erbe (2015): Woordenboek Afrikaans-Duits Wörterbuch Deutsch-Afrikaans. Gießen: VVB Laufersweiler. 
III Lexikografie 



\title{
Rufus H. Gouws \\ 9 Internet lexicography in the 21st century
}

\begin{abstract}
The era of internet lexicography confronts lexicographers with challenges and opportunities to enhance the quality of the lexicographic practice and to produce dictionaries that help in satisfying the lexicographic needs of the identified target user. This paper focuses on a few dictionary structures that play a significant role in internet dictionaries. The innovative value of dictionary portals as data structures is discussed, with specific reference to portalinternal and dictionary-external structures. Attention is also given to the role of the data distribution structure in allocating data and text types to their appropriate positions and to different ways to employ access structures to achieve rapid access to data. It is indicated that changes in the structures, compared to their occurrence in printed dictionaries, improve the success of dictionary consultation procedure. The paper adheres to the assumption that theoretical lexicography is needed to ensure a successful lexicographic practice.
\end{abstract}

Keywords: access basis, access domain, access routes, access structures data distribution structure, dictionary portal, internet lexicography, outer features, punctual access, systematic access, text compound

\section{Introduction}

The emergence and rapid development of the internet during the last decades of the previous century and the increased development in the early phase of the twenty first century had a comprehensive influence on the planning and production of reference works. Lexicography, a well-established practice with a strong theoretical basis, also became a target of this influence. Early influ-

\footnotetext{
Note: This work is based on research supported in part by the National Research Foundation of South Africa (Grant specific unique reference number (UID) 85434)). The Grant holder acknowledges that opinions, findings and conclusions or recommendations expressed in any publication generated by the NRF supported research are that of the author, and that the NRF accepts no liability whatsoever in this regard.
}

Rufus H. Gouws, Stellenbosch University, Private Bag X1, Matieland 7602, South Africa, E-Mail: rhg@sun.ac.za

Ә Open Access. (c) 2018 Rufus H. Gouws, publiziert von De Gruyter. (c) BY Dieses Werk ist lizenziert unter der Creative Commons Attribution 4.0 Lizenz. https://doi.org/10.1515/9783110538588-010 
ences of new technologies on the lexicographic practice became apparent in the last decades of the previous century with a movement in the field of printed dictionaries towards computational lexicography, machine-readable dictionaries and eventually dictionaries presented on CD-ROM. Although these dictionaries were mostly digital versions of printed dictionaries their occurrence introduced the transition from printed to digital dictionaries. The most significant phase in this transition came some years later with the introduction of internet dictionaries, heralding a wholly new era in the field of lexicography.

Internet lexicography, often also referred to as online lexicography, does not only represent a new medium for lexicographic work but it also offers a plethora of innovative possibilities to enhance the speed and success of dictionary consultation procedures and to enable lexicographers to improve the extent and presentation of data in their dictionaries. With regard to printed dictionaries it has been indicated that other reference sources often show specific similarities with dictionaries, cf. Wiegand (2013). Wiegand (2013a) already said that he knows no other scientific practice with so many interdisciplinary relations as lexicography. Internet lexicography also relies heavily on an interactive relation with other disciplines. Especially the relation with information science, cf. Fuertes-Olivera \& Nielsen (2012), is important when discussing the planning and production of internet dictionaries. As is the case with printed lexicography, internet lexicography should not be isolated from other reference sources but rather be situated within the broad spectrum of online information tools, cf. Bergenholtz, Bothma \& Gouws (2015).

The formulation of lexicographic theories had primarily been directed at printed dictionaries, cf. Gouws (2011). The question arises whether a new theory should be devised for online lexicography, resulting in separate theories for printed and online lexicography, or whether existing theory should be adapted to provide for both printed and online dictionaries. Gouws (2011: 24) argues in favor of a general unified theory that is not restricted in its scope to a single medium. It implies that certain aspects of a given unified theory will primarily or even exclusively be directed at printed dictionaries, other at internet dictionaries but there will also be features relevant to both printed and online dictionaries. This is the approach adhered to in the current paper.

\section{Internet lexicography: practice and theory?}

When discussing a broad topic like "Internet lexicography in the 21st century" one should not only focus on the lexicographic practice but should also pay 
attention to the role of lexicographic theory. With regard to printed dictionaries it cannot be denied that the lexicographic practice is much older than lexicographic theory. In the early phases of its development lexicographic theory had to catch up with centuries of development in the lexicographic practice. In the course of time the development in the theory of lexicography caught up with the lexicographic practice and reached a stage where metalexicographers did not only have to look at existing dictionaries to expand theoretical insights but the theory provided a basis from which suggestions could be made for future improvements and innovative procedures in the lexicographic practice.

The relation between theory and practice in the domain of printed dictionaries was balanced quite well when the transition to online dictionaries commenced. One would have expected that theory would have played a dominant role in the planning and eventual preparation of online dictionaries. Unfortunately, too few lexicographers of online dictionaries negotiated the possible assistance from the side of lexicographic theory and too few theoretical lexicographers pre-empted the needs brought about by a change in medium in the lexicographic practice. As a result, the lexicographic practice was once again isolated from influences from the field of lexicographic theory. As was the situation in the case of printed dictionaries, theory once again had to play a catching up game in order to provide a theoretical foundation for the online lexicographic practice and a point of departure in the planning and production of further online dictionaries. Fortunately, the last few years witnessed an extremely positive increase in the publication of theoretical contributions within the field of internet lexicography, e.g., De Schryver (2003), Engelberg \& Lemnitzer (2009), Fuertes-Olivera \& Bergenholtz (2011), Granger \& Paquot (2012), Müller-Spitzer (2014), Fuertes-Olivera \& Tarp (2014), a major component of the volume Gouws et al. (2013), Hildenbrandt \& Klosa (2016) and Klosa \& Müller-Spitzer (2016).

This paper will not try to reflect on all the salient issues discussed in these publications but will rather focus on some of the theoretically relevant features coming to the fore in some online dictionaries. The discussion will primarily be directed at various aspects of lexicographic structures. In this regard it has to be determined whether structures devised for printed dictionaries are relevant to internet dictionaries.

In the planning of any dictionary, irrespective of the medium, there will always be contents and the selection of the contents needs to be done in accordance with the needs and reference skills of the intended target users, culminating in the identification of the specific function(s) of the specific dictionary. Adhering to the identified function(s) of the dictionary, the contents should then be presented, and a prerequisite of these presentation procedures is the existence and proper utilization of different relevant dictionary structures. 
Functions, contents and structures can be regarded as three integral components of dictionaries.

If the trio constituted by functions, contents and structures should also form the basis of internet dictionaries few questions will be raised regarding the position of contents and functions because their role is self-evident. The same cannot be said about dictionary structures. A real metalexicographic issue in the transition from printed to internet dictionaries regards the notion of dictionary structures. Whereas contents and functions can maintain more or less the same role in internet dictionaries compared to their role in printed dictionaries, dictionary structures in the online medium often differ from those in the printed medium. Seminal work on dictionary structures in numerous articles by the leading German metalexicographer Herbert Ernst Wiegand, cf. Wiegand (1989a, 1989b, 1989c, 1996, 2003, 2005, 2008, 2009), Wiegand \& Beer (2013), Wiegand, Beer \& Gouws (2013), Wiegand \& Gouws (2013), Wiegand \& Smit (2013), focused exclusively on printed dictionaries - as explicitly stated in the titles of a number of these articles. A question that need to be answered is to what extent these structures can also prevail in online dictionaries.

This paper works with the assumption that certain structures remain more or less the same in internet dictionaries, some structures will be restricted to printed dictionaries, some structures will be significant in both printed and online dictionaries although certain adaptations need to be made for the online environment, whereas there will also be some new structures introduced for the online medium. The focus in this paper will only be on some of the structures relevant to internet dictionaries.

\section{Dictionary structures}

In this section some structures relevant to internet lexicography will be discussed. Some of these structures are taken from the domain of printed dictionaries and require a lesser or greater adaptation when used in online dictionaries. Some of the structures have emerged in internet dictionaries. Attention will be given to some aspects of dictionary portals, the data distribution structure and the access structure. Reference will also be made to the frame structure and the accompanying role of outer features. 


\subsection{Dictionary portals}

\subsubsection{A new type of lexicographic structure}

One of the new concepts in internet lexicography is that of dictionary portals as discussed in recent publications like Engelberg \& Müller-Spitzer (2013). Dictionary portals are collections of electronic dictionaries. According to Engelberg and Müller-Spitzer (2013: 1023) the word portal within the internet domain refers to "internet pages that are designed to serve as starting points for searches for information in particular thematic domains".

Engelberg \& Müller-Spitzer (2013: 1024) use the term dictionary portal to refer to a specific type of data structure:

(i) that is presented as a page or set of interlinked pages on a computer screen and

(ii) provides access to a set of electronic dictionaries,

(iii) where these dictionaries can also be consulted as standalone products.

The emergence of dictionary portals introduced a new type of lexicographic structure. Where printed dictionaries are standalone products with dictionaryinternal structures, the online environment offers the possibility of dictionaryexternal lexicographic structures, as seen in dictionary portals. The dictionary portal is not a dictionary structure but rather a lexicographic structure.

According to Bergenholtz, Tarp \& Wiegand (1999: 1778) the data distribution structure determines the venues of all data to be included in a dictionary as a text compound or "big text", also known as a carrier of text types, as suggested by Kammerer and Wiegand (1998: 224). In internet lexicography the notion of a dictionary as a carrier of text types can be complemented by the notion of a dictionary portal as a carrier of dictionaries. Structures do not only prevail within the individual dictionaries but also within the dictionary portal as such and these structures need to be accounted for in metalexicographic research in order to improve and possibly expand their use and function in the lexicographic practice.

Engelberg \& Müller-Spitzer (2013: 1027) distinguish between three types of dictionary portals, i.e. a dictionary net, a dictionary search engine and a dictionary collection. The present paper will not deal with the differences but will only refer to dictionary collections, according to Engelberg \& Müller-Spitzer (2013: 1028) the simplest example of dictionary portals. Dictionary collections often are websites with links to online dictionaries but Engelberg \& MüllerSpitzer $(2013: 1028,1029)$ also make provision for cases where the portal and the collection of dictionaries form a unit because the portal and the dictionaries have the same owner, with all dictionaries presented within the interface 
of the portal. This, the simplest type of dictionary collection, is the type of dictionary portal to be discussed in this paper.

\subsubsection{Structuring the opening page and ordering the selection of dictionaries of a dictionary portal}

As a data structure one of the significant features of dictionary portals is the presentation of a variety of dictionaries, usually on a single page. A dictionary portal functions as a point of departure from where different access routes can be employed that lead to different dictionaries and different items in these dictionaries. The user has the option to access either a single dictionary or to find the same search result in more than one or in all the dictionaries included in the portal.

The opening page of the portal of PharosAanlyn does not show the collection of dictionaries but merely indicates that the search will be executed in a collection of 31 dictionaries, as seen in Figure 9.1. A user has the opportunity to type the required word into the search box without knowing the different dictionaries to which this search option will guide him.

If the user requires information on the collection of dictionaries it can be obtained by clicking on the link "Verander" (= Change). The subsequent screenshot will display the full collection of dictionaries, a part of which can be seen in Figure 9.2.

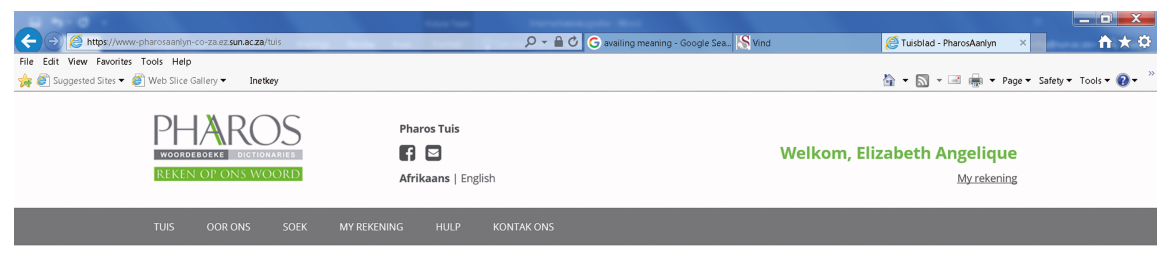

Soek op PharosAanlyn

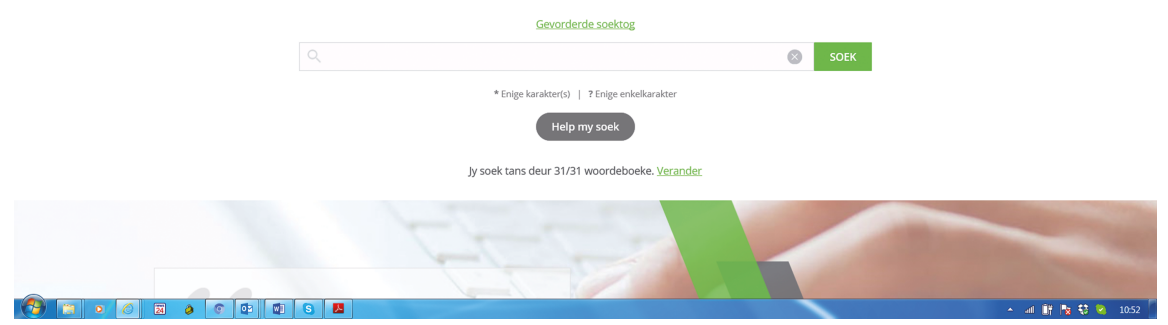

Fig. 9.1: Opening page of PharosAanlyn. 


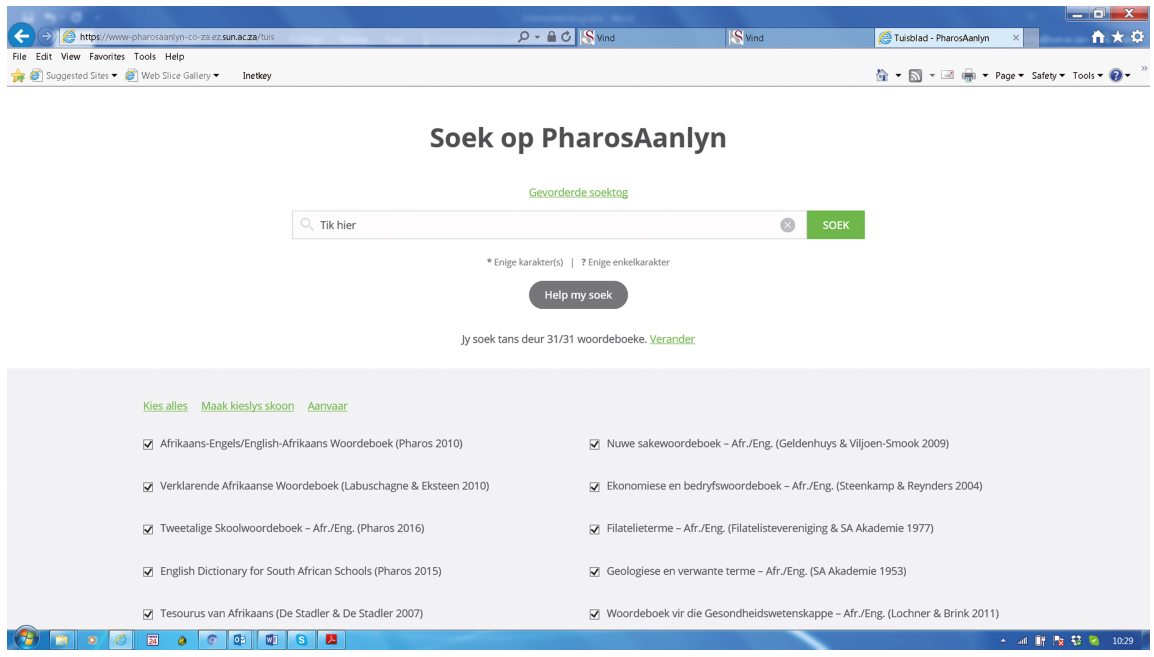

Fig. 9.2: Part of the dictionary collection in PharosAanlyn.

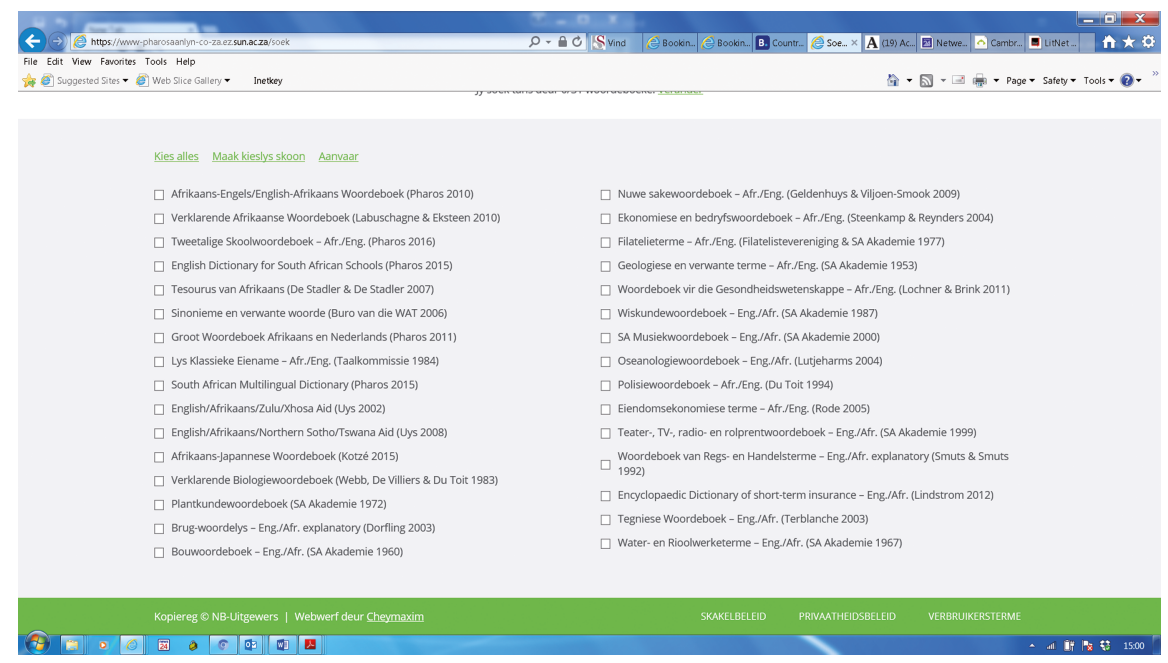

Fig. 9.3: The collection of dictionaries in PharosAanlyn.

The user can then opt to execute a given consultation within a single dictionary in the portal or to find the relevant data in all the dictionaries in the collection by ticking the appropriate boxes or by unticking the ticked boxes, cf. Figure 9.3.

A knowledgeable dictionary user who is familiar with the system used in PharosAanlyn will know that by typing the required word into the search box 


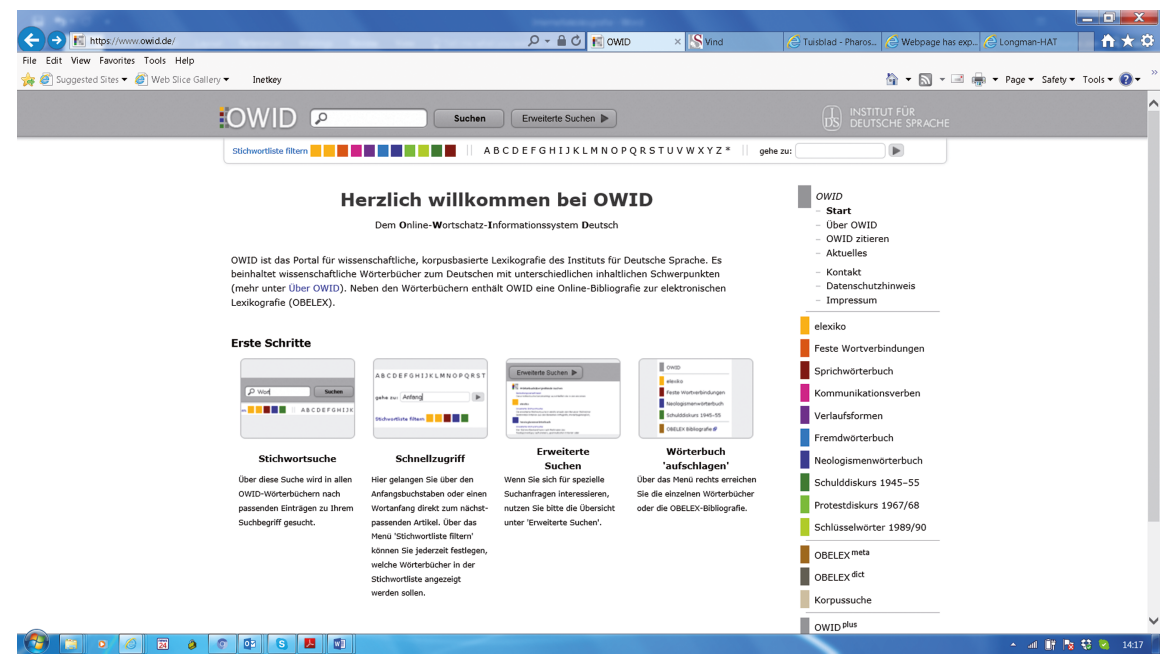

Fig. 9.4: Opening page of OWID.

on the opening page of the portal, Figure 9.1, he/she will be guided to the occurrence of that word in all the dictionaries in the portal's collection. This user will also know how to go about when in need of consulting only a specific single dictionary. The user consulting this portal for the first time or even infrequently might rather want to see the collection of dictionaries already displayed on the opening page of the portal. In terms of the data distribution structure of the dictionary portal the lexicographer needs to decide where what data will be presented. The portal OWID has the available dictionaries on the opening page, as seen in Figure 9.4, this gives the user an immediate indication of the available reference sources and allows him/her to make an informed decision regarding the dictionary he/she wants to consult:

Pictures of the collection of dictionaries in the portal can also accompany the list, as seen in Figure 9.5, on the opening page of the portal of Pearson Longman HAT. This could be helpful for users to identify or recognize specific dictionaries, but if all the dictionaries in the selection are not presented in this way, as unfortunately is the case in the Pearson Longman HAT portal, a user may assume that only the dictionaries of which the pictures are given are accessible via the portal.

The user can also employ a rapid access by entering the required word in the search box on the opening page. By clicking on the button "Soek/Search" an access route takes the user to the next page (shown in Figure 9.6) where the list of available dictionaries are given and which functions as a point of departure for further searches. 


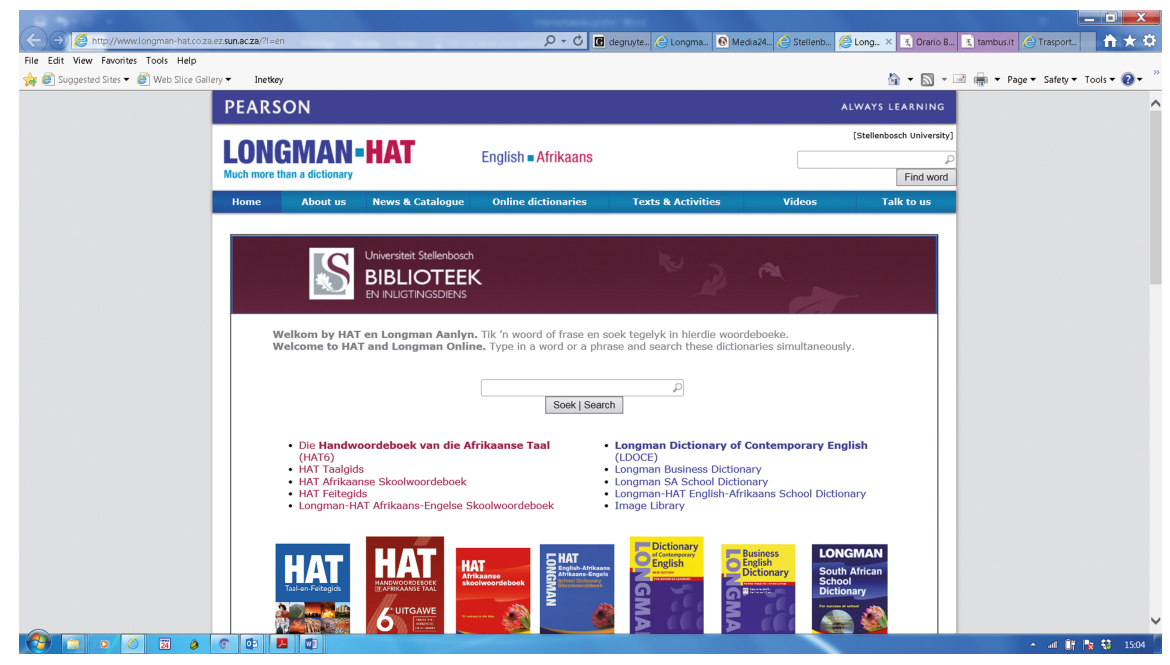

Fig. 9.5: Opening page of Pearson Longman HAT.

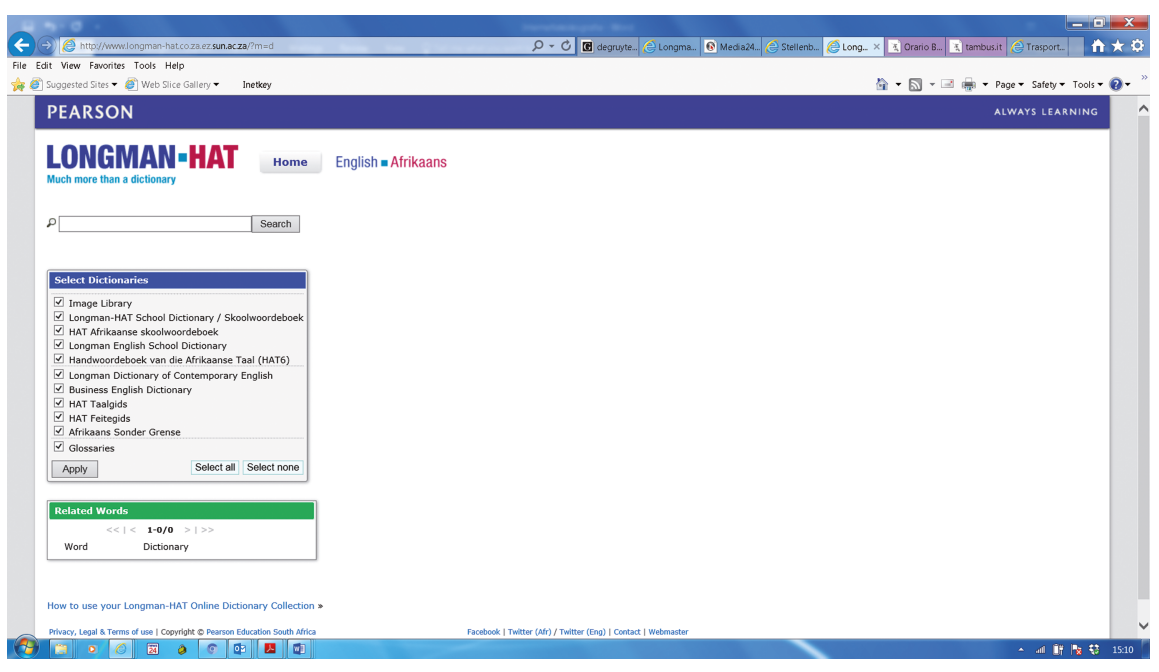

Fig. 9.6: List of dictionaries in Pearson Longman HAT.

As is the case in PharosAanlyn, the user has the option to access either a single dictionary, more than one dictionary or all the dictionaries in the portal's selection, as seen in Figure 9.7 and Figure 9.8.

Structures, e.g. the macro- and microstructures, are ordering structures, cf. Wiegand \& Gouws (2013: 78) and Wiegand \& Smit (2013: 159), and play a sig- 


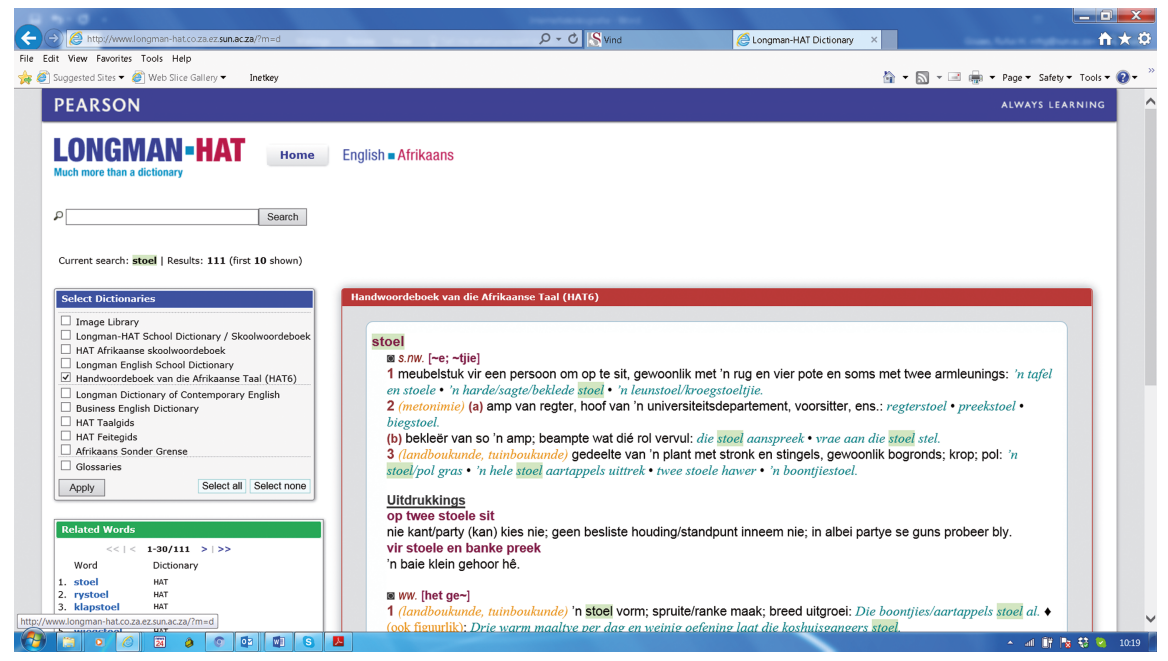

Fig. 9.7: Opting to access a single dictionary in Pearson Longman HAT.

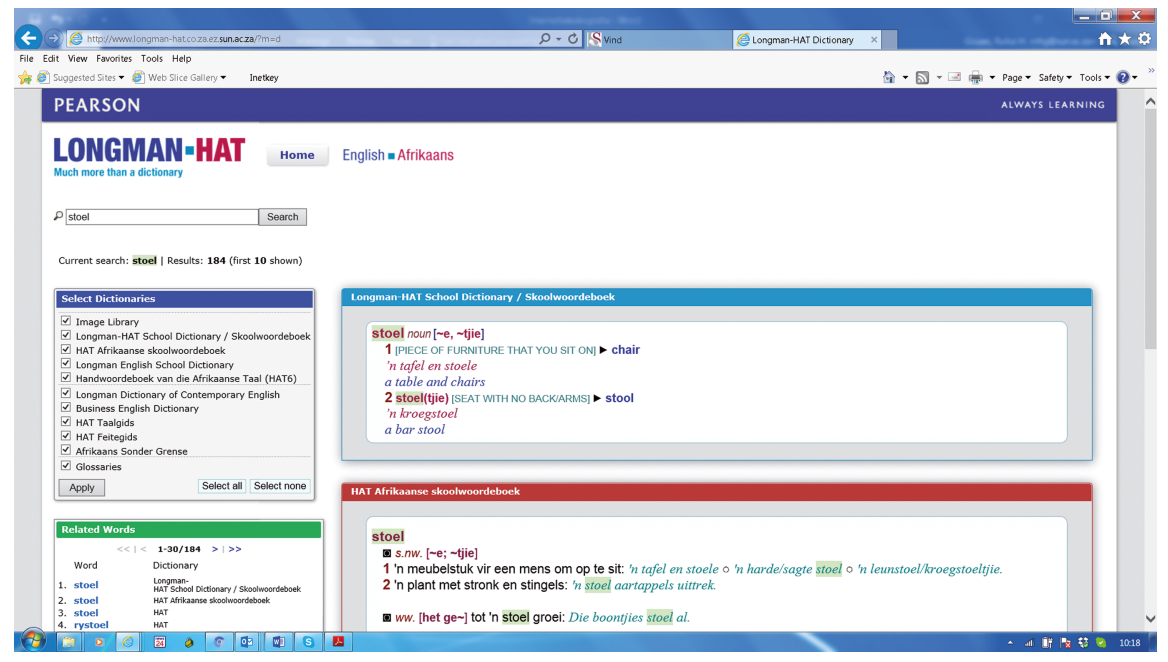

Fig. 9.8: Opting to access all the dictionaries in Pearson Longman HAT.

nificant role in the ordering of data. Dictionary portals are data structures and should also be ordering structures that prevent an arbitrary presentation of data in a dictionary portal. In PharosAanlyn the portal gives a haphazard view of the participating dictionaries because the user will struggle to identify a system according to which they are presented. The knowledgeable user will be 
able to see that general language dictionaries precede specialized dictionaries in the list but within these categories the ordering seems to be arbitrary. Also in the portal of Pearson Longman HAT no clear system of presenting the collection of dictionaries is noticeable. This is to the detriment of the user and shows a lack of planning of structures in these portals.

In a collection of dictionaries, the simplest type of dictionary portal, one would assume that the structuring of the opening page and the ordering of the selection of dictionaries would be done in a feasible, user-friendly and systematic way. The lack of ordering shows that, in spite of possible sophisticated dictionary-internal structures, these dictionary-external structures, i.e. the structures ordering the data on the opening page and the selected dictionaries, still require attention. Basic ordering and presentation procedures of the data in a dictionary portal are needed to enhance the consultation procedures of the target user.

\subsubsection{Data distribution in dictionary portals}

When planning a new dictionary one of the first structures to be decided on by the lexicographer is the data distribution structure. This applies to both printed and internet dictionaries. In printed or standalone online dictionaries the data distribution structure determines the data venues in the dictionary as a carrier of text types. The use of dictionary portals in internet lexicography demands an additional phase in the data distribution structure. In the planning of the individual dictionaries selected for inclusion in the portal lexicographers have to devise and employ the respective data distribution structures. Where lexicographers of standalone dictionaries have to decide on the overall structure, i.e. the textual book structure in printed dictionaries (Hausmann \& Wiegand 1989: 330) or the feature compound structure in online dictionaries (Klosa \& Gouws 2015: 149), the lexicographer devising a dictionary portal needs to decide on the dictionary-external venues to allocate data in the portal as a dictionary compound and text compound. Discussions of dictionary portals have focused on the portal as collection of dictionaries, a dictionary compound, but little or no attention has been directed at the outer features, i.e. the dictionary-external texts complementing the collection of dictionaries. These texts are needed to guide the identified target user of the dictionary portal and to help to enhance the success of his/her consultation experience.

When constructing a dictionary portal the lexicographer needs to employ a data distribution structure - not only to allocate the individual dictionaries to the portal but also to increase the accessibility of these dictionaries and to 
help the user to achieve an optimal retrieval of information from the data on offer in the collection of dictionaries included in the portal. Something similar to outer texts in printed dictionaries that display a partial or full frame structure or outer features in online dictionaries, cf. Klosa \& Gouws (2015), need to be presented in dictionary portals. It is the responsibility of the lexicographer to decide on the nature and extent of outer features to be included in dictionary portals. Hausmann \& Wiegand (1989: 331) already indicated that a printed dictionary should contain at least two texts, i.e. the central list and an outer text, usually found in the front matter section, giving the users' guidelines. A users' guidelines text, a key to the dictionary portal, can be regarded as a compulsory component of a dictionary portal, especially when a wide and diverse collection of dictionaries and dictionary types are included in the portal. This text complements the dictionary-internal user guidelines texts that should be part of any individual dictionary of the portal. Comparable to the distinction made by Gouws $(2001,2004)$ between respectively primary and secondary frames and primary and secondary outer texts in printed dictionaries, dictionary portals should have primary and secondary outer features. The secondary outer features should be dictionary-internal, functioning as outer features of individual dictionaries, and the primary outer features dictionary-external but portalinternal, functioning as outer features of the dictionary portal as a compound.

Portal-internal structures, including the data distribution structure, yet again give proof of the fact that in the advent of internet lexicography practice preceded theory. The practice of dictionary portals as a splendid way of improving internet lexicography needs to be supplemented by theoretical approaches that provide a better structuring of the dictionary portals. The primary outer features of dictionary portals will typically be unintegrated texts. However, depending on the subject matter of the dictionaries included in the collection, integrated outer texts, relevant to one or more dictionaries in the collection, could also be included in the outer section of the dictionary portal as a compound.

In the portal OWID, cf. Figure 9.4, the opening page offers the user not only a listing of the dictionary collection to ensure rapid access to a specific dictionary, but it also has a text in which the use of this portal is explained. This is an example of a typical primary outer feature.

When accessing a single dictionary one can either go directly to the required word or one can access a secondary outer feature, an outer text of the specific dictionary, to obtain guidelines for the use of the dictionary, as seen in Figure 9.9, an outer feature of the single dictionary elexiko.

This kind of assistance is lacking in the individual dictionaries in the portals of PharosAanlyn and Pearson Longman HAT although the portal of Pearson Longman HAT does have a user guidelines text as primary outer text. 


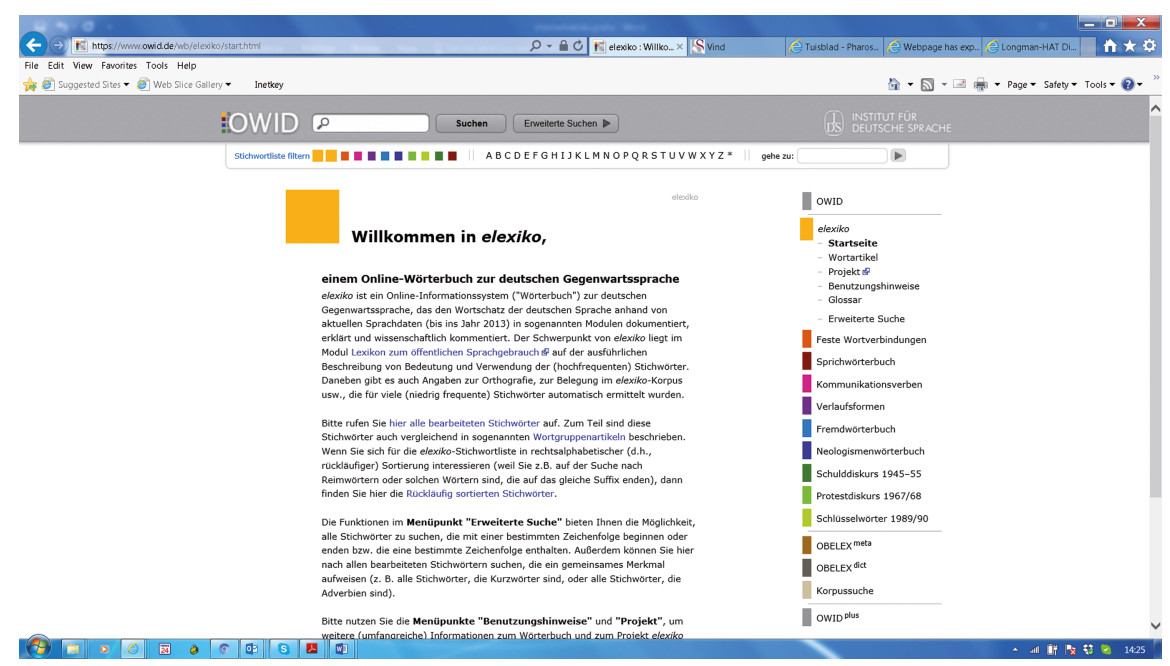

Fig. 9.9: Opening page of elexiko.

\subsection{Access}

Two remarks frequently made in discussions of internet lexicography regard the alleged unlimited space that is available and the increased speed of accessing online dictionaries. The issue of unrestricted space is not as unproblematic as it may sound because of the nature of the devices on which users have to access their online dictionaries. In this regard Lew (2004: 180) made the valid remark that we have to distinguish between unrestricted storage space and restricted presentation space. The dictionaries contained in dictionary portals are subjected to restricted presentation space but the dictionary portal as compound could have unrestricted storage space. The more dictionaries included in the selection the bigger is the challenge of the lexicographer to present the dictionaries and structure the dictionary portal in such a way that the potential of increased speed is not hampered by insufficient access structures. Speed of access to the required data can probably be regarded as one of the most significant advantages in the lexicographic practice.

In metalexicographic literature many publications have dealt with access structures, access routes and access processes, cf. Hausmann \& Wiegand (1989), Wiegand (2008), Engelberg \& Lemnitzer (2009), Svensén (2009). Bergenholtz \& Gouws (2010), Wiegand \& Beer (2013) Gouws (2017, in press; 2017a, in press). Access in online dictionaries has not yet received such a comprehensive coverage as access in printed dictionaries. With rapid, sophisticated access being one of the major advantages of internet dictionaries, lexicographers 
should employ innovative procedures to elevate the level of rapid access. Metalexicographers should follow a transformative and not a mere contemplative approach, cf. Tarp (2008: 10), in order to make theory-based suggestions for the improvement of access in the lexicographic practice.

An interactive relation should prevail between different structures in internet dictionaries. The interactive relation should, among others, help to improve the access of the target users of the dictionary to the relevant data and the subsequent retrieval of information from the data on offer. Structures that are candidates to participate in such an interactive relation to enhance access include the data distribution structure, the dictionary portal structure, the macrostructure, microstructure and article structure.

Internet lexicography compels metalexicographers to re-interpret and redefine certain concepts that have been conceptualized for printed dictionaries, e.g. that of the access domain. According to Wiegand \& Beer (2013: 110) data accessibility prevails because specific data are in the access domain of access structures. Gouws (2017, in press) already argued for a broader interpretation of the notion of the access domain. In internet lexicography the access domain can refer to the domain that accommodates the data to which access structures lead but also the domain within which access procedures are executed. This implies that both the starting point and destination of an access structure function as part of the access domain. In internet lexicography the dictionary portal as a whole, that is the dictionary portal as a data structure, constitutes an access domain.

Ordering is one of the features of access structures (Wiegand \& Beer 2013: 115) and internet dictionaries should employ access structures in such a way that systematic access to an ordered set of data is possible. Engelberg \& MüllerSpitzer (2013: 1024) regard dictionary portals as data structures that provide access to a collection of online dictionaries. Access is at the core of dictionary portals and they need to be planned in such a way that they promote optimal access to the dictionaries but also, by means of rapid access procedures, to specific items and search zones in the specific collection of dictionaries. With the portal as a whole functioning as access domain, the opening page of a dictionary portal is an access basis, i.e. the point of departure of one or more access routes and the subsequent access procedures. The access basis contains excurrent access structures that determine search routes that lead out of the access basis and into the dictionaries. These access routes can either lead to the list of all dictionaries in the collection, to the opening page of a single dictionary or to the required lemma or item in a specific dictionary. These different access possibilities need to be negotiated by the lexicographer. The planning and compilation of internet dictionaries should be seen as a team effort 
with metalexicographers, practical lexicographers and IT-experts joining hands to ensure the best possible product. Innovative access routes and procedures need to be formalized by metalexicographers so that they could be employed, adapted and improved in future dictionary projects.

\subsubsection{Types of access structures}

Engelberg \& Müller-Spitzer (2013: 1026) identify three main types of access structures within dictionary portals. These structures are external, outer and inner access structures. The external structures present links to the opening pages of the dictionaries - also links to dictionaries listed in the portal but functioning outside the portal. This is a new type of access structure, introduced for the online lexicographic environment. It is important to note that Engelberg \& Müller-Spitzer regard an external and outer access structure as two different types of access structure - and they motivate this distinction appropriately. This distinction that holds for dictionary portals is in contrast to the situation in printed dictionaries where the terms outer access structure and external access structure are regarded as synonyms (Wiegand \& Beer 2013: 113).

The outer access structure gives access to the lemmata in the individual dictionaries whereas the inner access structures help users to reach items within the dictionary articles. These last two types of access structures are comparable but not identical to those of printed dictionaries. The way in which these two structures are employed in internet dictionaries show some striking differences compared to the printed dictionary environment. Discussing access to data in information tools, Bergenholtz, Bothma \& Gouws (2015) distinguish between extra- and intra-lexicographical consultation phases. The access structures identified by Engelberg \& Müller-Spitzer (2013) fall within the domain of the intra-lexicographical consultation phase because each one of these access structures has its point of departure within the dictionary portal as access domain. Engelberg \& Müller-Spitzer's external access structure is an intra-lexicographical but dictionary-external structure whereas their outer and inner access structures are intra-lexicographical and dictionary-internal access structures. The dictionary portal as comprehensive access domain creates the opportunity for a wider range of access structures and these structures determine a vast possibility of access routes. As an example: the portal-internal but dictionary-external access route in PharosAanlyn, as determined by an external access structure, guides the user from the opening page (Figure 9.1) to the list of dictionaries (Figure 9.3) or to a single dictionary. This phase in the access procedure precedes the use of the dictionary-internal outer and inner access structures: 
Because of the innovative ways of accessing data internet dictionaries, whether standalone products or belonging to dictionary portals, need to present their identified target user with an explanation of the relevant access possibilities. This may demand that the typical access structures need to be explained in a user guidelines text - which emphasizes the need for an extended data distribution structure, i.e. a data distribution structure where the central list is complemented by outer features to accommodate data, cf. Bergenholtz, Tarp \& Wiegand (1999: 1779).

\subsubsection{New types of outer and inner access structures}

According to Wiegand \& Beer (2013: 113) an outer access structure in a printed dictionary is:

a linear textual structure, whose elements are outer access text elements, which can be accessed externally through the knowledge of general dictionary-external and specifically defined dictionary-internal principles for the linear sequence of the access text elements.

Where a printed dictionary has only a single wordlist the macrostructure as an ordering structure typically presents the lemmata as linearly ordered guiding elements to provide access to the dictionary article. Internet dictionaries do display certain macrostructural features, cf. Gouws (2014, 2017a, in press), but the default access to articles do nor proceed via the linearly ordered set of guiding elements. The occurrence of alphabet bars (on the left of the screenshot) and partial article stretches (to the right of the alphabet bar), cf. Figure 9.10, an opening page of the Dutch dictionary Algemeen Nederlands Woordenboek, gives the user who is used to an alphabetical way of searching for data in dictionaries a feeling of familiarity. Albeit not a traditional macrostructure the alphabet bar and the partial article stretches can assist with more traditional ways of accessing the data by means of lemmata as guiding elements, cf. Gouws (2014).

Gouws (2017a, in press) argues that in an internet dictionary that has such macrostructural features access to a given item can proceed via the full access structure - representing a systematic access procedure. In elexiko, cf. Figure 9.9, the opening page also contains an alphabet bar.

Looking for the word Stuhl a user can click on the letter $s$, and is then guided to a partial article stretch containing words from the article stretch "s", cf. Figure 9.11.

Subsequently the user can scroll down this partial article stretch until he/ she reaches the word Stuhl, cf. Figure 9.12. 


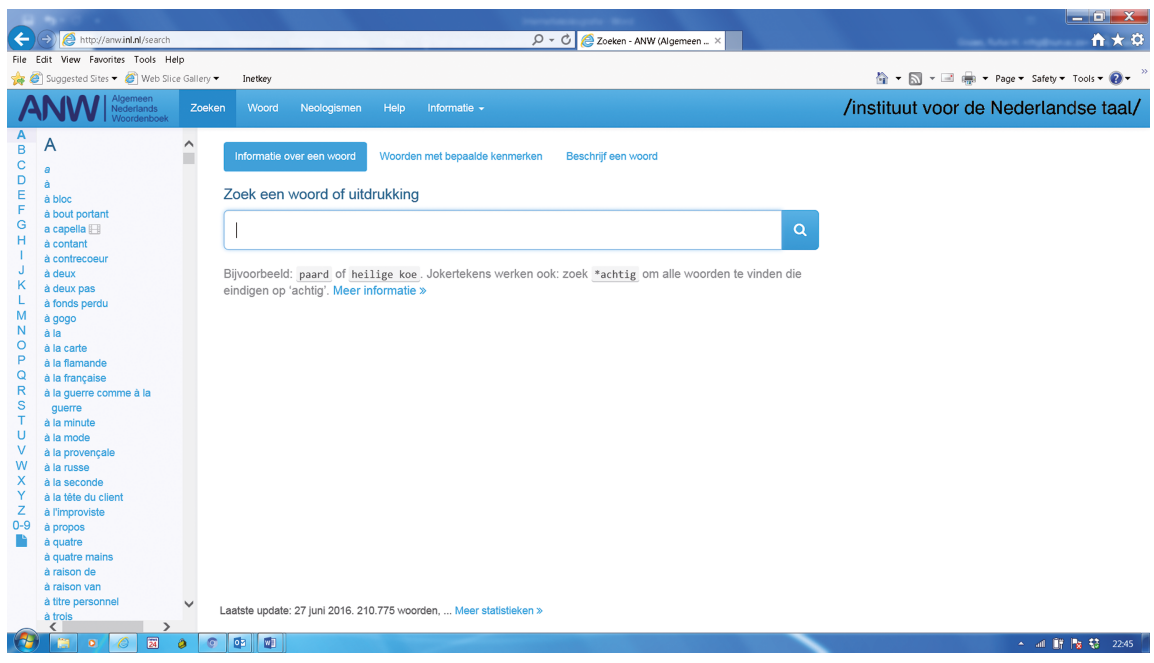

Fig. 9.10: Algemeen Nederlands Woordenboek.

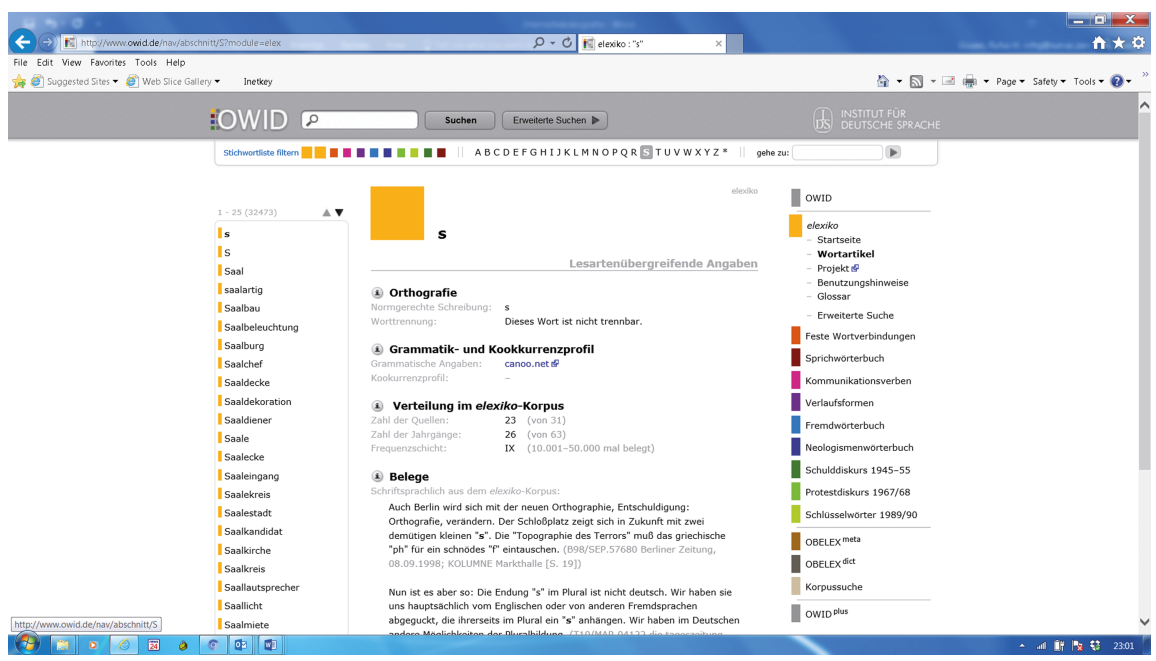

Fig. 9.11: Partial article stretch of " $\mathrm{S}$ " in elexiko.

By clicking on the word Stuhl in the partial article stretch the user is guided to a screenshot, Figure 9.13, representing the first layer of a multi-layered article, cf. Gouws (2017a, in press).

This slow and cumbersome access route resembles the way of accessing an item in an article in a printed dictionary - using the full access structure. 


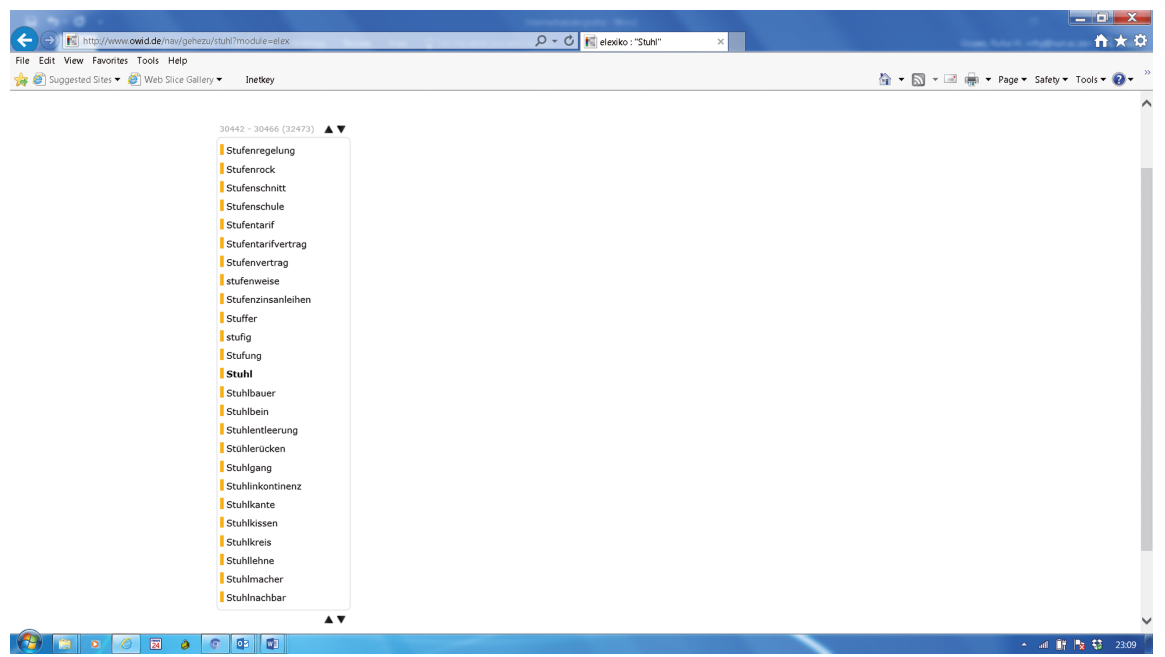

Fig. 9.12: Partial article stretch in elexiko.

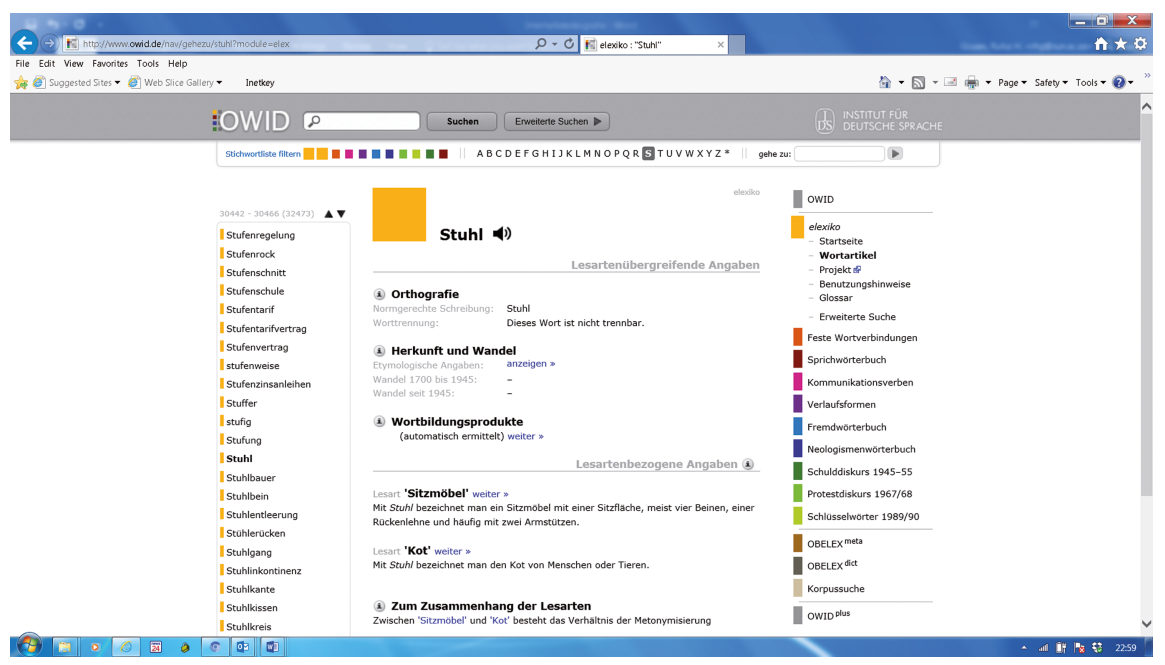

Fig. 9.13: First layer of the article of the lemma Stuhl in elexiko.

In internet dictionaries rapid access can be regarded as the default procedure. The knowledgeable user therefore has an alternative option, i.e. to embark on a punctual access procedure. Punctual access, cf. Gouws (2017a, in press), is an innovative type of rapid access in internet dictionaries where the user does not have to negotiate macrostructural features or a set of linearly ordered ac- 
cess elements to reach the required article. It prevails when the user types the required word into the search box on the opening screen and is immediately guided to the opening screenshot of the relevant dictionary article, as seen in Figure 9.13.

A dictionary like elexiko has a multi-layered article structure and the opening screenshot of a given article presents the first layer. By employing data identifying markers in the screenshot the user can follow different access routes to different types of data presented in different article layers.

The internet era confronts lexicographers with challenges - one of them is the challenge to increase the speed of access. The lexicographers of elexiko responded to this challenge and devised innovative ways of accessing the data. In the process they made changes to, among others, the access structure and the article structure.

\section{In conclusion}

Internet lexicography in the 21st century show certain resemblances with the paper dictionaries of the previous centuries. However, there are numerous changes and adaptations, especially with regard to the lexicographic structures. Metalexicography needs to contribute to the development of a theory that can be used to devise better models for better internet dictionaries. In order to have an optimal use of the contents in a dictionary and a full satisfaction of the functions of the dictionary the structures need to be planned and executed in a way that will assist the user in the best possible way to utilize the dictionary as a container of knowledge.

\section{References}

\section{Dictionaries}

Algemeen Nederlands Woordenboek: http://anw.inl.nl/search (Accessed on 30 May 2017). elexiko: http://www.owid.de/wb/elexiko/start.html (Accessed on 30 May 2017). OWID: https://www.owid.de/ (Accessed on 30 May 2017). Pearson Longman HAT: http://www.pearsonlongman.co.za/index.php (Accessed on 19 May 2017).

PharosAanlyn: https://www.pharosaanlyn.co.za/tuis (Accessed on 19 May 2017).

\section{Other literature}

Bergenholtz, Henning, Theo J. D. Bothma \& Rufus H. Gouws (2015): Phases and steps in the access to data in information tools. Lexikos 25, 1-30. 
Bergenholtz, Henning \& Rufus H. Gouws (2010): A new perspective on the access process. Hermes 44, 103-127.

Bergenholtz, Henning, Sven Tarp \& Herbert Ernst Wiegand (1999):

Datendistributionsstrukturen, Makro- und Mikrostrukturen in neueren

Fachwörterbüchern. In Lothar Hoffmann, Hartwig Kalverkämper \& Herbert Ernst Wiegand (eds.), Fachsprachen. Languages for special purposes. An international handbook of special-language and terminology research, 1762-1832. Berlin u. a.: de Gruyter.

Engelberg, Stefan \& Lothar Lemnitzer (2009): Lexikographie und Wörterbuchforschung. Tübingen: Stauffenburg.

Engelberg, Stefan \& Carolin Müller-Spitzer (2013): Dictionary portals. In Rufus H. Gouws et al. (eds.), Dictionaries. An International Encyclopedia of Lexicography. Supplementary volume: Recent developments with focus on electronic and computational lexicography, 1023-1035. Berlin, New York: de Gruyter.

Fuertes-Olivera, Pedro, A. \& Henning Bergenholtz (eds.) (2011): E-lexicography. London: Continuum.

Fuertes-Olivera, Pedro A. \& Sandro Nielsen (2012): Online dictionaries for assisting translators of LSP Texts: The accounting dictionaries. International Journal of Lexicography 25, 191-215.

Fuertes-Olivera, Pedro, A. \& Sven Tarp (2014): Theory and practice of specialised online dictionaries. Berlin: de Gruyter.

Gouws, Rufus H. (2001): Der Einfluß der neueren Wörterbuchforschung auf einen neuen lexikographischen Gesamtprozeß und den lexikographischen Herstellungsprozeß. In Andrea Lehr et al. (eds.), Sprache im Alltag, 521-531. Berlin u. a.: de Gruyter.

Gouws, Rufus H. (2004): Outer texts in bilingual dictionaries. Lexikos 14, 67-88.

Gouws, Rufus H. (2011): Learning, unlearning and innovation in the planning of electronic dictionaries. In Pedro A. Fuertes-Oliviera \& Henning Bergenholtz (eds.), E-lexicography, 17-29. London: Continuum.

Gouws, Rufus H. (2014): Makrostruktuuraanpassings vanaf gedrukte na e-woordeboeke. Tydskrif vir Geesteswetenskappe 54 (3), 481-504.

Gouws, Rufus H. (2017, in press): Dictionaries and access. In Pedro A. Fuertes-Olivera (ed.), The Routledge handbook of lexicography. Abingdon, Oxford: Routledge.

Gouws, Rufus H. (2017a, in press): Accessibility, access structures and access procedures. In Vida Jesenšek \& Milka Enčeva (eds.), Wörterbuchstrukturen zwischen Theorie und Praxis. Herbert Ernst Wiegand zum 80. Geburtstag gewidmet. Berlin u. a.: de Gruyter.

Gouws, Rufus H. et al. (eds.) (2013): Dictionaries. An International Encyclopedia of Lexicography. Supplementary volume: Recent developments with focus on electronic and computational lexicography. Berlin, New York: de Gruyter.

Granger, Sylviane \& Magali Paquot (eds.) (2012): Electronic lexicography. Oxford: Oxford University Press.

Hausmann, Franz J. \& Herbert Ernst Wiegand (1989): Component parts and structures of general monolingual dictionaries: A Survey. In Franz J. Hausmann et al. (eds.), Wörterbücher. Dictionaries. Dictionnaires. An International Encyclopedia of Lexicography, 328-360. Berlin u. a.: de Gruyter.

Hausmann, Franz J. et al. (eds.) (1989-1991): Wörterbücher. Dictionaries. Dictionnaires. An International Encyclopedia of Lexicography. Berlin, New York: De Gruyter.

Hildenbrandt, Vera \& Annette Klosa (eds.) (2016): Lexikographische Prozesse bei Internetwörterbüchern. OPAL Online publizierte Arbeiten zur Linguistik 1/2016 
(ISSN 1860-9422). Mannheim: IDS: http://pub.ids-mannheim.de/laufend/opal/pdf/ opal2016-1.pdf (Accessed on 30 April 2017)

Kammerer, Matthias \& Herbert Ernst Wiegand (1998): Über die textuelle Rahmenstruktur von Wörterbüchern. Präzisierungen und weiterführende Überlegungen. Lexicographica 14, 224-238.

Klosa, Annette \& Rufus H. Gouws (2015): Outer features in e-dictionaries. Lexicographica 31, 142-172.

Klosa, Annette \& Carolin Müller-Spitzer (eds.) (2016): Internetlexikografie. Ein Kompendium. Berlin u. a.: de Gruyter.

Lew, Robert (2004): Which dictionary for whom? Poland: Motivex.

Müller-Spitzer, Carolin (ed.) (2014): Using online dictionaries. Berlin u. a.: de Gruyter.

Schryver, Gilles-Maurice, de (2003): Lexicographer's dreams in the electronic age. International Journal of Lexicography 16 (2), 143-199.

Svensén, Bo (2009): A handbook of lexicography. Cambridge: Cambridge University Press.

Tarp, Sven (2008): Lexicography in the borderland between knowledge and non-knowledge. General lexicographical theory with particular focus on learner's lexicography. Tübingen: Niemeyer.

Wiegand, Herbert Ernst (1989a): Aspekte der Makrostruktur im allgemeinen einsprachigen Wörterbuch: alphabetische Anordnungsformen und ihre Probleme. In Franz J. Hausmann et al. (eds.) (1989-1991), Wörterbücher. Dictionaries. Dictionnaires. An International Encyclopedia of Lexicography, 371-409. Berlin u. a.: de Gruyter.

Wiegand, Herbert Ernst (1989b): Der Begriff der Mikrostruktur: Geschichte, Probleme, Perspektiven. In Franz J. Hausmann et al. (eds.) (1989-1991), Wörterbücher. Dictionaries. Dictionnaires. An International Encyclopedia of Lexicography, 409-462. Berlin u. a.: de Gruyter.

Wiegand, Herbert Ernst (1989c): Arten von Mikrostrukturen im allgemeinen einsprachigen Wörterbuch. In Franz J. Hausmann et al. (eds.) (1989-1991), Wörterbücher. Dictionaries. Dictionnaires. An International Encyclopedia of Lexicography, 462-501. Berlin u. a.: de Gruyter.

Wiegand, Herbert Ernst (1996): Das Konzept der semiintegrierten Mikrostrukturen. Ein Beitrag zur Theorie zweisprachiger Printwörterbücher. In Herbert Ernst Wiegand (ed.), Wörterbücher in der Diskussion II, 1-82. Tübingen: Max Niemeyer.

Wiegand, Herbert Ernst (2003): Überlegungen zur Typologie von Wörterbuchartikeln in Printwörterbüchern. Ein Beitrag zur Theorie der Wörterbuchform. Lexicographica 19, 169-313.

Wiegand, Herbert Ernst (2005): Über die Datenakzessivität in Printwörterbüchern. Lexikos 15, $1-35$.

Wiegand, Herbert Ernst (2008): Zugriffsstrukturen in Printwörterbüchern. Ein zusammenfassender Beitrag zu einem zentralen Ausschnitt einer Theorie der Wörterbuchform. Lexicographica 24, 209-315.

Wiegand, Herbert Ernst (2009): Wörterbuchregister. Grundlagen einer Theorie der Register in modernen Printwörterbüchern. Lexikos 18, 256-302.

Wiegand, Herbert Ernst (2013): Gedruckte Gebrauchsgegenstande mit lexikographischen Formeigenschaften. Lexicographica 29, 285-307.

Wiegand, Herbert Ernst (2013a): Lexikographie und Angewandte Linguistik. Zeitschrift für Angewandte Linguistik 58, 13-39.

Wiegand, Herbert Ernst \& Sandra Beer (2013): Access structures in printed dictionaries. In Rufus H. Gouws et al. (eds.), Dictionaries. An International Encyclopedia of 
Lexicography. Supplementary volume: Recent developments with focus on electronic and computational lexicography, 110-149. Berlin, New York: de Gruyter.

Wiegand, Herbert Ernst, Sandra Beer \& Rufus H. Gouws (2013): Textual structures in printed dictionaries: An overview. In Rufus H. Gouws et al. (eds.), Dictionaries. An International Encyclopedia of Lexicography. supplementary Volume: Recent developments with focus on electronic and computational Lexicography, 31-73. Berlin, New York: de Gruyter.

Wiegand, Herbert Ernst \& Rufus H. Gouws (2013): Macrostructures in printed dictionaries. In Rufus H. Gouws et al. (eds.), Dictionaries. An International Encyclopedia of Lexicography. Supplementary volume: Recent developments with focus on electronic and computational lexicography, 73-110. Berlin, New York: de Gruyter.

Wiegand, Herbert Ernst \& Maria Smit (2013): Microstructures in printed dictionaries. In Rufus H. Gouws et al. (eds.), Dictionaries. An International Encyclopedia of Lexicography. Supplementary volume: Recent developments with focus on electronic and computational lexicography, 149-214. Berlin, New York: de Gruyter. 


\title{
10 Methoden zur korpuslinguistischen Erforschung von semantischen Relationen
}

\begin{abstract}
Der Beitrag skizziert analytische Methoden, die zur Erforschung linguistischer Strukturen anhand sehr großer Korpora am IDS Mannheim im Rahmen des Forschungsbereichs Korpuslinguistik entwickelt wurden. Die Aspekte der Kookkurrenz, welche semantische Nähe verursacht, kommen in Modulen zum Tragen, die auf den Internetseiten ${ }^{1}$ des „Gläsernen Labors“ der Kookkurrenzdatenbank CCDB aufgeführt werden: Diese sind die Kookkurrenzanalyse, Verwandte Kookkurrenzprofile, Self-Organizing Maps, Contrasting Near Synonyms und Common Global Contexts. Alle dienen zur Beschreibung von Ausdrücken und ihren Beziehungen in lokalen und globalen Kontexten. Mit Fokus auf die aktuellsten Forschungsergebnisse zu semantischen Beziehungen schließt die Autorin mit der Forderung, diese im breiteren Sinn aufzufassen, z. B. deren Gegensätzlichkeit, aber auch mit Blick auf all diejenigen Strukturen, die in lokalen und globalen Kontexten aufgedeckt werden und introspektiv nicht zugänglich sind. Diese Perspektive wird den Wünschen der Lexikografen gerecht, die eine realistische Dokumentation semantischer Aspekte und manchmal auch subtiler Differenzierungen anstreben. Dies zeigt das dringende Bedürfnis nach Innovation bei der Beschreibung semantischer Relationen im authentischen Sprachgebrauch (parole), und nicht nur auf systemischer Ebene (langue).
\end{abstract}

Keywords: Antonymie, CCDB, Common Global Contexts, Contrasting Near Synonyms, Cooccurrence Profile, Lexikologie, Lexikographie, Self-Organizing Maps, semantische Relationen, Synonymie

\section{Zur Einführung}

Lexikologen und Lexikographen brauchen Korpora und vice versa. ${ }^{2}$ Für die meisten Lexikologen und Lexikographen präsentieren diese großen Daten-

1 Siehe unter http://corpora.ids-mannheim.de/ccdb/ (letzter Zugriff 20.5. 2017).

2 Vgl. den Titel des Beitrags von Eichinger (2007).

Marie Vachková, Karls-Universität in Prag, Institut für Germanische Studien, nám. Jana Palacha 2, Raum 320, CZ-116 38 Praha 1, Tschechien, E-Mail: marie.vachkova@ff.cuni.cz

Ә Open Access. (c) 2018 Marie Vachková, publiziert von De Gruyter. (c))BY Dieses Werk ist lizenziert unter der Creative Commons Attribution 4.0 Lizenz. 
umfänge vor allem Ressourcen von lexikalischen und grammatischen Strukturen, die bei deren Evidenz behilflich sind (z. B. auch im Rahmen der Kollokations-, Valenz-, Mehrwort- und jüngstens auch lexikographisch angewandten Konstruktionsforschung ${ }^{3}$ ) als Schauplatz der Dynamik syntaktischer Funktionen eines Wortes (z. B. im Falle neuer Adverbien, Konnektoren) oder als Material zum Nachweis metasemischer Erscheinungen aus der (mikro)diachronen Perspektive.

Eine einzigartige Sicht auf die very large corpora ${ }^{4}$ präsentiert für die Wortschatzforscher bzw. Wörterbuchmacher der Mannheimer Ansatz: ${ }^{5}$ Die im dynamischen Ganzen, hoher Vernetzung und in bisher noch nicht beschriebenen Relationen koexistierenden Worteinheiten, die aus der Perspektive der Informatik und der Linguistik mithilfe der hier entwickelten korpusanalytischen Methoden (siehe unter Abschnitt 3) analysiert werden, werden vor allem aus der Sicht der Rekurrenz, Kookkurrenznähe und Ähnlichkeit als theoretisch unbelastete Basiskriterien unter die Lupe genommen. Dies erfolgt im Rahmen der entwickelten Module, mittels derer neue Perspektiven auf die lexikalischen Aspekte des Sprachgebrauchs in allgemeineren Zusammenhängen eröffnet werden können.

Mit den hier im Weiteren präsentierten Analysemethoden ${ }^{6}$ kann auch das bisherige Design der systemischen Beziehungen im Wortschatz auf der Basis des schriftlichen Sprachgebrauchs hinterfragt bzw. auf bedeutende Weise vertieft werden. Das gängige Modell der lexikalisch-semantischen Beziehungen mit seiner Variation sollte wegen seiner bedeutenden Rolle in der germanistischen Ausbildung als Voraussetzung für Kontrastierung mit den Resultaten der korpuslinguistischen Analysen gesehen werden. Inwieweit die strukturalistische Auffassung das Beobachtungsvermögen bei der Datenanalyse die Sicht trübt, ist ein anderes Problem. Die linguistische Vorkenntnis muss in unseren Augen jedoch nicht immer einen Nachteil präsentieren:

3 Croft \& Sutton (im Erscheinen) unter 10.2.5 (http://www.unm.edu/ wcroft/Papers/Lexis-CxGFinal.pdf [letzter Zugriff 15.4. 2017]).

4 Das Deutsche Referenzkorpus DeReKo umfasste zum 8. 3. 2017 31,68 Milliarden Wörter; laufende Aktualisierung siehe http://www1.ids-mannheim.de/kl/projekte/korpora/ (letzter Zugriff 18. 4. 2017).

5 Zur Darlegung der theoretischen Prinzipien vgl. Belica et al. (2010). Zum aktuellen Stand vgl. unter http://www1.ids-mannheim.de/kl.html (letzter Zugriff 18.4. 2017). Eine didaktische Einführung siehe Perkuhn et al. (2012); siehe die Texte A, B, C und http://corpora.ids-mannheim.de/ccdb/ (letzter Zugriff 18.4. 2017).

6 Die in diesem Aufsatz besprochenen Analysemethoden wurden außer in den Texten ihres Urhebers Cyril Belica bereits mehrmals beschrieben und vorgestellt (siehe Literatur). Deswegen beschränkt sich die Autorin auf die Vermittlung des notwendigen Vorwissens, das für die Verwendung der einzelnen Module grundlegend ist. 


\begin{abstract}
Kategorien können hilfreich sein, um die Erkenntnisse zum Ausdruck zu bringen; ihre Existenz vorauszusetzen kann aber auch manchmal den Blick für das Wesentliche verschließen. Anders verhält es sich, wenn sich eine Systematik von ,Zusammengehörigem ‘ aus der Analyse und Interpretation der Daten ergibt. (Perkuhn \& Belica 2006: 6).
\end{abstract}

Die Grundtypen semantischer Relationen, die auf den herkömmlichen logischsemantischen Kategorisierungen beruhen bzw. die tradierten Definitionen der Synonymie, Antonymie usw., ${ }^{7}$ dienen den korpuslinguistisch orientierten Lexikologen und Lexikographen als hypothetische Grundlage und Vergleichsbasis $\mathrm{zu}$ den emergenten Strukturen in dem komplizierten parole-Gewebe und $\mathrm{zu}$ einer kontextbedingten Ad-hoc-Kategorisierung. ${ }^{8}$

In diesem Aufsatz wird in erster Reihe auf Erkenntnisse eingegangen, die zum großen Nutzen der Forschung und der Lehre ${ }^{9}$ in Kooperation mit der Mannheimer Arbeitsstätte für Korpuslinguistik gewonnen wurden und bis heute die lexikographischen Arbeiten prägen: Bei der Meisterung der Aufgaben, ${ }^{10}$ unter denen die lexikographisch umsetzbare Erforschung der antonymischen und synonymischen Relationen in der parole zentral ist, erscheint die Metapher der mikroskopischen Untersuchung bei Marková (2012) als zutreffend, weil sie die Anstrengungen der filigranen Datenanalysen als greifbare Empirie erscheinen lässt.

\title{
2 Die Erforschung semantischer Relationen und lexikographische Anregungen
}

Die korpusbasierte Lexikologie zielt heute vor allem auf eine gebrauchsbezogene Beschreibung der Bedeutungsbeziehungen ab, indem die Korpora unter Verwendung von recht unterschiedlich verankerten Herangehensweisen benutzt werden, wovon die Internetseiten der öffentlich zugänglichen Ressourcen mit ihren spezifischen Abfragemöglichkeiten zeugen. Die aktuellen Bemühungen der Lexikologen scheinen sich neuerdings vor allem auf die Gegensätzlichkeit

7 Vgl. den Vorspann bei Agricola \& Agricola (1992).

8 Vgl. Barsalou (2010: 87-88).

9 Zur Verwendung der Kookkurrenzdatenbank im Deutschunterricht vgl. Šemelík (2015). Zur lexikographischen Umsetzung siehe Lexikographische Sektion des Instituts für Germanische Studien; vgl. http://lexarchiv.ff.cuni.cz/lexikograficka-sekce/de/forschung-und-projekte/ (letzter Zugriff 27.2. 2017).

10 Zum Projekt des Großen akademischen Wörterbuchs Deutsch-Tschechisch vgl. Vachková (2017). 
bzw. Antonymie ${ }^{11} \mathrm{zu}$ konzentrieren, „the most robust of the semantic relations“, wie z. B. Murphy (2006: 170) schreibt, und zwar mit dem Ziel, die Natur dieser Relation auf die kognitiven Aspekte hin zu ergründen..$^{12}$ Neue Gesichtspunkte für die weitere Erforschung der Bedeutungsrelationen Synonymie und Antonymie sind bei Marková (2012) unter Verwendung der unten beschriebenen Methoden und in mehreren Arbeiten von Storjohann (siehe Literatur) zu finden. Wie die Vielfalt der in den letzten Jahren entstandenen Texte zeigt, kommen bei der Erforschung der Paradigmatik auch lexikographischdidaktische Perspektiven zur Geltung. ${ }^{13}$

An die Notwendigkeit der lexikographischen Darstellung der lexikalischsemantischen Beziehungen in sowohl allgemeinen als auch in Übersetzungswörterbüchern wird sowohl in der strukturalistischen Lexikographie (vgl. z. B. den Vorschlag von Herberg 1992), als auch heutzutage erinnert - handele es sich um Antonyme (vgl. Paradis \& Willners 2006) oder um Synonyme sowie auch um Antonyme (z. B. Storjohann 2005 ${ }^{14}$ ). Die Kritik an ungenügender Erfassung semantischer Relationen in ein- und zweisprachigen Werken lässt auf erhebliche Bearbeitungsprobleme schließen: Die Schwierigkeiten ${ }^{15}$ ergeben sich aus den recht veränderlichen ko- und kontextuellen Bezügen des bearbeiteten Lemmawortes und seiner variablen Gegenwörter. Die heutige Anforderung, sich bei der Wörterbuchschreibung (bzw. bei der Erstellung von OnlineDatenbanken und Online-Wörterbüchern) an authentische Textbelege zu halten, sollte ebenfalls auf die authentische Darstellung der Antonymie und der

11 Der Begriff Antonymie wird bei Agricola \& Agricola (1992) als Oberbegriff für (binäre) Gegensätzlichkeit verwendet (Konversivität, Komplementarität, Antonymie im eigentlichen Sinn, fakultative Gegenwortpaare). Anderseits ist der Terminus Antonymie in der Sekundärliteratur der Gegenwart schon usualisiert und wird häufig im weiten Sinn aufgefasst.

12 Vgl. die Publikationen von Paradis, Jones, Murphy, Willners in der Literaturliste.

13 Vgl. die Bibliographie von Kloudová (2015). Die klassische strukturalistisch begründete Forschung gilt noch als eine der Alternativen; vgl. z. B. Jones (2002), Jones et al. (2007), Paradis \& Willners (2001). Zur lexikographischen Bearbeitung in Lexikographie vgl. Paradis \& Willners (2007).

14 ,Judging by the relatively large number of dictionaries that cover paradigmatic items (pairs, triplets, or more complex word sets), dictionary users have a strong interest in this type of information. Such dictionaries are consulted in specific situations of text production when a user searches for alternative expressions in order to specify, to generalize or simply to vary in style or register [...]. However, in many monolingual German dictionaries the description of paradigmatic relations is often problematic and limited to a few types, such as synonymy and antonymy, and their presentation is inadequate“ Storjohann (2005: 2).

15 Deswegen werden die missglückten lexikographischen Lösungen auch angefochten; vgl. die Kritik an der Darstellung der Antonymie in der kommerziellen Übersetzungslexikographie Deutsch-Tschechisch von Paul \& Mareček (1997). 
Synonymie gerichtet sein. Außerdem werden umfassende Informationen in deren effektiver Verlinkung angestrebt, was unter anderem die Kompliziertheit der semantischen Beziehungen hervortreten lässt; vgl. z. B. bei Müller-Spitzer (2007). In eine lexikalische Datenbank sollten möglicherweise konstruktionsgebundene Antonyme und Synonyme, die jede Art der typisierten logischen Beziehung vertreten können, wie sie in den Sprachgebrauch eingehen, ${ }^{16}$ einkomponiert werden, soweit sie genug salient bzw. frequent sind und somit einen Grad an Verinnerlichung (entrenchment) signalisieren.

\section{Das Wort in lokalen und globalen Kontexten}

Die nächsten Ausführungen beziehen sich auf die Homepage des „gläsernen Labors“ der Kookkurrenzdatenbank CCDB. ${ }^{17}$ Die CCDB, die eine empirische Basis für die Eruierung sprachlicher Strukturen darstellt und ,auf der Grundlage eines Korpus geschriebener Gegenwartssprache von ca. 2,2 Milliarden laufenden Textwörtern“ aufgebaut wurde, umfasst

Kookkurrenzprofile zu ca. 220.000 verschiedenen Lemmata. Die Sammlung enthält zu jedem Lemma Ergebnisse von bis zu fünf verschiedenen Kookkurrenzanalysen in Form von Hierarchien von ähnlichen Verwendungen, mit bis zu 100.000 Verwendungsbeispielen pro Lemma und Analyse. (CCDB)

Die unter 3.1 besprochene Kookkurrenzanalyse funktionert auch im Rahmen des DeReKo, ${ }^{18}$ die weiter unten erwähnten Module RCP (Related Collocation Profile), SOM (Self Organizing Maps), CNS (Contrasting Near-Synonyms), CGC

16 Vgl. Vachková (2016) zur Umsetzung der vorgeschlagenen Suchanfragen für die GoogleSuche bei Jones et al. (2012: 60), mit web search strings (X steht für das zu kontrastierende Wort, * für den gesuchten Gegensatz): $\mathrm{X}$ and *, alike, from $\mathrm{X}$ to *, both $\mathrm{X}$ and *, $\mathrm{X}$ versus *, either $\mathrm{X}$ or*, between $\mathrm{X}$ and ${ }^{\star}$, whether $\mathrm{X}$ or ${ }^{\star}$, ${ }^{\star}$ and $\mathrm{X}$ alike*, from* to $\mathrm{X}$, both ${ }^{\star}$ and $\mathrm{X},{ }^{\star}$ versus $\mathrm{X}$, either * or $\mathrm{X}$, between ${ }^{\star}$ and $\mathrm{X}$, whether ${ }^{*}$ or $\mathrm{X}$.

17 Vgl. den Text des Urhebers auf der CCDB-Homepage unter http://corpora.ids-mannheim.de/ ccdb/; vgl. auch den Flyer unter http://www1.ids-mannheim.de/fileadmin/kl/dokumente/flyerccdb.pdf (letzter Zugriff 7.5. 2017).

18 Der statische Charakter der seit dem Jahr 2001 verfügbaren CCDB ist dem dynamischen, stets anwachsenden Korpus DeReKo gegenüberzustellen. Langsam zwingt sich die Frage nach einer Aktualisierung auf. Zur Kookkurrenzanalyse im Rahmen des DeReKo und deren Verwendung siehe auch die Texte von Steyer, vor allem zwischen 2000 und 2008 (http://pub.idsmannheim.de/autoren/ids/steyer.html (letzter Zugriff 20.5. 2017). 
(Common Global Contexts), und SPM (Semantic Proximity Model) ${ }^{19}$ sind jedoch nur auf der Basis der CCDB abrufbar.

Die Erforschung semantischer Relationen wird im Folgenden an kurzen Analysen erklärt, die sowohl für muttersprachliche als nichtmuttersprachliche Lexikographen von Interesse sein dürften.

\subsection{Kookkurrenzanalyse (KA): die Einbettung des Wortes in lokale Kontexte}

Die zur realistischen Darstellung der lexikalisch-semantischen Beziehungen dienenden Methoden haben statistisch relevante Ergebnisse zu liefern, die zu intersubjektiv nachvollziehbaren Relationen in deren lokaler Distribution bzw. ihrer konstruktionsgebundenen ${ }^{20}$ Rekurrenz führen.

Die lokalen syntagmatischen Verhältnisse bzw. alle syntaktischen Konstellationen, die im Einzelnen mittels der KA bloßgelegt werden können, bieten mittels des LLR-Wertes ${ }^{21}$ Information zur Festigkeit der Verbindung jeweiliger Kookkurrenzpartner und zugleich zur Wahrscheinlichkeit, mit welcher zwei Elemente gerade in dem gegebenen Datensatz (hier in der Kookkurrenzdatenbank CCDB) in den ermittelten syntagmatischen Mustern kookkurrieren, wie frequent sie sind (siehe die Angaben zur Trefferanzahl), und welchen Anteil das ermittelte syntaktische Muster an der jeweiligen Belegzahl aufweist (in Prozentzahl). Die KA erleichtert den Lexikographen die Identifizierung von typischen Satzeinbettungen eines Wortes anhand rekurrenter syntagmatischer Muster, die die lokalen Kontexte nahelegen, was z. B. auch bei den Gegenüberstellungen des syntaktischen Verhaltens der nahen Synonyme (siehe unten) nutzbar ist. Von den Lexikographen wird die KA als eine Analysemethode eingestuft, die das Informationsangebot der einsprachigen Wörterbücher weit übertrifft; vgl. http://www.duden.de/suchen/dudenonline/begreiflich (letzter Zugriff 2.5. 2017):

19 Das Semantic Proximity Model wird hier nicht im Einzelnen behandelt. Es stellt jedoch eine ergänzende Analysemethode dar, die zur Ausleuchtung von Diskrepanzen zwischen dem SOMModell und den KA-Ergebnissen beiträgt. Das Modell ist vor allem für die Abbildung ontologisch verankerter hierarchischer Strukturen geeignet (vgl. z. B. das generierte SPM-Skelett zu General).

20 Vgl. Murphy (2006) zur Antonymie, die sie als konstruktionsgebundene Erscheinung ansieht, und Storjohann (2010) über Konstruktionen, an denen Synonyme beteiligt sind.

21 Vgl. http://www1.ids-mannheim.de/kl/misc/tutorial.html bzw. https://www.bubenhofer.com/ korpuslinguistik/kurs/index.php?id=cosmas_client_kookk.html (letzter Zugriff 10. 5. 2017). 
[leicht] zu begreifen, zu verstehen; verständlich, nachvollziehbar

Beispiele

- in begreiflicher Erregung sein

- eine schwer begreifliche Nachlässigkeit

- es ist mir nicht begreiflich, wie das geschehen konnte

und http://www.duden.de/suchen/dudenonline/begreifbar (letzter Zugriff 2.5. 2017):

sich begreifen (1a) lassend

Beispiel

- das ist für andere nur schwer begreifbar

Die Überprüfung des vom Lexikographen aktuell bearbeiteten Materials durch die Ergebnisse der KA sichert die Repräsentativität der verwendungsspezifischen Konstruktionen in einem Wörterbuchartikel ab. Auf der Basis der generierten syntaktischen KA-Strukturen (vgl. Abb. 10.1 und Abb. 10.2) ist dann auch eine bessere Erarbeitung von Kontrasten möglich; die extrahierten typischen Verwendungsmuster sind außerdem mit den passenden Belegen immer im Hinblick auf das Zusammenspiel des LLR-Wertes und auf die Relevanz der Trefferanzahl auszuwerten (niedrige Trefferanzahl kann mit einem sehr hohen LLR-Wert zusammenfallen bzw. umgekehrt usw.).

Analysewort: begreiflich, Analysetyp 0

\begin{tabular}{|c|c|c|c|c|c|}
\hline+1 & 2 & 8198 & machen schwierig & $14100 \%$ & ist|sei es schwierig [ist ...] begreiflich zu machen daß ... \\
\hline+1 & 2 & 8198 & machen Kindern versuchen & $1100 \%$ & versuchen ... Kindern ... begreiflich ... machen \\
\hline$\mp 1$ & 2 & 8198 & machen Kindern & $1492 \%$ & den Kindern [die ...] begreiflich [zu] machen daß sie \\
\hline$\mp 1$ & 2 & 8198 & machen versuchen & $1080 \%$ & versuchen $[$ den ...] begreiflich zu machen dass \\
\hline+1 & 2 & 8198 & machen & $86596 \%$ & begreiflich $[z u]$ machen daß \\
\hline+-1 & -1 & 1706 & schwer Europäer & $333 \%$ & Europäer ... schwer begreiflichen \\
\hline$\mp-1$ & -1 & 1706 & schwer offenbar & $540 \%$ & offenbar $[\ldots]$ schwer begreiflich \\
\hline$\mp-1$ & -1 & 1706 & schwer & $18366 \%$ & ist|nur schwer [...] begreiflich zu machen \\
\hline+1 & 1 & 903 & Gründen nicht & $2588 \%$ & aus begreiflichen Gründen [...] nicht \\
\hline+1 & 1 & 903 & Gründen besonders stark & $4100 \%$ & Ländern aus begreiflichen Gründen besonders stark in Deutschland \\
\hline+1 & 11 & 903 & Gründen & $9194 \%$ & aus begreiflichen [...] Gründen nicht ... \\
\hline+-3 & -1 & 645 & ist durchaus & $1190 \%$ & ist [es] durchaus begreiflich daß|dass der \\
\hline+-3 & -1 & 645 & ist sehr & $1450 \%$ & ist $[\ldots]$ sehr $[\ldots]$ begreiflich \\
\hline \pm-3 & -1 & 645 & ist & $48176 \%$ & ist [...] begreiflich \\
\hline
\end{tabular}

Abb. 10.1: KA zum Analysewort begreiflich. 


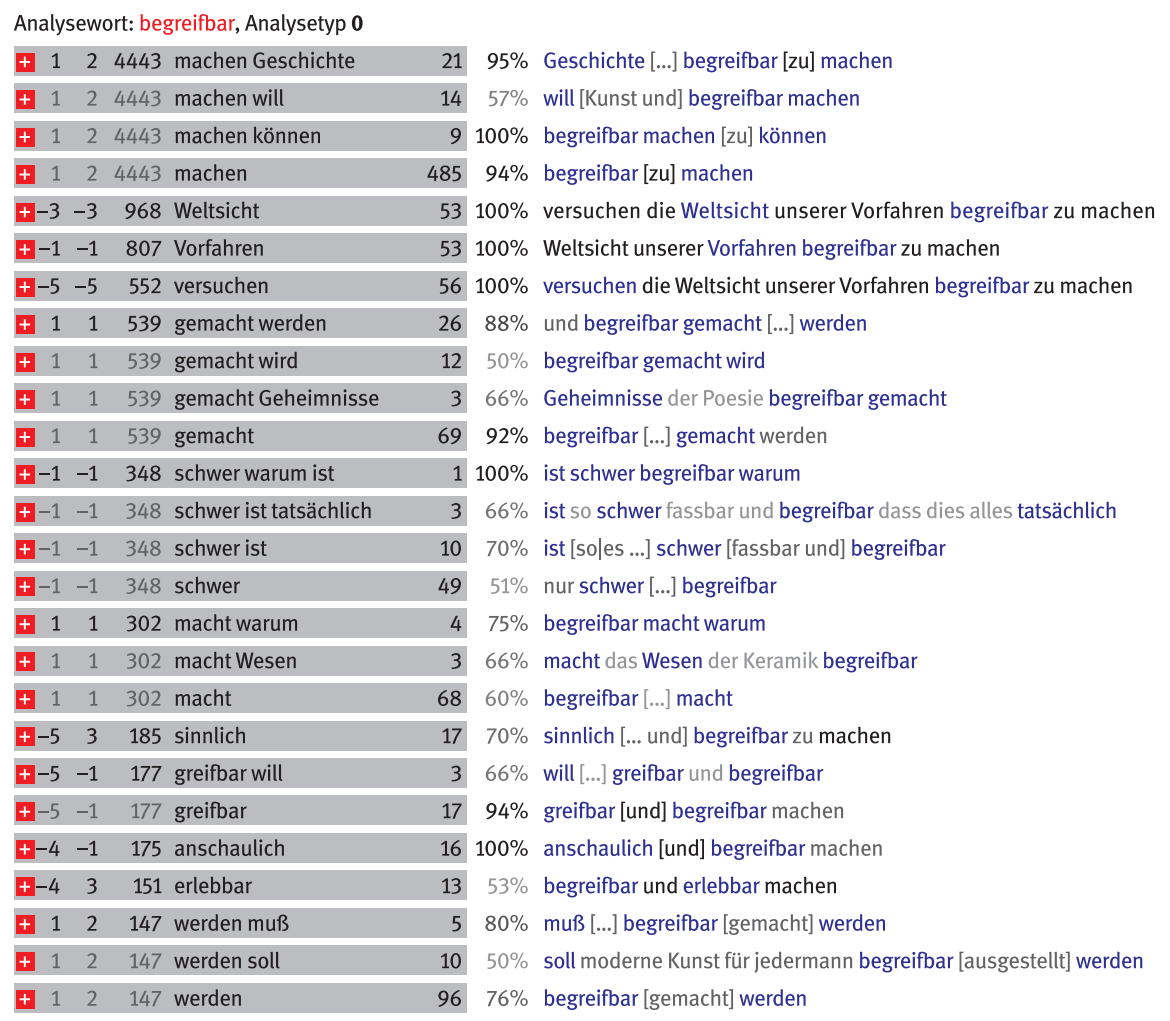

Abb. 10.2: KA zum Analysewort begreifbar.

Die Gegenüberstellung von extrahierten Strukturen (siehe unten) fällt dem geübten Auge eines Wörterbuchmachers nicht schwer; und das spezifische Verhalten des jeweiligen Adjektivs kann dabei umgehend identifiziert werden; vgl.

(es ist schwierig), (jmdm) etwas begreiflich (zu) machen + dass-Satz

aus schwer begreiflichen Gründen

es ist offenbar/nur schwer begreiflich + dass-Satz

es ist durchaus/sehr begreiflich + dass-Satz

(jemandem) etwas begreifbar (zu) machen

etwas wird begreifbar gemacht

es ist schwer begreifbar + dass-Satz

etwas macht etwas (nur) schwer begreifbar

etwas sinnlich/greifbar/anschaulich und begreifbar machen

begreifbar und erlebbar machen

etwas muss begreifbar gemacht/ausgestellt werden

Die KA kann mit Gewinn mit einer reziproken KA ergänzt werden: 
Textwort begreiflich tritt als Kookkurrenzpartner bei folgenden Analysewörtern und -typen auf:

\begin{tabular}{|c|c|c|}
\hline Wort & $\begin{array}{l}\text { Synser } \\
\text { ohne }\end{array}$ & $\begin{array}{c}\text { Iantika } \\
\text { mit }\end{array}$ \\
\hline machen $®$ & 289 & 289 \\
\hline warum $®$ & 251 & \\
\hline weshalb $®$ & 80 & \\
\hline Gemütsbewegung ${ }^{\circledR}$ & 79 & 79 \\
\hline wieso $\mathbb{R}$ & 43 & 43 \\
\hline unbegreiflich $®$ & 42 & 42 \\
\hline fassbar & 33 & 33 \\
\hline faßbar & 33 & 33 \\
\hline Ungeduld $®$ & 24 & \\
\hline
\end{tabular}

Abb. 10.3: Reziproke KA zum Analysewort begreiflich.

Textwort begreifbar tritt als Kookkurrenzpartner bei folgenden Analysewörtern und -typen auf:

\begin{tabular}{|c|c|c|}
\hline Wort & $\begin{array}{l}\text { Synser } \\
\text { ohne }\end{array}$ & $\begin{array}{c}\text { Iantika } \\
\text { mit }\end{array}$ \\
\hline Weltsicht ${ }^{\circledR}$ & 984 & 984 \\
\hline Vorfahr & 824 & 824 \\
\hline anschaulich $®$ & 167 & 167 \\
\hline greifbar $®$ & 159 & 159 \\
\hline erlebbar $®$ & 152 & 152 \\
\hline sinnlich $®$ & 128 & 128 \\
\hline erfahrbar ${ }^{\circledR}$ & 71 & 71 \\
\hline Wortsinn ${ }^{\circledR}$ & 67 & 67 \\
\hline buchstäblich $®$ & 65 & 65 \\
\hline jedermann $®$ & 64 & \\
\hline 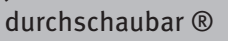 & 60 & 60 \\
\hline erzählbar ® & 54 & 54 \\
\hline Erdgeschichte ${ }^{\circledR}$ & 49 & 49 \\
\hline fassbar & 47 & 47 \\
\hline faßbar & 47 & 47 \\
\hline taktil & 38 & 38 \\
\hline
\end{tabular}

Abb. 10.4: Reziproke KA zum Analysewort begreifbar.

$\mathrm{Zu}$ weiteren Kontrastierungen dieses Paares anhand des CNS-Modells vgl. unter Abschnitt 3.4. ${ }^{22}$

22 Vgl. auch Vachková (2007). 


\subsection{Das Wort in globalen Kontexten: verwandte Kookkurrenzprofile (Related Cooccurrence Profiles) und lexikalische Merkmalskarten (SOM)}

Die unter Abschnitt 3.1 an zwei Adjektiven dargestellten Strukturen der „lokalen“ Ebene sagen aber noch nicht viel über die distributionellen Ähnlichkeiten bzw. die globalen Kontexte aus, d.h. über die Diskurseinbettungen der beobachteten Wörter. Der Weg von den lokalen zu den globalen Kontexten geht hier über die graduelle Ähnlichkeit der Kookkurrenzprofile: Das Kookkurrenzprofil (weiter im Text nur KP) präsentiert in kondensierter Form den typischen Usus eines Wortes; zur theoretischen Begründung vgl. Belica (2011: Kap. 2):

Die Gesamtheit aller quantitativen Ergebnisse der Kookkurrenzanalyse zu einem gegebenen Analyseobjekt (einem Lexem, einer Wortverbindung usw.) wird als Kookkurrenzprofil des Objektes bezeichnet und stellt - informell gesagt - ein Kondensat seines Gebrauchs dar. Es erfasst sowohl dominante Wortverbindungsstrukturen wie auch subtile Varianzphänomene im lokalen lexikalischen Kontext des analysierten Objektes, und bietet dadurch eine detaillierte Auskunft über die syntagmatische und paradigmatische Einbettung des Objekts im Sprachgebrauch aus präferenzrelationaler [...] Sicht. [In diesem Text wird auf die Version hingewiesen, die unter http://corpora.ids-mannheim.de/ SemProx.pdf zu finden ist. (http://corpora.ids-mannheim.de/SemProx.pdf (letzter Zugriff 20.4. 2017)]

Wie unter Abschnitt 3.1 skizziert, bieten die auf der Grundlage der KA und der CCDB entwickelten Module nicht nur die Möglichkeit, Reihen von verwandten KPen, die nach ihrem Verwandtschafts- bzw. Ähnlichkeitsgrad aufgelistet werden (vgl. das Modul Related Cooccurrence Profiles auf der Homepage der CCDB) zu sichten, sondern auch die RCP von zwei lexikalischen Einheiten anzusehen (siehe die Gegenüberstellung von zwei ähnlichen Kookkurrenzprofilen bei Keibel \& Belica (2007: Abschnitt 3.1). Die RCP geben Aufschluss über die paradigmatischen Beziehungen des Wortes; vgl. z. B. die RCP zu dem recht vagen Ausdruck interessant:

hochinteressant, spannend, aufschlussreich, reizvoll, bedeutsam, aufregen, bemerkenswert, unterhaltsam, attraktiv, informativ, kurzweilig, spannen, problematisch, uninteressant, beachtenswert, anspruchsvoll, faszinieren, brisant, lehrreich, erfreulich, unerfreulich, amüsant, spinnen, kurios, wichtig, niveauvoll, vergnüglich, vielseitig, originell, beunruhigen, überzeugend, begrüßenswert, vielfältig, toll, fragwürdig, wertvoll, verlocken, lesenswert, ansprechend, sinnvoll, merkwürdig, anregend, folgenreich, tollen, lustig, vielversprechend, schwierig, nützlich, beeindrucken, weiterbringen, vorteilhaft, bedeutungsvoll, erfolgsversprechend, lohnend, verblüffend, ausbaufähig, lobenswert, kulturgeschichtlich, heikel, anstrengend, lohnenswert, verdienstvoll, unattraktiv, aussagekräftig, fesselnd, ungewöhnlich, unangenehm, bedeutend, gewichtig, bedenklich, hochstehend, befremdlich, ergiebig, ärgerlich, wegweisend, lukrativ, beeindruckt, erstaunlich, bedenkenswert, abwechslungs- 
reich, unappetitlich, facettenreich, spaßig, diskussionswürdig, dankbar, zugkräftig, ethnologisch, fächern, seltsam, Fülle, anschaulich, ambitioniert, kunsthistorisch, lebensnah, löblich, diffizil, gefächert, faszinierend, herausfordernd, außergewöhnlich, grundverschieden, unwichtig, kenntnisreich, vielschichtig, erstrebenswert, angenehm, erwähnenswert, relevant, stringent, herausragen, gehaltvoll, akzeptabel, skurril, günstig, landschaftlich, vernünftig, absurd, kompliziert, bedrohlich, interessieren, betrüblich, zukunftsweisend, zeitgeschichtlich, realistisch, witzig, komisch, willkommen, pikant, kulturhistorisch ...

In diesem Zusammenhang ist Folgendes festzuhalten:

a) Der generierten KP-Liste ist intuitiv eine große Vielfalt an Verwendungen abzulesen, die Merkmale unterschiedlicher kommunikativer Situationen erahnen lassen.

b) Es lassen sich rekurrente Verwendungen der adverbial positionierten Adjektive ablesen, wie z. B. kulturgeschichtlich, ethnologisch interessant.

c) Der praktische Nutzen in stilistischer Hinsicht (die Liste der KPe kann als Fundgrube der Synonyme und Antonyme dienen) fußt auf der Annahme, dass es die gemeinsam geteilten ähnlichen Kontexte sind, auf deren Verinnerlichung unser Assoziationspotenzial basiert, das bei der Suche nach dem treffenden Ausdruck aktiviert wird.

d) Die Liste deutet auf Ähnlichkeiten in der Distribution von Synonymen und Antonymen: Deren häufige textuelle und konstruktionsgebundene Kookkurrenz trägt zur Ähnlichkeit deren KPe bei. Aus dieser Perspektive erscheint die Unterscheidung der Kategorien Synonym und Antonym als zweitrangig, was für die lexikologische Erkenntnis im Bereich der semantischen Beziehungen eine wichtige Erkenntnis ist: Antonyme und Synonyme sind dank deren Distribution durch Assoziationsfäden verbunden.

e) Die semantische Ähnlichkeit der Synonyme fußt auf deren rekurrent ähnlicher kontextueller Einbettung, die sich in der Ähnlichkeit der KPe manifestiert.

f) Letzten Endes ist die RCP-Liste bei der Reflexion des Synonym- und Antonymangebots in lexikographischer Hinsicht zu empfehlen, indem a) bis e) bei der Auswahl der aufzunehmenden Belege für den Wörterbuchartikel erwogen werden können.

\subsection{Lexikalische Merkmalskarten (Self Organizing Maps)}

Der auf der Grundlage informatischen und mathematischen Wissens bzw. Könnens umgesetzte Grundgedanke der semantischen Ähnlichkeit, die auf der Kookkurrenznähe gründet (näher dazu vgl. Belica 2011), ${ }^{23}$ kann an den selbst-

23 „Über die Analyse der Struktur der Kookkurrenzprofile und des multidimensionalen Vektorraumes, den diese Kookkurrenzprofile vermöge einer Ähnlichkeitsmetrik aufspannen, gelangt 
organisierenden lexikalischen Merkmalskarten entfaltet werden, indem das in den SOMs organisierte Wortmaterial interpretiert wird. ${ }^{24}$ Die SOMs sind im Prinzip für jeden nachvollziehbar, der die Anleitung, die grundsätzlich in zwei Schritten beruht, befolgt. Die intersubjektive Gültigkeit (vielfach im Rahmen des Hochschulunterrichts getestet ${ }^{25}$ ) beruht auf dem geteilten soziokulturellen Rahmen der Interpretierenden, die erschlossenen Lexemcluster (Supersigns) setzen Assoziationen des Betrachters in Bewegung und schrittweise erfolgt der Prozess der Semiose (siehe die Anlagen A.10.1, A.10.2 und die Interpretation am Paar begreiflich/begreifbar und Abschnitt 3.4):

Step one: Start in one of the SOM corners. Look at the words in a corner square (the representamen), and, following your mental associations, try to understand the underlying lexeme clustering principle (the interpretant) within that corner as compared to the clusters in the remaining corners. Do not limit your associations to the linguistic categories, and avoid aligning them with your lexicographic expectations in step one for as long as possible. Arriving at any meaningful interpretation of the organizing criterion in a corner, you have traced out your first SOM supersign. Now, try to extend that single square cluster by inspecting its neighbor squares in all directions and reflect on the gradual shift in your associations. Then, keep looking farther at the neighbors' neighbor squares in one particular direction, and imagine your associations converging to a new 'quilting point' (cf. Lacanian point de capiton), and, thus, the original sign morphing into a different one. Moving your focus forth and back, try to visualize the boundary where the initial sign eventually faded out, and where a notion of a new supersign entered your mind. Repeat for all corners and all directions. Try to assign each SOM square to at least one SOM supersign. Step Two: Interpreting SOM supersigns. Evaluate the identified SOM su-

man zu Erkenntnissen über die Struktur bestimmender Verwendungsaspekte von lexikalischen Einheiten, insbesondere über die Struktur ihrer lexikalisch-semantischer Relationen. Im Sinne der im Abschnitt 2 zitierten Maxime ,[...] sich dem Untersuchungsgegenstand Sprache mit möglichst wenigen Vorannahmen über diesen Gegenstand selbst zu nähern', wird dazu, d.h. zur Analyse bzw. Dimensionsreduktion des Vektorraumes, der Ansatz von neuronalen Netzen - in Form von selbstorganisierenden Merkmalskarten - aufgegriffen“ (Belica 2011: Kap. 4.3). Die SOMs wurden bereits in mehreren Arbeiten von Belica, Keibel, Perkuhn, Vachková, Marková, Šemelík und anderen (siehe Literatur) ausführlich vorgestellt. Auch aus Platzgründen wird in diesem Aufsatz auf leicht zugängliche Internetquellen mit bereits kommentierten Abbildungen hingewiesen.

24 Es erübrigt sich zu sagen, dass die Analysen und Fragestellungen aus der Position der Linguisten auch ein sinnvolles Feedback für das entwickelte Modell präsentieren. Auch hier gilt jedoch, dass die eingelebte linguistische Betrachtungsweise hie und da eine Barriere mit sich bringt; vgl. Keibel \& Belica (2007). So tendieren z. B. die strukturalistisch ausgerichteten Linguisten dazu, die SOM-Cluster als Wortfelder zu bezeichnen. Einerseits wird dadurch der schon sowieso unter verschiedenen theoretischen Voraussetzungen verwendete Begriff des Wortfeldes neu belastet, anderseits wird dadurch die Einsicht in das Zustandekommen der SOM-Karten geleugnet.

25 Seit 2006 bis heute im Rahmen der Lexikologieseminare; vgl. auch Šemelík (2015). 
persigns based on your specific lexicographic criteria of relevance. Then, map the relevant supersigns onto linguistic categories implied by your theoretical framework, e.g., on the paradigmatic level. [Leicht gekürzt nach Vachková \& Belica (2009), siehe http://corpora. ids-mannheim.de/IJGLSA.pdf (letzter Zugriff 20.4. 2017)]

Der Entzifferungsprozess der KP-Cluster, an denen die globalen Kontexte abzulesen sind, ${ }^{26}$ rückt in die Nähe der lexikographischen Praxis: Die Beschreibung der adäquaten Lesart, also der Bedeutungserfassung eines Wortes, fußt nicht nur auf der Spracherfahrung und dem Bildungshorizont des Bearbeiters, die nur schwierig von den assoziierten Sachverhalten wegzudenken sind (Vachková \& Belica 2009), ${ }^{27}$ sondern liefert auch zahlreiche Impulse für lexikologische, kognitiv ausgerichtete bzw. experimentelle Forschung (data priming). Von großem Gewinn ist die Verwendung der KPe in der germanistischen Lehre ${ }^{28}$ oder bei der Paraphrasierung bzw. Erfassung von Äquivalenzbeziehungen im Falle der abstrakten Nomina, bei denen die konstruierten lexikographischen Synonyme eher eine Barriere als Hilfe präsentieren, so z. B. das Substantiv Verklärung, das mit den konstruierten lexikographischen Synonymen das Verklären, das Verklärtwerden, das Verklärtsein ohne jedes lexikographische Beispiel umschrieben wird und erst in Bezug auf die Bedeutungsumschreibung des Verbs verklären und durch die vorhandenen Beispiele reflektiert werden kann. ${ }^{29}$

Der ikonische Wert der Karten kann aus dem lexikologischen Standpunkt folgendermaßen verstanden werden: Die möglichen Vernetzungsfäden der SOMCluster, die auf KPen beruhen, modellieren ein realistisches Geflecht von dynamischen, simultan existierenden lexikalischen Beziehungen unterschiedlicher Stabilität, die auf ihre Zusammengehörigkeit bzw. Festigkeit in der Verwendung

26 Der Benutzer der SOMs kann bei der Erschließung der einzelnen Cluster im Notfall (falls keine Assoziationen abgerufen werden) auf eine Internet-Suchmaschine zurückgreifen, indem man zwei oder mehrere Kookkurrenzprofile eingibt. Nicht selten tritt ein Aha-Effekt ein. Es ist ratsam, eine SOM-Karte mehrmals abzurufen, um die stabilen Kerne der identifizierten Cluster schneller voneinander abzugrenzen. Bei jedem Abrufen wird die SOM-Karte 10.000-mal neu berechnet. Bei der Analyse der SOM-Karte zu Tod wurden 20 Versionen verglichen.

27 In diesem Beitrag wird die elektronische Version des Aufsatzes unter http://corpora.idsmannheim.de/IJGLSA.pdf (letzter Zugriff 20.4. 2017) herangezogen.

28 Vgl. Vachková et al. (2008) über die Verwendung der SOM-Karten beim kreativen Schreiben, Šemelík (2015) zum interkulturell angelegten Unterricht und lexikalisch ausgerichteter Textarbeit, wo Reflexionen in Bezug auf Sprach- und Welterfahrung gefragt sind; vgl. auch die Anregungen bei der Übersetzung bzw. Interpretation der Schlagwörter, z. B. Freiheit (siehe die Analyse bei Vachková 2010).

29 Vgl. unter http://www.duden.de/suchen/dudenonline/verklären (letzter Zugriff 2. 5. 2017). Ein lexikographisches Bearbeitungsverfahren der Abstrakta im zweisprachigen Wörterbuch schlägt Vachková (2009) vor. 
hinweisen. Als Beispiel diene hier die Erfahrung mit der diskursbasierten Interpretation von Assoziationsnetzen bei der Bedeutungserfassung von Abstrakta, die vor allem im Fremdsprachenunterricht ein Deutungsproblem darstellen. ${ }^{30}$ Im Rahmen der Einbeziehung der KPe in die lexikographische Arbeit, bei der vor allem zentrale Wortinhalte und typische Verwendungen darzustellen sind, bleibt in der Regel kein Raum für die Darstellung der peripheren Verwendungen übrig, wo sich z. B. im Falle von zwei sinnverwandten Ausdrücken deren Gebrauchsdomänen zum Teil überlappen (mehr dazu unter Abschnitt 3.4).

Einer der bereits analysierten Fälle wäre z. B. das Substantiv Tod (vgl. die Abbildung der KP-Cluster in der SOM-Karte in Vachková \& Belica (2009) und http://corpora.ids-mannheim.de/IJGLSA.pdf (letzter Zugriff 20.4. 2017), wo folgende Diskurseinbettungen angedeutet werden:

\section{Sozialer Tod}

- Ausscheiden, Weggang, Pensionierung, Rausschmiss, Karriereende, Rauswurf

- künden, Abtritt

- ereilen, ereilt [X = etw. Unangenehmes hat jdn. ereilt]

\section{Kriminalität}

- Bluttat, Ermordung

- Entführung, Verhaftung, Festnahme, Gefangennahme, Verschleppung, Haftentlassung, Freilassung, Tat, Attentat, Umsturz

- Mord, Tötung, Enthauptung, Doppelmord, Vergewaltigung, Todesschuss

- Mörder, Exekution, exekutieren, exekutiert, Hinrichtung, hingerichtet, hinrichten

- Giftspritze, Galgen

- Haft, Haftstrafe, Gefängnis, Zuchthaus, Todeszelle

- Hochverrat, Freiheitsstrafe, Zuchthausstrafe, Todesstrafe, Verbannung

- verurteilen, verurteilt, lebenslang, lebenslänglich

- Haft, Delinquent, Ehebruch, Raubmord, Vergewaltiger, Schauprozess, Rehabilitierung

30 Vgl. Vachková (2010), zitiert in Belica (2011), wo die Schlussfolgerung von Schwanenflugel \& Gaviska (2005: 1738) herangezogen wird: „When abstract and concrete words are placed in highly supportive contexts [sprich: in Clustern von Kookkurrenzprofilen, M. V.], the preactivation of contextual knowledge [sprich: durch Assoziationen evoziertes Diskurswissen, das an Weltwissen gekoppelt ist, M. V.] should override processing [bzw. priming, M. V.] difficulties for abstract words.“ 


\section{Politik}

- Umschwung, Bestürzung, Entmachtung, Machtergreifung, Umschwung, Bestürzung, Ruhm, Scheitern

- Ankunft, Abreise, Heimkehr, Wintereinbruch

- Abgang (salopp), Ableben (gehoben), Abschied, Geheiß (gehoben), Abdankung (schweizerdeutsch: Trauerfeier)

Freitod

- Verschwinden, Gesundheitszustand, Psychogramm, Selbstmord, Schicksal, Verzweiflungstat

\section{KZ-Lager, Gefängnis}

- Verstümmelung, Misshandlung, Mörderin, Missbrauch, bestialisch, Mithäftling

- unschuldig, Gaskammer, Unschuldige, grausam

- Hungertod, Leid, geknebelt, verhungern, Einsamkeit

- schuldlos

- geschunden, misshandeln, misshandelt, verdursten, peinigen, gepeinigt

- Kopfschuss, Verhungern, Qual, Pein, Elend

Beerdigung

- Beisetzung, Begräbnis, Grab, Ruhestätte, Lebzeiten, Todestag, Beileid

Lebensgeschichte des Verstorbenen (VIPs)

- Thronbesteigung, Porträt, Geburtsort, Anwesenheit

- Erbe, Geburtshaus, Vermählung, Hochzeit

- Bildnis, Verehrer, Weggefährte, Geburtsstadt, Vermächtnis, Bewunderer

- verstorben, Heirat, Witwe, Trauer, Geliebte, Neffe, Sohn, Verlobung, Gefährtin

Tod in der Familie

- Gatte, herzensgut, mein, geliebt, betrauern, betrauert, trauern, allzufrüh, Abschiednehmen, Heimgang, Anteilnahme, Hinschied

- Patenkind, Bruder, Cousin, Onkel, Großvater, Schwägerin, Schwiegertochter, Enkelin, Schwiegervater

- beweinen, Sterbebett, fürsorglich

- Vater, Mutter, geschieden, untreu, Stiefmutter, Freundin, außerehelich, unehelich, verheiratet, verheiraten 
Heterogene Lebensgeschichten der Mitmenschen

- Begleitung, Vormund, unmündig

- Sterben, Zeugung, neugeboren, mitansehen, Nabelschnur

- Pubertät, Klassenkamerad, vierjährig, Lebensende, ertränken, ertränkt

- Einschulung, Stiefvater, drogensüchtig

- Todeskampf, qualvoll, geschrien, würgen, gewürgt

- Liebeskummer, Siechtum, Leiden

- Erstickungstod, Stromstoß, Verwundung

- Massensterben

Todesursachen u. Ä.

- Schicksalsschlag, tragisch

- Krankheit, Unfall, Unglück, Flugzeugunglück, Badeunfall, Verkehrsunfall, Autounfall, Feuertod, Todessturz, Fremdverschulden, Obduktion, Obduktionsbericht, Todesursache, Herzversagen, Herzstillstand, Herztod, Zwischenfall, Herzattacke, Hirnblutung, verbluten, Ersticken, Schwächeanfall, Hirntod, Gewalteinwirkung, Gehirnblutung, Vergiftung, Unterkühlung

- Einlieferung, Bewusstlosigkeit, bewusstlos, erblinden, Koma, versterben

- Drogentod, todkrank, herzkrank, schwerkrank, aidskrank, alkoholkrank

- Ertrinken, Fenstersturz, schwerverletzt, verblutet

- Frühgeburt, Totgeburt, Säugling

Der Kontrast $\mathrm{zu}$ einer lexikographischen Beschreibung [vgl. http://www. duden.de/suchen/dudenonline/Tod (letzter Zugriff 7.5. 2017)] kann wohl nicht größer sein. ${ }^{31}$ Während in den SOMs das Weltwissen aktiviert wird, baut ein klassisches Bedeutungswörterbuch auf einer abstrakten Bedeutungsumschreibung auf (Duden online):

1. Aufhören, Ende des Lebens; Augenblick des Aufhörens aller Lebensfunktionen eines Lebewesens

2. (oft dichterisch oder gehoben) in der Vorstellung als meist schaurige, düstere, grausame Gestalt gedachte Verkörperung des Todes; die Endlichkeit des Lebens versinnbildlichende Gestalt

Das sprachliche Wissen wird hier (in entsprechenden Gruppierungen) in den Beispielapparat in der Form von Kollokationen zusammen mit Wendungen,

31 Als großer Fortschritt ist das korpusbasierte Informationsangebot in dem entstehenden Online-Informationssystem (,Wörterbuch“) elexiko zu verzeichnen; vgl. http://www.owid.de/ artikel/254329 (letzter Zugriff 7.11. 2017), das eine Mittelstellung zwischen dem Online-Duden (Duden online) und dem lexikalischen SOM-Material einnimmt; vgl. den Eintrag zu Tod. Diesen Hinweis verdanke ich Petra Storjohann. 
Redensarten und Sprichwörtern verlagert. Beide in deren Konzeption und Anliegen unvergleichbaren Quellen leisten einen spezifischen Beitrag zur Bedeutungserfassung bzw. Bestimmung der möglichen Lesarten. Das SOMAnalyseverfahren liefert dabei einen greifbaren Beweis dafür, dass das im Korpus abgespeicherte Weltwissen über die momentanen Assoziationen eines Lexikographen bzw. das Angebot des Wörterbuchs in der Regel hinausgeht (vgl. z. B. oben den seltener assoziierten Kontext des sozialen Todes). Die Funktion der lexikalischen Merkmalskarten dient dazu, den aktuellen Skopus objektiv nachweisbarer und aktueller Lesarten zu ermitteln, was für die korpuslinguistisch basierte Wörterbucharbeit unabdingbar ist: Man sollte sich bei der Festlegung der Lesarten keineswegs auf statische Beschreibung der überlieferten Nachschlagewerke verlassen. ${ }^{32}$

\subsection{Von den SOMs zum Modul Contrasting Near Synonyms (CNS, Modellierung semantischer Nähe)}

Die Notwendigkeit, den bei der lexikographischen Beschreibung ermittelten Bedeutungsnuancen gerecht $\mathrm{zu}$ werden, die (nicht nur für den Nichtmuttersprachler) einen Stolperstein darstellen, hat die Entwicklung des Moduls CNS angeregt. Den ersten Anstoß bildeten das Paar genau - pünktlich (siehe unter anderem Vachková 2007: 18). Die Analysen haben damals unter Beweis gestellt, dass hier eine Methode vorliegt, die den schemenhaft konzipierten Lückentest $^{33}$ weit übertrifft. Die Gegenüberstellung zweier Ausdrücke wird durch den Vergleich von deren Gebrauchsdomänen ersetzt (siehe z. B. Vachková et al. 2007), wo das GWDS, Das Große Wörterbuch der Deutschen Sprache (1999), das, mit den Ergebnissen der von Schmidt 2007 erarbeiteten CNS-Analyse ${ }^{34}$ konfrontiert wird):

32 Damit will nicht behauptet werden, dass Analysen der Printwörterbücher bei der Erarbeitung von Datenbankeinträgen völlig ausgeschaltet werden sollten. Auch hier kann man auf periphere Lesarten stoßen, die erfahrungsgemäß vom gebildeten Benutzerkreis (Linguisten, Übersetzer) abgefragt werden.

33 Vgl. die Sets konstruierter Sätze, die Kontraste typischer Verwendungen veranschaulichen sollen, so z. B. bei Schippan (1992: 208). Die CNS-Methode wurde neulich in der Paronymforschung (http://www1.ids-mannheim.de/lexik/paronymwoerterbuch/dasparonymkorpus.html? $\mathrm{L}=1 \% 27 \mathrm{~A} \% 3 \mathrm{D} 0$ (letzter Zugriff 20.5. 2017) verwendet; vgl. die Interpretationen von SOM- und CNS-Karten zu sensitiv und sensibel bei Storjohann \& Schnörch (2017: 155, 157).

34 Die Tabelle ist in Vachková et al 2007 zu finden. Die Erstfassung der CNS-Karte ist unter der Adresse http://docplayer.org/17478276-Maerz-2007-sonderheft-d-14288-sonderheft-auslands kooperationen-des-instituts-fuer-deutsche-sprache.html abrufbar (letzter Zugriff 7.11. 2017). 
Tab. 10.1: CNS = Contrasting Near Synonyms, GWDS = Das Große Wörterbuch der Deutschen Sprache (1999).

\begin{tabular}{|c|c|c|c|c|}
\hline & \multicolumn{2}{|l|}{ genau } & \multicolumn{2}{|c|}{ pünktlich } \\
\hline & GWDS & CNS & GWDS & CNS \\
\hline $\begin{array}{l}\text { exakt, mit einem Muster, } \\
\text { Vorbild übereinstimmend }\end{array}$ & $\checkmark$ & $\checkmark$ & $x$ & $x$ \\
\hline $\begin{array}{l}\text { gewissenhaft ins Einzelne } \\
\text { gehend, korrekt }\end{array}$ & $\checkmark$ & $\checkmark$ & $\checkmark$ & $x$ \\
\hline $\begin{array}{l}\text { den Zeitpunkt genau } \\
\text { einhaltend }\end{array}$ & $x$ & $\checkmark$ & $\checkmark$ & $\checkmark$ \\
\hline $\begin{array}{l}\text { genau zur verabredeten, } \\
\text { festgesetzten Zeit }\end{array}$ & $x$ & $x$ & $\checkmark$ & $\checkmark$ \\
\hline sparsam, haushälterisch & $\checkmark$ & $x$ & $x$ & $x$ \\
\hline $\begin{array}{l}\text { die Exaktheit, Genauigkeit } \\
\text { einer Angabe betonend }\end{array}$ & $\checkmark$ & $\checkmark$ & $x$ & $x$ \\
\hline
\end{tabular}

Kommen wir nun auf die unter Abschnitt 3.1 mittels der KA kontrastierten Lexeme begreiflich und begreifbar zurück, indem die aufgefundenen Cluster der einzelnen SOMs (siehe Abb. A.10.1 und A.10.2 in der Anlage) mit der CNS-Karte zu dem kontrastierten Adjektivpaar (Anlage, Abb. A.10.3) kurz interpretiert und die wichtigsten Cluster angedeutet werden.

Sowohl die SOM zu begreiflich, als auch die SOM zu begreifbar lassen sich nicht von dem aus der KA gewonnenen Bild trennen, aus dem die Unterschiede zwischen der syntaktischen Beschaffenheit beider Lexeme (siehe unter Abschnitt 3.1) ersichtlich sind. Zusammenfassend lassen sich bei begreiflich Verben und Adjektive finden, die rational gesteuerte Prozesse benennen (A), sowie Substantive, die emotionale Zustände beschreiben (C). Diese müssen kommuniziert werden, wobei ihnen (Un)verständnis (= ([Nicht]Begreifen) entgegengebracht wird (D); sie sind in deren existenziellen Dimension berechtigt, worauf der komplexe Cluster F hindeutet: Gefühlslagen und psychische Prozesse nehmen ihre Erscheinungsformen an (untergründig, verhehlen, spüren, wachsen, verfliegen, hervorrufen). Cluster B fasst Aspekte des Nichtbegreifbaren als Gegensätze zum Cluster A zusammen: Die kummulierten Lexeme lassen auf die unbegreiflichen (weil verwunderlichen, suspekten, merkwürdigen, entsetzlichen usw.) Tatsachen schließen. Cluster D signalisiert das Begreiflichmachen als Vorstufe zu Empathie (D).

Die aktuelle Parametereinstellung im Rahmen der Kookkurrenzdatenbank macht es nicht möglich, das Bild in der identischen Version zu generieren. 
In der SOM-Karte zu begreifbar fasst man spontan die Cluster der drei Spalten links ins Auge, angefangen mit A: ${ }^{35}$ Hier geht es um die konkreten Bezüge des Begreifbaren; die Teilbereiche $B$ und $C$ in einem großen Cluster signalisieren den Prozess des Erklärens und nachfolgenden Begreifens und überlappen sich erheblich mit dem Cluster D, der auf die hochfrequenten Faktitiva (begreifbar machen) hinweist, zugleich aber auch die ausgeprägte Semantik von begreifbar nahelegt: Wohl deswegen ist das Adjektiv begreifbar in Kontexten zu finden, wo es um Veranschaulichung, Erlebbarkeit und hautnahe Erfahrung der komplizierten und abstrakten Sachverhalte $(\mathrm{H})$ geht (erfahrbar, erlebbar, darstellen, veranschaulichen, sinnlich, plastisch, lebendig, faszinieren, evozieren, fassbar, anschaulich, greifbar, sichtbar, allgemeinverständlich, lebensnah usw.). Außerdem sind zwei Momente zu erwähnen:

a) das (gegenüber -lich) semantisch ausgeprägte Suffix -bar, und

b) die in manchen Belegen evozierbare Metapher von be-greifen (z. B. Vielfalt aufgreifen und begreifbar machen ${ }^{36}$ ).

Unter E sind Ausdrücke kummuliert, die emotionale Wahrnehmungen beschreiben und einen Gegenpol zum Cluster A darstellen. Die Cluster F (abstrakte Sachverhalte, von denen der emotionale Bereich nicht $\mathrm{zu}$ trennen ist) und $\mathrm{G}$ (Grundfragen der menschlichen Existenz) stehen für schwierig deutbare Inhalte, die es zu begreifen gilt (vgl. z. B. greifbar und begreifbar machen ${ }^{37}$ ).

In der CNS-Karte (Abb. A.10.3 in der Anlage) wird der bereits angedeutete Kontrast zwischen den Kontext-Einbettungen der zwei Adjektive noch krasser ausgedrückt; gleichzeitig aber erscheinen deutlich die fließenden Grenzen des diskursspezifischen Gebrauchs: In diesen Zonen tauchen Lexeme auf, die auf gemeinsame Kontexte bzw. Bezüge der verglichenen Adjektive hinweisen bzw. auf die Konkurrenz von Strukturen (z. B. begreiflich/begreifbar machen). Diese Tatsache signalisieren die orangefarbenen Flächen unterschiedlicher Intensität. Zu den ausgeprägten Domänen sind die jeweiligen roten und gelben Berei-

35 Die Autorin folgt der Anleitung für die Interpretation unter Abschnitt 3.3. Es ist möglich, bei der Erschließung der Cluster auch andere Wege zu gehen, indem die Cluster in einer spontan erfolgenden Reihenfolge abgegrenzt werden können. Die individuelle Interpretation kann modifiziert werden: je nach dem momentanen Assoziationsskopus des interpretierenden Subjekts. Die Kernbereiche der Cluster sind jedoch als deren stabile Kerne anzusehen, was nach dem wiederholten Abrufen zum Vorschein kommt.

36 http://www.drliedtke.de/pdf/seminarangebot2016_vielfalt_aufgreifen_und_begreifbar_ machen.pdf (letzter Zugriff 20. 5. 2017).

37 https://verlage.westermanngruppe.de/veranstaltung/6966/Geometrie-greifbar-undbegreifbar-machen (letzter Zugriff 20.5. 2017). 
che zu zählen, die aus der jeweiligen SOM-Interpretation (siehe oben) dem Leser bereits bekannt sind und als zentral gelten können.

Der Gebrauch von zwei nahen Synonymen erscheint hiermit in einer realistischen Sicht: Die Erfassung zentraler Bereiche, die in den jeweiligen SOM-Karten bzw. der CNS-Abbildung angedeutet wurden, ${ }^{38}$ wäre anhand einer gängigen Bedeutungsumschreibung, wie unter Abschnitt 3.1 gezeigt, ein schwieriges Unternehmen: Der Lexikograph kann bei der Wahl eines Belegs bzw. lexikographischen Beispiels die semantische Relation zwischen den nahen Synonymen im Hinblick auf deren spezifische Bezüge nur schwer ignorieren. Diese sind nämlich schwierig in deren relativer Vollständigkeit abrufbar, wenn man introspektiv vorgeht.

\subsection{Common Global Contexts (CGC)}

Während mit der CNS-Methode in der ersten Phase auch bei der Ermittlung einer ganzen Skala an Kontrasten experimentiert wurde, ${ }^{39}$ ist in der nachfolgenden Forschung die Notwendigkeit entstanden, mittels der SOMs auf die Eruierung ausgeprägter gemeinsamer Gebrauchsdomänen vor allem bei Antonymen bzw. antonymisch gebrauchten Lexemen hinzuweisen (siehe Marková 2012). Schon seit den strukturalistischen Forschungen durch Filipec (vgl. Filipec 1961: 188), der sich der Notwendigkeit bewusst war, Kontexte - und nicht nur in denen vorkommende einzelne Lexeme - zu vergleichen, ist bekannt, dass Synonyme und Antonyme gemeinsames Vorkommen aufweisen (vgl. die Anm. 17), und dass nahe Synonyme (Plesionyme) häufig an Kontrastpaaren beteiligt sind (vgl. bei Cruse 2004: 157). Diese Tatsache konnte nun mit Hilfe der Kookkurrenzprofile aufgrund ihrer (graduellen) Ähnlichkeit quantitativ und statistisch nachgewiesen werden. Als extremes Beispiel für Nullkontrast der Gebrauchsdomänen kann der Fall von links vs. rechts dienen, die sich in globalen Kontexten völlig überlappen: Sie teilen zwei Domänen, nämlich Verkehr und Politik (sowohl die CNS- als auch die CGC-Karte ergeben in diesem Falle ein fast quadratloses orangenfarbenes Bild ${ }^{40}$ ).

38 Erfahrungsgemäß erfolgt die CNS-Analyse zügiger, falls die beiden SOM-Karten vorher herangezogen und reflektiert werden: Der Kontrast-Effekt wie auch die fließenden Grenzen der beiden Lesarten können auf der Basis einer präziseren Beobachtung tiefer nachvollzogen werden. In der aktuellen Fassung des CNS-Moduls sind auch die einzelnen Quadrate beim Anklicken mit Angaben zum prozentuellen Anteil der jeweiligen Gebrauchspräferenzen versehen.

39 Siehe dazu Vachková (2010), wo unter anderem die Begriffe Morgenland und Abendland gegenübergestellt wurden.

40 In diesem Bezug ist anzumerken, dass die Intensität der Farbflächen nicht nur durch den Faktor des Ähnlichkeitsgrades zwischen den jeweiligen KPen, sondern auch von der Frequenz bedingt ist. 
Die CGC-Methode regt die Erforschung beliebiger in Frage kommender Gegensatzpaare an; vgl. Abbilldung A.10.4 zum Gegenwortpaar kühl/kalt bzw. die Abbildung A.10.5 zu kühl/warm. Wie aus den CGC-Karten ersichtlich ist, sind die leicht erkennbaren Verwendungsdomänen nur als erste Orientierungshilfe zu verstehen. (Der Leser kann unmittelbar die Diskurse Wetter und Essen identifizieren.) Die feineren Schattierungen machten eingehende Analysen erforderlich (vgl. Marková 2012: 63-139). Zentrale Lesarten deuten unter anderem auf folgende Tendenzen: kalt neigt dazu, sich auf messbare Temperaturwerte zu beziehen; das Adjektiv kühl wird eher im Zusammenhang mit subjektiver Wahrnehmung schwankender Temperaturen verwendet. Außerdem sind zahlreiche periphere semantische Schattierungen ermittelt worden, wo beide Adjektive vorkommen. Die Visualisierung von CGC bzw. der Kontraste mithilfe des CNS-Moduls sind deswegen immer anhand von begleitenden punktuellen Analysen zu spezifizieren: Diese bestätigen immer wieder die Erkenntnis, dass aus der lexikographischen Sicht eine weite Auffassung der Antonymie praktischer ist: Mit dem Terminus Oppositionsbeziehungen ${ }^{41}$ wird eine breite Skala von Gegensätzlichkeitsrelationen in der parole angedeutet, die bei der korpuslinguistischen Untersuchung belegt und durch die hier genannten Methoden nachvollzogen werden können. Das CGC-Modul macht es möglich, das Bild der lexikalischen Kontraste durch deren gemeinsame kontextuelle Bezüge bzw. deren lexikalische Repräsentanten abzurunden.

\section{Forschungsdesiderate}

Die korpusanalytischen Methoden, die hier präsentiert wurden, liefern zahlreiche Anregungen für die Ermittlung von lexikalisch-semantischen Relationen sowohl auf der lokalen, als auch auf der globalen Ebene bzw. in deren Zusammenspiel. Während die Kookkurrenzanalyse ein zuverlässiges Mittel für die Eruierung der Kollokationsstrukturen und Kookkurrenzpartner eines Wortes auf der lokalen Ebene präsentiert, bieten die selbstorganisierenden Karten und deren Modifikationen in den vorhandenen Modulen des „gläsernen Labors“ auf der Homepage der CCDB wertvolle Einblicke in zentrale sowie periphere

41 Vgl. dazu auch Jones (2002: 1-2) und seinen treffenden Kommentar zu Schwierigkeiten mit dem terminologischen Gebrauch, wo von dem Standpunkt des Sprechers aus argumentiert wird: „Any native speaker would immediately identify the 'opposite' of words such as cold, legal and above without feeling the need to distinguish between gradable antonymy, complementarity and converseness. To deny the status of antonymy to any familiar pair of 'opposites' seems counterintuitive and likely to obscure the underlying uniformity of all such word pairs.“ 
Lesarten, die an das in den Korpora erhaltene Weltwissen gekoppelt sind. Die vorgestellten Analysemethoden sind keineswegs als bloßes „lexikographisches Spielzeug“ zu betrachten, sondern als Quelle von Anregungen für das Studium der Sprache in deren allgemeinen, vor allem kognitiven Bezügen, wo das assoziative Moment eine grundlegende Rolle spielt. Den bisher veröffentlichten Arbeiten sollten weitere Studien folgen, die außer Synonymie und Antonymie auch andere semantische Relationen zu eruieren hätten: Genannt sei in erster Reihe die gebrauchsbasierte Erschließung der Hypo- und Hyperonymie. Die bisherige Forschung hat bereits zahlreiche Anregungen bearbeiten können; viele neue sind jedoch noch erwartbar, wie z. B. Stichproben zu Gegensatzpaaren, an denen unterschiedliche Wortarten teilnehmen. Auch mit der didaktischen Verwendung der hier kurz vorgestellten Visualisierungen sind deren Umsetzungsbereiche wohl noch nicht erschöpft.

\section{Literatur}

Agricola, Christiane \& Erhard Agricola (1992): Wörter und Gegenwörter. Wörterbuch der sprachlichen Gegensätze. Mannheim u. a.: Bibliographisches Institut \& F. A. Brockhaus AG und Dudenverlag.

Barsalou, Lawrence W. (2010): Ad hoc categories. In Patrick C. Hogan (Hrsg.), The Cambridge encyclopedia of the language sciences, 87-88. New York: Cambridge University Press.

Belica, Cyril (2011): Semantische Nähe als Ähnlichkeit von Kookkurrenzprofilen. In Andrea Abel \& Renata Zanin (Hrsg.), Korpora in Lehre und Forschung, 155-178. Bozen-Bolzano University Press. Freie Universität Bozen-Bolzano. Online unter http://corpora.idsmannheim.de/SemProx.pdf (letzter Zugriff 20.5. 2017).

Belica, Cyril, Holger Keibel, Marc Kupietz, \& Rainer Perkuhn (2010): An empiricist's view of the ontology of lexical-semantic relations. In Petra Storjohann (Hrsg.), Lexical-Semantic Relations. Theoretical and practical perspectives, 115-144. Amsterdam: Benjamins.

CCDB: Belica, Cyril (2001-2017): Kookkurrenzdatenbank CCDB. Eine korpuslinguistische Denk- und Experimentierplattform für die Erforschung und theoretische Begründung von systemisch-strukturellen Eigenschaften von Kohäsionsrelationen zwischen den Konstituenten des Sprachgebrauchs. Institut für Deutsche Sprache Mannheim.

Croft, William \& Logan Sutton (im Erscheinen): Chapter 10. Construction grammar and lexicography. Draft. In Patrick Hanks \& Gilles-Maurice de Schryver (Hrsg.), International handbook of modern lexis and lexicography. http://www.unm.edu/ wcroft/Papers/ Lexis-CxG-Final.pdf [letzter Zugriff 15. 4. 2017]).

Cruse, David Alan (2004): Meaning in language: An introduction to semantics and pragmatics. Oxford: Oxford University Press.

Duden online: http://www.duden.de (letzter Zugriff 7.11. 2017)

Eichinger, Ludwig M. (2007): Linguisten brauchen Korpora und Korpora Linguisten - Wege zu wohl dokumentierten und verlässlichen Aussagen über Sprache. In Werner Kallmeyer \& Gisela Zifonun (Hrsg.), Sprachkorpora. Datenmengen und Erkenntnisfortschritt, 1-8. Berlin, New York: de Gruyter. 
Elexiko: siehe Projektinformationen u. http://www1.ids-mannheim.de/lexik/elexiko. (letzter Zugriff 7.11. 2017)

Filipec, Josef (1961): Česká synonyma z hlediska stylistiky a lexikologie. Praha: Nakladatelství Čekoslovenské akademie věd.

GWDS (1999): Das Große Wörterbuch der deutschen Sprache in 10 Bänden. Berlin: Bibliographisches Institut.

Herberg, Dieter (1992): Antonymische Beziehungen im Wortschatz und im Wörterbuch. In Karl Hyldgaard-Jensen \& Arne Zettersten (Hrsg.), Symposium on Lexicography V. Proceedings of the Fifth International Symposium on Lexicography May 3-5, 1990 at the University of Copenhagen., 245-264 (Lexicographica, Series maior 43). Niemeyer: Tübingen.

Jones, Steven (2002): Antonymy. A corpus-based perspective. London, New York: Routledge.

Jones, Steven, Carita Paradis, M. Lynne Murphy \& Caroline Willners (2007): Googling for 'opposites': a web-based study of antonym canonicity. In Corpora, Vol. 2 (2), 129-54.

Jones, Steven, M. Lynne Murphy, Carita Paradis \& Caroline Willners (2012): Antonyms in English. Construals, constructions and canonicity. Cambridge: Cambridge University Press.

Keibel, Holger \& Cyril Belica (2007): CCDB: A Corpus-Linguistic Research and Development Workbench. In Proceedings of Corpus Linguistics 2007, Birmingham. Online verfügbar unter http://corpora.ids-mannheim.de/cl2007-134.pdf (letzter Zugriff 20.5. 2017).

Kloudová, Věra (2015): Synonymie und Antonymie. Literaturhinweise zur Linguistik, Bd. 2. Mannheim: Institut für Deutsche Sprache.

Marková, Věra (2012): Synonyme unter dem Mikroskop. Eine korpuslinguistische Studie. Korpuslinguistik und interdisziplinäre Perspektiven auf Sprache, Bd. 2. Tübingen: Narr.

Müller-Spitzer, Carolin (2007): Der lexikografische Prozess. Konzeption für die Modellierung der Datenbasis. (Studien zur deutschen Sprache 42). Tübingen: Narr.

Murphy, M. Lynne (2006): Antonyms as lexical constructions: Or, why paradigmatic construction is not an oxymoron. Constructions, SV1 (8), 1-37.

Paradis, Carita \& Caroline Willners (2006): Selecting antonyms for dictionary entries: Methodological aspects. Working Papers 6, 95-106. Abrufbar unter http://bit.ly/1JLJ4pg (letzter Zugriff 4.5. 2015).

Paradis, Carita \& Caroline Willners (2007): Antonyms in dictionary entries: Selectional principles and corpus methodology. Studia Linguistica, 61(3), 261-277.

Paradis, Carita \& Caroline Willners (2011): Antonymy: From conventionalization to meaningmaking. Review of Cognitive Linguistics 9, 367-391. Amsterdam: John Benjamins.

Paul, Otto \& Zdeněk Mareček (1997): Německo-český slovník. Olomouc: Fin-publishing 1996. Česko-německý slovník. Olomouc: Fin Publishing 1996. [2. Ausgabe 1997]. In Brünner Beiträge zur Germanistik und Nordistik, Band XI, Sborník prací filozofické fakulty brněnské univerzity R 2, Studia minora facultatis philosophicae universitatis Brunnensis R 2, 105-107.

Perkuhn, Rainer \& Cyril Belica (2006): Korpuslinguistik - das unbekannte Wesen. Oder Mythen über Korpora und Korpuslinguistik. Sprachreport 1/2006, 2-8. http://pub.idsmannheim.de/laufend/sprachreport/pdf/sr06-1a.pdf (letzter Zugriff 18.4. 2017).

Perkuhn, Rainer, Keibel, Holger \& Marc Kupietz (2012): Korpuslinguistik. (= LIBAC Linguistik für Bachelor 3433). Paderborn: Fink.

Schippan, Thea (1992): Lexikologie der deutschen Gegenwartssprache. Tübingen: Niemeyer.

Schmidt, Marek (2010): Zur korpusgestützten Ermittlung naher Synonyme. In Alena Lejsková \& Jana Valdrová (Hrsg.), Die Grammatik, Semantik und Pragmatik des Wortes. Ihre Erforschung und Vermittlung, 140-149. Augsburg: Wißner. 
Schmidt, Marek (2012): Suche nach neuen Wegen der Bedeutungsvermittlung von nahen Synonymen im fortgeschrittenen Fremdsprachenunterricht - eine unendliche oder endlose Geschichte? In: Acta Universitatis Carolinae. Philologica 2/2012, Germanistica Pragensia XXII, 123-131. Praha: Karolinum.

Schwanenflugel, Paula J. \& David C. Gaviska (2005): Psycholinguistic Aspects of Word Meaning. In David Alan Cruse, Franz Hundsnurscher, Michael Job \& Peter Rolf Lutzeier (Hrsg.), Lexikologie. Lexicology. Ein internationales Handbuch zur Natur und Struktur von Wörtern und Wortschätzen. 2. Halbband. Handbücher für Sprache und Kommunikation, 1735-1748. Berlin, New York: de Gruyter. [HSK 21.2].

Šemelík, Martin (2015): Kookurrenzdatenbank CCDB im fortgeschrittenen DaF- und DaZUnterricht. Interkulturelle Sprachdidaktik im Fokus. In Věra Janíková \& Hana Andrášová (Hrsg.), Deutsch ohne Grenzen: Didaktik Deutsch als Fremdsprache, 290-303. Brno: Tribun EU.

Šemelík, Martin (2016): Noch zu Ge-...(-e) vs. -(er)ei. Eine korpuslinguistische und lexikographische Betrachtung. Deutsche Sprache 3/2016, 235-260.

Storjohann, Petra (2005): Sinnrelationen im Wörterbuch - Neue Ansätze und Perspektiven. In EliSe: Essener Linguistische Skripte elektronisch 5 (2), 35-61.

Storjohann, Petra (2005a): Corpus-driven vs. corpus-based approach to the study of relational patterns. In Proceedings of the Corpus Linguistics Conference 2005, Birmingham.1.(1). Birmingham: University of Birmingham. https://ids-pub.bsz-bw.de/ frontdoor/index/index/docld/5006 (letzter Zugriff 2.5. 2017).

Storjohann, Petra (2010): Lexico-semantic relations in theory and practice. In: Petra Storjohann, (Hrsg.), Lexical-semantic relations. Theoretical and practical perspectives, 5-13. Amsterdam: John Benjamins. Lingvisticae Investigationes Supplementa 28.

Storjohann, Petra (2014): Erweiterung von Wortschatzstrukturen durch die Verknüpfung von Synonymen bzw. Antonymen mit kontextgebundenen Kollokationsprofilen. In Annette Klosa (Hrsg.), Deutsche Sprache 4/14. Themenheft: Dateninterpretation und -präsentation in Onlinewörterbüchern am Beispiel von elexiko, 325-346. Berlin: Erich Schmidt.

Storjohann, Petra \& Ulrich Schnörch (2017): Sprachlicher Wandel der Gegenwart und seine Dokumentation in einem Wörterbuch. In Martin Šemelík, Marie Vachková \& Věra Kloudová (Hrsg.), Germanistica Pragensia, AUC Philologica, Germanistica Pragensia 4/ 2016, 133-172. Praha: Karolinum.

Vachková, Marie (2007): Adjektive auf -bar in kontrastiver und korpuslinguistischer Sicht. Eine metalexikographische Betrachtung. In Linguistica Pragensia 2, 57-74.

Vachková, Marie, Marek Schmidt \& Cyril Belica (2007): Prager Wanderungen durch die Manneheimer Quadrate. Sprachreport. Jg. 23, Sonderheft März 2007. IDS Mannheim, 16-21.

Vachková, Marie, Věra Marková \& Cyril Belica (2008): KOrpusbasierte Wortschatzarbeit im Rahmen des fortgeschrittenen Germanistikunterrichts. Zielsprache Deutsch. Zeitschrift für Unterrichtsmethodik und Angewandte Sprachwissenschaft. 3, 20-35.

Vachková, Marie (2009): Lexikografická synonyma, kookurenční profily a ekvivalentace německých abstrakt. Časopis pro moderní filologii 2, 78-89.

Vachková, Marie (2010): Zur Erforschung und Erfassung der diskursgebundenen semantischen Kontraste auf der Grundlage des SOM-Modells. In Germanistica Pragensia XX. AUC Philologica 2, 193-208. Praha: Karolinum.

Vachková, Marie (2017): 15 Jahre nach der Gründung der Lexikographischen Sektion (LS) des Instituts für Germanische Studien: Was haben wir gelernt? In Martin Šemelík, Marie 
Vachková \& Věra Kloudová (Hrsg.), Germanistica Pragensia, AUC Philologica, Germanistica Pragensia 4/2016, 11-20. Praha: Karolinum.

Vachková, Marie (2016): Paradigmatik in der großen lexikalischen Datenbank DeutschTschechisch. Linguistica Pragensia 25, 2015 (2), 103-115.

Vachková, Marie \& Cyril Belica (2009): Self-organizing lexical feature maps. Semiotic interpretation and possible application in lexicography. IJGLSA 13,2 [Intersiciplinary Journal for Germanic Linguistics and Semiotic Analysis. Berkeley. IJGLSA/University of California Press], 223-260. (http://corpora.ids-mannheim.de/IJGLSA.pdf [letzter Zugriff 7.11. 2017]) 


\section{Anlage}

(c) Cyril Belica: Modelling Semantic Proximity - Self-Organizing Map (version: 0.32, init tau: 0.04, dist: u, iter: 10000)

\section{begreiflich}

\begin{tabular}{|c|c|c|c|c|}
\hline $\begin{array}{l}\text { begreifbar } \\
\text { ergründen } \\
\text { vergegenwärtigen } \\
\text { fassbar } \\
\text { faßbar } \\
\text { erfahrbar } \\
\text { verdeutlichen } \\
\text { feststellbar }\end{array}$ & $\begin{array}{l}\text { erklärbar } \\
\text { aufrechterhalten } \\
\text { durchschaubar } \\
\text { vermittelbar } \\
\text { greifbar } \\
\text { berechenbar } \\
\text { herleiten } \\
\text { genießbar }\end{array}$ & $\begin{array}{l}\text { nachvollziehbar } \\
\text { einleuchten } \\
\text { einsichtig } \\
\text { plausibel } \\
\text { einleuchtend } \\
\text { rechtfertigen } \\
\text { vorstellbar } \\
\text { erträglich }\end{array}$ & $\begin{array}{l}\text { erklärlich } \\
\text { schwerfallen } \\
\text { unverständlich } \\
\text { begrüßenswert } \\
\text { unlogisch } \\
\text { ynmöglich } \\
\text { normal } \\
\text { unwahrscheinlich }\end{array}$ & $\begin{array}{l}\text { unbegreiflich } \\
\text { verwunderlich } \\
\text { suspekt } \\
\text { Außenstehende } \\
\text { rätselhaft } \\
\text { gleichgültig } \\
\text { vertraut } \\
\text { verwundern }\end{array}$ \\
\hline $\begin{array}{l}\text { begreifen } \\
\text { ermessen } \\
\text { durchschauen } \\
\text { erkennen } \\
\text { auseinanderhalten } \\
\text { ersichtlich } \\
\text { erahnen } \\
\text { wahr }\end{array}$ & nachvollziehen & $\begin{array}{l}\text { verständlich } \\
\text { zumal } \\
\text { evident } \\
\text { anraten } \\
\text { voraussehba }\end{array}$ & $\begin{array}{l}\text { übertrieben } \\
\text { übertreiben } \\
\text { unangebracht }\end{array}$ & $\begin{array}{l}\text { merkwürdig } \\
\text { eigenartig } \\
\text { seltsam } \\
\text { befremden } \\
\text { unfassbar } \\
\text { unfaßbar } \\
\text { entsetzlich } \\
\text { eigentümlich }\end{array}$ \\
\hline $\begin{array}{l}\text { begriffen } \\
\text { ahnep } \\
\text { wyndern } \\
\text { kennen } \\
\text { herausfinden } \\
\text { egal } \\
\text { eigentlich }\end{array}$ & $\begin{array}{l}\text { Aber } \\
\text { sowieso } \\
\text { jemand } \\
\text { wieso } \\
\text { denn } \\
\text { heutzutage } \\
\text { sowenig } \\
\text { Sprachbarriere }\end{array}$ & $\begin{array}{l}\text { gefühlsmäßig } \\
\text { kein } \\
\text { umtreiben } \\
\text { innewohnen } \\
\text { allzu } \\
\text { drängend }\end{array}$ & $\begin{array}{l}\text { berechtis } \\
\text { berechtis } \\
\text { entspring } \\
\text { innere }\end{array}$ & $\begin{array}{l}\text { irrat } \\
\text { bes }\end{array}$ \\
\hline $\begin{array}{l}\text { aufdrängen } \\
\text { geläufig } \\
\text { ungebildet } \\
\text { ankreiden } \\
\text { klarkommen }\end{array}$ & $\begin{array}{l}\text { rechtschaffen } \\
\text { ausreden } \\
\text { anecken }\end{array}$ & $\begin{array}{l}\text { instinktiv } \\
\text { Triebfeder } \\
\text { Zerstreuung } \\
\text { loswerden }\end{array}$ & $\begin{array}{l}\text { verhehlen } \\
\text { spüren } \\
\text { gewiss } \\
\text { gewiß } \\
\text { wachsen } \\
\text { verflogen } \\
\text { verfliegen } \\
\text { anfänglich }\end{array}$ & $\begin{array}{l}\text { Ungeduld } \\
\text { Zukunftsangst } \\
\text { Gereiztheit } \\
\text { Frustration } \\
\text { Unlust } \\
\text { Apathie } \\
\text { herrschen } \\
\text { Ängstlichkeit }\end{array}$ \\
\hline $\begin{array}{l}\text { nahebringen } \\
\text { klarmachen } \\
\text { näherbringen } \\
\text { weismachen } \\
\text { missfallen } \\
\text { mißfallen } \\
\text { goutiert } \\
\text { vertrauen }\end{array}$ & $\begin{array}{l}\text { vermitteln } \\
\text { beruhigen } \\
\text { ängstigen } \\
\text { Auskommen } \\
\text { gegenübertreten } \\
\text { hineinversetzen }\end{array}$ & $\begin{array}{l}\text { besänftigen } \\
\text { Zaum } \\
\text { einflößen } \\
\text { hergerissen } \\
\text { erwecken } \\
\text { artikuliert } \\
\text { wecken } \\
\text { Verständnis }\end{array}$ & $\begin{array}{l}\text { hervorrufen } \\
\text { hervorgerufen } \\
\text { versetzen } \\
\text { erregt } \\
\text { entgegenschlagen } \\
\text { ausgelöst } \\
\text { lösen } \\
\text { Affekt }\end{array}$ & $\begin{array}{l}\text { Widerwille } \\
\text { Verärgerung } \\
\text { Widerwillen } \\
\text { Aufregung } \\
\text { Befremden } \\
\text { Unverständnis } \\
\text { Unmut } \\
\text { Mißstimmung }\end{array}$ \\
\hline
\end{tabular}

Abb. A.10.1: SOM von begreiflich. 
(c) Cyril Belica: Modelling Semantic Proximity - Self-Organizing Map (version: 0.32, init tau: 0.04, dist: u, iter: 10000)

\section{begreifbar}

\begin{tabular}{|c|c|c|c|c|}
\hline $\begin{array}{l}\text { geschiettlich } \\
\text { physisch } \\
\text { ókonomisch } \\
\text { global } \\
\text { historisch } \\
\text { gesellschaftspolitisch } \\
\text { gesellschaftlich } \\
\text { gesantgesellschaftlich }\end{array}$ & $\begin{array}{l}\text { aufzeigen } \\
\text { determinieren } \\
\text { beeinflussen } \\
\text { Ökosystem } \\
\text { determiniery }\end{array}$ & $\begin{array}{l}\text { begreifen } \\
\text { ergründen } \\
\text { erforschen } \\
\text { verdeutlichen } \\
\text { interessieren } \\
\text { interessiert } \\
\text { ausbaden } \\
\text { einordnen }\end{array}$ & $\begin{array}{l}\text { begreiflich } \\
\text { verstehen } \\
\text { überblicken } \\
\text { klarmachen } \\
\text { enträtseln } \\
\text { ermessen } \\
\text { erahnen } \\
\text { begriffen }\end{array}$ & $\begin{array}{l}\text { nachvollziehbar } \\
\text { nachvollziehen } \\
\text { rational } \\
\text { erklärbar } \\
\text { einleuchten } \\
\text { überschaubar } \\
\text { einteuchtend } \\
\text { erkennbar }\end{array}$ \\
\hline $\begin{array}{l}\text { verorten } \\
\text { begrifflich } \\
\text { ausgeblendet } \\
\text { medjal } \\
\text { ausblenden } \\
\text { Einbettung } \\
\text { diskursiv } \\
\text { assthetisch }\end{array}$ & $\begin{array}{l}\text { auseihandersetzen } \\
\text { konfrontieren } \\
\text { konfrontiert } \\
\text { hineihwachsen } \\
\text { sensibilisieren } \\
\text { Zeitgeschehen } \\
\text { anpassen }\end{array}$ & $\begin{array}{l}\text { nahebringen } \\
\text { näherbringen } \\
\text { zurechtfinden } \\
\text { komplex } \\
\text { vermitteln } \\
\text { hinführen } \\
\text { wahrnehmen } \\
\text { Überblick }\end{array}$ & $\begin{array}{l}\text { auseinanderhalte } \\
\text { nachvollzogen } \\
\text { intuitiv } \\
\text { rüberbringen } \\
\text { erahnt }\end{array}$ & $\begin{array}{l}\text { verständlich } \\
\text { wahrnehmbal } \\
\text { erfassbar } \\
\text { erfaßbar } \\
\text { machen } \\
\text { Fachbegriff } \\
\text { lesbar } \\
\text { ablesbar }\end{array}$ \\
\hline $\begin{array}{l}\text { entfremden } \\
\text { entfremdet } \\
\text { wachhalten } \\
\text { Spannungsverhältnis } \\
\text { Grundbegriff } \\
\text { Instrumentalisierung } \\
\text { Nahtstelle } \\
\text { sondern }\end{array}$ & $\begin{array}{l}\text { Verschränkung } \\
\text { Lebenswirklichkeit } \\
\text { thematisieren } \\
\text { heranführen } \\
\text { Beziehungsgeflecht } \\
\text { LebensweIt } \\
\text { thematisiert } \\
\text { Vopstellungswelt }\end{array}$ & $\begin{array}{l}\text { widerspiegeln } \\
\text { spiegeln } \\
\text { Einblick } \\
\text { transformieren } \\
\text { vielgestaltig } \\
\text { hochkomplex } \\
\text { einfühlen } \\
\text { reflektieren }\end{array}$ & $\begin{array}{l}\text { erfahrbar } \\
\text { erlebbar } \\
\text { darstellen } \\
\text { veranschauliche } \\
\text { visualisieren } \\
\text { veranschaulicht } \\
\text { nachzeichnen }\end{array}$ & $\begin{array}{l}\text { fassbar } \\
\text { faßbar } \\
\text { ansehaulich } \\
\text { greifbar } \\
\text { sichtbar } \\
\text { identifizierbar } \\
\text { allgemeinverständlich } \\
\text { dargestellt }\end{array}$ \\
\hline $\begin{array}{l}\text { Totalität } \\
\text { näherkommen } \\
\text { genuin } \\
\text { untrennbar } \\
\text { Naturgeschichte } \\
\text { personal } \\
\text { impliziert } \\
\text { Grundfrage }\end{array}$ & $\begin{array}{l}\text { Widersprüchlichkeif } \\
\text { nachspüren } \\
\text { Deformation } \\
\text { Wahrnehmen } \\
\text { Artefakt } \\
\text { Zwiespalt } \\
\text { Dekonstruktion }\end{array}$ & $\begin{array}{l}\text { Gefühlswelt } \\
\text { transformiert } \\
\text { Vielschichtigkeit } \\
\text { Gleichzeitigkeit } \\
\text { Innenwelt } \\
\text { Facette } \\
\text { gespiegelt } \\
\text { Gegenwelt }\end{array}$ & $\begin{array}{l}\text { sinnlich } \\
\text { sinnfällig } \\
\text { bildlich } \\
\text { plastisch } \\
\text { lependig } \\
\text { faszinieren } \\
\text { Betrachter } \\
\text { bildhaft }\end{array}$ & $\begin{array}{l}\text { vergegenwärtig } \\
\text { hautnah } \\
\text { vergegenwärtig } \\
\text { realitätsnah } \\
\text { Einzelschicksál } \\
\text { lebensnah } \\
\text { fesselnd } \\
\text { schildern }\end{array}$ \\
\hline $\begin{array}{l}\text { wahren } \\
\text { yniversal } \\
\text { wahr } \\
\text { Ganzheit } \\
\text { Menschsein } \\
\text { schlechthin } \\
\text { göttlich }\end{array}$ & $\begin{array}{l}\text { transzendent } \\
\text { unvolkommen } \\
\text { transzendieren } \\
\text { vełsinnbildlichen } \\
\text { Transzendenz } \\
\text { Vergänglichkeit } \\
\text { Vergegenwärtigung }\end{array}$ & $\begin{array}{l}\text { idealisiert } \\
\text { metaphorisch } \\
\text { abstrahiert } \\
\text { archetypisch } \\
\text { mythisch } \\
\text { idealisieren } \\
\text { abstrahieren }\end{array}$ & $\begin{array}{l}\text { symbolhaft } \\
\text { Alltäglichkei } \\
\text { imaginieren } \\
\text { imaginiert } \\
\text { prosaisch } \\
\text { singulär } \\
\text { evozieren }\end{array}$ & $\begin{array}{l}\text { schrecklich } \\
\text { unbegreiflich } \\
\text { unfassbar } \\
\text { unfaßbar } \\
\text { anrührend } \\
\text { alltäglich } \\
\text { grauenvoll } \\
\text { bedauernswer }\end{array}$ \\
\hline
\end{tabular}

Abb. A.10.2: SOM von begreifbar. 
() Cyril Belica: Modelling Semantic Proximity - Contrasting Near-Synonyms (version: 0.21, init tau: 0.4, dist: x, iter: 10000)

\section{begreifbar begreiflich}

\begin{tabular}{|c|c|c|c|c|}
\hline $\begin{array}{l}\text { Widerwille } \\
\text { Verärgerung } \\
\text { Widerwillen } \\
\text { hervorrufen } \\
\text { hervorgerufen } \\
\text { Ungeduld } \\
\text { Aufregung } \\
\text { Befremden }\end{array}$ & Entfremdung & $\begin{array}{l}\text { anschaulich } \\
\text { sinnlich } \\
\text { sinnfällig } \\
\text { bildlich } \\
\text { visualisieren } \\
\text { plastisch } \\
\text { symbolhaft } \\
\text { Alltäglichkeit }\end{array}$ & $\begin{array}{l}\text { erlebbar } \\
\text { Widersprüchlichkeit } \\
\text { erfahrbar } \\
\text { Gefühlswelt } \\
\text { idealisiert } \\
\text { archetypisch } \\
\text { Deformation } \\
\text { transformiert }\end{array}$ & $\begin{array}{l}\text { transzendent } \\
\text { universal } \\
\text { unvollkommen } \\
\text { transzendieren } \\
\text { Ganzheit } \\
\text { Menschsein } \\
\text { schlechthin } \\
\text { göttlich }\end{array}$ \\
\hline $\begin{array}{l}\text { berechtigt } \\
\text { besänftigen } \\
\text { berechtigen } \\
\text { verhehlen } \\
\text { spüren } \\
\text { gewiß } \\
\text { gewiss } \\
\text { instinktiv }\end{array}$ & $\begin{array}{l}\text { Zaum } \\
\text { Zerstreuung } \\
\text { umtreiben } \\
\text { innere } \\
\text { bändigen } \\
\text { gepeinigt } \\
\text { existenziell } \\
\text { loswerden }\end{array}$ & $\begin{array}{l}\text { hautnah } \\
\text { vergegenwärtigt } \\
\text { unfassbar } \\
\text { unfaßbar } \\
\text { alltäglich } \\
\text { grauenvoll } \\
\text { bedauernswert } \\
\text { furchtbar }\end{array}$ & $\begin{array}{l}\text { widerspiegeln } \\
\text { Verschränkung } \\
\text { nachspüren } \\
\text { spiegeln } \\
\text { Lebenswirklichkeit } \\
\text { Einblick } \\
\text { thematisieren } \\
\text { heranführen }\end{array}$ & $\begin{array}{l}\text { begrifflich } \\
\text { Spannungsverhältnis } \\
\text { Grundbegriff } \\
\text { Wahrnehmen } \\
\text { Totalität } \\
\text { genuin } \\
\text { Einmaligkeit } \\
\text { Instrumentalisierung }\end{array}$ \\
\hline $\begin{array}{l}\text { beruhigen } \\
\text { missfallen } \\
\text { mißfallen } \\
\text { goutiert } \\
\text { ängstigen } \\
\text { goutieren } \\
\text { aufmerksam } \\
\text { mißtrauen }\end{array}$ & $\begin{array}{l}\text { klarmachen } \\
\text { weismachen } \\
\text { rechtschaffen } \\
\text { schmackhaft } \\
\text { ausreden } \\
\text { zumuten } \\
\text { suggerieren } \\
\text { Auskommen }\end{array}$ & $\begin{array}{l}\text { nahebringen } \\
\text { näherbringen } \\
\text { vermitteln } \\
\text { interessieren } \\
\text { ausbaden } \\
\text { interessiert } \\
\text { zurechtfinden } \\
\text { vetraut }\end{array}$ & $\begin{array}{l}\text { auseinandersetzen } \\
\text { verorten } \\
\text { konfrontieren } \\
\text { konfrontiert } \\
\text { ausgeblendet } \\
\text { hineinwachsen } \\
\text { wahrnehmen } \\
\text { näherkommen }\end{array}$ & $\begin{array}{l}\text { physisch } \\
\text { global } \\
\text { historisch } \\
\text { gesellschaftspolitisch } \\
\text { gesellschaftlich } \\
\text { gesamtgesellschaftlich } \\
\text { räumlich } \\
\text { determinieren }\end{array}$ \\
\hline $\begin{array}{l}\text { übertrieben } \\
\text { schwerfallen } \\
\text { zumal } \\
\text { gleichgültig } \\
\text { übertreiben } \\
\text { verwundern } \\
\text { geheuer } \\
\text { aufdrängen }\end{array}$ & $\begin{array}{l}\text { Aber } \\
\text { ahnen } \\
\text { wundern } \\
\text { sowieso } \\
\text { kein } \\
\text { jemand } \\
\text { wieso } \\
\text { denn }\end{array}$ & $\begin{array}{l}\text { verstehen } \\
\text { ermessen } \\
\text { auseinanderhalten } \\
\text { erahnen } \\
\text { kennen } \\
\text { begriffen } \\
\text { herausfinden } \\
\text { verdeutlicht }\end{array}$ & $\begin{array}{l}\text { begreifen } \\
\text { ergründen } \\
\text { vergegenwärtigen } \\
\text { verdeutlichen } \\
\text { doppeln } \\
\text { wahr } \\
\text { aufzeigen } \\
\text { wahren }\end{array}$ & $\begin{array}{l}\text { darstellen } \\
\text { veranschaulichen } \\
\text { erforschen } \\
\text { veranschaulicht } \\
\text { enträtseln } \\
\text { hochkomplex } \\
\text { Überblick } \\
\text { einordnen }\end{array}$ \\
\hline $\begin{array}{l}\text { merkwürdig } \\
\text { eigenartig } \\
\text { verwunderlich } \\
\text { suspekt } \\
\text { irratonal } \\
\text { seltsam } \\
\text { unverständlich } \\
\text { befremden }\end{array}$ & $\begin{array}{l}\text { rechtfertigen } \\
\text { vorstellbar } \\
\text { erträglich } \\
\text { erklärlich } \\
\text { entschuldbar } \\
\text { naheliegend } \\
\text { gerechtfertigt } \\
\text { vernünftig }\end{array}$ & $\begin{array}{l}\text { nachvollziehbar } \\
\text { verständlich } \\
\text { nachvollziehen } \\
\text { erklärbar } \\
\text { einleuchten } \\
\text { einleuchtend } \\
\text { feststellbar } \\
\text { ersichtlich }\end{array}$ & $\begin{array}{l}\text { faßbar } \\
\text { fassbar } \\
\text { rational } \\
\text { durchschaubar } \\
\text { machen } \\
\text { erkennbar } \\
\text { Laie } \\
\text { durchschauen }\end{array}$ & $\begin{array}{l}\text { erfassbar } \\
\text { erfaßbar } \\
\text { sichtbar } \\
\text { überschaubar } \\
\text { überblicken } \\
\text { nachvollzogen } \\
\text { identifizierbar } \\
\text { allgemeinverständlich }\end{array}$ \\
\hline
\end{tabular}

Abb. A.10.3: CNS von begreiflich - begreifbar. 
(c) Cyril Belica: Modelling Semantic Proximity - Common Global Contexts (version: 0.21, init tau: 0.4, dist: x, iter: 10000)

\section{kühl kalt}

\begin{tabular}{|c|c|c|c|c|}
\hline $\begin{array}{l}\text { laben } \\
\text { Erbsensuppe } \\
\text { Waffel } \\
\text { köstlich } \\
\text { Kaffee }\end{array}$ & $\begin{array}{l}\text { lauwarm } \\
\text { Tee }\end{array}$ & $\begin{array}{l}\text { gekühlt } \\
\text { dampfen } \\
\text { erkalten } \\
\text { sieden } \\
\text { Ofen }\end{array}$ & & \\
\hline \multirow[t]{2}{*}{$\begin{array}{l}\text { Punsch } \\
\text { Glühwein } \\
\text { Getränk }\end{array}$} & $\begin{array}{l}\text { Erfrischung } \\
\text { erfrischen } \\
\text { erfrischt }\end{array}$ & $\begin{array}{l}\text { heiß } \\
\text { wärmen } \\
\text { salzig } \\
\text { Wasser } \\
\text { Fön }\end{array}$ & & \\
\hline & $\begin{array}{l}\text { hochsommerlich } \\
\text { lau } \\
\text { Sommertag } \\
\text { Herbsttag } \\
\text { sommerlich } \\
\text { Frühlingstag } \\
\text { Sommer } \\
\text { Sommeranfang }\end{array}$ & $\begin{array}{l}\text { kühlen } \\
\text { warm } \\
\text { stickig } \\
\text { Warme } \\
\text { frösteln } \\
\text { drinnen } \\
\text { geheizt } \\
\text { temperiert }\end{array}$ & $\begin{array}{l}\text { naß } \\
\text { nass } \\
\text { zugig } \\
\text { staubig } \\
\text { frieren }\end{array}$ & $\begin{array}{l}\text { erwärmen } \\
\text { abkühlen } \\
\text { trocknen } \\
\text { gefrieren } \\
\text { glühen } \\
\text { abgekühlt } \\
\text { Wasserdampf } \\
\text { schmilzt }\end{array}$ \\
\hline $\begin{array}{l}\text { sonnig } \\
\text { unbeständig } \\
\text { wechselhaft } \\
\text { Föhn } \\
\text { tagsüber } \\
\text { Wetterfrosch } \\
\text { föhnig }\end{array}$ & $\begin{array}{l}\text { milden } \\
\text { Wetter } \\
\text { frühlingshaft } \\
\text { windstill } \\
\text { wolkenlos } \\
\text { verregnen } \\
\text { Sonnenschein } \\
\text { herbstlich }\end{array}$ & $\begin{array}{l}\text { schwül } \\
\text { trocken } \\
\text { frostig } \\
\text { ungemütlich } \\
\text { Wintertag } \\
\text { Hochsommer } \\
\text { Jahreszeit } \\
\text { Witterung }\end{array}$ & $\begin{array}{l}\text { feucht } \\
\text { feuchten } \\
\text { Kühle } \\
\text { Schwüle } \\
\text { Außentemperatur } \\
\text { Zugluft } \\
\text { Sonne } \\
\text { Kälte }\end{array}$ & $\begin{array}{l}\text { verdunsten } \\
\text { erwärmt } \\
\text { verdunstet } \\
\text { Luft } \\
\text { Feuchtigkeit } \\
\text { nährstoffreich } \\
\text { Verdunstung }\end{array}$ \\
\hline $\begin{array}{l}\text { wolkig } \\
\text { bewölkt } \\
\text { Regen } \\
\text { gebietsweise } \\
\text { Regenschauer } \\
\text { schneien } \\
\text { Vorhersage } \\
\text { Höchsttemperatur }\end{array}$ & $\begin{array}{l}\text { trüb } \\
\text { trübe } \\
\text { naßkalt } \\
\text { nasskalt } \\
\text { regnerisch } \\
\text { neblich } \\
\text { windig } \\
\text { trüben }\end{array}$ & $\begin{array}{l}\text { eiskalt } \\
\text { kälter } \\
\text { eisig } \\
\text { stürmisch } \\
\text { Schneeflocke } \\
\text { unwirtlich } \\
\text { Gefrierpunkt } \\
\text { Ostwind }\end{array}$ & $\begin{array}{l}\text { milde } \\
\text { mild } \\
\text { regenreich } \\
\text { strömen } \\
\text { feuchtkalt } \\
\text { Luftmasse } \\
\text { Westwind } \\
\text { Wetterlage }\end{array}$ & $\begin{array}{l}\text { Abkühlung } \\
\text { wärmer } \\
\text { subtropisch } \\
\text { Temperatur } \\
\text { Grad } \\
\text { Luftfeuchtigkeit } \\
\text { Temperaturunterschied } \\
\text { Luftschicht }\end{array}$ \\
\hline
\end{tabular}

Abb. A.10.4: CNS von kalt - kühl. 
() Cyril Belica: Modelling Semantic Proximity - Common Global Contexts (version: 0.21, init tau: 0.4, dist: x, iter: 10000)

\section{kühl warm}

\begin{tabular}{|c|c|c|c|c|}
\hline $\begin{array}{l}\text { Erfrischung } \\
\text { erfrischen } \\
\text { Kaffee } \\
\text { Getränk }\end{array}$ & genossen & $\begin{array}{l}\text { schattig } \\
\text { strahlend } \\
\text { Sommerabend } \\
\text { strahlen } \\
\text { lauschig } \\
\text { herrlich } \\
\text { Mittagszeit }\end{array}$ & $\begin{array}{l}\text { sonnig } \\
\text { wolkenlos } \\
\text { Herbstwetter } \\
\text { spätsommerlich } \\
\text { Winterwetter } \\
\text { Frühlingswetter }\end{array}$ & $\begin{array}{l}\text { wolkig } \\
\text { bewölkt } \\
\text { föhnig } \\
\text { Regenschauer } \\
\text { regnen } \\
\text { Höchsttemperatur } \\
\text { Vorhersage } \\
\text { Wetterfrosch }\end{array}$ \\
\hline lauwarm & $\begin{array}{l}\text { kühlen } \\
\text { mediterran }\end{array}$ & $\begin{array}{l}\text { lau } \\
\text { frühlingshaft } \\
\text { Hochsommer } \\
\text { Herbsttag } \\
\text { Wintertag } \\
\text { hochsommerlich } \\
\text { Jahreszeit } \\
\text { Sommertag }\end{array}$ & $\begin{array}{l}\text { milde } \\
\text { schwül } \\
\text { mild } \\
\text { Wetter } \\
\text { regnerisch } \\
\text { windig } \\
\text { milden } \\
\text { neblig }\end{array}$ & $\begin{array}{l}\text { wechselhaft } \\
\text { unbeständig } \\
\text { wärmer } \\
\text { Föhn } \\
\text { tagsüber } \\
\text { Westwind } \\
\text { Abkühlung } \\
\text { Ostwind }\end{array}$ \\
\hline \multirow[t]{2}{*}{$\begin{array}{l}\text { gekühlt } \\
\text { erfrischt } \\
\text { salzig } \\
\text { dampfen } \\
\text { sieden } \\
\text { Wasser }\end{array}$} & $\begin{array}{l}\text { heiß } \\
\text { temperiert } \\
\text { wärmen } \\
\text { temperieren } \\
\text { trocknen } \\
\text { geheizt }\end{array}$ & $\begin{array}{l}\text { kalt } \\
\text { trocken } \\
\text { feucht } \\
\text { feuchten } \\
\text { naß } \\
\text { nass } \\
\text { Warme } \\
\text { frösteln }\end{array}$ & $\begin{array}{l}\text { frostig } \\
\text { ungemütlich } \\
\text { kälter } \\
\text { eisig } \\
\text { eiskalt } \\
\text { Schneeflocke } \\
\text { unwirtlich } \\
\text { tauen }\end{array}$ & $\begin{array}{l}\text { Luftmasse } \\
\text { subtropisch } \\
\text { Feuchte } \\
\text { Hochdruckgebiet } \\
\text { Wetterlage } \\
\text { Biowetter } \\
\text { polar } \\
\text { strömen }\end{array}$ \\
\hline & behaglich & & $\begin{array}{l}\text { Witterung } \\
\text { Sommerhitze } \\
\text { Hitze } \\
\text { Temperatur } \\
\text { Grad } \\
\text { Außentemperatur } \\
\text { Gefrierpunkt } \\
\text { Kälte }\end{array}$ & $\begin{array}{l}\text { Luftfeuchtigkeit } \\
\text { verdunsten } \\
\text { verdunstet } \\
\text { Temperaturunterschied } \\
\text { Lufttemperatur } \\
\text { Luftschicht } \\
\text { Feuchtigkeit } \\
\text { Temperaturschwankung }\end{array}$ \\
\hline $\begin{array}{l}\text { luftig } \\
\text { mollig }\end{array}$ & & & & $\begin{array}{l}\text { erwärmen } \\
\text { erwärmt } \\
\text { abkühlen } \\
\text { aufgeheizt } \\
\text { Raumluft } \\
\text { abgekühlt }\end{array}$ \\
\hline
\end{tabular}

Abb. A.10.5: CNS von kühl - warm. 


\title{
Sven Staffeldt
}

\section{1 jetzt verstehen}

\section{Semantikopragmatische Bedeutungsaspekte (im Gebrauch) eines Temporaladverbs}

\begin{abstract}
Das Ziel dieses Aufsatzes besteht darin, ein lexikographisches Angebot für einen Eintrag zu jetzt im gesprochenen Deutsch zu unterbreiten. Dafür werden beispielhaft verschiedene temporale und nicht-temporale Verwendungen von jetzt auf der Basis von Belegen aus dem IDS-Korpus FOLK analysiert. Der Fokus liegt auf der Erfassung dessen, wie jetzt und die jetzt enthaltenden Äußerungen verstanden werden können (und zwar in semantischer und pragmatischer Hinsicht), was jetzt und Äußerungen mit jetzt also für Bedeutungen bzw. Funktionen haben. Bei den temporalen Verwendungen wird dabei ein Bedeutungsaspekt herausgearbeitet und für die Lexikographie stark gemacht, der in bisherigen Beschreibungen nur selten und eher versteckt zu finden ist: das Element des Adversativ-Kontrastiven. Schließlich wird ein Eintrag zu jetzt mit neun Lesarten modelliert, wobei die Anzahl der Lesarten nicht als vollständige Liste von Bedeutungspositionen angesehen werden darf und sich einzelne Lesarten auch überlagern können.
\end{abstract}

Keywords: Deixis, Gebrauchsbedeutung, Gesprochene Sprache, Lexikographie des gesprochenen Deutsch, Pragmatik, Semantik, Temporaladverbial

\section{1 jetzt-Lemmata}

„Was bedeutet jetzt?“, habe ich kürzlich (am 26.7.2017) Google gefragt und erhielt nach einer halben Sekunde die folgende Antwort:

Sven Staffeldt, Julius-Maximilians-Universität Würzburg, Institut für deutsche Philologie, Lehrstuhl für deutsche Sprachwissenschaft, Am Hubland, D-97074 Würzburg,

E-Mail: sven.staffeldt@uni-wuerzburg.de

Ә Open Access. (C) 2018 Sven Staffeldt, publiziert von De Gruyter. (बc) BY Dieses Werk ist lizenziert unter der Creative Commons Attribution 4.0 Lizenz. 


\section{Wörterbuch}

jetzt

\section{jẹtzt}

Adverb

1. verwendet, um auszudrücken, dass etwas im Moment des Sprechens der Fall ist oder sich ereignet.

„Bist du jetzt fertig/zufrieden?“

Partikel

1. verwendet, um eine Aussage oder Frage zu verstärken und eine gewisse Irritation auszudrücken.

„Wer hat jetzt meinen Schlüssel?“

Abb. 11.1: Erste Google-Anzeige auf die Frage „Was bedeutet jetzt?“.

Wie zu erwarten gewesen war, ist diese Google-Antwort netzparasitär. Sie ist beispielsweise gleichlautend mit dem entsprechenden Eintrag des nach Eingabe eines Codes aus dem Printwörterbuch frei zugänglichen digitalen PONS Deutsch als Fremdsprache (https://de.pons.com/), wobei lediglich die Menge der Beispiele reduziert wird:

I. jẹtzt $A D V$

\begin{tabular}{|c|c|}
\hline $\begin{array}{l}\text { verwendet, um auszudrücken, dass etwas } \\
\text { oder sich ereignet }\end{array}$ & \\
\hline Aist du jetzt fertig/zufrieden? & \\
\hline Jetzt können wir anfangen. & \\
\hline Früher war das anders als jetzt. & \\
\hline A II. jẹtzt PART umg $\approx$ nun, eigentlich & (i) \\
\hline $\begin{array}{l}\text { verwendet, um eine Aussage oder Frage z } \\
\text { Irritation auszudrücken }\end{array}$ & \\
\hline Wer hat jetzt meinen Schlüssel? & \\
\hline Jetzt habe ich das schon wieder vergessen! & \\
\hline
\end{tabular}

Abb. 11.2: Eintrag jetzt im digitalen Wörterbuch PONS Deutsch als Fremdsprache, s. v. jetzt; (https://de.pons.com/\%C3\%BCbersetzung?q=jetzt\&l=dedx\&in=\&lf= [letzter Zugriff 9.11. 2017]). ${ }^{1}$

1 Der Eintrag ist textidentisch zu dem Lemma jetzt in der neuesten Auflage des PONS Deutsch als Fremdsprache (vgl. Wolski 2015: 746). 
Wenn man die Beispiele in Bezug zu den gelieferten Verwendungsbeschreibungen setzt - und das ist ja genau die lexikographische Funktion von Beispielen: Sie sollen Verwendungen vor Augen führen, in denen klar wird, was die Verwendungsbeschreibung meint -, dann ergeben sich schnell einige Ungereimtheiten:

1. Einen Fragesatz hätte man nicht unbedingt gleich als erstes Beispiel erwartet, wenn es darum geht, dass mit der Verwendung von jetzt ausgedrückt wird, dass etwas zum Sprechzeitpunkt so ist oder geschieht. Aber sei es drum. Wichtiger ist, ob sich das jetzt in der Frage sinnvoll auf den Sprechzeitpunkt oder nur auf den Sprechzeitpunkt bezieht. Man könnte dann z. B. antworten: Ja, bin ich. Eben zumindest, als du ,jetzt“ gesagt hast, war ich das oder glaubte ich, es zu sein. Jetzt, da ich „jetzt“ sage, fällt mir ein, dass ich doch noch nicht fertig bin. Man will mit so einer Frage - das ist der Punkt gar nicht (nur) wissen, ob es zum Zeitpunkt des Sprechens so ist, sondern ob es darüber hinaus danach (auch noch) so ist. Das jetzt in der Frage bezieht also Zeitpunkte nach der Sprechzeit mit ein (also Zukünftiges). Ähnlich ist der Fall bei dem zweiten Beispiel: Wenn sich das jetzt nur auf die Sprechzeit bezöge, wäre der Zeitpunkt, zu dem man anfangen kann, verloren: Ist die Äußerung getätigt, ist der Moment weg. Das kann also nicht gemeint sein. Auch hier erstreckt sich der Zeitpunkt von jetzt in die direkte Zukunft hinein, was auch durch ab jetzt greifbar gemacht werden könnte.

2. Bei dem früher-Beispiel wird wohl auch nicht der bloße Sprechzeitpunkt gemeint sein. Wenn früher einen unbestimmten, aber wohl eher größeren Zeitraum in der nicht mehr ganz so nahen Vergangenheit erfasst, so wird auch mit jetzt nicht ein kleines Zeitfenster, sondern ein etwas größerer Zeitraum gemeint sein. Man wird davon ausgehen können, dass damit zeitliche Erstreckungen rund um die Jetzt-Zeit der Äußerung erfasst werden. Wenn klar wäre, dass es in nächster Zukunft oder Vergangenheit (sagen wir in oder vor drei Minuten) anders (gewesen) ist, wird man nicht sagen Früher war das anders als jetzt. Hier wird der Äußerungszeitpunkt also aufgebauscht zu einem Zeitraum, der sowohl Vergangenes als auch Zukünftiges mit einschließt.

3. Bei der Partikel jetzt soll es sich um Verstärkung oder den Ausdruck einer Irritation handeln. Was auch für die anderen Beispiele gilt, gilt für die Partikel in ganz besonderem Maße: Hier kommt es auf die Intonation an. Das PONS-Beispiel wird nun folgendermaßen automatisch verbalisiert, wenn man auf das Lautsprechersymbol drückt:

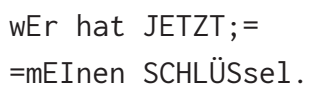


Durch die Realisierung mittels zweier Intonationsphrasen werden die potenziell unterscheidbaren Verwendungsvarianten unkenntlich und die Äußerung bedeutungsmäßig ungreifbar gemacht. Semantisch entscheidend ist doch - neben dem Intonationsverlauf - vor allem der Hauptakzent. Mit Betonung auf wer kann es sich z. B. um eine Verstehenssicherungsfrage handeln (z. B.: "Ich will, dass man noch einmal wiederholt, wer das ist, der/die meinen Schlüssel hat, denn ich kann kaum glauben, was ich gehört habe, oder ich habe es nur unzureichend gehört" usw.); Betonung auf hat ist wohl ein Verum-Fokusakzent (z. B.: "Ich will nicht wissen, wer ihn nicht hat, sondern wer ihn hat"); Betonung auf mei(nen) ist wohl ein Fokusakzent (z. B.: "Ich will nicht wissen, wer den Schlüssel meiner Frau hat, sondern wer meinen hat"); und Betonung auf Schlüs(sel) kann entweder ein unmarkierter Äußerungsakzent sein (z. B.: "Ich suche meinen Schlüssel und frage mich, wo er ist“ - hier würde die Partikelannahme vielleicht am ehesten greifen) oder ein Fokusakzent (z. B.: "Ich will nicht wissen, wer meinen Transponder hat, sondern wer meinen Schlüssel hat"). Bei Betonung auf jetzt kann es semantisch darauf hinauslaufen, dass der Schlüssel kürzlich schon einmal weg war, er sich zwischenzeitlich wieder angefunden hatte und nun (also eben: jetzt) erneut weg ist. Es ist nicht klar, warum zwischen dem Hauptakzent tragenden jetzt in dieser Frage und dem jetzt in der Frage zur ersten Bedeutungsposition (übrigens auch mit den beiden Hauptbetonungsmöglichkeiten auf jetzt oder auf fer(tig)) ein kategorialer Unterschied bestehen sollte dergestalt, dass es sich hier um eine Partikel und dort um ein Temporaladverb handelt.

Die sonderbaren zeitlichen Verhältnisse sind nicht in allen Wörterbüchern so verwischt, wie im PONS DaF. Im Langenscheidt Großwörterbuch Deutsch als Fremdsprache (Götz 2015) etwa findet man fünf Bedeutungspositionen für das Temporaladverb jetzt (siehe Abb. 11.3).

Während die Paraphrase 1 und die Verwendungsbeschreibung 3 auf Zeitpunkte abheben (während des Sprechens oder in der Vergangenheit), erweitert die Paraphrase 2 die zeitliche Ausdehnung auf einen Zeitraum. Bei den Paraphrasen 4 und 5 sind Punkt und Raum verschränkt. Auffällig bei den Beispielen sind die Gegensatzkonstruktionen: jetzt (nicht) vs. später (vielleicht), geregnet vs. Sonne sowie Hitzewelle vs. kühler explizit und früher wenige gejoggt vs. jetzt viele, sie hatte noch nicht alles vs. jetzt hatte sie alles, sie ist noch nicht verheiratet vs. sie wird jetzt heiraten implizit. Überblickt man die Beispiele zum Temporaladverb, so schält sich als ein wesentlicher Bedeutungszug also ein adversativ-kontrastives Element heraus, das aber von der Semantik eines aufs Temporale abzielenden Adverbs nicht ohne Weiteres gedeckt zu sein scheint.

Auch in elexiko findet sich diese Gegensatzbestimmung (siehe Abb. 11.4). 
Deutsch als Fremdsprache Wörterbuch

•jẹtzt

Adverb

1 genau zu dem Zeitpunkt, zu dem man spricht

Ich habe jetzt leider keine Zeit für dich. Komm bitte später wieder

Heute früh hat es geregnet, aber jetzt kommt die Sonne wieder heraus

2 im Zeitraum der Gegenwart

Viele Leute gehen jetzt joggen, um etwas für ihre Gesundheit zu tun

3 verwendet, um einen Zeitpunkt der Vergangenheit zu bezeichnen

Sie hatte jetzt alles, was sie wollte

4 drückt eine Veränderung zwischen einem Zeitpunkt in der Vergangenheit und der

Gegenwart aus $\approx$ inzwischen

Nach der Hitzewelle ist es jetzt wieder kühler geworden

5 in diesem Augenblich oder innerhalb sehr kurzer Zeit

Ich bin fertig, wir können jetzt gehen

Sie wird jetzt heiraten

Partikel betont und unbetont

6 gesprochen besonders in Fragesätzen verwendet, um Verärgerung, Ungeduld oder Verwunderung auszudrücken

Hast du das jetzt noch immer nicht verstanden?

Jetzt habe ich schon wieder vergessen, sie anzurufen!

Was ist denn jetzt schon wieder los?

von jetzt auf gleich gesprochen übertrieben schnell, sofort

Das kann ich nicht von jetzt auf gleich entscheiden

Abb. 11.3: Eintrag jetzt im digitalen Langenscheidt Großwörterbuch Deutsch als Fremdsprache (Götz 2015, s.v. jetzt).

Auch hier werden zunächst (vier) temporale Lesarten von (zwei) Partikellesarten unterschieden, wobei für „in diesem Moment“, „bald“ und „kürzlich“ im Abschnitt darunter festgehalten wird, dass sie Differenzierungen der Lesart „derzeit“" und mitunter schwer gegeneinander abgrenzbar sein sollen. Innerhalb der Bedeutungserklärung zur temporal übergeordneten Lesart „derzeit“ findet sich etwas versteckt - bei größeren Zeiträumen „im Gegensatz zu“. Meine These ist, und das werde ich im weiteren Verlauf dieses Aufsatzes zu belegen versuchen, dass dies als ein wesentlicher Bedeutungszug bestimmter jetzt-Verwendungen anzusehen ist. Dementsprechend müsste dieser dann lexikographisch prominenter behandelt werden.

2 Dieser übergeordneten temporalen Lesart entspricht in etwa das, was in Hoffmann (2016: 357) „Verweis auf einen Nahzeitraum“ genannt wird, wobei der ,jetzt-Zeitraum“ (ebd.) punktuell sein, den Äußerungszeitraum vorhergehender Turns einschließen, in eine erzählte Gegenwart verlagert werden oder sich auf die unmittelbare Zukunft erstrecken kann (vgl. Hoffmann 2016: 357 ff.). Ebenso ist in Zifonun, Hoffmann \& Strecker (1997: 340) von „unterschiedliche[n] Erstreckungen 
Lesart 'derzeit' weiter »

Mit jetzt wird auf einen Zeitraum verweisen, der zum Sprechzeitpunkt die Gegenwart mit einschließt. Auf einen mitunter größeren Zeitraum wird verwiesen, wenn beispielsweise dann (im Gegensatz zu einem abgrenzbaren vorausgehenden oder zukünftigen Zeitraum bzw. Zeitpunkt) eine Handlung abläuft. Auf einen mitunter auch sehr kurzen Zeitraum (oder Zeitpunkt) wird verwiesen, wenn inzwischen (im Zuge einer oft länger dauernden Entwicklung) beispielsweise ein Sachverhalt Gültigkeit erlangt hat bzw. weil nochmals ein Bezug zu Vergangenem hergestellt werden soll.

Lesart 'in diesem Moment' weiter»

Mit jetzt wird punktuell auf einen Zeitpunkt (oder sehr kurzen Zeitraum) verwiesen, der im unmittelbaren Moment der Sprechzeit liegt. Auf diesen Zeitpunkt (bzw. Zeitraum) wird verwiesen, wenn beispielsweise in diesem Moment eine Handlung abläuft oder eine Eigenschaft bzw. ein Sachverhalt Gültigkeit besitzt.

Lesart 'bald' weiter"

Mit jetzt wird auf einen Zeitraum (bzw. Zeitpunkt) verweisen, der relativ zum Sprechzeitpunkt in der (unmittelbaren) Zukunft liegt. Auf diesen Zeitpunkt (bzw. Zeitraum) wird verwiesen, wenn beispielsweise gerade dann eine Handlung abläuft oder ein Sachverhalt Gültigkeit besitzt.

Lesart 'kürzlich' weiter »

Mit jetzt wird auf einen Zeitraum (bzw. Zeitpunkt) verwiesen, der relativ zum Sprechzeitpunkt in der (näheren) Vergangenheit liegt, um diesen zu vergegenwärtigen. Auf diesen Zeitpunkt (bzw. Zeitraum) wird verwiesen, wenn beispielsweise gerade dann eine Handlung abläuft oder ein Sachverhalt Gültigkeit besitzt.

Lesart 'nun' weiter»

Mit der Verwendung von jetzt wird meist in Fragesätzen Ungeduld, Verärgerung, Erstaunen o. Ä. zum Ausdruck gebracht.

Lesart 'Verstärkung'

Eine Lesart Verstärkung in rhetorischen Fragen ist im elexiko-Korpus nicht belegt.

Abb. 11.4: Eintrag jetzt in elexiko (http://www.owid.de/artikel/279193?module=elex\&pos=3 [letzter Zugriff 27. 7. 2017]).

des Nahzeitraums, auf den mit jetzt verwiesen wird“, die Rede (fett und kursiv im Original). Bereits im Grimm'schen Wörterbuch (Deutsches Wörterbuch $=D W$ ) sind neben einigen weiteren Bedeutungen auch diese verschiedenen temporalen Erstreckungen von jetzt festgehalten: vgl. die Bedeutungsangaben 1, 3, 4 und 5 im Lemma jetzt; Deutsches Wörterbuch (1877: 2319 ff.). 


\section{2 jetzt-Verwendungen im gesprochenen Deutsch}

Die an den Wortarten Adverb und Partikel festgemachten Lemma-Gruppierungen in Wörterbüchern spiegeln eine Verwendungsbreite wider, die Wolfgang Imo (2010) in seinem Aufsatz zu jetzt als „zwischen Zeit- und Gesprächsdeixis“ (Imo 2010, Formulierung ist Teil des Titels) liegend beschreibt. Temporale und gesprächsdeiktische Verwendungen schließen sich zwar nicht notwendig aus (sie können sich verschieden stark überlagern, es handelt sich insofern um ein Kontinuum), aber es gibt auch klar nicht-temporale Verwendungen, bei denen jetzt ausschließlich gesprächsdeiktische Aufgaben übernimmt. Man könnte hier mit einer gewissen Vorsicht von einer (beginnenden) „Pragmatikalisierung "3 sprechen:

Der Begriff der Pragmatikalisierung wird hier als gradueller Prozess verstanden, bei dem ein Ausdruck eine diskursive Funktion übernimmt, dabei allmählich seine ursprüngliche Bedeutung verliert und eine sowohl syntaktische als auch phonetische Eigenständigkeit erfährt. (Mroczynski 2012: 123)

Im Folgenden möchte ich zunächst temporale Verwendungen von jetzt mit FOLK-Beispielen demonstrieren, anschließend einige nicht-temporale Verwendungen aufzeigen und anhand der Beispielanalysen dafür argumentieren, dass ein dritter Bedeutungsaspekt stark gemacht werden sollte, der insbesondere temporalen Verwendungen eigen ist oder sein kann: nämlich der adversativkontrastive Aspekt. Darüber hinaus zeigen sich bei den nicht-temporalen Verwendungen einige konversationelle Funktionen, bei denen das AdversativKontrastive aber nicht in den Vordergrund tritt.

Noch ein Wort zu dem empirischen Material. Es handelt sich dabei hauptsächlich um Belege aus dem Forschung- und Lehrkorpus der gesprochenen Sprache (FOLK), ${ }^{4}$ das vom IDS aufgebaut worden ist und mittlerweile zu einer

3 Diachron sind die Verhältnisse verwickelt, weil man mindestens zwei Stränge (jetzt und nun) miteinander vergleichen müsste. Vielleicht ließe sich die These aufstellen, dass eine Pragmatikalisierung von nun bereits im Mhd. zu erkennen war und jetzt im selben Zuge zunächst einmal die verloren gehenden temporalen Bedeutungen von nun aufgefangen hat (und damit einen Bedeutungszuwachs erfahren hat), seinerseits aber im Begriff ist, stärker text- und gesprächsstrukturelle Aufgaben zu übernehmen. Die Frage wäre dann (oder eben nun): Verliert jetzt an temporaler Bedeutung und gibt es eine sprachliche Einheit, die diese Verluste dann auffängt, falls es so sein sollte?

4 FOLK-Beispiele werden im Fließtext ohne eine weitergehende Angleichung an GAT2 so präsentiert, wie sie textuell in FOLK vorgefunden werden (es wird beispielsweise keine erweiternde Neutranskribierung nach Intonationsphrasen mit Hauptakzenten und Tonhöhenendbewegungen vorgenommen). Die Beitragszählungen werden für jedes FOLK-Beispiel bei 01 begonnen, entsprechen also nicht der FOLK-Zählung. 
Art Referenzkorpus für das gesprochene Deutsch zu werden beginnt (somit als kleinerer Bruder des schriftsprachlichen DeReKo angesehen werden kann). Die Suche nach dem Lemma jetzt ergibt dort derzeit (September 2017) knapp 17.500 Treffer. Damit zeigt sich auch in FOLK, was Imo für das damalige Münsteraner Korpus ${ }^{5}$ festgestellt hat, nämlich:

dass das Wort jetzt extrem häufig verwendet wird. [...] Die Hohe Rekurrenz weist darauf hin, dass jetzt offensichtlich wichtige Funktionen in der Alltagskommunikation übernimmt. (Imo 2010: 28)

Die Treffermenge wird von FOLK automatisch auf 10.000 reduziert, aber es dürfte auf der Hand liegen, dass auch diese Belegmenge im Rahmen einer qualitativen Studie nicht vollständig gesichtet werden kann. Für die Zwecke dieses Aufsatzes habe ich mich dagegen entschieden, eine umfassende systematische Studie durchzuführen. Insofern sind die folgenden Ausführungen auch nicht als Resultat eines induktiven Zugangs zu verstehen. Den Ausgangspunkt bilden vielmehr Beschreibungen der Bedeutungs- und Funktionsbreite von jetzt sowie grundsätzliche Überlegungen zur Temporalität von jetzt in solchen Belegen. Insbesondere greife ich hier auch zurück auf die Systematisierungen von Imo (2010), der den Stand der Dinge zu jetzt über die Rezeption einschlägiger Stellen aus der Literatur (darunter auch kanonisierter Literatur zur Deixis) entwickelt hat. ${ }^{6}$

\subsection{Vorrangig temporal beschreibbare jetzt-Verwendungen}

Was man wohl als erstes mit jetzt verbindet, ist die Markierung des Zeitpunkts, zu dem man jetzt sagt. Das Gemeinte ist als jetzt erweiternde Synonymisierung erfassbar mit der Paraphrase: ,jetzt in diesem Moment ${ }^{6}{ }^{7}$ Obwohl dies vielleicht als Grundbedeutung von jetzt angesehen werden kann, ist die Annahme dieser Bedeutung in zweierlei Hinsicht problematisch: Zum einen scheint sie, wie Imo (2010: 28) bemerkt, kaum vorzukommen. Zum anderen gibt es ein

5 Heute angesiedelt im Münsteraner „Centrum Sprache und Interaktion“ (http://audiolabor. uni-muenster.de/cesi/ [letzter Zugriff 26. 7. 2017]).

6 Vgl. unter anderem zu den nicht-formelhaften (bzw. nicht phraseologisch oder konstruktionsgrammatisch zu erfassenden) Verwendungen: Bühler (1982/1934: 102), Hennig (2000: 190 f.), Levinson (2000: 80 f.), Rauh (1978: 61), Rauh (1983: 18), Zifonun, Hoffmann \& Strecker (1997: 340 und 344).

7 Imo (2010: 28-32) erfasst diese Bedeutungsposition mit Bühler als Augenblicksmarke, bei deren Verwendung „der Zeitpunkt der Äußerung des Adverbs mit der Handlung zusammenfällt““ (Imo 2010: 28). 
Problem mit der Größe des Zeitpunktes. Wenn wirklich nur der Zeitpunkt der Äußerung des Adverbs gemeint ist, dann darf die außersprachliche Handlung nicht allzu lange dauern, um komplett mit der Äußerung von jetzt zusammenfallen zu können. Was für eine Handlung soll das sein bzw. was für Handlungen kommen dann überhaupt noch infrage, um mit der Äußerung von jetzt erfassbar zu sein? Man muss also den Zeitpunkt ausdehnen. Dann handelt es sich, pragmatisch gesprochen, im Grunde um eine Sinn-Auflockerung oder eine Sinn-Anreicherung: ${ }^{8}$ Gemeint ist nicht oder nicht nur der minimale, konkrete Zeitpunkt, zu dem ich jetzt sage, sondern vielmehr oder darüber hinaus ein ausgedehnterer, nur vage begrenzbarer Zeitpunkt meiner Äußerung, die jetzt enthält oder vielleicht auch der Zeitpunkt unseres ganzen Gesprächs, in dem eine Äußerung meinerseits vorkommt, die jetzt enthält. Es handelt sich also, bei Lichte besehen, nicht um einen Zeitpunkt, sondern um einen Zeitraum, der diesen Zeitpunkt enthält und in seiner konkreten Ausdehnung nicht auf sekundengenaue Abrechnung ausgelegt ist. ${ }^{9}$ Man könnte dies folgendermaßen visualisieren:

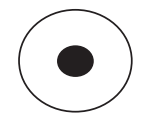

Abb. 11.5: Visualisierung der Bedeutungsposition ,jetzt in diesem Moment' (ausgedehnt). ${ }^{10}$

Der Kreis bezieht sich auf den Äußerungszeitraum, der mit dem denotierten Geschehenszeitraum zusammenfällt, der Punkt dagegen auf den Zeitpunkt der Äußerung des Wortes jetzt. Ein Beispiel für diese Bedeutung ist:

(1) Chance lassen

$01 \mathrm{AB} \quad$ am steuergerät hab ich jetzt keine spannungsversorgung ja

$02 \quad(0.21)$

03 LB mit ihrer messart

04 JM (schnalzt)

8 Also um eine Explikatur nach Sperber, Wilson \& Carston bzw. eine Implizitur nach Bach; vgl. dazu Finkbeiner (2015: 78-82) oder Rolf (2013: 167-171).

9 Levinson (2000: 80) erfasst diesen Zeitraum folgendermaßen: ,die Zeit, zu der der Sprecher die Äußerung produziert, die jetzt enthält‘.

10 Im Übrigen dürfte auch der scheinbar unausgedehnte Zeitpunkt psychologisch eine in Sekunden angebbare kurze Zeitspanne umfassen, und zwar nach Ernst Pöppel bis zu etwa 3 Sekunden (vgl. Pöppel 1997: 59-73) - vielen Dank an Stefan Engelberg für diesen Hinweis. 


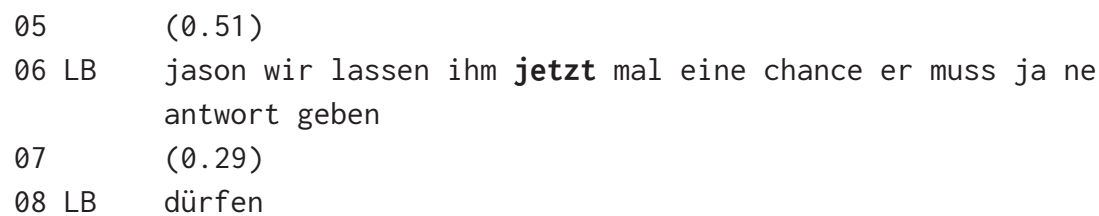

Dieses Beispiel enthält zwei jetzt-Verwendungen, die beide in unterschiedlicher Weise als Momenterfassung verstanden werden können. Im ersten Fall (AB in 01) kann die Handlung des Messens (oder zumindest die Erfassung des Ergebnisses der Handlung: das Messergebnis) in etwa mit dem Zeitpunkt der Äußerung zusammenfallen. Um das beurteilen zu können, bräuchte man allerdings Informationen über das ablaufende Geschehen, die aber leider nicht vorliegen. Bei einem Zusammenfall würde gelten: Es wird gemessen: ein Messergebnis liegt vor; und dieses wird bei Vorliegen verbalisiert.

Auch bei der zweiten jetzt-Verwendung fällt eine Handlung (die des ChanceLassens) mit der Äußerung (hier: von 06-08) zusammen. Es ließe sich im Unterschied zur ersten Verwendung daran denken, bei dieser Verwendung eine performative Struktur herauszuarbeiten. Performativität bedeutet: Indem man sagt, was man tut, tut man, was man sagt. Bezogen auf das Beispiel: Indem 06 bis 08 (oder zumindest: wir lassen ihm jetzt mal eine chance) gesagt wird, wird das vollzogen, was die Bedeutung von 06 bis 08 ist (nämlich: ihm die Chance $z u$ lassen) - mit der Folge, dass er (AB) nun seine Antwort geben darf.

Über diese temporale Bedeutung hinaus entfaltet jedenfalls die zweite jetzt-Äußerung als negativer Bescheid auf das Schnalzen von Jason (JM) aber auch eine adversativ-kontrastive Bedeutung. Es handelt sich bei diesem Ausschnitt nämlich um eine Lehr-Lern-Interaktion mit LB als dem Dozenten und JM und $A B$ (sowie weiteren) als Schülern. LB hat kurz zuvor $A B$ etwas gefragt. Der beginnt zu antworten, macht aber eine Pause, die LB für eine einschränkende Bemerkung (mit ihrer messart) nutzt. Es folgt wiederum eine Pause. Das anschließende Schnalzen von JM darf wohl als Geräusch beim oder zum Melden interpretiert werden (also als Erlaubnisanfrage, ob ihm das Rederecht erteilt werden könne). Auf dieses Schnalzen gibt es dann den an jason (= JM) adressierten negativen Bescheid, dass nicht JM jetzt reden, sondern AB seine Antwort weiter entwickeln darf. Sie machen also nicht, was JM jetzt gern machen würde, sondern das, was LB für richtig hält. Natürlich kann dieser adversativ-kontrastive Aspekt nicht unbedingt oder nicht nur als Bedeutungsaspekt von jetzt angesehen werden. Aber jetzt ist wichtiger Bestandteil einer Äußerung mit diesem Aspekt, denn es geht ja gerade darum geht, was man zu diesem Zeitpunkt macht.

Freilich sind auch punktuelle jetzt-Verwendungen zu finden, bei denen eine Grundbedeutung in etwa so paraphrasiert werden kann: ,jetzt, da ich jetzt 
sage‘. Das Levinson-Beispiel zur Verdeutlichung dieser Bedeutung, nämlich: Drück den Schalter jetzt! (vgl. Levinson 2000: 81) scheint mir aber - im Unterschied zu der Beurteilung von Imo (2010: 28) - nicht sonderlich gut geeignet, diese Verwendung zu illustrieren, und zwar aus folgenden Gründen: Mit der Äußerung von Drück den Schalter jetzt! ${ }^{11}$ wird standardmäßig (also, wenn nichts dagegen spricht) wohl ein direktiver Akt vollzogen. Für solche beziehen sich mehrere Gelingensbedingungen auf eine zukünftige $\mathrm{H}^{12}$-Handlung (z. B. dass $\mathrm{H}$ dazu in der Lage ist, eine zukünftige Handlung auszuführen, wozu auch gehören dürfte, dass $\mathrm{H}$ diese Handlung nicht bereits ausgeführt hat oder ausführt). Es ist also gerade nicht so, dass hier ein „extremes Zusammenfallen von sprachlicher und außersprachlicher Handlung oder außersprachlichem Geschehen“ (Imo 2010: 28) erwartbar oder verstehbar ist, sondern die Äußerung wird standardmäßig als der außersprachlichen Handlung vorausgehend interpretiert werden müssen. Ein vielleicht besseres Beispiel ist das folgende:

(2) Hallgeber

01 LB wer kann mir_n mal ganz kurz erklären (.) wie_s halve (.) hallgeberprinzip funktioniert (.) aber jetzt nur en grobe abriss

02

03 LB wo sin meine elektriker

$04 \quad(1.92)$

05 LB bitte die hand hoch

$06 \quad(2.47)$

07 LB el ei[ner wo sind die andern]

08

$[(($ Gemurmel, 1.3s $))]$

$09 \quad(0.21)$

10 XM ((schnieft))

$11 \quad(0.44)$

$12 \mathrm{AB}$ isch des de einigste elek ah kali [ganz schüch]tern (.) kommt die hand hoch (.) so nee jetzt wer kann mir kurz erklären wie hallgeber funktioniert

An diesem Beispiel (2) interessiert uns zunächst die jetzt-Verwendung in 12. Es handelt sich um eine syntaktisch desintegrierte Verwendung, bei der jetzt nicht

11 Levinson (2000: 81) beschreibt diese „gestische Verwendung“ temporal näher als Zeitspanne des Augenblicks, „in dem das Morphem selbst produziert wird“.

12 „H“ = Hörer/Hörerin oder Leser/Leserin. 
als Temporalangabe innerhalb einer satzförmigen Einheit fungiert. Vielmehr besetzt jetzt eine Scharnierposition zwischen nee (was seinerseits durch so im Diskurs verankert wird) ${ }^{13}$ und der zur Meldung auffordernden Wissensabfrage wer kann mir kurz erklären ... Temporal ließe sich sagen: Die Verwendung von jetzt markiert den Umschlagpunkt zwischen einer vorhergehenden und einer folgenden sprachlichen Aktivität. Und da dieser Umschlagpunkt ja genau in dem Moment vorliegt, in dem jetzt geäußert wird, ist die Bedeutung ,jetzt, da ich jetzt sage‘ voll eingelöst. Damit verbunden sind natürlich aufmerksamkeitssteuernde Funktionen. Wiederum ließe sich daran denken, hier Implizituren oder Explikaturen anzunehmen (etwa eine Vervollständigung des Inhalts mittels: Konzentration, aufpassen (jetzt) oder ähnliches), was auf den Vollzug direktiver Sprechakte hinausliefe. Bereits das bloße jetzt scheint aber hinreichend, genau diese Funktion zu übernehmen. Wiederum liegt hier eine Art performative Grundstruktur vor: Was immer ich sonst noch tue, indem ich jetzt sage, so gilt doch, ich meine genau den Augenblick jetzt, da ich jetzt sage. Indem ich jetzt sage, markiere ich also den Augenblick als das, was er ist und was zur Bedeutung von jetzt gehört. Ich hebe den jetzigen Augenblick heraus, indem ich jetzt sage.

Es zeigt sich aber auch hier eine adversativ-kontrastive Komponente. Mit so nee jetzt wird markiert, dass der Gesprächsabschnitt, in dem LB fragt und kaum jemand sich meldet, zu Ende ist und ein neuer beginnt, der von stärkerer Teilnahmebereitschaft geprägt sein soll. Es gibt in Bezug auf so nee jetzt in (12) also eine Vorher-Situation, die überwunden werden soll (= schwache, zögerliche Bereitschaft, sich zur Beantwortung der Frage in 01 zu melden), und eine Nachher-Situation, die eben diese Überwindung der Vorher-Situation sein soll (entschlossenes Melden und engagierte Antwortversuche). Damit wird der Druck auf die Schüler erhöht, endlich eine Antwort zu geben, und zugleich ist die Phase des Tipp-Gebens durch LB (mittels isch des de einigste elek ah kali in (12)) vorbei. In der Visualisierung erscheint einfach nur ein Punkt:

Abb. 11.6: Visualisierung der Bedeutungsposition ,jetzt in diesem Moment' (unausgedehnt), also: ,jetzt, da ich jetzt sage‘.

13 Prosodisch wird so nee jetzt - darüber gibt das Transkript allein leider keine genaue Auskunft - folgendermaßen realisiert: so NEE (. ) JETZT (. ). Es handelt sich hier also nicht, was man ohne Informationen zur Prosodie ansonsten auch annehmen könnte, um eine etwaige kollokative Einheit nee jetzt mit spaßigem Unterton. 
$\mathrm{Zu}$ den mehr oder weniger ausgedehnten Zeitpunktbedeutungen kommen nun weitere Zeitraumbedeutungen hinzu. Da haben wir zunächst die Lesart, nach der durch jetzt der Abschluss eines Geschehens markiert wird. Obwohl das Ergebnis bei dieser Lesart als statisch zu verstehen ist (ein Zustand ist erreicht, der im Moment jedenfalls gerade besteht), kann sich jetzt als Temporaladverbial nicht nur auf die Zeit des Ergebniszustandes beziehen, sondern als Temporaladverbial der zeitlichen Erstreckung auch auf die Dauer eines kürzlich stattgefundenen Geschehens. Prototypische Beispiele wären: Oh, jetzt habe ich einen Fehler gemacht ${ }^{14}$, Ah ja, jetzt weiß ich wieder ${ }^{15}$ oder $O$ ja, jetzt fällt es mir wieder ein. ${ }^{16}$ Synonymisch erweitert paraphrasieren lässt sich diese (von Imo zurückverweisend genannte) Bedeutungsposition mit ,jetzt gerade eben'. Hier ein FOLK-Beispiel:

(3) von hinten aufgezäumt

01 LB da hinne traut sich jemand herr fischer

$02 \mathrm{TF} \quad((\text { schmatzt }))^{\circ} \mathrm{h}($.$) ja wenn$

$03 \quad(0.21)$

04 TF wenn die blende im luftspalt isch dann ähm (.) isch des des signal für_s steuergerät dass ähm

$05 \quad(0.23)$

06 TF primärstrom fließen muss un[d dann wird der]

07 LB [her fischer ] sch

$08 \quad(0.29)$

09 LB stopp entschuldigung

$10 \quad(0.34)$

11 LB beginnen sie doch einfach mal (.) bei zündung ein mir fangen mal vorne an (.) ganz einfach

12 XM ${ }^{\circ}$ hh [((hustet)) ]

13 LB [sie hawwen je]tzt des ganze von hinten aufgezäumt

$14 \quad(0.42)$

15 LB vielleicht könn_mer des ganze mal von vorne mache (.)

16 US ((hustet))

17 LB wir zwei zusammen ich helfe ihnen

In 13 bewertet LB die in 01-05 gegebene Antwort von TF (= Herr Fischer) als von hinten aufgezäumt. Diese Bewertung ist als Begründung zu verstehen, wa-

$14 \mathrm{https} / / /$ books.google.de/books?isbn=3862745759 (letzter Zugriff 18. 9. 2017).

15 https://books.google.de/books?isbn=3844290354 (letzter Zugriff 18. 9. 2017).

16 https://books.google.de/books?isbn=3641090008 (letzter Zugriff 18. 9. 2017). 
rum LB nach einer erfolgreichen Unterbrechung Herrn Fischer auffordert, seine Antwort noch einmal so $\mathrm{zu}$ formulieren, dass er vorne anfängt (wofür LB schließlich seine Hilfe anbietet). 13 dürfte nun eine ambivalente Bewertung sein. Einerseits hat TF nichts oder nicht nur Falsches gesagt, andererseits lässt die Antwort für LB erkennen, dass TF das Erfragte noch nicht so verinnerlicht hat, dass er es in einer vielleicht sachlogischen Abfolge darstellen kann. Visualisiert werden kann dies folgendermaßen:

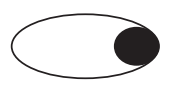

Abb. 11.7: Visualisierung der Bedeutungsposition ,jetzt gerade eben‘.

Und auch hier zeigt sich wieder ein adversativ-kontrastives Element. Was Herr Fischer gerade eben gemacht hat, soll gleich in Folge anders gemacht werden. Erschien die Antwort von Herrn Fischer gerade eben nicht in der richtigen, sachlogischen Reihenfolge, soll der Sachverhalt nun von vorn konzeptualisiert werden. Als Slogan dafür wäre geeignet: Eben so, jetzt aber so. Das nächste FOLKBeispiel kombiniert die gerade-eben-Lesart mit einer zukunftsbezogenen:

(4) Was passiert jetzt?

01 LB [ja] (.) gibt_s (.) en fachausdruck für diese schaldung

$02 \mathrm{RZ}$ darling[schalt ]

$03 \mathrm{XM} \quad[(($ hustet $))]$

04 LB ja (.) hier haben wir eine darlinschaltung sehr schön herr zopf (.) ja

$05 \quad(0.28)$

06 LB so (.) un jetzt sim_mer hier und jetzt kommt (.) der herr (.) fischer an den punkt an dem er ganz am anfang gewesen war herr fischer

$07 \quad(0.49)$

08 LB was passiert jetzt jetzt kommt der entscheidende

$09 \quad(0.31)$

10 LB die entscheidende schaltung

11 (1.26)

$12 \mathrm{TF}$ ja und äh

13 LB ja

$14 \mathrm{TF}$ (.) jetzt kann (.) von

$15 \quad(0.21)$ 
16 TF von fünfzehn auf ähm einunddreißig strom fließen also fließt primärstrom

17 LB (. ) genau jetzt haben wir unsern primärstrom fließen

$18 \quad(0.24)$

19 LB sehen se

$20 \quad(0.26)$

21 LB des war jetzt gar net so (.) kompliziert

Zunächst liegen wieder einige gerade-eben-Verwendungen vor, nämlich zweimal in 06 und resümmierend in 21. Darüber hinaus kann man aber auch vier Verwendungen (zweimal in 08, in 14 und in 17) feststellen, die sich in die Zukunft erstrecken. Diese (von Imo projizierend genannte) Bedeutungsposition kann charakterisiert werden mit ,ab jetzt' (wie in 14) oder, jetzt gleich sofort im nächsten Moment' (wie in 08). Prototypische Beispiele wären Komm, wir gehen jetzt, Herrgottnochmal!17 oder Jetzt wird er gleich wieder wütend sein. ${ }^{18}$ Ich spare mir an dieser Stelle das Vor-Augen-Führen der Interpretation dieser jetzt-Verwendungen in Beispiel (4), die folgendermaßen visualisiert werden können:

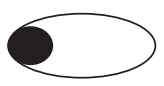

Abb. 11.8: Visualisierung der Bedeutungsposition ,ab jetzt', ,jetzt gleich sofort im nächsten Moment'.

Auch hier gibt es zum Teil Vorher-Nachher-Szenarien als adversativ-kontrastive Komponenten. Was vorher nicht möglich war, ist es nachher sehr wohl (dass z. B. ein bestimmter Strom fließen kann). Ob es sich dabei um ein inszeniertes Lehr-Lern-Szenario handelt (zum Beispiel mit Zeichnungen von Schaltungen statt mit konkreten, ausprobierbaren Schaltungen), kann anhand des Transkripts allein nicht entschieden werden. Man wird damit rechnen können, dass besonders deiktisch inszenierte jetzt-Verwendungen (also gewissermaßen „als Deixis am Phantasma“) über adversativ-kontrastive Elemente hinaus auch etwas Pointierendes haben. Jedenfalls weist die Einleitung des LB-Höhepunktes dieses Ausschnittes in 08 gleich zweimaliges (in Lehrfrage und Lehrantwort vorkommendes) jetzt auf. Es ist dies zugleich der Moment, in dem LB klärend

17 https://books.google.de/books?isbn=3257602324 (letzter Zugriff 18. 9. 2017). 18 https://books.google.de/books?isbn=364421901X (letzter Zugriff 18. 9. 2017). 
auf den früheren, von hinten aufgezäumten Beitrag von TF zurückkommt. Rückwirkend kann man (und soll man vielleicht auch) diesen Höhepunkt als didaktisch von langer Hand geplant ansehen. Man musste sich erst bis zu diesem Punkt vorkämpfen, den TF bei der früheren Meldung an den Anfang gestellt hatte; und erst ab jetzt, jetzt gleich sofort im nächsten Moment (und eben nicht am Anfang) kommt das an die Reihe, was TF in einer durch LB als sachlogisch unzutreffend charakterisierten Weise gleich als erstes geantwortet hatte.

Bei den bisherigen Verwendungen ist der durch jetzt erfasste Zeitraum prototypisch als eher kurz zu verstehen. Hoffman (2016: 357) nennt dies den „Nahzeitraum“. Es kann sich aber auch um größere Zeiträume oder um andersartige Temporalisierungen handeln. In den folgenden beiden Fällen ist die Bedeutungsposition paraphrasierbar mit ,dieses Mal'. Hier schwenkt die Temporalität um von Erstreckung eines Nahzeitraums auf Markierung eines nächsten Zeitpunkts/-raums innerhalb einer Abfolge von Zeitpunkten/-räumen:

(5) Weihnachtsgeld

$01 \mathrm{EM}$ oh ich bin mal gespannt wie viel weihnachtsgeld ich jetz krieg

$02 \quad(0.87)$

03 EM ende nächster woche müsst_es da sein

(6) die ganze Woche

01 NI un normal geht se nur zwei tage die woche arbeiten am (.) donnerstag freitag dann fährt se donnerstag

$03 \quad(0.24)$

$04 \mathrm{NI}$ morgens los

$05 \quad(0.34)$

$06 \mathrm{NI}$ übernachtet bei ihrer mutter kommt freitag nach der arbeit wieder heim ${ }^{\circ} \mathrm{h}($.$) aber jetz is die kollegin in urlaub$ jetz muss se die ganze woche arbeiten

(7) Hoffenheim

01 MT [welcher is des]

02 XM1 [+++++++++++++++]

$03 \mathrm{CH}$ günes

$04 \quad(0.59)$

$05 \mathrm{NI}{ }^{\circ} \mathrm{h}$ den den ich immer hab

$06 \quad(0.39)$ 
07 SK simuni[C ]

$08 \mathrm{NI} \quad$ [bloß] dass der (.) jetz in hoffenheim sitzt un nich mehr (.) bei hertha

In solchen jetzt-Verwendungen liegt der Kontrast bzw. der Gegensatz in dem Bruch mit Geschehenserwartungen aufgrund habitualisierter Abläufe. Der Slogan lautet: „Normalerweise ist es so, jetzt aber so“. In Beispiel (5) gibt es Grund zu der Annahme, dass sich das diesjährige Weihnachtsgeld in seiner Höhe von vorausgehenden Zahlungen unterscheidet. In Beispiel (6) nimmt das erste jetzt darauf Bezug, dass die normalerweise arbeitende Kollegin in der fraglichen bzw. in dieser Woche im Urlaub ist. Bei der zweiten jetzt-Verwendung in Beispiel (6) wurde die Geschehenserwartung zuvor explizit gemacht. Normalerweise gestaltet se ihre Arbeitswoche soundso, doch dieses Mal muss sie es soundso machen. Auch in Beispiel (7) ist die frühere Normalität (der spielt bei Hertha) nicht mehr gegeben (der sitzt in Hoffenheim). Den temporalen Kontrast bzw. Gegensatz zeigt jetzt an; ohne jetzt würde sich der durch bloß als solcher markierte Widerspruch eher nur auf die falsche Annahme beziehen.

Natürlich ist jetzt weder immer alleiniger Kontrast-/Gegensatzanzeiger und jetzt ist auch nicht ausschließlich adversativ-kontrastiv (eine temporale Semantik ist durchaus noch auszumachen). Aber es ist ein entscheidendes Mittel, um ein Vorher-Nachher-Szenario zu etablieren, und darin ist - das dürften und sollen die Beispiel zeigen - ein wichtiges Element der Bedeutung von temporalen jetzt-Verwendungen $\mathrm{zu}$ sehen.

Ein Übergangsbereich zu den nicht mehr vorrangig temporal zu verstehenden jetzt-Verwendungen ist in dem folgenden Fall festzumachen:

(8) Sperrmüll

01 AW hast deinen schon abgeholt

$02 \quad(0.21)$

$03 \mathrm{HM}$ nee meiner steht da noch den sch[meiße mer ] bitte net weg

$04 \mathrm{AW} \quad[(($ Lachansatz $))]$

$05 \mathrm{AW} \quad((\text { Lachansatz }))^{\circ} \mathrm{hh}$ (.) äh okay (.) dann ähm

$06 \mathrm{BS} \quad(($ lacht $))$

$07 \quad(1.07)$

08 AW den den wir nich mehr brauchen ab in_n keller für_n sperrmüll (.) ha ha mit den türen für_n sperrmüll (.) ${ }^{\circ} \mathrm{h}$

$09 \quad(1.22)$

10 HM hm_hm (.) 
11 AW weil des dauert jetz wieder_n halbes jahr oder jahr ne $01 \mathrm{HM} \quad{ }^{\circ} \mathrm{h}$

$13 \quad(0.64)$

$14 \mathrm{HM}$ na ja ich denk so

$15 \quad(0.99)$

16 HM bissel müsse mer noch warte ich denk zu de oschterferie meld i die noch_e mol a un dann schmeiße mer_s zeug weg

Bei diesem Ausschnitt handelt es sich um ein Stück aus einer Mitarbeiterbesprechung in einer sozialen Einrichtung, bei dem unter anderem auch Renovierungsarbeiten zu klären sind. In diesem Zusammenhang kommt die Frage auf, wie mit verschiedenen Putz-Utensilien zu verfahren ist. Konkret geht es hier um den Staubsauger von HM. In Segment 11 liegt zunächst einmal ein jetzt des Typs ,ab jetzt ' vor. Das Temporaladverbial jetzt und die Mensuralergänzung $n$ halbes jahr oder jahr zu dauern teilen sich hier das temporale Geschäft: jetzt markiert den Punkt, ab dem etwas zu laufen beginnt und in die Zukunft erstreckt, und $n$ halbes jahr oder jahr legt die zeitliche Erstreckung fest. Mit jetzt wieder wird aber zudem wohl eine Sprechereinstellung ins Spiel gebracht: AW scheint es nicht gut zu finden, wenn nicht weggeschmissen wird, denn dann steht es unerträglich lange rum. Das zeigt auch der entschlossen vorgetragene Kompromissvorschlag mit $a b$ in $n n$ keller. Wenn schon nicht weggeschmissen werden darf, so sollte doch wenigstens weggeräumt werden. Ein halbes oder ganzes Jahr soll das Zeug jedenfalls nicht mehr rumstehen.

\subsection{Vorrangig nicht-temporal beschreibbare jetzt-Verwendungen}

Imo (2010: 36-40) rekonstruiert gesprächsdeiktische Verwendungen von jetzt, die entweder etwas mit Inszenierungen bzw. Deixis am Phantasma, oder mit der (Re-) Organisation der Gesprächsstruktur („Übertragung des Zeitkonzepts von der ,realen“ Zeit auf die Gesprächszeit“; vgl. Imo 2010: 51) zu tun haben. Bei diesen Verwendungen gibt es einen Übergangsbereich, in dem temporal zu Verstehendes noch unterschiedlich großes Gewicht hat. Ich möchte an diese Überlegungen anschließen und weitere, aber immer weniger bis gar nicht mehr temporal zu verstehende Verwendungen anführen.

Bereits oben in Beispiel (2) (Segment 12) hatten wir eine kaum noch bis gar nicht mehr als temporal zu erfassende Verwendung von jetzt gesehen (obwohl diese Verwendung eigentlich dem Kern der wörtlichen Bedeutung entsprechen müsste). Vielmehr scheint eine diskursfunktionale Funktion zu dominieren, von der Imo sagen würde, dass die Zeitdeixis auf die Gesprächsdeixis übertra- 
gen wird. Der jetzt-Zeitpunkt markiert das Geschehen zu dem Zeitpunkt, an dem jetzt gesagt wird, als ein Geschehen mit Neustart: Bis hierhin haben wir dies gemacht, aber passt auf: nachfolgend macht ihr doch bitte das. Ein Beispiel zur Verdeutlichung (aus einem Prüfungsgespräch) - der Vergewisserung, dass ein bestimmter Themenbereich vorbereitet wurde, folgt die konkrete Prüfungsfrage, und jetzt markiert genau diesen Übergang:

(9) Luther

01 HS alles klar (.) so also sie ham sich befasst mit (.) äh martin luther und sprachgeschichte ja

$02 \quad(0.41)$

03 HS jetz

$04 \quad(0.32)$

05 HS es gibt zwei so landläufige

$06 \quad(0.83)$

07 HS ((schmatzt)) ja ich würd jetz nich sagen märchen aber meinungen ${ }^{\circ} \mathrm{h}$ äh die eine besagt martin luther ${ }^{\circ} \mathrm{h}$ hat als erster die bibel (.) ins deutsche übersetzt (.) und die zweite ${ }^{\circ} \mathrm{h}$ martin luther is (.) praktisch der erfinder ${ }^{\circ} \mathrm{h}$ der neuhochdeutschen

Diese Scharnierfunktion kann jetzt auch in Kombination mit weiteren, funktional an solchen Stellen ähnlich zu verwendenden Einheiten wie so oder also übernehmen:

(10) Achtung

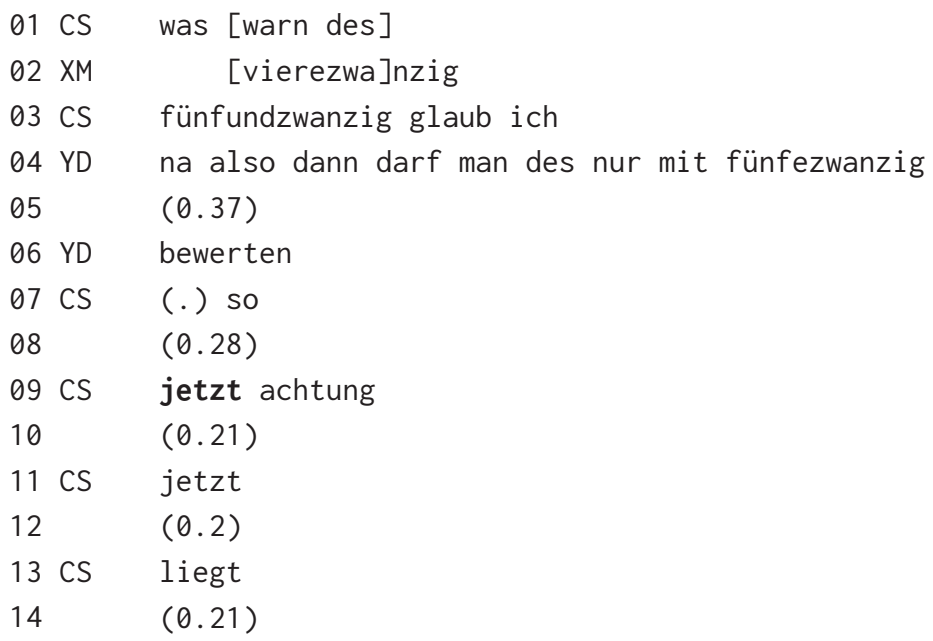




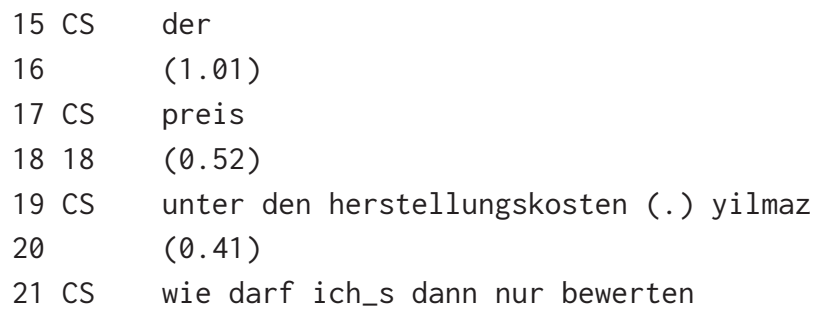

In Bezug auf die angenommene Funktion der Aufmerksamkeitssteuerung ist die Verwendung in 09 bei diesem Beispiel natürlich besonders schön. In 11 - eine im Grunde konditionale Verwendung des Typs ,jetzt in diesem Moment ${ }^{*}$ - tritt die Temporalität zugunsten des Kontrastes stark zurück. Yilmaz soll bedenken, was vorher nicht hinreichend oder einfach noch nicht bedacht wurde, dass der Preis nämlich unter den Herstellungskosten liegt. Nach preis hätte auch gut ein aber produziert werden können. Muss aber nicht; jetzt allein reicht aus.

Eine weitere diskursfunktionale Verwendung hat jetzt weiterhin auch in deliberativen Phasen der Sprachproduktion:

(11) Tabelle

$01 \mathrm{AM} \quad$ ja aber wie is es des (.) ähm (.) [vo ]m [äh
$02 \mathrm{PH}$
$03 \mathrm{PH}$
[des]

$04 \quad(0.35)$

05 AM äh insgesamt

$06 \quad(0.5)$

07 AM vom preisvergleich

$08 \quad(0.63)$

09 AM also wenn du alle

$10 \quad(0.24)$

11 AM äh jetzt mal sozusagen

$12 \quad(0.45)$

13 AM äh einreihen würdest und so e äh

$14 \mathrm{PH}$ was[_n ]

15 AM [ne ta]belle machen würdest wie viel (.) was kostet das welches (.) äh markenbier sozusagen is des günstigste

Es scheint sich auf den ersten Blick um eine Ausprägungsvariante der von Imo beschriebenen Konstruktion Sag ich (jetzt) mal (so) / ich sag (jetzt) mal bzw. [sagen wir mal so] zu handeln, die übrigens auch in Segment 07 in Beispiel (9) vorliegt (vgl. Imo 2010: 42-44; Imo 2007: 108). Während Imo (2007: 114) diese 
Konstruktion noch als Fragment ansieht, für das gilt „Die syntaktische Form ist [...] bereits stark verfestigt, die Funktion jedoch nicht“, legt sich Imo (2010: 44) hinsichtlich der Funktion auf die folgende Beschreibung fest:

Die Funktion der Floskeln, die die Wörter sagen und jetzt enthalten, besteht also generell in der Modalisierung von Äußerungen in dem Sinne, dass mögliche Kritikpunkte vorweg genommen werden und dass eine Äußerung als vorläufige Formulierung markiert wird. (Imo 2010: 44)

Mir scheint eine solche Funktionsbeschreibung für die von Imo beschriebenen spezifischen Konstruktionen (bzw. die von Imo untersuchten Konstrukte) mit ich, sag, mal und (optional) jetzt und so zwar zutreffend zu sein, eher nicht aber für die vorliegende Verwendung. Meines Erachtens liegt die Leistung von Segment 11 hier vielmehr darin, Zeit für die Überwindung einer sprachproduktionstechnischen Blockade zu gewinnen, die insgesamt gerahmt wird durch die beiden äh. Durch 11 wird klar, dass AM auf der Suche nach einer passenden Beschreibung (und letztlich eben nach einem passenden Wort) ist; 13 (und wohl insbesondere einreihen) ist dann das beste oder passendste, was AM produzieren kann. Man nimmt hier keinen Kritikpunkt vorweg oder markiert die Formulierung als vorläufig, sondern man markiert (bzw. projiziert) etwas als Kompromiss bei der Lösung eines Formulierungsproblems. Ohnehin handelt es sich ja schon um eine in 09 eingeleitete Reformulierung dessen, was zuvor in einer offenbar Probleme bereitenden Formulierungsarbeit nicht auf den Punkt gebracht werden konnte. Wenn das so ist, dann handelt es sich entweder nicht um eine Variante der von Imo beschriebenen Konstruktion, oder aber die Beschreibung dieser Konstruktion muss erweitert werden.

Die gleiche Funktion darf in dem folgenden Fall angenommen werden, in dem ja also jetzt also jetzt im im insgesamt den Prozess der Formulierungssuche anzeigen (wobei das zweite jetzt in 04 auch als Fokuspartikel zu einer Präpositionalgruppe mit im angesehen werden kann, die so aber in 05 nicht mehr bzw. mit dem für im grammatisch unpassenden paris erst in 06 realisiert wird):

(12) Paris

$\begin{array}{lll}01 & \text { AJ } & \text { ja } \\ 02 & \text { AJ } & \text { also } \\ 03 & \text { AJ } & \text { jetzt } \\ 04 & \text { AJ } & \text { also jetzt im im } \\ 05 & (0.97)\end{array}$

06 AJ öh (.) paris macht sie noch_n praktikum bei nem fotografen das is auch schon $h^{\circ}$ 
Nicht-temporale Verwendungen können insbesondere auch dann postuliert werden, wenn jetzt nicht als Adverbial innerhalb einer satzförmigen Einheit auftaucht, sondern direkt vor oder direkt nach Nominal- und Präpositionalgruppen, die als solche keine temporale Bedeutung haben. Man könnte auf die Idee kommen, wegen dieser Positionen hier von einer Fokuspartikel zu sprechen:

(13) Schreiben

$\begin{array}{ll}01 \mathrm{HN} & { }^{\circ} \mathrm{h} \text { das möcht ich jetz mit ihnen herausarbeiten } \\ 02 & (0.29) \\ 03 \mathrm{KW} & \text { ähm bei dem schreiben jetz } \\ 04 \mathrm{HN} & \text { genau }\end{array}$

An dieser Stelle eines Prüfungsgesprächs rückversichert sich die Studentin KW, ob sich 01 auf das schreiben bezieht. Es ist nicht leicht, für die Verwendung von jetz in 03 eine über die generelle Funktion der Heraushebung und damit der kontrastiv zu ansonsten denkbaren Einheiten erfolgenden Hochstufung kognitiv-rezeptiver Relevanz von bei dem schreiben hinausgehende Funktion zu erkennen. Meine Vermutung wäre, dass jetz signalisieren könnte, dass KW nicht einfach nachfragt, ob das schreiben gemeint sei (oder etwas anderes), sondern die starke Vermutung ausdrückt, HN könnte das schreiben gemeint haben (und nicht etwas anderes), diese Vermutung bzw. dieses Verständnis aber gern - ein begreiflicher Zug in einem Prüfungsgespräch - abgesichert wissen will. Dadurch erscheint bei dem schreiben nicht als hilflose Nachfrage, worauf sich $\mathrm{HN}$ in 01 bezogen haben könnte - was im Übrigen auch gegenüber HN ungeschickt wäre, denn so würde 01 als ziemlich vage herausgestellt; man läuft Gefahr, eine prüfungstaktisch problematische Gesichtsbedrohung zu begehen. Vielmehr zeigt KW eine aktive Präsenz. Sie versteht, was HN meint, auch wenn HN dies nicht voll expliziert, weshalb sie sich dann aber doch noch kurz rückversichert. Interessant an dieser konkreten Stelle ist noch zweierlei: Zum einen hat HN ja selbst zuvor jetz (des Typs ,jetzt gleich sofort im nächsten Moment') geäußert. Durch diese Rekurrenz schließt KW gewissermaßen kohärent an. Zum anderen - und das hängt damit zusammen - hat jetz hier auch eine gesprächsdeiktische Funktion des Rückverweises auf 01: Die Rückversicherung bezieht sich auf 01 und jetz zeigt gewissermaßen da hin (wodurch natürlich noch ein geringer semantischer Anteil an Temporalität des Typs ,jetzt gerade eben' erhalten bleibt).

(14) Im kleinen Stil (Überlappungen in 04-09 werden nicht angezeigt)

01 US ${ }^{\circ}$ hh und dann ähm (.) war sie halt so fair un wir ham halt wir ham halt ähm mit der (.) frau schlosser dann noch gere- 
det und sie meinte halt sie hätte sich eh für mich entschieden ${ }^{\circ} \mathrm{h}$ weil sie des halt auch sowieso fair findet wenn der

02 (0.36)

03 US wenn der zweite der (.) weil im ersten jahr is_es ja immer am meisten arbeit

04 AM (.) genau

05 US du machst die ganzen materialen des wie_n referendar und dann_n lehrer so nach dem motto also ${ }^{\circ} \mathrm{h}$ so $\operatorname{im}($.$) im$ kleinen stil jetz

$06 \mathrm{NH}$ jaja natürlich

$07 \mathrm{NH}$ du musst dich vorbereiten (.) ja

$08 \mathrm{AM} \quad \mathrm{hm}$

$09 \mathrm{NH} \quad \mathrm{klar}$

$10 \mathrm{NH} \quad \mathrm{hm}$

11 US ${ }^{\circ} \mathrm{h}$ und dann hat die andere auch gemeint irgendwie weil ich dann nachher dann eh ins ausland geh ${ }^{\circ} \mathrm{h}$ und sie jetz sowieso irgendwie en relativ stressiges semester hatm (.) is sie (.) eigentlich relativ einverstanden so[wieso damit] dass ich des jetzt mach und sie macht_s dann da[nach (.) wieder ]

Auch in 05 haben wir es mit einem nachgestellten jetz zu tun (nebenbei: Die beiden jetzt-Verwendungen des Typs, dieses Mal‘ in 11 zeigen sehr schön den Schwenk vom Nahzeitraum zur Zeitpunktabfolge). In 05 erscheint es aber zusammen mit so als Teil eines Rahmens, der insgesamt wohl fokussierende Funktion hat: so... jetzt. Die Kombination mit so kommt aber auch nicht-rahmend vor (wobei in 07 bei Beispiel (15) auch eine temporale Bedeutung vorliegen kann, nämlich dann, wenn zur Äußerungszeit Sommer ist und RK genau diesen Sommer meint und nicht den Fall, wenn Sommer ist):

(15) Im Sommer

01 RK wird das nich irgendwie sch[lecht we ]nn ihr das so

$02 \mathrm{EH}$

$[($ hustet $))]$

03

04 AK nö (.) kälte is do_gut $h^{\circ}$

$05 \quad(0.37)$

06 AK ((hustet))

07 RK [aber jetz so im im sommer]

$08 \mathrm{EH} \quad$ [aber im 


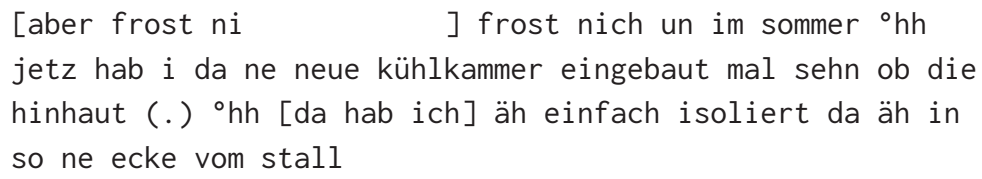

\section{Was ein LeGeDe-Eintrag jetzt berücksichtigen müsste}

An dieser Stelle möchte ich die beispielhafte Demonstration verschiedener Bedeutungspositionen von jetzt-Verwendungen im gesprochenen Deutsch abbrechen und zu einer Art Fazit kommen. Eins ist klar: Es bleibt noch viel zu tun. Das betrifft insbesondere die Identifizierung und Beschreibung von verfestigten, formelhaften Formen, von denen Imo (2010) bereits einige beschrieben hat (und die hier nicht weiter behandelt wurden). Elexiko kennt unter der Lesartkennzeichnung nun davon vor allem den ersten Typ (also den in Fragen wie Was (ist) jetzt? Was soll das jetzt? Wie jetzt? usw.). Darüber hinaus hat Imo noch beschrieben:

- sag ich (jetzt) mal (so)/ich sag (jetzt) mal;

hier scheint jetzt nach Imo allerdings semantisch verblasst $\mathrm{zu}$ sein und auch keine eigenständige Funktion zu haben,

und

- jetzt mal im Ernst/echt jetzt/wenn ich jetzt ganz ehrlich bin zur Anzeige der Interaktionsmodalität.

Hinzu kommen auf jeden Fall noch Verwendungen als Fokusoperator, aber sicher noch weitere. Das müssten etwas umfangreichere Studien ermitteln, etwa im Rahmen des LeGeDe-Projekts („Lexik des gesprochenen Deutsch“), das derzeit am IDS läuft. Die arbeitspraktische Frage wird bei notwendig qualitativem Vorgehen dabei aber sein: Wie lange darf man sich mit einer Einheit wie jetzt beschäftigen? Mal ganz davon abgesehen, ob man die Frage leicht beantworten kann, ob es sich überhaupt um die Einheit jetzt handelt oder um eine konstruktionsgrammatisch zu erfassende Einheit mit jetzt als einem Füllelement.

Der oben zitierte OWID-Eintrag zu jetzt (in elexiko) fächert die temporalen Verwendungen schon gut auf, kann aber noch optimiert werden und sollte um 
nicht-temporale Lesarten erweitert werden. Mit den in diesem Aufsatz verwendeten Lesarten-Beschreibungen würde man sich der Verwendungsbreite vielleicht so nähern können:

1. Temporale Lesarten

Alle temporalen Lesarten können über ein Bedeutungselement des Gegensatzes oder Kontrastes verfügen. Mit der Verwendung von jetzt wird dann ein Vorher-Nachher-Szenario aufgemacht. In dem oder vor dem Zeitpunkt oder Zeitraum, der durch jetzt erfasst wird, war es so, nach dem oder in dem durch jetzt erfassten Zeitpunkt oder Zeitraum aber so. Jetzt markiert in solchen Fällen also, dass etwas im Vergleich zu Zeitpunkten und Zeiträumen danach oder davor anders ist. Damit ist die Verwendung von jetzt gut geeignet, um einen Gegensatz oder Kontrast herzustellen. Nicht alle Verwendungen weisen diese Bedeutung auf, aber sie kann für viele Verwendungen als ein grundlegendes Merkmal angesehen werden.

a) Lesart ,jetzt, da ich jetzt sage،

Durch die Verwendung von jetzt markiert man den Zeitpunkt, zu dem das Wort jetzt geäußert wird. Äußerungen mit jetzt-Verwendungen dieses Typs beziehen sich inhaltlich auf sehr kurze Geschehnisse (Handlungen, Vorgänge usw.), die parallel zur Äußerung von jetzt ablaufen.

b) Lesart, jetzt in diesem Moment‘

Durch die Verwendung von jetzt markiert man mindestens den Zeitraum, innerhalb dessen die jetzt enthaltende Äußerung getätigt wird. Äußerungen mit jetzt-Verwendungen dieses Typs beziehen sich inhaltlich auf Geschehnisse (Handlungen, Vorgänge usw.), die parallel zur jetzt enthaltenden Äußerung stattfinden und eine gewisse zeitliche Ausdehnung haben, die nicht immer nur kurze Zeiträume umfassen muss.

c) Lesart ,jetzt gerade eben“

Durch die Verwendung von jetzt markiert man den Zeitraum, bevor jetzt geäußert wird und in dem der durch jetzt erfasste Zeitpunkt das Ende dieses Zeitraums darstellt. Äußerungen mit jetzt-Verwendungen dieses Typs beziehen sich inhaltlich auf Geschehnisse (Handlungen, Vorgänge usw.), die vor der Äußerung von jetzt abgelaufen sind. Diese Lesart geht einher mit der Betonung des Endzustandes eines Prozesses, der mit dem jetzt-Zeitpunkt zusammenfällt.

d) Lesart ,ab jetzt' bzw. ,jetzt gleich sofort im nächsten Moment‘ Durch die Verwendung von jetzt markiert man den Zeitraum, nach dem jetzt geäußert wird und in dem der durch jetzt erfasste Zeitpunkt den Anfang dieses Zeitraums darstellt. Äußerungen mit jetzt-Verwendungen dieses Typs beziehen sich inhaltlich auf Geschehnisse (Handlungen, Vorgänge usw.), die der Äußerung von jetzt mehr oder weniger 
direkt folgen oder folgen sollen (etwa Aufforderungen, etwas zu tun, oder Ankündigungen, dass etwas geschieht, oder Beschreibungen von Zuständen usw.). Bei dieser Lesart ist das Element des Gegensätzlichen besonders stark ausgeprägt.

e) Lesart ,dieses Mal

Durch die Verwendung von jetzt markiert man keinen Zeitpunkt oder Zeitraum, der in Bezug zu der Zeit zu setzen wäre, während derer man die jetzt enthaltende Äußerung tätigt. Vielmehr markiert man den nächsten oder einen zurückliegenden Zeitpunkt oder Zeitraum in einer Folge nacheinander ablaufender Zeitpunkte oder Zeiträume. Diese Verwendung nimmt also Bezug auf wiederholt ablaufende Geschehnisse. Auch hier ist das Element des Gegensätzlichen besonders stark ausgeprägt. Der durch jetzt markierte nächstfolgende oder zurückliegende Zeitpunkt oder Zeitraum unterscheidet sich in bestimmter Hinsicht von den anderen.

2. Nicht-Temporale Lesarten

a) Verwendung als Anzeiger für Wortsuchprozesse

funktionale Lesart: ,Warte, ich komme gleich drauf

Bei dieser Verwendung kann mit der Äußerung von jetzt (und evtl. weiteren Einheiten wie ja, also, so, äh usw.) die Formulierungstätigkeit unterstützt werden. In einem Äußerungsvorgang fällt einem das passende Wort nicht ein und während man nach dem Wort sucht, wird jetzt geäußert.

b) Verwendung als Einheit zur Gesprächsgliederung

funktionale Lesart: ,Pass auf!

Bei dieser Verwendung markiert jetzt den Beginn oder das Ende (bzw. beides) von Gesprächsteilen. Damit verbunden ist die Anzeige von etwas Neuem oder etwas besonders Pointiertem (Themawechsel, Reformulierungen, Aufforderungen usw.), wozu man besondere Aufmerksamkeit braucht. Jetzt kann in solchen Verwendungen als aufmerksamkeitssteuerndes Element gebraucht werden.

c) Verwendung als Fokuspartikel funktionale Lesart ,Hör genau hin, das ist wichtig!‘

Bei dieser Verwendung hebt jetzt insbesondere nominale oder präpositionale Einheiten hervor und setzt sie damit als für den Gesprächsverlauf an dieser Stelle besonders prominent. Dabei kann jetzt direkt vor oder direkt nach der durch jetzt fokussierten Einheit geäußert werden. Kombinierbar ist jetzt als Fokuspartikel mit so.

d) Verwendung als Abtönungspartikel funktionale Lesart: ,Was passiert (ist), nervt mich“ 
Bei dieser vor allem in Fragen vorkommenden Verwendung wird jetzt zum Ausdruck einer negativen Einstellung (z. B. Ärger, Unmut, genervt sein usw.) verwendet. Die jetzt enthaltenden Fragen mit $W$-Fragewörtern haben einen stark phraseologischen Charakter.

So, das war's jetzt erst einmal.

\section{Literatur}

Bühler, Karl (1982/1934): Sprachtheorie: Die Darstellungsfunktion der Sprache. 2. Aufl. 1934.Stuttgart: Lucius \& Lucius.

DW (1877): Deutsches Wörterbuch von Jacob Grimm und Wilhelm Grimm. Bd. 10: H - Juzen. Leipzig: Hirzel.

elexiko (2003 ff.): Online-Wörterbuch zur deutschen Gegenwartssprache. In OWID - Online Wortschatz-Informationssystem Deutsch, hrsg. vom Institut für Deutsche Sprache, Mannheim. http://www.owid.de/wb/elexiko/start.html (letzter Zugriff 27. 7. 2017).

Finkbeiner, Rita (2015): Einführung in die Pragmatik. Darmstadt: WBG.

FOLK: Forschungs- und Lehrkorpus Gesprochenes Deutsch, hrsg. vom Institut für Deutsche Sprache, Mannheim, http://agd.ids-mannheim.de/folk.shtml (letzter Zugriff 27. 7. 2017)

Götz, Dieter (Hrsg.) (2015): Langenscheidt Großwörterbuch Deutsch als Fremdsprache. Das einsprachige Wörterbuch für alle, die Deutsch lernen. Buch mit Online-Anbindung: Deutsch-Deutsch (Einsprachige Wörterbucher). Ausgabe 2015, herausgegeben von Dieter Götz, in Zusammenarbeit mit der Langenscheidt-Redaktion. München/Wien: Langenscheidt.

Hennig, Mathilde (2000): Tempus und Temporalität in geschriebenen und gesprochenen Texten. Tübingen: Niemeyer.

Hoffmann, Ludger (2016): Deutsche Grammatik. Grundlagen für Lehrerausbildung, Schule, Deutsch als Zweitsprache und Deutsch als Fremdsprache. 3. Aufl. Berlin: Schmidt.

Imo, Wolfgang (2007): Construction Grammar und Gesprochene-Sprache-Forschung. Konstruktionen mit zehn matrixsatzfähigen Verben im gesprochenen Deutsch. Tübingen: Niemeyer.

Imo, Wolfgang (2010): Das Adverb jetzt zwischen Zeit- und Gesprächsdeixis. ZGL 3, 25-58. Levinson, Stephen C. (2000): Pragmatik. Tübingen: Niemeyer.

Mroczynski, Robert (2012): Grammatikalisierung und Pragmatikalisierung. Zur Herausbildung der Diskursmarker wobei, weil und ja im gesprochenen Deutsch. Tübingen: Narr.

PONS DaF: PONS Deutsch als Fremdsprache. http://de.pons.com/Übersetzung/deutsch-alsFremdsprache (letzter Zugriff 26.7. 2017).

Pöppel, Ernst (1997): Grenzen des Bewußtseins. Wie kommen wir zur Zeit, und wie entsteht Wirklichkeit? Frankfurt a. M./Leipzig: Fischer.

Rauh, Gisa (1983): Aspects of Deixis. In Gisa Rauh (Hrsg.), Essays on Deixis, 9-60. Tübingen: Narr.

Rauh, Gisa (1978): Linguistische Beschreibung deiktischer Komplexität in narrativen Texten. Tübingen: Narr.

Rolf, Eckard (2013): Inferentielle Pragmatik. Zur Theorie der Sprecher-Bedeutung. Berlin: Schmidt. 
Wolski, Werner (2015): PONS Großwörterbuch Deutsch als Fremdsprache. Mit OnlineWörterbuch und Drehscheibe „unregelmäßige Verben“. Stuttgart: PONS.

Zifonun, Gisela, Ludger Hoffmann \& Bruno Strecker (1997): Grammatik der deutschen Sprache. Bd. 1. Berlin/New York: de Gruyter (= Schriften des IDS 7.1). 


\title{
12 Deutsch als Fremdsprache und die deutsch-englische Lexikographie bis 1900
}

\begin{abstract}
Die Geschichte der deutsch-englischen Lexikographie aus der Perspektive Deutsch als Fremdsprache wurde bis jetzt kaum beachtet. Hier wird diese Geschichte von ihren Anfängen im 17. Jahrhundert bis ca. 1900 skizziert. Drei Phasen werden unterschieden: 1. eine erste Phase von polyglotten Vokabularen bis gegen Ende des 17. Jahrhunderts; 2. die Anfänge einer bilingualen deutsch-englischen Lexikographie, 1687-1800; und 3. die Entstehung und Herausbildung einer deutsch-englischen Lexikographie mit englischsprachigen Lernern als expliziter Zielgruppe, 1800 bis ca. 1900. Neben einer zu erwartenden zunehmenden Reife in der Präsentation und in den angebotenen Informationen zur Grammatik, Phraseologie und Aussprache, kann auch ein wachsendes Interesse nachgewiesen werden, praktische Hilfe für die Übersetzung aus der Muttersprache ins Deutsche anzubieten sowie eine zunehmende Berücksichtigung der Fachterminologie. An der deutsch-englischen Lexikographie lassen sich auch zwei andere Entwicklungen der deutschen Sprachgeschichte
\end{abstract}

\footnotetext{
Anmerkung: Es ist mir eine Freude und eine Ehre, mit diesem Beitrag Professor Ludwig Eichingers unermüdliches Interesse an der Vielfältigkeit der deutschen Sprache zu würdigen. Gleichzeitig ergreife ich die Gelegenheit, den Aufsatz auch meinem britischen Kollegen William Jervis (Bill) Jones (geb. 1941) in seinem 77. Lebensjahr zu widmen, der mit Herrn Eichinger das rege Interesse an der Geschichte der Sprachwissenschaft und der Sprachreflexion (vgl. Jones 1999) sowie an der Entwicklung und der Varietät der deutschen Lexik teilt - Themen, die in meinem Beitrag zusammenkommen. Bill Jones habe ich die akribische Geschichte der Farbbezeichnungen zu verdanken (vgl. Jones 2013), auf die ich mich hier zum Teil stütze, aber noch grundsätzlicher: Seinem Vorbild verdanke ich die Inspiration, sich als britische Germanistin überhaupt erst auf das Feld der Geschichte der deutschen Lexikographie zu wagen (vgl. Jones 2000). Die Bedeutung der Lexikographie und der Metalexikographie für die Gegenwart zeigt sich in besonderem Maße anhand zahlreicher Wörterbuchprojekte der Abteilung Lexik am IDS, die Professor Eichinger seit vielen Jahren begleitet. Die Bemühungen um die Pflege der Geschichte und um die Dokumentation der deutschen Sprache auf beiden Seiten des Kanals durch diese beiden Männer sollen hiermit gewürdigt werden.
}

Nicola McLelland, University of Nottingham, School of Cultures, Languages and Area Studies, University Park, Nottingham NG7 2RD, Great Britain,

E-Mail: nicola.mclelland@nottingham.ac.uk

Ә Open Access. (c) 2018 Nicola McLelland, publiziert von De Gruyter. (c) BY Dieses Werk ist lizenziert unter der Creative Commons Attribution 4.0 Lizenz. 
und Geschichte der Sprachwissenschaft ablesen: sprachpuristische Tendenzen und die historisch-vergleichende Wissenschaft. Beachtenswert ist - auf diesem sonst rein männlichen Feld - auch der Beitrag einer bisher völlig unbekannten Lexikographin, Elizabeth Weir (1888).

Keywords: Adolphus Bernays, Karl Breul, Deutsch als Fremdsprache, deutschenglische Lexikographie, Georg Heinrich Noehden, James Oehlschäger, Sprachgeschichte, Elizabeth Weir, Gebhard Wendeborn, Wörterbücher

\section{Einleitung}

Dieser Beitrag untersucht die Geschichte der deutsch-englischen Lexikographie bis 1900 aus der Perspektive der Geschichte des Deutschlernens durch englischsprachige Lerner. Die Geschichte der Lexikographie - der Wörterbücher und Vokabulare - wurde in meinem Beitrag zur Geschichte des Deutsch als Fremdsprache (DaF) in England 1600-2000 explizit ausgeklammert (McLelland 2015). Auch in der Geschichte der deutschen und der bilingualen Lexikographie wurden diejenigen Werke, die eine englischsprachige Leserschaft im Auge hatten, bisher kaum beachtet. Abgesehen von Stein (1985), die die Anfänge der deutsch-englischen und englisch-deutschen Lexikographie bis 1716 darstellt, werden die lexikographischen Werke, die englischsprachigen Lernern zur Verfügung standen, in der Forschung selten erwähnt. Eine der wenige Ausnahmen ist Hartmann (2007), dessen Hauptinteresse bei der Übersetzungsäquivalenz, der Übersetzung von kulturellen Spezifika (z. B. cider, cricket) und der kontrastiven Lexikologie liegt. Im Folgenden gilt es also, diese Forschungslücke für den Zeitraum bis 1900 zumindest ansatzweise zu schließen. Drei Phasen sind zu unterscheiden: 1. eine erste Phase von polyglotten Vokabularen bis gegen Ende des 17. Jahrhunderts; 2. die Anfänge einer bilingualen deutsch-englischen Lexikographie, 1687-1800; und 3. die Entstehung und Herausbildung einer deutsch-englischen Lexikographie mit englischsprachigen Lernern als expliziter Zielgruppe, 1800 bis ca. 1900. Wie wir sehen werden, spiegelt diese Geschichte der deutsch-englischen Lexikographie die Geschichte von Deutsch als Fremdsprache in Großbritannien und Amerika wider: von einem kaum beachtetem Kuriosum zu einer prestigeträchtigen Sprache und Kultur gegen 1800, schließlich zur Etablierung bis 1900 von Deutsch als Sprache der Wissenschaft und als Schul- und Universitätsfach. 


\section{Die polyglottische Tradition im 16. und 17. Jahrhundert}

Wie Stein (1985) schon vor dreißig Jahren feststellte, ist es nicht einfach, die Anfänge der deutsch-englischen Lexikographie zeitlich zu verorten, zum Teil, weil Bibliographien wie Claes (1977) und Kühn (1978) nicht konsequent sind in dem, was sie als Wörterbuch erkennen, bzw. inwiefern zwei- oder mehrsprachige Werke systematisch berücksichtigt werden. Den ersten Kandidaten für den Anfang der deutsch-englischen Lexikographie stellt für Stein ein undatiertes sechssprachiges Vokabular dar, das entweder ca. 1530 oder aber ca. 1550 erschien (Stein 1985: 136) und das neben Deutsch und English vier weitere Sprachen (Latein, Französisch, Spanisch und Italienisch) einschließt. Die Lemmata (auf Latein) sind nicht alphabetisch, sondern thematisch angeordnet. In der Einleitung einer anderen Ausgabe aus dem Jahr 1541 wird das Werk eine „entrig and gate“ (Stein 1985: 138) genannt; es gehört also zur Introito e portaTradition, die auf Adam von Rottweil (1477) zurückgeht (Giustiniani 1987 als Herausgeber; siehe auch Hüllen 2005). Lediglich ein Vertreter dieser Tradition, der sich auf das Sprachpaar Deutsch-Englisch bezog, wurde in England gedruckt (1537; siehe Stein 1985: 156). Das Vokabular ist scheinbar für diejenigen gedacht, die keine Schulbildung genossen haben, darunter „artificers and women" (der Wortlaut ähnelt der Formulierung in einer Ausgabe aus dem Jahr 1531, allerdings ohne Englisch; siehe Stein 1985: 138). Demzufolge hätte also das frühste lexikographische Werk mit deutschem und englischem Sprachmaterial zumindest auch eine weibliche Zielgruppe anvisiert; aber Stein findet keine Indizien dafür, dass der Inhalt tatsächlich auf ein Frauenpublikum zugeschnitten wäre. Der nächste lexikographische Text, der Deutsch und Englisch zusammenführt, ist ein sechssprachiger Vertreter des zweiten bedeutenden praktischen Sprachlehrmittels, das im sechzehnten und siebzehnten Jahrhundert in ganz Europa weit verbreitet war: die Colloques des Antwerper Sprachmeisters Noel de Berlaimont/Barlement, eine Sammlung von zweisprachigen Dialogen, auf die eine Wortliste folgt. Am eher gelehrteren Ende des lexikographischen Spektrums im 16. Jahrhundert finden wir die polyglotten Wörterbücher von Adrianus Junius (1577) and Ambrogio Calepino (1585). Alle vier Werke wiesen viele Auflagen auf (Stein 1985: 157-164), aber nur in dem undatierten entrig and gate-Text stehen Deutsch und Englisch nebeneinander, was ihren systematischen Vergleich und/oder ihre gegenseitige Erlernung erleichtert hätte; weder Deutsch noch Englisch dienten als Lemmasprache. Theoretisch hätten solche Werke dennoch von englischsprachigen Lernern des Deutschen oder umgekehrt gebraucht werden können, aber angesichts der Tatsache, dass die ersten englischen Lehrwerke des Deutschen und DaF-Lehrbücher für englisch- 
sprachige Lerner erst im siebzehnten Jahrhundert erschienen (siehe unten), wird dies sehr selten der Fall gewesen sein.

Das erste Wörterbuch mit deutschen Lemmata ist das unvollständige Werk von Henisch (1616), das als erstes deutsches Wörterbuch bekannt ist, welches aber in der Tat mehrsprachig war. Englisch wird hier berücksichtigt, wie der folgende (von Stein 1985: 145 zitierte) Eintrag veranschaulicht:

Biß, mundvoll, maulvoll, ein Stuck [sic] von einem jeden ding, bolus, rei cuisqu; frustū, buccea, pars, particula था ANG. a morsell.

Ein Jahr nach dem ersten und einzigen Band des Wörterbuchs von Henisch (1616, Nachdruck 1973) erscheint in England John Minsheus Guide into the Tongues, das erste mehrsprachige (elfsprachige) Wörterbuch mit englischen Lemmata (Minsheu 1617, Nachdruck 1978). Das Werk wird im Bibliothekskatalog der Universität Cambridge zurecht als "etymological dictionary of the English language" beschrieben; aber der Kompilator hoffte offenbar auch auf das Interesse derjenigen zu stoßen, die Sprachen für praktische Zwecke erlernen wollten, denn er präsentiert es als „a practical guide for merchants 'that are in person to traficke in forreien Countreys and Tongues' " (so lautet der volle Titel des Werkes; Schäfer 1978: vii). ${ }^{1}$ In der Praxis war Minsheus Werk kaum zum Deutschlernen nutzbar, wenn wir dabei mehr verstehen als die Auflistung einzelner Lexeme, um gelehrten Nutzern den interlingualen Vergleich zu ermöglichen (McLelland 2015: 26-27). Dies muss betont werden, denn das Werk hatte bis 1627 vier Auflagen. Offenbar war dieses Werk von großem Interesse, ${ }^{2}$ aber nicht zum Zweck der Kommunikation. Minsheus Werk spiegelt damit den Status des Deutschen in England des frühen 17. Jahrhunderts wider: Es weckte ein beschränktes Interesse unter Gelehrten, aber ein allgemeiner Markt für ein praktisches deutsch-englisches Wörterbuch existierte offenbar noch nicht.

\section{Die zweisprachige Lexikographie 1687 bis 1790}

Wörterbücher von eher gelehrten Autoren wie Henisch und Minsheu förderten das Studium neu bewerteter Landessprachen, aber es ist unwahrscheinlich, dass sie von deutschlernenden Engländern, oder umgekehrt, gebraucht wurden. Das erste, tatsächlich für Lerner konzipierte, bilinguale deutsch-englische

1 Unklar bleibt, was für Quellen Minsheu für sein deutsches Material zu Rate zog, aber Schäfer hält das Deutsche für „remarkably idiomatic“ (Schäfer 1973: 35).

2 Die Royal Society scheint ein Exemplar angeschafft zu haben (Van der Lubbe 2007: 39). 
Vokabular erscheint am Ende von Heinrich Offelens Zwey-fache gründliche Sprach-Lehr (Offelen 1687), nach einer deutschsprachigen Grammatik des Englischen, einer englischsprachigen Grammatik des Deutschen sowie zweisprachigen Dialogen, die von beiden Lernergruppen gleichermaßen verwendet werden konnten. Mit diesem Werk beginnt für Hartmann (2007: 172) die deutschenglische Lexikographie. Offelens Vokabular bestand aus zwei Wortlisten: zum einen Wörter „that are almost alike in spelling, yet signifie different things; and some others that have the same Sound, and yet are different in spelling“ (Offelen 1687: 140, Part II), z. B. Elend ,misery‘ und Elend ,exile‘, und Ehe ,marriage‘, ehe ,before‘ (Offelen 1687: 141); darüber hinaus bot Offelen seinen Nutzern eine nach Themen geordnete Wortliste. Als praktizierender Sprachlehrer mit einem Auge für die Bedürfnisse seiner Lerner $^{3}$ verwendete er systematisch die Abkürzungen $M, F, N$ zur Markierung des Genus und * für Lemmata im Plural (Offelen 1687: 159-188). Sein Wortschatz, den er als Namen-buch bezeichnete, beginnt mit dem Wortfeld Von Gott, dann Von den Geschöpfen, Von den Theilen des Menschenleibs, [...], spiegelt also die damalige Konzeption der Weltordnung wider; unter den darauffolgenden Themen sind praktische Inhaltsbereiche vorzufinden, wie etwa von den Krankeiten, von den Sinnen, von Kleidern, on Speisen [...] Von der Schul. Wie der Titel Namen-buch suggeriert, besteht die Liste fast ausschließlich aus Nomina, obwohl auch einige adjektivische Farbbezeichnungen in der Liste unter der Rubrik Von den Sinnen auftauchen.

Königs A Royal Compleat Grammar (König 1715; und viele spätere Auflagen $^{4}$ ) ist wie Offelens Werk ein gattungstypisches Sprachlehrbuch der Epoche. Ähnlich zu Offelens Struktur (Offelen 1687) bot es ebenfalls ein Wörterbuch, das aber mit 2.429 Einträgen auf über 39 Seiten viel umfangreicher ist als Offelens Wortliste. Wie bei Offelen sind semantisch verwandte Wörter nach Themen gruppiert; diese weitverbreitete Praxis in der Sprachenpädagogik basierte auf der Annahme, dass „a 'natural' arrangement of words, which follows the 'natural' order of things, is helpful for the memory of the language learner" (Hüllen 1995: 3). ${ }^{5}$ Eine semantische Gruppierung war auch deswegen praktisch, weil sie die Wortlisten sowohl für den englischsprachigen als auch für den deutschsprachigen Markt gleichermaßen verwendbar machte. Wenn auch

3 Den vollständigen Titel stellt Offelen als „Professor of Seven languages, (viz.) English, French, Spanish, Italian, Latin, and High- and Low-Dutch“ vor (McLelland 2015: 35-36).

4 Siehe McLelland (2015: 44).

5 Königs Themenbereiche lauten wie folgt: God, the elements, time (i.e. the world as a whole); Man, society; body, senses, clothes, food; family relationships; offices; war; arts, professions; church, court; tools; house, husbandry; school; metals; animals; precious stones; agriculture, plants; countries, towns; measures, numbers. 
diese onomasiologische lexikographische Tradition gegen Ende des 18. Jahrhunderts mehr oder weniger ausstirbt, so bleibt sie im Sprachunterricht noch durchaus präsent, und gewinnt in anderer Form einen erneut wichtigen Platz im Deutsch als Fachsprache-Unterricht (oder „for specific purposes“) im späten 19. und im frühen 20. Jahrhundert. ${ }^{6}$

Klippel (1994: 71) stellt zahlreiche Ähnlichkeiten zwischen König (1715) und der späteren Kurzverfasste Anleitung zur englischen Sprache von Matthias Kramer (1746) fest; beide greifen auf eine frühere, in Amsterdam erschienene englische Grammatik von Sewel (1705) zurück. Kramers Werk besteht aus einer Grammatik, Dialogen, Briefen, Redewendungen und einem Vokabular „of the most needful words \& c.“ (Kramer 1746: 349-411). Kramer - der offenbar nicht an englischsprachige Lerner des Deutschen dachte - listet seinen Wortschatz aber lediglich nach englischen Lemmata (vgl. Abb. 12.1).

Für etwaige englischsprachige Lerner des Deutschen ist die grammatische Information $\mathrm{zu}$ den deutschen Wörtern unsystematisch. Zum Beispiel finden wir in drei aufeinanderfolgen Einträgen wind, window und wine das Genus einmal klar markiert (der), einmal zweideutig indiziert (ein), und einmal unmarkiert (ohne Artikelwort): wind, der Wind, etwas winden; window, ein Fenster; wine, Wein.

Das erste eigenständige deutsch-englische Wörterbuch erschien 1716 in Leipzig von Christian Ludwig (Ludwig 1716; vgl. Stein 1985; Glück 2002: 336), erheblich später als die ersten Nachschlagewerke dieser Art für die Sprachkombinationen Französisch-Englisch, Spanisch-Englisch und Italienisch-Englisch, die, wie Stein 1985 bemerkt, alle schon bis Ende des 16. Jahrhunderts erschienen waren. Das späte Datum entspricht dem relativ spät entfachten Interesse an Deutsch als Fremdsprache in England - das erste deutsch-englische Lehrwerk für englischsprachige Lerner ist Aedlers High Dutch Minerva (Aedler 1680; der bereits oben erwähnte Offelen bot das zweite im Jahre 1687) - im Vergleich zum späten 15. Jahrhundert für Englisch-Französisch und Mitte des 16. Jahr-

6 Siehe zum Beispiel Anonymus (1899) und Whitfield (1903), A course of commercial German mit seinen „systematic English-German mercantile vocabularies”. Zum Beispiel beginnt der Teil „systematic vocabulary” zum Thema Arbeit wie folgt: „Economics (Political economy): die Nationalökonomie; economist, der Ökonom; wants, die Bedürfnisse (sing. das Bedürfnis); wealth (goods), die Güter (sing. das Gut) Career, die Laufban; industrial, industriell; commercial, kaufmännisch; pursuit (calling, occupation, profession), der Beruf, die Beschäftigung; speciality; das Fach (pl. die Fächer); trade (as a status), der Kaufmannsstand; to choose (select), erwählen” (Whitfield 1903: 53). Generell wuchs im späten 19. und im frühen 20. Jahrhhundert das Interesse an der deutschen Sprache für kommerzielle Zwecke. Erwähnt sei hier auch Bithells Commercial German Dictionary (Bithell 1922), siehe McLelland (2015: 79-81). Zur onomasiologischen Wörterbuchtradition vgl. Hüllen (2002: 89-101). 
$\stackrel{A}{B}$ ULA R Y Ofithe moft needfull Words \&c.<smiles>[14CH3][14C]([14CH3])[Mg]</smiles>

Det notbiventigften Slyci(e beræiede (Part. orat.)

A, Docr An, wiro bro Den Engetlátidern für ein B̈ef(blectots. nort (2lrtilel) gebrouldt, unb bebentet cin, als: a Man, cin DRenid); in Arm tin IIrm 26 .

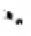

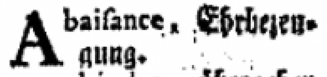
to abandon, yiferaeben, verlafferi, fabrea Ioffen. to abate, abbreøeen, min. Derit.

to abide, bleiben, wobnen. Alility, Fabbialcit, 23 ermo, gent, भradst.

Alyuration, 216/diverung 2lbjanuma.

Able, făbig, tủdjtig verms. getio.

to abolish, abjibaffen, ab. thun.

A bridgment, furyer'begriff,

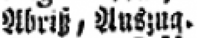

Abfolution, Qoîturedung, Erlajung, Ftblafs.

to abforb, veridiludien, eintrintent.

Abftinence, Entbaltung, शboruc).

Abiud, ungereimt, fhotridt.
A.

Acceflion, 3agabe, 3nfats. Accomplice, Wittbdter, iditgefullite.

to accomplish, etfullet.

Account, Biecturung, פec. cjeufdaft, Ninfeben, Er.

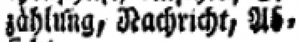
fint.

to accuifom, getwelonen.

Acrbity, Serbigtcit, Eduste.

Acknowledgmeat, ßిthint. nis, Erkenntlidsteit.

Acorn, cine Eiøjl.

Acquzintance, Belannt.

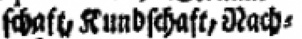
rid)t.

Acquiefsence, ,̧ufsiebengcit, Beopfichtunts.

Asquitance, Quitung.

Activity, Sraft, Étirtle, Sourtigleit.

to adapt, bequem madien.

Abb. 12.1: Ausschnitt aus dem Nachschlagewerk Kramers (Kramer 1746).

hunderts für Englisch-Spanisch (siehe z. B. McLelland 2017: 11, 14). Ludwigs deutsch-englisches Wörterbuch erschien zehn Jahre nach seinem dreisprachigen Wörterbuch Englisch-Französisch-Deutsch (Ludwig 1706), das der Prinzessin Sophia, Kurfürstin von Hannover, gewidmet war. In den Vorworten zu beiden Werken spielt Ludwig auf die Hannoversche Thronfolge in der britischen Monarchie an (Stein 1985: 149). Aber es ist kein Zufall, dass dieses erste Wörter- 
buch nicht in England, sondern in Deutschland erscheint, dessen Interesse deutlich größer ist als das englische - man vergleiche die bis 1770 insgesamt 27 neu veröffentlichten Werke für deutsche Lerner des Englischen (Klippel 1994: 59) mit einem halben Dutzend für Deutschlerner in England (McLelland 2015: 342-345). Zwar hoffte Ludwig, dass sein Wörterbuch von beiden Lernergruppen genutzt werden könnte - zur Aussprache bemerkte er, dass es „beyderseits“ notwendig wäre, „eine lebendige Stimme“ zu hören, um die Aussprache korrekt zu lernen (Vorwort, zitiert in Stein 1985: 155) -, aber in Wirklichkeit dachte er vor allem an einen deutschsprachigen Markt. Die Wortbetonung hat er nur für englische Stichwörter, nicht aber für deutsche Wörter angegeben.

Abgesehen von der Aussprache ist Ludwigs Wörterbuch jedoch für englischsprachige Deutschlerner gut zu nutzen, jedenfalls in der vierten, verbesserten Auflage von 1789 (zuerst 1706), die schon fast alle wichtigen Charakteristika eines modernen bilingualen Wörterbuches bietet. Unterschiedliche Bedeutungen werden separat und nummeriert aufgelistet; praktische Hinweise zum Gebrauch der Lemmata werden angegeben, etwa Kasusrektion der Verben (z. B. sagen (einem etwas); Ludwig (1789: 79) sowie detaillierte Veranschaulichungen des Gebrauchs von Kollokationen und Redewendungen. Die Lemmata sind strikt alphabetisch angeordnet, ohne Berücksichtigung der Umlaut-Vokale $\ddot{a}, \ddot{o}, \ddot{u}$, was allerdings manchmal dazu führt, dass verwandte Lemmata nicht direkt aufeinanderfolgen, geschweige denn, dass alle Ableitungen und Zusammensetzungen eines Stammworts zusammen gruppiert wären. So finden wir etwa die Sage a common saying or talk; a rumour; a report durch sieben SägeZusammensetzungen von dem Verb sagen getrennt (z. B. Sägegrube, saw-pit); Sage selbst trennt die Säge von diesen sieben Säge-Lemmata. Diese Anordnung erleichtert das Nachschlagen von unbekannten Wörtern, ohne Wissen über die Verwandtschaft der einzelnen Einträge weder vorauszusetzen noch vermitteln $\mathrm{zu}$ wollen. Es steht damit im Kontrast zum Stammwortprinzip im ersten vollständigen deutschen Wörterbuch von Stieler (1691), dessen Ziel es war, Der Teutschen Sprache Stammbaum oder Fortwachs oder Teutscher Sprachschatz zu dokumentieren, wie der Titel schon besagt (Stieler 1691); dementsprechend sind in diesem Fall bei ihm alle Säge und sage(n)-Ableitungen und Zusammensetzungen jeweils systematisch zusammengruppiert.

Das Vorwort zu Ludwig (1789) verspricht, dass Aktiv- und Passivformen angegeben werden; dies geschieht im Wörterbuch allerdings nicht explizit, vielmehr wird die korrekte Verwendung der Form mit Belegen illustriert, ebenfalls die Formen unregelmäßiger und starker Verben, etwa s. v. rufen, Es ward gerufen: zu pferde!, Sie riefen: ins gewehr! (Ludwig 1789: 65). Nicht immer ist die Information eindeutig: für nennen finden wir - ohne weitere Erläuterung sowohl ich bin nach ihm genannt wie wir beide sind nach einem namen genen- 
net; die Präteritumsform wird nicht angegeben. Laut dem Vorwort werden „Abweichungen der Verben [...] meistens“ angezeigt, aber eigentlich sollten sie dem Lesepublikum schon bekannt sein; bei etwaigen Zweifelsfällen wird geraten, in einer Grammatik nachzusehen (Ludwig 1789: 3, Vorwort). Das Wörterbuch sollte nutzerfreundlich sein, aber erhob nicht den Anspruch, eine Grammatik zu ersetzen.

Mehrere deutsch-englische Wörterbücher des 18. Jahrhunderts basierten auf dem Universal Etymological English Dictionary von Nathan Bailey (Bailey 1721, 1. Ausgabe). Als erster hat Theodor Arnold dieses Wörterbuch für deutschsprachige Englischlerner adaptiert in seinem Vollständig[en] kleine[n] WörterBuch, englisch und deutsch worinnen alle in der Englischen Sprache gebrauchliche Wörter, mit ihrem so wohl geschwinden als auch langen Accent bezeichnet, ingleichen mit ihrer Pronunciation und Bedeutung enthalten [...] (Arnold 1752, spätere Auflagen 1757, 1761, 1783, 1790). ${ }^{7}$ Bailey hatte lediglich „Haupt = und Stammwörter“ als Lemmata aufgenommen, was zur Folge hatte, dass, wie Arnold erklärte, viele geläufige englische Wörter im Wörterbuch fehlten. Arnold fügte diese Wörter sowie Hinweise zur korrekten Aussprache hinzu (beispielsweise Ábbot (abbot from franz. abbé und latein abbas), the Governour of an $A b b y$, ein $A b t^{8}$ ), aber - wie bei Ludwig - ausschließlich für deutschsprachige Lerner des Englischen und nicht andersherum. Nach Arnold adaptierte ebenfalls Fahrenkrüger (1797) Baileys Wörterbuch. Fahrenkrüger bemerkte stolz in seinem Vorwort, dass er die englische Aussprache bei zahlreichen Informanden aus verschiedenen englischen Provinzen habe überprüfen können, aber er stellte fest, dass „Pronunciation eben nicht die glänzendste Seite der englischen Sprache ist“ (Fahrenkrüger 1797: v, Vorwort). Er warnte vor den zahlreichen Wörtern, deren identische Aussprache leicht verwechselt werden könnte, wie etwa hoar (,Reif') und whore (,Hure‘) sowie rite, write, right und wright (,Rite, schreiben, richtig, der -macher'). Sogar englischsprachige Muttersprachler würden über die hohe Anzahl solcher Dubletten staunen.

Am Ende dieser zweiten Phase in der deutsch-englischen Lexikographie in der die Bedürfnisse englischsprachiger Deutschlerner immer nur sekundär waren - steht Rabenhorsts New pocket-dictionary of the German and English

7 Theodor Arnold hatte auch schon 1736 eine erfolgreiche englische Grammatik für deutschsprachige Lerner veröffentlicht, die bis 183816 weitere Neuauflagen und Adaptationen aufwies (Klippel 1994: 168).

8 Ein Vergleich dieses Eintrags mit dem entsprechenden Eintrag bei Bailey (Ausgabe 1728) (Abbot אבא, the chief Ruler of an Abby, \& c. - noch fast identisch in der 13. Ausgabe 1749) lässt erkennen, dass Arnold offenbar auch zusätzliche Informationen aus anderen Quellen hinzugefügt hat. 
languages (Rabenhorst 1800), „composed chiefly after the dictionaries of Adelung, Johnson, and others of the best authorities“. Die Titelseite verspricht:

The Parts of Speech, and the Genders of the German Nouns, are properly distinguished; each Word accented according to its just and natural Pronunciation; and the Irregularities of the English and German Verbs duly marked. (Rabenhorst 1800: Titelseite)

Tatsächlich aber werden Betonung und Aussprache für das Deutsche nicht angegeben, genau wie bei Ludwig, Arnold und Fahrenkrüger. Das Genus der Substantive wird angegeben, starke und unregelmäßige Verben sind mit einem Sternchen markiert; Verbformen werden dagegen nicht verzeichnet - das Werk ist schließlich als nur $14 \mathrm{~cm}$ großes Taschenwörterbuch konzipiert.

\section{Die Anfänge einer zweisprachigen Lexikographie für Englischsprachige, 1790 bis 1800}

Bis zum Ende des 18. Jahrhunderts gab es also noch keine für englischsprachige Deutschlerner gedachten zweisprachigen Wörterbücher. Dennoch entwickelte sich dieser Markt langsam. Beilers New German Grammar (Beiler [1731] 1736) ist das erste für englischsprachige Lerner konzipierte Deutschbuch, das mehrere Auflagen erhielt; das Werk von Bachmair wurde sogar dreimal neu aufgelegt (Bachmair 1751, 1752, 1771; auch zwei amerikanische Ausgaben 1793, 1811; vgl. McLelland 2015: 52-53). Das erste selbstständige und exklusiv für englischsprachige Deutschlerner verfasste Vokabular war Hesses alphabetisches Vocabulary of the German Tongue (Hesse 1794). Das in der Bibliothek der Universität von Cambridge aufbewahrte Exemplar dieses Werkes ist zusammen mit George Crabbs Elements of German Conversation und Easy and entertaining selection of German prose and poetry (Crabb 1800a, b) zu einem einzigen Band zusammengebunden. Crabb war einer der allerersten nicht-deutschen Autoren eines englisch-deutschen Lehrwerks (McLelland 2015: 67). Wie Hesses Einleitung erklärt,

the nouns are arranged in alphabetical order, and their genders and declensions carefully pointed out, so that any doubt in their cases may be easily removed.

This being the principal object of this work; all Substantives terminating in ung, heit, keit and schaft, are omitted, being always of the feminine gender and third declension. (Hesse 1794: [iii]) 
Es sei ebenfalls davon auszugehen, dass Substantive auf $-e$, wenn nicht aufgelistet, weiblich sind, z. B. die Rede. Hesse habe einige ,primitive verbs and their derivatives“ inkludiert, ,which will make the attainement of them easy and expeditious“ (Hesse 1794: A2 r-v = Seiten [ii.-iii.]). Da aber „the Verbs have many different significations, these examples may be enlarged upon with the assistance of a good dictionary“. Vollständig sollte das Vokabular also nicht sein; es ging Hesse darum, Derviationsprozesse zu veranschaulichen. Das erste verbale Stichwort achten hat die Sub-Lemmata verachten, erachten, nicht aber etwa beachten. ${ }^{9}$

Im Jahre 1797, drei Jahre nach Hesse, veröffentlichte Gebhard Wendeborn, ein wie Crabb in London wohnhafter Deutschlehrer, ein Vokabular als Teil seines Deutsch-Lehrbuches, das sonst auch zweisprachige Dialoge und eine Grammatik umfasste und somit sich der in Europa weitverbreiteten SprachbuchTradition anschloss, in der Offelen, König, Kramer und Crabb auch standen. Wendeborn (1797) listete mehr als dreitausend nach Thema geordnete Wörter, die „frequently occur in conversation“. ${ }^{10}$ Die Liste endete mit einer alphabetischen Auflistung über neun Seiten von Adjektiven sowie einer substantiellen alphabetischen Liste von Verben in ihren Infinitivformen. Wendeborn listete auch sogenannte „peculiar expressions“ auf, denn „every language has some peculiar modes of expression, or idiotisms“; er führte also die „hauptsächlichen“ („principal“) Ausdrücke auf, „which occur in familiar conversation“ rund 85 an der Zahl, wie etwa ins Gras beißen oder Er nimmt kein Blatt vors Maul (Wendeborn 1797: 112-117). Solche Idiome erschienen Wendeborn deswegen wichtig, weil sie sich nicht leicht aus einer Sprache in die andere übersetzen ließen, was bereits der Verfasser des ersten Deutschlehrbuchs für englischsprachige Lerner beobachtet hatte (McLelland 2015: 34-35). Wie wir sehen werden, wird das Problem der sprachspezifischen Redewendungen und Idiome wiederholt in deutsch-englischen Wörterbüchern für englischsprachige Lerner thematisiert, und ihre Berücksichtigung wird oft als besonderer Vorteil des jeweiligen Werkes erwähnt (vgl. Anonym. 1886; und dazu nachfolgend in Abschnitt 5; Weir 1888; Breul 1906).

9 Während in vielen deutsch-englischen Wörterbüchern alle Lemmata ohne Rücksicht auf die grammtische Kategorie systematisch großgeschrieben werden, sind bei Hesse nicht einmal die Substantive großgeschrieben, z. B. abend m.2 [...] abbruch, m.2.

10 Themen waren: on time and seasons, the universe, stones, metals and minerals, man, human faculties and passions, human imperfections and maladies, human events and accidents, relationship, dress and things belonging to it, victuals, utensils for the kitchen and table, states and governments, military, religion and church, ,things relating to sciences, and liberal arts“, trade and professions, town and buildings, etc., country and fields, animals, birds, reptiles and insects, fish and amphibious animals. 
Neben seinem thematischen Vokabular hat Wendeborn als erster in der englischsprachigen DaF-Tradition seinen Lesern auch bestimmte Wörterbücher empfohlen. Bemerkenswert ist, dass seine beiden ersten Empfehlungen einsprachige deutsche Wörterbücher sind: die zweite Ausgabe von Adelungs Wörterbuch sowie eine gekürzte Version:

This new edition is corrected and improved by the author, and it can justly be said, that this dictionary may rival any of its kind, among other European nations. - Mr. Adelung has likewise just published a very useful Abridgement of this greater dictionary, in 4 Vols. (Wendeborn 1797: 199; gemeint ist wohl Adelung (1793-1802))

Wendeborn erwähnt ebenfalls die zweisprachigen Wörterbücher von Arnold und Ludwig, allerdings jeweils ohne weiteren Kommentar. Besonders stark empfohlen hat Wendeborn das Wörterbuch von Johann(es) Ebers (1796-1799) „composed chiefly after the German dictionaries of Mr. Adelung and Mr. Schwan“ (Wendeborn 1797: 199). Wendeborn fand dieses Wörterbuch in drei Bänden „particularly, to be compiled with a view to assist the English to learn the German language“ (Wendeborn 1797: 200).

Die erste Ausgabe von Johannes Ebers’ 1793 Vollständiges Wörterbuch der englischen Sprache für die Deutschen hatte sich - wie der Titel schon besagt in erster Linie an deutschsprachige Englischlerner gerichtet. ${ }^{11}$ Offenbar existierte aber inzwischen ein beachtenswerter Markt englischsprachiger Deutschlerner, und eine spätere Auflage berücksichtigte diesen explizit:

A new hand-dictionary of the English language for the Germans and of the German language for Englishmen. Every word of either language being accurately accented and the pronunciation of every English word added. (Ebers 1800-1802)

Zum ersten Mal wird hier die Hauptbetonung bei jedem deutschen Wort markiert (allerdings nicht ganz konsequent, sondern mit variierenden Konventionen, manchmal mit einem Akut, manchmal mit einem Gravis, oder aber mit einem hochgesetzten Strichlein). Darüber hinaus bietet Ebers zahlreiche zusätzliche Angaben, die einem Lerner nützlich waren: Vergangenheitsformen (Präteritum und Partizipien) der unregelmäßigen und starken Verben erscheinen als Lemmata, was dem Lerner, der sie nicht unbedingt als solche erkennt,

11 Für diesen Markt ist es ein signifikanter Fortschritt, dass detaillierte Hinweise zur Aussprache eines jeden Wortes angegeben werden, nicht nur die Betonung, z. B. college kal'-ledsch. In der Vorrede zum zweiten Band der zweiten Ausgabe (1802) wehrt sich Ebers gegen die Kritik, er hätte Fehler bei der Angabe der englischen Aussprache gemacht. Beim Wort abstract sei es beispielsweise absolut korrekt, dass zwei unterschiedliche Betonungen angegeben seien: 'abstract [Substantiv], ab’stract [Verb]. 
sehr helfen würde, z. B. Begáb, the Imperf. or Pret. of begeben, which see. [...] Begánn, see Beginnen. Die Abkürzungen v.r.a. und v.r.n. (verbum regulare activum und verbum regulare neutrum) geben grammatische Informationen $\mathrm{zu}$ den Verblemmata: z. B. behàgen v.r.a. to inviron, incompas, inclose, surround with a Hedge, to fence; vgl. behàgen v.r.n. to please one, to like, to delight in; begrauen v.r.n. to grow old, hoary, auch begegnen v.r.n. ${ }^{12}$ Das Genus wird, wenn auch nicht immer auf dieselbe Weise, konsequent markiert. Beim Buchstaben $A$ finden wir also Einträge wie Abàrt, (die); aber bei $B$ wird das Artikelwort dem Lemma vorangestellt: der Baum.

\section{Vokabulare und Wörterbücher für englischsprachige Lerner des Deutschen, 1800 bis ca. 1900}

Im 19. Jahrhundert wächst die Zahl der zweisprachigen englisch-deutschen Wörterbücher; im Folgenden können etliche bedeutende, in Deutschland entstandene Wörterbücher nicht diskutiert werden (z. B. Flügel \& Sporschil 1830, Muret \& Sanders 1891), und ich beschränke mich auf einige in Großbritannien und in den USA erschienenen Werke, die - während sie durchaus aus der deutschen Tradition schöpften - sich mehr auf englischsprachige Lerner richteten und ihre Bedürfnisse besonders berücksichtigen wollten. Als erste sei Noehdens Bearbeitung des 1800 erschienenen Taschenwörterbuches von Rabenhorst erwähnt (Noehden 1814). Wie Noehden, der bereits eine Grammatik und Übungen verfasst hatte, in seinem Vorwort schrieb, habe er die Revision von Rabenhorsts Werk zunächst für eine leichte Aufgabe gehalten, aber er sei „more and more impressed with the defectiveness of the original“ gewesen (Noehden 1814: ii, Vorwort Bd. I.) und habe schließlich zwei Jahre für die Bearbeitung gebraucht. Rabenhorsts Werk leide unter dem typischen Problem, dass es vor allem für deutschsprachige Leser konzipiert wurde, was im DeutschEnglischen-Teil am deutlichsten zum Vorschein kommt: Es gebe nur wenige Wörter („,but few words“), die keiner „new definition and interpretation“ bedurften. Bei Noehden wird zum ersten Mal das Genus der deutschen Substantive in beiden Hälften des Wörterbuches angegeben, z. B. im 1. Band Cup, s. [d. h. Substantiv] der Becher, Kelch, die Schale, und im 2. Band Becher, m. a cup,

12 Die Abkürzungen werden nicht aufgeschlüsselt, waren aber wohl geläufig; siehe beispielsweise Thomas (1761: 88). Mein Dank gilt Klaus Fischer für diesen Hinweis. 
goblet. Dadurch wird das Wörterbuch benutzerfreundlich, nicht nur für die Übersetzungen aus dem Deutschen, sondern auch für die seit den 1790er Jahren im Sprachunterricht zunehmend an Bedeutung gewinnende Aufgabe des Übersetzens aus der Muttersprache in die Fremdsprache (prose composition). ${ }^{13}$

Noehden war der erste unter den deutsch-englischen Lexikographen des 19. Jahrhunderts, der zum Thema Fremdwörter, Entlehnungen und Sprachpurismus Stellung nahm. Sein Standpunkt war ziemlich puristisch:

It is a characteristick of the German language, that it possesses the power to create words out of it's [sic!] own elements, for any new ideas. This, in a great measure, obviates the necessity of having recourse to foreign terms and expressions, and have [sic!] given rise to the attempts of purifying it from every thing extraneous. The principle, on which such attempts have been made, is not to be controverted. It tends to call forth, and develop, all the energies of which the language is capable: it increases, as it were, it's [sic!] substance, and widens it's [sic] compass: it renders it more apt and serviceable for the operations of the minds. And if it is better to form new words, out of the funds of the language itself, (provided, this can be done) than to borrow them from strangers; it must also be desirable to replace such foreign terms, as successively have crept into the language, by substitutes of a native and genuine stamp. [...] I think myself warranted in recommending to the student a scrupulous adherence to the laws of purity. (Noeden 1814: iii, Hervorhebung N. McL.)

In einigen Fällen muss aber trotz allem, „however unwillingly“ anerkannt werden, „that a foreign, or borrowed, term would be preferable“; aber;

It will be remarked, that in this dictionary, the licence of employing foreign (that is to say, impure and not genuine German) expressions has been studiously abstained from. (Noeden 1814: iii)

Nicht immer aber sind solche „foreign expressions“ ganz zu vermeiden: „those, which it was necessary to exhibit, in the second part, for the purpose of explanation“ sind mit dem Zeichen † markiert, um so den Leser zu warnen „of the degree of estimation to which they are entitled“ (Noehden 1814: iii). Beispiele für so gekennzeichnete Lemmata sind Reservat, Resident, Residenz, Resonanz, die alle im deutsch-englischen-Band erscheinen. Sie werden aber nicht als mögliche Übersetzungen im englisch-deutschen Teil vorgeschlagen; vgl. etwa Residence, s. der Aufenthalt, die Wohnung; der Wohnort, Aufenthaltsort; Bodensatz (nicht: die Residenz!). Das semantische Feld der Farben ist hier aufschlussreich, denn hier hat das Deutsche im Laufe der Jahrhunderte viele Fremdwörter aufgenommen. Für die Farbe zwischen rot und gelb waren laut Jones (2013:

13 Zur Geschichte der Übersetzung aus der L1 in die Fremdsprache, die besonders im 19. Jahrhundert mit der Etablierung der Fremdsprachen in den Schulen und in den öffentlichen Prüfungen einherging, siehe McLelland (2017: 99-103, 135-137). 
355) im 18. Jahrhundert die pomeranz-basierten Farbbezeichnungen noch fast so häufig wie orange(n)-Bezeichnungen, aber ab 1750 ist orangen nach Jones der häufigste Ausdruck - er bietet ein „spätes Beispiel“ für die pomeranzFarbbezeichnungen aus dem Jahr 1783. Für das englische Stichwort orange finden wir bei Noehden (1814) die Pomeranze, Apfelsine, und als Adjektiv pomeranzengelb. Das Wort Orange erscheint zwar im Sinne von ,Obst' im deutsch-englischen Teil und wird als Fremdwort markiert, aber eine Farbbezeichnung orangen fehlt. Im Jahr 1814 scheint Noehden also bewusst und als eher konservativer Purist gegen den Strom zu schwimmen.

Eine weitere Bearbeitung des Rabenhorst-Noehden-Wörterbuches verdanken wir Hannibal Evans Lloyd (1827, Reprint 1829). Wie Lloyd in seinem Vorwort sagt (Lloyd 1829: v), gibt er die Genitiv- und Mehrzahlendungen für jedes Substantiv an, damit der Leser die Deklination des jeweiligen Wortes erkennen kann; für die verschiedenen Deklinationen wird auf die Grammatik von Noehden hingewiesen - eine Zusammenfassung erscheint auf den Seiten v-vii. Für die unregelmäßigen Verben wird über die von Noehden gelieferten Details hinaus auch das Präsens (1., 2. und 3. Person), das Präteritum sowie das Partizip Perfekt (weder aber für Derivationen noch für Zusammensetzungen der Grundverben) angegeben. Lloyd erklärt weiter:

The method adopted in the last edition [d. h. Noehden 1814], of omitting the root, in the very numerous german [sic! ] compounds, has been retained in the present, as this contrivance, while it saves a great deal of room, has not been found to occasion any inconvenience in the use of the work. (Lloyd 1829: vii-viii)

Dieselbe Strategie wurde auch - wo möglich - im englisch-deutschen Teil angewendet (z. B. Stone; -blind, -break, -cutter, -fruit \& c.); vgl. Lloyd (1829: viii).

Kaltschmidts New and complete dictionary of the English and German languages: with two sketches of grammar, English and German (Kaltschmidt 1837) liegt in mancherlei Hinsicht noch hinter Noehden zurück. Das Genus der deutschen Wörter wird, wie bei Noehden, in beiden Teilen des Nachschlagewerkes angegeben, sowie die Plural-, nicht aber die Genitivformen der deutschen Lemmata. Die platzsparende Verwendung des Bindestrichs bei Noehden und Lloyd kommt hier nicht vor. Kaltschmidts Wörterbuch ist aber wegen seiner Berücksichtigung des Fachwortschatzes von Interesse, einer Lexik, die neben Fremdwörtern im 19. Jahrhundert ebenfalls an Bedeutung gewinnt. ${ }^{14}$ Abkürzungen

14 Sporschil wies explizit auf die „exceedingly numerous technical words“ hin, die sein Wörterbuch berücksichtigte und als solche markierte (Flügel \& Sporschil 1830: VII, Bd. 2). Beispiele für aus dem Bergbau stammende Termini in diesem Wörterbuch sind etwa abbauen - to pay off (with the produce of a mine) the expense of working it; abschneiden: die Erze schneiden sich $a b$ - the vein of ore ends suddenly; auflassen - eine Erzgrube to abandon a mine; Befahren: 
zur Kennzeichnung von rund 60 Fachbereichen werden angegeben (Kaltschmidt 1837: Bd. 1, xxiii-xxiv), z. B. für die Landwirtschaft, den Bergbau, die Botanik, das Tanzen, das Militär und die Hutmacherei, wenn auch diese Markierungen in der Praxis im Wörterbuch nicht sehr häufig auftreten. Kaltschmidt scheint Noehdens sprachpuristische Tendenzen nicht zu teilen - viele der Wörter, die Noehden vermied, finden wir bei Kaltschmidt, wie etwa im englisch-deutschen Teil Resident [...] s. (ein Geschäftsbetrauter in fremden Hauptstädten) der Resident.

In diesem vierten Jahrzehnt des 19. Jahrhunderts beginnt der Deutschunterricht im englischen Bildungssystem institutionalisiert zu werden, sowohl an den sogenannten Public Schools, den elitären Privatschulen (siehe Proescholdt 1991; McLelland 2015: 78-79), als auch an den Universitäten. Adolphus Bernays war 1831 bis 1863 Professor für deutsche Sprache und Literatur am King's College London (Flood 1999). ${ }^{15} \mathrm{Zu}$ den zahlreichen Werken, die er veröffentlichte, zählt sein German word-book: a comparative vocabulary displaying the close affinity between the German and English languages (Bernays 1852). Das wordbook war kein vollständiges Wörterbuch, sollte aber drei Zwecke erfüllen:

[...] to encourage the student of the German language by showing him the great affinity that exists between this language and his own [...] to facilitate his labour by supplying him with a large number of words, so arranged as to be easily remembered. And thirdly, to draw his attention to etymology in general, by displaying before him in the simple form of a vocabulary, the development of a language from a comparatively few roots and stems, and the formation of words by internal changes, or by the addition of prefixes or affixes. (Bernays 1852: vii)

Das heißt, Bernays hoffte, wie schon Offelen und seine Vorläufer in den Sprachbüchern des 16. Jahrhunderts, durch sinnvolles Gruppieren das Erlernen von Vokabeln zu erleichtern. Sein System war aber nicht thematisch, sondern etymologisch und philologisch motiviert und nutzte die genetische Verwandtschaft und die neu erkannten systematischen, phonologischen Unterschiede zwischen Englisch und Deutsch aus. Nach einer Einführung in deutsche Ausspracheregeln führt Bernays ab Seite 26 ,words of the same origin, with altered consonants, systematically arranged“ auf. Als erste kommen etwa Stichwörter im Englischen mit $<\mathrm{ch}>$, die mit einem $<\mathrm{c}>$ oder $<\mathrm{k}>$ im Deutschen korrespondieren: Birch

to get into a mine; Berghalde - heap of earth thrown out of mine; einfahren - to descend the mine; Flage - vein of solid stone crossing the mine.

15 University College London hatte Mai 1828 als erste britische Universität eine deutsche Professur etabliert; der erste Inhaber war Ludwig von Mühlenfels, der allerdings schon drei Jahre später die Universität verließ (Flood 1999: 105, Anm. 12). 
die Birke, chamber die Kammer. Bernays' Einstellung Fremdwörtern gegenüber war eine ganz andere als Noehdens. Er listete bewusst viele Wörter auf, die aus dem Lateinischen, Griechischen, Französischen oder Italienischen stammen, weil sie für englischsprachige Lernende leicht erkennbar sind. Viele

[...] have been objected to by purists, and many have been happily replaced by words of genuine German origin; yet they have been, and most of them still are employed by the best German writers. (Bernays 1852: iv)

Bernays dokumentiert also ab Seite 55 ,words nearly similar in form, and identical in meaning, many of them borrowings from other languages common to English and German“, von absolut, addressiren, Advent bis Zoolog. Auf diese Aufreihung folgen dann, was wir heute false friends nennen, Wörter unterschiedlicher Sprachen, die in ihrer Schreibung ähnlich sind, aber in diesen teilweise oder vollständig unterschiedliche Bedeutungen haben, z. B. accordiren - to bargain, vs to accord übereinstimmen, gestatten (Bernays 1852: 138159). In diesem Sinne rekurriert Bernays auf eine ähnliche Strategie wie die von Offelen (1687), der Wörter aufnahm, ,that are almost alike in spelling, yet signifie different things; and some others that have the same Sound, and yet are different in spelling“ (Offelen 1687: 140, Teil II).

Ein weiterer wichtiger Fortschritt in der Lexikographie für englischsprachige Deutschlernende waren die ersten detaillierten Ausspracheangaben der Lemmata, die bei Oehlschläger (1861) zu finden sind - mehr als 150 Jahre, nachdem Ebers bereits ähnliche Hilfestellungen zur Aussprache für Englischlerner geliefert hatte. Oehlschläger, der laut der Titelseite Professor für moderne Fremdsprachen in Philadelphia war, hatte bereits 1850 ein Aussprachewörterbuch des Englischen für deutschsprachige Lerner sowie eine deutsche Aussprachefibel (Pronouncing German Reader; Oehlschläger 1852) veröffentlicht. Auch seine Ausgabe von Griebs deutsch-englischem Wörterbuch bot seinen Lesern im Vorwort allgemeine, aber detaillierte Hinweise zur deutschen Aussprache und Lesestücke mit interlinearen Hinweisen zur Aussprache (siehe Grieb 1857 mit einem supplement von Oehlschläger: X-XXIII). Oehlschlägers englisches Aussprachewörterbuch richtete sich nicht an Gebildete, sondern an seine Landsleute, die als Einwanderer „in Amerika eine neue Heimath suchen“, ob Handwerker, Landsmann, oder Geschäftsmann (Oehlschläger 1850: v) - englischsprachige Deutschlerner waren also nicht ursprünglich seine Zielgruppe gewesen. Dennoch hat Oehlschläger (1861) als erster endlich auch Ausspracheangaben zu den deutschen Lemmata gemacht ,in English characters and English sounds“ (wie die Titelseite von Oehlschläger 1861 es versprach). Für das Englische konnte Oehlschläger auf bereits existierende Anweisungen zurückgreifen; für das Deutsche gab es diese nicht, obwohl deutsche Laute für Fremd- 
sprachenlerner in Sprachlehrbüchern mehr oder weniger präzise beschrieben waren, oft durch Hinweis auf Laute anderer Sprachen wie Latein, Französisch oder Griechisch (McLelland 2015: 209-218). Oehlschläger strebte eine Aussprache an, die ,as free of provincialisms as possible“ sei, „[...] generally the pronunciation of the North of Germany“ (Oehlschläger 1861: iv). Im Vorwort wies er auf die Effekte der Auslautverhärtung hin, sodass die Auslaute bei Wort und Nord trotz unterschiedlicher Schreibung identisch sind (Oehlschläger 1861: iv). Zur deutschen Wortbetonung erklärte er, dass „Compound words have generally two accents“; der erste, stärkere Akzent sei im Wörterbuch durch " markiert, der zweite oder schwächere durch ' (Oehlschläger 1861: vii). Vermutlich war Oehlschläger dieser Art der Beschreibung in Walkers Aussprachewörterbuch des Englischen begegnet, der schrieb:

[...] there is also, in several words consisting of many syllables, a secondary accent, which contributes in so small degree to the distinctness, force and harmony of the pronunciation. (Walker 1820: 15)

Wie Oehlschlägers Anweisungen zur Aussprache der deutschen Lemmata aussahen, sei anhand folgender Beispiele veranschaulicht:

Aal, ahl, m. (-es; pl. -e) eel.

Abbild, âp'-bĭlt, n. (-es; pl. -er) copy, image

Abbildung, âp“-bǐll-dǒŏnk, f. (pl -en) representation, copy

Bei der Angabe der Aussprache handhabte Oehlschläger die Worttrennung bewusst pragmatisch, um nicht zu einer falschen Aussprache zu verleiten:

In a few cases the more correct division of words into syllables has been sacrificed to obtain a more accurate pronunciation: thus lenken, Lenkung, should be divided in len'ken, len'-kŏŏnk, but as the pronunciation would be inaccurate, it has been divided into lenk'-en, lenk'-ǒŏnk. (Oehlschläger 1861: vii)

Auf Oehlschläger (1861) folgte das anonyme Aussprachewörterbuch vom Cassell-Verlag (Anonymus 1886). Es richtete sich an „the numerous class of self-educating students who do not enjoy the benefit of oral instruction from a native of Germany“. (Man vergleiche eine ähnlich zunehmende Berücksichtigung des selbständigen 'teach-yourself'-Lerners in britischen DaF-Lehrbüchern, wenn auch erst gegen Ende des Jahrhunderts; vgl. McLelland 2015: 180). Der anonyme Kompilator warnt vor regionaler Variation in der deutschen Aussprache und vor der daraus resultierenden Unmöglichkeit absoluter Genauigkeit in deutschen Ausspracheangaben. Aber:

But all that the English student of German really requires, or can reasonably hope to accomplish, is to be able to pronounce the language in such a way as to make himself 
understood by natives; and this he may attain by the use of the present dictionary. (Anonymus 1886: iii)

Die in der Einleitung gebotenen Hinweise zur Aussprache deutscher Laute und zur Wortbetonung basieren offenbar auf Oehlschlägers Prinzipien in seiner Ausgabe von Griebs Wörterbuch (Grieb 1857), etwa für $\ddot{u}$ : „produce the oo in the word oozy, retain this position of the organs and then try to pronounce the ee in eel“ (Anonymus 1886: 1), ebenfalls die Beschreibung von primärem und sekundärem Akzent. Eines der ausgezeichneten Merkmale („excellencies“) des Wörterbuches sei laut dem Vorwort der bewusste Verzicht auf diejenigen Wörter, deren Bedeutung für englischsprachige Benutzer leicht $\mathrm{zu}$ erraten sind, auch wenn in der Praxis leicht verständliche Fremdwörter, wie Residenz, republikanisch - von Noehden bewusst vermieden - durchaus als Lemmata mitaufgenommen wurden. Der Lexikograf war - wie andere vor und nach ihm im 19. Jahrhundert - auch stolz darauf, ,a rich store of idiomatic phrases which embody the genius of the language“ sowie fachspezifische Termini geliefert zu haben:

Various technical terms relating to commerce, sciences, the arts, \&c. [sic!], have also been inserted, all of them being adapted to the present advanced state of knowledge and civilization. (Anonymus 1886: iv)

\section{Die erste deutsch-englische Lexikografin: Elizabeth Weir}

Zwei Jahre nach dem anonymen Cassell-Wörterbuch erscheint das wohl erste von einer Frau kompilierte deutsche Wörterbuch, Heath's New German Dictionary von Elizabeth Weir (1888), meines Wissens ein Unikum in der deutschen Lexikographie bis 1900 und eine Rarität in der Geschichte der deutschen Sprachwissenschaft bis 1900 insgesamt (siehe McLelland 2018). Das Vorwort zum Wörterbuch wurde 1888 in Stuttgart verfasst, und Weir erwähnt dankend die vielen deutschen Freunde, die ihr bei der Berücksichtigung von zahlreichen technischen Ausdrücken sowie bei Idiomen geholfen hätten, die, „though of common occurrence in every-day life, are not generally found in dictionaries“. Die Umsetzung dieses Prinzips veranschaulicht etwa der Eintrag für das Lemma nichts (Abb. 12.2).

Sie hat aber auch wichtige deutsche Wörterbücher zu Rate gezogen; genannt werden die Werke von Hilpert (1828), Flügel (wohl Flügel \& Sporschil 1830), Lucas (1854), und Köhler (vermutlich Köhler 1880), sowie Daniel Sanders (1852) und Meyers Conversations-Lexikon (1840-1855); vgl. Weir (1888: vi). 


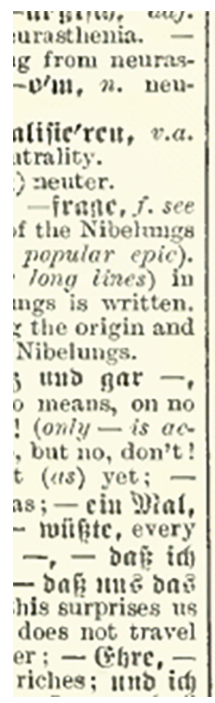

non-payment; - jailung cutcs wed)\}ess, dishonoring a bill. -3ulafiuts, $f$. non-admission.

9 id $y^{\prime} t c, f .(p l ., n)$ niece.

19 ights, I. ind. \& indec. prom. (orig. genit. of gitid : cp. Didj foll - nefiiiten, you shall not be desirous of anything (obs.)) naught, nothing; adverb'ly: in nothing, nowis ; not at all; - a1s, - anfier, nothing but, nothing short of; - Der 21rt, nothing of the kind, no such thing; - oerefeidien, no such thing; $\rightarrow$ ditos wortiger, nevertleless, untwithstanding, however; gau3 ub gax -, onrdjaus -, idiled). terding 6 , rein, lauter - - in Der $201 t$, nothing at all, nothing whatever, not at all; unefr, nothing more, no more. mir - Dir-. quite coolly, witlıont more ado; or that eg mir - bir - , he did it without 80 much as

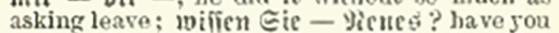
no news? Das hat - 3n iageu, fdadet -, that is of no consequence; io vict ruic -, next to nothing; - weniger als, anything but: fvert, of no value; wenu es neiter - ift if that is or be all; ouv ift ihm wic -, he does not mind that ; fout - ! nothing more! is that all! foier ift - 3k forfen, there is nothing to laugh at in this; cG iit - (Butes ant ifm, there is no good in him; es ift - baran, an ter

Abb. 12.2: Wörterbucheintrag nichts bei Weir (1888).

Aber trotz redlicher Bemühungen seitens Husbands (2001), konnte bisher nicht ermittelt werden, wieso sich Elizabeth Weir in Deutschland aufhielt, woher sie stammte, und wie sie zu ihrer Expertise in der deutsch-englischen Lexikographie gelangte. Ihr Wörterbuch sollte dem „young student“ ein handliches Werk (,a handy volume“) bieten, mit „a collection of idioms, proverbs, and quotations [...], which is larger and more varied" als in anderen Wörterbüchern (Weir 1888: [v]). Als erste in der Geschichte der deutsch-englischen Lexikographie thematisiert Weir die Frage nach Übersetzungsäquivalenz, indem sie ihre Nutzer daran erinnert, dass ,a word in one language seldom answers entirely to any single word in another, so that what are called synonyms are really only approximately such“. Aus diesem Grunde - und mit einem Gespür für das, was im 20. Jahrhundert als kontrastive Lexikologie theorisiert werden sollte - habe sie Beispiele für den Gebrauch der Wörter angeführt, „thoroughly illustrative of the points in the two languages in which they differ from one another" (Weir 1888: v). Weir bemerkt zu Recht, dass die Mehrheit bisheriger deutschenglischer Wörterbücher von Deutschen geschrieben wurde; sie hätten deswegen

[...] not provided for the difficulty which the English student feels when called to select from some dozen German words the special one which answers to the special sense in which the English word is to be used. The arrangement adopted here, while very simple, will be found to obviate this difficulty. (Weir 1888: v) 
Zum Schluss sei hier noch kurz auf die Bearbeitung von Weirs Wörterbuch durch Karl Breul (1906) hingewiesen. Breul - ein, wenn nicht der, führende Germanist und Pionier einer noch in Kinderschuhen steckenden Disziplin von Modern Languages in Großbritannien (Paulin 2010) - zog, wie Weir, ,all the best dictionaries“ zu Rate, vor allem „the admirable Muret-Sanders [1891, N. McL.] in the compilation of which I myself had for some time a modest share“ (Breul 1906: iii). Er nahm viele Dialektwörter auf (als Beispiele nannte er „Deern, Marjell, Fluh etc.“), sowie viele umgangssprachliche Ausdrücke. Außerdem wollte Breul, wie Weir, der als Cambridger Universitätsdozent auch die Cambridge Syndicate Junior- und Senior-Prüfungen für englische Schüler und Schülerinnen im Alter von 16 und 18 Jahren benotete (McLelland 2015: 111), Übersetzungen aus dem Englischen ins Deutsche möglichst unterstützen, eine der Hauptaufgaben in den Prüfungen sowie auch an der Universität: „All reasonable assistance for writing German composition [d.h. hier die Übersetzung ins Deutsche], up to an advanced stage, has [...] been given.“ (Breul 1906: iiii). Eine besonders hilfreiche Innovation im Vergleich $\mathrm{zu}$ Weir (1888) sei Breuls Meinung nach die Angabe des korrekten Kasus für Idiome mit Wechselpräpositionen in Klammern, denn dies sei „the frequent cause of errors in composition“; die Kasusrektion der Präpositionen nach bestimmten Substantiven sei ebenfalls eine wichige Neuheit, wie etwa Hoffnung (auf). Breul behauptet, den „moderaten Prinzipien“ („moderate principles“) des Allgemeinen Deutschen Sprachvereins gefolgt zu sein, indem er bevorzugt deutsche Entsprechungen anstatt englischstammiger Fremdwörter angibt: „German renderings are generally given alone or in the first place, where in the previous edition the English word had merely been rendered by a German homonym“ (Breul 1906: iv). Was die Fachbegriffe betrifft, so ist aus kulturgeschichtlicher Sicht interessant zu beobachten, welche Sachbereiche Breul explizit neu berücksichtigt, darunter neben Militärwesen und Postwesen auch Radfahren, Autofahren und Tennis. Manche Zusammensetzungen habe Breul, wie er sagt, deswegen als eigenständige Lemmata behandeln müssen, weil ihr erstes Element flektiert sei; als Beispiele nennt er Frauenhasser (nicht etwa Frau-hasser) und Weiberfeind - beides relativ neue Wörter, die auch den Zeitgeist widerspiegeln.

\section{Schlussbemerkungen und Ausblick}

Von dieser zwar skizzenhaften und unvollständigen Geschichte der Lexikographie aus der Perspektive Deutsch als Fremdsprache sind dennoch einige neue Einsichten zu gewinnen. Erstens ist neben einem Prozess der Spezialisierung zunächst auf die Sprachkombination Deutsch-Englisch, dann ab dem späten 
18. Jahrhundert auf die besonderen Bedürfnisse der englischsprachigen Deutschlerner bezogen - eine zunehmende lexikografische Professionalisierung festzustellen: in der Präsentation (etwa der platzsparende Gebrauch des Bindestrichs) wie in der zunehmend konsequenten Darstellung und Veranschaulichung praktischer grammatischer und phraseologischer Merkmale der Lemmata. Auch Hinweise zur Aussprache der einzelnen deutschen Stichwörter tauchen Mitte des 19. Jahrhunderts auf, allerdings gut 150 Jahre später als in den bilingualen Wörterbüchern für deutsche Englischlerner - eine Verzögerung, die das erst viel später aufkommende Interesse an DaF unter Englischsprachigen widerspiegelt. Bemerkenswert ist auch die zunehmende Berücksichtigung jener Wörterbuchbenutzer, die Hilfe bei der Wortwahl suchen, einer ab ca. 1800 typischen Schulaufgabe der Übersetzung aus der Muttersprache ins Deutsche. Es handelt sich dabei um eine Entwicklung, die besonders bei Noehden (1814), Weir (1888), und Breul (1906) festzustellen ist. Die Berücksichtigung von Fachterminologie im 19. Jahrhundert zeigt auch die wachsende Bedeutung des Deutschen als Wissenschaftssprache und - was hier nur nebenher erwähnt werden konnte - als Wirtschaftssprache. An der Lexikographie des 19. Jahrhunderts lassen sich andere Themen in der deutschen Sprachgeschichte und Geschichte der Sprachwissenschaft ablesen, was hier einerseits am Beispiel des Sprachpurismus, andererseits der historisch-vergleichenden Grammatik veranschaulicht werden konnte. Sprachgeschichte ist auch Kulturgeschichte; und eine genauere Analyse der neu aufgenommenen Lemmata (wie etwa die hier zitierten Beispiele aus der Frauenbewegung) und der neu berücksichtigten Fachbereiche wäre aufschlussreich. Schließlich stellt - in dieser sonst rein männlichen Geschichte - der Beitrag zur englisch-deutschen Lexikographie der völlig unbekannten Elizabeth Weir einen zusätzlichen Baustein in der noch unvollständigen Geschichte der Frauen in der deutschen Sprachwissenschaft dar (McLelland 2018).

\section{Literatur}

Adelung, Johann Christoph (1793-1802): Auszug aus dem grammatischkritischen Wörterbuche der hochdeutschen Mundart. 4 Bde. Leipzig: Breitkopf.

Adrianus Junius (1577): Nomenclator, omnium rerum propia nomina variis linguis explicata indicans [...]. Antverpiae, ex off. Christophori Plantini

Aedler, Martin [anonym erschienen] (1680): The Hig [sic] Dutch Minerva // a-la-mode [...] London: Printed for the author. Nachdruck Menston 1972. England: Scolar Press.

Anonymus (1886): German pronouncing dictionary in 2 parts: German-English-EnglishGerman with an appendix. [S.I.]: Cassell.

Arnold, Theodor (1752): A compleat English dictionary: Oder vollständiges englisch-deutsches Wörterbuch. Leipzig: Großische Handlung. 
Bachmair, John James (1771): A complete German grammar [...]. London: Printed for G. Keith, B. Law, E. and C. Dilly, and Robinson and Roberts.

Bailey, Nathan (1721): An universal etymological English dictionary [...]. London: Printed for E. Bell [and 9 others].

Beiler, Benedictus (1731): A NEW German Grammar. Whereby and ENGLISHMAN May easily attain to the KNOWLEDGE of the German Language, especially useful for MERCHANTS and TRAVELLERS. [...]. 2. Aufl. 1736. London: J. Downing for the author.

[Berlaimont, Noel de] (1576): Colloqvia Et Dictionariolvm Sex Linguarvm [...]. Antuerpiæ: Apud Henricum Henricium [...]. Die von Hüllen (2005: 54) erwähnte erste überkommene erwähnte Ausgabe von 1536 habe ich nicht nachweisen können. Bernays, Adolphus (1852): German word-book: a comparative vocabulary displaying the close affinity between the German and English languages. London: J. W. Parker.

Bithell, Jethro (1922): Commercial German dictionary. London: Sir I. Pitman \& Sons.

Breul, Karl [\& Elizabeth Weir] (1906): A new German and English dictionary. Revised by Karl Breul. [S. I.]: Cassell.

Calepino, Ambrosius (1585): Dictionarium decem linguarum [...]. Lyon: Etienne Michel.

Claes, Franz (1977): Bibliographisches Verzeichnis der deutschen Vokabulare und Wörterbücher, gedruckt bis 1600 . Hildesheim: Olms.

Crabb, George (1800a): Elements of German conversation: Upon the plan of Perrin's Elements. London: Printed by C. Whittingham, for T. Boosey.

Crabb, George (1800b): An easy and entertaining selection of German prose and poetry. [...]. London: printed for the author, by C. Whittingham, and sold by J. Johnson, T. Boosey, C. Geisweiler, De Boffe, and Esher, and Wilson and Spence, York.

Ebers, Johannes (1793): Vollständiges Wörterbuch der englischen Sprache für die Deutschen: Nach den neuesten und besten Hülfsmitteln mit richtig bezeichneter Aussprache eines jeden Wortes bearbeitet von Johannes Ebers. Leipzig: Breitkopf, Sohn und Compagnie.

Ebers, Johannes (1796-1799): The new and complete dictionary of the German and English languages: composed chiefly after the German dictionaries of Mr. Adelung and of Mr. Schwan [...]. 3 Bde. Leipzig: Printed for Breitkopf and Haertel.

Ebers, Johann (1800-1802): A new hand-dictionary of the English language for the Germans and of the German language for Englishmen. Every word of either language being accurately accented and the pronunciation of every English word added. Halle: Renger. 2 Bde.

Fahrenkrüger, Johann Anton (1796): Nathan Bailey Dictionary, English-German and GermanEnglish oder Englisch-deutsches und deutsch-englisches Wörterbuch. 9 Aufl., umgearbeitet von J. A. Fahrenkrüger. Leipzig: Frommann.

Flood, John L. (1999): Ginger beer and sugared cauliflower. Adolphus Bernays and language teaching in nineteenth-century London. In Rüdiger Gorner \& Helen Kelly-Holmes (Hrsg.), Vermittlungen. German studies at the turn of the century, 101-115. Munich: Iudicium.

Flügel, Johann Gottfried \& Johann Sporschil (1830): Vollständiges Englisch-Deutsches und Deutsch-Englisches Wörterbuch, etc. (A complete Dictionary of the English and German and German and English Languages. TI. 1. ... von J. G. Flügel. Tl. II. ... von J. Sporschil.). Leipzig: Liebeskind.

Giustiniani, Vito R. (1987): Adam von Rottweil, Deutsch-Italienischer Sprachführer. Tübingen: Narr.

Glück, Helmut (2002): Deutsch als Fremdsprache in Europa vom Mittelalter bis zur Barockzeit. Berlin u. a.: de Gruyter. 
Grieb, Christoph Friedrich (1842): English-Deutsches und Deutsch-Englisches Wörterbuch mit einer tabellarischen Uebersicht der von den neuern englischen Orthoepisten verschieden ausgesprochenen Wörter. Stuttgart: Hoffmann.

Grieb, Christoph Friedrich (1857): A dictionary of the English and German languages, with a synopsis of English words differently pronounced by different orthoëpists to which is added a supplement [...]. Philadelphia: Kohler.

Hartmann, Reinhard Rudolf Karl (2007): 300 years of English-German language contact and contrast: The translation of culture-specific information in the general bilingual dictionary. In Reinhard Rudolf Karl Hartmann (Hrsg.), Interlingual lexicography: Selected essays on translation equivalence, constrative linguistics and the bilingual dictionary, 175-184. Tübingen: Niemeyer.

Hartmann, Reinhard Rudolf Karl (2007): Interlingual lexicography: Selected essays on translation equivalence, constrative linguistics and the bilingual dictionary. Tübingen: Niemeyer.

Henisch, Georg (1549-1618) (1616 [1973]): Teütsche Sprach und Weißheit: Thesaurus linguae et sapientiae Germanicae, A-G [mehr nicht erschienen]. Augsburg: Francus 1616.

[Nachdruck Hildesheim: Olms 1973].

Hesse, E. (1794): A vocabulary of the German tongue: With a collection of familiar phrases. London: Printed for T. Boosey, No 4, Old Broad-Street; and R. Cheeswright, No 39, KingStreet. The Cambridge UL copy I consulted is bound together with Crabb's Elements of German conversation (1800) and with his Selection of german prose and poetry to translate (1800).

Hilpert, Joseph Leonhard (1828): Englisch-deutsches und deutsch-englisches Woerterbuch. Karlsruhe: Braun.

Hüllen, Werner (1995): The path through an undergrowth: A Royal Compleat Grammar, English and High German (1715). Paradigm 17, http://faculty.education.illinois.edu/ westbury/paradigm/hullen.html (letzter Zugriff 4.4. 2018)

Hüllen, Werner (2002): Collected papers on the history of linguistic ideas, hrsg. von Michael Isermann. Münster: Nodus.

Hüllen, Werner (2005): Kleine Geschichte des Fremdsprachenlernerns. Berlin: Schmidt.

Husbands, Christopher T. (2001): Who was Elizabeth P. Weir?: Gender visibility and female invisibility in the world of lexicography. The Linguist 40 (2), 48-51.

Jones, William Jervis (2013): German colour terms: A study in their historical evolution from earliest times to the present. Amsterdam: Benjamins.

Jones, William Jervis (2000): German lexicography in the European context: A descriptive bibliography of printed dictionaries and word lists containing German language (16001700). (= Studia Linguistica Germanica 58). Berlin u. a.: de Gruyter.

Jones, William Jervis (1999): Images of language. German attitudes to European languages from 1500 to 1800. Amsterdam: Benjamins.

Kaltschmidt, Jacob Heinrich (1837): A new and complete dictionary of the English and German languages: With two sketches of grammar, English and German. Leipsic: Tauchnitz.

Klippel, Friederike (1994): Englischlernen im 18. und 19. Jahrhundert. Die Geschichte der Lehrbücher und Unterrichtsmethoden. Münster: Nodus.

Köhler, Friedrich (1880): Englisch-Deutsches und Deutsch-Englisches Taschen-Wörterbuch. Leipzig: Reclam.

König, Johann (1715): A royal compleat grammar, English and High-German [...]. London: gedruckt für Wilhelm Frieman. 
Kramer, Johann Matthias (1746): Kurzverfasste Anleitung zur englischen Sprache, worinnen die nöthigsten, und wesentlichsten grammatikalischen Regeln, nach der ... Lehrart ... Wilhelm Sewels ... angewiesen werden. Hamburg: Hertelische Handlung.

Kühn, Peter (1978): Deutsche Wörterbücher: eine systematische Bibliographie. Tübingen: Niemeyer.

Lloyd, Hannibal Evans (1829): Rabenhorst's pocket dictionary of the German and English languages, in two parts. London: printed for Longman, Rees, Orme, Brown, \& Green [...]. [1. Ausgabe 1827].

Lucas, Newton Ivory (1854): Englisch-Deutsches und Deutsch-Englisches Wörterbuch: mit besonderer Rücksicht auf den gegenwärtigen Standpunkt der Literatur und Wissenschaft. Bremen: Schünemann.

Ludwig, Christian (1706): A dictionary English, German, and French [...]. Leipzig: Fritschen. Ludwig, Christian (1716): Teutsch-Englisches Lexicon [...]. Leipzig: Bey Thomas Fritschen.

Ludwig, Christian (1789): Christian Ludwig's teutschenglisches Lexikon [...]. Leipzig: In Johann Friedrich Gleditschens Buchhandlung. Online vergügbar unter https://archive.org/ details/christianludwigs00ludwuoft (letzter Zugriff 4. 4. 2018)

McLelland, Nicola (2015): German through English eyes. A history of language teaching and learning in Britain, 1500-2000. Wiesbaden: Harrassowitz.

McLelland, Nicola (2017): Teaching and learning foreign languages: A History of language education, assessment and policy in Britain. London: Routledge.

McLelland, Nicola (2019): That subtle influence for which women are best suited: Women and German language studies, 1700-1920. In Wendy Ayres-Bennett \& Helena Sanson (Hrsg.), Distant and neglected voices. Women in the history of linguistics. Oxford: OUP.

Meyer, Joseph (1840-1855): Das große Conversations-Lexicon für die gebildeten Stände. Hildburghausen: Bibliographisches Institut.

Minsheu, John (1617 [1978]): Ductor in linguas = Guide into the tongues; and, Vocabularium hispanicolatinum $=$ A most copious Spanish dictionary. Delmar: Scholars' Facsimiles and Reprints.

Muret, Eduard \& Daniel Sanders (1891): Encyklopädisches englisch-deutsches und deutschenglisches Wörterbuch. Berlin, New York: Langenscheidt, International News Co.

Noehden, Georg Heinrich (1814): Rabenhorst's dictionary of the German and English languages: In two parts. London: Printed for Longman, Hurst, Rees, Orme and Brown; Cadell and Davies; T. Boosey, and J. Mawman.

Offelen, Heinrich (1687): A double grammar for Germans to learn English and for Englishmen to learn the German tongue. Zwey-fache gründliche Sprach-Lehr, für Hochteutsche, englisch, und für Engelländer hochteutsch zu lernen. London: Old Spring Garden by Charing Cross.

Oehlschläger, James C. (1850): English German and German English pocket-dictionary: With a pronunciation of the English part in German characters and German sounds. Philadelphia: Weik.

Oehlschläger, James C. (1852): Pronouncing German reader. New York: Appleton \& Co.

Oehlschläger, James C. (1861): English German and German-English pocket-dictionary, with the pronunciation of the English words in German sounds and signs. New York: Steiger.

Paulin, Roger (2010): Breul, Karl Hermann (1860-1932). Oxford dictionary of national biography. Oxford University Press, Mai 2010; Online-Ausgabe, https://doi.org./ 10.1093/ref:odnb/61616 (letzter Zugriff 4. 4. 2018).

Proescholdt, C. W. (1991): The introduction of German language teaching into England. German Life and Letters 44(2), 93-102. 
Rabenhorst, C. T. (1800): The new pocket-dictionary of the German and English languages. Leipsic: Printed for C. T. Rabenhorst.

Sanders, Daniel (1852): Das deutsche Wörterbuch von J. Grimm. Hamburg: Hoffmann und Campe.

Schäfer, Jürgen (1973): John Minsheu: Scholar or charlatan? Renaissance Quarterly 26 (1), 23-35.

Schäfer, Jürgen (1978): Introduction. In Jürgen Schäfer (Hrsg.), John Minsheu, Guide into the tongues, v-xxi. New York: Scholars' facsimiles and reprints.

Sewel, Wilhelm (1705): A compendious guide to the English language. Amsterdam: printed for the widdow [sic] of Stephen Swart. [2. Ausgabe 1706].

Stein, Gabriele (1985): Englisch-German/German-English Lexicography: Its early beginnings. Lexicographica. International Annual for Lexicography / Revue Internationale de Lexicographie / Internationales Jahrbuch für Lexikographie, 134-164.

Stieler, Kaspar (1691 [1968]): Der Teutschen Sprache Stammbaum und Fortwachs oder Teutscher Sprachschatz [...]. Nürnberg: Johann Hoffmann. Nachdruck 1968 mit einem Nachwort von Stefan Sonderegger. München: Kösel.

Tafel, Leonhard \& Louis Hermann Tafel (1870): Neues, vollständiges englisch-deutsches und deutsch-englisches Taschen-Wörterbuch, mit der Aussprache der deutschen und der englischen Wörter, und mit besonderer Berücksichtigung der technischen Ausdrücke der Künste und Wissenschaften, für Geschäftsleute und Schulen. Philadelphia: McVey.

Thomas, Jean (1761): Le Sincere Maitre de Langue en Autriche. Der aufrichtige Sprach-Meister in Oesterreich. Das ist: Kurze, gründliche ... Anweisung zu der Französischen Sprach (etc.). Steyr: Menhardt.

Van der Lubbe, Fredericka (2007): Martin Aedler and the High Dutch Minerva. The first German grammar for the English. Frankfurt am Main: Lang.

Walker, John (1820): A critical pronouncing dictionary, and expositor of the English language [...] London: Cadell \& Davies.

Weir, Elizabeth (1888): Heath's new German dictionary: In two parts, German-English/ English-German. Boston: Heath.

Wendeborn, Gebhard Friedrich August (1797): Exercises to Dr. Wendeborn's introduction to German grammar. London: Printed for the Author.

Whitfield, Edward Elihu \& Carl Kaiser (1903): A course of commercial German. London: Longmans. 


\section{Register}

access basis 215, 228

access domain 215, 228-229

access route 215, 220, 222, 227-229, 231, 233

access structure 215, 218, 227-231, 233

access, systematic $215,228,230$

Ähnlichkeitsbeziehung 61

Amtssprache 4, 189-190, 192, 194, 201202, 208-209

Analytizität 91, 94, 113, 116-117

Antonymie / antonymy 14-15, 237, 239240, 242, 247, 257-258

Äquikomplexitätsthese 91-92, 119

Bedeutungsanalyse, wahrheitsfunktionale 21

Bedeutungsaspekt 26, 267, 273, 276

Bedeutungsbeziehung 9, 61, 86, 239

Bedeutungsbeziehung, semantic relation 237, 240

Bedeutungsnähe 61,83

Begriffsgeschichte 31, 33-43, 45, 47, 50, 55-56

Bernays, Adolphus 296, 310-311

Beziehung, lexikalisch-semantische 238, 240, 242, 248, 257

Breul, Karl 296, 305, 315-316

CCDB (Kookkurrenzdatenbank) 71-72, 7779, 83-85, 237, 241-242, 246, 257

Common Global Contexts (CGC) 237, 241242, 256-257

Contrasting Near Synonyms (CNS) 237, 241, 253-254, 264-266

data distribution structure $215,218-219$, 222, 225-226, 228, 230

Datenanalyse 238-239

Definition 4, 171-173, 178-186, 190, 192, 239

Deixis 25, 267, 273-274, 281, 284, 288

Dekomposition 9, 21

Deutsch als Fremdsprache (DaF) 268, 270271, 295-296, 300, 315-316

Deutsch, gesprochenes 5, 267, 273, 290

Deutsch, namibisches 189, 203
Diskursanalyse 2, 31, 34-35, 37, 41-43, 45, $47,49-50,52-53,55-56$

Distribution 61, 72, 77, 83, 242, 247

Eigentlichkeit 171-173, 179, 182-185

Eindeutigkeit 171-173, 179, 183-185

Epistemologie 2, 31-35, 37-38, 40, 42-44, $46-47,51,53,55-56,178$

Exaktheit 171-173, 179, 182-185, 254

Fachsprache 4, 171, 178, 180, 182, 184-185

Formsynkretismus 91, 110, 118-119

Foucault, Michel 31-33, 36-38, 41-43, 45, 47, 50-51, 55

Frame 27, 31, 33, 36, 40, 45-53, 155-156

Frame-Semantik 31, 45, 47, 50, 54

Gebrauchsbedeutung 267

Gendering 3, 127, 130, 147

Genderlinguistik 127

Genusverhalten 3, 127, 132

Geschlechterforschung 127

Geschlechtsbinarität 128

Homonymie $61,78,80,83,134,315$

Identität 4, 129, 189, 196-197, 206-208, 210

Idiom 3, 11, 24, 151-167, 305, 313, 315

Idiomatizität 153

Idiomforschung, kontrastive 151

Internetlexikographie / internet lexicography 216

Kasus 91, 96-97, 99-100, 105, 110, 118-119, 315

Komplexität, grammatische 3, 91-92, 9495, 104, 107, 119

Kompositionalitätsprinzip 9-11

Kontext 3, 23-25, 33-34, 38, 44-45, 50, 61, 63-65, 68, 70-71, 80-81, 83-84, 9899, 103, 108, 111, 134, 157-159, 164, $166,179,242,246-247,249,253,255-$ 256

Kontrast, deutsch-englisch 91, 118

Ә Open Access. (C) 2018 publiziert von De Gruyter. (c) BY Dieses Werk ist lizenziert unter der Creative Commons Attribution 4.0 Lizenz.

https://doi.org/10.1515/9783110538588-014 
Kookkurrenz / coocurrence 4, 61, 70, 72, $80,83-84,247$

Kookurrenzprofil / cooccurrence profile 237

Korpus 1, 3-5, 61, 63-64, 66, 70, 74, 83, $134,151,154,159-160,168,237,239$, 241, 253, 258, 267, 274

Korpuslinguistik 239

Koselleck, Reinhart 31, 34, 36-42, 45, 55

Lesart 5, 13, 22, 74, 78, 80-81, 84-85, 103 , $108,153-154,164,166,249,253,256-$ 258, 267, 271, 279-280, 291-292

Lexikographie / lexicography 2, 4, 63, 215219, 225-228, 233, 237, 240, 267, 295298, 303-304, 311, 313-316

Lexikographie, deutsch-englische 295-296, 299

Lexikologie / lexicology 1, 5, 237, 239, 296, 314

Linguistik, kognitive 152, 154, 168, 171-172, 182, 185

Mennonitendeutsch 189

Methode, kognitive 151, 160

Noehden, Georg Heinrich 296, 307-310, 313, 316

Oehlschäger, James 296

outer features 215, 218, 225-226, 230

Personennamen 3, 127, 130-131, 145

Phrasem 151-152

Phraseologie 151, 154, 160, 167-168, 293, 295, 316

Polylexikalität 152-153

Polysemie $3,61,80,83,153,173,182$

Pragmalinguistik 4, 171-172, 178-179, 182, 184-185

Pragmatik 17, 45, 91-92, 94, 99, 101-102, $113,119,146,152,183,185-186,190$, 267, 275, 312

punctual access 215, 232

Rumäniendeutsch 189

Russisch 140, 151, 153-159

Russisch, Idiom 157
Satzsemantik 9-10, 36, 45

Satzstruktur 91-92, 94, 101, 113

Schema 27, 46, 54, 75

Self-Organizing Map 237

Semantik 2-4, 9-27, 31, 33-40, 42-51, 53, 55-56, 84, 91-101, 104-105, 107-111, 113, 117-119, 130-132, 144, 146-147, $151-156,158-161,163-166,168,172-$ $173,175,178,184-185,192,237,239-$ $242,247,253,255-258,267,270,283$, 288, 290, 299, 308

Semantik, historische 2, 37-39, 47, 50, 56

Spracherhalt 189, 206-209

Sprachgeschichte 295-296, 316

Sprachvergleich 5, 91-92, 100

Sprachzentrum 189-190, 193-195, 197, 209

Standardvarietät 4, 189-195, 197, 199-200, 203-204, 206-210

Synonymie / synonymy 14, 130, 155-156, 173, 182, 192, 237, 239-241, 247, 258

Synthetizität 91, 94, 113, 116-117, 119

System, terminologisches 4, 171-172, 178182, 184-185

Systemlinguistik 4, 171-173, 175, 184-186

Temporaladverbial 267, 279, 284

Terminologisierung 171, 173, 179-180, 183, 186

Terminus 4, 36, 40, 46, 48, 140, 153, 158, 171-173, 175, 178-186, 194-195, 240, 257, 309, 313

text compound 215, 219, 225

Tiernamen 127, 130, 139-141, 143-145, 147

Transgender 127

Transparenz, semantische 3, 91-92, 94-95, 99, 104-105, 107-110, 113, 117-119

Typologie 3, 33-34, 39, 91-93, 96, 117, 119, 167,183

Unisexnamen 127-129, 138, 140, 145

Variante, nationale 189, 192-193, 195

vector space model 61,80

Vokalubar 296, 307

Wahrheitsbedingungen 9-10, 13, 16, 23, 25-26

Wahrheitswert 9-11, 25

Weir, Elizabeth 296, 305, 313-316

Wendeborn, Gebhard 296, 305-306 
Wissen 21, 27, 31-35, 38, 41, 44-47, 52-56, 136, 183-184, 252, 302

Wissen, verstehensrelevantes $31,34,36$, $38,46-47,56$
Wissenselement $32,42-43,47-52$

word embeddings $61,82-83$

Wörterbuchportal / dictionary portal 215, 218-220, 222, 224 



\section{Autorinnen und Autoren}

Dietrich Busse, Heinrich Heine Universität Düsseldorf, Institut für Germanistik, Universitätsstraße 1, D-40225 Düsseldorf, E-Mail: d.busse@uni-duesseldorf.de

Dmitrij Dobrovol'skij, Russische Akademie der Wissenschaften, Institut für russische Sprache, Volkhonka 18/2, 119019 Moskau, Russland, E-Mail: dobrovolskij@gmail.com

Stefan Engelberg, Institut für Deutsche Sprache Mannheim, R5, 6-13, D-68161 Mannheim, E-Mail: engelberg@ids-mannheim.de

Klaus Fischer, London Metropolitan University, 166-220 Holloway Road, London N7 8DB, Great Britain, E-Mail: k.fischer@londonmet.ac.uk

Rufus H. Gouws, Stellenbosch University, Private Bag X1, Matieland 7602, South Africa, E-Mail: rhg@sun.ac.za

Heidrun Kämper, Institut für Deutsche Sprache Mannheim, R5, 6-13, D-68161 Mannheim, E-Mail: kaemper@ids-mannheim.de

Birte Kellermeier-Rehbein, Bergische Universität Wuppertal, Fakultät für Geistes- und Kulturwissenschaften, Gaußstraße 20, D-42113 Wuppertal, E-Mail: kellermeier-rehbein@uni-wuppertal.de

Manfred Krifka, Leibniz-Zentrum Allgemeine Sprachwissenschaft (ZAS) und HumboldtUniversität zu Berlin, Schützenstraße 18, D-10117 Berlin, E-Mail: krifka@leibniz-zas.de

Nicola McLelland, University of Nottingham, School of Cultures, Languages and Area Studies, University Park, Nottingham NG7 2RD, Great Britain,

E-Mail: nicola.mclelland@nottingham.ac.uk

Damaris Nübling, Johannes Gutenberg-Universität Mainz, FB 05 - Philosophie und Philologie, Deutsches Institut, Historische Sprachwissenschaft des Deutschen, Jakob-Welder-Weg 18, D-55128 Mainz, E-Mail: nuebling@uni-mainz.de

Rainer Perkuhn, IDS Mannheim, R5, 6-13, D-68161 Mannheim, E-Mail: perkuhn@ids-mannheim.de

Thorsten Roelcke, Institut für Sprache und Kommunikation, Technische Universität Berlin, Sekr. HBS 2, Hardenbergstraße 16-18, D-10623 Berlin, E-Mail: roelcke@tu-berlin.de

Sven Staffeldt, Julius-Maximilians-Universität Würzburg, Institut für deutsche Philologie, Lehrstuhl für deutsche Sprachwissenschaft, Am Hubland, D-97074 Würzburg, E-Mail: sven.staffeldt@uni-wuerzburg.de

Petra Storjohann, Institut für Deutsche Sprache Mannheim, R5, 6-13, D-68161 Mannheim, E-Mail: storjohann@ids-mannheim.de

Marie Vachková, Karls-Universität in Prag, Institut für Germanische Studien, nám. Jana Palacha 2, Raum 320, CZ-116 38 Praha 1, Tschechien, E-Mail: marie.vachkova@ff.cuni.cz 
